\title{
Measurements of Branching Ratios And Search for CP Violation in the Modes Bo --> Rho Pi, Rho K
}

(in French)

\author{
Sandrine Laplace
}

SLAC-R-835

Prepared for the Department of Energy

under contract number DE-AC02-76SF00515

Printed in the United States of America. Available from the National Technical Information Service, U.S. Department of Commerce, 5285 Port Royal Road, Springfield, VA 22161. 
This document, and the material and data contained therein, was developed under sponsorship of the United States Government. Neither the United States nor the Department of Energy, nor the Leland Stanford Junior University, nor their employees, nor their respective contractors, subcontractors, or their employees, makes an warranty, express or implied, or assumes any liability of responsibility for accuracy, completeness or usefulness of any information, apparatus, product or process disclosed, or represents that its use will not infringe privately owned rights. Mention of any product, its manufacturer, or suppliers shall not, nor is it intended to, imply approval, disapproval, or fitness of any particular use. A royalty-free, nonexclusive right to use and disseminate same of whatsoever, is expressly reserved to the United States and the University. 
UNIVERSITE PARIS 7 - DENIS DIDEROT

THESE de DOCTORAT

Spécialité: Physique des particules

Présentée par

\section{Sandrine LAPLACE}

pour obtenir le grade de DOCTEUR de l'UNIVERSITE PARIS 7

\section{Mesure des rapports d'embranchement et recherche de la violation de CP dans les modes $B^{0} \rightarrow \rho \pi, \rho K$}

Soutenue le 30 avril 2003

JURY

M. B. D'ALMAGNE Président

MME B. BLOCH-DEVAUX Rapporteur

M. A. HÖCKER

M. F. LE DIBERDER

MME M-N. MINARD Rapporteur

MME H. QUINN 


\section{Remerciements}

"La reconnaissance silencieuse ne sert à personne." Gladys Bronwyn Stern, Ecrivain américain.

L'ascension de cette montagne appelée "thèse" aura duré pratiquement trois ans. Comme toute nouvelle ascension, on ne sait pas ce qu'on trouvera en chemin: il y aura les épines, les éboulis, les crevasses, et même pire, mais il y aura aussi bien des plaisirs. Néanmoins, on aura beau mobiliser toute son énergie et toute sa force, le succès de l'expédition dépend grandement des compagnons de cordée. Sur le sommet enfin atteint, je souhaite rendre hommage à ma cordée dont la longueur dépasse largement la règle des trois personnes maximum.

Je commencerai par remercier François Richard et Bernard D'Almagne qui m'ont ouvert les portes du LAL. En plus d'avoir hérité du meilleur bureau du bâtiment 208 (je vois déjà une queue qui se forme devant la porte pour se ruer sur ma chaise dès que j'aurai tourné le dos), j'ai largement usé et abusé des ordinateurs et imprimantes. A ce sujet, je voudrais saluer la patience de Christian Becam que j'ai souvent séquestré le vendredi soir à 19 heures pour la résolution de divers problèmes toujours "super-urgents".

Constituer un jury de thèse consiste en la minimisation d'un $\chi^{2}$ bien plus compliqué que ceux rencontrés dans mes analyses: comme s'il était encore nécessaire de vérifier les lois de la statistique, la solution finale correspond bien à un optimal. Je voudrais donc remercier les membres qui constituent mon jury: Bernard D'Almagne, François Le Diberder et Helen Quinn (pour qui ce dut être une sacrée tâche de lire ces 270 pages de français), ainsi que Brigitte Bloch-Devaux et Marie-Noëlle Minard, mes deux rapporteurs, qui m'ont prodigué conseils éclairés et encouragements pour mon manuscrit et la fin de thèse.

Il existe tout un groupe de personnes au LAL couramment regroupées sous le terme de "jeunes" (la définition de ce terme étant variable, et devant permettre d'évincer tous les permanents par rapport aux thésards et post-doc). C'est entre "jeunes" que nous avons partagé nos talents culinaires durant les pauses gâteaux, et que plus récemment nous avons discuté des neutrinos et bientôt du concept de tutorat au LAL. Merci donc aux "jeunes" (entre autres, entendons nous bien, les "vieux" y sont aussi pour quelque chose) pour ces ponctuations joyeuses du quotidien. Certains d'entre eux ont côtoyé les mêmes couloirs que moi au bâtiment 208: Tuan, qui m'a régulièrement rapporté l'état de mes kilos (et de mon âge relativement au sien) par un “tu as grossi, ma vieille” (notez qu'à certains moments, j'ai quand même eu droit à la remarque inverse - sur mes kilos, pas sur mon age); Alexandre à qui j'ai eu la joie d'apprendre le vocabulaire de bouseuse récemment; Troels, qui à force de venir me demander une lettre à thé chaque matin finira sûrement par apprendre que "tea bag" se traduit par sachet de thé; Laurent, qui m'a permis de doubler mes connaissances sur la langue française depuis qu'il partage mon bureau; et enfin Benjamin, mon translaté dans l'espace à l'autre bout du couloir, mais pas dans le temps puisque nous avons partagé pas mal d'heures studieuses indues.

Parmi les moins "jeunes" (qui sait comment il va interpréter cela), je voudrais remercier Laurent Duflot, dit Dudu, qui fut mon tuteur effectif avant que le concept n'apparaisse au LAL: j'ai reçu sa visite quasi-quotidienne lorsqu'il allait vérifier son absence de courrier.

Enfin, le bâtiment 208, c'est surtout le groupe BABAR, placé sous l'égide d'Anne-Marie Lutz qui sème continuellement une bonne atmosphère sur notre groupe. Merci donc à elle, 
ainsi qu'à Andreas, François, Gilbert, Guy, Heiko, Laurent, Marie-Hélène, Michel, Stéphane, Sophie, Troels, Valeria et Vincent. Merci à Heiko qui m'a accueillie dans son bureau avec toute sa gentillesse et ses connaissances intarissables. Son "il faut bosser" résonne encore dans ce bureau...

Le moins qu'on puisse dire, c'est que ces désintégrations soit-disant sans aucun charme attirent la foule! Et c'est tant mieux, puisque cela m'a permis de partager mon quotidien avec le "super cool group" parisien (copyright LPNHE-LAL) composé d'Andreas, François, Heiko, José, Lydia, Muriel: un grand merci à eux d'être en effet aussi "cool" et d'avoir su créer une ambiance de travail si agréable.

Quelle sacrée épopée que celle de CKMfitter! Qui aurait pu présager du destin de ce projet de DEA? Il m'est difficile de résumer toutes les idées qui me viennent à l'esprit à l'évocation de CKMfitter tant la vie de ce projet fut ponctuée de mille événements heureux (je garde un souvenir extraordinaire du workshop au Michigan) ou plus délicats (les murs du bâtiment 280 de SLAC se souviennent encore de la joute verbale lors de ce fameux meeting $\sin 2 \beta$ de l'été 2000 ). J'irai donc directement au but en remerciant infiniment les trois autres mousquetaires qui ont créé puis élevé ce projet au niveau qu'il a atteint aujourd'hui: Andreas, Heiko et François. Merci pour cette belle expérience. Le clan des gardiens de CKMfitter s'est récemment agrandi avec Jérôme Charles (ce qui donne la preuve que CKMfitter est un code "user-friendly" puisqu'il tourne mêmesur les machines d'un théoricien), José, Lydia et Muriel: le futur foisonnant de CKMfitter est donc tout assuré.

Je tire mon chapeau bien bas au cuisinier Patrick Janot et à son ramasseur de bûches Marumi Kado pour l'accueil qu'ils m'ont fait lors de ma visite au CERN durant ma première année de thèse. A quand le Rutor?

Mon séjour prolongé à Berkeley, puis SLAC durant ma première année de thèse fut l'occasion de nombreuse rencontres qui, j'espère, survivront malgré les distances. Je remercie Moishe Pripstein pour son chaleureux accueil à LBL. J'ai travaillé là-bas avec Vasia Shelkov, dont la créativité professionnelle est débordante, et que je souhaite remercier pour son enthousiasme et sa générosité. Un grand merci à Bob Cahn qui est régulièrement venu me sortir de mon bureau pour aller partager le "thé des théoriciens", à qui nous avons parlé de toutes nos azéropi-treries déclenchant ainsi la rédaction du papier, et pour son soutien constant. Enfin, merci à Eric Charles et Alex Romosan pour leur talent d'imitateur de mon "freedom accent".

Puisque j'évoque le monde des théoriciens, je voudrais remercier Yossi Nir avec qui j'ai eu la chance de collaborer à deux reprises: merci à lui, ainsi qu'à Zoltan Ligeti et Gilad Perez, de m'avoir offert une incursion dans le monde de la nouvelle physique, et de la confiance dont ils m'ont témoignée.

Ma première rencontre à SLAC fut celle de Shahram Rahatlou (dont j'ai miraculeusement su écrire le nom correctement depuis le début, ce qui a sûrement contribué à notre amitié). Installée dans le bureau en face du sien, j'ai ainsi pu profiter de sa musique de U2 tard le soir, mais surtout de ses talents culinaires: personne ne fait les lasagnes comme Shahram. Les soirées SLACiennes furent animées par, entre autres, Haleh et Amir (avec qui j'ai partagé mes premiers sushis), Sylvie et David (les Québécois fous), Owen (LE spécialiste des clubs à San Francisco) et Wouter (que je remercie aussi pour sa patience à m'expliquer RooFit). Pour m'aider à appréhender le système SVTRAD, j'ai reçu l'aide précieuse de Tim Meyer et Michael Wilson, que je remercie, ainsi que Patricia Burchat qui m'a introduite dans l'équipe SVTRAD.

Je souhaite aussi remercier vivement Stew Smith, Hassan Jawalhery et Sau Lan Wu pour 
leur appui actif, en particulier durant ces derniers mois.

Devant le déplacement de masse généré par l'analyse $\rho \pi$, un groupe de travail a été créé, probablement parmi les plus actifs de BABAR: merci à Andreas, Christophe, François, Haibo, Jan, Jinwei, Julian, Paul, Pierre-François, Ran, Roy, Sau Lan, Vasia, Yibin et Zhitang pour le climat d'émulation qui a régné dans ce groupe. Pendant les mois précédant la première sortie de notre résultat à ICHEP 2002, j'ai partagé mon bureau de SLAC avec Jan Stark, avec qui j'ai beaucoup appris. Je tiens donc à remercier chaleureusement Jan pour la sacré équipe que nous avons formée, pour la relecture de ma thèse dans son état primitif (et sa maman pour la traque des fautes restantes sur la première page de texte de ma thèse), et pour tous les bons conseils qu'il m'a prodigués ces derniers mois.

Enfin, last but not least, mes chefs de cordée qui se sont investis coeurs et âmes dans l'encadrement dans cette expédition... J'aimerais trouver les mots justes pour leur rendre un hommage sincère à la hauteur de ce qu'ils m'ont apporté durant ces trois dernières années.

François Le Diberder, d'abord, "l'ancien" qui veille au grain, celui qui a insufflé de nombreuses idées de projets à réaliser durant la thèse, qui a réalisé de nombreux remontages de moral dans les moments difficiles, et qui a contribué à rendre mon séjour à SLAC à la fois joyeux et productif.

Et puis ma Ferrari de thèse (comme me l'a présenté François la première fois), Andreas Höcker, incarnant un enthousiasme débordant et donc communicatif pour la physique, qui m'a appris la rigueur et le travail soigné tout en créant une atmosphère de travail joviale. Ce ne fut pas toujours une tâche facile de suivre une Ferrari dans ma petite deux-chevaux, mais puisque cette dernière tient encore debout, c'est que j'ai dî y parvenir.

Merci donc à vous deux, Andreas et François, pour la qualité de votre encadrement, pour m'avoir fait découvrir une si large palette de la physique des particules, et pour la grande dose de bonne humeur distillée durant ces 3 années.

Pour finir, en dehors du cadre professionnel, je me dois de rendre hommage aux "amis parisiens" qui m'ont pardonné ma monomanie de la physique et mon alzheimer récurrent, et qui n'ont pas leur pareil pour promettre qu'on se couchera tôt, tout en finissant la soirée à 4 heures du matin (en moyenne): il s'agit de Colin, Cyrille, Florent et Jérôme. Je voudrais aussi envoyer une petite pensée aux "non-parisiennes": Nathalie, pour être simplement là depuis des années, et Jessica (bonne chance à toi), pour ses encouragements qui m'ont fait chaud au coeur, surtout les dimanches après-midi au travail malgré le soleil resplendissant dehors...

A ma mère et ma soeur,

"Lorsqu'elle eut terminé de vider son coeur, quelqu'un éteignit la lune." Gabriel Garcia Marquez, "L'amour au temps du choléra". 


\section{Table des matières}

$\begin{array}{lr}\text { Introduction } & 1\end{array}$

I Eléments de théorie $\quad 5$

$1 \quad$ Introduction à la violation de $C P \quad 7$

1.1 Le Modèle Standard et ses limites . . . . . . . . . . . . . . . 7

1.2 La matrice CKM et la violation de $C P \ldots \ldots . \ldots 7$

1.2.1 La brisure spontanée de la symétrie électrofaible et la matrice CKM 8

1.2.2 Conditions menant à la violation de $C P \ldots \ldots$. . . . . . . . . 9

1.2.3 Paramétrisation de Wolfenstein de la matrice CKM . . . . . . . . 10

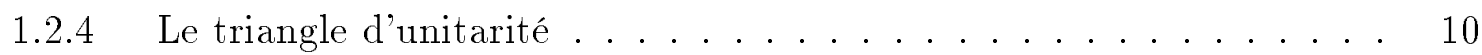

1.2.5 Ordres de grandeur de la violation de $C P \ldots \ldots \ldots 11$

1.3 Le système des mésons $B \ldots \ldots \ldots \ldots \ldots \ldots$

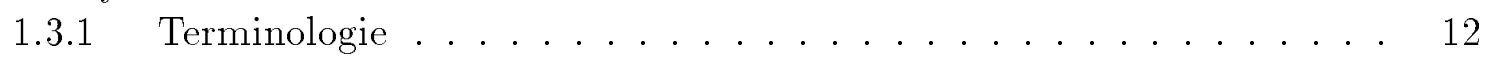

1.3.2 Les oscillations de $B^{0} \ldots \ldots \ldots \ldots \ldots$

1.3.3 Production d'une paire $B^{0} \bar{B}^{0}$ cohérente à $B A B A R$. . . . . . . . . 14

1.3.4 Désintégration d'un méson $B$ en un état propre de saveur . . . . . 15

1.3.5 Désintégration d'un méson $B$ en un état propre de $C P \ldots \ldots$

1.4 Les trois types de violation de $C P \ldots \ldots \ldots \ldots$. . . . . . . . . . . . . . .

1.4.1 La violation de $C P$ dans la désintégration $\left(\left|\bar{A}_{\bar{f}} / A_{f}\right| \neq 1\right) \ldots \ldots$

1.4.2 La violation de $C P$ dans le mélange $B^{0}-\bar{B}^{0}(|q / p| \neq 1) \ldots \ldots$

1.4.3 La violation de $C P$ due à l'interférence entre les désintégrations avec et sans mélange $(\operatorname{Im} \lambda \neq 0) \ldots \ldots \ldots 18$

1.5 Les trois niveaux de complexité d'une analyse $C P \ldots \ldots$. . . . . . . 18

1.5.1 $|\lambda|=1$, état final propre de $C P(\sin 2 \beta$ dans les "modes en or" $b \rightarrow c \bar{c} s) 18$

1.5.2 $|\lambda| \neq 1$ et état final propre de $C P\left(\sin 2 \alpha_{\text {eff }}\right.$ dans le mode $\left.B^{0} \rightarrow \pi^{+} \pi^{-}\right) \quad 19$

1.5.3 $|\lambda| \neq 1$ et état final non propre de $C P\left(\sin 2 \alpha_{\text {eff }}\right.$ dans le mode $B^{0} \rightarrow$ $\left.\rho^{ \pm} \pi^{\mp}\right) \ldots \ldots \ldots \ldots \ldots 21$

2 Eléments de théorie pour les modes $B^{0} \rightarrow \rho \pi \quad 27$

2.1 Mesurer l'angle $\alpha$ : où est la difficulté? . . . . . . . . . . . 27

2.2 Hypothèse théorique de base: la symétrie $S U(2)$ d'isospin . . . . . . . . 28

2.2.1 Amplitudes des désintégrations $B \rightarrow \rho \pi \ldots \ldots . \ldots 28$

2.2.2 Les relations d'isospin . . . . . . . . . . . . . . . . 29

2.2.3 L'analyse d'isospin de $B \rightarrow \rho \pi$ en (quasi) deux corps . . . . . . . . 30

2.2.4 L'analyse Dalitz de $B^{0} \rightarrow \pi^{+} \pi^{-} \pi^{0} \ldots \ldots \ldots$. . . . . . . . . . . . . . . . 32

2.2.5 Limite sur $\left|\alpha-\alpha_{\text {eff }}^{ \pm}\right|$utilisant $S U(2) \ldots \ldots . \ldots . \ldots 33$ 
2.3 Hypothèse théorique plus poussée: la symétrie $S U(3) \ldots \ldots$. . . . . . . . 34

\section{Etude de la violation de CP dans l'expérience $B A B A R$}

$\begin{array}{ll}\text { Survol des chapitres à venir } & 37\end{array}$

3 Dispositif expérimental $\quad 39$

3.1 L'accélérateur et l'anneau de stockage PEP-II . . . . . . . . . . . . . . 39

3.1.1 L'accélérateur linéaire . . . . . . . . . . . . . . . 40

3.1.2 Le collisionneur PEP-II . . . . . . . . . . . . . . . . . . . . . . . . . . 40

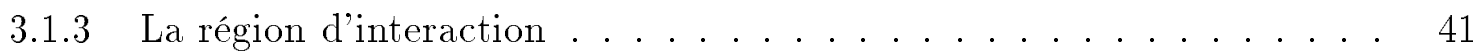

3.1.4 Le système de coordonnées . . . . . . . . . . . . . . . . . 42

3.2 Le détecteur $B A B A R \ldots \ldots \ldots \ldots$

3.2.1 Détecteur de vertex au silicium (SVT) . . . . . . . . . . . . 43

3.2 .2 Chambre à dérive $(\mathrm{DCH}) \ldots \ldots \ldots \ldots$

3.2.3 Détecteur à lumière Cherenkov (DIRC) . . . . . . . . . . . . . . 49

3.2.4 Calorimètre électromagnétique (EMC) . . . . . . . . . . . 53

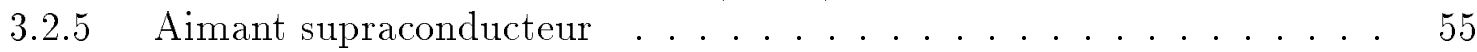

3.2.6 Retour de flux instrumenté (IFR) . . . . . . . . . . . . . . 55

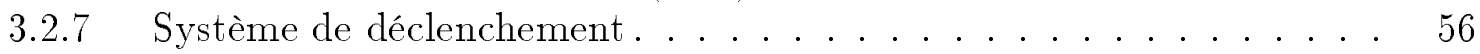

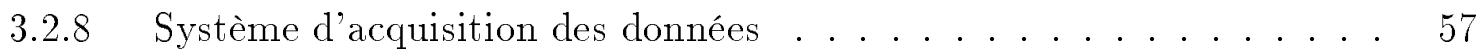

3.3 Contrôle et protection du SVT . . . . . . . . . . . . . . . 58

3.3.1 Rôles du système de protection du SVT (SVTRAD) . . . . . . . . . 58

3.3.1.1 Le bruit de fond de PEP-II ............. 58

3.3.1.2 Les risques encourus par le SVT . . . . . . . . . 59

3.3.2 Description du système SVTRAD . . . . . . . . . . . . . . . . 59

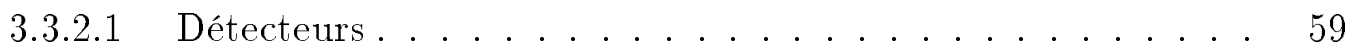

3.3.2.2 Electronique de lecture et algorithme de protection associé . 61

3.3.2.3 Logiciel en temps réel, obtention des taux de radiation et algorithmes de protection associés . . . . . . . . 62

3.3.3 Performances du système, doses accumulées . . . . . . . . . . . 64

3.3.4 Amélioration du logiciel en temps réel pour les périodes d'injection . 65

3.3.4.1 Définition d'un nouveau critère de qualité d'injection . . . . 66

3.3.4.2 Nouveaux algorithmes de protection pendant l'injection . . . 66

3.3.4.3 Bilan du nouveau algorithme de protection activé pendant

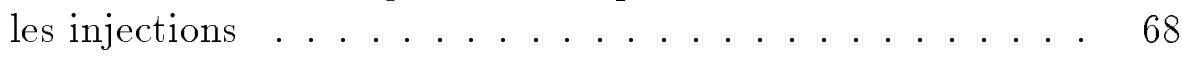

3.4 Les données. . . . . . . . . . . . . . . . . 70

3.4.1 Données on-peak et off-peak . . . . . . . . . . . . . . 70

3.4.2 Les événements Breco . . . . . . . . . . . . . . . . . . . 72

3.5 La simulation Monte Carlo . . . . . . . . . . . . . . . 72

4 Analyse temporelle et mesure des rapports d'embranchement de $B^{0} \rightarrow$ $\rho^{ \pm} \pi^{\mp}$ et $B^{0} \rightarrow \rho^{-} K^{+} \quad 77$

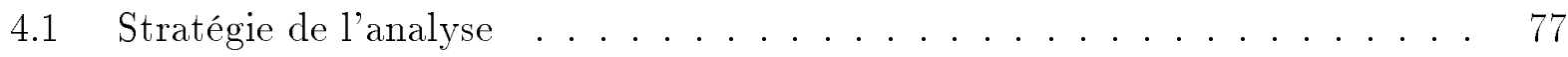

4.2 Topologie des différents types d'événements . . . . . . . . . . . . . . 78

4.3 Reconstruction et sélection du $B_{\text {rec }}: B^{0} \rightarrow \rho h(h=\pi, K) \ldots \ldots$. . . . 79

4.3.1 Sélection des kaons et pions chargés ............ 80 
4.3.1.1 Reconstruction des traces ........................ 80

4.3.1.2 Identification des traces . . . . . . . . . . . . 81

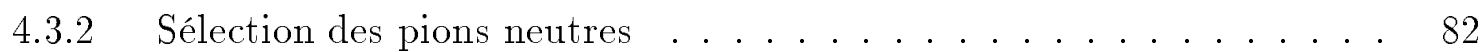

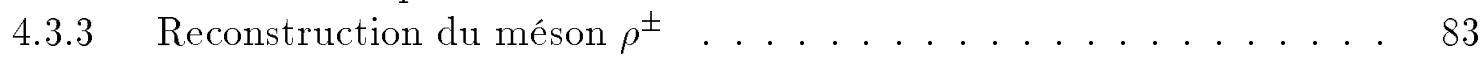

4.3.3.1 Reconstruction et sélection ............. 83

4.3.3.2 Interférences $\rho^{+} \rho^{-} \ldots \ldots \ldots \ldots$............ 84

4.3.3.3 Choix de la charge du $\rho \ldots \ldots . \ldots . \ldots . \ldots 5$

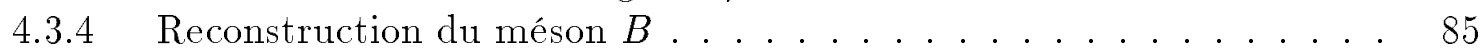

4.3.5 Réjection des bruits de fond $B$ en 2-corps . . . . . . . . . . 87

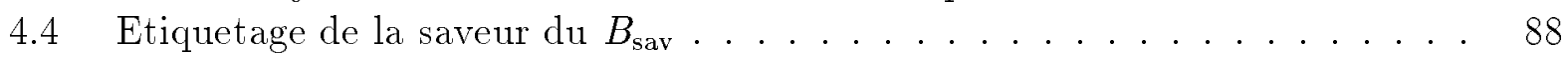

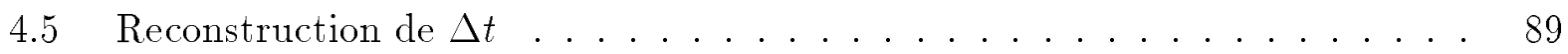

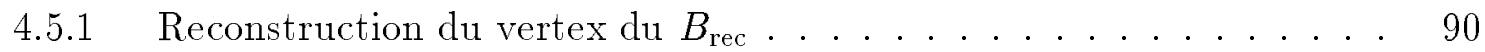

4.5.2 Reconstruction $\mathrm{d} u$ vertex $\mathrm{d} u B_{\mathrm{sav}} \ldots \ldots \ldots . \ldots . \ldots 90$

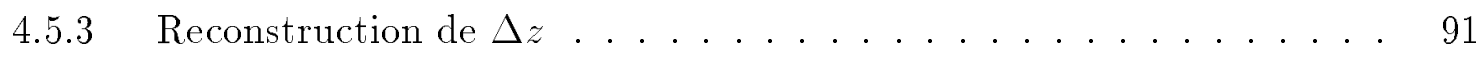

4.5.4 Conversion entre $\Delta z$ et $\Delta t \ldots \ldots \ldots \ldots$

4.5 .5 Coupures sur $\Delta t \ldots \ldots \ldots . \ldots \ldots 2$

4.6 Lutte contre le bruit de fond $q \bar{q} \ldots \ldots \ldots \ldots$

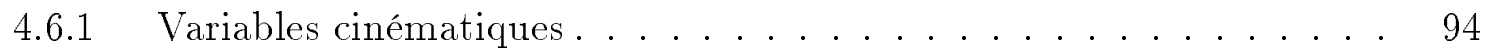

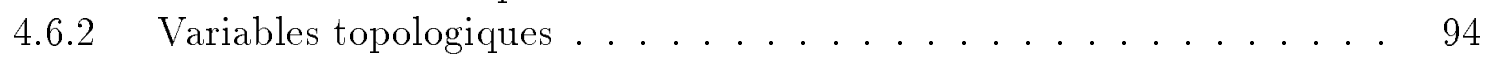

4.6.3 Corrélations linéaires entre les variables . . . . . . . . . . . . . 98

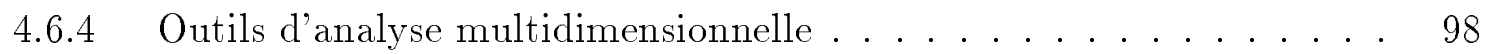

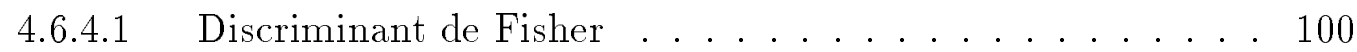

4.6.4.2 Réseau de neurones ..................... 100

4.6.5 Optimisation de la variable multidimensionnelle . . . . . . . . 100

4.6.5.1 Performances des trois configurations .......... 101

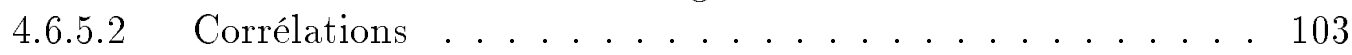

4.6.5.3 Choix de la configuration finale............ 104

4.7 Sélection finale et choix $d u$ meilleur candidat . . . . . . . . . . . . . 104

4.7.1 Efficacité finale de sélection . . . . . . . . . . . . . . . . 104

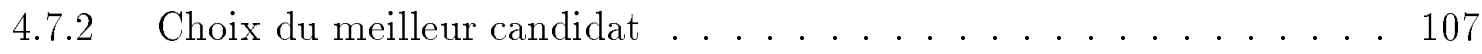

4.8 Bruit de fond provenant d'autres modes $B \ldots \ldots \ldots$. . . . . . 108

4.8.1 Modes $B$ non charmés . . . . . . . . . . . . . . . 108

4.8.2 Modes $B$ charmés . . . . . . . . . . . . . . . 110

4.8.2.1 Monte Carlo générique $b \rightarrow c \ldots . . . . . . . . . .110$

4.8.2.2 Déterminer la contamination de $b \rightarrow c$ à partir des données 114

4.8.3 Classification des modes $B$ charmés et non charmés . . . . . . . . 115

4.9 Distributions de référence . . . . . . . . . . . . . . 116

4.9.1 Signal et bruit de fond continurum . . . . . . . . . . . . . 117

4.9.1.1 $m_{\mathrm{ES}} \ldots \ldots \ldots \ldots \ldots \ldots \ldots$

$4.9 .1 .2 \quad \Delta E \ldots \ldots \ldots \ldots \ldots \ldots \ldots$

4.9.1.3 $x_{\mathrm{NN}} \ldots \ldots \ldots \ldots \ldots \ldots \ldots$

$4.9 .1 .4 \theta_{C} \ldots \ldots \ldots \ldots \ldots$

4.9.1.5 $\Delta t \ldots \ldots \ldots \ldots \ldots \ldots$

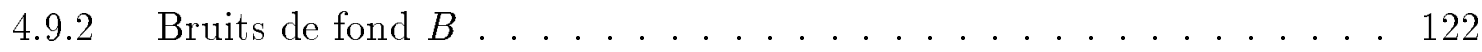

4.9.2.1 $m_{\mathrm{ES}}, \Delta E$ et $x_{\mathrm{NN}} \ldots \ldots \ldots \ldots \ldots . \ldots \ldots 122$

4.9.2.2 Corrélations entre $m_{\mathrm{ES}}$ et $\Delta E$ dans le bruit de fond $B$. . 122

4.9.2.3 Distributions de $\Delta t$ et corrélations charge-étiquette . . . 123 
4.10 Fonction de vraisemblance . . . . . . . . . . . . . . . 124

4.10.1 Composition de la fonction de vraisemblance . . . . . . . . . . . 124

4.10.2 Paramètres libres de l'ajustement final . . . . . . . . . . . . . . 126

4.11 Validation de l'analyse en avengle . . . . . . . . . . . . . . . 127

4.11.1 Validation par des simulations rapides . . . . . . . . . . 127

4.11.2 Ajustements sur des échantillons connus (MC, simulation rapide, off-

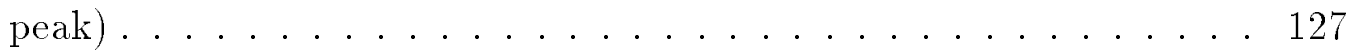

4.11.2.1 Monte Carlo $\rho \pi$ et $\rho K$ (grande statistique) . . . . . . . 128

4.11.2.2 Monte Carlo Continuum et données off-peak (grande statis-

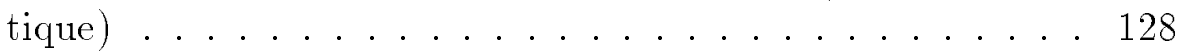

4.11.2.3 Cocktails de MC signal et bruit de fond $B$, et de continuum de la simulation rapide (échantillons de taille réaliste) . . . 128

4.11.2.4 Discussion des résultats . . . . . . . . . . . . 129

4.12 Ajustement final des données (dévoilement du résultat) . . . . . . . . . 130

4.12.1 Résultat de l'ajustement final sur les données on-peak . . . . . . . . 130

4.12 .2 Niveau de confiance des résultats . . . . . . . . . . . . . . . 132

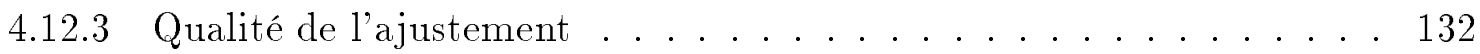

4.12.4 Nombre d'événements $\rho \pi$ pour une charge de $\rho$ et une saveur de $B^{0}$

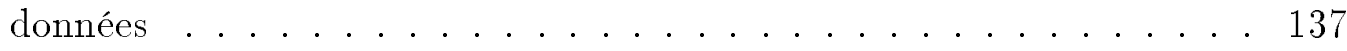

4.12.5 Paramétrisation physique de la violation de $C P$ directe . . . . . 138

4.13 Validations a posteriori . . . . . . . . . . . . . 138

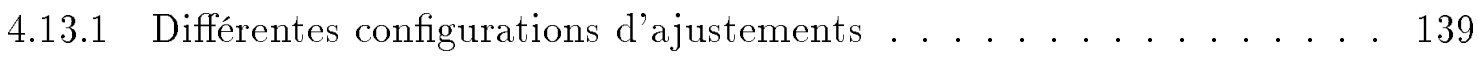

4.13.2 Vérifications sur $\Delta C_{K}, \Delta S_{K}$ et $C_{K} \ldots \ldots \ldots \ldots$. . . . . . . . . . . . . . . . 139

4.13.3 Information contenue dans $m(\rho)$ et $\cos \theta_{H}(\rho) \ldots \ldots \ldots$. . . . . 139

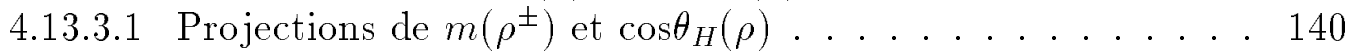

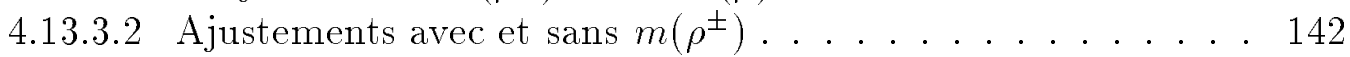

4.14 Etudes des erreurs systématiques . . . . . . . . . . . . . . 142

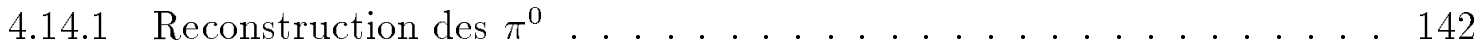

4.14.2 Validations du signal en utilisant le mode $B^{0} \rightarrow D^{-} \rho^{+} \ldots$. . . . . 143

4.14.2.1 Distribution de $x_{\mathrm{NN}} \ldots \ldots \ldots \ldots$. . . . . . . . . . . . . . . . . 143

4.14.2.2 Distributions de $\Delta E$ et $m_{\mathrm{ES}} \ldots \ldots \ldots \ldots . \ldots . \ldots 143$

4.14.2.3 Fraction de signal mal reconstruit ............ 144

4.14.3 Fonction de résolution et étiquetage . . . . . . . . . . . . . 145

4.14.4 Désintégrations doublement supprimées de Cabibbo . . . . . . . . . 146

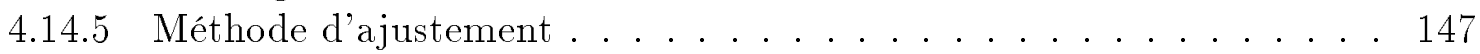

4.14.6 Identification de la trace célibataire . . . . . . . . . . . . . 147

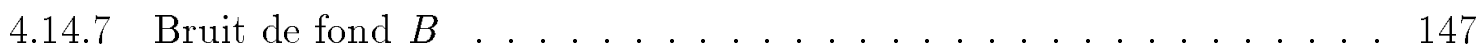

4.14.7.1 Incertitudes dues aux rapports d'embranchement . . . . . 147

4.14.7.2 Incertitudes reliées aux paramètres $C P$ et de dilution . . . 148

4.14.7.3 Incertitudes reliées aux autres hypothèses . . . . . . . . . . 148

4.14.7.4 Résumé des incertitudes systématiques liées aux bruits de

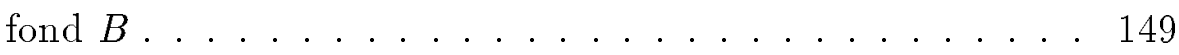

4.14.8 Résumé des incertitudes systématiques . . . . . . . . . . . . 149

4.15 Rapports d'embranchement de $B^{0} \rightarrow \rho^{ \pm} \pi^{\mp}$ et $B^{0} \rightarrow \rho^{-} K^{+} \ldots \ldots$. . . . 150

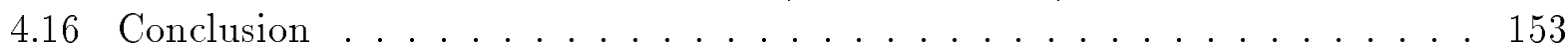


$5 \quad$ Mesure du rapport d'embranchement de $B^{0} \rightarrow \rho^{0} \pi^{0}$

5.1 Stratégie de l'analyse et différences avec $B^{0} \rightarrow \rho^{ \pm} \pi^{\mp} \ldots \ldots$. . . . . . . 155

5.2 Sélection de $B^{0} \rightarrow \rho^{0} \pi^{0} \ldots \ldots \ldots \ldots \ldots \ldots$. . . . . . . . . . . . . . . . . . . . . . . . . . . 156

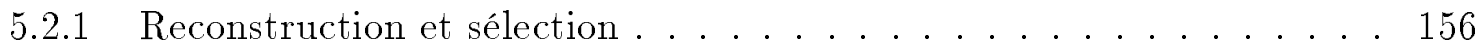

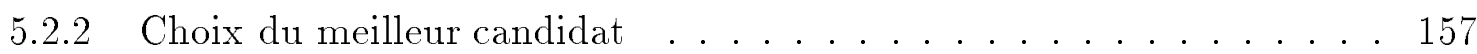

5.2.3 Statistiques finales de la sélection . . . . . . . . . . . 157

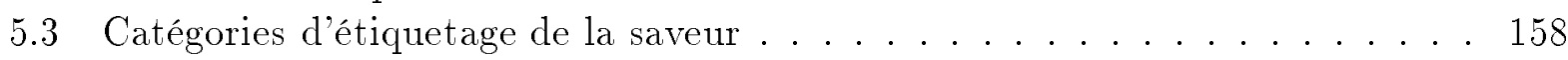

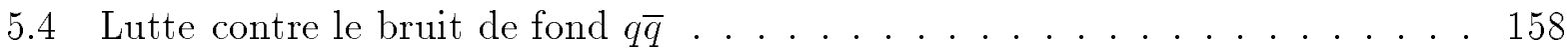

5.4.1 Configurations de variables . . . . . . . . . . . . 158

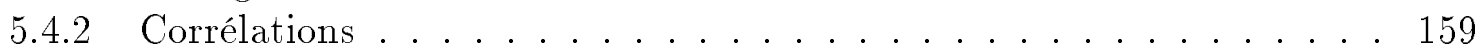

5.5 Bruit de fond provenant d'autres modes $B \ldots \ldots \ldots$. . . . . . . . 159

5.6 Fonction de vraisemblance . . . . . . . . . . . . . . . 161

5.6.1 Composition de la fonction de vraisemblance . . . . . . . . . 161

5.6.2 Corrélations entre les variables entrant la fonction de vraisemblance . 162

5.7 Validation de l'analyse en avengle . . . . . . . . . . . . . 162

5.7.1 Validation par des simulations rapides .................... 162

5.7.1.1 Erreurs sur $N_{\rho^{0} \pi^{0}}$ pour différentes configurations . . . . . . 162

5.7.1.2 Pulls des variables libres dans l'ajustement final . . . . . . 162

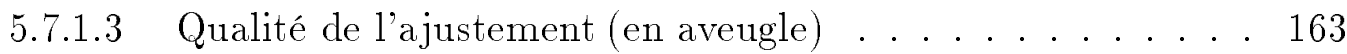

5.7.2 Ajustements sur des échantillons connus . . . . . . . . . . . 164

5.7.2.1 Monte Carlo $B^{0} \rightarrow \rho^{0} \pi^{0}$ et données off-peak (grande statis-

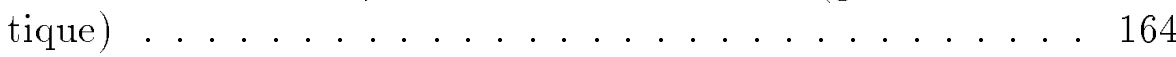

5.7.2.2 Cocktails de MC signal et bruits de fond $B$, et de continuum

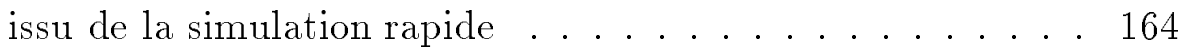

5.8 Ajustement final des données . . . . . . . . . . . . . . . . 165

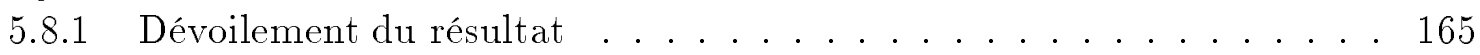

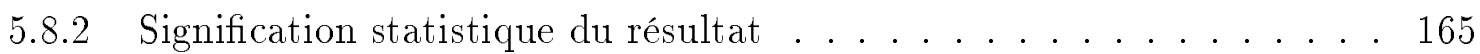

5.8.3 Limite supérieure à $90 \%$ de niveau de confiance sur le nombre d'évé-

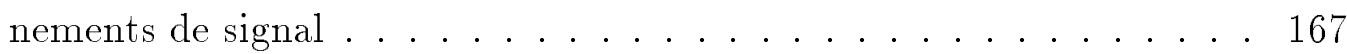

5.9 Etudes des erreurs systématiques . . . . . . . . . . . . . . 168

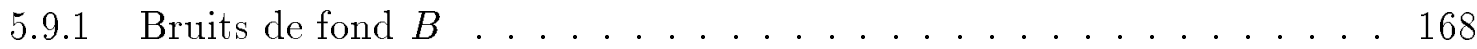

5.9.2 Validations des distributions du signal en utilisant le mode $B^{0} \rightarrow D^{-} \rho^{+} 168$

5.9.2.1 Distribution de $\Delta E$ et $m_{\mathrm{ES}} \ldots \ldots \ldots . . \ldots . . \ldots 168$

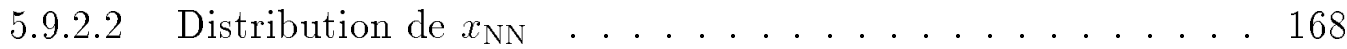

5.9.2.3 Interférences résiduelles $\rho^{0}-f^{0} \ldots \ldots \ldots \ldots$. . . . . . 168

5.9.2.4 Interférences résiduelles $\rho^{0}-\rho^{ \pm}$et avec $\pi^{+} \pi^{-} \pi^{0}$ non résonant 170

5.10 Limite supérieure à $90 \% \mathrm{CL} \operatorname{sur} \mathcal{B}\left(B^{0} \rightarrow \rho^{0} \pi^{0}\right)$ incluant les incertitudes sys-

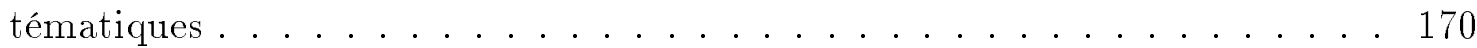

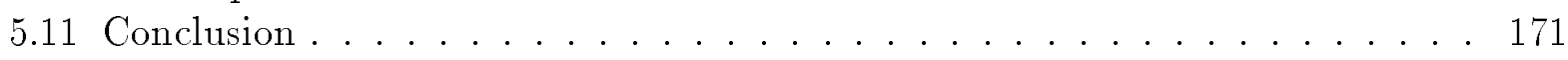

6 Premiers pas dans l'analyse Dalitz de $B^{0} \rightarrow \pi^{+} \pi^{-} \pi^{0} \quad 173$

6.1 Introduction . . . . . . . . . . . . . . . . 173

6.2 Reconstruction de $B^{0} \rightarrow \pi^{+} \pi^{-} \pi^{0} \ldots \ldots \ldots \ldots \ldots \ldots$. . . . . . . . . . . . . . . . . . . 174

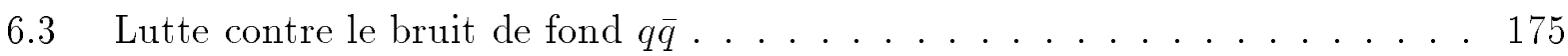

6.4 Bruit de fond provenant d'autres modes $B \ldots \ldots \ldots$. . . . . . 176

6.5 Distributions de $B^{0} \rightarrow \pi^{+} \pi^{-} \pi^{0}$ dans le diagramme de Dalitz . . . . . . . 176

6.5.1 Facteur de forme du $\pi \ldots \ldots \ldots \ldots$ 
6.5.1.1 Les résonances qui se désintègrent en $\pi \pi$. . . . . . . . 179

6.5.1.2 Les données $e^{+} e^{-} \rightarrow \pi^{+} \pi^{-}$et les désintégrations des $\tau^{ \pm}$. . 180

6.5.1.3 Paramétrisation de Gounaris-Sakurai du facteur de forme . 181

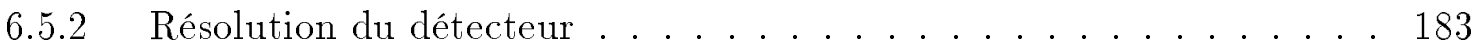

6.5.3 Efficacité de sélection dans le diagramme de Dalitz . . . . . . . . 184

6.5.4 Fraction d'événements mal reconstruits dans le diagramme de Dalitz 184

6.6 Fonction de vraisemblance et paramétrisations . . . . . . . . . . . 185

6.6.1 Fonction de vraisemblance . . . . . . . . . . . . . 185

6.6.2 Paramétrisations de $m_{\mathrm{ES}}, \Delta E, x_{\mathrm{NN}} \ldots \ldots \ldots \ldots \ldots$

6.6.3 Paramétrisations de $\mathcal{P}^{3 \pi}\left(\Delta t, m^{2}\left(\pi^{+} \pi^{0}\right), m^{2}\left(\pi^{-} \pi^{0}\right)\right) \ldots \ldots \ldots$. . . 186

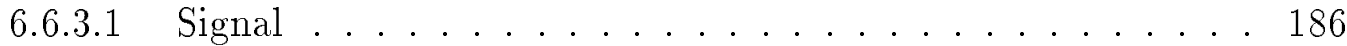

6.6.3.2 Bruit de fond continuum .............. 187

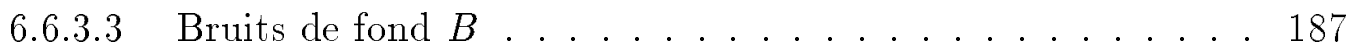

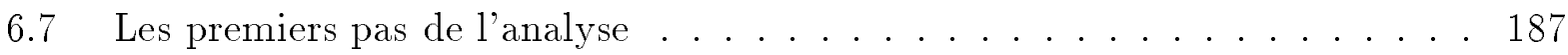

6.7.1 Etude de sensibilité . . . . . . . . . . . . 187

6.7.2 Impact des phases fortes des résonances $\rho(1450)$ et $\rho(1700) \ldots \ldots 187$

6.7.3 Impact de la résolution sur $m^{2}\left(\pi^{ \pm} \pi^{0}\right) \ldots \ldots \ldots \ldots$. . . . . . 189

6.7.4 Impact des bruits de fond . . . . . . . . . . . . . 189

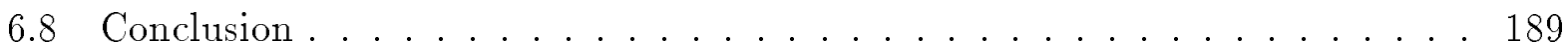

III Contraintes sur la matrice CKM 191

$\begin{array}{lr}\text { Introduction } & 193\end{array}$

$7 \quad$ Méthode statistique $\quad 195$

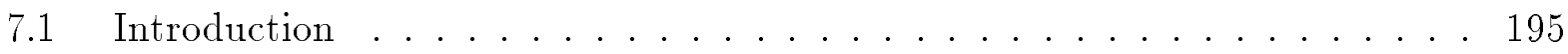

7.2 La fonction de vraisemblance . . . . . . . . . . . . . . . 197

7.2.1 La fonction de vraisemblance expérimentale $\mathcal{L}_{\exp } \ldots \ldots$. . . . . . 197

7.2.2 La fonction de vraisemblance théorique $\mathcal{L}_{\text {theo }} \ldots \ldots \ldots$. . . . . 198

7.3 Test du Modèle Standard . . . . . . . . . . . . . . . . . 198

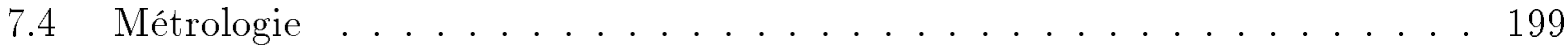

7.5 Physique au delà du Modèle Standard . . . . . . . . . . . . . . 199

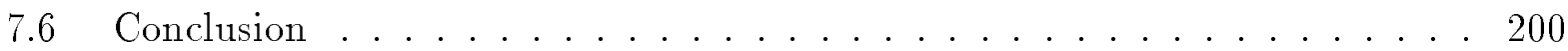

8 Ajustement standard des paramètres CKM 201

8.1 Ingrédients de l'ajustement standard . . . . . . . . . . . . . . . . 201

8.1.1 Les éléments de matrice CKM . . . . . . . . . . . . 201

8.1.2 Les observables $C P \ldots \ldots \ldots \ldots$

8.1.3 Résumé des ingrédients . . . . . . . . . . . . . 205

8.2 Niveau de confiance global de l'ajustement standard . . . . . . . . . . 207

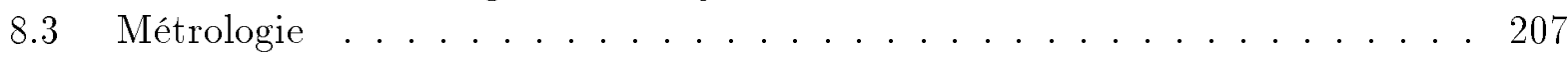

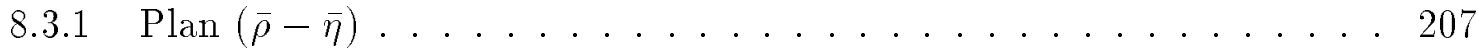

8.3.2 Plan $(\sin 2 \alpha-\sin 2 \beta)$ et $(\sin 2 \beta-\gamma) \ldots \ldots . \ldots 207$

8.3.3 Contraintes uni-dimensionnelles . . . . . . . . . . 209

8.4 Conclusions . . . . . . . . . . . . . . . . . 209 
$9 \quad$ Interprétation des résultats expérimentaux sur $B \rightarrow \rho h \quad 213$

9.1 Introduction . . . . . . . . . . . . . . . . . . 213

9.2 Ingrédients pour les analyses $S U(2)$ et $S U(3) \ldots \ldots \ldots \ldots$

9.3 Les pingouins dans $B^{0} \rightarrow \rho^{ \pm} \pi^{\mp} \ldots \ldots \ldots \ldots \ldots \ldots$

9.3.1 Hypothèse de pingouins nuls . . . . . . . . . . . . . . . 215

9.3.2 Contraintes sur les rapports pingouin/arbre utilisant l'ajustement stan-

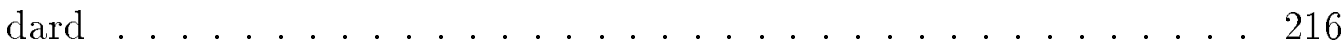

9.4 Contraintes sur $\alpha$ utilisant $S U(2) \ldots \ldots \ldots \ldots \ldots$

9.4.1 Limites sur $\left|\alpha-\alpha_{\text {eff }}^{ \pm}\right|$utilisant $S U(2) \ldots \ldots \ldots . \ldots . \ldots 216$

9.4.2 Extrapolations futures de l'analyse d'isospin complète . . . . . . 218

9.5 Contraintes sur $\alpha$ utilisant $S U(3) \ldots \ldots \ldots \ldots \ldots \ldots$

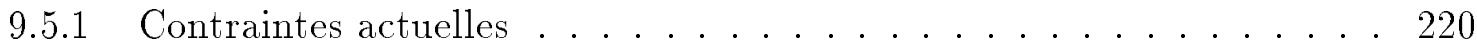

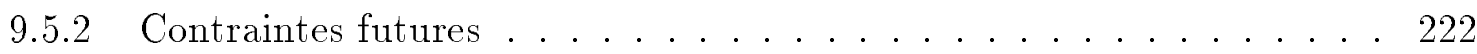

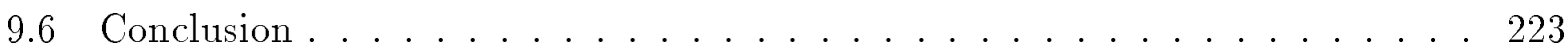

10 La matrice CKM au delà du Modèle Standard 227

10.1 Introduction . . . . . . . . . . . . . . . . . 227

10.2 Prédiction de $A_{\mathrm{SL}}$ dans le Modèle Standard . . . . . . . . . . . . . . 228

$10.3 A_{\mathrm{SL}}$ dans les modèles de nouvelle physique . . . . . . . . . . . . . . 229

10.3.1 Modèle de nouvelle physique dans le mélange $B^{0}-\bar{B}^{0} \ldots$. . . . . . . 229

10.3.2 Modèles avec violation de saveur minimale . . . . . . . . . . 231

10.4 Conclusion . . . . . . . . . . . . . . . . . 233

$\begin{array}{lr}\text { Conclusion } & 235\end{array}$

A Le mode $B^{0} \rightarrow a_{0}^{ \pm} \pi^{\mp}$ : théorie et mesure du rapport d'embranchement 239

A.1 Théorie relative au mode $B^{0} \rightarrow a_{0}^{ \pm} \pi^{\mp} \ldots \ldots$. . . . . . . . . . 239

A.1.1 Analyse Dalitz de $B^{0} \rightarrow a_{0}^{ \pm} \pi^{\mp} \ldots \ldots \ldots \ldots . \ldots \ldots 239$

A.1.2 Analyse en deux-corps de $B^{0} \rightarrow a_{0}^{ \pm} \pi^{\mp}$. . . . . . . . . . . . 240

A.1.2.1 Absence des courants de seconde classe dans le Modèle Stan-

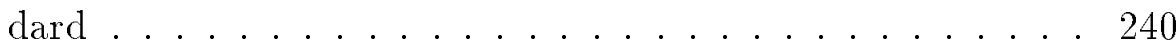

A.1.2.2 Simplification de l'analyse en deux-corps de $B^{0} \rightarrow a_{0}^{ \pm} \pi^{\mp}$. . 241

A.1.2.3 Au delà de la factorisation naïve . . . . . . . . . . . . 241

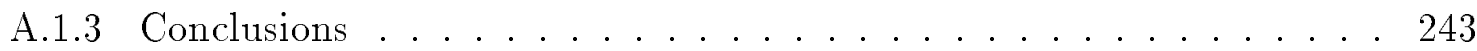

A.2 Mesure du rapport d'embranchement . . . . . . . . . . . . . 243

B Variables pour la lutte contre le bruit de fond $q \bar{q} \quad \mathbf{2 4 7}$

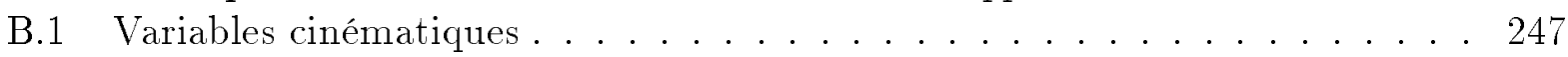

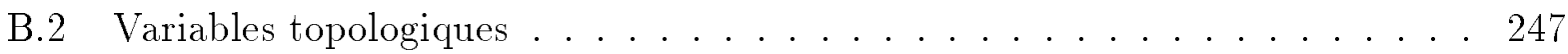

C Lutte contre le bruit de fond $B$ non charmé $\quad 249$

C.1 Elaboration des réseaux de neurones . . . . . . . . . . . . . . . 249

C.2 Incertitudes systématiques. . . . . . . . . . . . . . . 252

D Rapport d'embranchement des bruits de fond $B$ non charmés 255 


\section{Introduction}

L'édification du modèle permettant d'expliquer les phénomènes agissant à l'échelle fondamentale des particules est un travail de longue haleine: cela fait 38 ans que la violation de la symétrie $C P$ entre matière et anti-matière a été découverte dans le système des mésons $K$ [1], et 29 ans que le mécanisme de Kobayashi-Maskawa (KM) [2] a été proposé pour l'expliquer. Mais la preuve expérimentale que le mécanisme KM constitue bien la source principale de la violation de $C P$ dans le Modèle Standard n'a été apportée que très récemment.

Par ce mécanisme, la matrice de Cabibbo-Kobayashi-Maskawa (CKM) de couplage entre les courants chargés et les quarks possède une phase irréductible dès lors qu'il existe trois familles de quarks de masses non-dégénérées. L'unitarité de la matrice CKM se traduit par des relations entre les éléments de la matrice dont certaines peuvent se représenter graphiquement dans le plan complexe par un triangle dit d'unitarité. L'un de ces triangles, mettant en jeu les couplages reliés au quark $b$, est particulièrement adapté à l'étude de la violation de $C P$ dans le système des mésons $B$. Le but est de sur-contraindre le sommet de ce triangle par plusieurs mesures dans le système des mésons $B$ et des mésons $K$, reliées aux côtés ou aux angles du triangle. On espère ainsi mettre en évidence un désaccord entre les différentes déterminations indépendantes $d u$ sommet, ce qui serait une preuve de physique au delà du Modèle Standard. Est-on en droit d'espérer observer des effets indirects de nouvelle physique dans le secteur de la physique des saveurs et de la violation de $C P$ ? La réponse est affirmative puisque, d'une part, de nombreux modèles au-delà du Modèle Standard contiennent de nouvelles sources de violation de $C P$, et d'autre part, l'ampleur de la violation de $C P$ dans le Modèle Standard ne peut expliquer l'asymétrie entre matière et anti-matière dans l'Univers [3].

La validité du mécanisme KM a été établie récemment dans le système des mésons $B_{d}$ grâce à la mesure précise de $\sin 2 \beta$, où $\beta$ est l'un des angles du triangle d'unitarité. Cette mesure a été réalisée par les deux usines à $B: \quad B A B A R$ (auprès du collisionneur PEP-II à SLAC, Etats-Unis) et Belle (auprès du collisionneur KEKB à KEK, Japon), qui accumulent des données depuis 1999. En plus d'observer pour la première fois la violation de la symétrie $C P$ dans un système autre que les mésons $K$, cette mesure s'est surtout avérée en parfait accord avec les autres contraintes du triangle d'unitarité issues des mésons $B$ (conservant $C P)$ et $K($ violant $C P)$.

La recherche de preuves indirectes de physique au-delà du Modèle Standard n'est pas pour autant terminée: dans le système des mésons $B_{d}$, on ne peut néanmoins plus envisager d'alternatives au mécanisme KM, mais plutôt des corrections à ce mécanisme. Dans le système des mésons $B_{s}$, tout est encore envisageable.

Ainsi, les usines à $B$, de même que d'autres projets en début de prise de données tels que le Tevatron, ou en préparation, tels que le LHC, ont pour objectif de mesurer de nouvelles observables permettant de sur-contraindre le triangle d'unitarité et la matrice CKM.

Le travail de thèse présenté dans ce document porte sur l'une de ces nouvelles observables: 
l'angle $\alpha$ du triangle d'unitarité. Plusieurs désintégrations permettent en principe de mesurer cet angle, et nous nous concentrons ici sur les désintégrations non-charmées du $B$ en 3 corps: $B^{0} \rightarrow \rho^{ \pm} \pi^{\mp}, B^{0} \rightarrow \rho^{-} K^{+}, B^{0} \rightarrow a_{0}^{ \pm} \pi^{\mp}$.

Dans la première partie de ce document (chapitres 1 et 2), la phénoménologie de la matrice $C K M$ et de la violation de $C P$ dans le système des mésons $B$ est exposée, ainsi que les éléments de théorie se rapportant aux modes analysés dans cette thèse.

Dans la deuxième partie, le travail expérimental réalisé auprès de l'expérience $B A B A R$ est présenté. Il s'agit d'une part, d'un travail proche du dispositif expérimental, et d'autre part, d'un travail d'analyse de données.

Le dispositif expérimental, comprenant l'accélérateur linéaire, le collisionneur et le détecteur, est décrit dans le chapitre 3 , suivi de la description de mon travail réalisé auprès du système de protection du SVT contre les radiations. Alors responsable de ce système pendant plusieurs mois à SLAC, j'ai amélioré le logiciel en temps réel de ce système activé durant les périodes d'injection, ces dernières contituant une période critique en terme d'irradiations.

Le chapitre 4 décrit la principale analyse de cette thèse: utilisant une statistique d'environ $90 \cdot 10^{6}$ paires $B \bar{B}$, une analyse $C P$ dépendante du temps des modes $B^{0} \rightarrow \rho^{ \pm} \pi^{\mp}$ et $B^{0} \rightarrow \rho^{-} K^{+}$est menée, et leurs rapports d'embranchement sont mesurés. Il s'agit d'une analyse complexe, car ces modes souffrent de nombreuses sources de bruits de fond parfois irréductibles et mal connues. Néanmoins, le développement d'outils de lutte contre le bruit de fond issu de la production de paires quark-antiquark, et l'étude minutieuse de ceux issus des autres désintégrations du $B$ permettent leur contrôle. Un ajustement par maximum de vraisemblance mène à la séparation des différentes composantes de signal et de bruits de fond des données, et à l'extraction des paramètres reliés à violation de la symétrie $C P$ ainsi qu'aux propriétés des désintégrations considérées. Cette analyse mobilise une douzaine de personnes issues de 5 laboratoires en France et aux Etats-Unis: ainsi, quelques parties de l'analyse présentée ici sont réalisées par d'autres collaborateurs, et sont résumées succinctement pour aider à la compréhension. Dans ce document, j'insiste tout particulièrement sur mes contributions personnelles à toutes les étapes de l'analyse.

Le rapport d'embranchement du mode supprimé de couleur $B^{0} \rightarrow \rho^{0} \pi^{0}$ est aussi mesuré (chapitre 5). Il s'agit d'un ingrédient essentiel de l'analyse du diagramme de Dalitz de $B^{0} \rightarrow$ $\pi^{+} \pi^{-} \pi^{0}$ devant mener, à terme, à la mesure de l'angle $\alpha$.

Nous bâtissons enfin les fondations de l'analyse du diagramme de Dalitz, et réalisons des estimations de sensibilité expérimentale pour $\alpha$, décrites dans le chapitre 6 . Il s'agit des premiers pas dans cette analyse très prometteuse - et ambitieuse - pour la mesure de l'angle $\alpha$ qui va être intensément poursuivie dans les mois à venir.

Sur une statistique plus limitée de $22 \cdot 10^{6}$ paires $\overline{B B}$, nous mesurons le rapport d'embranchement du mode $B^{0} \rightarrow a_{0}^{ \pm} \pi^{\mp}$, initialement proposé comme candidat à une analyse de diagramme de Dalitz similaire à celle de $B^{0} \rightarrow \rho^{ \pm} \pi^{\mp}$ pour la mesure de l'angle $\alpha$. Je montre que pour des raisons cinématiques, cette analyse n'est en fait pas réalisable, mais des études phénoménologiques supplémentaires concernant la faisabilité d'une analyse $C P$ en deux corps mènent à d'intéressants résultats décrits dans l'annexe $\mathrm{A}$.

Enfin, la troisième partie de ce document expose les contraintes apportées sur la matrice CKM par les mesures réalisées dans cette thèse, ainsi que par d'autres mesures dans le système des mésons $B$ et $K$. Ces interprétations se font essentiellement à l'aide d'un logiciel dénommé CKMfitter (développé en dehors de la collaboration BABAR, au cours de cette thèse) permettant de réaliser une analyse globale de la matrice CKM dans le cadre de diverses approches statistiques, dont celle que nous utilisons principalement: l'approche fréquentiste. 
Après avoir présenté, dans les chapitres 7 et 8 , l'analyse standard de la matrice CKM ( $c$-à$d$ utilisant des observables dont l'interprétation au sein du Modèle Standard ne souffre pas d'erreurs théoriques très controversées), nous passons à l'interprétation des résultats obtenus par l'analyse des modes $B^{0} \rightarrow \rho \pi$ exposés dans cette thèse en terme de contraintes sur l'angle $\alpha$ dans le cadre des symétries de saveur $S U(2)$ et $S U(3)$ (chapitre 9). 


\section{Partie I}

\section{Eléments de théorie}





\section{Chapitre 1}

\section{Introduction à la violation de $C P$}

\subsection{Le Modèle Standard et ses limites}

La théorie de jauge basée sur le groupe de symétrie $S U(3)_{\text {couleur }} \otimes S U(2)_{\text {gauche }} \otimes U(1)_{\text {hypercharge }}$ est capable d'expliquer l'ensemble des observations en physique des particules faites à ce jour. En ce sens, elle remplit parfaitement son rôle de théorie (du grec theorein, "observation" [4]) et justifie la présence du mot "Standard" dans le nom qui lui a été attribué, le "Modèle Standard". La présence du mot "Modèle" traduit néanmoins l'idée que les scientifiques s'en font: il ne s'agit que d'une "représentation simplifiée" [4] d'une théorie plus fondamentale. La plupart des efforts de la physique des particules se concentrent donc dans la recherche de failles dans ce Modèle Standard, qui seraient autant d'indices des propriétés de cette théorie plus fondamentale.

Nous possédons déjà, par ailleurs, un bon nombre d'indices: pour n'en citer que quelquesuns, d'un point de vue formel, la gravitation est absente de ce modèle, le secteur scalaire du modèle (qui n'a d'ailleurs pas encore été directement confirmé expérimentalement) souffre du problème dit de naturalité ( $c$ - $\grave{a}-d$, les corrections radiatives à la masse $d u$ Higgs divergent quadratiquement avec l'énergie); d'un point de vue expérimental, le modèle ne peut ni expliquer l'asymétrie baryonique observée dans l'univers [3], ni pourquoi les masses des fermions s'étalent sur plusieurs ordres de grandeurs, etc.

En continuant à explorer le spectre de création de particules au sein de collisionneurs d'énergie de plus en plus élevée, on espère découvrir les preuves directes de physique au delà du Modèle Standard. Une autre manière de procéder est de tenter de détecter indirectement, grâce à des mesures de haute précision, les effets de ces nouvelles particules créées virtuellement. Le secteur de la violation de $C P$ est de ce point de vue attractif: d'une part, le mécanisme de violation de $C P$ au sein du Modèle Standard est entièrement décrit par la matrice Cabibbo-Kobayashi-Maskawa, ou matrice CKM [5, 2], et en particulier par la présence d'une unique phase dans cette matrice. D'autre part, la majorité des extensions du Modèle Standard possèdent de nouvelles sources de violation de $C P$.

\subsection{La matrice CKM et la violation de $C P$}

L'origine de la matrice CKM est intimement liée à la génération de la masse des quarks via la brisure spontanée de la symétrie électrofaible $S U(2)_{\text {gauche }} \otimes U(1)_{\text {hypercharge }}$ en $U(1)_{\text {e.m. par le }}$ mécanisme de Higgs. 


\subsubsection{La brisure spontanée de la symétrie électrofaible et la matrice CKM}

L'introduction "à la main" d'un terme de masse pour les bosons de jauge ( $W \pm$ et $Z^{0}$ ) et les fermions dans le Lagrangien du Modèle Standard brise l'invariance de jauge locale de ce dernier. Pour y remédier, on introduit le doublet de champs scalaires de Higgs:

$$
\phi=\left(\begin{array}{c}
\phi_{1} \\
\phi_{2}
\end{array}\right)
$$

de potentiel $V(\phi)=\lambda / 3\left(\phi^{\dagger} \phi-v^{2} / 2\right)$. La forme de ce potentiel est telle que la valeur moyenne d'énergie du vide du champs de Higgs est non nulle:

$$
\langle\phi\rangle=\frac{1}{\sqrt{2}}\left(\begin{array}{l}
0 \\
v
\end{array}\right) .
$$

Cet état fondamental a perdu la symétrie de jauge $S U(2)_{\text {gauche }} \otimes U(1)_{\text {hypercharge }}$

En récrivant le Lagrangien en fonction de petites excitations autour de l'état fondamental, on obtient des termes de masse pour les bosons de jauge. Les termes de masse des fermions, quant à eux, s'obtiennent en introduisant un couplage de Yukawa entre le champ de Higgs et les champs de quarks:

$$
\mathcal{L}_{\mathrm{Y}}=-Y_{i j}^{u} \bar{Q}_{L}^{i} \phi u_{R}^{j}-Y_{i j}^{d} \bar{Q}_{L}^{i} \phi d_{R}^{j}
$$

où

$$
Q_{L}^{i}=\left(\begin{array}{c}
u_{L}^{i} \\
d_{L}^{i}
\end{array}\right),
$$

est le doublet de quarks "gauches" de la $i$-ème famille, $u(d)_{R}^{j}$ sont les singlets de quarks "droits" de type "up" ("down") de la $j$-ème famille, et $Y_{i j}^{u(d)}$ sont les matrices $3 \times 3$ (dans le cas de 3 générations) complexes de couplages Yukawa pour les quarks de type "up" ("down").

Après développement autour de l'état fondamental, le Lagrangien du couplage de Yukawa Higgs-quarks s'écrit alors:

$$
\mathcal{L}_{\mathrm{Y}}=-M_{i j}^{u} \bar{Q}_{L}^{i} u_{R}^{j}-M_{i j}^{d} \bar{Q}_{L}^{i} d_{R}^{j},
$$

où $M_{i j}^{u(d)}=Y_{i j}^{u(d)} v / \sqrt{2}$ sont les matrices de masse des quarks de type "up" ("down").

Les champs de quarks présents dans le Lagrangien sont états propres de l'interaction électrofaible, mais ne sont pas états propres de masse (qui sont les états physiques se propageant dans l'espace-temps). On peut obtenir les matrices de masse dans la base propre de masse par la transformation utilisant les quatre matrices complexes et unitaires $V_{L, R}^{u, d}$ :

$$
M^{u, \operatorname{diag}}=V_{L}^{u} M^{u} V_{R}^{u} \text {, et } M^{d, \operatorname{diag}}=V_{L}^{d} M^{d} V_{R}^{d},
$$

où les matrices de masse $M^{u(d) \text {,diag }}$ sont désormais réelles (et diagonales).

Dans la base propre de masse, le Lagrangien d'interaction des quarks avec les courants chargés $W^{ \pm}$s'écrit alors:

$$
\mathcal{L}_{\text {c.c. }}=\frac{g}{\sqrt{2}} \bar{u}_{L}^{i} V_{L, i}^{u} \gamma^{\mu} V_{L, j}^{d, \dagger} d_{L}^{j} W_{\mu}+\text { conjugé hermitique. }
$$

On définit la matrice CKM par $V_{\mathrm{CKM}}=V_{L}^{u} V_{L}^{d \dagger}$. Les couplages des courants chargés $W$ sont ainsi proportionnels aux éléments de la matrice CKM:

$$
V_{\mathrm{CKM}}=\left(\begin{array}{ccc}
V_{u d} & V_{u s} & V_{u b} \\
V_{c d} & V_{c s} & V_{c b} \\
V_{t d} & V_{t s} & V_{t b}
\end{array}\right) .
$$




\subsubsection{Conditions menant à la violation de $C P$}

Dénombrons le nombre de paramètres indépendants dans la matrice CKM dans le cas général de $n$ générations de quarks: la condition d'unitarité entraîne les $n^{2}$ relations suivantes entre les éléments de la matrice:

$$
\sum_{j=1}^{n} V_{i j} V_{k j}^{*}=\delta_{i k} .
$$

Ces relations s'obtiennent en multipliant les éléments des colonnes (resp. des lignes) prises 2 à 2.

Une matrice complexe $n \times n$ unitaire possède ainsi $2 n^{2}-n^{2}$ (relations d'unitarité) $=n^{2}$ paramètres réels indépendants. Chacune des phases des $2 n$ champs de quarks peut être modifiée:

$$
\begin{aligned}
& u_{\alpha}=e^{i \psi_{\alpha}} u_{\alpha}^{\prime}, \\
& d_{i}=e^{i \psi_{i}} d_{i}^{\prime},
\end{aligned}
$$

ce qui entraîne la transformation suivante de $V=V_{\mathrm{CKM}}$ :

$$
V_{\alpha i}^{\prime}=e^{i\left(\psi_{\alpha}-\psi_{i}\right)} V_{\alpha i}
$$

Seules les différences de phases sont des paramètres physiques et on peut donc soustraire $2 n-1$ phases non physiques. Parmi les $n^{2}-(2 n-1)=(n-1)^{2}$ paramètres restants, $\frac{1}{2} n(n-1)$ sont des angles d'Euler et $\frac{1}{2}(n-1)(n-2)$ sont des phases.

Ainsi, avec deux familles, la matrice est réelle et paramétrée par un seul angle de rotation: l'angle de Cabibbo $\theta_{C}$ [5], introduit en 1963 par Cabibbo pour expliquer les désintégrations des particules étranges. L'idée de Kobayashi et Maskawa [2] fut de remarquer, en 1973 ( $c$-à- $d$, avant la découverte de la troisième génération de quarks), qu'avec une troisième famille, la matrice devient complexe et est paramétrée par trois angles d'Euler et une unique phase permettant d'introduire la violation de la symétrie $C P^{1}$ : on réfère souvent à cette idée par "le mécanisme KM", devenant un "paradigme" dans le Modèle Standard où seule la phase CKM génère la violation de $C P$.

Cette unique phase est irréductible à la condition que les masses des quarks de même charge soient non dégénérées. Le cas échéant, une nouvelle symétrie apparaît dans le Lagrangien (les rotations unitaires dans l'espace des deux quarks de masse dégénérée) supprimant l'unique phase présente.

Les conditions pour avoir violation de $C P$ sont résumées par Jarlskog [6] dans l'expression suivante:

$$
\operatorname{det}\left[\mathrm{M}^{\mathrm{u}}, \mathrm{M}^{\mathrm{d}}\right]=-2 F F^{\prime} J \neq 0 \Rightarrow C P \text { est violée, }
$$

\footnotetext{
${ }^{1} C^{\prime}$ est en effet la présence d'un couplage complexe dans le Lagrangien qui permet de générer la violation de $C P$ : en effet, la symétrie $C P$ transforme un opérateur en son conjugué hermitique adjoint:

$$
\mathcal{O}(\vec{x}, t) \stackrel{\mathrm{CP}}{\longrightarrow} \mathcal{O}^{\dagger}(-\vec{x}, t) .
$$

Ainsi, si l'on schématise le Lagrangien par la forme $\mathcal{L}=a \mathcal{O}+a^{*} \mathcal{O}^{\dagger}$ (où $a$ est une constante de couplage), il est clair que si $a=a^{*}$, le Lagrangien est invariant sous $C P$, et réciproquement que la présence de couplages complexes entraîne une violation de la symétrie $C P$.
} 
avec

$$
\begin{aligned}
& F=\left(m_{t}-m_{c}\right)\left(m_{t}-m_{u}\right)\left(m_{c}-m_{u}\right) / m_{t}^{3} \\
& F^{\prime}=\left(m_{b}-m_{s}\right)\left(m_{b}-m_{d}\right)\left(m_{s}-m_{d}\right) / m_{b}^{3} \\
& \operatorname{Im}\left[V_{\alpha j} V_{\beta k} V_{\alpha k} V_{\beta j}\right]=J \sum_{\gamma, l} \epsilon_{\alpha \beta \gamma} \epsilon_{j k l},
\end{aligned}
$$

où $F$ et $F^{\prime}$ sont reliés à la condition de non dégénérescence des masses des quarks, et $J$ à la condition de complexité de la matrice CKM. Le paramètre $J$ est appelé paramètre de Jarlskog [6]. Il est invariant de phase, $c-\grave{a}-d$ sous la transformation 1.12. Nous reviendrons sur l'interprétation géométrique de ce paramètre dans la section 1.2.4.

\subsubsection{Paramétrisation de Wolfenstein de la matrice CKM}

On observe que les éléments de la matrice CKM sont hiérarchisés: les transitions électrofaibles entre les différentes familles de quarks sont de plus en plus petites lorsque les familles sont éloignées: on a donc une brisure de la symétrie de saveur. Wolfenstein [7] a proposé une paramétrisation de la matrice tenant compte de cette hiérarchie en développant les éléments en puissance du sinus de l'angle de Cabibbo, $\lambda=\sin \theta_{C} \simeq 0.22$. En négligeant les termes d'ordre supérieur à $\lambda^{5}[8]$, on obtient:

$$
V_{\mathrm{CKM}} \simeq\left(\begin{array}{ccc}
1-\frac{1}{2} \lambda^{2}-\frac{1}{8} \lambda^{4} & \lambda & A \lambda^{3}(\rho-i \eta) \\
-\lambda\left[1+A^{2} \lambda^{4}\left(\rho+i \eta-\frac{1}{2}\right)\right] & 1-\frac{1}{2} \lambda^{2}-\frac{1}{8} \lambda^{4}\left(1+4 A^{2}\right) & A \lambda^{2} \\
A \lambda^{3}\left[1-(\rho+i \eta)\left(1-\frac{1}{2} \lambda^{2}\right)\right] & -A \lambda^{2}\left[1+\lambda^{2}\left(\rho+i \eta-\frac{1}{2}\right)\right] & 1-\frac{1}{2} A^{2} \lambda^{4}
\end{array}\right) \text { (1.16) }
$$

Il est intéressant de noter que les quatre paramètres $A, \lambda, \rho$ et $\eta$ (ou plus généralement, les éléments de la matrice CKM) ne sont pas prédits par le Modèle Standard. Ils sont contraints par des mesures dont les prédictions théoriques sont reliées aux éléments de la matrice CKM. A ce jour, $\lambda$ et $A$ sont ainsi respectivement connus avec une précision de $1 \%$ et $2 \%$ environ, alors que $\rho$ et $\eta$ sont les paramètres les moins connus.

Dans la paramétrisation de Wolfenstein, le paramètre de Jarlskog $J$ est égal à:

$$
J=A^{2} \lambda^{6} \eta \simeq 10^{-5}
$$

et la condition de violation de $C P J \neq 0$ se traduit par $\eta \neq 0$.

\subsubsection{Le triangle d'unitarité}

On peut représenter les six relations 1.9 pour lesquelles $i \neq k$ par des triangles dans le plan complexe. A cause de la hiérarchie des éléments de la matrice CKM, quatre de ces triangles sont quasiment plats et possèdent un angle difficilement mesurable. Plus intéressants expérimentalement sont les deux triangles restants, dont les trois cotés sont d'ordre $\lambda^{3}$ :

$$
\begin{aligned}
& V_{u d} V_{u b}^{*}+V_{t d} V_{t b}^{*}+V_{c d} V_{c b}^{*}=0 \\
& V_{u b} V_{t b}^{*}+V_{u d} V_{t d}^{*}+V_{u s} V_{t s}^{*}=0 .
\end{aligned}
$$

Ces deux triangles sont quasiment identiques, puisque les trois termes possèdent la même phase et le même module, à l'ordre $\lambda^{2}$ près. Les désintégrations des mésons $B_{d}$ permettent de contraindre les cotés et angles du premier de ces deux triangles, que l'on appellera par la suite 
LE Triangle d'Unitarité. Le schéma du haut de la Fig. 1.1 montre ce Triangle d'Unitarité, dont les trois angles sont dénommés $\alpha, \beta$ et $\gamma^{2}$, et valent:

$$
\alpha=\arg \left[-\frac{V_{t d} V_{t b}^{*}}{V_{u d} V_{u b}^{*}}\right], \beta=\arg \left[-\frac{V_{c d} V_{c b}^{*}}{V_{t d} V_{t b}^{*}}\right], \quad \gamma=\arg \left[-\frac{V_{u d} V_{u b}^{*}}{V_{c d} V_{c b}^{*}}\right] .
$$

On normalise l'expression 1.18 par $V_{c d} V_{c b}^{*}$ pour obtenir le schéma du bas de la même figure. Dans ce cas, le sommet du triangle a pour coordonnées $(\rho, \eta)^{3}$.

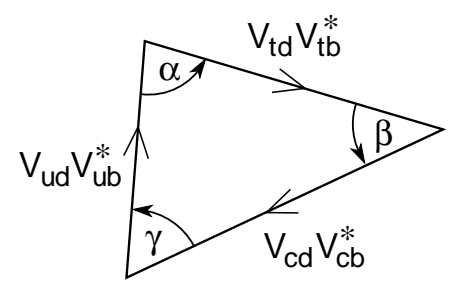

(a)

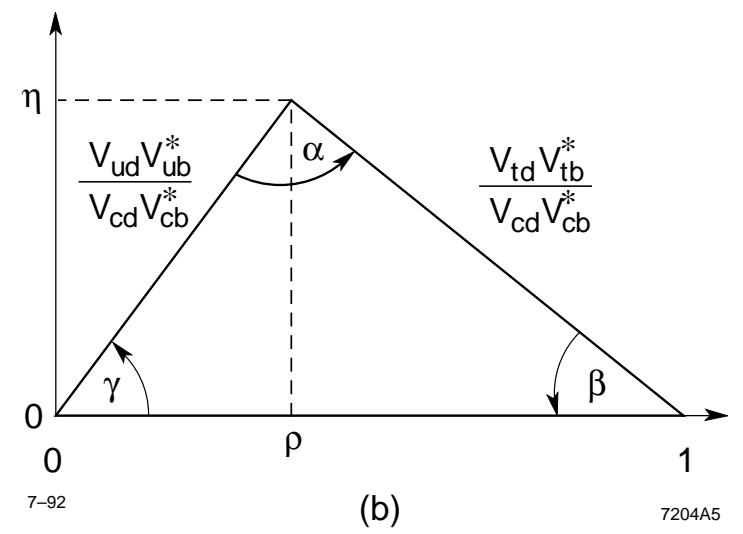

Figure 1.1: (a): représentation dans le plan complexe de la relation d'unitarité reliant la première et dernière colonnes de la matrice CKM. (b): une fois le triangle en (a) normalisé par $V_{c d} V_{c b}^{*}$, on obtient Le Triangle d'Unitarité de base 1 et de sommet $(\rho, \eta)$.

Les aires de tous les triangles d'unitarité sont égales à $J / 2$ : ceci est lié à la présence d'une unique phase dans le cas de 3 générations de quark. L'invariance de phase de $J$ peut ainsi s'exprimer de façon géométrique: un rephasage des champs de quarks (comme dans l'éq. 1.11) se traduit par une rotation du triangle d'unitarité dans le plan complexe, ce qui laisse l'aire $(J)$ du triangle invariante. Cette transformation laisse aussi les cotés du triangle invariants: ainsi, les modules des éléments de matrice $\left|V_{i j}\right|^{2}$ sont aussi invariants de phase.

\subsubsection{Ordres de grandeur de la violation de $C P$}

Le paramètre $J$ donne la taille absolue de la violation de $C P$ dans le Modèle Standard. On mesure sa valeur à environ $10^{-5}$ (Eq. 1.17), ce qui est petit par rapport à sa valeur maximale possible $\left(J_{\max }=1 /(6 \sqrt{3}) \simeq 0.1\right)$. Ainsi, la valeur absolue de la violation de $C P$ dans le Modèle Standard est petite.

\footnotetext{
${ }^{2}$ Pour des raisons historiques, ces angles sont dénommés $\phi_{1}=\beta, \phi_{2}=\alpha$ et $\phi_{3}=\gamma$ dans la communauté japonaise.

${ }^{3}$ Le remplacement $\rho \rightarrow \bar{\rho}=\rho\left(1-\lambda^{2} / 2\right)$ et $\eta \rightarrow \bar{\eta}=\eta\left(1-\lambda^{2} / 2\right)$ améliore la précision des coordonnées du sommet du triangle.
} 
Néanmoins les effets engendrés par la violation de $C P$ peuvent être importants: pour avoir une idée de la taille de ces effets, il convient de comparer $J$ à la largeur de désintégration du système considéré. Ainsi, plus les désintégrations considérées sont supprimées, plus l'effet de la violation de $C P$ est relativement important. La violation de $C P$ est en effet intimement liée à la brisure de la symétrie de saveur. Le tableau 1.1 (issu de la ref. [9]) donne la taille de l'effet relatif de la violation de $C P$ pour les systèmes de mésons $K, D$ et $B$ obtenue en divisant le paramètre de Jarlskog par la largeur de désintégration.

\begin{tabular}{cccc}
\hline $\begin{array}{c}\text { Système } \\
\text { considéré }\end{array}$ & $\begin{array}{c}\text { Contribution dominante } \\
\text { au niveau des quarks }\end{array}$ & $\begin{array}{c}\text { Largeur de } \\
\text { désintégration }\end{array}$ & $\begin{array}{c}\text { Effet de violation } \\
\text { de } C P\end{array}$ \\
\hline$K$ & $s \longrightarrow u$ & $\propto \lambda^{2} \times$ espace de phase & $\propto A^{2} \lambda^{4} \eta$ \\
$D$ & $c \longrightarrow s$ & $\propto 1 \times$ espace de phase & $\propto A^{2} \lambda^{6} \eta$ \\
$B$ & $b \longrightarrow c$ & $\propto A^{2} \lambda^{4} \times$ espace de phase & $\propto \lambda^{2} \eta$ \\
\hline
\end{tabular}

Tableau 1.1: Importance relative des effets de violation de CP dans les systèmes des mésons $K, D$ et $B$ obtenue en divisant le paramètre de Jarlskog par la largeur de la désintégration dominante mise en jeu. [9]

C'est dans le système des mésons $B$ que l'on attend les asymétries $C P$ les plus importantes. Le système des mésons $B$ présente en outre un intérêt théorique dû à la présence du quark $b$ de grande masse, propriété simplifiant les calculs de QCD nécessaires à l'interprétation de certaines mesures au sein du Modèle Standard. A l'opposé, les systèmes des mésons $K$ et $D$ souffrent de grandes incertitudes théoriques.

La section suivante décrit plus en détail le système des mésons $B$.

\subsection{Le système des mésons $B$}

\subsubsection{Terminologie}

Sont regroupés sous le terme de mésons $B$ les mésons possédant un quark $b$. L'autre quark peut être un quark léger de première génération $(u, d)$ ou un quark plus lourd de la seconde génération $(s, c)$. Le tableau 1.2 donne les quatre types de mésons $B$ existant, ainsi que leur masse et durée de vie.

\begin{tabular}{|c|cc|}
\hline Méson & Masse $\left(\mathrm{MeV} / \mathrm{c}^{2}\right)$ & Durée de vie $(\mathrm{ps})$ \\
\hline$B_{d}^{0} \equiv b d$ & $5279.4 \pm 0.5$ & $1.542 \pm 0.016$ \\
$B_{u}^{+} \equiv \bar{b} u$ & $5279.0 \pm 0.5$ & $1.674 \pm 0.018$ \\
\hline$B_{s}^{0} \equiv b s$ & $5369.6 \pm 2.4$ & $1.461 \pm 0.057$ \\
$B_{c}^{+} \equiv \bar{b} c$ & $6400 \pm 400$ & $0.46 \pm 0.18$ \\
\hline
\end{tabular}

Tableau 1.2: Les différents mésons $B$, leur masse et leur durée de vie.

A $B A B A R$, seuls les $B_{d}^{0}$ et $B_{u}^{+}$sont produits, et nous les dénotons $B^{0}$ et $B^{+}$. On utilise par la suite trois bases différentes pour décrire les mésons $B^{0}$ :

- La base de saveur, d'états propres $\left|B^{0}\right\rangle$ et $\left|\bar{B}^{0}\right\rangle$, correspondant aux états avec une structure de quarks bien définie. 
- La base de $C P$, d'états propres $\left|B_{\mathrm{CP}=+1}\right\rangle$ et $\left|B_{\mathrm{CP}=-1}\right\rangle$ vérifiant:

$$
\begin{aligned}
& C P\left|B_{\mathrm{CP}=+1}\right\rangle=+\left|B_{\mathrm{CP}=+1}\right\rangle, \\
& C P\left|B_{\mathrm{CP}=-1}\right\rangle=-\left|B_{\mathrm{CP}=-1}\right\rangle .
\end{aligned}
$$

- La base de masse, d'états propres $\left|B_{L}\right\rangle$ et $\left|B_{H}\right\rangle$ (états propres du Lagrangien total), correspondant à des états ayant une masse $M$ et une durée de vie $\Gamma$ définies.

Pour les $B$ neutres, le fait que ces trois bases soient différentes est à l'origine de la riche phénoménologie des oscillations de $B$ exploitée à $B A B A R$.

\subsubsection{Les oscillations de $B^{0}$}

Les mésons $B$ "physiques" se propageant dans l'espace et dans le temps (c-à-d, les états propres de masse) sont une superposition linéaire des états propres de saveur $B^{0}=\bar{b} d$ et $\bar{B}^{0}=b \bar{d}$. Appelant $B^{0}(t)\left(\right.$ resp. $\left.\bar{B}^{0}(t)\right)$ un méson de saveur $B^{0}\left(\right.$ resp. $\left.\bar{B}^{0}\right)$ à $t=0$, l'évolution temporelle de ces états est gouvernée par l'équation de Schrödinger:

$$
i \frac{d}{d t}\left(\begin{array}{l}
\left|B^{0}(t)\right\rangle \\
\left|\bar{B}^{0}(t)\right\rangle
\end{array}\right)=\left(\begin{array}{c}
M-i \frac{\Gamma}{2}
\end{array}\right)\left(\begin{array}{l}
\left|B^{0}(t)\right\rangle \\
\left|\bar{B}^{0}(t)\right\rangle
\end{array}\right),
$$

où $M$ est la matrice de masse (partie dispersive correspondant à la présence d'états intermédiaires virtuels dans l'oscillation $B^{0}-\bar{B}^{0}$ ) et $\Gamma$ la matrice de désintégration (partie absorptive mettant en jeu des états intermédiaires réels). Les états propres de masse sont les vecteurs propres de $M-i \Gamma / 2$. Les états propres de masse plus léger $B_{L}$ et plus lourd $B_{H}$ ( $L, H$ comme "light" et "heavy") s'expriment en fonction des états propres de saveur au temps $t=0$ comme:

$$
\begin{aligned}
& \left|B_{L}\right\rangle=p\left|B^{0}\right\rangle+q\left|\bar{B}^{0}\right\rangle, \\
& \left|B_{H}\right\rangle=p\left|B^{0}\right\rangle-q\left|\bar{B}^{0}\right\rangle,
\end{aligned}
$$

où $p^{2}+q^{2}=1$. L'évolution temporelle de $B_{H, L}$ est gouvernée par les valeurs propres $M_{H}-i \Gamma_{H}$ et $M_{L}-i \Gamma_{L}$ :

$$
\left|B_{H, L}(t)\right\rangle=e^{-\left(i M_{H, L}+\Gamma_{H, L} / 2\right) t}\left|B_{H, L}\right\rangle .
$$

Dans la suite, on utilise les conventions de masse et durée de vie suivantes:

$$
\begin{aligned}
m_{B} & =\frac{M_{H}+M_{L}}{2}, & \Gamma_{B} & =\frac{\Gamma_{H}+\Gamma_{L}}{2}, \\
\Delta m_{B} & =M_{H}-M_{L}, & \Delta \Gamma_{B} & =\Gamma_{H}-\Gamma_{L} .
\end{aligned}
$$

$\Delta m_{B}$ est défini positif. En inversant l'éq. 1.24, en utilisant la dépendance en temps de $B^{0}$ et $\bar{B}^{0}$ décrite en 1.25 , puis enfin en exprimant les états propres de masse en terme des états propres de saveur grâce à l'éq. 1.24, on obtient l'évolution temporelle des états $\left|B^{0}(t)\right\rangle$ et $\left|\bar{B}^{0}(t)\right\rangle$ :

$$
\begin{aligned}
& \left|B^{0}(t)\right\rangle=g_{+}(t)\left|B^{0}\right\rangle \quad+\frac{q}{p} g_{-}(t)\left|\bar{B}^{0}\right\rangle, \\
& \left|\bar{B}^{0}(t)\right\rangle=\frac{p}{q} g_{-}(t)\left|B^{0}\right\rangle+g_{+}(t)\left|\bar{B}^{0}\right\rangle,
\end{aligned}
$$


où

$$
\begin{aligned}
& g_{+}(t)=e^{-i m t} e^{-\Gamma_{B} t / 2}\left[\cosh \frac{\Delta \Gamma_{B}}{4} \cos \frac{\Delta m_{B} t}{2}-i \sinh \frac{\Delta \Gamma_{B}}{4} \sin \frac{\Delta m_{B} t}{2}\right], \\
& g_{-}(t)=e^{-i m t} e^{-\Gamma_{B} t / 2}\left[-\sinh \frac{\Delta \Gamma_{B}}{4} \cos \frac{\Delta m_{B} t}{2}+i \cosh \frac{\Delta \Gamma_{B}}{4} \sin \frac{\Delta m_{B} t}{2}\right] .
\end{aligned}
$$

Les propriétés de l'oscillation décrite par l'éq. 1.28 dépendent des valeurs de $\Delta m_{B}$ et $\Delta \Gamma_{B}$ qui diffèrent selon le système de mésons considéré. Rappelons que $\Delta \Gamma_{B}$ dépend des états intermédiaires réels accessibles aux deux saveurs de mésons: dans le cas des mésons $B$, il s'agit de transitions $b \rightarrow c \bar{c} d$ ou $b \rightarrow u \bar{u} d$ qui sont supprimées de Cabibbo, ce qui n'est pas le cas dans les systèmes des $B_{s}, K$ et $D$. La valeur de $\Delta m_{B}$ dépend de la présence éventuelle de transitions supprimées de Cabibbo ou supprimées par le mécanisme de GIM [10] (à cause de la valeur des masses des quarks): c'est ce qui se produit dans les systèmes des $K$ où l'on a donc $\Delta m_{K} \ll \Delta \Gamma_{K}$. Dans le système des $B$, par contre, $\Delta m_{B}$ ne souffre d'aucune de ces suppressions, et l'on a donc $\Delta m_{B} \gg \Delta \Gamma_{B}$, ainsi que $\Delta m_{B_{s}} \gg \Delta \Gamma_{B_{s}}$. Enfin, dans le système des $D$, on s'attend à $\Delta m_{D} \simeq \Delta \Gamma_{D}$.

On néglige donc $\Delta \Gamma_{B}$ dans le système des mésons $B$ ce qui simplifie l'éq. 1.27:

$$
\begin{aligned}
& \left|B^{0}(t)\right\rangle=e^{-i m t} e^{-\Gamma_{B} t / 2}\left[\cos \frac{\Delta m_{B} t}{2}\left|B^{0}\right\rangle+i \frac{q}{p} \sin \frac{\Delta m_{B} t}{2}\left|\bar{B}^{0}\right\rangle\right], \\
& \left|\bar{B}^{0}(t)\right\rangle=e^{-i m t} e^{-\Gamma_{B} t / 2}\left[\cos \frac{\Delta m_{B} t}{2}\left|\bar{B}^{0}\right\rangle+i \frac{p}{q} \sin \frac{\Delta m_{B} t}{2}\left|B^{0}\right\rangle\right] .
\end{aligned}
$$

\subsubsection{Production d'une paire $B^{0} \bar{B}^{0}$ cohérente à $B A B A R$}

A $B A B A R$, les mésons $B(J=0)$ sont produits en paire à partir de la résonance $\Upsilon(4 S)$ $(J=1)$ : cette résonance est un état lié $b \bar{b}$ de masse $10.58 \mathrm{Gev} / c^{2}$ située juste au delà du seuil de production d'une paire de mésons $B$. La figure 1.2 montre la production d'une paire $B^{0} \bar{B}^{0}$ ou $B^{+} B^{-}$par le $\Upsilon(4 S)$.

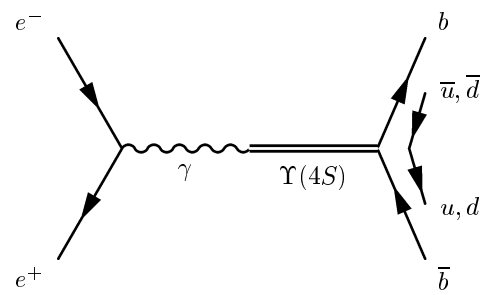

Figure 1.2: Diagramme de production d'une paire $B^{0} \bar{B}^{0}$ ou $B^{+} B^{-}$par un $\Upsilon(4 S)$.

La fonction d'onde totale $|\psi\rangle$ de la paire $B^{0} \bar{B}^{0}$ est le produit de la fonction d'onde spatiale $\left|\psi_{\text {spatiale }}\right\rangle$ et de la fonction d'onde de saveur $\left|\psi_{\text {saveur }}\right\rangle$. Selon la statistique de Bose-Einstein, $|\psi\rangle$ doit être à chaque instant symétrique sous l'échange des deux mésons $B$. La paire de mésons $B$ est produite dans un moment angulaire $L=1$, ce qui implique que $\left|\psi_{\text {spatiale }}\right\rangle$ soit anti-symétrique. Il faut donc que $\left|\psi_{\text {saveur }}\right\rangle$ soit aussi anti-symétrique: en conséquence, à chaque instant, il y a toujours un méson de saveur $B^{0}$ et l'autre de saveur $\bar{B}^{0}$. Il s'agit d'un exemple de cohérence quantique menant au paradoxe d'Einstein-Polosky-Rosen [11].

Chaque $B$ évolue selon l'équation 1.29, mais en phase avec l'autre $B$. Une fois qu'un des deux mésons $B$ se désintègre ${ }^{4}$, l'autre méson $B$ continue à évoluer selon la même équation 1.29. On peut ainsi, connaissant la saveur d'un des deux $B$ relier cette dernière à la

\footnotetext{
${ }^{4}$ La cohérence quantique du système est alors bien entendue détruite.
} 
saveur de l'autre $B$ grâce au temps $\Delta t$ écoulé entre les deux désintégrations. La figure 1.3 montre la production de la paire de $B$ par le $\Upsilon(4 S)$. Pour rendre $\Delta t$ mesurable, la paire est boostée (le faisceau des $e^{-}$est plus énergétique que celui des $e^{+}$) selon l'axe des faisceaux.

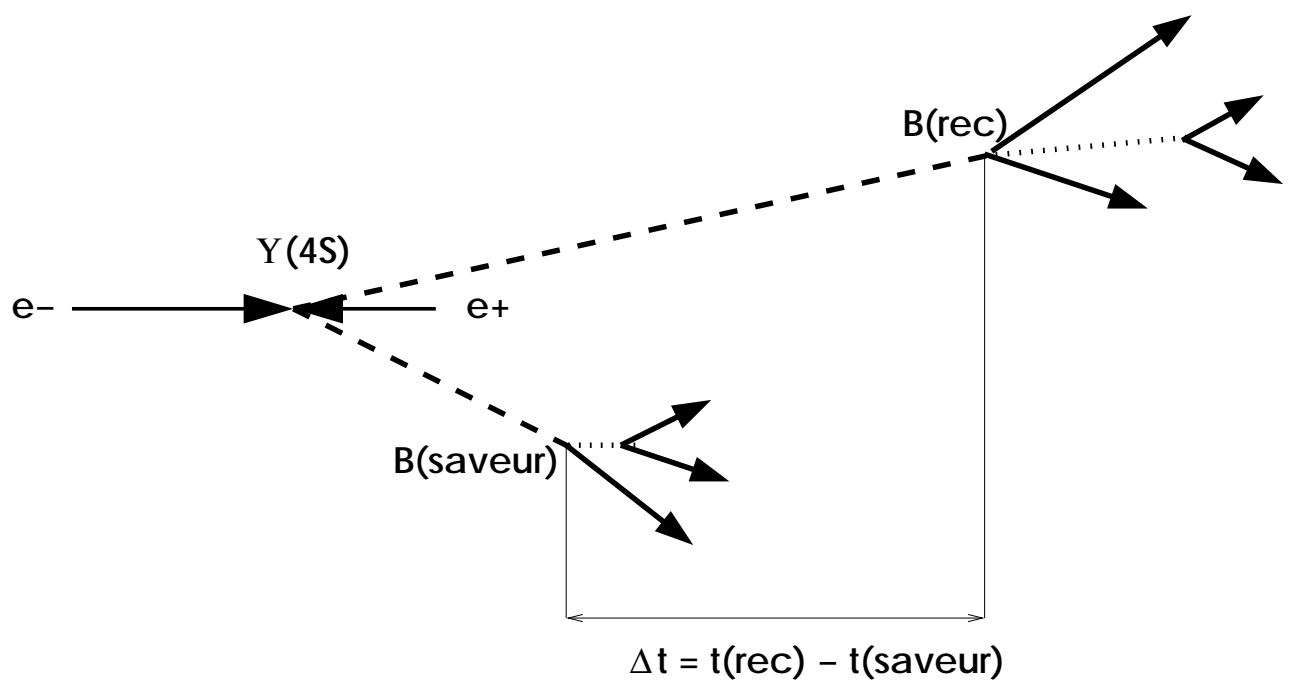

Figure 1.3: Schéma de la production d'une paire $B^{0} \bar{B}^{0}$ ou $B^{+} B^{-}$par un $\Upsilon(4 S)$.

Le méson $B_{\text {rec }}$ est un $B$ exclusivement reconstruit et $B_{\text {sav }}$ est un $B$ état propre de saveur dont les produits de désintégrations permettent de reconstituer la saveur.

Deux catégories d'événements $B_{\text {rec }}$ sont étudiés à BABAR:

- le méson $B_{\text {rec }}$ est un état propre de saveur: on connaît ainsi la saveur des deux $B$, et on peut étudier les propriétés des oscillations des $B$ (en particulier, la fréquence d'oscillation donnée par $\Delta m_{d}$ ).

- le méson $B_{\text {rec }}$ n'est pas un état propre de saveur et peut être produit à la fois par un $B^{0}$ et un $\bar{B}^{0}$. L'état final peut être un état propre de $C P$ ou pas. la saveur de $B_{\text {rec }}$ est déduite à partir de celle de $B_{\text {sav }}$ et $\Delta t$. Avec cet échantillon, on peut étudier la violation de $C P$.

Nous détaillons dans la suite la distribution en temps de ces deux échantillons.

\subsubsection{Désintégration d'un méson $B$ en un état propre de saveur}

Ce type d'événement est divisé en deux catégories:

- Evénements mélangés: $B_{\text {rec }}$ et $B_{\text {sav }}$ ont la même saveur $\left(B^{0} B^{0}\right.$ ou $\left.\bar{B}^{0} \bar{B}^{0}\right)$.

- Evénements non mélangés: $B_{\text {rec }}$ et $B_{\text {sav }}$ ont une saveur opposée $\left(B^{0} \bar{B}^{0}\right)$.

Les taux de désintégrations de ces deux types d'événements en fonction du temps sont donnés par:

$$
\begin{aligned}
f_{\text {non mélangé }}(\Delta t) & \propto e^{-\Delta t / \tau_{B}}\left(1+\cos \Delta m_{B} \Delta t\right), \\
f_{\text {mélangé }}(\Delta t) & \propto e^{-\Delta t / \tau_{B}}\left(1-\cos \Delta m_{B} \Delta t\right),
\end{aligned}
$$

où $\tau_{B}=1 / \Gamma_{B}$. Ainsi, à $\Delta t=0$, il n'y a aucun événement mélangé. La figure 1.4 montre les distributions données dans l'éq. 1.30.

Un exemple d'une telle désintégration est $B^{0} \rightarrow \rho^{-} K^{+}$dont l'analyse est décrite dans le chapitre 4. 


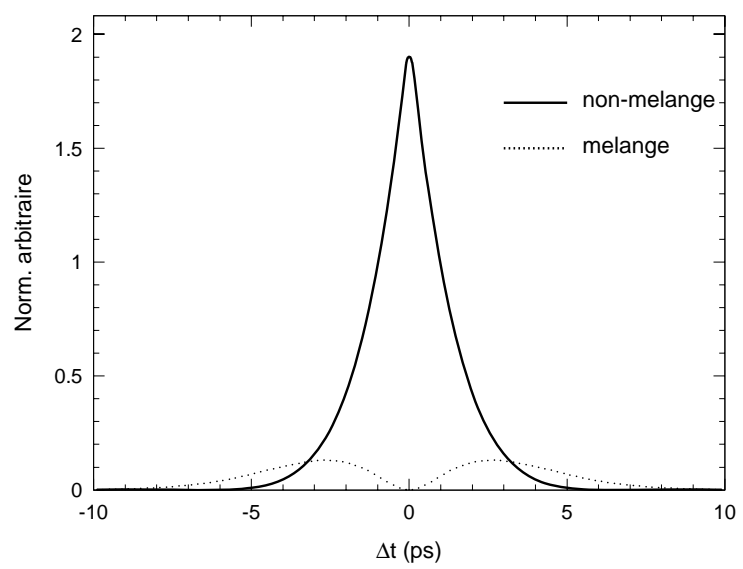

Figure 1.4: Distribution des taux dépendant du temps des événements états propres de saveur mélangés et non mélangés.

\subsubsection{Désintégration d'un méson $B$ en un état propre de $C P$}

Nous nous plaçons dans le cas où l'un des deux $B$ se désintègre dans un état propre de $C P\left(\mathrm{f}_{\mathrm{CP}}\right)$ au temps $t_{1}$, et où l'autre méson $B_{\mathrm{sav}}$ se désintègre en un état propre de saveur au temps $t_{2}$. Nous définissons la valeur propre $\eta_{f}$ de $C P$ de l'état final $\mathrm{f}_{\mathrm{CP}}$ par $C P\left|\mathrm{f}_{\mathrm{CP}}\right\rangle=\eta_{\mathrm{f}}\left|\mathrm{f}_{\mathrm{CP}}\right\rangle$, et dénotons les amplitudes de désintégration du $B^{0}$ et $\bar{B}^{0}$ en $\mathrm{f}_{\mathrm{CP}}$ par:

$$
\begin{aligned}
& A_{f}=\left\langle\mathrm{f}_{\mathrm{CP}}|\mathcal{H}| \mathrm{B}^{0}\right\rangle \\
& \bar{A}_{f}=\left\langle\mathrm{f}_{\mathrm{CP}}|\mathcal{H}| \overline{\mathrm{B}}^{0}\right\rangle
\end{aligned}
$$

où $\mathcal{H}$ est l'Hamiltonien de la désintégration $B \rightarrow \mathrm{f}_{\mathrm{CP}}$. Si au temps $t_{2}$, le $B_{\text {sav }}$ est un $\bar{B}^{0}$, alors au même moment $B_{\text {rec }}$ est un $B^{0}$, et par la suite, le taux de désintégration $\Gamma\left(B^{0} \rightarrow \mathrm{f}_{\mathrm{CP}}\right)(\Delta t)=\left|\left\langle\mathrm{f}_{\mathrm{CP}}|\mathcal{H}| \mathrm{B}^{0}(\Delta t)\right\rangle\right|^{2}$ d'avoir un $B^{0}$ se désintégrant en $\mathrm{f}_{\mathrm{CP}}$ en fonction de $\Delta t=t_{2}-t_{1}$ est obtenue à partir de l'éq. 1.29 (nous abandonnons la constante de normalisation et le terme exponentiel momentanément):

$$
\begin{aligned}
\left|\left\langle\mathrm{f}_{\mathrm{CP}}|\mathcal{H}| \mathrm{B}^{0}(\Delta \mathrm{t})\right\rangle\right|^{2} & \propto\left|A_{f} \cos \frac{\Delta m_{B} \Delta t}{2}+i \frac{q}{p} \bar{A}_{f} \sin \frac{\Delta m_{B} \Delta t}{2}\right|^{2}, \\
& \propto\left|A_{f}\right|^{2}\left|\cos \frac{\Delta m_{B} \Delta t}{2}+i \frac{q}{p} \frac{\bar{A}_{f}}{A_{f}} \sin \frac{\Delta m_{B} \Delta t}{2}\right|^{2}, \\
& \propto\left|A_{f}\right|^{2}\left[\frac{1+|\lambda|^{2}}{2}+\frac{1-|\lambda|^{2}}{2} \cos \left(\Delta m_{B} \Delta t\right)-\operatorname{Im} \lambda \sin \left(\Delta m_{B} \Delta t\right)\right],
\end{aligned}
$$

où

$$
\lambda=\frac{q}{p} \frac{\bar{A}_{\bar{f}}}{A_{f}}=\eta_{f} \frac{q}{p} \frac{\bar{A}_{f}}{A_{f}} .
$$

De façon similaire, le taux de désintégration $\Gamma\left(\bar{B}^{0} \rightarrow \mathrm{f}_{\mathrm{CP}}\right)(\Delta t)=\left|\left\langle\mathrm{f}_{\mathrm{CP}}|\mathcal{H}| \overline{\mathrm{B}}^{0}(\Delta t)\right\rangle\right|^{2}$ d'un $\bar{B}^{0}$ en $f_{\mathrm{CP}}$ en fonction de $\Delta t$ s'écrit

$$
\left|\left\langle\mathrm{f}_{\mathrm{CP}}|\mathcal{H}| \overline{\mathrm{B}}^{0}(\Delta \mathrm{t})\right\rangle\right|^{2} \propto\left|A_{f}\right|^{2}\left[\frac{1+|\lambda|^{2}}{2}-\frac{1-|\lambda|^{2}}{2} \cos \left(\Delta m_{B} \Delta t\right)+\operatorname{Im} \lambda \sin \left(\Delta m_{B} \Delta t\right)\right](1 .
$$

Nous pouvons alors calculer l'asymétrie $C P$ entre $B^{0} \rightarrow \mathrm{f}_{\mathrm{CP}}$ et $\bar{B}^{0} \rightarrow \mathrm{f}_{\mathrm{CP}}$ en fonction du temps $\Delta t$ par:

$$
a_{\mathrm{CP}}(\Delta t)=\frac{\Gamma\left(B^{0} \rightarrow \mathrm{f}_{\mathrm{CP}}\right)(\Delta t)-\Gamma\left(\bar{B}^{0} \rightarrow \mathrm{f}_{\mathrm{CP}}\right)(\Delta t)}{\Gamma\left(B^{0} \rightarrow \mathrm{f}_{\mathrm{CP}}\right)(\Delta t)+\Gamma\left(\bar{B}^{0} \rightarrow \mathrm{f}_{\mathrm{CP}}\right)(\Delta t)},
$$




$$
=\frac{\left(1-|\lambda|^{2}\right) \cos \Delta m_{B} \Delta t-2 \operatorname{Im} \lambda \sin \Delta m_{B} \Delta t}{1+|\lambda|^{2}} .
$$

On voit que l'asymétrie $C P$ est non nulle si l'une de ces trois conditions est vérifiée:

- $|\lambda| \neq 1$, c'est-à-dire $|q / p| \neq 1$ ou $\left|\bar{A}_{\bar{f}} / A_{f}\right| \neq 1$.

- $\operatorname{Im} \lambda \neq 0$.

Il est intéressant de noter que la condition $|\lambda| \neq 1$ apparaît devant un cosinus (fonction paire dont l'intégrale est non nulle): on peut donc l'observer en mesurant des taux dépendant du temps, ou non. Par contre, pour observer la dernière condition $\operatorname{Im} \lambda \neq 0$, il faut tenir compte de la dépendance en temps, puisque cette dernière est une fonction sinus (fonction impaire dont l'intégrale est nulle).

Nous décrivons maintenant plus en détails les trois types de violation de $C P$ découlant de ces trois conditions: chacun de ces types est traité dans cette thèse.

\subsection{Les trois types de violation de $C P$}

\subsubsection{La violation de $C P$ dans la désintégration $\left(\left|\bar{A}_{\bar{f}} / A_{f}\right| \neq 1\right)$}

La violation de $C P$ dans la désintégration correspond à la condition $|\bar{A} / A| \neq 1$, c'est-à-dire que l'amplitude d'une désintégration n'est pas égale à celle de sa conjuguée $C P$.

De façon générale, il peut y avoir plusieurs diagrammes contribuant à $B^{0} \rightarrow f$ et $\bar{B}^{0} \rightarrow \bar{f}$. Les amplitudes totales $A_{f}$ et $\bar{A}_{\bar{f}}$ s'écrivent donc:

$$
\begin{aligned}
& A_{f}=\sum_{i} A_{i} e^{i \delta_{i}} e^{i \phi_{i}}, \\
& \bar{A}_{\bar{f}}=\sum_{i} A_{i} e^{i \delta_{i}} e^{-i \phi_{i}},
\end{aligned}
$$

où les amplitudes $A_{i}$ sont réelles, et l'indice $i$ correspond aux différents processus participant à la désintégration. Chaque processus possède deux types distincts de phases: les phases faibles $\phi_{i}$ sont dues à la complexité du couplage électrofaible, et violent donc $C P$. Les phases fortes $\delta_{i}$ sont dues aux interactions dans l'état final, dominées par l'interaction forte, et sont invariantes sous $C P^{5}$. Nous ne savons pas à l'heure actuelle calculer ces phases fortes au sein de QCD de façon indépendante de modèle.

Dans le cas de deux processus contribuant à l'amplitude totale, l'asymétrie entre $\left|A_{f}\right|$ et $\left|\bar{A}_{\bar{f}}\right|$ s'écrit:

$$
\left|A_{f}\right|^{2}-\left|\bar{A}_{\bar{f}}\right|^{2}=-2 \sum_{i, j} A_{i} A_{j} \sin \left(\phi_{i}-\phi_{j}\right) \sin \left(\delta_{i}-\delta_{j}\right) .
$$

Pour avoir une asymétrie non-nulle, à la fois les phases faibles et les phases fortes des deux processus doivent être différentes. Cette asymétrie peut se produire aussi bien dans les $B$ neutres que dans les $B$ chargés.

\footnotetext{
${ }^{5}$ Ce n'est pas rigoureusement correct: le Lagrangien de QCD doit en fait contenir un terme qui viole $C P$, dont une signature expérimentale serait la présence d'un moment dipolaire du neutron non nul. Les limites supérieures actuelles sur ce dipôle montre que la violation de $C P$ dans QCD est très petite, pour une raison encore incomprise: ceci est connu sous le nom de "strong $C P$ problem" [12].
} 
Ce type de violation de $C P$ souffre des incertitudes théoriques reliées à la présence des phases fortes. La violation de $C P$ directe a été observée dans le système des mésons $K$ (mesure de $\epsilon^{\prime} / \epsilon[13]$ ), mais aucun signal significatif n'a encore été vu dans le système des mésons $B$.

\subsubsection{La violation de $C P$ dans le mélange $B^{0}-\bar{B}^{0}(|q / p| \neq 1)$}

Si les taux des deux processus $C P$-conjugués $B^{0} \rightarrow \bar{B}^{0}$ et $\bar{B}^{0} \rightarrow B^{0}$ ne sont pas égaux, on a une violation de $C P$ dans le mélange $B^{0}-\bar{B}^{0}$. La première observation d'une violation de la symétrie $C P$ dans le système des mésons $K$ en 1964 [1] concernait ce type de violation. Dans le système des mésons $B$, on attend cette violation de $C P$ de l'ordre de $10^{-3}$, et elle n'a pas encore été observée. On peut la mesurer grâce à l'asymétrie semi-leptonique $A_{\mathrm{SL}}$ avec des leptons de "mauvais signe" (qui constituent une signature de l'oscillation du $B^{0}$ ou $\bar{B}^{0}$ ):

$$
A_{\mathrm{SL}}=\frac{\Gamma\left[\bar{B}^{0}(t) \rightarrow \ell^{+} X\right]-\Gamma\left[B^{0}(t) \rightarrow \ell^{-} X\right]}{\Gamma\left[\bar{B}^{0}(t) \rightarrow \ell^{+} X\right]+\Gamma\left[B^{0}(t) \rightarrow \ell^{-} X\right]}=\frac{1-|q / p|^{4}}{1+|q / p|^{4}}
$$

\subsubsection{La violation de $C P$ due à l'interférence entre les désintégra- tions avec et sans mélange $(\operatorname{Im} \lambda \neq 0)$}

On voit grâce à l'équation 1.35 que la violation de $C P$ peut aussi se manifester si $|\lambda|=1$, lorsque la phase relative entre $q / p$ et $\bar{A} / A$ est non nulle, correspondant à une interférence entre les désintégrations de $B$ ayant ou n'ayant pas oscillé (on dit de façon abrégée interférence entre désintégration et mélange), c'est à dire entre $B^{0} \rightarrow f$ et $B^{0} \rightarrow \bar{B}^{0} \rightarrow f$.

Deux cas sont à distinguer (on suppose $|q / p|=1$ ):

- si $|\lambda|=1$ (une seule phase faible intervient, et donc l'éq. 1.38 est nulle), alors l'éq. 1.35 se simplifie au terme $\mathrm{d} u$ sinus, permettant ainsi d'isoler ce dernier type de violation de $C P$ :

$$
a_{\mathrm{CP}}(\Delta t)=-\operatorname{Im} \lambda \sin \Delta m_{B} \Delta t
$$

- si $|\lambda| \neq 1$ (plusieurs phases faibles interviennent, et donc l'éq. 1.38 est potentiellement non nulle), les autres types de violation de $C P$ sont aussi à considérer.

\subsection{Les trois niveaux de complexité d'une analyse $C P$}

\subsection{1 $|\lambda|=1$, état final propre de $C P(\sin 2 \beta$ dans les "modes en or" $b \rightarrow c \bar{c} s)$}

Nous décrivons dans cette section la mesure de $\sin 2 \beta$ dans les "modes en or" $b \rightarrow c \bar{c} s$.

Les deux diagrammes dominants montrés sur la figure 1.5 contribuent aux modes $b \rightarrow c \bar{c} s$ : un diagramme en arbre supprimé de couleur ${ }^{6}$ et un diagramme pingouin fort.

Dans $B A B A R$, les modes $b \rightarrow c \bar{c} s$ considérés pour la mesure de $\sin 2 \beta$ sont $J / \psi K_{S}^{0}$, $\psi(2 S) K_{S}^{0}, \xi_{c 1} K_{S}^{0}, \eta_{c} K_{S}^{0}$ et $J / \psi K_{L}^{0}$.

\footnotetext{
${ }^{6}$ Les diagrammes supprimés de couleur viennent avec un facteur $1 / N_{c}$, où $N_{c}$ est le nombre de couleurs. Certains modèles utilisent l'approximation $N_{c} \rightarrow \infty$ au sein de laquelle ces diagrammes sont négligeables.
} 

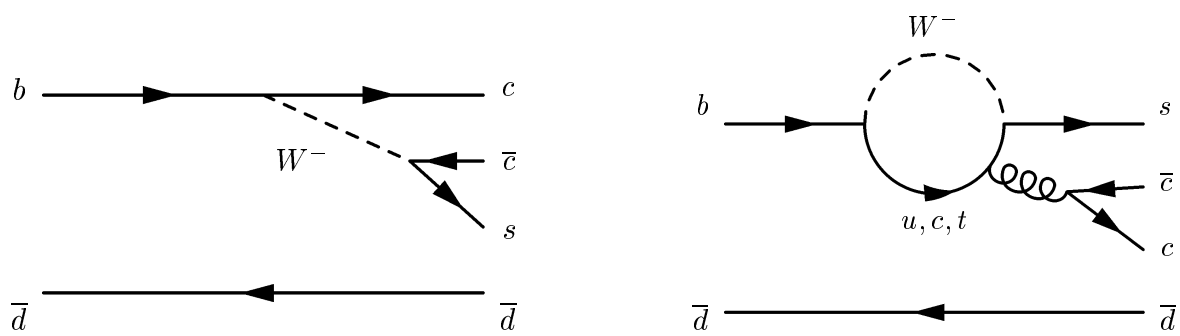

Figure 1.5: Diagrammes des modes $b \rightarrow c \bar{c} s$. Gauche: diagramme en arbre supprimé de couleur, droite: diagramme pingouin fort.

Dénotant l'amplitude du diagramme en arbre par $T_{c \bar{c} s}$, et celles des pingouins par $P^{u, c, t}$ (où $u, c, t$ sont les quarks présents dans la boucle), on écrit l'amplitude totale de $b \rightarrow c \bar{c} s$ comme:

$$
A=V_{u s} V_{u b}^{*} P^{u}+V_{c s} V_{c b}^{*}\left(T_{c \bar{c} s}+P^{c}\right)+V_{t s} V_{t b}^{*} P^{t} .
$$

Grâce à la relation d'unitarité $V_{t s} V_{t b}^{*}=-V_{c s} V_{c b}^{*}-V_{u s} V_{u d}^{*}$, on se ramène à deux phases faibles:

$$
A=V_{u s} V_{u b}^{*}\left(P^{u}-P^{t}\right)+V_{c s} V_{c b}^{*}\left(T_{c \bar{c} s}+P^{c}-P^{t}\right) .
$$

On a $V_{u s} V_{u b}^{*}=\mathcal{O}\left(\lambda^{4}\right)$, contribution petite par rapport à $V_{c s} V_{c b}^{*}=\mathcal{O}\left(\lambda^{2}\right)$. Ainsi, une unique phase faible domine dans ce processus, et $\lambda$ s'écrit:

$$
\lambda(b \rightarrow c \bar{c} s)=\eta_{\mathrm{CP}} \frac{V_{t d}^{*} V_{t b}}{V_{t d} V_{t b}^{*}} \frac{V_{c s}^{*} V_{c b}}{V_{c s} V_{c b}^{*}} \frac{V_{c d}^{*} V_{c s}}{V_{c d} V_{c s}^{*}},
$$

où le premier terme provient du mélange $B^{0}-\bar{B}^{0}(q / p)$, le second de l'amplitude dominante de la désintégration $(\bar{A} / A)$ et le troisième au mélange $K^{0}-\overline{K^{0}}$ due à la présence d'un $K^{0}$ dans l'état final considéré. Le paramètre $\eta_{\mathrm{CP}}$ vaut -1 pour les états finals possédant un $K_{S}^{0}$, et +1 pour ceux possédant un $K_{L}^{0}$, sauf pour l'état final $\eta_{c} K_{S}^{0}$ où $\eta_{\mathrm{CP}}=+1$.

Ainsi, grâce à l'éq. 1.20, on obtient:

$$
\operatorname{Im} \lambda(b \rightarrow c \bar{c} s)=\sin 2 \beta+\mathcal{O}\left(10^{-3}\right) .
$$

Ce paramètre a été mesuré précisément par les collaborations BABAR et Belle. La valeur moyenne de leurs résultats vaut $\sin 2 \beta=0.734 \pm 0.054$ [14], en parfait accord avec la prédiction du Modèle Standard obtenue dans un ajustement global de la matrice CKM [15] ( $c f$. chapitre 8), démontrant que le mécanisme KM est bien la source dominante de violation de $C P$ dans les courants chargés électrofaibles.

Le paramètres $\sin 2 \beta$ peut aussi, entre autres, être mesuré dans les modes $b \rightarrow s \bar{s} s$, comme $B^{0} \rightarrow \phi K_{s}^{0}$, dont le diagramme dominant est un diagramme pingouin ( $c f$. figure 1.6), particulièrement sensible à la nouvelle physique.

Les mesures de BABAR et Belle [16] ne sont pas encore significatives: $\sin 2 \beta\left(\phi K_{s}^{0}\right)=$ $-0.39 \pm 0.41$, mais sont à $2.7 \sigma$ de la valeur mesurée dans les modes cités précédemment. Une discussion sur la présence éventuelle de nouvelle physique dans ce mode est donnée en Réf. [17].

\subsection{2 $|\lambda| \neq 1$ et état final propre de $C P\left(\sin 2 \alpha_{\text {eff }}\right.$ dans le mode $\left.B^{0} \rightarrow \pi^{+} \pi^{-}\right)$}

Les diagrammes dominants contribuant à la désintégration $B^{0} \rightarrow \pi^{+} \pi^{-}$(du type $\bar{b} \rightarrow \bar{u} d u$ ) sont donnés sur la figure 1.7 . 


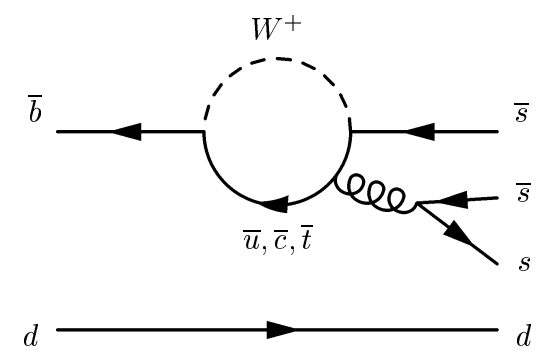

Figure 1.6: Diagramme pingouin fort des modes $b \rightarrow s \bar{s} s$. La nouvelle physique peut entrer dans la boucle de fermions de ce diagramme, et donc mener à une mesure de sin $2 \beta$ différente de celle mesurée dans les processus dominés par les diagrammes en arbre.

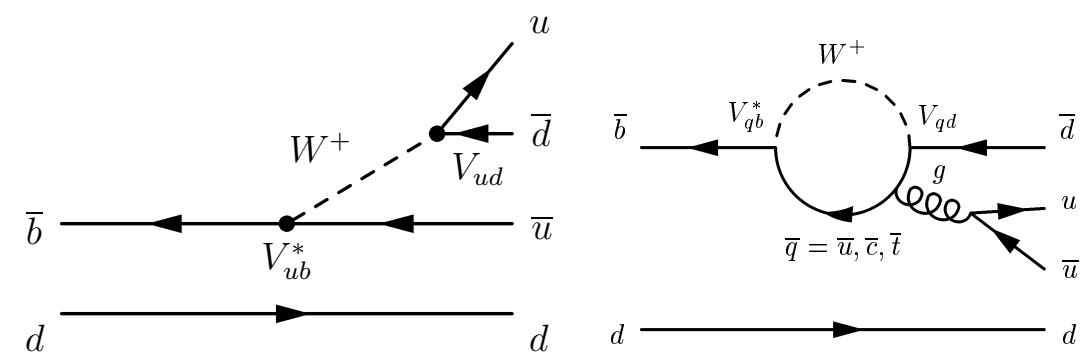

Figure 1.7: Diagrammes des modes $\bar{b} \rightarrow \bar{u} d u$. Gauche: diagramme en arbre, droite: diagramme pingouin fort.

Grâce à la relation d'unitarité $V_{t d} V_{t b}^{*}=-V_{c d} V_{c b}^{*}-V_{u d} V_{u d}^{*}$, on se ramène là encore de trois contributions de phases faibles différentes

$$
A=V_{u d} V_{u b}^{*}\left(T_{\bar{u} d u}+P^{u}\right)+V_{c d} V_{c b}^{*} P^{c}+V_{t d} V_{t b}^{*} P^{t}
$$

à deux contributions:

$$
\begin{aligned}
A & =V_{u d} V_{u b}^{*}\left(T_{\bar{u} d u}+P^{u}-P^{c}\right)+V_{t d} V_{t b}^{*}\left(P^{t}-P^{c}\right), \\
& =\left|V_{u d} V_{u b}^{*}\right| e^{i \gamma}\left(T_{\bar{u} d u}+P^{u}-P^{c}\right)+\left|V_{t d} V_{t b}^{*}\right| e^{i \beta}\left(P^{t}-P^{c}\right), \\
& =e^{i \gamma} T+e^{-i \beta} P,
\end{aligned}
$$

où $T \equiv\left|V_{u d} V_{u b}^{*}\right|\left(T_{\bar{u} d u}+P^{u}-P^{c}\right)$ et $P \equiv-\left|V_{t d} V_{t b}^{*}\right|\left(P^{t}-P^{c}\right)$. Contrairement au cas exposé dans la section 1.5.1, dans la décomposition ci-dessus, il n'y a pas d'amplitude dominante $\left(\left|V_{u d} V_{u b}^{*}\right|\right.$ et $\left|V_{t d} V_{t b}^{*}\right|$ sont tous les deux de l'ordre $\left.\lambda^{3}\right)$ : on se retrouve donc dans le cas où potentiellement $|\lambda| \neq 1$, et où $\operatorname{Im} \lambda$ ne mesure pas directement une phase faible. En supposant $|q / p|=1$, on a:

$$
\lambda=e^{2 i \alpha} \frac{1+P / T e^{-i \alpha}}{1+P / T e^{+i \alpha}}=|\lambda| e^{i 2 \alpha_{\mathrm{eff}}} .
$$

Les taux de désintégrations de $B^{0} \rightarrow \pi^{+} \pi^{-}$en fonction du temps sont donnés par les eqs. 1.32 et 1.34. Nous définissons les coefficients des cosinus et sinus par:

$$
C_{\pi^{+} \pi^{-}}=\frac{1-|\lambda|^{2}}{1+|\lambda|^{2}}, \quad S_{\pi^{+} \pi^{-}}=\frac{2 \operatorname{Im} \lambda}{1+|\lambda|^{2}}
$$

soit

$$
C_{\pi^{+} \pi^{-}}=\frac{N\left(B^{0} \rightarrow \pi^{+} \pi^{-}\right)-N\left(\bar{B}^{0} \rightarrow \pi^{+} \pi^{-}\right)}{N\left(B^{0} \rightarrow \pi^{+} \pi^{-}\right)+N\left(\bar{B}^{0} \rightarrow \pi^{+} \pi^{-}\right)}, \quad S_{\pi^{+} \pi^{-}}=\sqrt{1-C_{\pi^{+} \pi^{-}}^{2}} \sin 2 \alpha_{\mathrm{eff}} .
$$


Nous changeons les signes des coefficients des cosinus et sinus des Eq. 1.32 et 1.34 afin que ces dernières se réfèrent à la saveur du $B_{\text {sav }}$, et non pas du $B_{\text {rec }}$ (puisque c'est ainsi que l'on procède expérimentalement). Les taux de désintégration sont alors donnés par:

$$
\begin{aligned}
f_{B_{\text {gaveur }}^{\circ}}^{\pi^{+} \pi^{-}} & =\frac{e^{-|\Delta t| / \tau_{B}}}{4 \tau_{B}}\left[1-C_{\pi^{+} \pi^{-}} \cos \left(\Delta m_{B} \Delta t\right)+S_{\pi^{+} \pi^{-}} \sin \left(\Delta m_{B} \Delta t\right)\right], \\
f_{\bar{B}_{\text {gaveur }}^{\circ}}^{\pi^{+} \pi^{-}} & =\frac{e^{-|\Delta t| / \tau_{B}}}{4 \tau_{B}}\left[1+C_{\pi^{+} \pi^{-}} \cos \left(\Delta m_{B} \Delta t\right)-S_{\pi^{+} \pi^{-}} \sin \left(\Delta m_{B} \Delta t\right)\right] .
\end{aligned}
$$

En l'absence de pingouins, $\sin 2 \alpha_{\text {eff }}=\sin 2 \alpha$, ce qui mène à une détermination de $\alpha$ avec 2 solutions miroirs dans l'intervalle $[0, \pi]$.

Les coefficients $C_{\pi^{+} \pi^{-}}$et $S_{\pi^{+} \pi^{-}}$ont été mesurés par BABAR [19] et Belle [18], et la valeur moyenne vaut $C_{\pi^{+} \pi^{-}}=-0.49 \pm 0.19$ et $S_{\pi^{+} \pi^{-}}=-0.46 \pm 0.26$ (une interprétation de ces résultats se trouve dans la réf. [20]).

\subsection{3 $|\lambda| \neq 1$ et état final non propre de $C P\left(\sin 2 \alpha_{\text {eff }}\right.$ dans le mode $\left.B^{0} \rightarrow \rho^{ \pm} \pi^{\mp}\right)$}

Nous nous intéressons finalement au mode $B^{0} \rightarrow \rho^{ \pm} \pi^{\mp}$, qui est le sujet de cette thèse. L'état final n'est pas état propre de $C P$. Les diagrammes de la désintégration $B^{0} \rightarrow \rho^{ \pm} \pi^{\mp}$ sont les mêmes que ceux de $B^{0} \rightarrow \pi^{+} \pi^{-}$(cf. figure 1.7). Dans le diagramme en arbre, le $\rho$ peut être créé soit par le quark spectateur du $B$ (cela est noté par l'exposant ${ }^{-+}$par la suite), soit par le $W$ (cela est noté par l'exposant ${ }^{+-}$). Dans cette section d'introduction, on fait l'hypothèse simplificatrice que le $\rho$ est une résonance de petite largeur, de telle sorte que l'on puisse négliger les interférences entre $\rho^{+}$et $\rho^{-}$.

Dans ce mode, nous n'avons plus deux amplitudes de désintégration comme dans l'éq. 1.50, mais quatre amplitudes:

$$
\begin{array}{llll}
A_{f}=\left\langle f|\mathcal{H}| B^{0}\right\rangle & \longrightarrow & A^{+-}\left(B^{0} \rightarrow \rho^{+} \pi^{-}\right)=e^{+i \gamma} T^{+-}+e^{-i \beta} P^{+-}, \\
\bar{A}_{f}=\left\langle f|\mathcal{H}| \bar{B}^{0}\right\rangle & \longrightarrow & \bar{A}^{+-}\left(\bar{B}^{0} \rightarrow \rho^{+} \pi^{-}\right)=e^{-i \gamma} T^{-+}+e^{+i \beta} P^{-+}, \\
A_{\bar{f}}=\left\langle\bar{f}|\mathcal{H}| B^{0}\right\rangle & \longrightarrow & A^{-+}\left(B^{0} \rightarrow \rho^{-} \pi^{+}\right)=e^{+i \gamma} T^{-+}+e^{-i \beta} P^{-+}, \\
\bar{A}_{\bar{f}}=\left\langle\bar{f}|\mathcal{H}| \bar{B}^{0}\right\rangle & \longrightarrow & \bar{A}^{-+}\left(\bar{B}^{0} \rightarrow \rho^{-} \pi^{+}\right)=e^{-i \gamma} T^{+-}+e^{+i \beta} P^{+-} .
\end{array}
$$

Nous définissons ${ }^{7}$ :

$$
\lambda^{+-} \equiv \frac{q}{p} \frac{\bar{A}^{+-}}{A^{+-}}, \quad \lambda^{-+} \equiv \frac{q}{p} \frac{\bar{A}^{-+}}{A^{-+}}, \quad \kappa^{-+} \equiv \frac{q}{p} \frac{\bar{A}^{+-}}{A^{-+}}, \quad \kappa^{+-} \equiv \frac{q}{p} \frac{\bar{A}^{-+}}{A^{+-}} .
$$

Les paramètres $\lambda^{+-(-+)}$n'impliquent qu'une seule combinaison de charge de $\rho \pi$, et les deux types d'amplitudes $T(P)^{+-}$et $T(P)^{-+}$. Ils ne sont pas sensibles à la violation de $C P$ directe. Les paramètres $\kappa^{+-(-+)}$impliquent les deux combinaisons de charges de $\rho \pi$, mais seulement un seul type d'amplitude. La phase de $\kappa^{+-(-+)}$est liée aux angles effectifs $\alpha_{\text {eff }}^{+-(-)}$définis dans les Eq. 1.73 et 1.73, et le module à la violation de $C P$ directe.

Les paramètres $\lambda^{+-}$et $\lambda^{-+}$n'ont pas les bonnes propriétés sous la transformation $C P$, en particulier $\lambda^{+-} \neq \pm 1$ ou $\lambda^{-+} \neq \pm 1$ ne signifie pas automatiquement que la symétrie $C P$

\footnotetext{
${ }^{7}$ A cause de l'identité $\lambda^{+-} \lambda^{-+}=\kappa^{+-} \kappa^{-+}$, les quatre paramètres de l'éq. 1.52 ne sont pas indépendants, et sont introduits pour des raisons pratiques.
} 
soit violée. Nous pouvons construire des $\lambda$ reflétant les propriétés $C P$ et de saveur de la désintégration $B^{0} \rightarrow \rho^{ \pm} \pi^{\mp}$. Nous définissons ainsi:

$$
\begin{aligned}
& \left|\lambda_{\mathrm{CP}}\right|^{2} \equiv \frac{\left|\lambda^{+-}\right|^{2}+\left|\lambda^{-+}\right|^{2}+2\left|\lambda^{+-}\right|^{2}\left|\lambda^{-+}\right|^{2}}{2+\left|\lambda^{+-}\right|^{2}+\left|\lambda^{-+}\right|^{2}}, \\
& \left|\lambda_{\mathrm{tag}}\right|^{2} \equiv \frac{1+2\left|\lambda^{+-}\right|^{2}+\left|\lambda^{+-}\right|^{2}\left|\lambda^{-+}\right|^{2}}{1+2\left|\lambda^{-+}\right|^{2}+\left|\lambda^{-+}\right|^{2}\left|\lambda^{+-}\right|^{2}}, \\
& \operatorname{Im} \lambda_{\mathrm{CP}} \equiv \frac{\operatorname{Im} \lambda^{+-}\left(1+\left|\lambda^{-+}\right|^{2}\right)+\operatorname{Im} \lambda^{-+}\left(1+\left|\lambda^{+-}\right|^{2}\right)}{2+\left|\lambda^{+-}\right|^{2}+\left|\lambda^{-+}\right|^{2}}, \\
& \operatorname{Im} \lambda_{\operatorname{tag}} \equiv \frac{\operatorname{Im} \lambda^{+-}\left(1+\left|\lambda^{-+}\right|^{2}\right)-\operatorname{Im} \lambda^{-+}\left(1+\left|\lambda^{+-}\right|^{2}\right)}{1+2\left|\lambda^{-+}\right|^{2}+\left|\lambda^{-+}\right|^{2}\left|\lambda^{+-}\right|^{2}} .
\end{aligned}
$$

La signification de $\lambda_{\text {tag }}$ et $\lambda_{\mathrm{CP}}$ se comprend à travers les exemples suivants:

- Si $B^{0} \rightarrow \rho^{ \pm} \pi^{\mp}$ est un état propre de saveur, par exemple $A^{-+}=\bar{A}^{+-}=0$ de telle sorte que $\lambda^{+-}=0$ et $\lambda^{-+}=\infty$, alors $\left|\lambda_{\text {tag }}\right|=0$, ce qui correspond à une dilution maximale pour la mesure de $\alpha$ (puisque l'on ne peut mesurer la violation de $C P$ due à l'interférence entre désintégration et mélange dans un mode auto-étiquetant).

Le paramètre $\lambda_{\text {tag }}$ est relié à l'abondance relative de $\rho$ produits par le $W$ et par le quark spectateur (le cas extrême d'un état propre de saveur correspondant à l'une de ces productions étant nulle).

- Si $B^{0} \rightarrow \rho^{ \pm} \pi^{\mp}$ se comporte comme un état propre de $C P$, c'est-à-dire que $\left|A^{+-}\right|=$ $\left|A^{-+}\right|$et $\left|\bar{A}^{+-}\right|=\left|\bar{A}^{-+}\right|$de telle sorte que $\left|\lambda^{+-}\right|=\left|\lambda^{-+}\right|=|\lambda|$, alors $\left|\lambda_{\text {tag }}\right|=1$ (soit une dilution minimale) et $\left|\lambda_{\mathrm{CP}}\right|=|\lambda|$. Cela revient à avoir autant de $\rho$ produits par le $W$ que par le quark spectateur: on peut alors "oublier" la charge du $\rho$, et ne regarder que les asymétries entre $B^{0} \rightarrow \rho \pi$ et $\bar{B}^{0} \rightarrow \rho \pi$, comme pour un état propre de $C P$.

Les taux de désintégration de $B^{0} \rightarrow \rho^{ \pm} \pi^{\mp}$ dépendants du temps sont donnés par:

$$
\begin{aligned}
& \left|\left\langle\rho^{+} \pi^{-}|\mathcal{H}| B^{0}(\Delta t)\right\rangle\right|^{2} \propto\left|A^{+-}\right|^{2}\left[\frac{1+\left|\lambda^{+-}\right|^{2}}{2}+\frac{1-\left|\lambda^{+-}\right|^{2}}{2} \cos \left(\Delta m_{B} \Delta t\right)-\operatorname{Im} \lambda^{+-} \sin \left(\Delta m_{B} \Delta t\right)\right], \\
& \left|\left\langle\rho^{+} \pi^{-}|\mathcal{H}| \bar{B}^{0}(\Delta t)\right\rangle\right|^{2} \propto\left|\bar{A}^{+-}\right|^{2}\left[\frac{1+\left|\lambda^{+-}\right|^{2}}{2}-\frac{1-\left|\lambda^{+-}\right|^{2}}{2} \cos \left(\Delta m_{B} \Delta t\right)+\operatorname{Im} \lambda^{+-} \sin \left(\Delta m_{B} \Delta t\right)\right], \\
& \left|\left\langle\rho^{-} \pi^{+}|\mathcal{H}| B^{0}(\Delta t)\right\rangle\right|^{2} \propto\left|A^{-+}\right|^{2}\left[\frac{1+\left|\lambda^{-+}\right|^{2}}{2}+\frac{1-\left|\lambda^{-+}\right|^{2}}{2} \cos \left(\Delta m_{B} \Delta t\right)-\operatorname{Im} \lambda^{-+} \sin \left(\Delta m_{B} \Delta t\right)\right], \\
& \left|\left\langle\rho^{-} \pi^{+}|\mathcal{H}| \bar{B}^{0}(\Delta t)\right\rangle\right|^{2} \propto\left|\bar{A}^{-+}\right|^{2}\left[\frac{1+\left|\lambda^{-+}\right|^{2}}{2}-\frac{1-\left|\lambda^{-+}\right|^{2}}{2} \cos \left(\Delta m_{B} \Delta t\right)+\operatorname{Im} \lambda^{-+} \sin \left(\Delta m_{B} \Delta t\right)\right] .
\end{aligned}
$$

On factorise $\left(1+\left|\lambda^{+-}\right|^{2}\right) / 2$ et $\left(1+\left|\lambda^{-+}\right|^{2}\right) / 2$ dans les eqs. 1.54 et 1.55 , et l'on définit $C^{ \pm}$, $S^{ \pm}$, ainsi que $C, \Delta C, S, \Delta S$ et $A_{\mathrm{CP}}$ par:

$$
\begin{gathered}
C^{+} \equiv C+\Delta C \equiv \frac{1-\left|\lambda^{+-}\right|^{2}}{1+\left|\lambda^{+-}\right|^{2}}, \quad C^{-} \equiv C-\Delta C \equiv \frac{1-\left|\lambda^{-+}\right|^{2}}{1+\left|\lambda^{-+}\right|^{2}}, \\
S^{+} \equiv S+\Delta S \equiv \frac{2 \operatorname{Im} \lambda^{+-}}{1+\left|\lambda^{+-}\right|^{2}}, \quad S^{-} \equiv S-\Delta S \equiv \frac{2 \operatorname{Im} \lambda^{-+}}{1+\left|\lambda^{-+}\right|^{2}}, \\
A_{\mathrm{CP}}=\frac{\left|A^{+-}\right|^{2}+\left|\bar{A}^{+-}\right|^{2}-\left|A^{-+}\right|^{2}-\left|\bar{A}^{-+}\right|^{2}}{\left|A^{+-}\right|^{2}+\left|\bar{A}^{+-}\right|^{2}+\left|A^{-+}\right|^{2}+\left|\bar{A}^{-+}\right|^{2}}
\end{gathered}
$$


On insère ces paramètres dans les eqs. 1.54 et 1.55, où l'on change aussi le signe des cosinus et sinus pour que la saveur se réfère à $B_{\text {sav }}$. On obtient ainsi les taux de désintégration de $\rho^{ \pm} \pi^{\mp}$ en fonction du temps $\Delta t$ et de la saveur de $B_{\text {sav }}$ :

$$
\begin{aligned}
& f_{B_{\mathrm{avv}}^{\mathrm{o}}}^{\rho^{ \pm} \pi^{\mp}}=\left(1 \pm A_{\mathrm{CP}}\right) \frac{e^{-|\Delta t| / \tau_{B}}}{4 \tau_{B}}\left[1-C^{ \pm} \cos \Delta m_{B} t \Delta t+S^{ \pm} \sin \Delta m_{B} t \Delta t\right], \\
& f_{\bar{B}_{\mathrm{gav}}^{\mathrm{o}}}^{\rho^{ \pm} \pi^{\mp}}=\left(1 \pm A_{\mathrm{CP}}\right) \frac{e^{-|\Delta t| / \tau_{B}}}{4 \tau_{B}}\left[1+C^{ \pm} \cos \Delta m_{B} t \Delta t-S^{ \pm} \sin \Delta m_{B} t \Delta t\right] .
\end{aligned}
$$

Les coefficients $C, \Delta C, S, \Delta S$ et $A_{\mathrm{CP}}$ s'expriment en fonction des paramètres précédemment définis de la manière suivante:

$$
\begin{aligned}
& C=\frac{1-\left|\lambda_{\mathrm{CP}}\right|^{2}}{1+\left|\lambda_{\mathrm{CP}}\right|^{2}}, \Delta C=\frac{1-\left|\lambda_{\mathrm{tag}}\right|^{2}}{1+\left|\lambda_{\mathrm{tag}}\right|^{2}}, \\
& S=\frac{2 \operatorname{Im} \lambda_{\mathrm{CP}}}{1+\left|\lambda_{\mathrm{CP}}\right|^{2}}, \Delta S=\frac{2 \operatorname{Im} \lambda_{\mathrm{tag}}}{1+\left|\lambda_{\mathrm{tag}}\right|^{2}}, \\
& A_{\mathrm{CP}}=\frac{\left(1+\left|\lambda^{+-}\right|^{2}-\left|\kappa^{+-}\right|^{2}\right)\left|\lambda^{-+}\right|^{2}-\left|\kappa^{+-}\right|^{2}}{\left(1+\left|\lambda^{+-}\right|^{2}+\left|\kappa^{+-}\right|^{2}\right)\left|\lambda^{-+}\right|^{2}+\left|\kappa^{+-}\right|^{2}} .
\end{aligned}
$$

D'après les définitions de l'éq. 1.53, on voit que la mise en évidence de la violation de $C P$ se fait via les paramètres $C$ (violation de $C P$ dans la désintégration) et $S$ (violation de $C P$ due à l'interférence entre désintégrations avec et sans mélange). Les paramètres $\Delta C$ et $\Delta S$, quant à eux, mesurent l'asymétrie entre les diagrammes où le $\rho$ est produit par le $W$ ou à l'aide du quark spectateur, et sont donc reliés aux facteurs de forme hadroniques. Enfin, $A_{\mathrm{CP}}$ mesure l'asymétrie de charge entre les $\rho^{+} \pi^{-}$et $\rho^{-} \pi^{+}$, quantité reliée à la violation de $C P$ dans la désintégration. On nommera par la suite $C, S$ et $A_{\mathrm{CP}}$ paramètres $C P$, et $\Delta C$, $\Delta S$ paramètres de dilution.

Les paramètres $\lambda^{+-}$et $\lambda^{-+}$dépendent de $\alpha$, ainsi que des amplitudes des diagrammes en arbre et pingouins:

$$
\begin{aligned}
& \lambda^{+-}=e^{2 i \alpha}\left(\frac{T^{-+}}{T^{+-}}\right)\left(\frac{1+P^{-+} / T^{-+} e^{-i \alpha}}{1+P^{+-} / T^{+-} e^{+i \alpha}}\right), \\
& \lambda^{-+}=e^{2 i \alpha}\left(\frac{T^{+-}}{T^{-+}}\right)\left(\frac{1+P^{+-} / T^{+-} e^{-i \alpha}}{1+P^{-+} / T^{-+} e^{+i \alpha}}\right) .
\end{aligned}
$$

En l'absence de pingouins, et en définissant $r_{T^{+-}}=\left|T^{-+} / T^{+-}\right|$et $\hat{\delta}_{T}=\arg \left(T^{-+} T^{+-*}\right)$, on a:

$$
\begin{aligned}
& \lambda^{+-}=r_{T^{+-}} e^{i\left(2 \alpha-\hat{\delta}_{T}\right)}, \quad \text { soit } \operatorname{Im}\left(\lambda^{+-}\right)=r_{T^{+-}} \sin \left(2 \alpha-\hat{\delta}_{T}\right), \\
& \lambda^{-+}=\frac{1}{r_{T^{+-}}} e^{i\left(2 \alpha+\hat{\delta}_{T}\right)}, \quad \text { soit } \quad \operatorname{Im}\left(\lambda^{-+}\right)=\frac{1}{r_{T^{+-}}} \sin \left(2 \alpha+\hat{\delta}_{T}\right),
\end{aligned}
$$

ou de façon équivalente,

$$
\begin{aligned}
S_{\rho \pi} & =\frac{2 r_{T^{+-}}}{1+r_{T^{+-}}^{2}} \sin 2 \alpha \cos \hat{\delta}_{T} \\
\Delta S_{\rho \pi} & =\frac{2 r_{T^{+-}}}{1+r_{T^{+-}}^{2}} \cos 2 \alpha \sin \hat{\delta}_{T}
\end{aligned}
$$




$$
\begin{aligned}
C_{\rho \pi} & =0, \\
\Delta C_{\rho \pi} & =\frac{1-r_{T^{+-}}^{2}}{1+r_{T^{+-}}^{2}}, \\
A_{\mathrm{CP}} & =0,
\end{aligned}
$$

soit pas de violation de $C P$ directe. D'autre part, la mesure de $\sin \left(2 \alpha \pm \delta_{T}\right)$ mène à 8 solutions miroirs pour $\alpha$ entre $[0, \pi]$. On se retrouve donc dans un cas plus défavorable que pour $B^{0} \rightarrow \pi^{+} \pi^{-}$qui permet de mesurer $\alpha$ avec 2 solutions miroirs en l'absence de pingouins (cf. Sec. 1.5.2).

Charles [24] a introduit les paramètres $A_{\rho \pi}^{+-}, A_{\rho \pi}^{-+}$reliés à la violation de $C P$ directe définis comme suit:

$$
\begin{aligned}
& A_{\rho \pi}^{+-} \equiv \frac{\left|\bar{A}^{-+}\right|^{2}-\left|A^{+-}\right|^{2}}{\left|\bar{A}^{-+}\right|^{2}+\left|A^{+-}\right|^{2}}=\frac{\left|\kappa^{+-}\right|^{2}-1}{\left|\kappa^{+-}\right|^{2}+1}, \\
& A_{\rho \pi}^{-+} \equiv \frac{\left|\bar{A}^{+-}\right|^{2}-\left|A^{-+}\right|^{2}}{\left|\bar{A}^{+-}\right|^{2}+\left|A^{-+}\right|^{2}}=\frac{\left|\kappa^{-+}\right|^{2}-1}{\left|\kappa^{-+}\right|^{2}+1},
\end{aligned}
$$

où nous avons suivi la convention de signe de $B A B A R$, soit une convention opposée à celle de la référence [24].

En la présence de pingouins, $A_{\rho \pi}^{+-}$et $A_{\rho \pi}^{-+}$sont donnés par:

$$
\begin{aligned}
& A_{\rho \pi}^{+-}=-\frac{2 r_{P T^{+-}} \sin \alpha \sin \delta_{P T^{+-}}}{1+r_{P T^{+-}}^{2}-2 r_{P T^{+-}} \cos \alpha \cos \delta_{P T^{+-}}}, \\
& A_{\rho \pi}^{-+}=-\frac{2 r_{P T^{-+}} \sin \alpha \sin \delta_{P T^{-+}}}{1+r_{P T^{-+}}^{2}-2 r_{P T^{-+}} \cos \alpha \cos \delta_{P T^{-+}}},
\end{aligned}
$$

où $r_{P T^{+-}}=\left|P^{+-} / T^{+-}\right|, \delta_{P T^{+-}}=\arg \left(P^{+-} T^{+-*}\right)$, et similairement pour l'autre combinaison de charge. On voit que l'on peut avoir violation de $C P$ directe. Nous définissons des angles faibles effectifs $\alpha_{\text {eff }}^{+-}$et $\alpha_{\text {eff }}^{-+}$par:

$$
\arg \kappa^{-+}=2 \alpha_{\text {eff }}^{+-}, \quad \arg \kappa^{+-}=2 \alpha_{\text {eff }}^{-+} .
$$

Les paramètres $\lambda^{+-}$et $\lambda^{-+}$s'écrivent alors en fonction de $\alpha_{\text {eff }}^{+-}$et $\alpha_{\text {eff }}^{-+}$, ainsi que de $\hat{\delta}=$ $\arg \left(\lambda^{+-} \kappa^{-+^{*}}\right)=\arg \left(\lambda^{-+^{*}} \kappa^{+-}\right)=\arg \left(A^{-+} A^{+-*}\right)$ de la manière suivante:

$$
\frac{\operatorname{Im} \lambda^{+-}}{\left|\lambda^{+-}\right|}=\sin \left(2 \alpha_{\mathrm{eff}}^{+-}+\hat{\delta}\right), \quad \frac{\operatorname{Im} \lambda^{-+}}{\left|\lambda^{-+}\right|}=\sin \left(2 \alpha_{\mathrm{eff}}^{-+}-\hat{\delta}\right),
$$

et les paramètres $S$ et $\Delta S$ comme:

$$
\begin{aligned}
& S+\Delta S_{\rho \pi}=\sqrt{1-\left(C+\Delta C_{\rho \pi}\right)^{2}} \sin \left(2 \alpha_{\text {eff }}^{+-}+\hat{\delta}\right), \\
& S-\Delta S_{\rho \pi}=\sqrt{1-\left(C-\Delta C_{\rho \pi}\right)^{2}} \sin \left(2 \alpha_{\text {eff }}^{-+}-\hat{\delta}\right) .
\end{aligned}
$$

Afin d'illustrer la signification des paramètres $C, S, \Delta C, \Delta S, A_{\mathrm{CP}}$, le tableau 1.3 donne les valeurs de ces paramètres pour différentes configurations d'amplitudes arbre et pingouin. 


\begin{tabular}{|c|c|c|c|c|c|c|c|c|c|}
\hline \multicolumn{5}{|c|}{ Configuration } & \multirow{2}{*}{$A_{\mathrm{CP}}$} & \multirow{2}{*}{$C$} & \multirow{2}{*}{$S$} & \multirow{2}{*}{$\Delta C$} & \multirow{2}{*}{$\Delta S$} \\
\hline$\alpha\left(^{\circ}\right)$ & $T^{+-}$ & $T^{-+}$ & $P^{+-}$ & $P^{-+}$ & & & & & \\
\hline 100 & 1 & 1 & 0 & 0 & 0 & 0 & -0.34 & 0 & 0 \\
\hline 100 & 1 & 0.5 & 0 & 0 & 0 & 0 & -0.23 & +0.60 & 0 \\
\hline 100 & 1 & 1 & 0.5 & 0.5 & 0 & 0 & +0.60 & 0 & 0 \\
\hline 100 & 1 & 1 & 0.5 & 0.1 & 0 & 0 & +0.24 & +0.05 & 0 \\
\hline 100 & 1 & 1 & 1 & 1 & 0 & 0 & +0.99 & 0 & 0 \\
\hline 100 & $1 e^{i 10^{\circ}}$ & 1 & 0 & 0 & 0 & 0 & -0.34 & 0 & +0.16 \\
\hline 100 & $1 e^{i 10^{\circ}}$ & 0.5 & 0 & 0 & 0 & 0 & -0.27 & +0.60 & +0.13 \\
\hline 0 & $1 e^{i 10^{\circ}}$ & 1 & 0 & 0 & 0 & 0 & 0 & 0 & -0.17 \\
\hline 100 & 1 & 1 & $1 e^{i 10^{\circ}}$ & 1 & -0.10 & -0.10 & +0.98 & -0.001 & +0.01 \\
\hline 0 & 1 & 1 & $1 e^{i 10^{\circ}}$ & 1 & 0 & 0 & 0 & -0.004 & -0.01 \\
\hline 100 & 1 & $1 e^{i 10^{\circ}}$ & $1 e^{i 10^{\circ}}$ & 1 & -0.21 & -0.10 & +0.99 & 0 & 0 \\
\hline 100 & 1 & 0.5 & $0.2 e^{i 60^{\circ}}$ & $0.2 e^{-i 140^{\circ}}$ & -0.35 & +0.03 & -0.44 & +0.53 & -0.08 \\
\hline
\end{tabular}

Tableau 1.3: Valeurs de $C, S, \delta C, \delta S, A_{\mathrm{CP}}$ pour différentes configurations d'amplitudes arbre et pingouin. 


\section{Chapitre 2}

\section{Eléments de théorie pour les modes $B^{0} \rightarrow \rho \pi$}

Le chapitre précédent a introduit les notions générales relatives à la violation de $C P$ et le système des mésons $B$. Nous nous concentrons dans ce chapitre sur les méthodes permettant d'extraire l'angle $\alpha$ à partir du mode $B^{0} \rightarrow \rho \pi$.

\subsection{Mesurer l'angle $\alpha$ : où est la difficulté?}

En l'absence de diagrammes pingouins, la mesure des taux dépendant du temps de $B^{0} \rightarrow$ $\rho^{ \pm} \pi^{\mp}$ ( $c f$. Eq. 1.57 et 1.58) permet de mesurer $\sin \left(2 \alpha \mp \delta_{T}\right)$ (où $\delta_{T}$ est la différence des phases fortes entre les diagrammes en arbre $T^{+-}$et $T^{-+}$), soit $\alpha$ avec 8 ambiguïtés dans l'intervalle $[0, \pi]$ (cf. Eq. 1.63). En la présence de diagrammes pingouins, cette mesure ne fournit pas assez d'observables pour extraire $\alpha$. Il existe néanmoins des méthodes utilisant des observables supplémentaires permettant de mesurer $\alpha$ dans ce cas de figure. Comme on peut le voir dans les eqs. 1.61, ces méthodes doivent permettre de mesurer le module et la phase des rapports $P^{+-} / T^{+-}$et $P^{-+} / T^{-+}$.

Il existe des méthodes théoriques permettant de calculer ces rapports [26], mais nous ne nous intéressons ici qu'aux méthodes expérimentales. Celles-ci nécessitent néanmoins toujours des hypothèses plus ou moins indépendantes de modèle:

- Symétrie d'isospin SU(2): il s'agit de l'hypothèse de base, bien vérifiée expérimentalement ${ }^{1}$. Deux analyses utilisent cette hypothèse:

- l'analyse dite d'isospin $[27,28]$ utilisant l'ensemble des modes $\rho \pi$ issus des $B$ chargés et neutres (dont certains modes sont difficiles à mesurer). Elle permet de mesurer $\sin \left(2 \alpha \pm \hat{\delta}_{T}\right)$.

- l'analyse dans le diagramme Dalitz [29] qui ne requiert que les $B$ neutres, mais une compréhension détaillée du diagramme de Dalitz $\pi^{+} \pi^{-} \pi^{0}$. Elle permet en principe de déterminer toutes les amplitudes en arbre et pingouins, et donc de mesurer $\alpha$ sans ambiguïté entre 0 et $\pi$.

- Symétrie SU(3): cette hypothèse plus poussée ${ }^{2}$ permet de relier les diagrammes pingouins de $B^{0} \rightarrow \rho^{-} \pi^{+}, B^{0} \rightarrow \rho^{-} K^{+}$et $B^{0} \rightarrow K^{*+} \pi^{-}$qui sont les amplitudes

\footnotetext{
${ }^{1}$ De possibles problèmes sont néanmoins mentionnés dans la réf. [21].

${ }^{2}$ Les corrections à la symétrie SU(3) sont typiquement de l'ordre de $\left(f_{K}-f_{\pi}\right) / f_{\pi} \simeq 23 \%$ [24].
} 
dominantes de ces deux derniers modes. C'est la raison pour laquelle nous mesurons simultanément $B^{0} \rightarrow \rho^{ \pm} \pi^{\mp}$ et $B^{0} \rightarrow \rho^{-} K^{+}$dans l'analyse présentée dans le chapitre 4 .

\subsection{Hypothèse théorique de base: la symétrie $S U(2)$ d'isospin}

\subsubsection{Amplitudes des désintégrations $B \rightarrow \rho \pi$}

Les diagrammes dominants (arbre et pingouin fort) contribuant à la désintégration $B^{0} \rightarrow$ $\rho^{ \pm} \pi^{\mp}$ sont montrés sur la figure 1.7. Pour obtenir les diagrammes dominants des désintégrations des $B$ chargés, on échange le quark spectateur $d$ par $u$. Les diagrammes en arbre supprimés de couleur (schéma de gauche de la figure 2.1) sont présents dans les désintégrations possédant un $\pi^{0}$ dans l'état final (et constitue l'unique diagramme en arbre pour $\left.B^{0} \rightarrow \rho^{0} \pi^{0}\right)$. Ils sont attendus supprimés par un facteur d'environ $1 /(3 \sqrt{2})$ par rapport aux arbres non-supprimés de couleur ${ }^{3}$.

Les diagrammes sous-dominants sont montrés sur la figure 2.1: il s'agit des diagrammes pingouins électrofaibles ${ }^{4}$ ou supprimés par la règle Okubo-Zweig-Iizuka ${ }^{5}$ (OZI) [25]. Nous négligeons ces diagrammes par la suite.
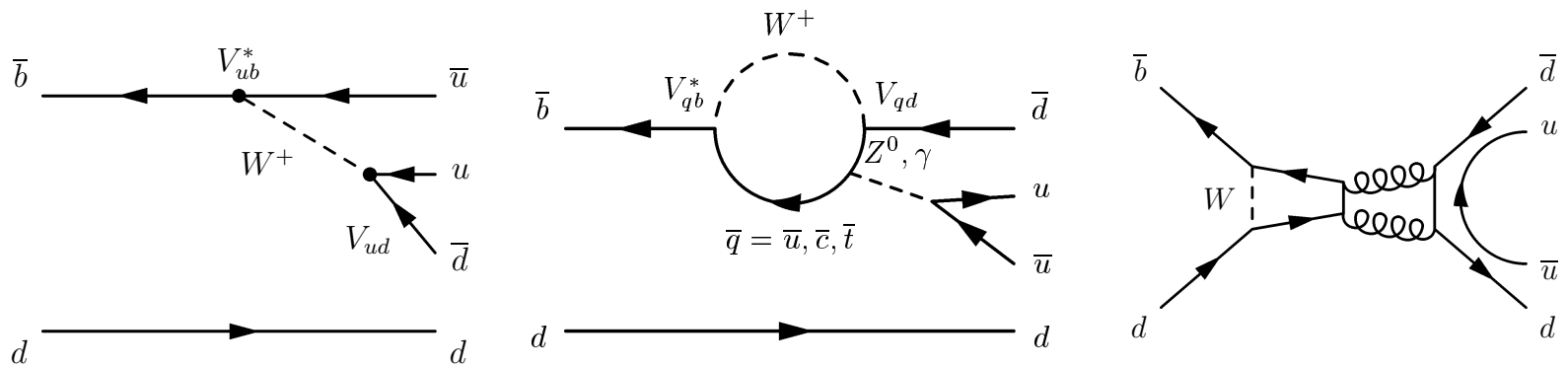

Figure 2.1: Gauche: diagramme en arbre supprimé de couleur. Milieu: diagramme pingouin électrofaible. Droite: diagramme pingouin supprimé $O Z I$.

Les amplitudes des désintégrations $B \rightarrow \rho \pi$ sont dérivées dans l'éq. 1.46. En absorbant le facteur $q / p$ dans les amplitudes correspondantes au $\bar{B}^{0}$ nous pouvons éliminer une phase globale $e^{-i \beta}$. Nous obtenons alors les expressions suivantes:

$$
\begin{array}{ll}
A^{+-}\left(B^{0} \rightarrow \rho^{+} \pi^{-}\right) & =e^{-i \alpha} T^{+-}+P^{+-}, \\
A^{-+}\left(B^{0} \rightarrow \rho^{-} \pi^{+}\right) & =e^{-i \alpha} T^{-+}+P^{-+}, \\
A^{00}\left(B^{0} \rightarrow \rho^{0} \pi^{0}\right) & =e^{-i \alpha} T^{00}+P^{00}, \\
q / p \bar{A}^{+-}\left(\bar{B}^{0} \rightarrow \rho^{+} \pi^{-}\right) & =e^{+i \alpha} T^{-+}+P^{-+}, \\
q / p \bar{A}^{-+}\left(\bar{B}^{0} \rightarrow \rho^{-} \pi^{+}\right) & =e^{+i \alpha} T^{+-}+P^{+-}, \\
q / p \bar{A}^{00}\left(\bar{B}^{0} \rightarrow \rho^{0} \pi^{0}\right) & =e^{+i \alpha} T^{00}+P^{00} .
\end{array}
$$

${ }^{3}$ Le facteur $1 / 3$ pour la suppression de couleur, et le facteur $1 / \sqrt{2}$ pour le facteur d'isospin

${ }^{4}$ Le rapport de l'amplitude des pingouins électrofaibles sur celle des pingouins forts est de l'ordre de:

$$
\frac{\alpha_{Q E D}\left(m_{b}\right) \times m_{t}^{2} / m_{W}^{2}}{\alpha_{Q C D}\left(m_{b}\right) \times \ln \left(m_{t}^{2} / m_{W}^{2}\right)} \simeq \frac{1 / 133 \times 4.7}{0.22 \times \ln (4.7)} \simeq 0.1
$$

${ }^{5}$ Cette règle interdit la création ou l'annihilation de paires de quark-antiquark à l’intérieur d'un hadron. 
De même, pour les $B$ chargés:

$$
\begin{aligned}
& A^{+0}\left(B^{+} \rightarrow \rho^{+} \pi^{0}\right)=1 / \sqrt{2}\left(e^{-i \alpha} T^{+0}+P^{+0}\right), \\
& A^{0+}\left(B^{+} \rightarrow \rho^{0} \pi^{+}\right)=1 / \sqrt{2}\left(e^{-i \alpha} T^{0+}+P^{0+}\right), \\
& A^{-0}\left(B^{-} \rightarrow \rho^{-} \pi^{0}\right)=\bar{A}^{+0}=1 / \sqrt{2}\left(e^{+i \alpha} T^{+0}+P^{+0}\right), \\
& A^{0-}\left(B^{-} \rightarrow \rho^{0} \pi^{-}\right)=\bar{A}^{0+}=1 / \sqrt{2}\left(e^{+i \alpha} T^{0+}+P^{0+}\right),
\end{aligned}
$$

où le facteur $1 / \sqrt{2}$ est dî au fait que deux combinaisons parmi les trois pions de l'état final $\pi^{+} \pi^{-} \pi^{ \pm}$) peuvent créer le $\rho$. On peut dériver des relations entre ces amplitudes grâce à la symétrie d'isospin.

\subsubsection{Les relations d'isospin}

On peut utiliser la symétrie d'isospin $S U(2)$ pour isoler la contribution des diagrammes en arbre à l'amplitude totale [27, 28], permettant ainsi de mesurer $\sin \left(2 \alpha \pm \delta_{T}\right)$ tel que décrit dans l'éq. 1.63.

Dans la désintégration $B \rightarrow \rho \pi$, on peut distinguer deux étapes se comportant différemment aux yeux de la symétrie d'isospin:

- la première étape correspond à celle représentée sur les diagrammes de la figure 2.1, c'est-à-dire à la production via un processus arbre ou pingouin des quatre quarks de l'état final $(\{\bar{u}, \bar{d}, u\}$, et le quark spectateur $d$ (resp. $u$ ) pour les désintégrations des $B$ neutres (resp. chargés)). Cette étape fait intervenir les interactions électrofaibles et engendre donc des sauts d'isospin. Nous commençons par exclure le quark spectateur $d$, et déterminons les valeurs d'isospin possible pour l'état final $\{\bar{u}, \bar{d}, u\}$ :

- diagramme en arbre: le $W$ émet un iso-vecteur $\left(I_{3}(u \bar{d})=1\right)$, qui se combine avec le quark $\bar{u}\left(I_{3}(\bar{u})=1 / 2\right)$ issu du quark $\bar{b}$. L'état final peut donc avoir les valeurs d'isospin $I_{f}(\bar{u}, \bar{d}, u)=3 / 2$ ou $I_{f}(\bar{u}, \bar{d}, u)=1 / 2$.

- diagramme pingouin fort: le quark $d$ sortant de la boucle a un isospin $I_{3}=1 / 2$. Le gluon émis de la boucle est un iso-singlet (il couple de façon égale à $u$ et $d$ ). L'état final a donc la valeur d'isospin $I_{f}(\bar{u}, \bar{d}, u)=1 / 2$.

En incluant le quark spectateur $d$ d'isospin $I_{f}(d)=-1 / 2$, on obtient finalement que les diagrammes en arbre génèrent $I_{f}=0,1,2$ par des transitions $\Delta I=1 / 2$ et $3 / 2^{6}$, et que les diagrammes pingouins forts génèrent $I_{f}=0,1$ par des transitions $\Delta I=1 / 2$.

Dans une deuxième étape, on dérive des relations entre les amplitudes de différentes désintégrations $B \rightarrow \rho \pi$ afin d'isoler la composante $\Delta I=3 / 2$ où seuls les diagrammes en arbre contribuent ${ }^{7}$.

- la seconde étape correspond à l'hadronisation en $\rho^{+} \pi^{-}$des quatre quarks produits dans la première étape via l'interaction forte: cette deuxième étape conserve donc l'isospin. On peut ainsi décomposer l'amplitude $\left\langle\rho^{+} \pi^{-}\left|H_{\text {hadr. }}\right| \bar{u} d u, \bar{d}\right\rangle$ en amplitudes de différents

\footnotetext{
${ }^{6}$ Les diagrammes pingouins électrofaibles mènent au même résultat: le quark $d$ sortant de la boucle a un isospin $I_{3}=1 / 2$. Le $Z^{0}$ ou le $\gamma$ émis de la boucle peut être un iso-singlet ou un iso-vecteur, puisque la charge des quarks intervient dans le couplage du $\gamma / Z^{0}$ à la paire $\bar{q} q$. L'état final peut donc avoir les valeurs d'isospin $I_{f}(\bar{u}, \bar{d}, u)=3 / 2$ ou $I_{f}(\bar{u}, \bar{d}, u)=1 / 2$.

${ }^{7}$ Puisqu'on néglige les pingouins électrofaibles.
} 
$\Delta I$ et $I_{f}$. Le calcul est détaillé dans la réf. [33], et l'on obtient la décomposition suivante:

$$
\begin{aligned}
& A^{+0}=\frac{1}{2} \sqrt{\frac{3}{2}} A_{3 / 2,2}-\frac{1}{2} \frac{1}{\sqrt{2}} A_{3 / 2,1}+\frac{1}{\sqrt{2}} A_{1 / 2,1} \\
& A^{0+}=\frac{1}{2} \sqrt{\frac{3}{2}} A_{3 / 2,2}+\frac{1}{2} \frac{1}{\sqrt{2}} A_{3 / 2,1}-\frac{1}{\sqrt{2}} A_{1 / 2,1} \\
& A^{+-}=\frac{1}{2} \frac{1}{\sqrt{3}} A_{3 / 2,2}+\frac{1}{2} A_{3 / 2,1}+\frac{1}{2} A_{1 / 2,1}+\frac{1}{\sqrt{6}} A_{1 / 2,0} \\
& A^{-+}=\frac{1}{2} \frac{1}{\sqrt{3}} A_{3 / 2,2}-\frac{1}{2} A_{3 / 2,1}-\frac{1}{2} A_{1 / 2,1}+\frac{1}{\sqrt{6}} A_{1 / 2,0} \\
& A^{00}=\frac{1}{\sqrt{3}} A_{3 / 2,2}-\frac{1}{\sqrt{6}} A_{1 / 2,0}
\end{aligned}
$$

où les amplitudes $A_{\Delta I, I_{f}}$ correspondent à la transition des quarks $\bar{u} d u, \bar{d}$ ayant un nombre d'isospin $\Delta I$ (c'est-à-dire que la transition de la première étape a engendré un changement d'isospin $\Delta I$ ) vers le système $\rho^{+} \pi^{-}$ayant un nombre d'isospin $I_{f}$.

On voit que l'on dispose de 5 amplitudes $A_{i j}$ pour 4 amplitudes d'isospin $A_{\Delta I, I_{f}}$, et l'on peut donc dériver les deux relations pentagonales suivantes entre les désintégrations des $B$ neutres et $B$ chargés:

$$
\begin{aligned}
& \sqrt{2}\left[A^{+0}+A^{0+}\right]=A^{+-}+A^{-+}+2 A^{00} \\
& \sqrt{2}\left[\bar{A}^{+0}+\bar{A}^{0+}\right]=\bar{A}^{+-}+\bar{A}^{-+}+2 \bar{A}^{00} .
\end{aligned}
$$

D'autre part, si l'on injecte le fait que les diagrammes en arbre correspondent à $\left(\Delta I, I_{f}\right)=$ $(3 / 2,2),(3 / 2,1),(1 / 2,1),(1 / 2,0)$ et les pingouins forts à $\left(\Delta I, I_{f}\right)=(1 / 2,1),(1 / 2,0)$, on en déduit les relations $d$ 'isospin suivantes:

$$
\begin{aligned}
\sqrt{2}\left[T^{+0}+T^{0+}\right] & =T^{+-}+T^{-+}+2 T^{00}, \\
P^{00} & =-1 / 2\left(P^{+-}+P^{-+}\right), \\
P^{+0} & =+\left(P^{+-}-P^{-+}\right), \\
P^{0+} & =-\left(P^{+-}-P^{-+}\right) .
\end{aligned}
$$

Les deux analyses utilisant les relations d'isospin sont détaillées dans les sections suivantes.

\subsubsection{L'analyse d'isospin de $B \rightarrow \rho \pi$ en (quasi) deux corps}

L'analyse d'isospin [27, 28] consiste à mesurer les rapports d'embranchement et asymétries $C P$ des modes $\rho \pi$ issus des $B$ neutres et chargés.

Par la suite, on utilisera les notations suivantes pour les rapports d'embranchement $\mathcal{B}$ et asymétries $C P$ directes non dépendantes du temps $\mathcal{A}$ des différents modes $h h^{\prime}(h=$ $\left.\rho, \pi, K, K^{*}\right)$ :

$$
\begin{aligned}
\mathcal{B}^{h h^{\prime}} & \equiv \mathcal{B}\left(B \rightarrow h h^{\prime}\right)=\frac{1}{2}\left(\left|A_{h h^{\prime}}\right|^{2}+\left|\bar{A}_{h h^{\prime}}\right|^{2}\right), \\
\mathcal{A}^{h h^{\prime}} & \equiv \frac{\left|\bar{A}_{h h^{\prime}}\right|^{2}-\left|A_{h h^{\prime}}\right|^{2}}{\left|\bar{A}_{h h^{\prime}}\right|^{2}+\left|A_{h h^{\prime}}\right|^{2}} .
\end{aligned}
$$


Le tableau 2.1 fait le compte des observables apportées par ces différentes mesures: l'analyse dépendante du temps des modes issus des $B$ neutres amène 3 observables par charge du $\rho$ : les coefficients des cosinus et sinus, et le taux total d'événements pour une charge donnée. Pour le mode $B^{0} \rightarrow \rho^{ \pm} \pi^{\mp}$, ceci correspond aux paramètres $\mathcal{B}\left(B^{0} \rightarrow \rho^{ \pm} \pi^{\mp}\right), C, S$, $\Delta C, \Delta S$ et $A_{\mathrm{CP}}\left(B^{0} \rightarrow \rho^{ \pm} \pi^{\mp}\right)$, et pour le mode $B^{0} \rightarrow \rho^{0} \pi^{0}$, aux paramètres $\mathcal{B}\left(B^{0} \rightarrow \rho^{0} \pi^{0}\right)$, $C_{\rho^{0} \pi^{0}}$ et $S_{\rho^{0} \pi^{0}}$. Les mesures reliées aux modes issus des $B$ chargés apportent 2 observables par charge de $\rho$ : il s'agit des rapports d'embranchement $\mathcal{B}\left(B^{+} \rightarrow \rho^{+} \pi^{0}\right)$ et $\mathcal{B}\left(B^{+} \rightarrow \rho^{0} \pi^{+}\right)$, et des asymétries $C P$ directe $\mathcal{A}\left(B^{+} \rightarrow \rho^{+} \pi^{0}\right)$ et $\mathcal{A}\left(B^{+} \rightarrow \rho^{0} \pi^{+}\right)$.

Comme indiqué dans le tableau, une fois que l'on a tenu compte des relations d'isospin liant certaines de ces inconnues, toutes ces mesures apportent suffisamment d'observables pour déterminer toutes les inconnues entrant en jeu, dont $\alpha$ à 8 ambiguïtés près.

\begin{tabular}{|c|c|c|c|c|}
\hline \multirow{2}{*}{ Mode } & \multirow{2}{*}{ Amplitudes } & \multirow{2}{*}{ Inconnues } & \multicolumn{2}{|c|}{ Observables } \\
\hline & & & Analyse $d$ 'isospin & Analyse Dalitz \\
\hline$B^{0} \rightarrow \rho^{+} \pi^{-}$ & $e^{-i \alpha} T^{+-}+P^{+-}$ & 5 & 3 & 2 \\
\hline $\bar{B}^{0} \rightarrow \rho^{+} \pi^{-}$ & $e^{+i \alpha} T^{-+}+P^{-+}$ & 4 & - & 2 \\
\hline$B^{0} \rightarrow \rho^{-} \pi^{+}$ & $e^{-i \alpha} T^{-+}+P^{-+}$ & - & 3 & 2 \\
\hline $\bar{B}^{0} \rightarrow \rho^{-} \pi^{+}$ & $e^{+i \alpha} T^{+-}+P^{+-}$ & - & - & 2 \\
\hline \multicolumn{2}{|c|}{ Phase globale } & -1 & - & -1 \\
\hline \multicolumn{2}{|c|}{ Total utilisant $B^{0} \rightarrow \rho^{ \pm} \pi^{\mp}$} & 7 & 5 & 6 \\
\hline$B^{0} \rightarrow \rho^{0} \pi^{0}$ & $e^{-i \alpha} T^{00}+P^{00}$ & 4 & 3 & 2 \\
\hline $\bar{B}^{0} \rightarrow \rho^{0} \pi^{0}$ & $e^{+i \alpha} T^{00}+P^{00}$ & - & - & 2 \\
\hline \multicolumn{2}{|c|}{ Relations d'isospin } & -2 & - & - \\
\hline \multicolumn{2}{|c|}{ Total utilisant $B^{0} \rightarrow \rho^{ \pm, 0} \pi^{\mp, 0}$} & 9 & 8 & 10 \\
\hline$B^{+} \rightarrow \rho^{+} \pi^{0}$ & $e^{-i \alpha} T^{+0}+P^{+0}$ & 4 & 1 & 1 \\
\hline$B^{+} \rightarrow \rho^{0} \pi^{+}$ & $e^{-i \alpha} T^{0+}+P^{0+}$ & - & 1 & 1 \\
\hline$B^{-} \rightarrow \rho^{-} \pi^{0}$ & $e^{-i \alpha} T^{-0}+P^{-0}$ & 4 & 1 & 1 \\
\hline$B^{-} \rightarrow \rho^{0} \pi^{-}$ & $e^{-i \alpha} T^{0-}+P^{0-}$ & - & 1 & 1 \\
\hline \multicolumn{2}{|c|}{ Relations d'isospin } & -6 & - & - \\
\hline Total utilisa & 111ssi les $B$ chargés & 11 & 12 & 14 \\
\hline
\end{tabular}

Tablea1 2.1: Nombre d'inconnues et d'observables apportées par les analyses d'isospin et Dalitz. Une phase globale est soustraite des inconnues ainsi que des observables de l'analyse Dalitz (qui sont des amplitudes, et donc des nombres complexes. Les autres observables, rapports d'embranchement et paramètres temporels sont réels).

Deux niveaux d'hypothèses expérimentales sont envisageables dans cette analyse: on peut considérer la désintégration $B \rightarrow \rho \pi$ en deux corps, ou en quasi deux corps:

- dans l'approche en deux corps, qui est l'approche suivie dans l'analyse détaillée dans le chapitre 4 , les résonances $\rho^{ \pm}$sont supposées de largeur négligeable. Cela implique que les états finals $\rho^{+} \pi^{-}$et $\rho^{-} \pi^{+}$n'interfèrent pas, et que l'on peut donc les reconstruire séparément par des coupures dans l'espace de phase. En réalité, la largeur de la résonance $\rho$ est grande $(150 \mathrm{MeV})$, et pour réaliser cette analyse, il faut donc couper expérimentalement les principales interférences, et évaluer l'effet des interférences résiduelles. 
- Dans l'approche en quasi-deux corps, on tient compte des interférences entre $\rho^{+} \pi^{-}$et $\rho^{-} \pi^{+}$grâce à une intégration sur l'espace de phase. Les détails de cette analyse sont exposés dans la réf. [33], et un résumé est donné ici:

- Un événement est associé à la catégorie $\rho^{+} \pi^{-}$ou $\rho^{-} \pi^{+}$selon un certain critère (qui peut être celui de la réf. [33], ou celui utilisé dans l'analyse en deux corps décrite dans le chapitre 4 de la partie II) .

- Dans la catégorie $\rho^{+} \pi^{-}$, l'amplitude est la somme de Breit-Wigner du $\rho^{+}$(de même signe), du $\rho^{-}$(de signe opposé) et de l'interférence entre les deux. Le poids de chacun de ces termes est donné par les intégrales des Breit-Wigner correspondantes modulées par l'efficacité de sélection dans la catégorie $\rho^{+} \pi^{-}$pour chaque type.

- Les coefficients $C, \Delta C, S, \Delta S$ et $A_{\mathrm{CP}}$ de la section 1.5.3 sont maintenant fonction de ces sommes pondérées, alors que dans l'analyse en deux corps, on négligeait les événements de signes opposés et les interférences.

Certains ingrédients nécessaires à l'analyse d'isospin de $B \rightarrow \rho \pi$ sont déjà mesurés, d'autres non: l'analyse temporelle de $B^{0} \rightarrow \rho^{ \pm} \pi^{\mp}$ est décrite dans le chapitre 4 et la réf. [39], les limites actuelles sur $B^{0} \rightarrow \rho^{0} \pi^{0}$ sont données dans les réf. [46, 47, 48], le rapport d'embranchement de $B^{+} \rightarrow \pi^{+} \rho^{0}$ (trois pions chargés dans l'état final) a été mesuré [142, 143,144 ], et enfin celui de $B^{+} \rightarrow \pi^{0} \rho^{+}$(deux pions neutres dans l'état final) n'a pas encore été mesuré.

\subsubsection{L'analyse Dalitz de $B^{0} \rightarrow \pi^{+} \pi^{-} \pi^{0}$}

Dans l'analyse Dalitz (dépendante du temps et de l'espace de phase), on considère désormais la désintégration en trois-corps $B^{0} \rightarrow \pi^{+} \pi^{-} \pi^{0}$ : en plus d'utiliser la distribution temporelle des événements (comme dans l'analyse d'isospin), on utilise la distribution dans l'espace de phase (c'est-à-dire dans le plan Dalitz $\left(m_{\pi^{+} \pi^{0}}^{2}, m_{\pi^{-} \pi^{0}}^{2}\right)$ ). Ceci nous permet de profiter des interférences entre les différentes résonances $\rho$ intermédiaires. L'avantage de cette méthode est de pouvoir accéder à la mesure de $\alpha$ sans ambiguïté entre 0 et $\pi$ sans avoir besoin de mesurer toutes les désintégrations des $B$ chargés. Mais c'est une analyse complexe, car elle requiert la maîtrise expérimentale et phénoménologique du diagramme Dalitz.

On définit l'amplitude de $B^{0}\left(\bar{B}^{0}\right) \rightarrow \pi^{+} \pi^{-} \pi^{0}$ comme la somme de résonances intermédiaires se désintégrant en paires de pions:

$$
\begin{aligned}
& A_{3 \pi} \equiv f_{+} A^{+-}+f_{-} A^{-+}+f_{0} A^{00} \\
& \bar{A}_{3 \pi} \equiv f_{+} \bar{A}^{+-}+f_{-} \bar{A}^{-+}+f_{0} \bar{A}^{00},
\end{aligned}
$$

oì $f_{i}$ est le facteur de forme du $\pi$ incluant la dépendance angulaire des produits de désintégration.

En considérant la violation $C P$ dans le mélange négligeable $(|q / p|=1)$, les taux dépendants du temps $f_{+}\left(f_{-}\right)$quand le méson étiquetant a une saveur $B^{0}\left(\bar{B}^{0}\right)$ sont donnés par:

$$
f_{ \pm}(\Delta t)=\frac{e^{-|\Delta t| / \tau_{B^{0}}}}{4 \tau_{B^{0}} N}\left[1 \pm \frac{2 \operatorname{Im}\left[\bar{A}_{3 \pi} A_{3 \pi}^{*}\right]}{\left|A_{3 \pi}\right|^{2}+\left|\bar{A}_{3 \pi}\right|^{2}} \sin \left(\Delta m_{B} \Delta t\right) \mp \frac{\left|A_{3 \pi}\right|^{2}-\left|\bar{A}_{3 \pi}\right|^{2}}{\left|A_{3 \pi}\right|^{2}+\left|\bar{A}_{3 \pi}\right|^{2}} \cos \left(\Delta m_{B} \Delta t\right)\right]
$$


où la normalisation $N$ est donnée par:

$$
\begin{aligned}
N & =\frac{1}{2}\left[\left(\sum_{\kappa \in\{+,-, 0\}}\left|A^{\kappa}\right|^{2}\right)\left\langle\left|\left(f_{+}+f_{-}+f_{0}\right) / 3\right|^{2}\right\rangle+\sum_{\kappa<\sigma \in\{+,-, 0\}} \operatorname{Re} 2 A^{\kappa} A^{\sigma *}\left\langle f_{\kappa} f_{\sigma}^{*}\right\rangle\right. \\
& \left.+\left(\sum_{\kappa \in\{+,-, 0\}}\left|\bar{A}^{\kappa}\right|^{2}\right)\left\langle\left|\left(f_{+}+f_{-}+f_{0}\right) / 3\right|^{2}\right\rangle+\sum_{\kappa<\sigma \in\{+,-, 0\}} \operatorname{Re} 2 \bar{A}^{\kappa} \bar{A}^{\sigma *}\left\langle f_{\kappa} f_{\sigma}^{*}\right\rangle\right] .
\end{aligned}
$$

En insérant les amplitudes 2.12, on obtient pour les expressions suivantes pour les coefficients de l'éq. 2.13:

$$
\begin{aligned}
\left|A_{3 \pi}\right|^{2} \pm\left|\bar{A}_{3 \pi}\right|^{2} & =\sum_{\kappa \in\{+,-, 0\}}\left|f_{\kappa}\right|^{2} U_{\kappa}^{ \pm}+2 \sum_{\kappa<\sigma \in\{+,-, 0\}}\left(\operatorname{Re}\left[f_{\kappa} f_{\sigma}^{*}\right] U_{\kappa \sigma}^{ \pm, \operatorname{Re}}-\operatorname{Im}\left[f_{\kappa} f_{\sigma}^{*}\right] U_{\kappa \sigma}^{ \pm, \operatorname{Im}}\right) \\
\operatorname{Im}\left(\bar{A}_{3 \pi} A_{3 \pi}^{\star}\right) & =\sum_{\kappa \in\{+,-, 0\}}\left|f_{\kappa}\right|^{2} I_{\kappa}+\sum_{\kappa<\sigma \in\{+,-, 0\}}\left(\operatorname{Re}\left[f_{\kappa} f_{\sigma}^{*}\right] I_{\kappa \sigma}^{\operatorname{Im}}+\operatorname{Im}\left[f_{\kappa} f_{\sigma}^{*}\right] I_{\kappa \sigma}^{\mathrm{Re}}\right), \quad(2.15)
\end{aligned}
$$

avec:

$$
\begin{aligned}
U_{\kappa}^{ \pm} & =\left|A^{\kappa}\right|^{2} \pm\left|\bar{A}^{\kappa}\right|^{2}, \\
U_{\kappa \sigma}^{ \pm, \operatorname{Re}(\operatorname{Im})} & =\operatorname{Re}(\operatorname{Im})\left[A^{\kappa} A^{\sigma *} \pm \bar{A}^{\kappa} \bar{A}^{\sigma *}\right], \\
I_{\kappa} & =\operatorname{Im}\left[\bar{A}^{\kappa} A^{\kappa *}\right], \\
I_{\kappa \sigma}^{\operatorname{Re}} & =\operatorname{Re}\left[\bar{A}^{\kappa} A^{\sigma *}-\bar{A}^{\sigma} A^{\kappa *}\right], \\
I_{\kappa \sigma}^{\operatorname{Im}} & =\operatorname{Im}\left[\bar{A}^{\kappa} A^{\sigma *}+\bar{A}^{\sigma} A^{\kappa *}\right],
\end{aligned}
$$

où l'on a utilisé les notations raccourcies $A^{+} \equiv A^{+-}, A^{-} \equiv A^{-+}$et $A^{0} \equiv A^{00}$.

Les Eqs. 2.16-2.20 correspondent à 27 observables inter-dépendantes permettant de mesurer le module et la phase de chaque amplitude $A^{x y}$ et $\bar{A}^{x y}$ (soit 12 observables indépendantes). Comme montré dans le tableau 2.1, ceci apporte suffisamment d'observables pour contraindre les 11 inconnues des $B$ neutres et mesurer ainsi $\alpha$. L'ajout des $B$ chargés permet de sur-contraindre l'ajustement, mais n'est plus absolument nécessaire.

En pratique, on a le choix entre ajuster les 27 coefficients $U$ et $I$, ou les modules et phases des amplitudes $A^{i j}{ }^{8}$, ou enfin directement les modules et phases des amplitudes arbre et pingouin, et $\alpha$. Il existe des avantages et des inconvénients pour chaque méthode: la fonction de vraisemblance est Gaussienne pour les deux premières, mais ne l'est pas forcément pour la dernière. D'autre part, les deux premières méthodes ne sont pas sensibles aux solutions miroirs en $\alpha$ (définies dans le tableau 9.4 de la référence [33]), alors que la dernière l'est.

\subsubsection{Limite sur $\left|\alpha-\alpha_{\text {eff }}^{ \pm}\right|$utilisant $S U(2)$}

Charles [24] a dérivé la limite suivante sur $\alpha$ en utilisant la symétrie $S U(2)$ et les modes $B^{0} \rightarrow \rho^{0} \pi^{0}$ et $B^{0} \rightarrow \rho^{ \pm} \pi^{\mp}:$

$$
\left|\alpha-\alpha_{\text {eff }}^{ \pm}\right| \leq \frac{1}{2} \arccos \left[\frac{1}{\sqrt{1-A_{\rho \pi}^{ \pm}}}\left(1-4 \frac{\mathcal{B}^{00}}{\mathcal{B}^{ \pm}}\right)\right],
$$

\footnotetext{
${ }^{8} \mathrm{Si}$ l'une des ces deux premières méthodes est choisie, la détermination de l'angle $\alpha$ se fait alors dans une deuxième étape
} 
avec:

$$
\begin{aligned}
2 \alpha_{\text {eff }}^{ \pm} & \equiv \arg \left(\frac{q}{p} \frac{\bar{A}^{+-}+\bar{A}^{-+}}{A^{+-}+A^{-+}}\right), \\
\mathcal{B}^{00} & \equiv \frac{1}{2}\left(\left|A^{00}\right|^{2}+\left|\bar{A}^{00}\right|^{2}\right), \\
A_{\rho \pi}^{ \pm} & \equiv \frac{\left|\bar{A}^{+-}+\bar{A}^{-+}\right|^{2}-\left|A^{-+}+A^{+-}\right|^{2}}{\left|\bar{A}^{+-}+\bar{A}^{-+}\right|^{2}+\left|A^{-+}+A^{+-}\right|^{2}}, \\
\mathcal{B}^{ \pm} & \equiv \frac{1}{4}\left(\left|A^{+-}+A^{-+}\right|^{2}+\left|\bar{A}^{-+}+\bar{A}^{+-}\right|^{2}\right) .
\end{aligned}
$$

Les deux derniers paramètres sont liés aux interférences entre $\rho^{+} \pi^{-}$et $\rho^{-} \pi^{+}$et ne sont donc accessibles que par l'analyse Dalitz. Cette limite peut être utilisée dans le cas où $\mathcal{B}^{00}$ est trop faible pour contribuer à l'analyse Dalitz.

\subsection{Hypothèse théorique plus poussée: la symétrie $S U(3)$}

On peut contraindre les amplitudes pingouins de $B^{0} \rightarrow \rho^{ \pm} \pi^{\mp}$ en utilisant les modes $B^{0} \rightarrow$ $\rho^{-} K^{+}$et $B^{0} \rightarrow \mathrm{K}^{*+} \pi^{-}$et la symétrie $S U(3)$ comme suggéré par Charles [24]. Les amplitudes pour ces deux modes s'écrivent:

$$
\begin{aligned}
A_{K}^{+-} \equiv A\left(B^{0} \rightarrow K^{*+} \pi^{-}\right) & =\left|V_{u s} V_{u b}^{*}\right| e^{i \gamma} T_{K}^{+-}+\left|V_{t s} V_{t b}^{*}\right| P_{K}^{+-}, \\
A_{K}^{+} \equiv A\left(B^{0} \rightarrow \rho^{-} K^{+}\right) & =\left|V_{u s} V_{u b}^{*}\right| e^{i \gamma} T_{K}^{+}+\left|V_{t s} V_{t b}^{*}\right| P_{K}^{-+}
\end{aligned}
$$

Puisque $\left|V_{u s} V_{u b}^{*}\right| \ll\left|V_{t s} V_{t b}^{*}\right|$, l'amplitude de ces modes est dominée par la contribution des diagrammes pingouins.

En utilisant la symétrie $S U(3)$ et en négligeant les contributions supprimées par $1 / N_{c}$ et $1 / m_{b}$ (du type pingouin OZI montré sur le diagramme de droite sur la figure 2.1 ), on a ${ }^{9}$ :

$$
\left|P_{\rho \pi}^{+-}\right|=\left|P_{K}^{+-}\right|, \quad\left|P_{\rho \pi}^{-+}\right|=\left|P_{K}^{-+}\right| .
$$

Ceci entraîne les limites suivantes sur $\alpha$ :

$$
\begin{aligned}
\left|\alpha-\alpha_{\text {eff }}^{+-}\right| \leq \frac{1}{2} \arccos \left[\frac{1}{\sqrt{1-A_{\rho \pi}^{+-2}}}\left(1-2 \lambda^{2} \frac{\mathcal{B}_{K^{*} \pi}^{+-}}{\mathcal{B}^{+-}}\right)\right], \\
\left|\alpha-\alpha_{\text {eff }}^{-+}\right| \leq \frac{1}{2} \arccos \left[\frac{1}{\sqrt{1-A_{\rho \pi}^{-+2}}}\left(1-2 \lambda^{2} \frac{\mathcal{B}_{\rho K}^{-+}}{\mathcal{B}^{-+}}\right)\right] .
\end{aligned}
$$

où

$$
\begin{aligned}
\mathcal{B}^{+-} & \equiv \frac{1}{2}\left(\left|A^{+-}\right|^{2}+\left|\bar{A}^{-+}\right|^{2}\right), \\
\mathcal{B}^{-+} & \equiv \frac{1}{2}\left(\left|A^{-+}\right|^{2}+\left|\bar{A}^{+-}\right|^{2}\right) .
\end{aligned}
$$

\footnotetext{
${ }^{9}$ Par rapport à la définition originale de $P^{+-}$et $P^{-+}$où ces paramètres contiennent le facteur $\left|V_{t d} V_{t b}^{*}\right|$ (cf. définition juste après l'éq. 1.46), on définit $P_{\rho \pi}^{+-}=P^{+-} /\left|V_{t d} V_{t b}^{*}\right|$ et $P_{\rho \pi}^{-+}=P^{-+} /\left|V_{t d} V_{t b}^{*}\right|$ indépendants des éléments de matrice CKM.
} 


\section{Partie II}

\section{Etude de la violation de CP dans l'expérience $B A B A R$}





\section{Survol des chapitres à venir}

Comme expliqué en introduction, le système des mésons $B$ fournit un environnement propice à l'étude de la violation de $C P$. Il permet, entre autres (et du moins, en principe), de mesurer les angles $\alpha, \beta$ et $\gamma$ du triangle d'unitarité. Nous nous intéressons ici à la mesure de l'angle $\alpha$ (cf. Sec. 1.5.3) grâce aux modes $B^{0} \rightarrow \rho^{ \pm} \pi^{\mp}, B^{0} \rightarrow \rho^{-} K^{+}, B^{0} \rightarrow \rho^{0} \pi^{0}$ et $B^{0} \rightarrow a_{0}^{ \pm} \pi^{\mp}$, ainsi qu'aux mesures des rapports d'embranchement $\mathcal{B}$ de ces modes. Les analyses réalisées, ainsi que la statistique utilisée, sont résumées dans le tableau.

\begin{tabular}{l|cll}
\hline Mode & $\# \overline{B B}\left(10^{6}\right)$ & Type d'analyse & Ref. \\
\hline$B^{0} \rightarrow \rho^{ \pm} \pi^{\mp}$ & 88.86 & Analyse temporelle et mesure de $\mathcal{B}$ & {$[38],[39]$} \\
$B^{0} \rightarrow \rho^{-} K^{+}$ & 88.86 & Analyse temporelle et mesure de $\mathcal{B}$ & {$[38],[39]$} \\
$B^{0} \rightarrow \rho^{0} \pi^{0}$ & 88.86 & Mesure de $\mathcal{B}$ & {$[40]$} \\
$B^{0} \rightarrow \pi^{+} \pi^{-} \pi^{0}$ & 88.86 & Analyse Dalitz (premiers pas) & {$[41]$} \\
$B^{0} \rightarrow a_{0}^{ \pm} \pi^{\mp}$ & 22.74 & Mesure de $\mathcal{B}$ & {$[42],[43]$} \\
\hline
\end{tabular}

Tablea1 2.2: Analyses présentées dans ce document: on donne la statistique utilisée, le type d'analyse réalisée, et les références à consulter.

Les transitions non-charmées $b \rightarrow u$ sont supprimées par rapport aux transitions charmées $b \rightarrow c$ par au moins un facteur $\left(\left|V_{u b}\right| /\left|V_{c b}\right|\right)^{2} \simeq 1 \%$ [44]. Il s'agit donc de modes rares dont les rapports d'embranchement sont aux alentours de $10^{-5}$ à $10^{-6}$. Les mesures précédemment réalisées sur ces modes sont résumées dans le tableau 2.3.

\begin{tabular}{llccc}
\hline Mode & Collab. & $\mathcal{B}\left(10^{-6}\right)$ & $\# \overline{B B}\left(10^{6}\right)$ & Ref. \\
\hline$B^{0} \rightarrow \rho^{ \pm} \pi^{\mp}$ & CLEO & $27.6_{-7.4}^{+8.4} \pm 4.2$ & 7.0 & {$[45]$} \\
& BABAR & $28.9 \pm 5.4 \pm 4.3$ & 22.7 & {$[43]$} \\
& Belle & $20.8_{-6.3}^{+6.0}+2.8$ & 31.9 & {$[46]$} \\
\hline$B^{0} \rightarrow \rho^{0} \pi^{0}$ & CLEO & $<5.1 @ 90 \%$ CL (stat. seulement) & 8.3 & {$[47]$} \\
& Belle & $<5.3 @ 90 \%$ CL (stat. seulement) & 31.9 & {$[46]$} \\
& BABAR & $<10.6 @ 90 \%$ CL (stat. et syst.) & 22.7 & {$[48]$} \\
\hline$B^{0} \rightarrow \rho^{-} K^{+}$ & BELLE & $15.8_{-4.6}^{+5.1 .7}+1.7$ & 23 & {$[49]$} \\
\hline$B^{0} \rightarrow a_{0}^{ \pm} \pi^{\mp}$ & \multicolumn{5}{c}{ Non mesuré } \\
\hline
\end{tabular}

Tableau 2.3: Mesure des rapports d'embranchement $\mathcal{B}$ des modes étudiés réalisée avant ou pendant le travail présenté ici. La statistique utilisée est donnée dans la colonne \# $\overline{B B}$.

Dans l'expérience $B A B A R$, les mésons $B$ sont produits par paires à la résonance du $\Upsilon(4 S)$. Dans le centre de masse de ce dernier, les deux $B$ ne volent en moyenne que sur $30 \mu \mathrm{m}$, distance expérimentalement difficile à mesurer. Or la distance entre les deux $B$ est un ingrédient 
crucial pour l'étude de la violation de $C P$ car elle fournit la mesure du temps $\Delta E$ écoulé entre les deux désintégrations nécessaire aux analyses temporelles (voir Eq. 1.57 et 1.58). Ce problème est résolu en boostant la paire de $B$ : les faisceaux $e^{+}$et $e^{-}$de PEP-II ont respectivement une énergie de 3.1 et $9 \mathrm{GeV}$, menant à un facteur de boost $\beta \gamma=0.55$, et à une séparation moyenne entre les deux $B$ d'environ $250 \mu \mathrm{m}$ dans le laboratoire.

La figure 1.3 montre la topologie d'une désintégration de $\Upsilon(4 S)$ à $B A B A R$ : on reconstruit exclusivement la désintégration d'un des deux $B$ (noté $B_{\text {rec }}$ ) en un état final accessible à la fois par un $B^{0}$ et par un $\bar{B}^{0}$ (par exemple $\rho^{ \pm} \pi^{\mp}$ ). Le $B_{\text {rec }}$ est utilisé pour l'étude de la violation de $C P$. L'autre $B$ (noté $B_{\text {sav }}$ ) est utilisé pour déterminer la saveur de $B_{\text {rec }}$ à $\Delta t=0$. Nous exploitons les corrélations entre produits de désintégration et saveur du $B$ pour étiqueter la saveur de $B_{\mathrm{sav}}$.

Puisque les modes analysés sont des modes rares, ils sont noyés dans du bruit de fond. Nous développons donc une analyse multidimensionnelle (utilisant plusieurs variables) de lutte contre le bruit de fond pour parvenir à extraire le signal.

Enfin, les paramètres $C P$, les taux de signal et de bruit de fond, et les autres paramètres sont extraits des données grâce à un ajustement par maximum de vraisemblance.

Toutes ces étapes sont exposées dans la suite pour les analyses des modes $B^{0} \rightarrow \rho^{ \pm} h^{\mp}$, $B^{0} \rightarrow \rho^{0} \pi^{0}$ et $B^{0} \rightarrow a_{0}^{ \pm} \pi^{\mp}$ : je détaillerai plus particulièrement les parties des analyses que j'ai personnellement réalisées, et passerai plus rapidement sur les parties accomplies par des collaborateurs ${ }^{10}$.

Le plan de cette partie s'organise comme suit: le dispositif expérimental (PEP-II et $B A B A R$ ) est présenté dans le chapitre 3 , puis l'analyse dépendante du temps de $B^{0} \rightarrow \rho^{ \pm} h^{\mp}$ est décrite en détails dans le chapitre 4 . La mesure du rapport d'embranchement de $B^{0} \rightarrow \rho^{0} \pi^{0}$ et les premiers pas dans l'analyse Dalitz de $B^{0} \rightarrow \pi^{+} \pi^{-} \pi^{0}$ sont exposés dans les chapitre 5 et 6 . Enfin, la mesure du rapport d'embranchement de $B^{0} \rightarrow a_{0}^{ \pm} \pi^{\mp}$ est résumée dans l'annexe A.

\footnotetext{
${ }^{10}$ Ces parties sont les suivantes: tests sur le bruit de fond charmé (section 4.8.2.2), validations a posteriori sur le signal $B^{0} \rightarrow \rho^{-} K^{+}$(section 4.13.2), vérification de la présence de $\rho$ dans le signal (section 4.13.3) et quelques unes des erreurs systématiques (section 4.14). D’autre part, certains développements utilisés dans l'analyse sont issus d'autres analyses $\left(\sin 2 \beta\right.$ et $\left.B^{0} \rightarrow \pi^{+} \pi^{-}\right)$, comme l'étiquetage (section 4.4), la fonction de résolution de $\Delta t$ (section 4.9.1.5) et les distributions de l'angle Cherenkov $\theta_{C}$ (section 4.9.1.4).
} 


\section{Chapitre 3}

\section{Dispositif expérimental}

Ce chapitre expose le dispositif expérimental utilisé par les analyses décrites dans cette thèse: l'accélérateur et l'anneau de stockage PEP-II, ainsi que le détecteur BABAR sont décrits dans les sections 3.1 et 3.2. Ma participation au dispositif expérimental concerne le système de protection contre les radiations du détecteur de traces et de vertex au silicium. La description de ce système, ainsi que de mon travail s'y rapportant, est décrite dans la section 3.3. Enfin, les données et la simulation Monte Carlo utilisées dans les analyses présentées ultérieurement sont détaillées dans les sections 3.4 et 3.5.

\subsection{L'accélérateur et l'anneau de stockage PEP-II}

PEP-II est un collisionneur spécialement construit pour fournir au détecteur BABAR une grande quantité de paires de mésons $B$ (d'où son nom d'usine à $B$ ). Ces paires sont boostées afin que la distance entre les points de désintégrations des deux mésons soit suffisamment grande pour être précisément mesurée.

Dans la phase de prise de données "on-peak", le collisionneur fonctionne avec une énergie dans le centre de masse égale à la masse du $\Upsilon(4 S)$ ( $c f$. figure 3.1 ), soit $10.58 \mathrm{GeV} / \mathrm{c}^{2}$, qui se situe juste au delà du seuil de production de paires $\overline{B B}$. Dans la phase de prise de données "off-peak", l'énergie dans le centre de masse se situe $40 \mathrm{MeV}$ en deçà de la masse du $\Upsilon(4 S)$, où la production de paires $\overline{B B}$ est nulle.

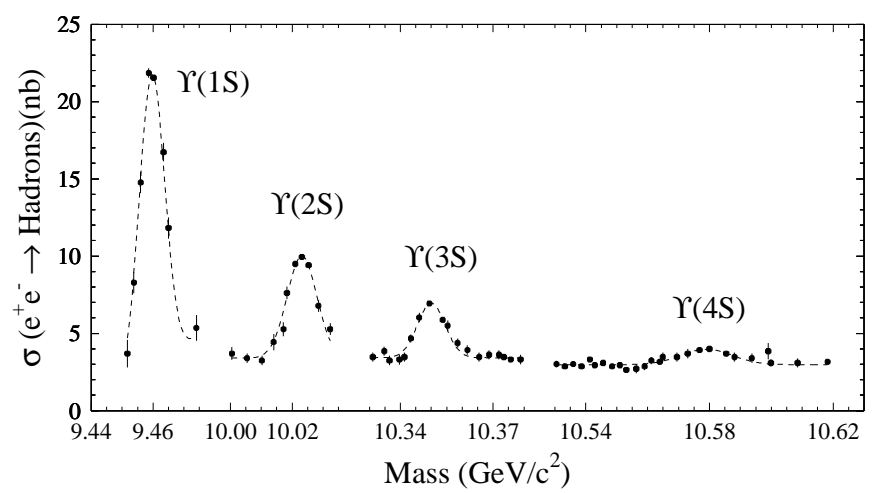

Figure 3.1: Section efficace de production des résonances $\Upsilon$. PEP-II opère sur la résonance $\Upsilon(4 S)\left(10.58 \mathrm{GeV} / \mathrm{c}^{2}\right)$ pour enregistrer les données on-peak, et légèrement en deçà $\left(10.54 \mathrm{GeV} / \mathrm{c}^{2}\right)$ pour les données off-peak. 


\subsubsection{L'accélérateur linéaire}

La figure 3.2 montre l'accélérateur linéaire ("LINear ACcelerator" en anglais, LINAC) et les anneaux du collisionneur PEP-II. Les électrons sont produits au début du LINAC (dans "l'egun"), puis accélérés jusqu'à la section 19 où ils servent à la génération des positrons (dans la "positron source"). Les positrons sont ramenés en section 1 du LINAC, puis sont accélérés jusqu'à la section 4 où ils atteignent une énergie de $3.1 \mathrm{GeV}$ et sont envoyés vers l'anneau de stockage de basse énergie ("Low Energy Ring" en anglais, LER) du collisionneur PEP-II. Quand les électrons ne sont pas utilisés pour la production des positrons, ils sont accélérés jusqu'à la section 8 du LINAC où ils atteignent une énergie de $9 \mathrm{GeV}$ et sont envoyés vers l'anneau de stockage de haute énergie ("High Energy Ring" en anglais, HER).

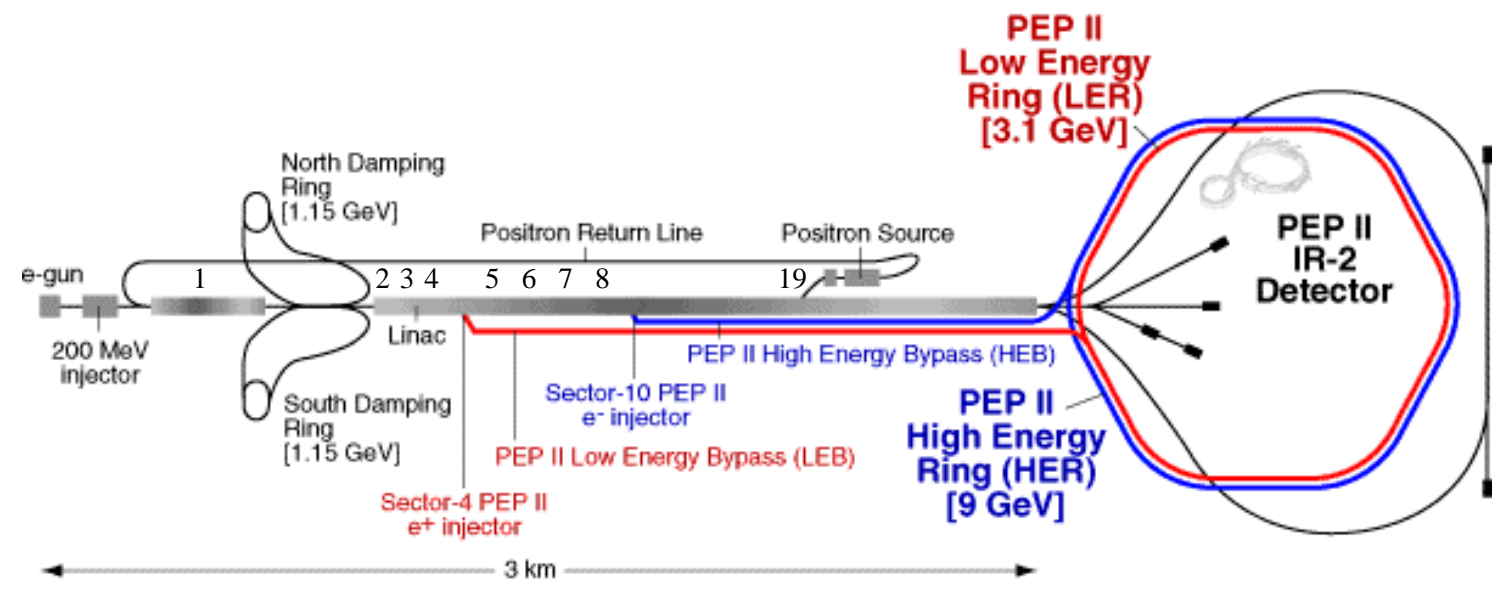

Figure 3.2: L'accélérateur linéaire (LINAC), et le collisionneur PEP-II (voir texte).

Les faisceaux d'électrons et de positrons sont injectés dans les anneaux de PEP-II avec une fréquence de $60 \mathrm{~Hz}$. L'injection se fait verticalement: les orbites des faisceaux déjà en place dans PEP-II sont modifiées par des aimants de façon à ce que les trajectoires des faisceaux injectés soient quasiment parallèles à celles des faisceaux en place. En pratique, l'angle entre ces trajectoires n'est pas nul, ce qui engendre une oscillation bétatron des particules injectées. Les particules se trouvant dans les queues des faisceaux sont alors plus proches des parois des tubes à vide, entraînant la création de gerbes électromagnétiques. Ces oscillations s'amortissent en émettant des radiations synchrotrons au bout de quelques tours dans le collisionneur. Durant ces brèves phases d'injection (environ 5 minutes), le bruit de fond au niveau du détecteur $B A B A R$ est suffisamment intense pour nuire à la prise de données: les hautes-tensions de certains détecteurs sont abaissées par prudence.

\subsubsection{Le collisionneur PEP-II}

La figure 3.3 montre le collisionneur PEP-II: le détecteur BABAR se trouve dans la région 2, où les deux anneaux se croisent (il s'agit de l'unique point de croisement). Les électrons circulent dans le sens des aiguilles d'une montre sur ce schéma, et les positrons dans le sens opposé.

Des collimateurs sont placés sur les orbites des deux faisceaux aux endroits adéquats afin de limiter la quantité de bruit de fond arrivant dans le détecteur BABAR. Ces collimateurs consistent en des mâchoires placées à $10 \sigma$ (où $\sigma$ est la largeur verticale ou horizontale du 


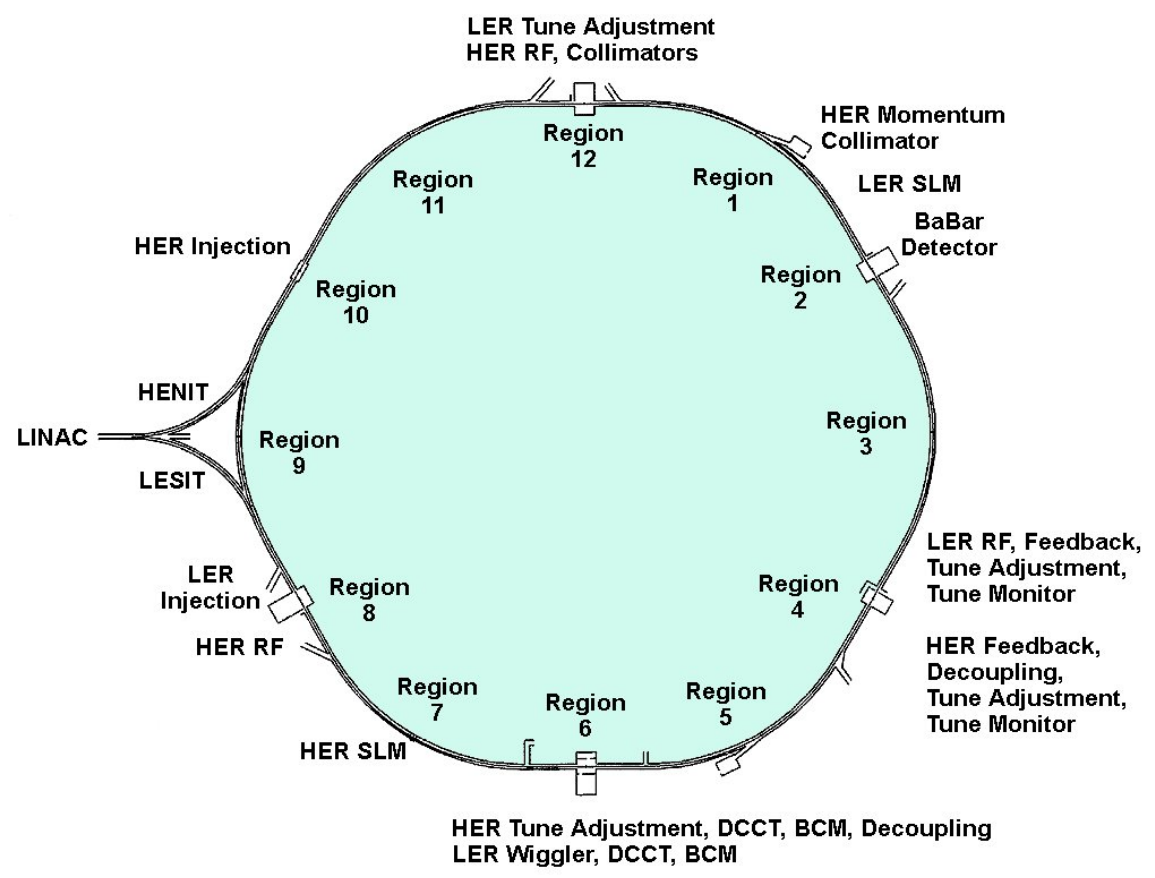

Figure 3.3: Le collisionneur PEP-II (voir texte).

faisceau) de l'orbite, coupant ainsi les queues trop longues des faisceaux engendrées par les oscillations bétatrons et les interactions faisceaux-gaz.

Les paramètres nominaux du collisionneur PEP-II sont donnés dans le tableau 3.1, et les paramètres records (évoluant chaque jour) pour la luminosité instantanée et intégrée dans le tableau 3.2.

\begin{tabular}{l|c}
\hline \hline Paramètre & Valeur nominale \\
\hline Circonférence (m) & 2199,318 \\
Type de particule HER/LER & $e^{-} / e^{+}$ \\
Energie HER/LER $(\mathrm{GeV})$ & $9,0 / 3,1$ \\
Courant HER/LER (A) & $0,75 / 2,15$ \\
Nombre de paquets par faisceau & 1658 \\
Espacement entre paquets (ns) & 4,2 \\
$\sigma_{x}(\mu \mathrm{m})$ & 110 \\
$\sigma_{y}(\mu \mathrm{m})$ & 3,3 \\
$\sigma_{z}(\mathrm{~mm})$ & 9 \\
Angle de croisement (mrad) & 0 \\
Luminosité instantanée $\left(10^{33} \mathrm{~cm}^{-2} \mathrm{~s}^{-1}\right)$ & 3 \\
Luminosité intégrée $\left(p b^{-1} / \mathrm{jour}^{-}\right)$ & 135 \\
\hline \hline
\end{tabular}

Tableau 3.1: Paramètres du collisionneur PEP-II.

\subsubsection{La région d'interaction}

La figure 3.4 montre une vue dans le plan horizontal de la région d'interaction des faisceaux, avec la position des divers aimants présents dans cette région: les aimants quadripolaires 


\begin{tabular}{l|c}
\hline Luminosité instantanée $\left(10^{33} \mathrm{~cm}^{-2} \mathrm{~s}^{-1}\right)$ & 5.11 \\
\hline Luminosité intégrée $\left(\mathrm{pb} \mathrm{b}^{-1}\right):$ & \\
par tour de garde $(8$ heures $)$ & 116.5 \\
par jour & 321.4 \\
par semaine & 1876.3 \\
par mois & 6952.3 \\
\hline
\end{tabular}

Tableau 3.2: Valeurs records pour la luminosité instantanée et intégrée.

Q1 à Q5 servent à focaliser les faisceaux. Les faisceaux entrent en collision de front (avec un angle nul), puis sont séparés immédiatement par les aimants dipolaires B1 (car le premier point de croisement parasite se trouve seulement à $63 \mathrm{~cm}$ du point d'interaction). Les aimants B1 et Q1 sont à l'intérieur du champ magnétique de $B A B A R$, et pénètrent dans le détecteur.

\section{Interaction Region}

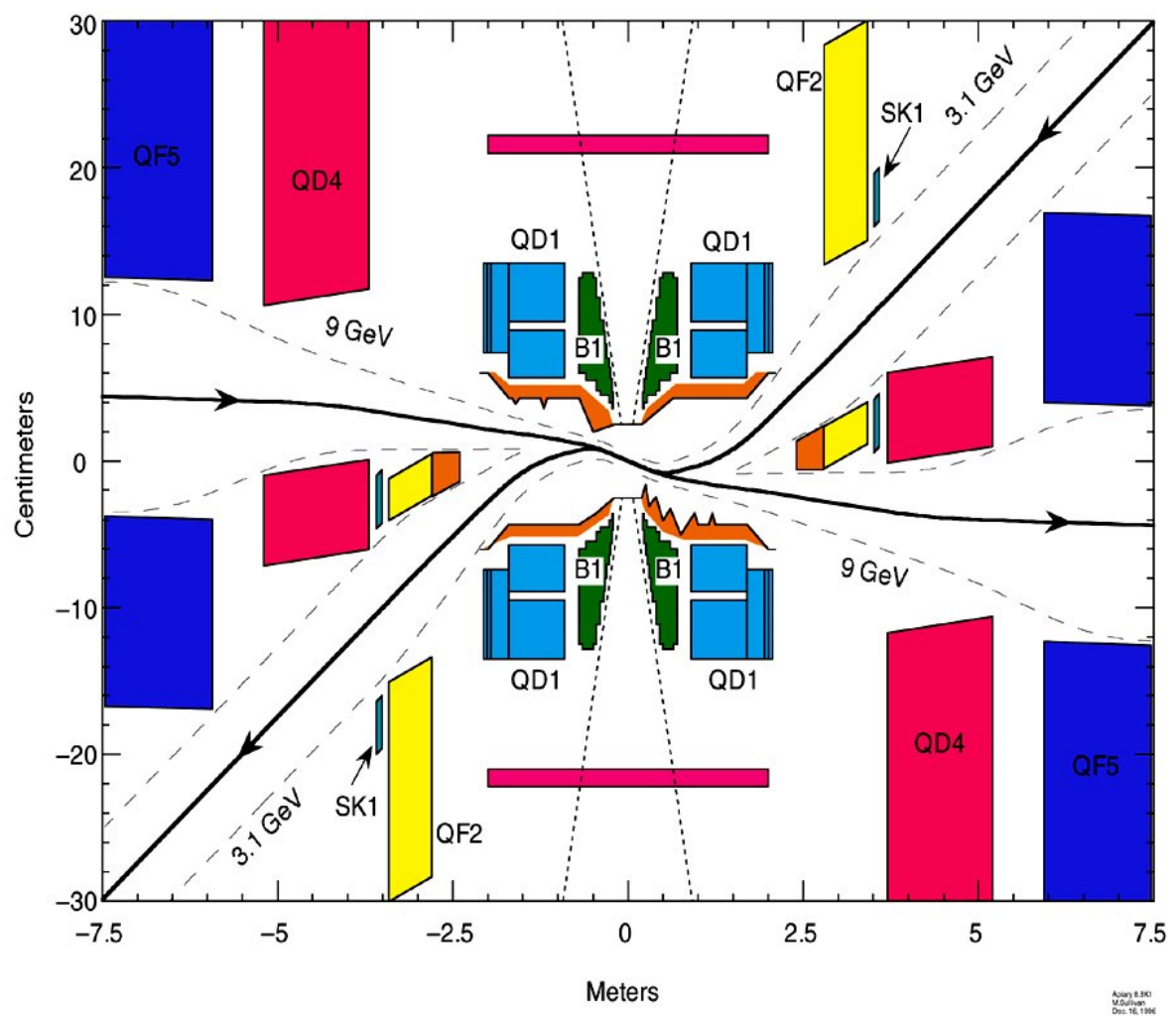

Figure 3.4: Vue dans le plan horizontal de la région d'interaction des faisceaux. L'échelle en $x$ est 25 fois plus grande que l'échelle en z. Les électrons arrivent par la gauche et sortent par la droite. Les lignes en tirets quasi-verticales indiquent les limites de l'acceptance du détecteur BABAR. Le rôle des divers aimants est explicité dans le texte.

\subsubsection{Le système de coordonnées}

Le système de coordonnées utilisé dans BABAR est le suivant:

- l'axe z pointe horizontalement dans la direction du faisceau d'électrons. 
- l'axe y pointe verticalement vers le haut.

- l'axe $x$ point horizontalement vers l'extérieur du collisionneur PEP-II.

La direction du faisceau d'électrons est souvent appelée la direction "avant" ( $c$-à- $d$, vers les z positifs).

\subsection{Le détecteur BABAR}

L'étude de la violation de $C P$ nécessite trois ingrédients: la possibilité de reconstruire des états finals exclusifs, la détermination de la saveur du $B$, et la mesure du temps entre les désintégrations des deux $B$. Ceci pose des contraintes fortes sur la constitution du détecteur $B A B A R$. Il s'agit tout d'abord d'un détecteur asymétrique (puisque les paires $B^{0} \bar{B}^{0}$ sont boostées) devant posséder un détecteur de vertex avec une bonne résolution spatiale, en particulier dans la direction $z$ du boost. Le détecteur doit pouvoir distinguer les différents types de particules chargées $(e, \mu, \pi, K$ et $p)$ dans une grande gamme cinématique (utile à la reconstruction exclusive de plusieurs modes, et à l'étiquetage de la saveur qui utilise des électrons, muons et kaons). Il doit pouvoir détecter des traces chargées sur une large gamme d'impulsion transverse $60 \mathrm{MeV} \lesssim p_{T} \lesssim 4 \mathrm{GeV}$ et les objets neutres $\left(\pi^{0}, \gamma\right.$, hadrons neutres) dans la gamme d'énergie $20 \mathrm{MeV} \lesssim E \lesssim 5 \mathrm{GeV}$.

Le détecteur BABAR (dont une description précise se trouve dans la référence [50]) est représenté sur la figure 3.5. Il est composé, en partant radialement du point d'interaction, d'un détecteur de vertex au silicium (Silicon Vertex Detector, SVT) qui donne des informations sur les traces chargées, d'une chambre à dérive (Drift CHamber, DCH) qui est le principal instrument de mesure de l'impulsion des traces chargées, d'un détecteur à lumière Cherenkov (Detector of Internally Reflected Cherenkov light, DIRC) qui permet l'identification des particules, d'un calorimètre électromagnétique (ElectroMagnetic Calorimeter, EMC) qui donne des informations sur les particules neutres, ainsi que sur les électrons, d'un aimant supraconducteur, et enfin d'un retour de flux instrumenté (Instrumented Return Flux, IFR) qui permet l'identification des muons et des hadrons neutres.

Nous détaillons chacun de ces sous-détecteurs dans la suite.

\subsubsection{Détecteur de vertex au silicium (SVT)}

Le SVT [51] est un détecteur de vertex au silicium permettant la mesure précise des vertex de désintégration des $B$, ainsi que la reconstruction totale (pour les traces de faible impulsion transverse $p_{T}<120 \mathrm{MeV} / \mathrm{c}$ ) ou partielle (en association avec la DCH, pour les traces de plus grande impulsion transverse) des traces chargées. Il permet aussi d'aider à l'identification des particules à partir de leur perte d'énergie par ionisation $d E / d x$.

La figure 3.6 montre la coupe transversale et longitudinale du SVT. Ce dernier est un détecteur cylindrique dont l'angle polaire (dans le référentiel du laboratoire) couvre une acceptance comprise entre 17 et $150^{\circ}$ (l'acceptance étant renforcée dans la direction avant à cause du boost) comprenant 5 couches de détecteurs (340 éléments au total) constitués de micro-pistes semi-conductrices au silicium. Chaque couche contient entre 6 (pour les trois couches internes) et 16(18) (pour les $4(5)^{e}$ couches) modules s'étendant sur toute la longueur du SVT. Chaque module possède son électronique de lecture propre située à son extrémité (à l'extérieur du volume d'acceptance afin de limiter la présence de matériel inactif dans cette région), et contient entre 4 et 8 détecteurs au silicium. Les deux faces des couches sont 


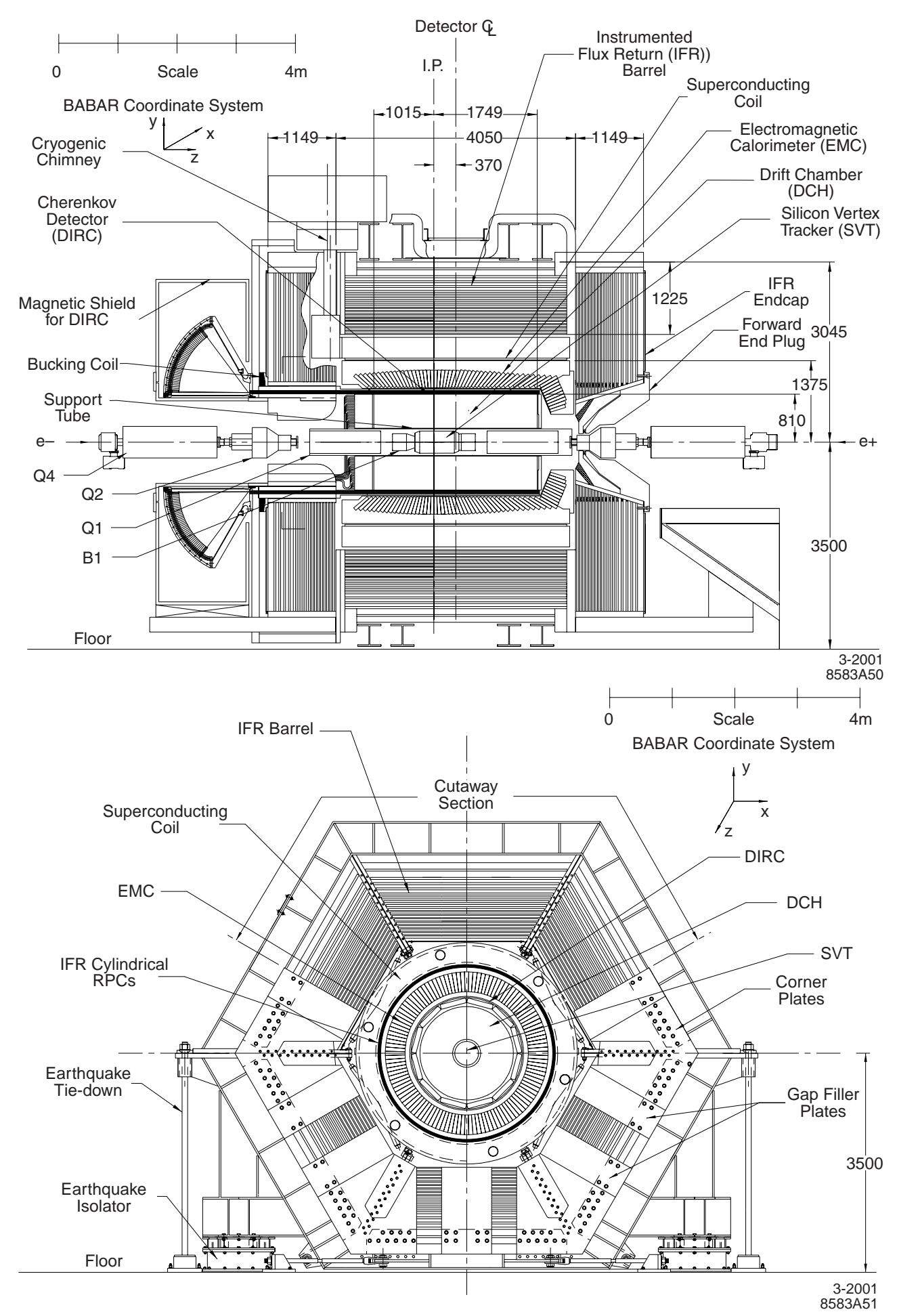

Figure 3.5: Coupe longitudinale (figure du haut) et transversale (figure du bas) du détecteur BABAR. Il est composé, en partant radialement du point d'interaction, du SVT, de la DCH, du DIRC, de l'EMC, d'un aimant supraconducteur, et de l'IFR (voir texte). 
instrumentées: une face possède des micro-pistes orientées parallèlement à la direction $z$ pour la mesure de l'angle azimutal $\phi$ des traces, et l'autre face des micro-pistes orientées perpendiculairement à l'axe $z$ pour la mesure de la coordonnée $z$.

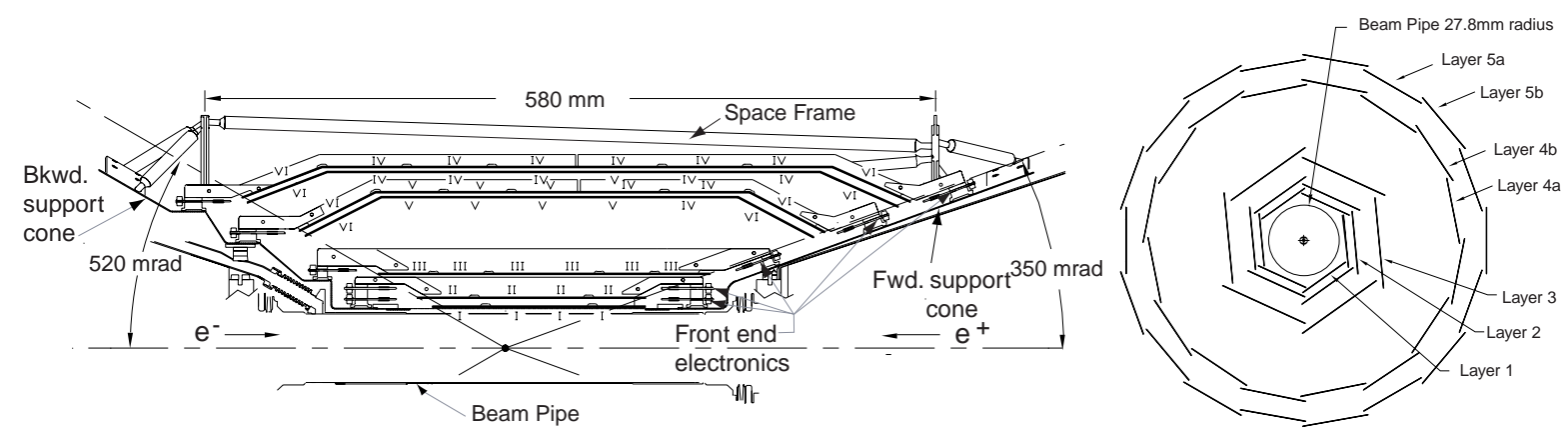

Figure 3.6: Coupe longitudinale (figure de gauche) et transversale (figure de droite) du SVT, composé de 5 couches de détecteurs en silicium. C'est un détecteur asymétrique (avec une meilleure acceptance vers l'avant) disposé juste autour du tube à vide en béryllium.

Les résolutions en $z$ et $\phi$ pour les 5 couches en fonction de l'angle d'incidence de la trace sont données sur la figure 3.7.
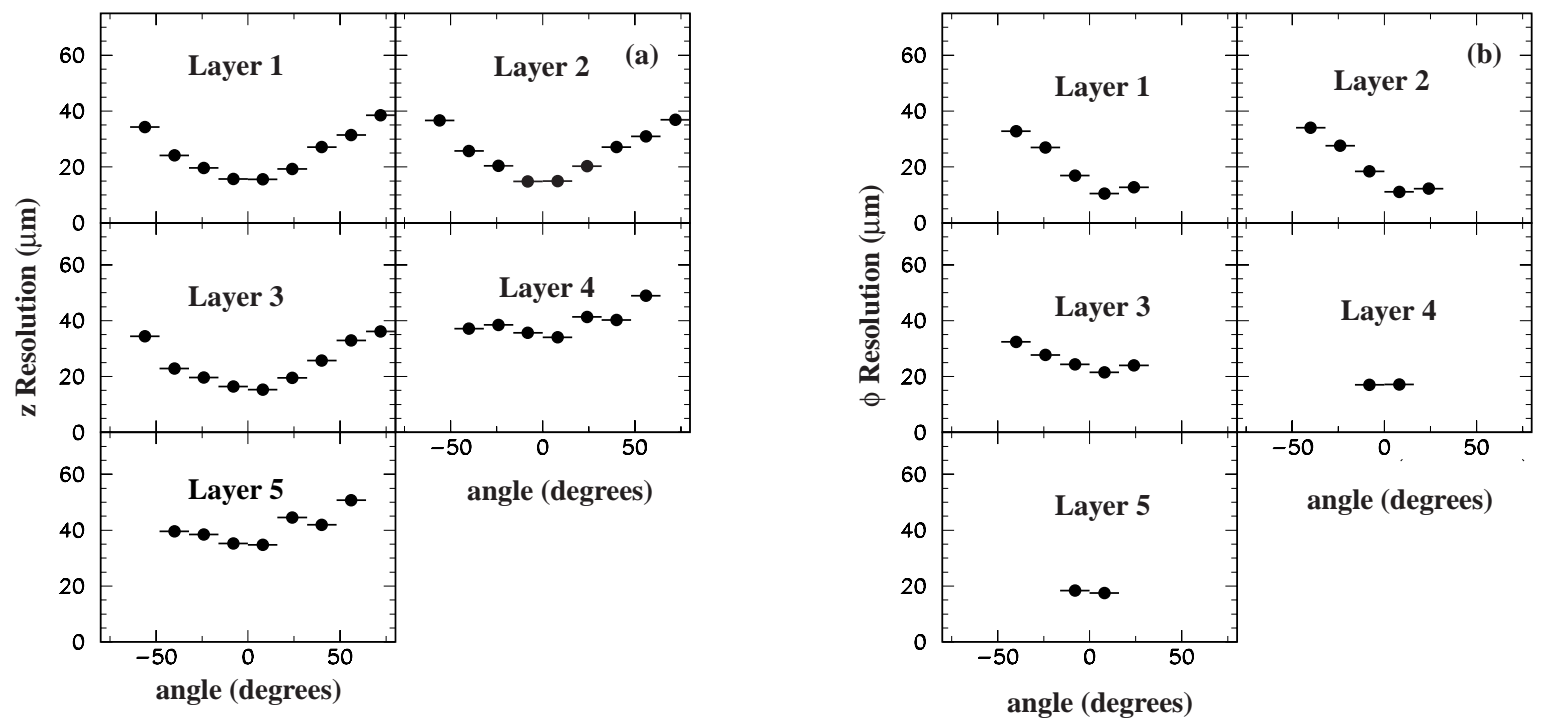

Figure 3.7: Résolution en z (a) et $\phi(b)$ en fonction de l'angle d'incidence de la trace pour chacune des 5 couches du SVT.

Les trois couches internes du SVT servent à la reconstruction du vertex: quand le $B$ est reconstruit exclusivement (c'est le cas du $B_{\text {rec }}$ ), la résolution sur son vertex de désintégration est de $\simeq 60 \mu \mathrm{m}$, alors que la résolution se dégrade à $\simeq 120 \mu \mathrm{m}$ pour le $B_{\text {sav }}$ dont le vertex est reconstruit itérativement à partir des traces restantes dans l'événement une fois celles du

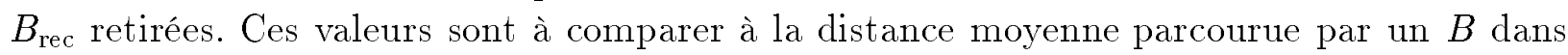
le détecteur, qui est de $250 \mu \mathrm{m}$. Des simulations Monte-Carlo ont montré qu'en gardant la résolution des vertex à la moitié de la distance moyenne parcourue, la dégradation sur l'asymétrie CP mesurée n'est que de 10\% [52]. 
Puisque le SVT est le détecteur le plus proche des faisceaux, il est particulièrement exposé et sensible aux radiations. La section 3.3 décrit plus en détails la résistance du SVT aux radiations, et surtout le système de protection qui lui est associé, dénommé SVTRad.

\subsubsection{Chambre à dérive $(\mathrm{DCH})$}

La chambre à dérive permet de détecter les trajectoires des traces chargées et de mesurer leur impulsion, leur charge et leur position. Elle sert aussi à l'identification des particules pour les faibles impulsions $(\lesssim 1 \mathrm{GeV} / \mathrm{c}$ pour la séparation $\pi / p$ et $\lesssim 0.6 \mathrm{GeV} / \mathrm{c}$ pour $\pi / K$ ) grâce à la mesure $d u d E / d x$. Pour les impulsions plus élevées, cette fonction est remplie en association avec le DIRC, lui fournissant la mesure de l'impulsion et de la position de point d'impact des traces sur le radiateur du DIRC: associé à la mesure de l'angle Cherenkov par le DIRC, ceci permet de déduire la masse de la particule.

La figure 3.8 montre la coupe longitudinale de la $\mathrm{DCH}$ : le détecteur possède une acceptance comprise entre 17 et $156^{\circ}$ (comme pour le SVT, plus importante vers l'avant). Il est composé de 40 couches cylindriques de cellules de fils hexagonales (7104 au total). La paroi interne du détecteur mesure $1 \mathrm{~mm}$ d'épaisseur et est en béryllium ( $0.2 \%$ de longueur de radiation), alors que la paroi externe, qui doit supporter la tension des fils appliquée sur les deux bouchons, mesure $1.5 \mathrm{~mm}$ d'épaisseur et est en carbone $(1.5 \%$ de longueur de radiation). La lecture électronique des couches se fait sur le bouchon arrière afin, encore une fois, de limiter la présence de matériel inactif dans la zone d'acceptance. La chambre est remplie avec un mélange d'hélium et d'isobutane (ce dernier ayant un excellent pouvoir d'ionisation) en proportion $80: 20 \%$. Cette proportion est choisie pour minimiser la longueur de radiation du mélange de gaz.

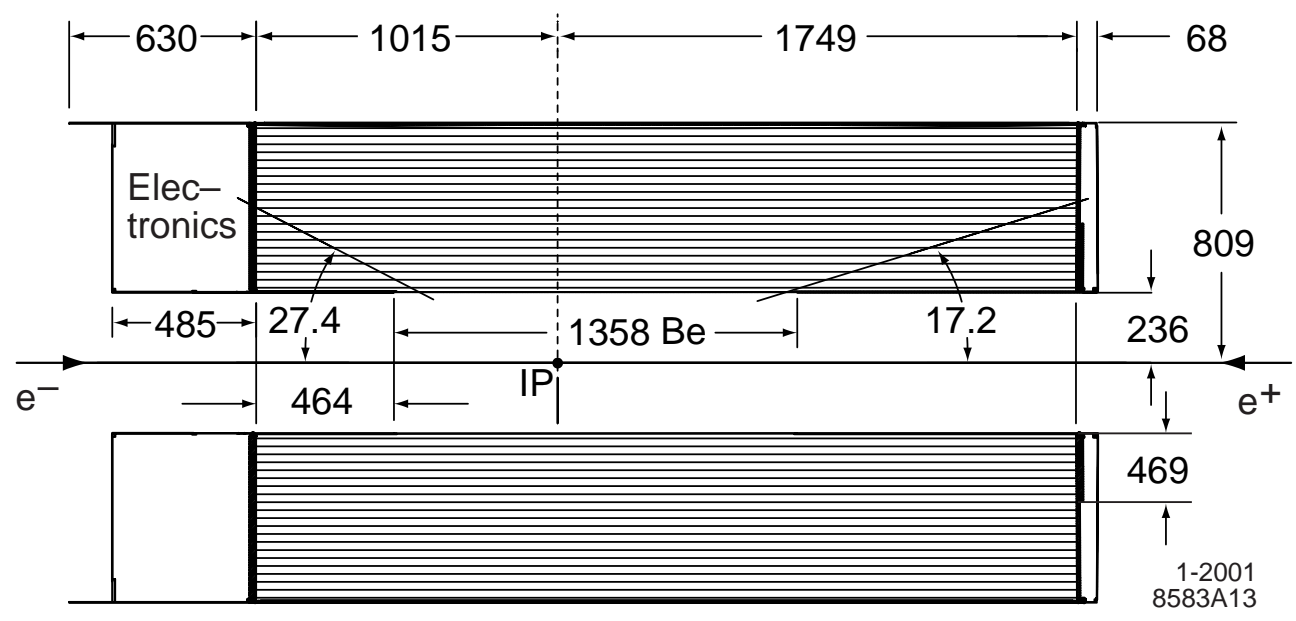

Figure 3.8: Coupe longitudinale de la DCH: comme le SVT, le détecteur est asymétrique vers l'avant. L'électronique est située sur le bouchon arrière.

Les 40 couches sont divisées en 10 super-couches d'angles stéréos ${ }^{1}$ différents. Les couches ayant un angle stéréo nul sont appelées couches radiales et mesurent l'angle de courbure des traces permettant de remonter à leur impulsion. Les couches ayant un angle stéréo nonnul sont appelées couches stéréo et mesurent la position longitudinale des traces avec une résolution d'environ $1 \mathrm{~mm}$.

\footnotetext{
${ }^{1}$ L'angle stéréo est défini par l'angle formé entre les fils de la cellule et l'axe $z$ dans le plan de révolution autour de l'axe $z$.
} 


\begin{tabular}{lcccc}
\hline Type & Matériaul & $\begin{array}{c}\text { Diamètre } \\
(\mu \mathrm{m})\end{array}$ & $\begin{array}{c}\text { Tension } \\
\text { électrique }(\mathrm{V})\end{array}$ & $\begin{array}{c}\text { Tension } \\
\text { mécanique }(\mathrm{g})\end{array}$ \\
\hline sensible & $\mathrm{W}-\mathrm{Re}$ & 20 & 1960 & 30 \\
champ & $\mathrm{Al}$ & 120 & 0 & 155 \\
garde & $\mathrm{Al}$ & 80 & 340 & 74 \\
compensation & $\mathrm{Al}$ & 120 & 825 & 155 \\
\hline
\end{tabular}

Tableau 3.3: Type, matériau, diamètre, tension électrique et mécanique des fils composants les cellules de la DCH (voir texte).

La figure 3.9 montre la structure des super-couches ainsi que des cellules qui les composent: une cellule correspond à un fil central, dit fil sensible, en tungstène rhénié (qui est un matériau suffisamment solide pour supporter la force de traction des deux bouchons de la DCH) recouvert d'or (afin d'éviter la corrosion), entouré de 6 fils de champ en aluminium (matériau léger limitant ainsi la longueur de radiation). Les fils sensibles sont portés à un potentiel de $1960 \mathrm{~V}$ de 1999 à 2001 (une partie des données a été prise avec un potentiel de $1900 \mathrm{~V}$ afin de déterminer le point de fonctionnement optimal) et $1930 \mathrm{~V}$ en 2002 . Les fils de champs ont un potentiel nul. A l'interstice des super-couches, les fils de champ sont remplacés par des fils de garde portés à $340 \mathrm{~V}$, afin d'éviter que les lignes de champ ne se déforment aux frontières des super-couches. Enfin, entre les première et dernière couches et les parois de la $\mathrm{DCH}$, des fils de compensation portés à un potentiel de $825 \mathrm{~V}$ collectent les charges créées dans les parois.

Les tensions sont choisies pour optimiser le gain du gaz ( $c-\grave{a}-d$ le nombre d'électrons voyageant dans une cellule), faire en sorte que le champ soit le plus uniforme possible dans une cellule, et éviter le vieillissement prématuré de la DCH (plus la tension est haute, plus les fils attirent des poussières).

La figure 3.10 montre les performances de la DCH pour l'identification des particules. Le graphe de gauche représente la mesure $\mathrm{d} u d E / d x$ en fonction de l'impulsion transverse. Les points représentent les données, qui sont comparées aux prédictions utilisant la fonction de Bethe-Bloch pour différents types et masses de particules. Le graphe de droite montre la différence entre le $d E / d x$ mesuré dans la $\mathrm{DCH}$ (à une haute tension de $1900 \mathrm{~V}$ ) et attendu pour des événements Bhabha: la résolution est de $7.5 \%$. Pour des muons cosmiques, la résolution est de $6.5 \%$.

La reconstruction des traces chargées passe par un algorithme de filtre de Kalman [53] utilisant des points de la DCH et du SVT: l'algorithme recherche d'abord des traces dans la DCH à partir de segments de traces. Puis par extrapolation, il recherche les segments de traces correspondants dans le SVT. Un ajustement est ensuite réalisé sur la trace complète du SVT et de la DCH, tenant compte de la perte d'énergie des particules dans les détecteurs. Les traces trouvées seulement par un des deux détecteurs sont comparées pour tenter de reconstituer les traces déviées par le matériel se trouvant entre le SVT et la DCH. Enfin, les points restants dans le SVT procèdent par deux algorithmes de traces complémentaires.

La figure 3.11 illustre les performances de la reconstruction des traces par le SVT et la DCH: le graphe de gauche représente l'efficacité de reconstruction des traces en fonction de l'impulsion transverse et de l'angle polaire. L'efficacité est la fraction d'événements pour lesquels la portion dans la DCH d'une trace trouvée dans le SVT a bien été reconstruite. Elle se moyenne à $98 \pm 1 \%$ pour $p_{T}>200 \mathrm{MeV} / \mathrm{c}$ et un angle polaire supérieur à $500 \mathrm{mrad}$. Le graphe de droite en haut montre la résolution sur les paramètres d'impact $d_{0}$ et $z_{0}$ dans les plans $(x, y)$ et $z$ respectivement en fonction de l'impulsion transverse. Enfin, le graphe 

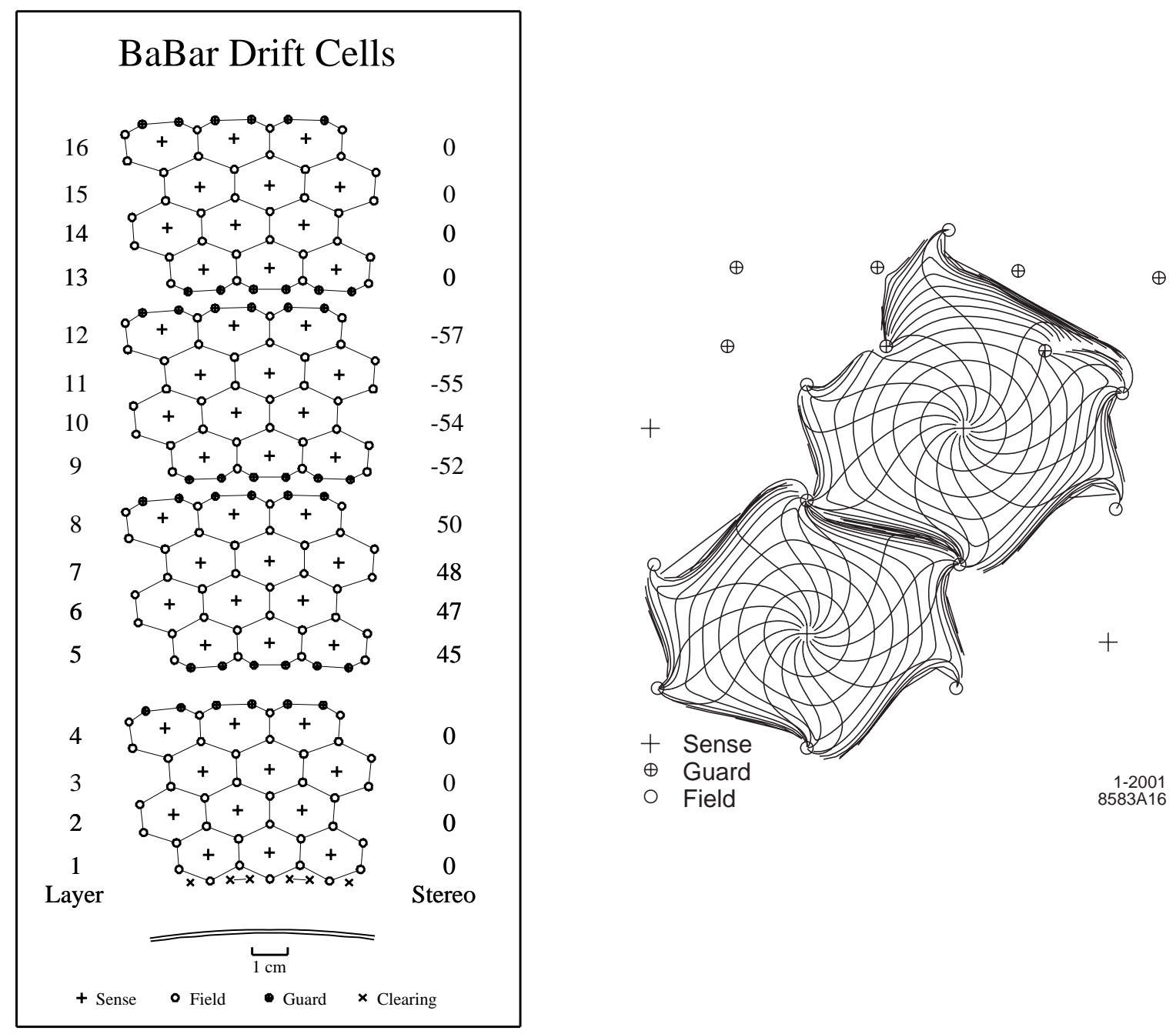

Figure 3.9: Figure de gauche: coupe transversale d'un ensemble de super-couches de la DCH. Une super-couche est composée de 4 couches de cellules axiales (avec un angle stéréo nul) ou stéréo (avec un angle stéréo non-nul). La figure montre aussi la composition d'une cellule: au centre se trouve un fil sensible, entouré de 6 fils de champ. Figure de droite: isochrones de dérive (contours de temps de dérive égaux) des cellules situées sur les couches 3 et 4 d'une super-couche axiale. Les isochrones sont séparés de 100 ns. 

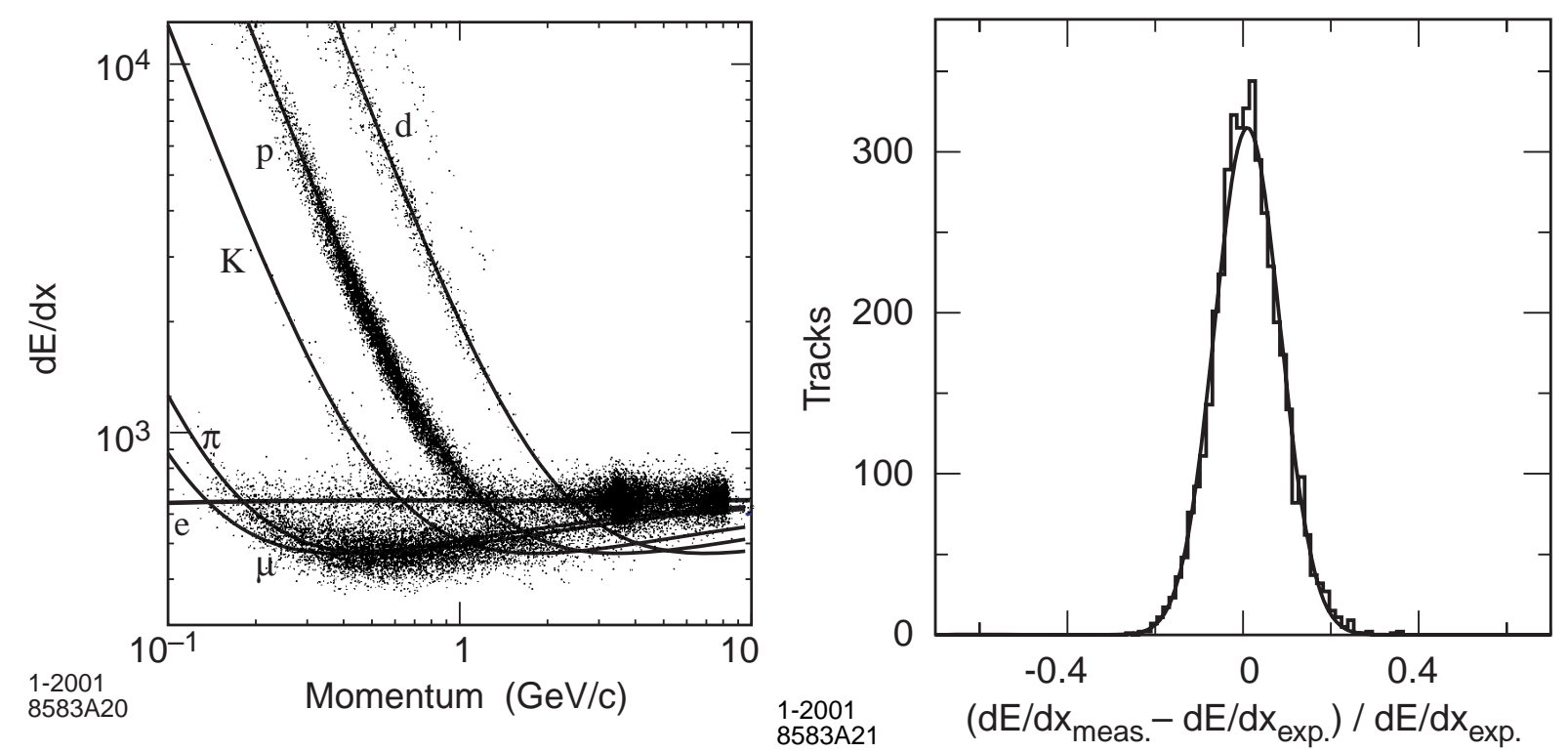

Figure 3.10: Graphe de gauche: mesure $d u d E / d x$ dans la $D C H$ en fonction de l'impulsion. Les données (points) sont comparées aux prédictions de Bethe-Bloch pour diverses hypothèses de particules chargées. Graphe de droite: différence entre le dE/dx mesuré dans la DCH et attendu: la résolution est de $7.5 \%$.

de droite en bas donne la résolution sur l'impulsion transverse en fonction de l'impulsion transverse, qui est modélisée par:

$$
\frac{\sigma_{p_{T}}}{p_{T}}=(0.13 \pm 0.01) \% \times \frac{p_{T}}{(1 \mathrm{GeV} / \mathrm{c})} \oplus(0.45 \pm 0.03) \% .
$$

\subsubsection{Détecteur à lumière Cherenkov (DIRC)}

Le DIRC est destiné à l'identification des hadrons chargés: c'est un détecteur à lumière Cherenkov de conception nouvelle qui permet, connaissant la position et l'angle de l'impact de la trace chargée dans le DIRC, de remonter à l'angle Cherenkov $\theta_{C}$ des particules chargées traversant son radiateur. L'angle Cherenkov est relié à la vitesse $\beta=p / E$ de cette particule par:

$$
\cos \theta_{C}=\frac{1}{\beta n} .
$$

Connaissant l'indice de réfraction du matériau constituant le radiateur, ici le quartz possédant un indice $n=1.473$, et grâce à la mesure de l'impulsion $p$ de la trace par la DCH et le SVT, on peut finalement remonter à la masse $m$ de la particule:

$$
\beta=\frac{p}{E}=\frac{p}{\sqrt{p^{2}+m^{2}}}, \text { soit } m=p \sqrt{n^{2} \cos ^{2} \theta_{C}-1}
$$

Son principe novateur est illustré sur la figure 3.12: le radiateur du détecteur est composé de 144 barres de quartz artificiel (qui résistent mieux aux radiations que le quartz naturel), regroupées en 12 boîtes disposées en anneau autour de la DCH, couvrant ainsi $93 \%$ de l'angle azimutal et un angle polaire comprit entre 25.5 et $147^{\circ}$. La perte d'acceptance azimutale est due à l'espace entre les boîtes. Le rayon interne du DIRC étant de $81 \mathrm{~cm}$ et le champ 

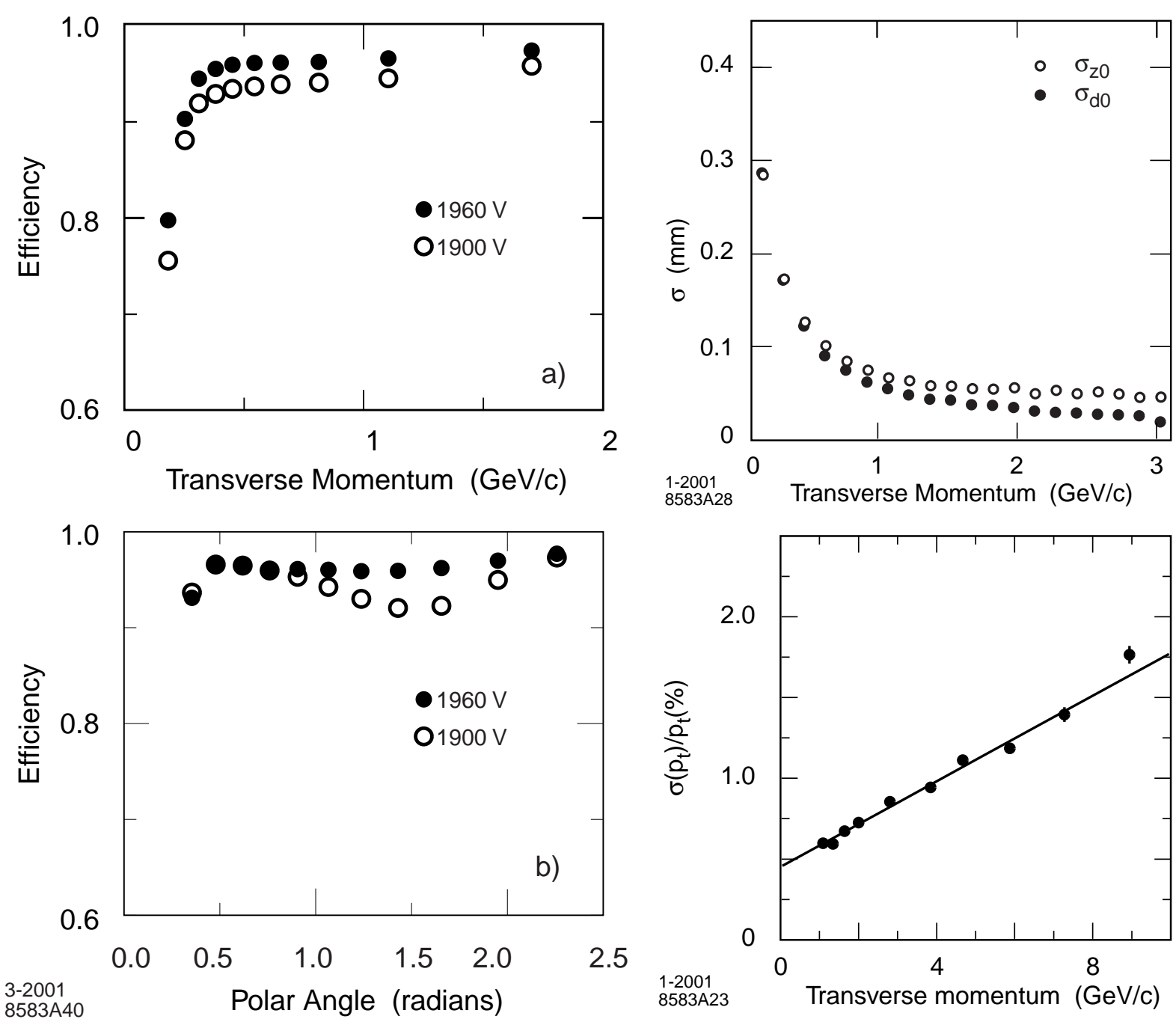

Figure 3.11: Performances de la reconstruction des traces par le SVT et la DCH: Graphes de gauche: efficacité de reconstruction des traces dans la DCH en fonction de a) $\bar{l}$ 'impulsion transverse et b) l'angle polaire, lorsque la DCH fonctionne avec une haute tension de 1900 et $1960 \mathrm{~V}$. L'efficacité est la fraction d'événements pour lesquels la portion dans la DCH d'une trace trouvée dans le SVT a bien été reconstruite. Graphe de droite, haut: ré-

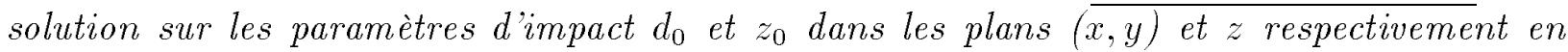
fonction de l'impulsion transverse. Graphe de droite, bas: résolution sur l'impulsion transverse en fonction de l'impulsion transverse. 
magnétique de BABAR de $1.5 \mathrm{~T}$, seules les particules avec une impulsion supérieure à 250 $\mathrm{MeV} / \mathrm{c}$ peuvent atteindre le DIRC.

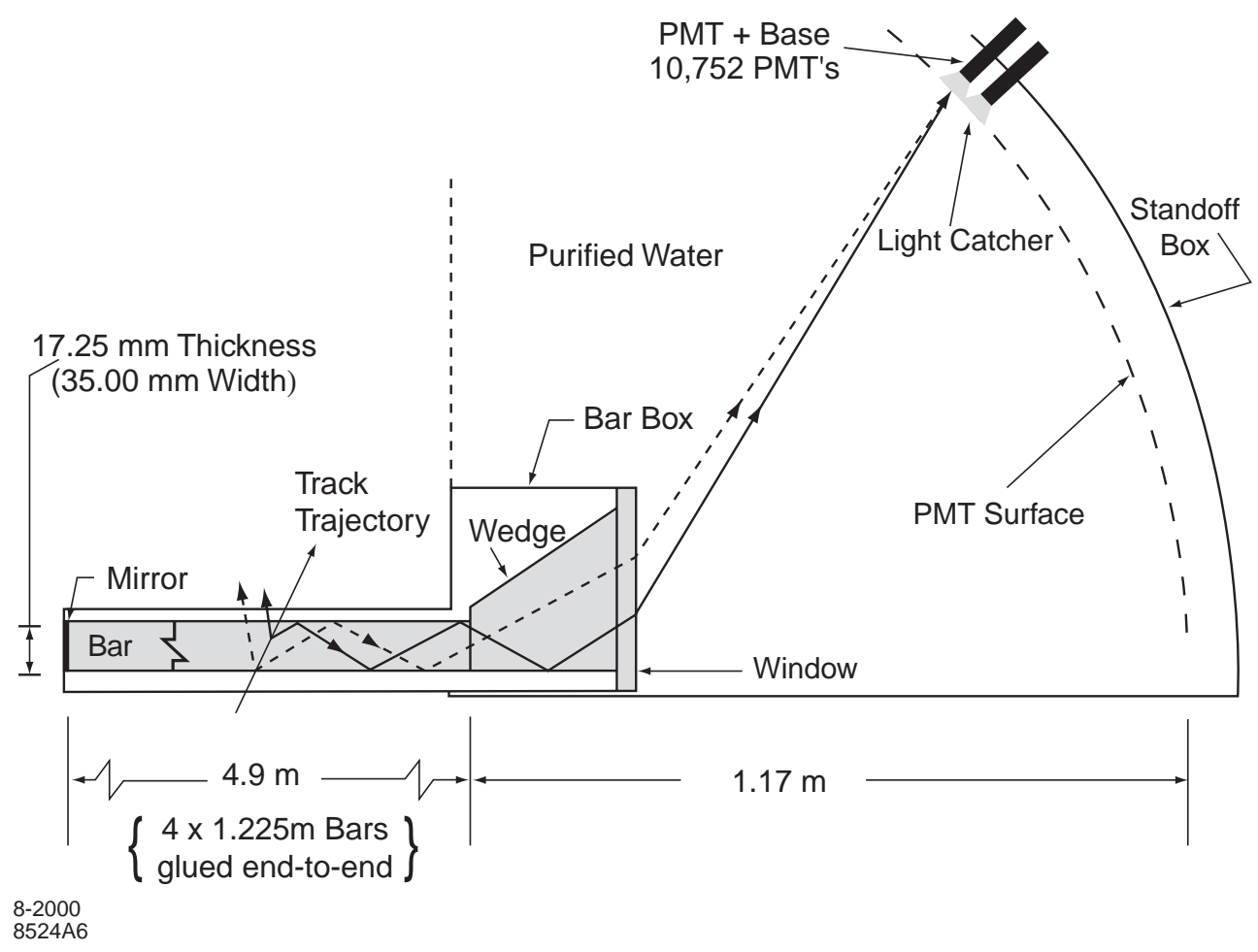

Figure 3.12: Schéma illustrant le principe du DIRC: les photons Cherenkov émis par une particule traversant la barre de quartz (en gris clair) sont réfléchis sur les parois de la barre jusqu'à l'extrémité arrière, où ils passent dans la boîte d'expansion remplie d'eau, puis atteignent les photomultiplicateurs qui tapissent la surface de cette boîte.

Lorsqu'une particule chargée traverse une barre, elle émet un cône de photons Cherenkov d'angle $\theta_{C}$ par rapport à la direction d'incidence de la trace. Le nombre moyen de photons émis en fonction de l'angle polaire est montré sur le graphe de droite de la figure 3.14: entre 20 et 65 photons Cherenkov sont émis selon l'angle. Ce nombre est minimal quand la trace arrive à $90^{\circ}$ dans le radiateur, puisque c'est dans cette configuration qu'elle traverse le moins de quartz. Ces photons sont réfléchis sur les faces internes de la barre jusqu'à son extrémité arrière (les photons émis vers l'avant sont réfléchis sur des miroirs placés à l'extrémité avant des barres). L'angle $\theta_{C}$ est conservé lors des multiples réflexions (entre $\simeq 50$ et $\simeq 300$ ) grâce à l'orthogonalité et la qualité des faces des barres. Afin d'augmenter la résolution de détection, l'extrémité arrière des barres de quartz se termine dans une boîte d'expansion remplie de $6 \mathrm{~m}^{3}$ d'eau (dont l'indice de 1.33 est proche de celui du quartz, limitant ainsi les réflexions entre les deux milieux), dont la paroi externe sphérique, située à $1.2 \mathrm{~m}$ des barres de quartz, est tapissée de 10752 photomultiplicateurs de $2.5 \mathrm{~cm}$ de diamètre (un blindage magnétique protège les photomultiplicateurs du champ magnétique de BABAR). L'image obtenue sur la surface de détection représente une fraction du cône Cherenkov, éventuellement dédoublée à cause des ambiguïtés. En effet, les différentes réflexions sur les faces créent au maximum 16 ambiguiités.

La reconstruction de l'angle Cherenkov se fait par une méthode de maximum de vraisemblance utilisant les informations spatiales (les angles polaire et azimutal de la trace) et temporelles $\left(\Delta t_{\gamma}\right.$, l'intervalle entre le temps d'arrivée attendu et mesuré du photon) mesurées 
par le DIRC. Pour chaque trace ayant traversé le volume fiduciel du DIRC, cette méthode calcule de façon itérative une valeur de vraisemblance pour chaque type de particule $(e, \mu$, $\pi, K, p)$.

L'avantage du DIRC réside dans le fait qu'il n'occupe que $8 \mathrm{~cm}$ radialement (correspondant à $19 \%$ de longueur de radiation), puisque la surface de détection se trouve à l'extérieur du détecteur ${ }^{2}$, permettant ainsi de construire une DCH de grand rayon, tout en limitant celui de l'EMC, et par la même son cô̂t.

Enfin, la résolution en temps $\Delta t_{\gamma} \mathrm{d} u$ DIRC permet de rejeter le bruit de fond provenant des faisceaux, et d'exclure les autres traces de l'événement comme source possible du photon considéré. Elle permet aussi de faire passer le nombre d'ambiguïtés de 16 à typiquement 3 . La résolution en temps est de $1.7 \mathrm{~ns}$, proche de la résolution intrinsèque des photomultiplicateurs qui est de $1.5 \mathrm{~ns}$.

La figure 3.13 illustre les performances du DIRC: le graphe de gauche montre la résolution sur l'angle Cherenkov pour des muons issus d'événements di-muons $e^{+} e^{-} \rightarrow \mu^{+} \mu^{-}$. La résolution est donnée par $\sigma_{C, t r a c k}=\frac{\sigma_{C, \gamma}}{\sqrt{N_{p e}}}$, où $\sigma_{C, \gamma}$ est la résolution angulaire pour un photon qui vaut $10.2 \mathrm{mrad}$ et $N_{p e}$ est le nombre de photons détectés (voir figure 3.14). La résolution $\sigma_{C, \text { track }}$ moyenne est de $2.5 \mathrm{mrad}$. Le graphe de droite montre la séparation $\pi$ - $K$ attendue pour des événements $B \rightarrow \pi^{+} \pi^{-}$calculée à partir de la résolution de l'angle Cherenkov et du nombre de photons Cherenkov obtenus dans les événements di-muons. La séparation reste aussi bonne que $3 \sigma$ pour une impulsion de $4 \mathrm{GeV} / \mathrm{c}$.
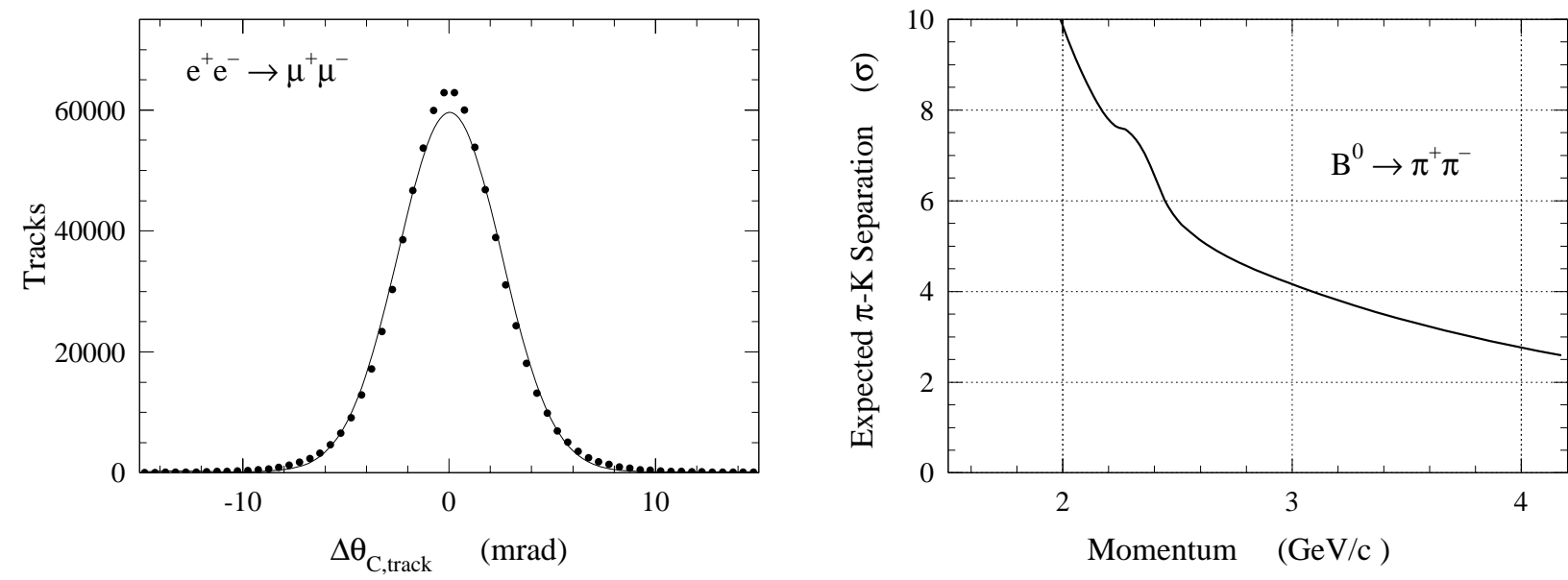

Figure 3.13: Graphe de gauche: différence entre l'angle Cherenkov mesuré et attendu dans des événements di-muons. La résolution est de 2.5 mrad. Graphe de droite: la séparation $\pi-K$ attendue pour des événements $B \rightarrow \pi^{+} \pi^{-}$(voir texte). La séparation reste aussi bonne que $3 \sigma$ pour une impulsion de $4 \mathrm{GeV} / \mathrm{c}$.

Le graphe de gauche de la figure 3.14 montre l'angle Cherenkov en fonction de l'impulsion de la trace pour des kaons issus de la désintégration d'un $D^{*+} \rightarrow D^{0}\left(K^{-} \pi^{+}\right) \pi^{+}$.

\footnotetext{
${ }^{2}$ Dans le cas des détecteurs RIHC (RIng CHerenkov), la lumière détectée est celle réfractée par la surface du radiateur, et donc les récepteurs de lumière se trouvent dans la direction d’incidence de la trace, et occupent ainsi un plus grand volume au sein du détecteur. L'originalité du DIRC réside dans le fait que la lumière est réfléchie à l’intérieur du radiateur.
} 

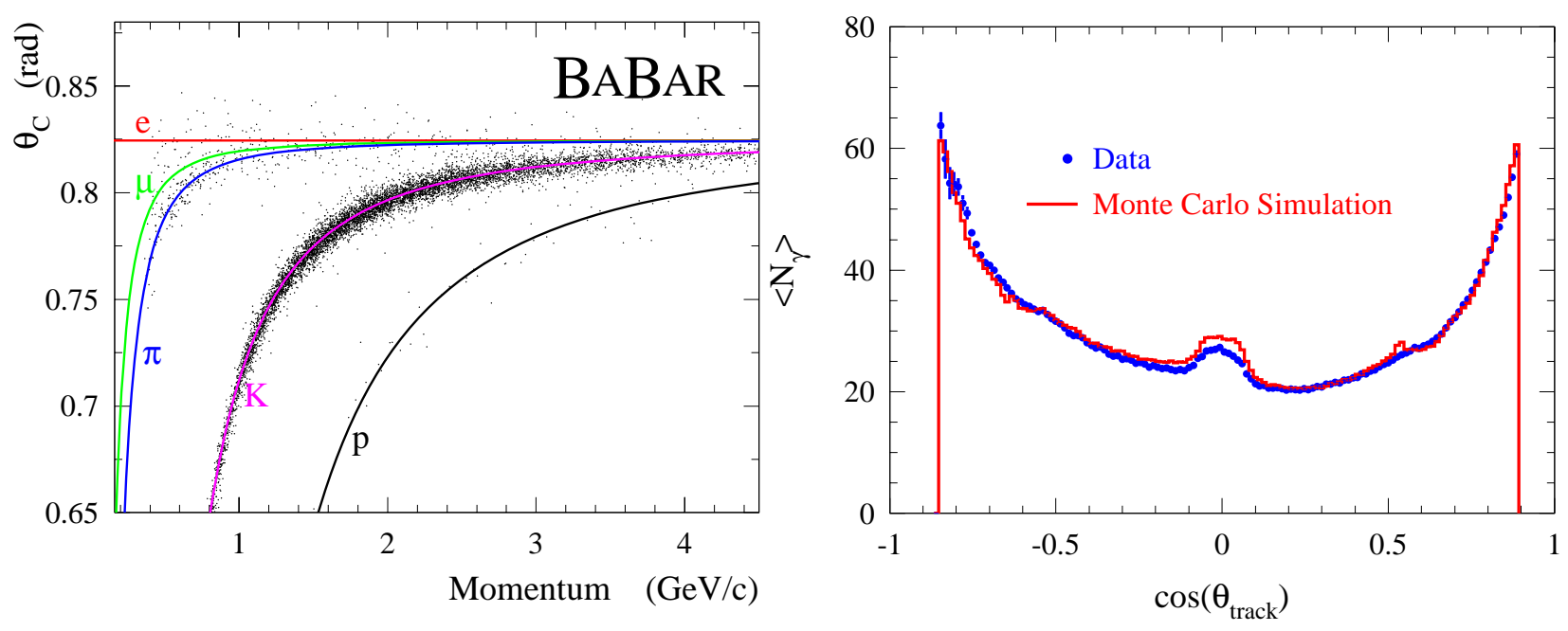

Figure 3.14: Figure de gauche: angle Cherenkov en fonction de l'impulsion de la trace pour des kaons issus de la désintégration d'un $D^{*+} \rightarrow D^{0}\left(K^{-} \pi^{+}\right) \pi^{+}$. Les lignes de couleur correspondent à différentes hypothèses de particules. Figure de droite: nombre de photons Cherenkov détectés en fonction de l'angle polaire de la trace pour des traces d'événements di-muons, et la simulation Monte Carlo correspondante. Le nombre moyen de photons dans la simulation a été modifié pour correspondre aux données.

\subsubsection{Calorimètre électromagnétique (EMC)}

Le calorimètre électromagnétique mesure l'énergie contenue dans les gerbes électromagnétiques, ainsi que leur position. Ceci permet la détection des photons issus des désintégrations des $\pi^{0}$ et $\eta$, ainsi que des processus électromagnétiques et radiatifs. Associé à la DCH, l'EMC permet aussi principalement l'identification des électrons. L'EMC est composé de 6580 cristaux d'iodure de césium dopés avec $0.1 \%$ de thallium ( $\mathrm{Cs}(\mathrm{Tl}))^{3}$ disposés en 48 anneaux de 120 cristaux dans le tonneau, et 8 anneaux de 80 à 120 cristaux dans le bouchon situé à l'avant, comme indiqué sur la figure 3.15 . Il couvre $90 \%$ de l'angle azimutal, et entre 15.8 et $140.8^{\circ}$ de l'angle polaire. Les cristaux sont disposés de façon légèrement non-projective le long de l'axe $z$ (l'angle de dépointage des cristaux varie entre 15 et $45 \mathrm{mrad}$ selon l'angle polaire) pour éviter l'inefficacité de détection due aux espaces entre les cristaux. Dans le plan perpendiculaire à l'axe $z$, par contre, les cristaux sont disposés de façon projective, ce qui mène à une perte en acceptance de 2.5\%. Malgré une efficacité intrinsèque de détection quasi-parfaite pour les photons de basse énergie (quelques $\mathrm{MeV}$ ), seuls les photons de plus de $20 \mathrm{MeV}$ sont réellement détectables à cause de la présence de bruits de fond élevés à ces faibles énergies provenant à la fois des faisceaux et des événements de physique.

Les cristaux sont de forme trapézoïdale, et représentent entre 16.1 et 17.6 longueurs de radiation. Ils sont enveloppés dans des réflecteurs pour maximiser la lumière collectée. A l'arrière de chaque cristal sont apposées deux photodiodes collectant la lumière émise.

De fins tubes sont disposés sur la face avant du calorimètre. Dans ces tubes circule du fluocarbone excité par irradiation de neutrons, et émettant donc des photons de $6.13 \mathrm{MeV}$, ce qui permet de calibrer et contrôler le calorimètre (ceci est réalisé environ une fois par semaine). Une autre source de calibration sont les événements Bhabhas.

\footnotetext{
${ }^{3}$ Le thallium est un activateur qui permet de modifier les niveaux d'énergie du cristal afin d'augmenter la probabilité et la longueur d'onde de la lumière de scintillation.
} 


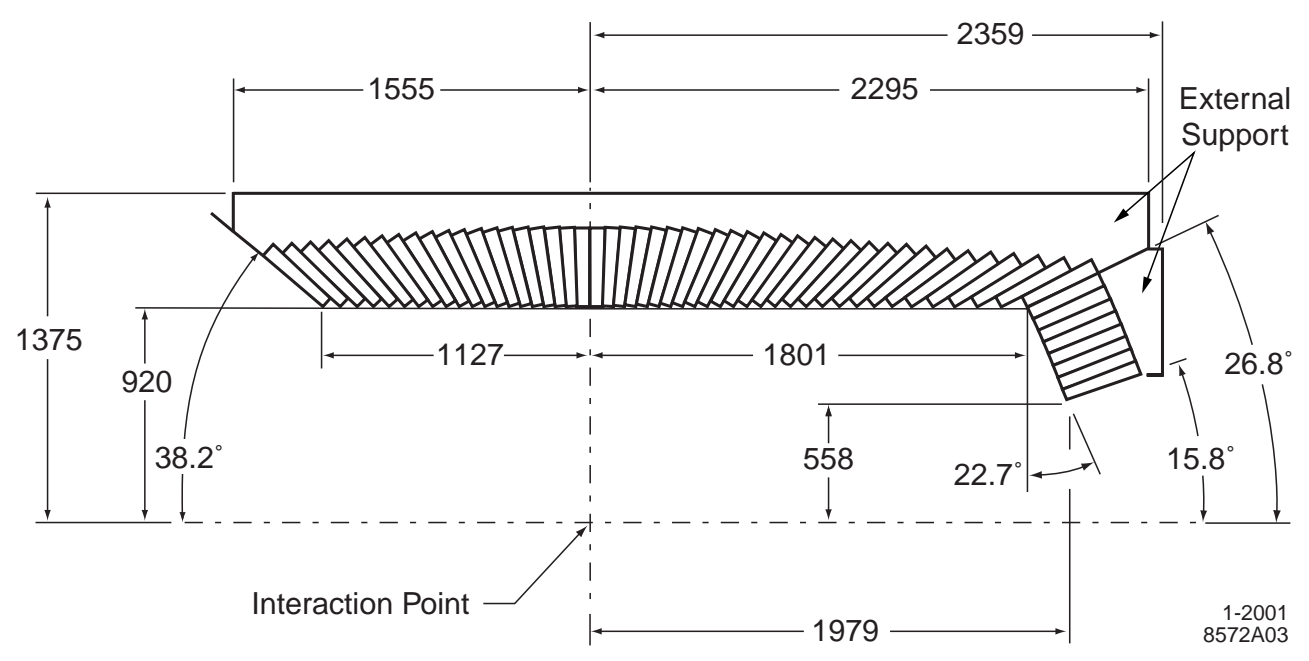

Figure 3.15: Coupe longitudinale de l'EMC.

Une gerbe électromagnétique s'étale sur un amas de plusieurs cristaux adjacents: un algorithme de reconnaissance de forme isole les amas célibataires (avec un seul maximum d'énergie) et les amas composites (avec plusieurs maxima d'énergie). Un amas est reconstruit à partir d'un cristal principal ayant une énergie de plus de $10 \mathrm{MeV}$. L'énergie des éventuels maxima secondaires est calculée de façon itérative, et leur position est déterminée par une méthode des centres de gravité [54]. Enfin, un maximum peut être associé à une trace chargée si sa position est consistante avec l'impact de la trace sur le calorimètre.

Pour distinguer les gerbes dues aux hadrons neutres des gerbes électromagnétiques (dues aux photons ou électrons), plusieurs variables de forme de gerbe sont construites. La première variable est le moment latéral [55] (LAT) défini comme

$$
\mathrm{LAT}=\frac{\sum_{i=3}^{n} E_{i} r_{i}^{2}}{E_{1} R_{0}^{2}+E_{2} R_{0}^{2}+\sum_{i=3}^{n} E_{i} r_{i}^{2}},
$$

où $n$ est le nombre cristaux dans l'amas, $E_{i}$ est l'énergie du cristal $i$ (les cristaux sont rangés par ordre d'énergie décroissante, donc $i=1$ correspond au cristal avec l'énergie maximale), $r_{i}, \phi_{i}$ sont les coordonnées polaires du centre $\mathrm{d} u$ cristal $i$, et $R_{0}$ est la distance moyenne entre deux cristaux. Le LAT est compris entre 0 et 1 . La gerbe d'un hadron s'étend sur un plus grand nombre de cristaux (grand LAT) qu'une gerbe provenant d'un photon (LAT < 0.8). La deuxième variable exploite la régularité de la gerbe électromagnétique, qui est moins importante pour les hadrons que pour les photons: il s'agit des moments de Zernike décrits dans la référence [56].

La résolution en énergie et angles $\theta$ et $\phi$ de l'EMC est la suivante:

$$
\begin{aligned}
\frac{\sigma_{E}}{E} & =\left(\frac{2.32 \pm 0.30}{4 \sqrt{E /(1 \mathrm{GeV})}} \oplus 1.85 \pm 0.12\right) \% \\
\sigma_{\theta, \phi} & =\left(\frac{3.87 \pm 0.07}{\sqrt{E /(1 \mathrm{GeV})}} \oplus 0.00 \pm 0.04\right) \mathrm{mrad}
\end{aligned}
$$

La figure 3.16 montre divers graphes associés aux performances de l'EMC: le graphe de gauche représente la résolution en énergie en fonction de l'énergie pour différents types 
d'événements, modélisée par l'éq. 3.5. Le graphe de droite montre la masse invariante de deux photons dans des événements $B^{0} \bar{B}^{0}$ pour des photons d'énergie supérieure à $30 \mathrm{MeV}$ et des $\pi^{0}$ d'énergie supérieure à $300 \mathrm{MeV}$. La ligne pleine est un ajustement des données: la résolution obtenue est de $6.9 \mathrm{MeV} / \mathrm{c}^{2}$.
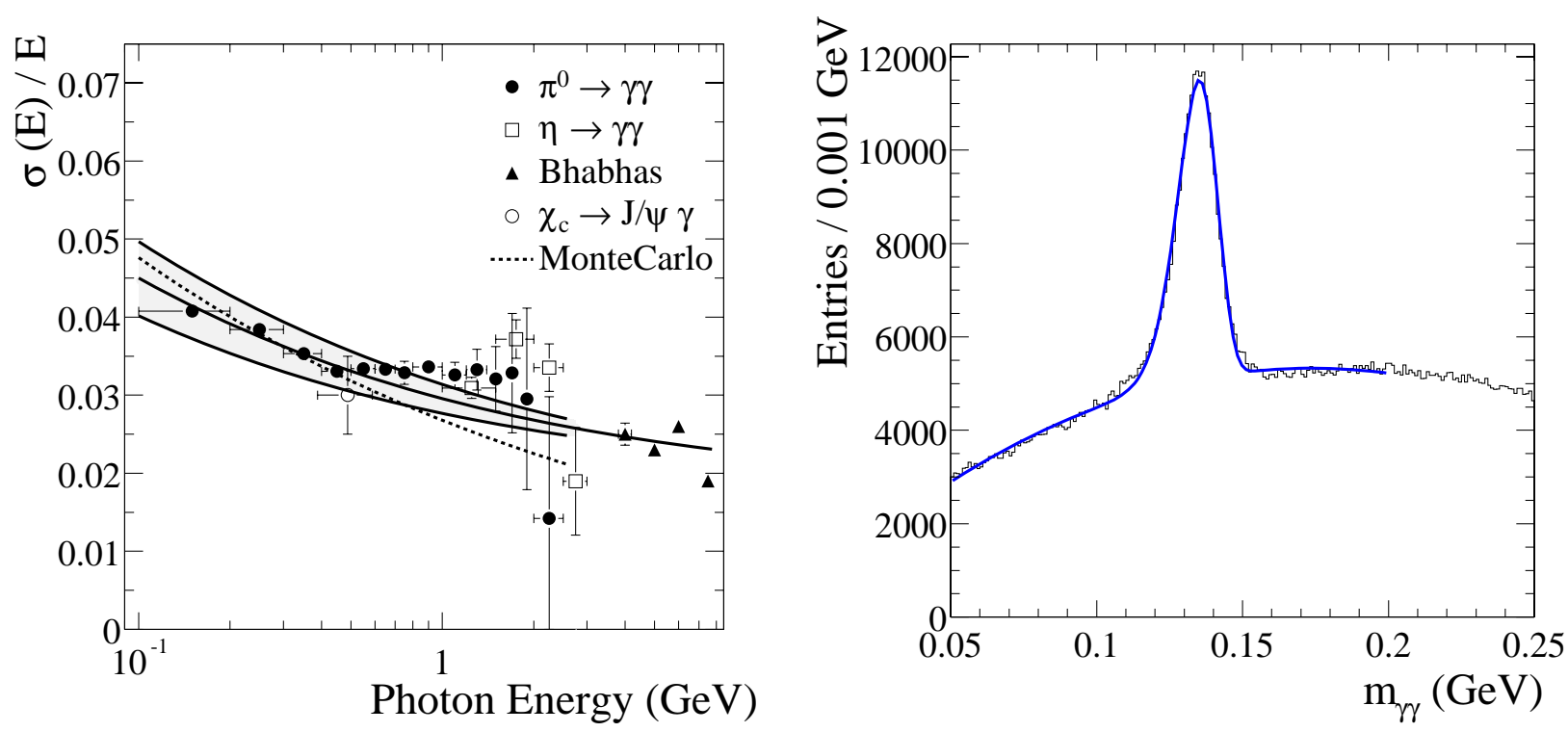

Figure 3.16: Graphe de gauche: résolution en énergie en fonction de l'énergie pour différents types d'événements Graphe de droite: masse invariante de deux photons dans des événements $B^{0} \bar{B}^{0}$ pour des photons d'énergie supérieure à $30 \mathrm{MeV}$ et des $\pi^{0}$ d'énergie supérieure à $300 \mathrm{MeV}$. La ligne pleine est un ajustement des données: la résolution obtenue est de $6.9 \mathrm{MeV} / \mathrm{c}^{2}$.

\subsubsection{Aimant supraconducteur}

Pour permettre la mesure de l'impulsion et la charge des traces chargées, un champ magnétique constant de $1.5 \mathrm{~T}( \pm 0.2 \mathrm{mT})$ est appliqué sur le détecteur $B A B A R$, principalement le long de l'axe $z$, grâce à un solénoïde supraconducteur. Le matériau supraconducteur est composé d'un câble de Rutherford en filaments de niobium-titanium (NbTi) incrusté dans de l'aluminium, d'une longueur totale de $10.3 \mathrm{~km}$, et dans lequel circule un courant de 4600 A. Le solénoïde est refroidi à une température de $4.5 \mathrm{~K}$ grâce à de l'hélium liquide. Une structure en acier sert pour le retour du flux magnétique ainsi qu'à la résistance structurale du détecteur aux forces magnétiques provenant du champ appliqué, et aux forces mécaniques provenant d'un éventuel tremblement de terre. Cette structure d'acier est instrumentée, comme décrit dans le paragraphe suivant.

\subsubsection{Retour de flux instrumenté (IFR)}

Le retour de flux instrumenté a pour but l'identification des muons (importants pour l'étiquetage de la saveur des $B$ ), ainsi que des hadrons neutres (principalement $K_{L}^{0}$ et neutrons). Il est composé d'une structure en acier (pour le retour du flux magnétique du solénoïde de $B A B A R$ ) servant de filtre à muons. La figure 3.17 représente le tonneau et les deux bouchons hexagonaux avant et arrière de l'IFR: l'acier est segmenté en plaques d'épaisseur croissante avec le rayon du détecteur et variant entre 2 et $10 \mathrm{~cm}$. 


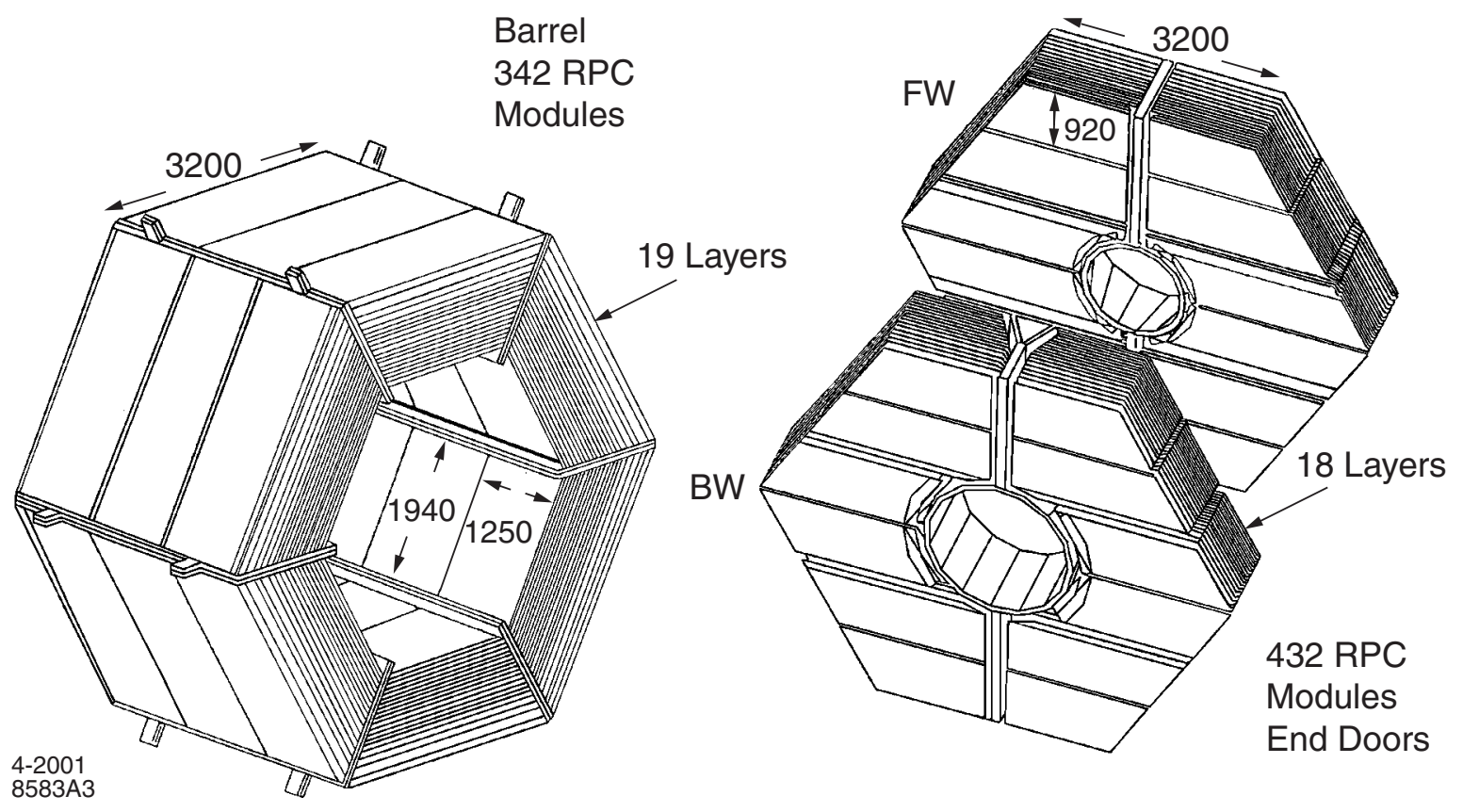

Figure 3.17: Figure de gauche: tonneau de l'IFR. Figure de droite: bouchons avant (FW) et arrière $(B \overline{W)}$ de l'IFR.

Des compteurs à plaques résistives (Resistive Plate Chamber, RPC) sont insérés entre ces segments d'acier: le tonneau contient 19 couches de RPC, et les bouchons 18. D'autre part, deux couches de RPC cylindriques sont installées entre l'EMC et le cryostat du solénoïde L'acceptance angulaire polaire de l'ensemble de ces compteurs est comprise entre 17.2 et $157.1^{\circ}$, et l'acceptance azimutale est de $87 \%$.

Le concept des RPC, qu'elles soient plates ou cylindriques, est le même: la figure 3.18 montre la coupe d'un tel détecteur. Il consiste en deux feuilles de bakélite de $2 \mathrm{~mm}$ d'épaisseur séparées de $2 \mathrm{~mm}$ et portées à une différence de potentiel de $8 \mathrm{kV}$. Un gaz composé principalement d'argon et de fréon remplit l'espace entre les deux feuilles. Une particule traversant le détecteur engendre un signal lu par des électrodes d'aluminium placées sur chaque face de la RPC. Il y a 806 modules de RPC, pour une surface totale active de $2000 \mathrm{~m}^{2}$, ce qui constitue le plus grand détecteur RPC construit jusqu'ici.

Les signaux dans l'IFR associés avec une trace chargée peuvent correspondre soit à des muons, soit à des hadrons chargés. Des variables discriminantes reliées aux quantités mesurées par l'IFR permettent l'identification des muons avec une efficacité de $90 \%$ sur une gamme d'impulsion allant de 1.5 à $3 \mathrm{GeV}$. Les signaux dans l'IFR non-associés avec une trace chargée correspondent aux $K_{L}^{0}$ et autres hadrons neutres. Puisqu'une fraction significative des hadrons neutres interagissent avant d'atteindre l'IFR, les informations provenant de l'EMC sont aussi utilisées pour leur identification: ainsi, l'efficacité de reconstruction des $K_{L}^{0}$ varie entre 20 et $40 \%$ pour une impulsion entre 1 et $4 \mathrm{GeV} / \mathrm{c}$ respectivement (IFR et EMC combinés).

\subsubsection{Système de déclenchement}

Le système de déclenchement a pour but de sélectionner les événements de physique avec une très bonne efficacité (plus de $99 \%$ pour les événements $B^{0} \bar{B}^{0}$, et aux alentours de $90 \%$ 


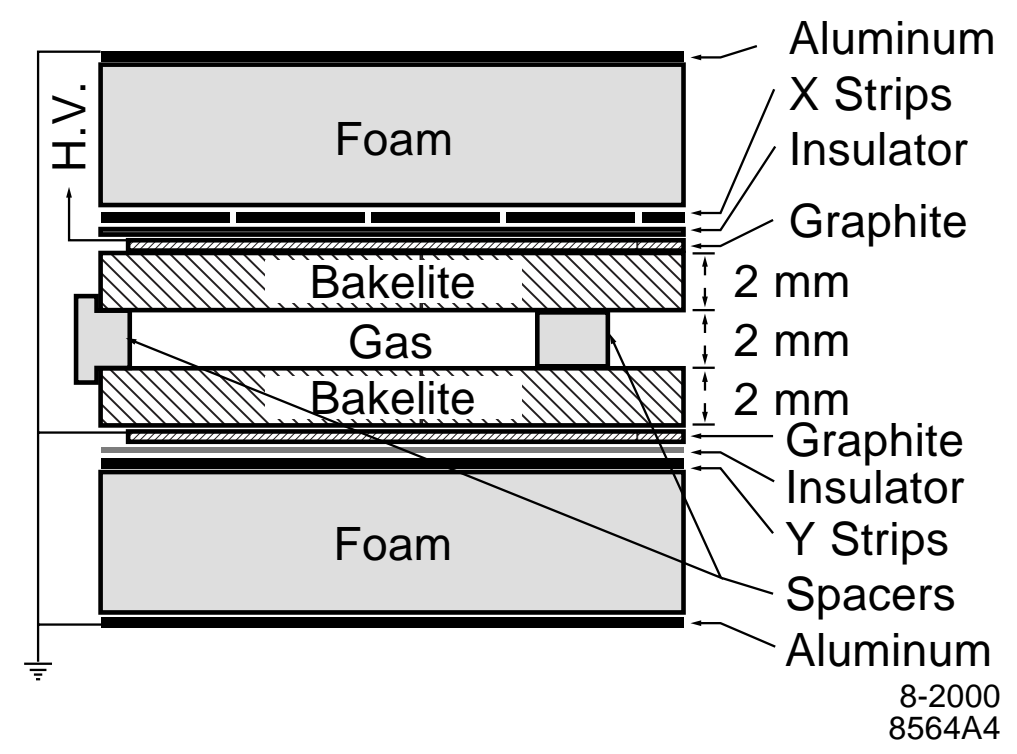

Figure 3.18: Coupe d'une RPC: deux feuilles de bakélite, entre lesquelles est emprisonné un gaz, sont portées à une différence de potentiel de $8 \mathrm{kV}$. Une particule traversant le détecteur provoque un signal lu par des électrodes d'aluminium placées sur chaque face de la RPC

pour les $\tau^{+} \tau^{-}$et $\mu^{+} \mu^{-}$), tout en rejetant les événements de bruit de fond et en gardant le taux d'enregistrement des événements à moins de $120 \mathrm{~Hz}$. A luminosité courante, le taux de bruit de fond provenant des faisceaux est typiquement de $20 \mathrm{kHz}$ chacun, avec une ou plusieurs traces dans la DCH (de $p_{T}>120 \mathrm{MeV} / \mathrm{c}$ ) ou au moins un amas dans l'EMC (de $E>100 \mathrm{MeV})$.

Deux niveaux de déclenchement sont utilisés: L1 et L3, respectivement basés sur des algorithmes au niveau de l'électronique, et informatiques. La décision prise par le niveau L1 s'appuie sur les traces chargées reconstruites dans la DCH, et/ou les amas de l'EMC, ainsi que les traces détectées dans l'IFR pour certains types d'événements. Le niveau L3 affine les méthodes de décision de L1, utilisant par exemple de meilleurs algorithmes de reconstruction de traces (ainsi que de vertex) et de reconstruction d'amas dans 1'EMC. L3 est intégré dans l'environnement du traitement des événements en ligne (Online Event Processing, OEP).

\subsubsection{Système d'acquisition des données}

Le système d'acquisition des données de BABAR supporte un taux d'acquisition de niveau L1 de $2 \mathrm{kHz}$ maximum, pour une taille d'événement de 32 kbytes. Il est composé de 157 modules de lecture (ReadOut Modules, ROM) qui reçoivent les informations venant des différents sous-détecteurs (classés en partitions) via des fibres optiques, et les transmettent aux "fermes" informatiques en-ligne qui reconstruisent le signal total. Ces fermes contiennent entre autres OEP et le niveau L3 du système de déclenchement décrits dans la section 3.2.7. Les conditions des sous-détecteurs (haute tensions, pureté des gaz, ...) sont aussi enregistrées pour chaque événement afin d'assurer la qualité des données et de permettre les calibrations. L'efficacité du système d'acquisition des données est de $98 \%$.

Les données enregistrées sont sectionnées en "runs", c'est-à-dire en périodes de prise de données de deux heures maximum. 


\subsection{Contrôle et protection du SVT}

\subsubsection{Rôles du système de protection du SVT (SVTRAD)}

La nécessité pour une usine à $B$ d'avoir des faisceaux de grand courant pour atteindre une luminosité élevée représente un défi pour le détecteur $B A B A R$ en terme de radiations engendrées par les bruits de fond de la machine. En particulier, la région d'interaction est très complexe, et le fait que des éléments de PEP-II se situent à l'intérieur du détecteur $B A B A R$ constitue un souci potentiel quant aux radiations.

Dans ce contexte, le système de protection du SVT (SVTRAD) [57] poursuit deux objectifs:

- mesurer les radiations dues au bruit de fond de PEP-II: ces mesures sont utiles pour le SVT, mais aussi pour tous les autres sous-détecteurs de BABAR.

- protéger le SVT des dommages dus aux radiations: dans des conditions atypiques (dégradation du vide du tube à vide, mauvais contrôle des faisceaux, etc), le bruit de fond dû aux faisceaux peut atteindre des proportions telles que le SVT pourrait être sérieusement endommagé. Un système pouvant couper les faisceaux si les radiations sont trop élevées est donc nécessaire. Dans SVTRAD, il existe en fait deux procédés pour couper les faisceaux: un au niveau de l'électronique (appelé "logique câblée"), et un au niveau du logiciel en temps réel (appelé "logique logicielle").

Un équilibre doit néanmoins être trouvé entre les besoins de l'expérience d'accumuler rapidement la luminosité, et la protection du SVT contre les dommages dus aux radiations. En effet, après une interruption de faisceaux, il faut injecter ces derniers à nouveau à partir de zéro, entraînant une perte d'efficacité et un surcroît de radiations (car deux injections de suite sont plus cô̂teuses qu'une seule radiation, même mauvaise).

Les bruits de fond de PEP-II, ainsi que les risques encourus par le SVT, sont détaillés dans cette section.

\subsubsection{Le bruit de fond de PEP-II}

En mode de fonctionnement normal, les trois types de bruits de fond présents dans PEP-II sont les suivants, par ordre d'importance croissante:

- La radiation synchrotron générée dans les aimants de courbure, et dans les quadripôles focalisant. L'agencement de la région d'interaction, et la mise en place de masques sur l'anneau PEP-II réduisent ce bruit de fond à un niveau très faible.

- Les particules issues des interactions faisceau-faisceau par des processus Bhabha.

- Les particules (photons, électrons ou positrons) issues d'interactions avec les atomes de gaz résiduel. Seul ce dernier bruit de fond est significatif.

D'autre part, durant les injections des faisceaux, d'autres bruits de fond significatifs sont présents ( $c f$. sections 3.1.1 et 3.1.2) lorsque les faisceaux s'approchent trop près des parois.

Les bruits de fond dus au gaz résiduel et aux injections constituent la principale source de radiation entraînant des dommages potentiels, et des taux d'occupation des détecteurs conduisant à des temps morts (et donc à une perte de données). 


\subsubsection{Les risques encourus par le SVT}

Malgré l'utilisation de technologies résistantes aux radiations pour la construction du SVT, celui-ci est le détecteur le plus vulnérable aux radiations à cause de sa proximité avec les faisceaux (la couche la plus intérieure est à $3 \mathrm{~cm}$ de l'axe des faisceaux).

Les dommages dus aux radiations se situent:

- dans les détecteurs en silicium. Les dommages se produisent principalement dans la structure cristalline du silicium: les atomes de silicium heurtés par une particule incidente peuvent se déplacer, créant ainsi un trou et un interstice. Les effets de ces déplacements sont de deux types: l'augmentation du courant de fuite du détecteur et donc du bruit, et une variation de la tension de déplétion pouvant avoir un impact sur les conditions opérationnelles et la résolution du détecteur.

- dans l'électronique de premier niveau: les radiations causent une augmentation du bruit, et une perte de gain des composants électroniques.

Des études quantitatives sur les dommages dus aux radiations ont été réalisées à la fois sur les détecteurs en silicium et sur l'électronique de premier niveau [58]. Leurs conclusions sont que l'on ne s'attend pas à une dégradation significative des performances du SVT jusqu'à des doses intégrées de $5 \mathrm{Mrad}$, soit au moins jusqu'en 2005. Les parties les plus exposées du détecteur ont déjà reçu 1.6 Mrad de radiations.

\subsubsection{Description du système SVTRAD}

Le système SVTRAD comporte 12 détecteurs composés de photodiodes PIN, d'une électronique de traitement conçue spécifiquement pour ce système, et d'un logiciel de lecture en temps réel. Nous décrivons brièvement ces composants dans cette section.

\subsubsection{Détecteurs}

Pour satisfaire aux contraintes spatiales et de coûts, et afin d'obtenir une sensibilité similaire à celle du SVT, les détecteurs choisis sont des photodiodes PIN de grande surface $(1 \mathrm{~cm} \times$ $1 \mathrm{~cm}$ ). Au total, 12 diodes sont installées à $z=+12.1 \mathrm{~cm}$ et $-8.5 \mathrm{~cm}$, sur un rayon de 3 $\mathrm{cm}$. L'agencement des diodes est montré sur la figure 3.19: elles sont disposées sur 3 plans horizontaux situés au même niveau que le faisceau (MID), au-dessous (BTM) et au-dessus (TOP). Le bruit de fond est présent principalement dans le plan horizontal des faisceaux: ainsi les diodes MID sont les plus sensibles aux radiations (on observe un facteur 10 entre les doses enregistrées par les diodes MID par rapport aux diodes TOP et BTM).

Les diodes sont dénommées selon leur position géographique: à l'avant ("Forward", F) ou à l'arrière ("Backward", B) pour la position en $z$, et à l'est ("East", E) ou à l'ouest ("West", W) pour la position en $x$ : ainsi, les quatre diodes situées sur le plan du faisceau sont appelées BW: MID, BE: MID, FW: MID et FE: MID. A cause de l'action des aimants dipolaires B1 ( $c f$. section 3.1.3), BW: MID est la diode la plus sensible au faisceau de haute énergie (HER), et FE: MID au faisceau de basse énergie (LER).

Les diodes PIN consistent en une couche intrinsèque épaisse $(300 \mu \mathrm{m})$ de silicium de type $N$, entourée d'une fine couche de type $P$ du coté actif, et d'une fine couche de type $N^{+}$à l'arrière. La capacité de la diode ainsi formée est de $50 \mathrm{pF}$. La diode est polarisée en inverse avec une tension de $50 \mathrm{~V}$, de telle sorte que la région centrale désertée couvre toute la couche intrinsèque (maximisant ainsi le volume de détection). 


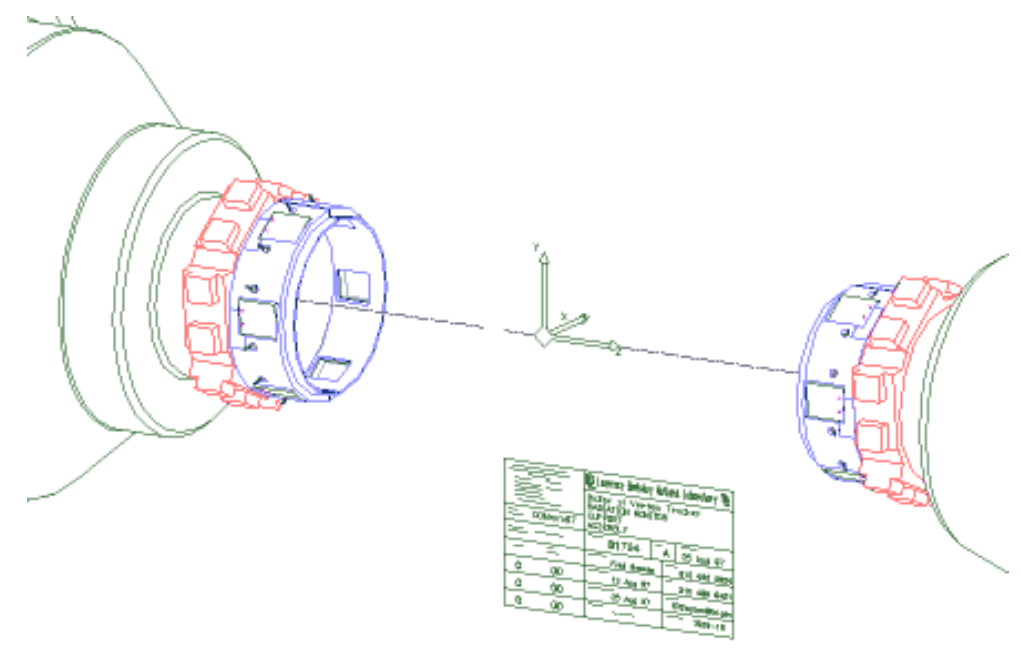

Figure 3.19: Agencement des 12 diodes PIN du système SVTRAD. Elles sont situées à l'extrémité des dipoles B1, sur les deux anneaux de part et d'autre de l'origine des coordonnées sur ce schéma.

Les populations d'électrons et trous suivent les distributions de Fermi-Dirac, en conséquence, le courant de fuite de la diode est très dépendant de la température. Il s'écrit:

$$
I(T)=I\left(T_{0}\right) \times\left(\frac{T}{T_{0}}\right)^{2} e^{-\frac{E}{k}\left(\frac{1}{T}-\frac{1}{T_{0}}\right)},
$$

où $E$ est l'énergie de la bande interdite pour le silicium $(1.2 \mathrm{eV}), T_{0}$ est la température de référence $\left(20^{\circ}\right)$, et $k$ est la constante de Boltzmann. Les changements de température étant attendus petits en mode de fonctionnement normal, on peut développer l'éq. 3.7 pour obtenir le modèle simplifié suivant:

$$
I(T)=I\left(T_{0}\right) e^{\alpha\left(T-T_{0}\right)}
$$

où $\alpha$ est un coefficient de température, valant typiquement $10 \% /{ }^{\circ} \mathrm{C}$. Pour mesurer la température au niveau des diodes, 24 thermistances sont installées à proximité.

Néanmoins, la température n'est pas le seul facteur déterminant l'évolution du courant de fuite: il faut aussi tenir compte des dommages dus aux radiations (qui sont du même type que ceux causés dans le silicium du SVT). Le courant de fuite augmente en effet linéairement avec la dose intégrée. Ainsi, l'augmentation du courant de fuite entre les temps $t_{1}$ et $t_{2}$ est donnée par:

$$
\Delta I\left(t_{1}, t_{2}\right)=\kappa \int_{t_{1}}^{t_{2}} \operatorname{Dose}(t) d t
$$

où $\kappa$ est un coefficient typiquement de l'ordre de quelques $\mathrm{nA} / \mathrm{krad}$ et où Dose( $\mathrm{t}$ ) est le taux de radiation en fonction $d u$ temps.

Quand une particule chargée passe à travers la région désertée de la diode, des paires électron-trou sont créees le long de sa trajectoire (en nombre proportionnel à l'énergie perdue par la particule). Grâce à la tension appliquée, les électrons et trous se déplacent, augmentant ainsi le courant de fuite de la diode. Une dose de 1 Mrad correspond à une charge d'environ $0.2 \mathrm{pC}$.

Pour extraire les doses dues aux radiations, il faut donc précisément mesurer le courant de fuite total (somme du piédestal et du signal dû aux radiations), et évaluer la valeur du piédestal à soustraire. 


\subsubsection{Electronique de lecture et algorithme de protection associê}

La figure 3.20 montre la composition d'un module électronique pour SVTRAD, traitant 3 diodes et leurs 6 thermistances. Un module possède deux secteurs distincts: un pour la mesure de la radiation (secteur nommé "MON", comme "monitoring", sur le schéma), et un implémentant un premier procédé menant à interrompre les faisceaux en cas de dose trop élevée (secteur nommé "ABORT" sur le schéma). Les signaux des 12 diodes passent à travers le secteur MON, mais seules les diodes MID passent à travers le secteur ABORT, puisque ces dernières sont les plus sensibles aux radiations.

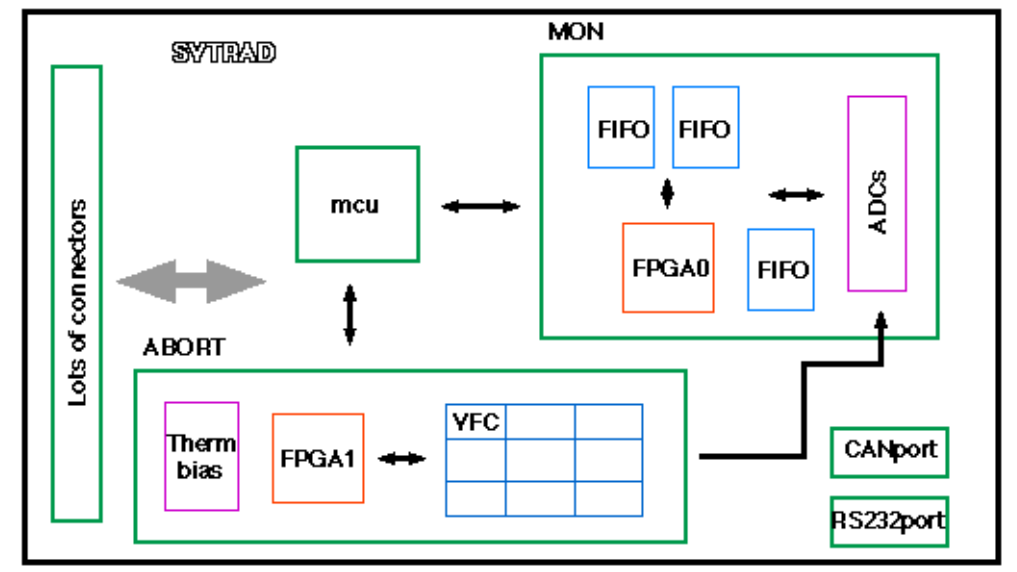

Figure 3.20: Schéma d'un module électronique SVTRAD. Un module possède deux parties: la partie "MON" permettant de mesurer les radiations, et la partie "ABORT" permettant l'interruption des faisceaux en cas de dose trop élevée.

- Logique câblée de coupure de faisceaux: cette logique a pour objectif de répondre rapidement à une forte dose reçue dans un temps court. Elle est implémentée de la façon suivante: le courant de fuite issu des diodes passe d'abord par un convertisseur tension-fréquence ("Voltage to Frequency Converter", VFC), puis le signal suit deux voies: d'un coté, il est transmis au secteur "MON" en repassant par un convertisseur fréquence-tension (FVC), et d'un autre coté, il est transmis à un circuit à réseaux logiques programmables ("Field Programmable Gate Array", FPGA) dans lequel est programmé la logique menant à interrompre les faisceaux.

Cette logique est illustrée sur la figure 3.21, son principe est celui d'un "seau qui fuit": le signal issu des diodes $\left(I_{\text {diode }}\right)$ remplit le seau. On tient compte de la dépendance en température $\left(I_{\text {temp }}\right)$ du courant de fuite de la diode par un trou dans le seau plus ou moins gros selon la température. Une horloge $\left(I_{\text {timer }}\right)$ a le pouvoir de modifier le niveau (vers le haut ou vers le bas) dans le seau selon des valeurs de seuil programmables. Ces seuils sont illustrés sur la figure 3.22: il s'agit du seuil $d_{0}$ sur le taux de radiation au-delà duquel on intègre ce taux, et du seuil $D_{0}$ sur l'intégrale au-delà duquel on considère la dose comme trop élevée. Lorsque le seau est plein, un signal d'alarme de 250 ns est envoyé. Si ce signal est en coïncidence avec le signal d'injection de PEP-II (qui a une fréquence de $30 \mathrm{~Hz}$ et dure $67 \mathrm{~ns}$ ) à l'intérieur d'une porte de $1 \mathrm{~ms}$, on considère que cette dose élevée est due à l'injection: l'alarme ne déclenche alors qu'une inhibition de l'injection. Dans le cas contraire, on coupe les faisceaux. 
1. Diode and Themistor are voltage biased.

2. f_diode increases with Temperature.

3. f_temp increases wth Temperature, too.

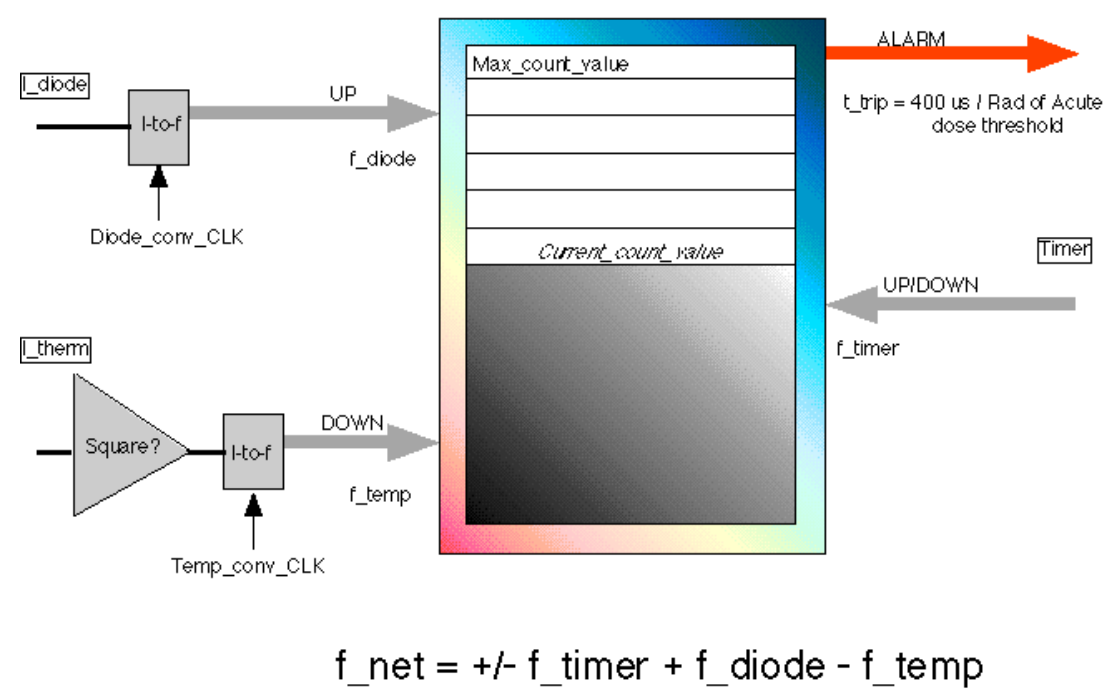

Figure 3.21: Logique du "seau qui fuit" programmée dans le FPGA du secteur servant à interrompre les faisceaux en cas de dose trop élevée (voir texte).

Puisque l'algorithme du "seau qui fuit" ne tient compte que de la dépendance en température du piédestal, mais pas de la dépendance dans les doses précédemment reçues (Eq. 3.9), il faut calibrer les paramètres du "seau qui fuit" régulièrement pour tenir compte de ce dernier effet.

- Mesure de la radiation: le signal analogique issu du secteur ABORT est traduit à une fréquence de $1 \mathrm{kHz}$ en signal numérique par un ADC ("Analogic to Digital Converter"), qui passe ensuite par un FPGA accumulant le signal dans un registre. Le signal est alors partagé entre deux voies: une voie "lente" à $0.47 \mathrm{~Hz}$ (qui moyenne le signal sur $N$ échantillons, où $N$ est programmable), qui est celle continuellement lue et transmise au logiciel en temps réel, et une voie "rapide" à $1 \mathrm{kHz}$, qui n'est lue que sur demande extérieure.

\subsubsection{Logiciel en temps réel, obtention des taux de radiation et algorithmes de protection associês}

Chaque module communique via un bus CAN ("Controller Area Network") avec un logiciel de contrôle en temps réel nommé EPICS ("Experimental Physics and Industrial Controle System"). Ce logiciel (aussi utilisé pour les autres sous-détecteurs, ainsi que pour PEPII) fourni un certain nombre d'applications pour utiliser les données issues du module (par exemple, pour créer des panneaux interactifs, disponibles dans la salle de contrôle de $B A B A R$, montrant des chiffres, graphes, alarmes reliés au système considéré) et contrôler ce dernier depuis des stations UNIX. Des exemples de photos de panneaux EPICS sont montrées sur la figure 3.23 .

Le logiciel extrait les signaux issus des ADC à une fréquence de $0.5 \mathrm{~Hz}$. L'algorithme implémenté dans EPICS pour obtenir les taux de radiation à partir de ces signaux d'ADC comporte deux temps: 


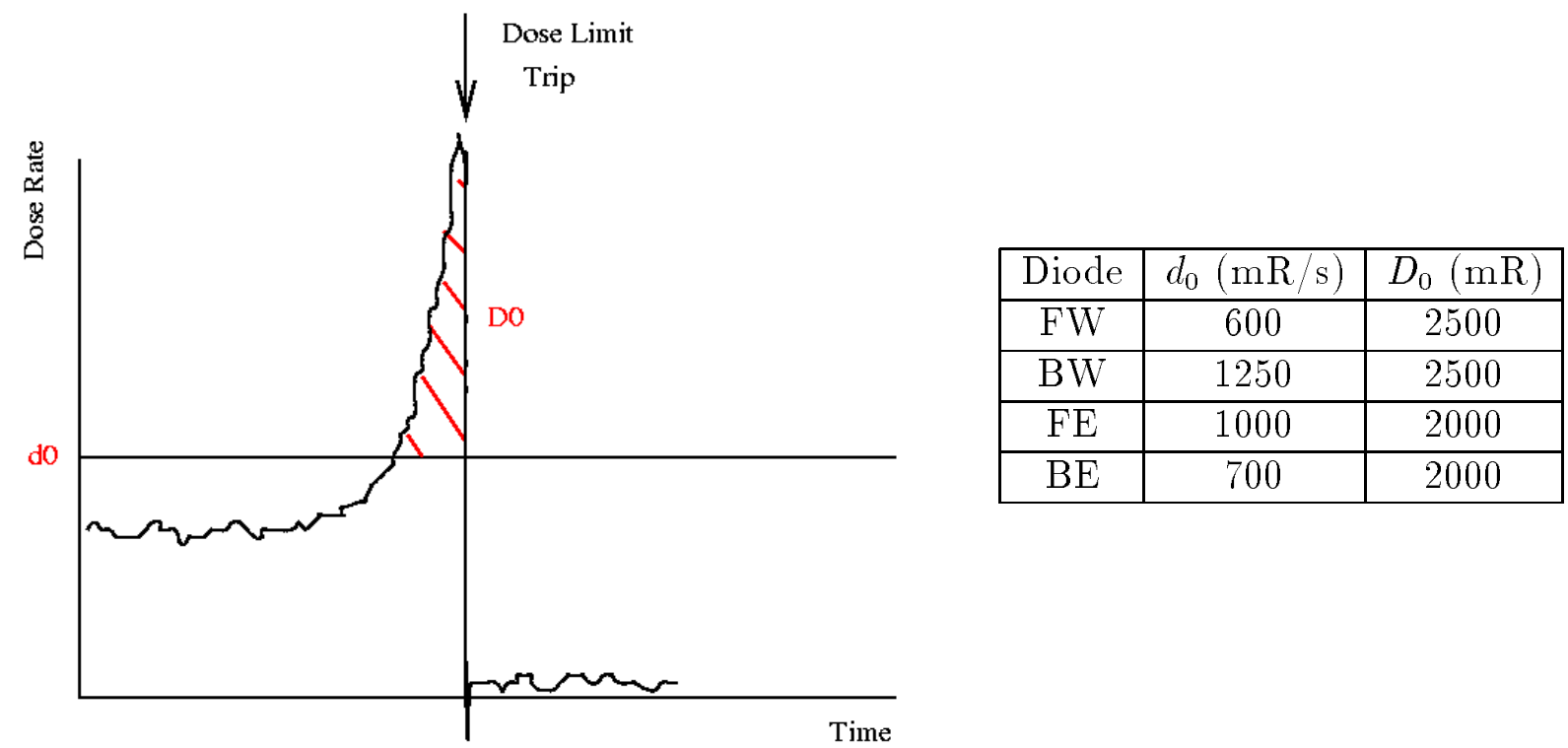

Figure 3.22: Gauche: défnition des seuils $d_{0}$ et $D_{0}$ : quand le taux de radiation dépasse le seuil $d_{0}$, on intègre la courbe. Si la valeur de l'intégrale dépasse le seuil $D_{0}$, alors la dose est considérée comme trop élevée. Droite: valeur des seuils pour les quatre diodes MID.

- Lorsqu'il n'y a pas de faisceaux, et donc pas de radiations, les signaux d'ADC mesurent le piédestal des diodes. On réalise alors une calibration en accumulant entre 10 et 15 échantillons de signaux d'ADC et de mesures de température (à une fréquence de 0.5 $\mathrm{Hz}$, cela prend environ 20-30 secondes ${ }^{4}$ ), ce qui permet de déterminer le piédestal avec une précision de $0.25 \mathrm{nA}$, menant à une distribution des taux de radiation centrée en 0 avec un écart-type de $1 \mathrm{mR} / \mathrm{s}$.

- Lorsqu'il y a des faisceaux, et donc de la radiation, les signaux d'ADC contiennent une composante de piédestal et une composante de signal. On utilise alors la dernière mesure du piédestal en mode de calibration pour l'extrapoler à la température courante (en utilisant l'éq. 3.8), et au niveau d'irradiation actuel (en utilisant l'éq. 3.9).

Des effets supplémentaires dus aux dommages causés par les radiations dans les diodes viennent compliquer l'extraction des taux de radiation: il s'agit des phénomènes de recuit et de contre-recuit. Une grande partie des vides intersticiels crées par irradiation se comblent par effet de diffusion. En fonction de la température, certains des défauts restants peuvent encore être comblés (effet de recuit), ou aggravés (effet de contre-recuit). Cet effet est particulièrement visible dans $B A B A R$ quand les faisceaux sont interrompus pendant des périodes assez longues: pendant la période sans faisceaux, l'effet de recuit entraîne une diminution du courant de fuite, puis quand les faisceaux reprennent, l'effet de contre-recuit entraîne une dégradation du piédestal plus importante que la simple loi linéaire de l'éq. 3.9. Cet effet n'est pas pris en compte dans les modèles de prédiction du piédestal, car il est instable et donc difficile à modéliser.

En plus de l'algorithme de protection au niveau de l'électronique décrit dans la section 3.3.2.2, il existe un algorithme de protection au niveau d'EPICS visant à limiter la dose reçue par le SVT sur le long terme (en opposition à celui au niveau de l'électronique visant

\footnotetext{
${ }^{4}$ Le système SVTRAD empêche alors PEP-II de pouvoir injecter les faisceaux jusqu’à ce que la calibration soit terminée.
} 

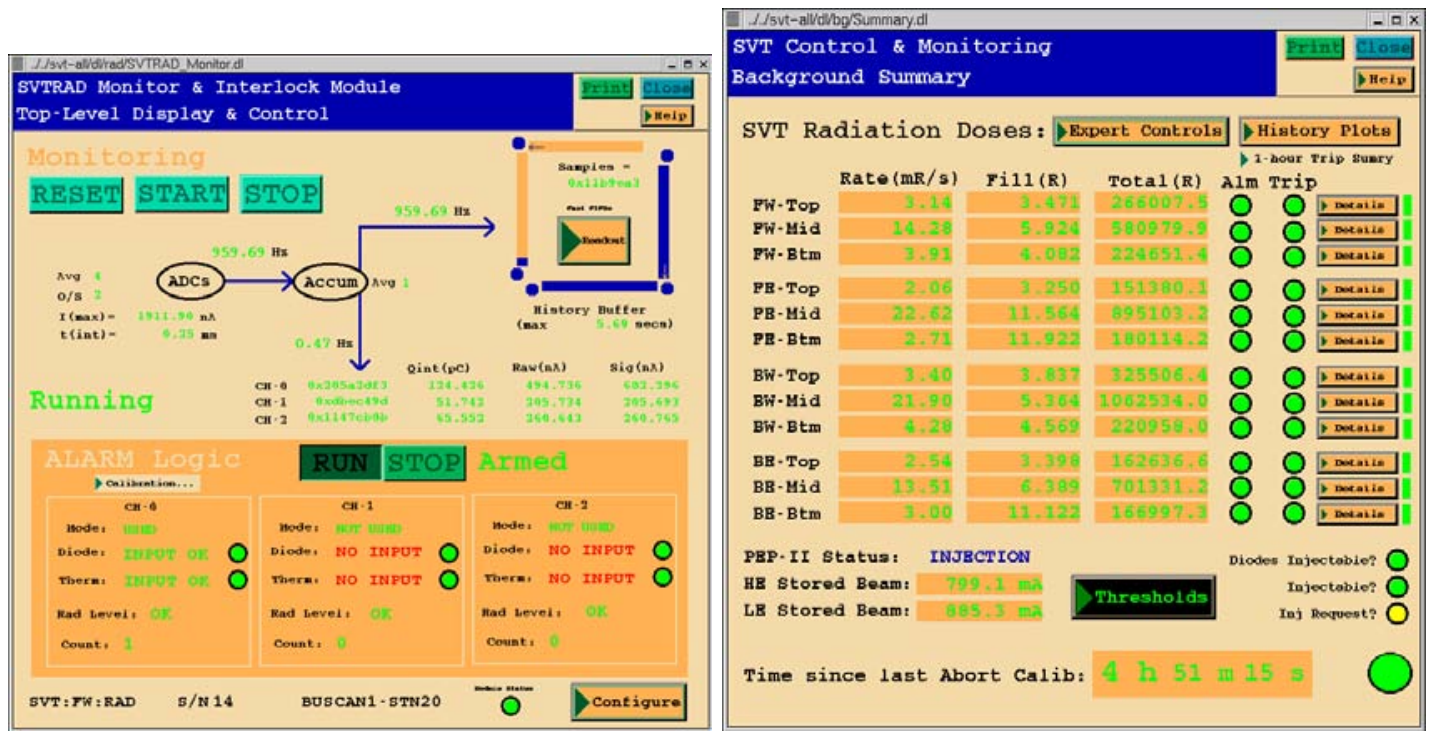

Figure 3.23: Photos de panneaux d'EPICS: le panneau de gauche montre le proxy EPICS pour un module SVTRAD, avec, entre autres, les signaux d'ADC utilisés pour le calcule des taux de radiation. Le panneau de droite est un résumé des informations relatives à chaque diode, incluant les taux de radiation, les doses intégrées, les statuts d'alarme, etc.

à limiter une dose importante sur un temps court). Le principe est simple: si le taux de radiation reste au dessus d'un seuil pendant plus de 5 minutes (augmenté à 10 minutes pour le run 3), un signal est envoyé au module électronique SVTRAD entraînant l'interruption des faisceaux. Les seuils actuels sont donnés dans le tableau 3.4. Avant mon intervention dans le logiciel en temps réel, cet algorithme fonctionnait à la fois pendant les périodes d'injection et de faisceaux stables. Depuis, un autre algorithme opère pendant les périodes d'injection, et cet "algorithme des 5 minutes" n'opère plus que durant les périodes de faisceaux stables.

\begin{tabular}{|c|c|}
\hline Diode & Seuil $(\mathrm{mR} / \mathrm{s})$ \\
\hline FW & 70 \\
\hline BW & 1000 \\
\hline FE & 80 \\
\hline BE & 50 \\
\hline
\end{tabular}

Tableau 3.4: Seuils actuels pour l'algorithme de coupure des faisceaux par le logiciel en temps réel: la diode BW: MID a été retirée du système car elle est désormais trop endommagée par les radiations.

\subsubsection{Performances du système, doses accumulées}

La figure 3.24 à gauche montre la dose accumulée par certaines diodes depuis le début de la prise de données de BABAR: toutes les diodes à la fois MID et TOP/BTM se situent au-dessous du bugget prévu pour le SVT. La figure à droite montre le rapport des doses accumulées pendant les périodes d'injection de faisceaux stables pendant l'année 2001: bien que les périodes d'injection ne représentent qu'une petite fraction du temps, le rapport est 
environ de 1. Ceci montre que les périodes d'injection sont particulièrement cruciales en terme de radiations.
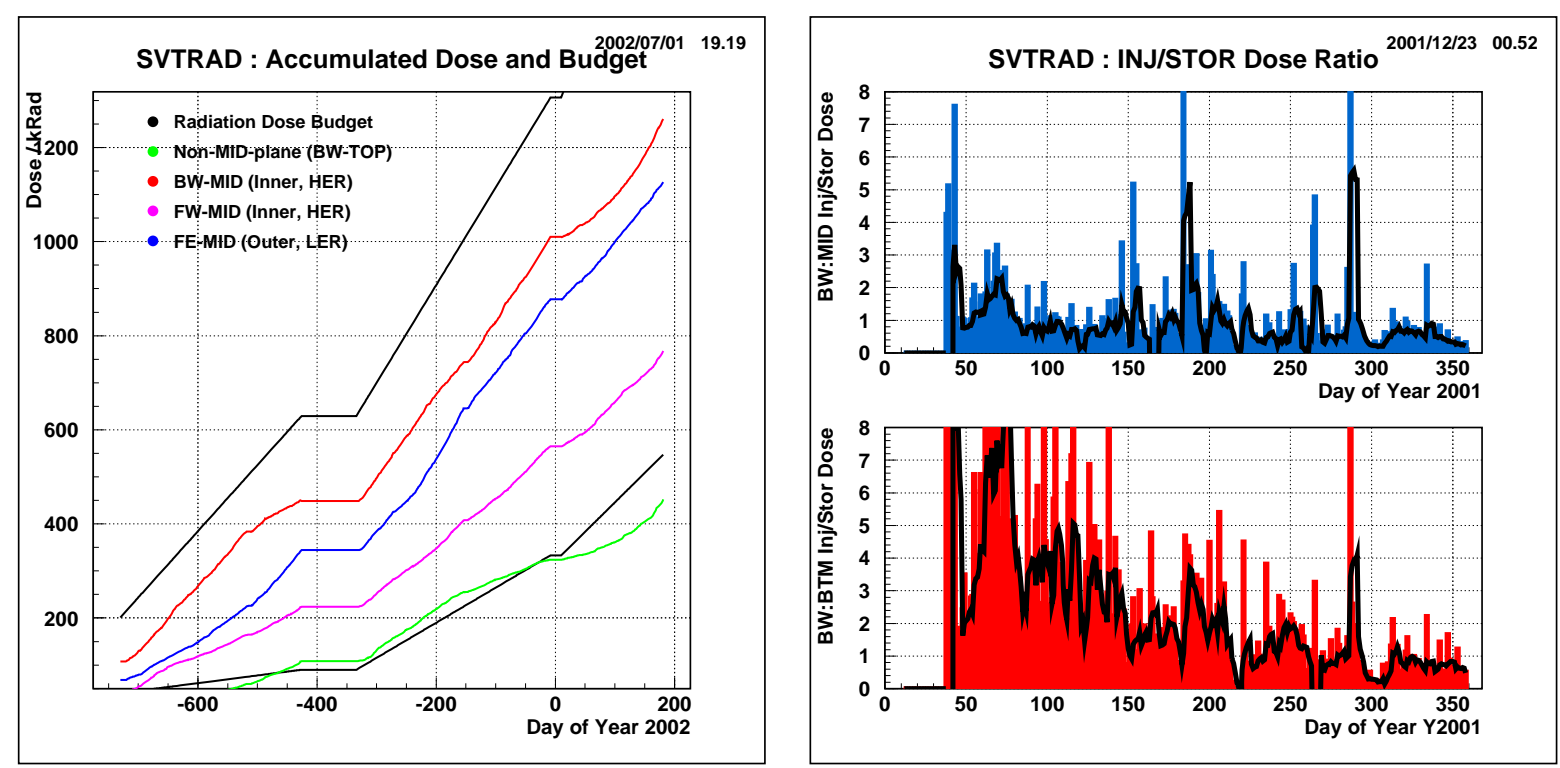

Figure 3.24: Figure de gauche: dose accumulée par certaines diodes depuis le début de la prise de données de BABAR. Les lignes noires indiquent le budget de doses de SVT. Toutes les diodes à la fois MID et TOP/BTM se situent au-dessous de ces lignes de bugget. Figure de droite: rapport des doses accumulées pendant les périodes d'injection de faisceaux stables pendant l'année 2001: bien que les périodes d'injection ne représentent qu'une petite fraction du temps, le rapport est environ de 1. Ceci montre que les périodes d'injection sont particulièrement cruciales en terme de radiations.

\subsubsection{Amélioration du logiciel en temps réel pour les périodes d'in- jection}

Parmi les deux systèmes de protection activés pendant ces périodes, le fonctionnement de la logique câblée n'est pas à remettre en cause, alors que celle au niveau d'EPICS peut l'être. En effet, les opérateurs de PEP-II contournent souvent ce système en stoppant l'injection pendant quelques secondes lorsque la quatrième minute d'alarme est atteinte, repassant ainsi au-dessous du seuil au-delà duquel le système de protection est enclenché, et remettant le compteur des minutes à zéro. Une autre critique à apporter à ce système est sa logique binaire: l'algorithme entraînera ou pas une interruption des faisceaux (et seulement une interruption des faisceaux alors que d'autres actions sont possibles, comme inhiber l'injection, ou limiter le taux d'injection) quelque soit le stade de l'injection: il arrive ainsi parfois que les faisceaux soient coupés alors que l'injection était sur le point de finir, et que la dose accumulée sur cette injection avortée et la suivante soit bien plus élevée que si le système avait laissé l'injection se terminer, même avec des taux de radiation élevés.

Après avoir noté ces points faibles dans le système de protection EPICS durant les injections, j'ai implémenté une nouvelle logique pour améliorer ce système. Elle est décrite ci-dessous. 


\subsubsection{Définition d'un nouveau critère de qualité d'injection}

Comme on l'a vu ci-dessus, le critère de la quantité de dose intégrée en 5 minutes n'est pas un critère optimal pour les périodes d'injection (alors qu'il l'est pour les périodes de faisceaux stables): si les 5 dernières minutes d'une injection donnent lieu à des radiations élevées, ce n'est pas grave tant que le reste de l'injection est "propre". Ainsi, ce qui compte, c'est la quantité de dose accumulée depuis le début de l'injection à un certain stade de l'injection, c'est-à-dire, pour une valeur donnée des courants de faisceaux.

SVT Diodes - SoftABORT studies

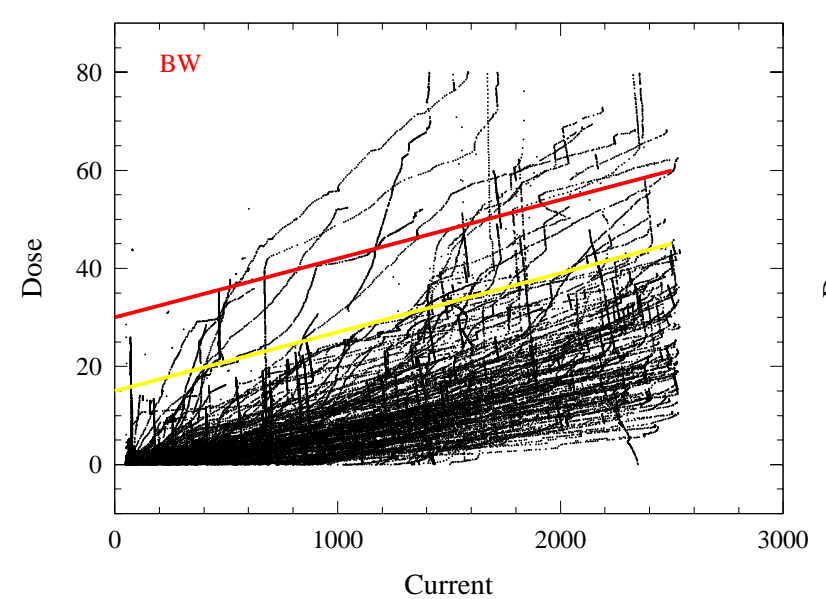

SVT Diodes - SoftABORT studies

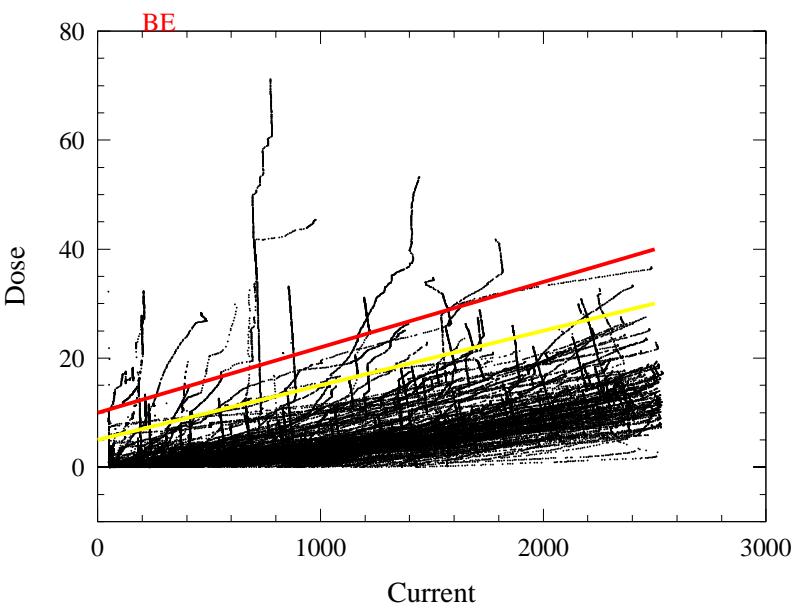

Figure 3.25: Dose intégrée depuis le début des injections par les diodes BW: MID (gauche) et BE: MID (droite) en fonction de la somme des courants des faisceaux. Les injections de bonne qualité sont définies comme ayant une pente douce et constante, menant ainsi à une faible dose totale (lorsque les faisceaux sont complètement injectés).

La figure 3.25 montre des courbes de dose intégrée par les diodes BW: MID et BE: MID depuis le début de l'injection en fonction de la somme des courants des faisceaux. On distingue clairement deux types d'injections: celles dont la courbe possède une pente douce et constante jusqu'à la fin de l'injection (menant à une dose totale par injection de moins de $40 \mathrm{rad}$ ), et celles présentant une forte pente, ou des sauts brusques (et menant à une dose totale entre 40 et plus de $100 \mathrm{rad}$ ).

\subsubsection{Nouveaux algorithmes de protection pendant l'injection}

Cette observation a mené à l'implémentation dans EPICS de la logique illustrée sur la figure 3.26:

- Nous définissons une courbe "dose-courant" seuil avec une pente correspondant à celle d'une bonne injection typique, et avec une ordonnée à l'origine ("Off1" sur le schéma) suffisamment élevée pour contenir l'ensemble des bonnes injections (pour l'exemple précédent de BW: MID, menant à moins de $40 \mathrm{rad}$ de dose totale). Les pentes et ordonnées à l'origine actuellement utilisées dans BABAR sont données dans le tableau 3.5.

- Une bonne injection restera constamment au-dessous de la courbe seuil. Si ce n'est pas le cas, l'injection est inhibée pendant quelques secondes afin de laisser le temps aux opérateurs de PEP-II de réaliser le problème et de tenter d'y remédier. On définit 


\begin{tabular}{c|cc}
\hline Diode & Pente $(\mathrm{mR} / \mathrm{mA})$ & Ordonnée $(\mathrm{R})$ \\
\hline FW & 301 & 29.9 \\
FE & 50 & 10.0 \\
BW & 269 & 26.6 \\
BE & 42 & 8.3 \\
\hline
\end{tabular}

Tableau 3.5: Pentes et ordonnées à l'origine des courbes seuils initales pour le système (voir texte).

ensuite une nouvelle courbe seuil dont la pente est la même que la première, mais dont l'ordonnée à l'origine est décalée vers le haut à partir du point où l'injection a été inhibée (voir la figure. Le coefficient $C \leq 1$ permet éventuellement de réduire le seuil de tolérance à chaque fois que le précédent seuil a été dépassé).

- Si à partir de là, l'injection est à nouveau propre, alors elle restera au-dessous de la nouvelle courbe seuil. Sinon, le même scénario se répète.

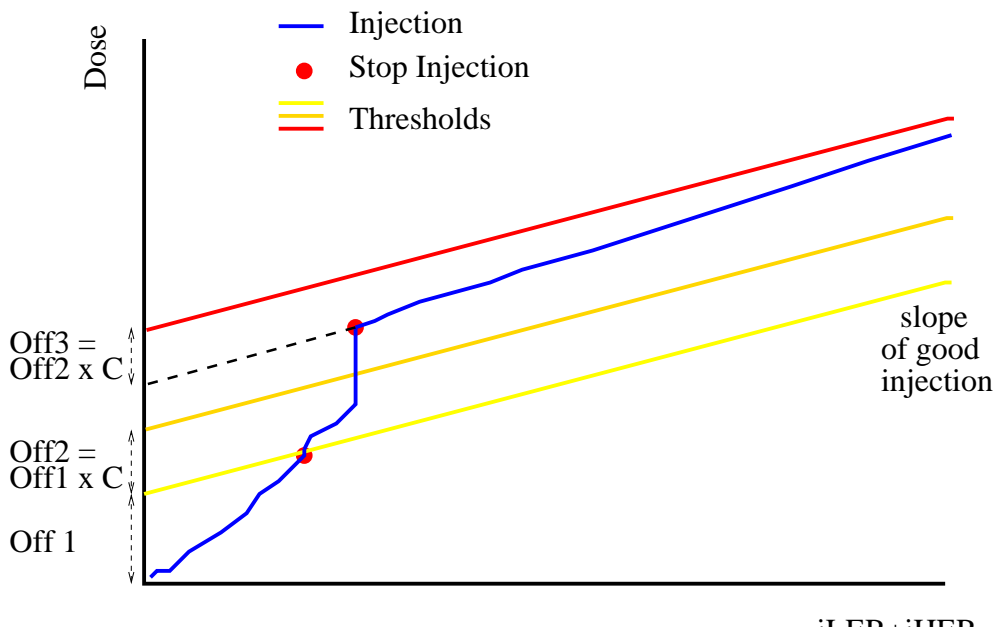

Figure 3.26: Le nouvel algorithme de contrôle des radiations programmé dans EPICS: ce schéma représente la dose intégrée depuis le début de l'injection en fonction de la somme des courants des faisceau. Voir le texte pour l'explication de l'algorithme.

Les personnes en garde à BABAR et surtout les opérateurs de PEP-II ont à leur disposition un certain nombre de panneaux leur indiquant l'évolution des performances de l'injection et du statut du système de protection décrit dans cette section. Des exemples d'activation du système de protection sont montrés sur la figure 3.27: il s'agit de photos d'écrans prises lors de la prise de données de BABAR courant 2002. On y voit une courbe "dose-courant" montrant deux inhibitions de faisceaux, ainsi que les alarmes déclenchées en présence d'une inhibition imminente.

Si la dose intégrée devient trop élevée, l'algorithme envisage ${ }^{5}$ alors de interrompre les faisceaux en utilisant l'algorithme dit de "coupure intelligente" illustré sur la figure 3.28.

\footnotetext{
${ }^{5}$ Cette partie de l'algorithme a été utilisé durant toute l'année 2002, mais a été retiré du système en 2003. En effet, en pratique, il s'avère qu’il est toujours plus cô̂teux en terme de radiations de interrompre les faisceaux que de les injecter jusqu'au bout.
} 


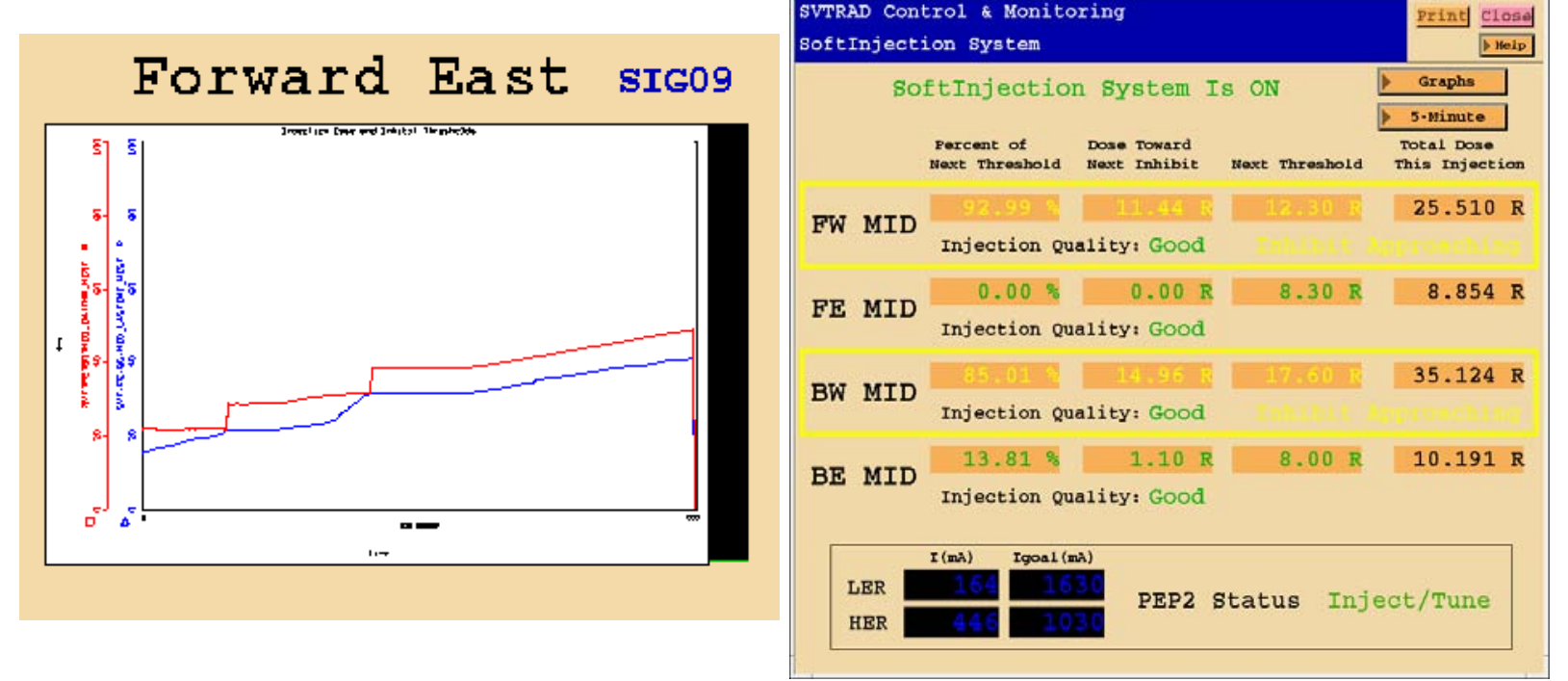

Figure 3.27: Panneau de gauche: exemple de graphe "dose-courant" pendant une injection. La courbe rouge montre le seuil, et la courbe bleue correspond à l'injection en cours. On voit que la courbe bleue a recontré le seuil à deux reprises, avant de reprendre une pente de "bonne injection". Panneau de droite: signaux d'alarme à l'approche d'une inhibition. Le problème

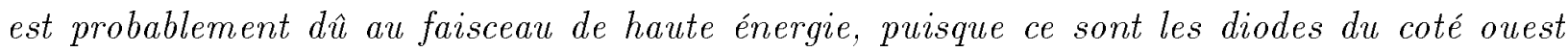
( $F W$ et $B W)$ qui déclenchent l'alarme.

On définit la dose totale $D$ comme la moyenne des doses totales des bonnes injections. On appelle $R$ la dose intégrée depuis le début de l'injection en cours. Lors d'une injection, les opérateurs de PEP-II prévoient les courants finaux à atteindre: nous dénommons la somme de ces courants par " $I_{\text {goal }}$ ", et la somme des courants déjà injectés par $I$. Le rapport $I$ sur

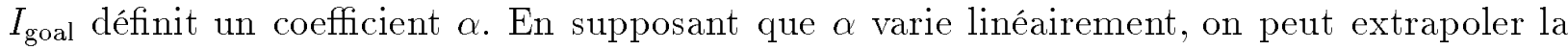
dose totale que l'on atteindra pour l'injection en cours comme étant $R / \alpha$.

L'algorithme de "coupure intelligente" consiste à interrompre les faisceaux si la dose extrapolée $R / \alpha$ est plus grande que la dose qu'on accumulerait en coupant les faisceaux maintenant et en ré-injectant depuis le début (la dose obtenue serait alors $R+D$, en admettant que la prochaine injection soit bonne): on choisit ainsi la solution la plus économique en terme de radiations entre finir l'injection en cours, ou la recommencer.

\subsubsection{Bilan du nouveau algorithme de protection activé pendant les injections}

Le bilan du nouvel algorithme de protection activé pendant les injections est positif:

- les opérateurs de PEP-II apprécient les informations transmises par ce nouveau système durant les injections: elles leur permettent de suivre les performances des injections, et le statut du système de protection très précisément.

- ce système visait entre autres à limiter le nombre de coupures de faisceaux par le logiciel en temps réel pendant les injections, car le cô̂t en radiations est souvent plus élevé quand il faut injecter à nouveau les faisceaux depuis zéro. Ce point de vue est maintenant complètement adopté par BABAR dont la nouvelle politique de protection durant les injections en 2003 est de ne plus interrompre les faisceaux du tout avec le 


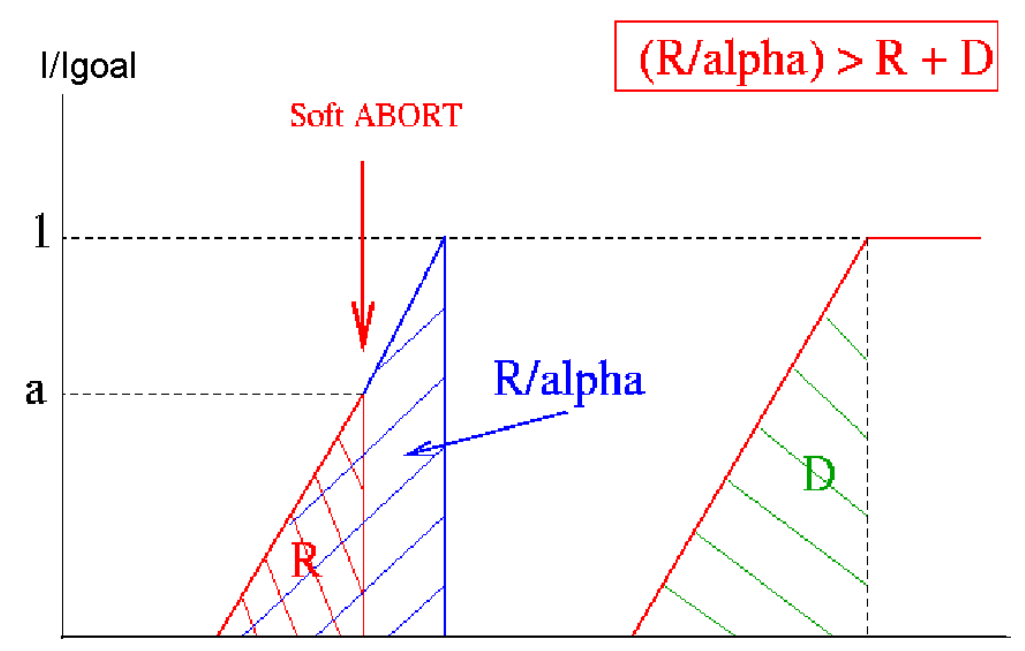

Figure 3.28: Schéma expliquant l'algorithme de "coupure intelligente" implémenté dans EPICS: le schéma représente des séquences d'injection en fonction du temps. Voir le texte pour l'explication de l'algorithme.

logiciel (mais bien entendu, la partie principale du logiciel concernant les inhibitions d'injection reste quant à elle en place).

- des améliorations ont été apportées à ce système depuis sa mise en service en 2002: par exemple, au lieu de considérer les quatre diodes séparément comme cela a été implémenté en premier lieu, le système utilise désormais la diode avec le plus grand taux de radiation, et lorsque l'injection est inhibée par cette diode, les seuils des quatre diodes sont augmentés tous à la fois, limitant ainsi le nombre d'inhibitions en coïncidence.

La dose accumulée par les diodes en fonction de la luminosité intégrée est montrée sur la figure 3.29 pour les années 2001 (avant la mise en service du nouveau système de protection décrit ici) et 2002 (après sa mise en service). Grâce à ce système ainsi qu'aux autres systèmes de protection implémentés dans SVTRAD, la dose intégrée par $\mathrm{fb}^{-1}$ est restée au-dessous des limites correspondantes à une demi-durée de vie du SVT. 


\section{Silicon Vertex Tracker: Radiation Monitoring}

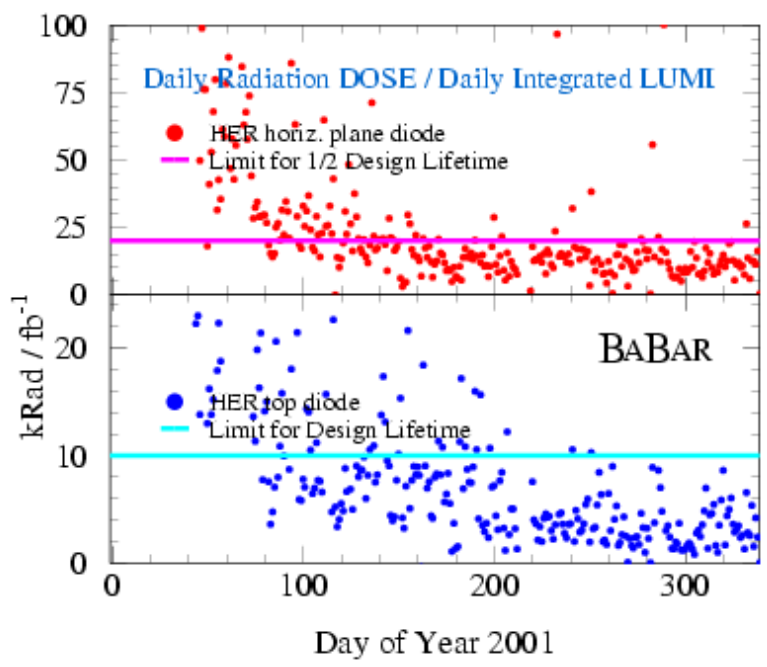

Silicon Vertex Tracker: Radiation Monitoring

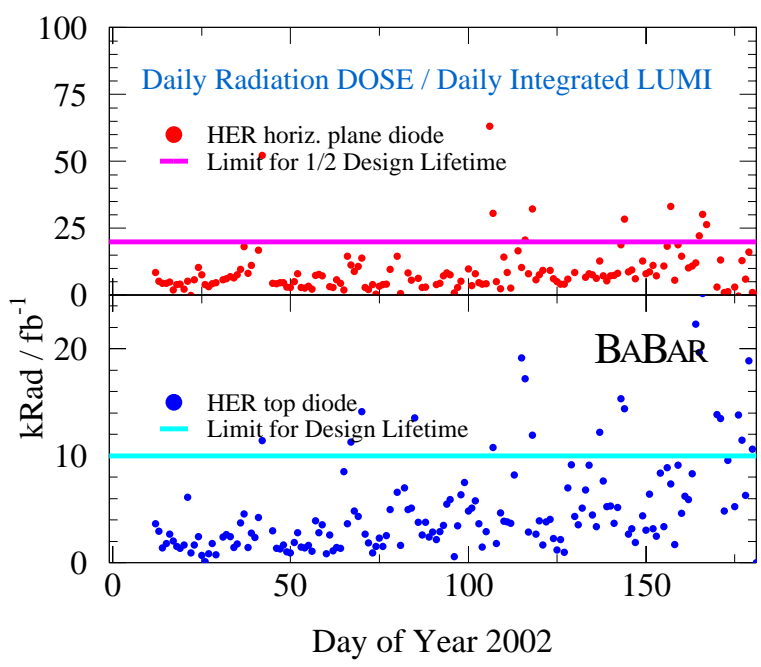

Figure 3.29: Dose (krad) en fonction de la luminosité intégrée ( $\mathrm{fb}^{-1}$ ) pour les années 2001 (gauche) et 2002 (droite): le nouveau système de protection pendant les injections a été installé au début de l'année 2002: grâce à ce système, ainsi qu'aux autres systèmes de protection implémentés dans SVTRAD, la quantité de dose par $\mathrm{fb}^{-1}$ est restée au-dessous des limites correspondantes à une demi-durée de vie du SVT.

\subsection{Les données}

\subsubsection{Données on-peak et off-peak}

Les données utilisées dans cette thèse ont été prises entre les mois d'octobre 1999 et octobre 2000 ("Run 1"), et entre les mois de février 2001 et juin 2002 ("Run 2"). Le "Run 3" a débuté en novembre 2002, mais n'est pas pris en compte ici. La figure 3.30 montre la luminosité intégrée depuis les débuts de BABAR en 1999 jusqu'en janvier 2003.

Environ $90 \%$ des données sont enregistrée sur le pic du $\Upsilon(4 S)(M(\Upsilon(4 S))=10.58 \mathrm{GeV})$ : on dénomme ces événements "on-peak". Les sections efficaces de $e^{+} e^{-}$à cette énergie sont données dans le tableau 3.6: on y voit le poids relatif des événements de physique du $B$ $\left(e^{+} e^{-} \rightarrow \Upsilon(4 S)(b \bar{b})\right)$ par rapport aux événements de continuum $e^{+} e^{-} \rightarrow c \bar{c}, s \bar{s}, u \bar{u}, d \bar{d}$ et aux événements leptoniques $e^{+} e^{\rightarrow} \tau^{+} \tau^{-}, \mu^{+} \mu^{-}$, et $e^{+} e^{-}$.

Le reste des données est pris $40 \mathrm{MeV}$ au-dessous du pic du $\Upsilon(4 S)$ (dénommé "off-peak"), afin de disposer d'un ensemble événements continuum et leptoniques uniquement. Ces événements constituent en effet un bruit de fond pour les analyses des événements $B$, et en particulier des modes rares non-charmés comme ceux étudiés dans cette thèse.

Le tableau 3.7 résume l'ensemble des données on-peak et off-peak utilisées dans cette thèse. 


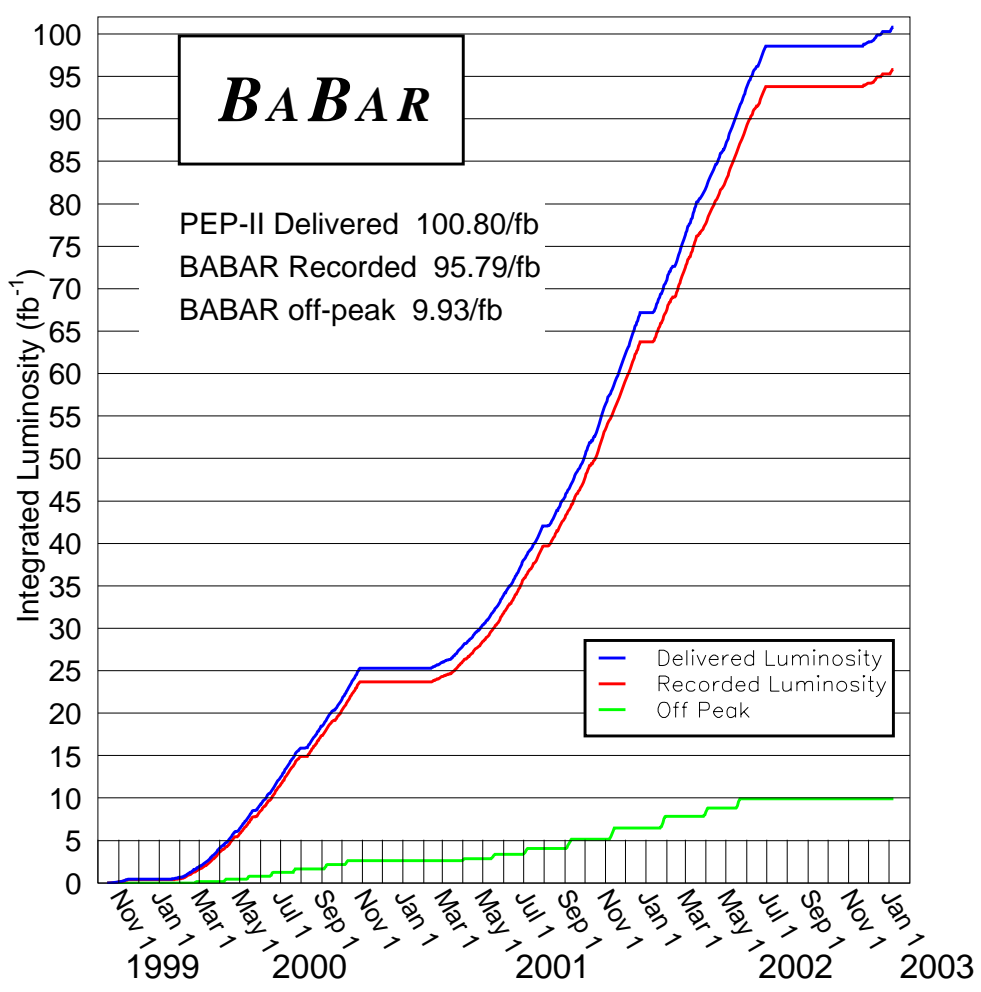

Figure 3.30: Luminosité sur le pic du $\Upsilon(4 S)$ délivrée par PEP-2 (courbe bleue) et enregistrée par BABAR (courbe rouge). La courbe verte indique la luminosité enregistrée $40 \mathrm{MeV}$ en-deçà du pic du $\Upsilon(4 S)$.

\begin{tabular}{cc}
\hline$e^{+} e^{-} \rightarrow$ & Section efficace (nb) \\
\hline$b b(\Upsilon(4 S))$ & 1.13 \\
$c \bar{c}$ & 1.30 \\
$s \bar{s}$ & 0.35 \\
$u \bar{u}$ & 1.39 \\
$d \bar{d}$ & 0.35 \\
\hline$\tau^{+} \tau^{-}$ & 0.94 \\
$\mu^{+} \mu^{-}$ & 1.16 \\
$e^{+} e^{-}$ & $\simeq 40$ \\
\hline
\end{tabular}

Tableau 3.6: Section efficace de production [59] quand l'énergie dans le centre de masse des faisceaux est égale à la masse du $\Upsilon(4 S)$ ("on-peak").

\begin{tabular}{ccc}
\hline Année & Luminosité on-peak $\left(\mathrm{fb}^{-1}\right)$ & Luminosité off-peak $\left(\mathrm{fb}^{-1}\right)$ \\
\hline $1999-2000$ & 20.721 & 2.602 \\
2001 & 35.605 & 3.786 \\
2002 & 25.553 & 3.192 \\
\hline Total & 81.879 & 9.58 \\
\hline
\end{tabular}

Tableau 3.7: Luminosité on-peak et off-peak correspondante aux données utilisées dans cette thèse. 


\subsubsection{Les événements Breco}

Les désintégrations des $B$ en états propres de saveur $D^{(*)-} \pi^{+} / \rho^{+} \mathrm{a}_{1}^{+}{ }^{6}$ sont regroupées sous le terme de Breco. Produits en quantité abondante à $B A B A R$, et reconstruits avec une pureté élevée, ces modes sont utilisés dans la plupart des analyses $C P$ de $B A B A R$ pour mesurer les paramètres des oscillations des $B$, comme $\Delta m_{d}$, mais aussi les paramètres de la fonction de résolution de $\Delta t$, les taux de mauvais étiquetage, etc.( $c f$. Sec. 4.4 et 4.9.1.5). La figure 3.31 montre les distributions en $m_{\mathrm{ES}}$ ( $c f$. déf. dans l'éq. 4.4) des échantillons Breco des $B$ neutres et chargés. Le tableau 3.8 donne les statistiques relatives à ces échantillons [60].
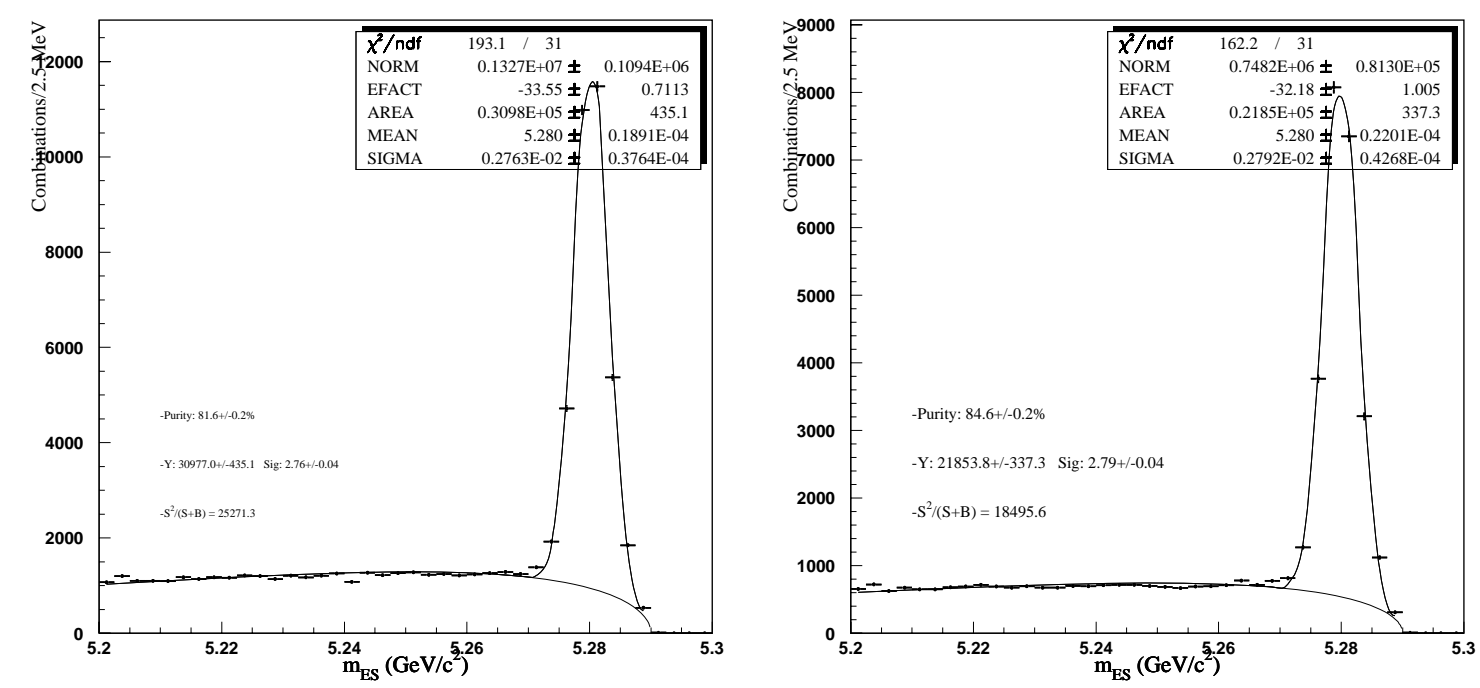

Figure 3.31: Distributions en $m_{\mathrm{ES}}$ (voir def. dans Eq. 4.4) des échantillons Breco des $B$ neutres et chargés [60].

\begin{tabular}{cccc}
\hline Mode & $\begin{array}{c}\text { Nombre d' } \\
\text { événements }\end{array}$ & $\begin{array}{c}\text { Pureté } \\
(\%)\end{array}$ & $\sigma\left(m_{\mathrm{ES}}\right)$ \\
\hline$B^{0}$ & $30977.0 \pm 435.1$ & $81.6 \pm 0.2$ & $2.76 \pm 0.04$ \\
\hline$B^{+}$ & $21853.8 \pm 337.3$ & $84.6 \pm 0.2$ & $2.79 \pm 0.04$ \\
\hline
\end{tabular}

Tablea1 3.8: Nombres d'événements, puretés et largeurs de la distribution de $m_{\mathrm{ES}}$ des modes Breco neutres et chargés [60].

\subsection{La simulation Monte Carlo}

Les analyses réalisées dans BABAR reposent en partie sur les simulations Monte Carlo: les efficacités de sélection, les distributions des différentes variables, les validations des algorithmes d'analyse, etc., sont obtenues grâce au Monte Carlo.

La simulation est réalisée en trois étapes:

\footnotetext{
${ }^{6}$ Les modes de production de charme $B \rightarrow D^{(*)}$ constituent la majorité des modes Breco. D'autres modes charmonium tels que $J / \psi K^{* 0}\left(K^{+} \pi^{-}\right)$sont aussi inclus dans cet échantillon, mais contribuent seulement dans une moindre mesure.
} 
- la simulation de la physique: les quadri-vecteurs des particules de la désintégration considérée sont produits aléatoirement en tenant compte des contraintes spécifiques aux collisions $e^{+} e^{-}$permises par PEP-II, telles que les fluctuations d'énergie des particules des faisceaux, la taille de la région lumineuse et l'angle entre les axes des deux faisceaux. Deux programmes sont utilisés pour ceci: JETSET [61] (pour le continuum, et certaines désintégrations des $B$ ) et EvtGen [62] (pour les désintégrations $B$, dont les modes de signal considérés dans cette thèse).

- la propagation des particules dans la matière du détecteur: cette étape est réalisée par le programme GEANT [63] (la plupart des simulations utilisées dans cette thèse utilisent la version GEANT4 [64]), et simule les interactions des particules avec la matière du détecteur, incluant leur perte d'énergie, les modifications de trajectoire, la production de particules secondaires, etc. Cette étape requiert la modélisation précise de la géométrie de BABAR et des matériaux qui le composent.

- la réponse du détecteur aux évênements simulés: cette dernière étape repose aussi sur le programme GEANT, car la réponse du détecteur est déterminée par les interactions des particules avec les matériaux des détecteurs de BABAR. La réponse de l'électronique de lecture est aussi simulée. Cette étape inclut les différentes conditions de fonctionnement des sous-détecteurs (par exemple, les tensions de la DCH, la composition des gaz, etc., enregistrés lors des prises de données, cf. Sec. 3.2.8). D'autre part, des événements de bruit de fond provenant de l'accélérateur sont mélangés en proportion réaliste aux événements générés par la simulation. Néanmoins, la description de la réponse des sous-détecteurs n'est pas parfaite. Par exemple, l'efficacité de reconstruction des $\pi^{0}$ est surestimée dans les simulations (voir Sec. 4.14.1). Nous aurons donc parfois besoin de corriger les simulations utilisées.

Pour les analyses réalisées dans cette thèse, nous utilisons les simulations Monte Carlo listées ci-dessous. Pour chacune de ces simulations, une version non corrigée et une version corrigée pour la reconstruction des $\pi^{0}$ (voir Sec. 4.14.1) sont disponibles. Par défaut, les versions corrigées sont utilisées tout au long des analyses exposées, sauf mention contraire.

\section{- Simulations communes à toutes les analyses:}

$-e^{+} e^{-} \rightarrow \tau^{+} \tau^{-}$générique: $8.5 \times 10^{6}$ événements.

- $B^{0} \bar{B}^{0}$ générique: $79.3 \times 10^{6}$ événements.

- $B^{+} B^{-}$générique: $90.8 \times 10^{6}$ événements.

- Plus d'une centaine de modes de désintégrations non-charmées du $B$.

- Simulations spécifiques à l'analyse de $B^{0} \rightarrow \rho^{ \pm} \pi^{\mp}$ et $B^{0} \rightarrow \rho^{-} K^{+}$:

- $B^{0} \rightarrow \rho^{ \pm} \pi^{\mp}$ non interférant: $464 \times 10^{3}$ événements. Deux ensembles d'événements sont générés séparément pour $B^{0} / \bar{B}^{0} \rightarrow \rho^{-} \pi^{+}$et $B^{0} / \bar{B}^{0} \rightarrow \rho^{+} \pi^{-}$: les interférences entre ces deux modes sont ignorées. Le facteur de forme du $\pi$ utilisé dans cette simulation est une Breit-Wigner relativiste. C'est le Monte Carlo du signal $B^{0} \rightarrow \rho^{ \pm} \pi^{\mp}$ utilisé par défaut dans l'analyse de $B^{0} \rightarrow \rho^{ \pm} \pi^{\mp}$. 
- $B^{0} \rightarrow \rho^{ \pm} \pi^{\mp}$ interférant: $239 \times 10^{3}$ événements. On génère les résonances $\rho(770)$, $\rho(1450)$ et $\rho(1700)$ en prenant en compte les interférences. Le facteur de forme utilisé dans le Monte Carlo utilise la paramétrisation de Kühn-Santamaria [65]:

$$
F_{\pi}(s)=\frac{\mathrm{BW}_{\rho(770)}(s)+\beta \mathrm{BW}_{\rho(1450)}(s)+\gamma \mathrm{BW}_{\rho(1700)}(s)}{1+\beta+\gamma}
$$

où les propagateurs des Breit-Wigner BW sont donnés par:

$$
\mathrm{BW}_{\rho\left(M_{\rho}\right)}^{\mathrm{KS}}(s)=\frac{M_{\rho}^{2}}{M_{\rho}^{2}-s-i \sqrt{s} \Gamma_{\rho}(s)}
$$

et les largeurs par:

$$
\Gamma_{\rho}(s)=\Gamma_{\rho}\left(M_{\rho}^{2}\right)\left(\frac{M_{\rho}^{2}}{s}\right)^{1 / 2}\left(\frac{k(s)}{k\left(M_{\rho}^{2}\right)}\right)^{3},
$$

pour des pions dans l'onde P. Le paramètre $k(s)=\frac{1}{2} \sqrt{s} \beta_{\pi}(s)$ et $k\left(M_{\rho}^{2}\right)$ est l'impulsion du pion dans le référentiel du $\rho$. Selon la référence [65], les amplitudes $\beta$ et $\gamma$ sont supposées réelles.

La paramétrisation de Kühn-Santamaria a été ajustée sur les données $\tau$ de ALEPH [66]. Néanmoins, l'espace de phase des désintégrations $\tau$ est tel qu'il supprime la contribution de la résonance $\rho(1700)$. Les données des annihilations $e^{+} e^{-}$de la collaboration DM2 [67] sont utilisées pour corriger ceci. L'ajustement total donne $\beta=-0.23 \pm 0.02$ et $\gamma=0.08 \pm 0.02$. Les masses et largeur des $\rho$ se trouvent dans la référence [66].

$-B^{0} \rightarrow \rho^{-} K^{+}$(non interférant): $236 \times 10^{3}$ événements (le facteur de forme du $\pi$ est une Breit-Wigner relativiste).

La figure 3.32 montre la distribution de la masse invariante de $\pi^{ \pm} \pi^{0}$ dans le Monte Carlo interférant et non interférant $B^{0} \rightarrow \rho^{ \pm} \pi^{\mp}$.

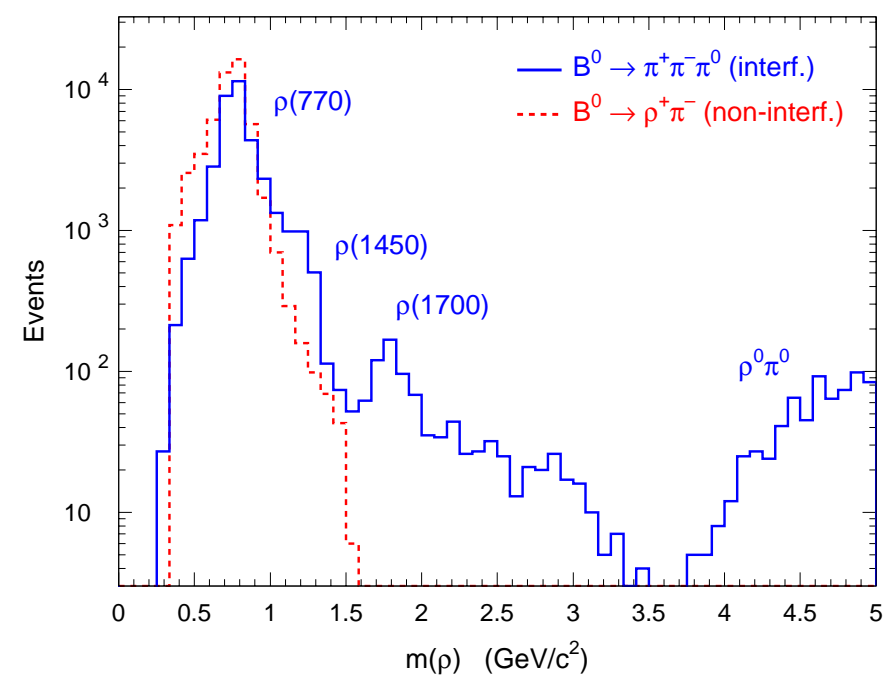

Figure 3.32: Distribution de la masse invariante de $\pi^{ \pm} \pi^{0}$ dans le Monte Carlo interférant (ligne bleue) et non interférant (ligne rouge) $B^{0} \rightarrow \rho^{ \pm} \pi^{\mp}$. 
- Simulations spécifiques à l'analyse de $B^{0} \rightarrow \rho^{0} \pi^{0}$ :

- $B^{0} \rightarrow \rho^{0} \pi^{0}$ non interférant: $224 \times 10^{3}$ événements (le facteur de forme du $\pi$ est aussi une Breit-Wigner relativiste).

- Simulations spécifiques à l'analyse de $B^{0} \rightarrow a_{0}^{ \pm} \pi^{\mp}$

- $B^{0} \rightarrow a_{0}^{ \pm} \pi^{\mp}$ non interférant: 48000 événements. 


\section{Chapitre 4}

\section{Analyse temporelle et mesure des rapports d'embranchement de $B^{0} \rightarrow \rho^{ \pm} \pi^{\mp}$ et $B^{0} \rightarrow \rho^{-} K^{+}$}

\subsection{Stratégie de l'analyse}

L'analyse dépendante du temps en deux corps de $B^{0} \rightarrow \rho^{ \pm} \pi^{\mp}$ et $B^{0} \rightarrow \rho^{-} K^{+}$exposée dans ce chapitre se donne deux objectifs:

1. déterminer les nombres d'événements de $B^{0} \rightarrow \rho^{ \pm} \pi^{\mp}$ et $B^{0} \rightarrow \rho^{-} K^{+}$, et calculer les rapports d'embranchement correspondants.

2. mesurer les asymétries $C P$ et les paramètres de dilution dans $B^{0} \rightarrow \rho^{ \pm} \pi^{\mp}$ et $B^{0} \rightarrow$ $\rho^{-} K^{+}$(cf. Eq. 1.57 et 1.58):

- asymétrie $C P: C, S$ et $A_{C P}$ dans $B^{0} \rightarrow \rho^{ \pm} \pi^{\mp}$, et $A_{\mathrm{CP}}^{K}$ dans $B^{0} \rightarrow \rho^{-} K^{+}$.

- paramètres de dilution: $\Delta C$ et $\Delta S$ dans $B^{0} \rightarrow \rho^{ \pm} \pi^{\mp}$.

Ces paramètres sont extraits grâce à un ajustement de maximum de vraisemblance sur des événements sélectionnés parmi les données on-peak. Dans cet échantillon, nous trouvons différents types d'événements:

- le signal $B^{0} \rightarrow \rho^{ \pm} \pi^{\mp}$ et $B^{0} \rightarrow \rho^{-} K^{+}$, où l'on distingue les trois catégories suivantes possédant des caractéristiques différentes ${ }^{1}$ :

- le signal correctement reconstruit (où toutes les particules de l'état final ont été retrouvées).

- le signal mal reconstruit (où une ou plusieurs particules de l'état final sont issues du bruit de fond) avec un $\rho$ de charge correcte (qui ne biaise pas les asymétries de charge).

- le signal mal reconstruit avec un $\rho$ de charge incorrecte (qui biaise les asymétries de charge).

\footnotetext{
${ }^{1}$ Certaines figures de ce chapitre ont une légende en anglais, et l'on réfère alors à ces trois catégories par respectivement "true signal", "Self-Cross Feed (SCF) right charge" et "Self-Cross Feed (SCF) wrong charge".
} 
- le bruit de fond $e^{+} e^{-} \rightarrow q \bar{q}$ continuum, séparé en deux catégories: celui contaminant $B^{0} \rightarrow \rho^{ \pm} \pi^{\mp}$ et celui contaminant $B^{0} \rightarrow \rho^{-} K^{+}$.

- le bruit de fond provenant d'autres désintégrations de $B$, aussi séparé dans les catégories $B^{0} \rightarrow \rho^{ \pm} \pi^{\mp}$ et $B^{0} \rightarrow \rho^{-} K^{+}$.

La section 4.2 décrit la topologie de ces différents types d'événements. La sélection du signal est décrite dans la section 4.3, l'étiquetage du $B_{\text {sav }}$ dans la section 4.4, et la reconstruction de $\Delta t$ dans la section 4.5. La lutte contre le bruit de fond continuum, et le traitement du bruit de fond $B$ sont respectivement détaillés dans les sections 4.6 et 4.8. La sélection finale est résumée dans la section 4.7 .

L'ajustement final des données, et les tests de validation qui lui sont associés, sont décrits dans les sections 4.10.1 à 4.13. Les études des incertitudes systématiques sont exposées dans la section 4.14. Enfin, le calcul des rapports d'embranchement de $B^{0} \rightarrow \rho^{ \pm} \pi^{\mp}$ et $B^{0} \rightarrow \rho^{-} K^{+}$ est réalisé dans la section 4.15 .

L'élaboration de l'analyse telle qu'exposée dans ce chapitre s'est faite de façon itérative: en particulier, les choix réalisés pour la lutte contre le bruit de fond continuum ou le traitement du bruit de fond $B$ ont nécessité des analyses intermédiaires complètes. De ce fait, on trouvera des simulations rapides ${ }^{2}$ dans les sections dédiées aux bruits de fond, alors que l'ajustement final n'est décrit qu'ultérieurement.

L'analyse est réalisée "en aveugle", ce qui signifie que les valeurs des paramètres mesurés sont cachées pendant les phases d'élaboration et de validation de l'analyse. Nous dévoilons les résultats une fois que toutes les études de validation en aveugle donnent des résultats satisfaisants.

\subsection{Topologie des différents types d'événements}

Les différents types d'événements énoncés ci-dessus possèdent des topologies distinctes, qui se différencient sur 2 échelles:

- $e^{+} e^{-} \rightarrow X$ : dans le cas des événements de signal $e^{+} e^{-} \rightarrow \Upsilon(4 S) \rightarrow B \bar{B}$, la désintégration du $\Upsilon(4 S)$ de spin en 1 en deux mésons $B$ de spin 0 donne lieu à une distribution angulaire du $B$ dans le centre de masse de $e^{+} e^{-}(\mathrm{cdm})$ en $\sin ^{2} \theta$ (où $\theta$ est l'angle entre la direction du $B$ et l'axe $z$ ). Par contre, pour les événements de bruit de fond $e^{+} e^{-} \rightarrow f \bar{f}$ où $f$ est un fermion de spin $1 / 2$ (lepton ou quark), la distribution angulaire est en $1+\cos ^{2} \theta$ (où $\theta$ est l'angle entre la direction du "jet" de quarks et l'axe $z$ ).

- $X \rightarrow$ état final: en ce qui concerne le signal, une paire $B^{0} \bar{B}^{0}$ étant produite quasiment au repos dans le centre de masse de $e^{+} e^{-}$, il reste peu d'énergie $(\simeq 300 \mathrm{MeV})$ pour booster la paire de $B$, et la désintégration de chaque $B$ est donc quasi-isotrope. D'autre part, il n'y a pas de corrélation entre les directions des produits de désintégration de chaque $B$. Lors de la production de quarks légers $q \bar{q}$ où $q=u, d, s$ (appelé bruit de fond "continuum"), par contre, il existe une structure en deux "jets" dos à dos, et donc une direction privilégiée caractérisant l'état final. Il y a par la même une corrélation entre les produits de désintégration de l'éventuel candidat qui a tendance à se trouver dans l'axe des deux "jets". Lors de la production du quark plus lourd $c$ (appartenant aussi au continuum), cette structure est toujours présente, mais atténuée.

\footnotetext{
${ }^{2}$ Une simulation rapide consiste en la génération et l'ajustement d'événements selon les mêmes distributions de probabilité.
} 
Le tableau 4.1 résume les topologies des différents types d'événements, et la figure $4.1^{3}$ montre, pour chacun de ces types d'événements, le nombre de traces chargées et l'énergie visible (définie comme la somme des énergies des traces chargées et neutres).

\begin{tabular}{lll}
\hline \hline Type d'événement & Topologie & Contamination du signal \\
\hline$e^{+} e^{-} \rightarrow \Upsilon(4 S) \rightarrow B \bar{B}$ & $\begin{array}{l}\text { Nombreux hadrons } \\
\text { Désintégration isotrope dans cdm }\end{array}$ & $\begin{array}{l}\text { significative par d'autres } \\
\text { états finals que le signal }\end{array}$ \\
\hline$e^{+} e^{-} \rightarrow q \bar{q}$ où $q=u, d, s, c$ & $\begin{array}{l}\text { Nombreux hadrons } \\
\text { Désintégration en 2 “jets" dos à dos }\end{array}$ & principale \\
\hline$e^{+} e^{-} \rightarrow \tau^{+} \tau^{-}$ & $\begin{array}{l}\text { Peu de hadrons } \\
\text { Configuration dos à dos }\end{array}$ & $\begin{array}{l}\text { peu importante } \\
\left.\text { (assimilé à } e^{+} e^{-} \rightarrow q \bar{q}\right)\end{array}$ \\
\hline$e^{+} e^{-} \rightarrow e^{+} e^{-}, \mu^{+} \mu^{-}$ & $\begin{array}{l}2 \text { traces de grande impulsion } \\
\text { dos à dos }\end{array}$ & négligeable \\
\hline$e^{+} e^{-} \rightarrow \gamma \gamma$ & $\begin{array}{l}2 \text { photons de grande impulsion } \\
\text { dos à dos }\end{array}$ & négligeable \\
\hline \hline
\end{tabular}

Tablea1 4.1: Topologie des différents types d'événements
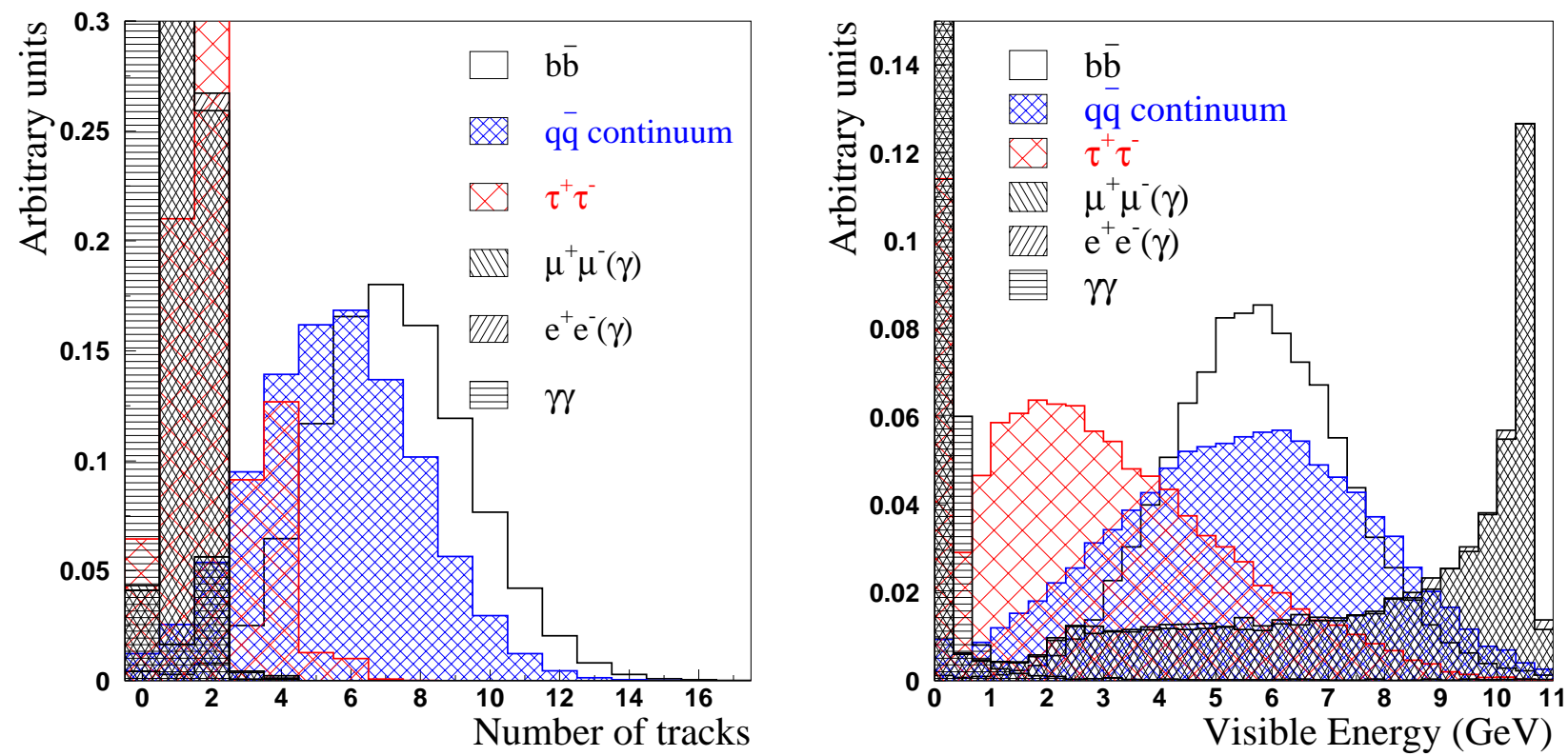

Figure 4.1: Nombre de traces chargées (gauche) et énergie visible (droite, voir texte pour la définition) pour les différents types d'événements.

Ces caractéristiques seront utilisées par la suite pour discriminer les événements de signal de ceux du bruit de fond.

\subsection{Reconstruction et sélection du $B_{\text {rec }} \quad B^{0} \rightarrow \rho h(h=$ $\pi, K)$}

$B^{0} \rightarrow \rho h$ est une désintégration en deux corps: le $\rho$ et le $h$ sont émis dos à dos dans le centre de masse du $B$, avec (en négligeant les masses du $\rho$ et du hadron $h=\pi, K$ par rapport à

\footnotetext{
${ }^{3}$ Ces figures sont issues de la référence [68].
} 
celle du $B$ ) $p_{\rho, h}^{*} \simeq E_{\rho, h}^{*} \simeq m_{B} / 2 \simeq 2.6 \mathrm{GeV} / c$. Dans le référentiel du laboratoire, on a, après avoir boosté les particules par $\gamma \simeq 1.144$ et $\beta \simeq 0.48$ :

$$
1.6<p_{\rho, h} \simeq E_{\rho, h}<4.1 \mathrm{GeV} / \mathrm{c} .
$$

Cet intervalle est en pratique un peu plus large à cause de l'impulsion non-nulle du $B$ et de la résolution du détecteur.

Dans cette section, nous décrivons la reconstruction et la sélection de $B^{0} \rightarrow \rho h$. L'efficacité finale de sélection ainsi que les efficacités relatives de chaque coupure décrite dans cette section sont donnée dans le tableau 4.7. Les figures se trouvant dans cette section sont basées sur le Monte Carlo incluant les effets de détecteur, sauf mention contraire. La plupart des figures sont faites pour le signal $B^{0} \rightarrow \rho^{ \pm} \pi^{\mp}$, et ne sont pas répétées pour $B^{0} \rightarrow \rho^{-} K^{+}$ puisque sa cinématique est très similaire.

\subsubsection{Sélection des kaons et pions chargés}

L'état final contient deux traces chargées: un pion issu de la résonance $\rho$, et un hadron $h$ issu du $B$ (appelé trace célibataire) qui peut être soit un pion, soit un kaon.

\subsubsection{Reconstruction des traces}
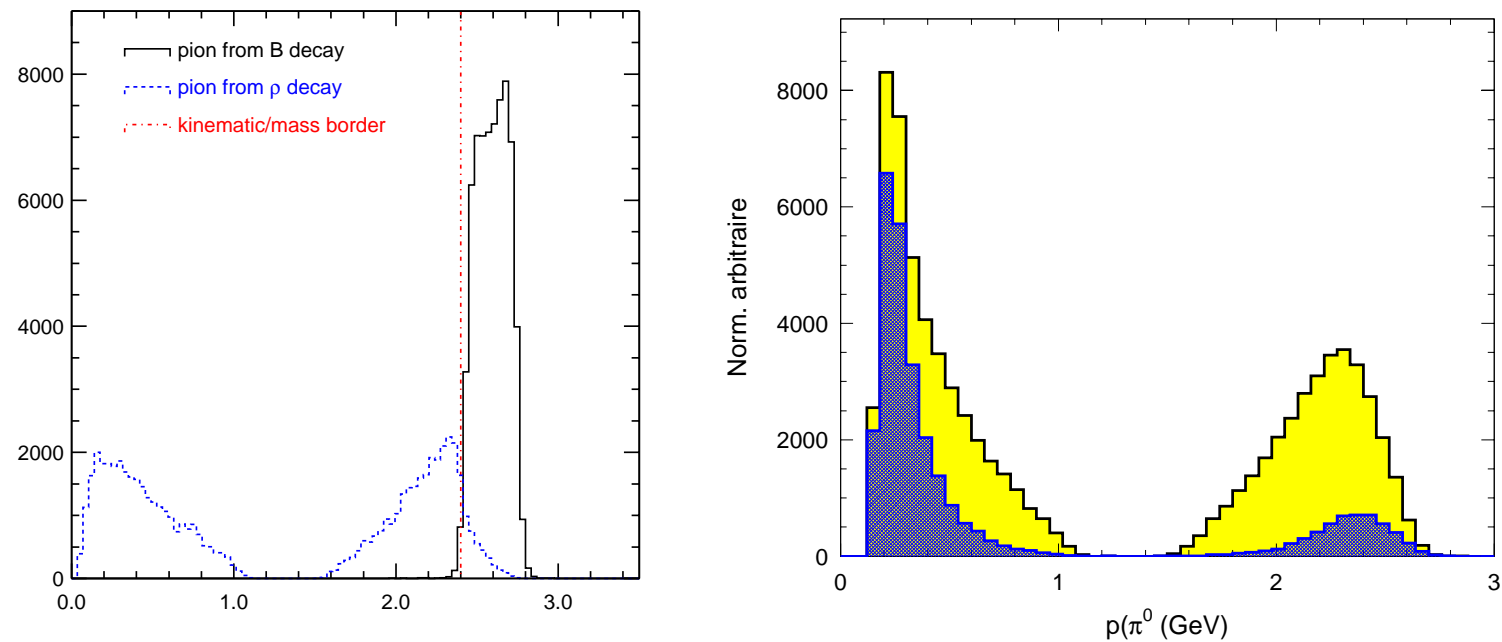

Figure 4.2: Figure de gauche: impulsion dans le référentiel du centre de masse du $\Upsilon(4 S)$ des deux traces chargées dans le Monte Carlo $B^{0} \rightarrow \rho^{ \pm} \pi^{\mp}$. La courbe en pointillé correspond au pion issu de la résonance du $\rho$, et la courbe en trait plein, au pion célibataire. Figure de droite: impulsion du pion neutre dans le centre de masse. La partie colorée en bleue correspond aux événements mal reconstruits (cf. Sec. 4.3.2). La cinématique du $\pi^{0}$ est identique à celle du $\pi$ chargé issu du $\rho$ (figure de gauche), mais il y a accumulation de $\pi^{0}$ mal reconstruits à faibles impulsions.

Le graphe de gauche de la figure 4.2 montre la distribution de l'impulsion des deux pions chargés dans la désintégration $B^{0} \rightarrow \rho^{ \pm} \pi^{\mp}$ dans le référentiel du centre de masse du $\Upsilon(4 S)$ : l'impulsion du pion célibataire pique à $2.6 \mathrm{GeV} / \mathrm{c}$. Celle du pion issu du $\rho$ est comprise entre 0 et $2.6 \mathrm{GeV} / \mathrm{c}$ et pique à faible et haute impulsion: en effet, l'hélicité du $\rho^{ \pm}$étant nulle (puisque les hélicités du $B^{0}$ et du $h^{\mp}$ dans $B^{0} \rightarrow \rho h$ sont nulles), et le $\rho$ étant une particule 
vecteur, la désintégration du $\rho^{ \pm}$en $\pi^{ \pm} \pi^{0}$ suit une distribution angulaire en $\cos ^{2} \theta_{H}(\rho)$, où $\theta_{H}(\rho)$ est l'angle polaire dans le centre de masse du $\rho^{ \pm} \mathrm{du} \pi^{ \pm}$issu du $\rho$ par rapport à la direction de vol du $\rho$ (cf. Fig. 4.3).
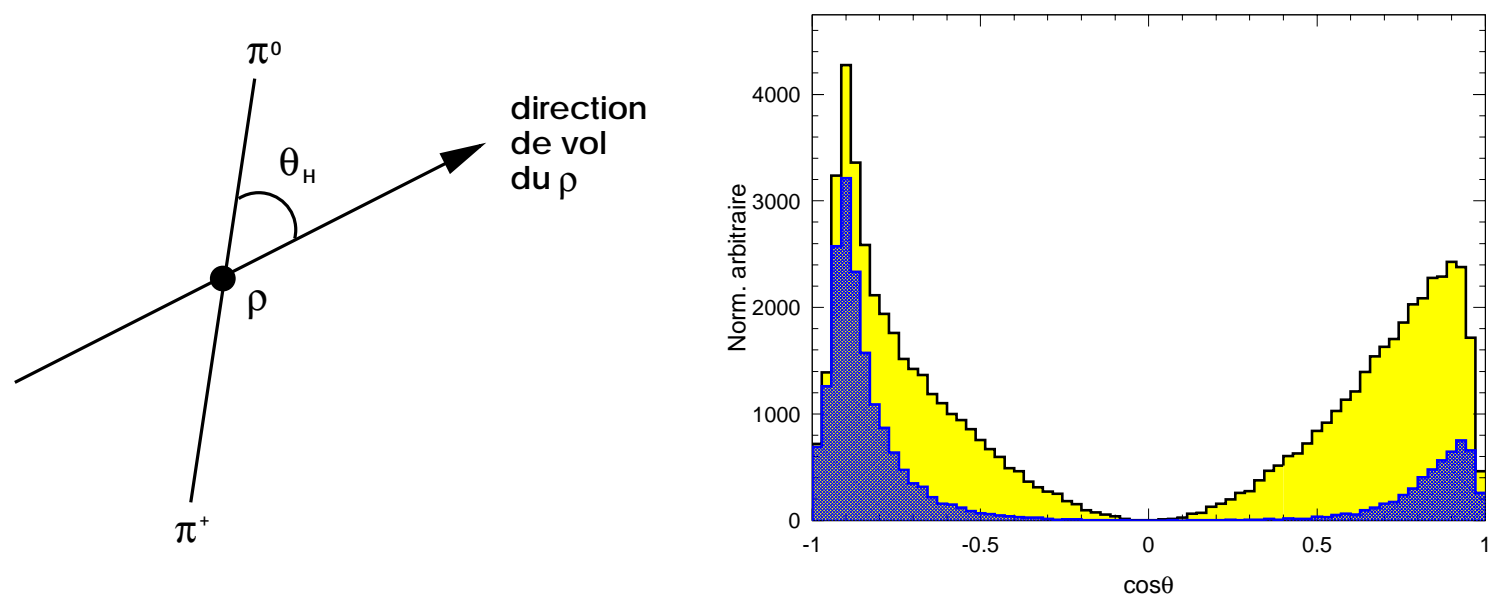

Figure 4.3: Gauche: schéma illustrant la définition de $\theta_{H}(\rho)$. Droite: distribution de $\cos \theta_{H}(\rho)$ (voir texte) de tous les événements de signal $B^{0} \rightarrow \rho^{ \pm} \pi^{\mp}$ (courbe jaune) et ceux événements mal reconstruits (courbe bleue).

Comme décrit dans la section 3.2.2, les traces chargées sont reconstruites à partir des points détectés par le SVT (Sec. 3.2.1) et/ou la DCH (Sec. 3.2.2). Il existe différents niveaux de sélection: pour la reconstruction de l'état final $\pi^{+} \pi^{-} \pi^{0}$, nous utilisons la sélection qui correspond aux critères suivants:

- impulsion dans le centre de masse inférieure à $10 \mathrm{GeV} / \mathrm{c}$.

- impulsion transverse dans le laboratoire supérieure à $100 \mathrm{MeV} / \mathrm{c}$.

- paramètre d'impact $d_{0}$ dans le plan $(x, y)$ inférieur à $1.5 \mathrm{~cm}$, et paramètre d'impact $z_{0}$ selon l'axe $z$ inférieur à $10 \mathrm{~cm}$.

- au moins 12 points dans la DCH.

\subsubsection{Identification des traces}

Les traces identifiées comme des électrons sont rejetées en appliquant en mode veto le sélecteur d'électrons basé sur les coupures suivantes [69]: $500<d E / d x<1000$ (unité arbitraire), $0.75<E / p<5$, le profil latéral $0<$ LAT $<0.6$ ( $c f$. Eq. 3.4), le moment de Zernike $\left|A_{42}\right|<10$ [56], et nombre de cristaux $>3$.

Le pion est sélectionné [70] en rejetant l'hypothèse qu'il puisse s'agir d'un kaon: ces derniers sont identifiés en utilisant une fonction de vraisemblance d'identification calculée en comparant la réponse du SVT, de la DCH et du DIRC au passage d'une particule à la réponse attendue pour un type de particule donné. Pour le SVT et la DCH, on utilise la mesure de la perte d'énergie par ionisation $d E / d x$. En ce qui concerne le DIRC, la fonction de vraisemblance est le produit de deux composantes: la première est celle associée à l'angle Cherenkov pour une hypothèse de particule $i$, et la deuxième est la probabilité Poissonnienne liée au nombre de photons Cherenkov détectés comparé au nombre de photons attendus (ce 
dernier dépend de l'impulsion et de l'angle d'incidence de la trace dans les barres de quartz: cf. figure 3.14).

La fonction de vraisemblance totale $\mathcal{L}_{i}$, pour une hypothèse de type de particule $i$, est le produit des vraisemblance du SVT, de la DCH et du DIRC. Il existe plusieurs algorithmes de sélection des kaons avec différents niveaux de coupures. L'algorithme utilisé en mode veto dans cette analyse est tel que le nombre de pions restants dans l'ensemble des kaons identifiés est en dessous de $5 \%$.

Nous rejetons aussi les traces compatibles avec l'hypothèse d'un proton en utilisant la même fonction de vraisemblance avec des coupures différentes [71].

La séparation entre la trace célibataire $h=\pi$ et $h=K$ se fait au niveau de l'ajustement final en utilisant l'angle Cherenkov mesuré pour cette trace dans le DIRC. Pour pouvoir utiliser cette information, on demande que le nombre de photons Cherenkov associée à la trace chargée $h^{ \pm}$soit supérieur à 5 , ce qui implique que la trace soit dans l'acceptance du DIRC. De plus, nous demandons à ce que l'angle Cherenkov mesuré soit à moins de $4 \sigma$ de l'angle attendu pour un $\pi$ ou un $K$ (cf. Eq. 3.14).

\subsubsection{Sélection des pions neutres}

Le pion neutre se désintègre en deux photons dans $98.8 \%$ des cas [44]. La figure 4.4 montre l'énergie et le profil latéral $L A T$ (défini dans l'eq. 3.4) des photons issus du $\pi^{0}$ dans le Monte Carlo $B^{0} \rightarrow \rho^{ \pm} \pi^{\mp}$.
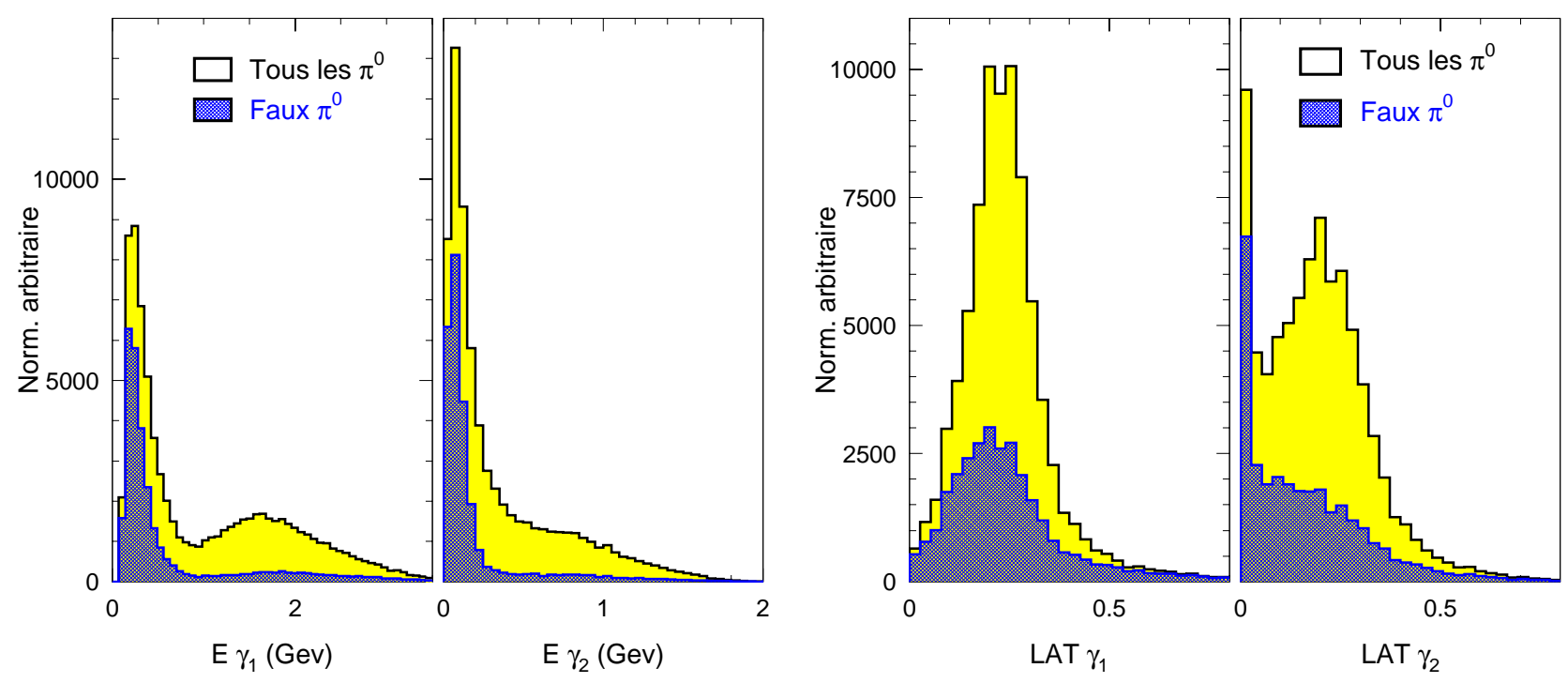

Figure 4.4: Energie dans le laboratoire (figure de gauche) et LAT (cf. Eq. 3.4) (figure de droite) des photons issus du $\pi^{0}$ dans le Monte Carlo $B^{0} \rightarrow \rho^{ \pm} \pi^{\mp}$ : la courbe jaune représente tous les $\pi^{0}$, et la courbe bleue les $\pi^{0}$ dont au moins l'un des photons a été mal reconstruit ("faux" $\left.\pi^{0}\right)$. Ces événements mal reconstruits comportent des photons de faible énergie. Le photon $\gamma_{1}$ est par définition celui de plus grande énergie.

Il arrive parfois qu'au moins un des deux photons utilisé pour la reconstruction du $\pi^{0}$ ne soit pas issu de la désintégration $B^{0} \rightarrow \rho h$, mais soit issu du bruit de fond ${ }^{4}$ : ceci constitue la principale source d'événements mal reconstruits. On voit sur la figure que ces événements

\footnotetext{
${ }^{4}$ Il peut s'agir du bruit de fond de PEP-II, ou d'un $\gamma$ pris de l'autre $B$.
} 
se concentrent à basse énergie et petit LAT: pour réduire le nombre d'événements mal reconstruits, on applique les coupures $E_{\gamma}>50 \mathrm{MeV}$ et $0.01<$ LAT $<0.6$. La masse invariante des deux photons doit être comprise entre 100 et $160 \mathrm{MeV}$, et la somme de leur énergie doit être supérieure à $200 \mathrm{MeV}^{5}$.
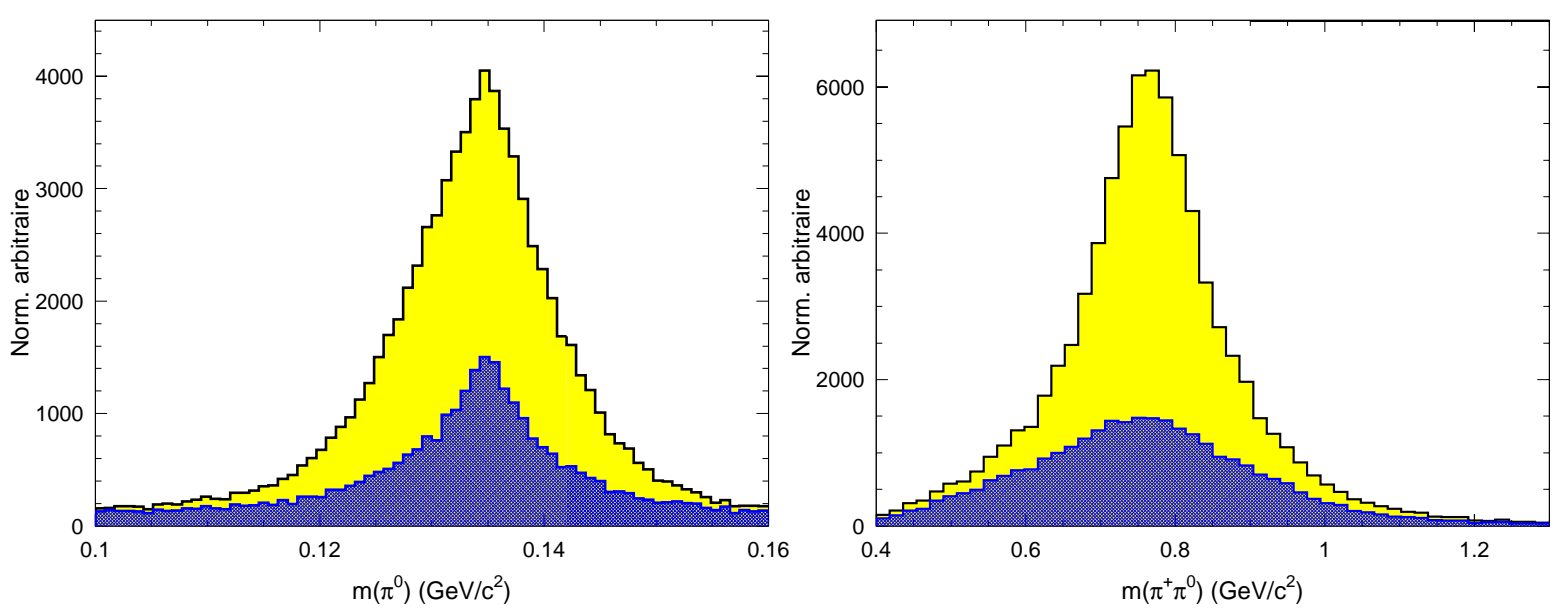

Figure 4.5: Masse invariante de la paire $\gamma \gamma$ (figure de gauche) et de la paire $\pi^{ \pm} \pi^{0}$ (figure de droite) dans le Monte Carlo $B^{0} \rightarrow \rho^{ \pm} \pi^{\mp}$. La courbe bleue montre les événements mal reconstruits.

\subsubsection{Reconstruction du méson $\rho^{ \pm}$}

\subsubsection{Reconstruction et sélection}

Le méson $\rho^{ \pm}$est reconstruit en associant deux pions $\pi^{ \pm} \pi^{0}$. Le graphe de gauche sur la figure 3.32 montre la masse invariante des deux pions $\pi^{ \pm} \pi^{0}$ dans le Monte Carlo $B^{0} \rightarrow \rho^{ \pm} \pi^{\mp}$ interférant qui inclue les résonances $\rho(770), \rho(1450)$ et $\rho(1700)$. Est superposée la distribution équivalente dans le Monte Carlo non-interférant n'incluant que le $\rho(770)$. Notre objectif est de reconstruire majoritairement le $\rho(770)$. Pour ceci, on demande à ce que la masse invariante de la paire de pions soit comprise entre 0.4 et $1.3 \mathrm{GeV} / \mathrm{c}^{2}$.

Les impulsions des trois traces sont ajustées à l'intérieur de leurs erreurs de reconstruction en tenant compte des contraintes suivantes:

- l'impulsion du $\pi^{0}$ est ajustée (voir Sec. 4.3.2) pour que la masse du $\pi^{0}$ soit égale à sa valeur nominale [44].

- les trajectoires des deux traces chargées sont ajustées pour former un vertex commun.

- les impulsions des trois particules de l'état final sont ajustées pour satisfaire les contraintes de masse et d'énergie du candidat $B$ (en faisant l'hypothèse que les trois traces sont des pions).

\footnotetext{
${ }^{5}$ Pour les $\pi^{0}$ d’impulsion dans le centre de masse supérieure à environ $2 \mathrm{GeV}$, il arrive que les deux photons issus du $\pi^{0}$ forment un unique amas dans le calorimètre, avec deux maxima locaux d'énergie, voir un seul maximum pour des $\pi^{0}$ de plus haute énergie. Si les deux maxima sont trop rapprochés, nous ne pouvons plus reconstruire la masse du $\pi^{0}$ donnée par $m_{\pi^{0}}^{2}=2 E_{\gamma_{1}} E_{\gamma_{2}}\left(1-\cos \theta_{\gamma_{1} \gamma_{2}}\right)$. Dans cette analyse, nous excluons ce type d'événements, ce qui mène à une perte absolue d'efficacité de moins de $1 \%$.
} 
La distribution de la masse du $\rho(770)$ ainsi obtenue est montrée sur la figure 4.5 à droite.

Du fait de la distribution de l'angle d'hélicité en $\cos ^{2} \theta_{H}(\rho)$ (cf. section 4.3.1.1), il y a très peu d'événements produits pour des angles $\theta_{H}(\rho)$ autour de $90^{\circ}$, alors que cette zone est peuplée par les événements de bruit de fond: on exclut donc les événements ayant $\left|\cos \theta_{H}(\rho)\right|<0.25$.

\subsubsection{Interférences $\rho^{+}-\rho^{-}$}

La figure 4.6 montre le diagramme de Dalitz de $B^{0} \rightarrow \rho^{ \pm} \pi^{\mp}$ : les bandes du $\rho$ se croisent (et interfèrent) dans la région de petites masses $m^{2}\left(\pi^{ \pm} \pi^{0}\right)$. Nous voulons effectuer une analyse de $B^{0} \rightarrow \rho^{ \pm} \pi^{\mp}$ en deux corps (en ne tenant donc pas compte des interférences), ce qui nous amène à exclure les événements pour lesquels à la fois les masses $m\left(\pi^{+} \pi^{0}\right)$ et $m\left(\pi^{-} \pi^{0}\right)$ sont comprises entre 0.4 et $1.3 \mathrm{GeV} / \mathrm{c}^{2}$ (indiqué par la zone rouge sur la figure, et dénommée zone d'interférence). L'effet des interférences résiduelles existant en dehors de cette région d'exclusion est traité comme une incertitude systématique.

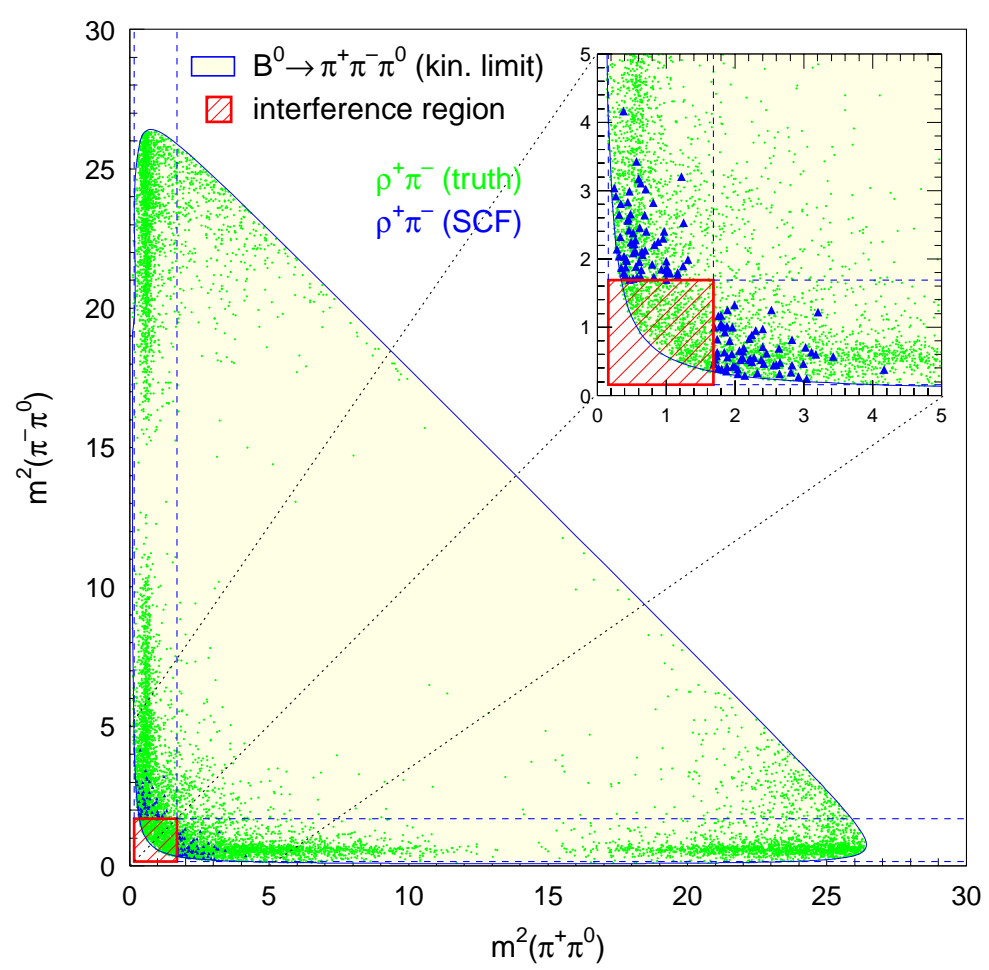

Figure 4.6: Diagramme de Dalitz de $B^{0} \rightarrow \rho^{ \pm} \pi^{\mp}$ pour les événements Monte Carlo interférant. Les points verts indiquent les événements correctement reconstruits, et les triangles bleus les événements mal reconstruits. Les coupures appliquées sur la masse invariante des paires $\pi^{ \pm} \pi^{0}$ sont indiquées par les lignes pointillées bleues. La zone d'interférence exclue est hachurée en rouge.

Exclure la zone d'interférence permet d'autre part de rejeter une fraction importante des événements mal reconstruits, puisque ces derniers s'accumulent dans cette région où les masses $m^{2}\left(\pi^{+} \pi^{0}\right)$ et $m^{2}\left(\pi^{-} \pi^{0}\right)$ sont proches, $c$ - $\grave{a}-d$ l'impulsion $d u \pi^{0}$ est faible ( $c f$. figures 4.2 et 4.4$)$. 


\subsubsection{Choix de la charge du $\rho$}

Les événements mal reconstruits possédant un $\pi^{0}$ de faible impulsion, il se peut que l'on associe ce $\pi^{0}$ à la trace célibataire, plutôt qu'à la trace originellement issue du $\rho$, changeant ainsi le signe du $\rho$. Il est important de limiter ces événements de mauvaise charge, et de savoir les distinguer de ceux de bonne charge, puisqu'ils peuvent affecter les asymétries de charge.

La charge du $\rho$ est choisie comme étant celle de la trace chargée de plus basse impulsion: le graphe de gauche de la figure 4.2 montre en effet que dans $86 \%$ des cas $(c$-à- $d$ pour les événements situés en deçà de la ligne rouge verticale à $2.4 \mathrm{GeV} / \mathrm{c}$ ), cette trace correspond au pion issu du $\rho$. Pour les événements au delà de $2.4 \mathrm{GeV} / \mathrm{c}$, les distributions des impulsions des deux traces chargées se chevauchent. Dans ce cas, on choisi le $\rho$ correspondant à la paire de pions de masse invariante la plus proche de la masse nominale du $\rho$.

Cette méthode permet de diminuer la fraction d'événements de mauvaise charge $w_{Q}$ de $55 \%$ par rapport à un choix basé simplement sur la combinaison de pions possédant la masse invariante la plus proche de la masse nominale du $\rho$.

\subsubsection{Reconstruction du méson $B$}

Le méson $B$ est reconstruit à partir des deux traces chargées et du pion neutre. La vraie valeur de l'énergie-impulsion du $B$ dépend de l'énergie des faisceaux $E_{b}$ : pour ne pas être sensible à la dispersion en énergie de ces derniers, on définit les deux variables suivantes [72]:

- La variable $\Delta E$ est définie comme

$$
\Delta E=E_{B}^{*}-\sqrt{s} / 2,
$$

où $E_{B}^{*}$ est l'énergie du candidat $B$ dans le centre de masse et $\sqrt{s}$ est l'énergie des faisceaux dans le centre de masse. Les événements de signal $B^{0} \rightarrow \rho^{ \pm} \pi^{\mp}$ piquent autour de $\Delta E=0$, avec une résolution de $32 \mathrm{MeV}$. La résolution sur $\Delta E$ est dominée par l'incertitude sur l'énergie mesurée du candidat $B$.

Pour $B^{0} \rightarrow \rho^{-} K^{+}$, la distribution de $\Delta E$ n'est pas centrée en zéro, car $\Delta E$ est calculé en faisant l'hypothèse que toutes les traces chargées sont des pions. Avec un kaon dans l'état final, le décalage vaut:

$$
\delta(\Delta E)=\gamma\left(\sqrt{m_{\pi^{ \pm}}^{2}+p_{\text {cél. }}^{2}}-\sqrt{m_{K^{ \pm}}^{2}+p_{\text {cél. }}^{2}}\right),
$$

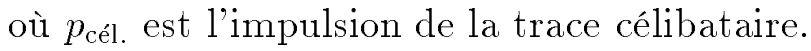

On sélectionne les événements pour lesquels $-120<\Delta E<150 \mathrm{MeV}$ (la borne inférieure de cette coupure est resserrée pour supprimer le bruit de fond $B$ en 4 -corps: voir section 4.8.1).

- La masse avec énergie substituée, $m_{\mathrm{ES}}$, est définie comme

$$
m_{\mathrm{ES}}=\sqrt{\left(s / 2+p_{i} \cdot p_{B}\right)^{2} / E_{i}^{2}-p_{B}^{2}}
$$

où $E_{i}$ et $p_{i}$ sont respectivement l'énergie totale et le vecteur d'impulsion de la paire $e^{+} e^{-}$dans le référentiel du laboratoire, et $p_{B}$ est le vecteur d'impulsion du candidat $B$ reconstruit. Les événements de signal piquent autour de la masse du méson $B$ 
$\left(5.279 \mathrm{GeV} / \mathrm{c}^{2}\right)$ avec une résolution de $2.6 \mathrm{MeV} / \mathrm{c}^{2}$. La résolution de $m_{\mathrm{ES}}$ est dominée par la dispersion en énergie des faisceaux.

On sélectionne les événements pour lesquels $5.23<m_{\mathrm{ES}}<5.2895 \mathrm{GeV} / \mathrm{c}^{2}$. La borne inférieure de cette coupure est suffisamment lâche pour garder des événements de bruit de fond continuum afin d'aider à la détermination des paramètres des distributions de bruit de fond dans l'ajustement final (nous reviendrons sur ce point dans section 4.10.2).
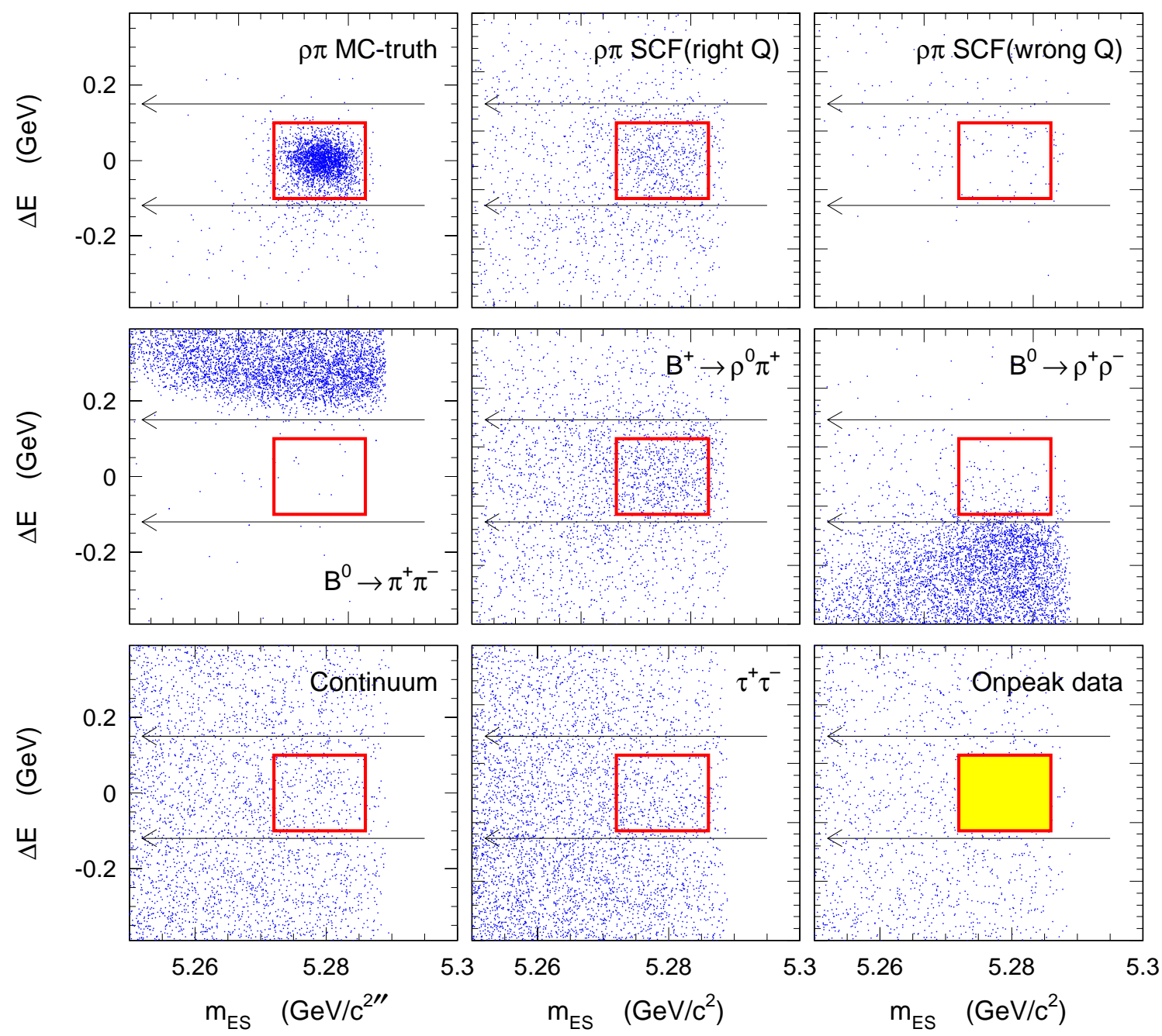

Figure 4.7: Plans $\Delta E-m_{\mathrm{ES}}$ pour divers types d'événements. De haut en bas, et de gauche à droite: signal correctement reconstruit (" $M C$-truth"), mal reconstruit avec une charge du $\rho$ correcte ("SCF right $Q ")$ et incorrecte ("SCF wrong $Q ")$; trois exemples de bruits de fond provenant d'autres désintégrations $d u B$ en deux-corps $B^{0} \rightarrow \pi^{+} \pi^{-}$, en trois-corps $B^{+} \rightarrow \rho^{0} \pi^{+}$, en quatre-corps $B^{0} \rightarrow \rho^{+} \rho^{-}$; le bruit de fond continuum off-peak, le bruit de fond $e^{+} e^{-} \rightarrow \tau^{+} \tau^{-}$, et enfin les données on-peak. Les flèches indiquent les coupures sur $\Delta E$.

Ces deux variables présentent l'avantage d'être peu corrélées pour le signal et les principales sources de bruit de fond comme montré sur la figure 4.8. Elles sont faibles et de forme linéaire pour le continuum. Pour le signal correctement reconstruit, elles sont positives $(+7 \%)$ pour $\Delta E<0$ et négatives $(-12 \%)$ pour $\Delta E>0$. Enfin, elles sont globalement positives $(+12 \%)$ pour le signal mal reconstruit. 

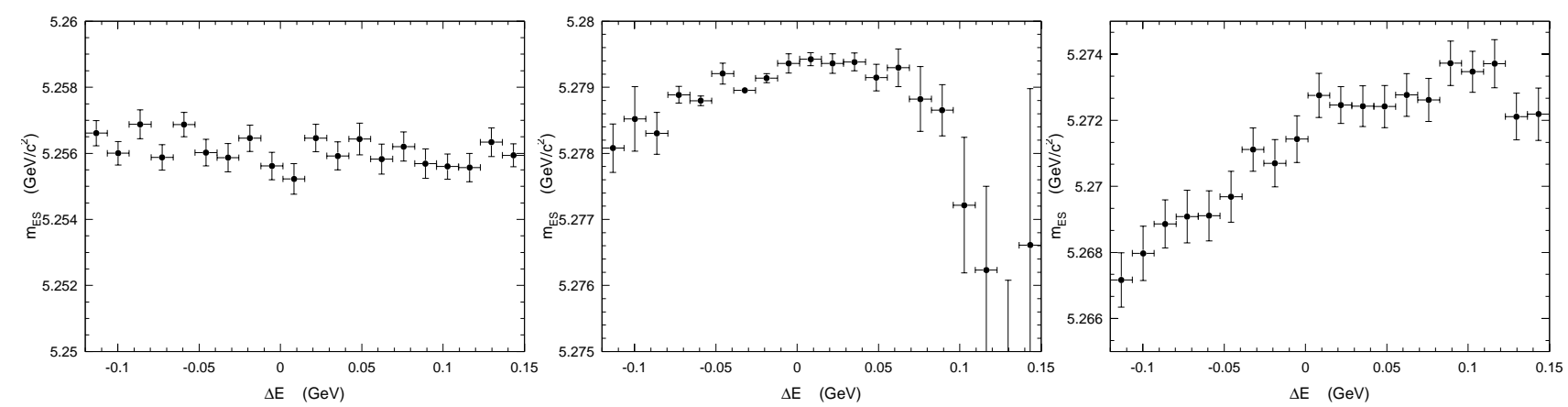

Figure 4.8: Corrélations entre $\left\langle m_{\mathrm{ES}}\right\rangle$ et $\Delta E$ pour le bruit de fond continuum (gauche), signal correctement reconstruit (milieu) et signal mal reconstruit (droite).

La figure 4.7 montre les plans $\Delta E-m_{\mathrm{ES}}$ pour différents types d'événements: le signal correctement reconstruit ("MC-truth"), mal reconstruit avec une charge du $\rho$ correcte ("SCF right Q") et incorrecte ("SCF wrong Q"); trois exemples de bruits de fond provenant d'autres désintégrations du $B$ en deux-corps $B^{0} \rightarrow \pi^{+} \pi^{-}$, en trois-corps $B^{+} \rightarrow \rho^{0} \pi^{+}$, en quatre-corps $B^{0} \rightarrow \rho^{+} \rho^{-}$; le bruit de fond continuum off-peak, le bruit de fond $e^{+} e^{-} \rightarrow \tau^{+} \tau^{-}$, et enfin les données on-peak (dominées par le continuum).

\subsubsection{Réjection des bruits de fond $B$ en 2-corps}

La figure 4.9 montre la masse invariante $m\left(\pi^{+} \pi^{-}\right)$en fonction de $\Delta E$ pour le signal $B^{0} \rightarrow$ $\rho^{ \pm} \pi^{\mp}$ ainsi que les bruits de fond $B^{0} \rightarrow \pi^{+} \pi^{-}$et $B^{0} \rightarrow K^{+} \pi^{-}$: on voit qu'une coupure sur ces deux variables permet de rejeter presque intégralement ces deux bruits de fond. En plus de la coupure sur $\Delta E$ à $150 \mathrm{MeV}$, nous appliquons donc la coupure $m\left(\pi^{+} \pi^{-}\right)<5.14 \mathrm{GeV} / \mathrm{c}^{2}$.

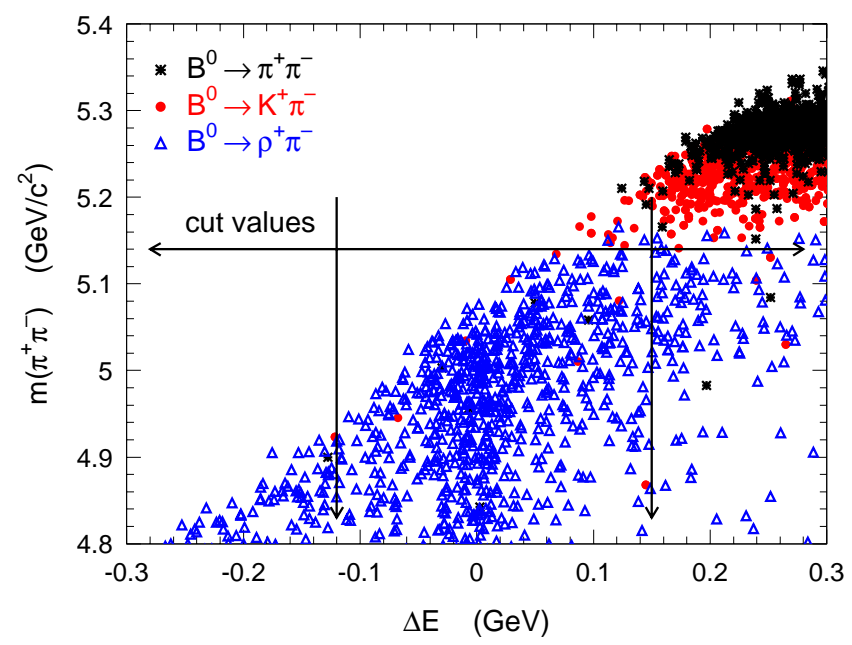

Figure 4.9: Masse invariante $m\left(\pi^{+} \pi^{-}\right)$en fonction de $\Delta E$ pour le signal $B^{0} \rightarrow \rho^{ \pm} \pi^{\mp}$, ainsi que les bruits de fond $B^{0} \rightarrow \pi^{+} \pi^{-}$et $B^{0} \rightarrow K^{+} \pi^{-}$. Les flèches indiquent les coupures réalisées. 


\subsection{Etiquetage de la saveur du $B_{\text {sav }}$}

Après avoir retiré les traces du $B_{\text {rec }}\left(B^{0} \rightarrow \rho h\right)$, nous analysons les traces restantes de l'événement (dénommées "reste de l'événement", en anglais Rest-Of-the-Event, ROE) pour étiqueter la saveur du $B_{\mathrm{sav}}$. L'étiquetage exploite les corrélations existant entre la saveur du $B_{\text {sav }}$ et la charge de leptons, kaons, et pions mous présents dans ses produits de désintégration.

La source des principales corrélations exploitables est la désintégration en cascade $b \rightarrow$ $c \rightarrow s$, montrée sur la figure 4.10. Le premier vertex de cette désintégration peut produire un lepton primaire: le signe de la charge de ce dernier est directement corrélé à la saveur du $B$ (un lepton de charge négative correspond à un $\bar{B}^{0}$ ). Le deuxième vertex peut produire un lepton secondaire, d'impulsion plus faible: le signe de sa charge correspond à une saveur opposée. Lorsque confondu avec ce dernier, le lepton secondaire donne donc lieu à un mauvais étiquetage. Enfin, 1'hadronisation en kaon du quark $s$ situé à la fin de la cascade $b \rightarrow c \rightarrow s$ fournit la principale source d'étiquetage utilisant le signe des kaons (où un $K^{-}$correspond à $\left.\operatorname{un~} \bar{B}^{0}\right)$.

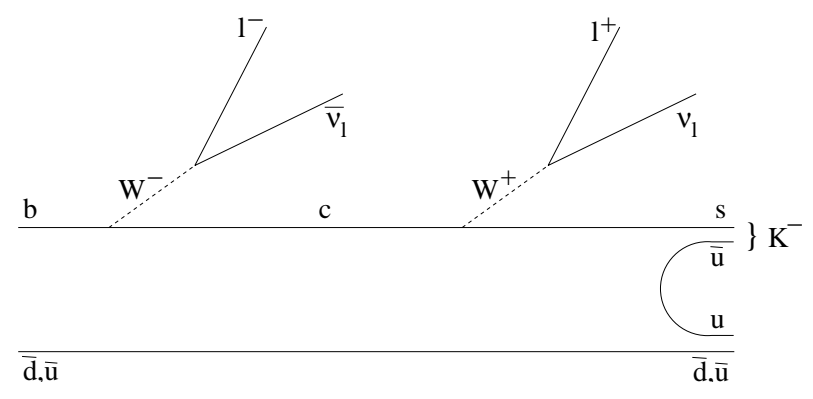

Figure 4.10: Désintégration en cascade $b \rightarrow c \rightarrow s$ constituant la source des principaux signaux d'étiquetage.

L'algorithme d'étiquetage [73] utilisé dans les analyses de cette thèse est composé de deux sous-algorithmes: l'un est basé sur des coupures [74] et l'autre sur un réseau de neurones [75].

Les événements sont classés dans une des 5 catégories mutuellement exclusives suivantes (énoncées par taux de mauvais étiquetage décroissant):

- Leptons: le ROE possède un électron ou un muon de grande impulsion $\left(p^{*}>1.0 \mathrm{GeV} / \mathrm{c}\right.$ pour un $e^{ \pm}$et $p^{*}>1.1 \mathrm{GeV} / \mathrm{c}$ pour un $\left.\mu^{ \pm}\right)$. Le signe de la charge du lepton est utilisé pour déterminer la saveur de $B_{\text {sav }}$, à moins que la somme des charges des kaons présents dans le ROE ne mène à un signe contraire: dans ce cas, cet événement est classé dans la catégorie suivante.

- Kaons: possède un ou plusieurs kaons. La somme des signes des kaons est utilisée pour déterminer la saveur de $B_{\mathrm{sav}}$.

- NT1 et NT2 (réseau de neurones): si l'événement n'est pas classé comme lepton ou kaon, il est traité par un réseau de neurones ${ }^{6}$. Suivant la valeur en sortie, l'événement est classé NT1 (bonne indication de saveur) ou NT2 (indication médiocre).

- NoTag: si un événement ne rentre dans aucune des catégories ci-dessus, il est nonétiqueté.

\footnotetext{
${ }^{6}$ Le réseau de neurones utilise d'autres propriétés de la désintégration du $B_{\text {sav }}$ : les pions mous issus d'un $D^{*}$, les leptons non-identifiés par les algorithmes de sélection des électrons et muons, les leptons de plus basse impulsion, les kaons chargés non-sélectionnés par l’identificateur de kaons, ...
} 
La fraction de mauvais étiquetage d'une saveur de $B$ donnée, pour une catégorie d'étiquetage $i$, est mesurée par les paramètres $\omega_{i}$ pour un $B^{0}$ et $\bar{\omega}_{i}$ pour un $\bar{B}^{0}$. Nous parlerons plutôt de la fraction moyenne de ces paramètres, $\left\langle\omega_{i}\right\rangle=\left(\omega_{i}+\bar{\omega}_{i}\right) / 2$, et de la différence $\Delta w_{i}=\left(w_{i}-\bar{w}_{i}\right) / 2$. On définit la dilution par $D_{i}=1-2 \omega_{i}\left(\bar{D}_{i}=1-2 \bar{\omega}_{i}\right)$, ainsi que la dilution moyenne $\langle D\rangle$ et la différence des dilutions $\Delta D$, définies comme pour le paramètre $\omega$. On dénote $\epsilon_{i}$ la fraction d'événements assignés à la catégorie d'étiquetage $i$. La qualité de l'étiquetage dans une catégorie $i$ est mesurée par le facteur de qualité ${ }^{7} Q_{i}=\epsilon_{i}\left(1-2\langle w\rangle_{i}\right)^{2}=\epsilon_{i} D_{i}^{2}$.

Le tableau 4.2 donne les valeurs de ces paramètres pour chaque catégorie d'étiquetage mesurées dans l'échantillon Breco ( $c f$. Sec. 3.4.2) [52]. Ce sont celles utilisées pour les événements de signal correctement reconstruit lors de l'ajustement final. Au total, $67.5 \%$ des événements sont étiquetés, avec un facteur de qualité global de $25.1 \%$. La catégorie des Leptons a le plus faible taux de mauvais étiquetage, et celle des Kaons le meilleur facteur de qualité.

\begin{tabular}{|ccccccc|}
\hline Catégorie & $\varepsilon(\%)$ & $\langle w\rangle(\%)$ & $\Delta\langle w\rangle(\%)$ & $D(\%)$ & $\Delta D(\%)$ & $Q(\%)$ \\
\hline \hline Lepton & $11.1 \pm 0.2$ & $8.6 \pm 0.9$ & $0.6 \pm 1.5$ & $82.8 \pm 1.8$ & $-1.1 \pm 3.0$ & $7.6 \pm 0.4$ \\
Kaon & $34.7 \pm 0.4$ & $18.1 \pm 0.7$ & $-0.9 \pm 1.1$ & $63.8 \pm 1.4$ & $1.8 \pm 2.1$ & $14.1 \pm 0.6$ \\
NT1 & $7.7 \pm 0.2$ & $22.0 \pm 1.5$ & $1.4 \pm 2.3$ & $55.9 \pm 3.0$ & $-2.7 \pm 4.7$ & $2.4 \pm 0.3$ \\
NT2 & $14.0 \pm 0.3$ & $37.3 \pm 1.3$ & $-4.7 \pm 1.9$ & $25.4 \pm 2.5$ & $9.4 \pm 3.8$ & $0.9 \pm 0.2$ \\
\hline Total & $67.5 \pm 0.5$ & & & & & $25.1 \pm 0.8$ \\
\hline
\end{tabular}

Tableau 4.2: Valeurs des paramètres d'étiquetage $\varepsilon,\langle w\rangle, \Delta\langle w\rangle, D, \Delta D$ et $Q$ pour chaque catégorie de tagging, mesurées dans l'échantillon Breco [52].

Le tableau 4.3 donne les valeurs des paramètres d'étiquetage obtenues dans les Monte Carlo de signal $B^{0} \rightarrow \rho^{ \pm} \pi^{\mp}$ et $B^{0} \rightarrow \rho^{-} K^{+}$. Les efficacités d'étiquetage sont différentes pour les événements correctement et mal reconstruits, en particulier dans la catégorie Leptons. En effet, la multiplicité des produits de désintégration du $B_{\text {sav }}$ dans la catégorie Leptons étant plus faible que dans les autres catégories, la possibilité d'avoir un événement de signal mal reconstruit empruntant un $\gamma$ au $B_{\text {sav }}$ est plus limitée. Lors de l'ajustement final, nous utilisons les valeurs issues du Monte Carlo pour les événements mal reconstruits.

Le tableau 4.4 donne la répartition des événements de bruit de fond continuum et $\tau^{+} \tau^{-}$ dans les différentes catégories d'étiquetage. On peut noter le faible taux de bruit de fond dans la catégorie Leptons.

\subsection{Reconstruction de $\Delta t$}

Le $B_{\text {rec }}$ se propageant dans l'espace (se désintégrant en $\rho^{ \pm} h^{\mp}$ ) est une superposition (variant avec le temps) des états propres de saveur $B^{0}$ et $\bar{B}^{0}$. Comme la saveur des deux $B$ est opposée, la saveur de $B_{\text {rec }}$ est connue au moment de la désintégration de $B_{\text {sav }}$ (puisque l'on étiquète la saveur de ce dernier). Le contenu en saveur de $B_{\text {rec }}$ au moment de sa propre désintégration est fonction du temps $\Delta t$ qui le sépare de la désintégration de $B_{\text {sav }}$, c'est à dire de la distance $\Delta z$ séparant les vertex de désintégration des deux $B$, comme indiqué sur le schéma 4.11. La zone en gris clair du schéma indique la zone d'interaction ("beam spot")

\footnotetext{
${ }^{7} \mathrm{~L}$ 'erreur statistique sur les asymétries $C P$ étiquetées est inversement proportionnelle à $\sqrt{\sum_{i=1}^{\mathrm{Ntag}} Q_{i}}$.
} 


\begin{tabular}{|c|c|c|c|c|c|c|c|}
\hline \multicolumn{3}{|l|}{ Mode } & Lepton (\%) & Kaon (\%) & NT1 $(\%)$ & NT2 $(\%)$ & No tag $(\%)$ \\
\hline & & $\overline{f_{c}}$ & $10.5 \pm 0.2$ & $34.9 \pm 0.4$ & $6.9 \pm 0.2$ & $14.3 \pm 0.3$ & $33.5 \pm 0.4$ \\
\hline \multirow[t]{3}{*}{$B^{0} \rightarrow \rho^{ \pm} \pi^{\mp}$} & & $\langle w\rangle$ & $7.2 \pm 0.6$ & $18.5 \pm 0.5$ & $21.7 \pm 1.2$ & $33.5 \pm 1.0$ & - \\
\hline & & $\Delta\langle w\rangle$ & $1.5 \pm 1.2$ & $0.8 \pm 1.0$ & $3.9 \pm 2.4$ & $1.6 \pm 1.9$ & - \\
\hline & & 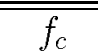 & $\overline{c 10.9 \pm 0.3}$ & 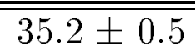 & 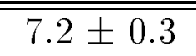 & 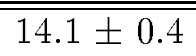 & $32.7 \pm 0.5$ \\
\hline \multirow[t]{4}{*}{$B^{0} \rightarrow \rho^{ \pm} K^{\mp}$} & & $\langle w\rangle$ & $6.3 \pm 0.8$ & $17.6 \pm 0.7$ & $20.8 \pm 1.6$ & $31.1 \pm 1.3$ & - \\
\hline & & $\Delta\langle w\rangle$ & $-2.4 \pm 1.5$ & $-0.5 \pm 1.3$ & $-0.6 \pm 3.2$ & $-0.3 \pm 2.6$ & - \\
\hline & Corr. & $f$ & 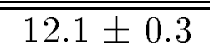 & 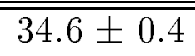 & 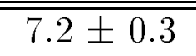 & 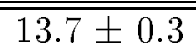 & $32.2 \pm 0.4$ \\
\hline & Comb. & $J_{c}$ & $6.7 \pm 0.3$ & $35.5 \pm 0.6$ & $6.2 \pm 0.3$ & $15.3 \pm 0.5$ & $36.2 \pm 0.7$ \\
\hline \multirow[t]{4}{*}{$B^{0} \rightarrow \rho^{ \pm} \pi^{\mp}$} & Corr. & & $7.0 \pm 0.7$ & $17.8 \pm 0.6$ & $21.6 \pm 1.4$ & $32.1 \pm 1.2$ & - \\
\hline & Comb. & & $8.3 \pm 1.5$ & $20.0 \pm 0.1$ & $21.8 \pm 2.3$ & $36.0 \pm 1.7$ & - \\
\hline & Corr. & & $0.6 \pm 1.3$ & $0.5 \pm 1.2$ & $0.5 \pm 2.8$ & $0.4 \pm 2.3$ & - \\
\hline & Comb. & & $5.0 \pm 2.9$ & $1.2 \pm 1.8$ & $1.1 \pm 4.5$ & $3.6 \pm 3.3$ & - \\
\hline
\end{tabular}

Tablea1 4.3: Fractions d'événements par catégorie d'étiquetage, fractions de mauvais étiquetage moyennes, et différences de ces fractions entre un $B^{0}$ et un $\bar{B}^{0}$, dans les Monte Carlo $\rho \pi$ et $\rho K$. La dernière partie du tableau donne ces chiffres pour les événements correctement reconstruits ("corr.") et mal reconstruits ("comb.") du Monte Carlo $\rho \pi$ non-interférant.

\begin{tabular}{|c|c|c|c|c|c|}
\hline & Lepton (\%) & Kaon (\%) & NT1 (\%) & NT2 (\%) & NoTag (\%) \\
\hline "MC Continu1um & $\overline{0.5 \pm 0.1}$ & $20.2 \pm 0.6$ & $\overline{4} 4.0 \pm 0.3$ & 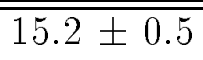 & 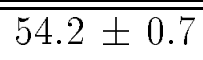 \\
\hline $\mathrm{MC} u d s$ & $0.4 \pm 0.1$ & $22.1 \pm 0.6$ & $4.2 \pm 0.3$ & $15.5 \pm 0.5$ & $57.8 \pm 0.7$ \\
\hline $\mathrm{MC} c \bar{c}$ & $0.7 \pm 0.2$ & $36.8 \pm 1.4$ & $3.5 \pm 0.5$ & $14.2 \pm 1.0$ & $44.9 \pm 1.4$ \\
\hline$\tau^{+} \tau^{-} \mathrm{MC}$ & $0.5 \pm 0.5$ & $6.6 \pm 1.8$ & $7.1 \pm 1.8$ & $8.6 \pm 2.0$ & $77.3 \pm 3.0$ \\
\hline Données off-peak & $0.4 \pm 0.1$ & $26.6 \pm 0.6$ & $4.6 \pm 0.3$ & $14.9 \pm 0.5$ & $53.4 \pm 0.7$ \\
\hline
\end{tabular}

Tableau 4.4: Répartition des événements de bruit de fond continuum et $\tau^{+} \tau^{-}$dans les différentes catégories d'étiquetage.

des faisceaux de dimensions [76, 77]:

$$
\left(\sigma_{x} \times \sigma_{y} \times \sigma_{z}\right)=150 \mu \mathrm{m} \times 5 \mu \mathrm{m} \times 1 \mathrm{~cm} .
$$

\subsubsection{Reconstruction du vertex du $B_{\text {rec }}$}

Le vertex du $B_{\text {rec }}\left(B^{0} \rightarrow \rho^{ \pm} \pi^{\mp}\right)$ est reconstruit à partir des deux traces chargées présentes dans l'état final. La résolution sur la reconstruction de ce vertex est typiquement de $60 \mu \mathrm{m}$.

\subsubsection{Reconstruction du vertex du $B_{\text {sav }}$}

Le vertex du $B_{\text {sav }}$ est reconstruit à partir des traces du ROE. Le processus d'ajustement du vertex est itératif: après avoir exclu les traces du $B_{\text {rec }}$ et remplacé les traces chargées issues de candidats $K_{s}^{0}$ ou $\Lambda$ (dénommés $V^{0}$ ) par leur mère, l'algorithme ajuste le vertex des traces restantes en appliquant la contrainte que toutes les traces considérées doivent provenir de la zone d'interaction des faisceaux: la position et dimension de ce dernier sont déterminées pour chaque run. Etant donnée la distance moyenne de vol d'un $B$ de $250 \mu \mathrm{m}$, 


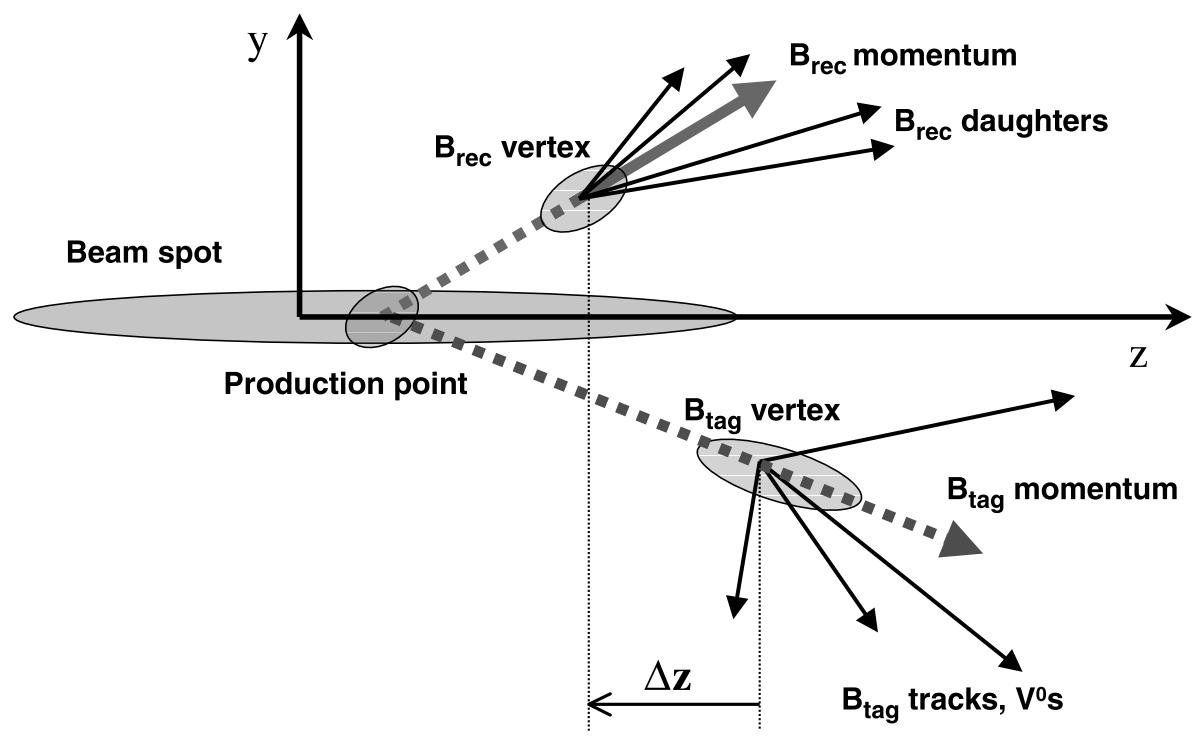

Figure 4.11: Schéma représentant la production, le vol et la désintégration des deux B. La zone en gris clair montre la zone d'interaction ("beam spot") des faisceaux dont les dimensions sont données dans l'eq. 4.5. La distance $\Delta z$ est définie comme la distance entre $B_{\mathrm{rec}}$ et $B_{\mathrm{sav}}$. Sur ce schéma, les distances selon l'axe y sont surdimensionnées: l'angle entre les deux mésons $B$ est en fait plus petit que 214 mrad.

c'est principalement la dimension selon l'axe $y$ de la zone d'interaction des faisceaux qui contraint la détermination du vertex. Cette contrainte permet de retirer les traces ayant volé trop loin (supposées provenir d'un vertex secondaire). Si l'une des traces du ROE contribue au $\chi^{2}$ total par plus d'une valeur seuil (égale à 6), ce candidat est retiré, l'ajustement est réitéré, et ainsi de suite jusqu'au moment où tous les candidats restants ont une contribution au $\chi^{2}$ en deçà du seuil.

La résolution de reconstruction sur ce vertex est typiquement $110 \mu \mathrm{m}$ (soit deux fois plus grande que pour le vertex de $B_{\text {rec }}$ ).

\subsubsection{Reconstruction de $\Delta z$}

La différence $\Delta z$ entre la position du vertex selon l'axe $z$ de $B_{\text {sav }}$ et celle de $B_{\text {rec }}$ est définie comme:

$$
\Delta z=z_{B_{\mathrm{rec}}}-z_{B_{\mathrm{sav}}} .
$$

La figure 4.12 montre la distribution de $\Delta z$, son erreur et le résidu $\Delta z_{\text {géné }}-\Delta z_{\text {mes. }}$ dans le Monte Carlo $B^{0} \rightarrow \rho^{ \pm} \pi^{\mp}$. La résolution sur $\Delta z$ est de $200 \mu \mathrm{m}$, et est dominée par la résolution du vertex de $B_{\text {sav }}$

\subsubsection{Conversion entre $\Delta z$ et $\Delta t$}

Si l'on néglige l'impulsion dans le centre de masse du $B_{\text {rec }}$, la conversion entre $\Delta z$ et $\Delta t$ s'écrit:

$$
\Delta z=\beta \gamma c \Delta t
$$

Le facteur $\beta \gamma$ est calculé à partir des énergies de faisceaux mesurées toutes les 5 secondes, et possède une incertitude de $0.1 \%$. 

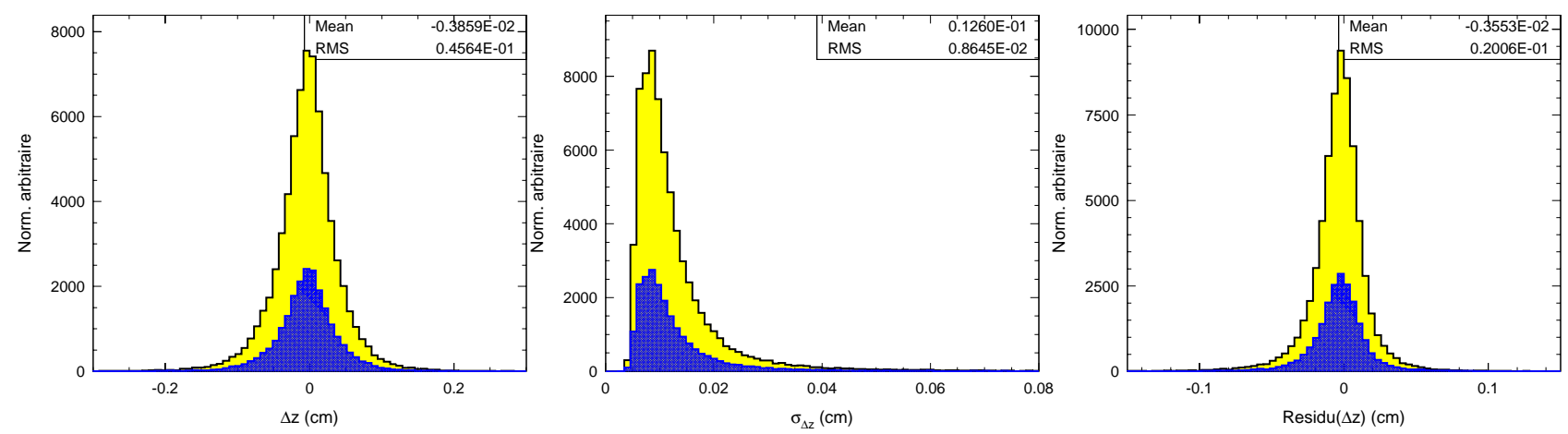

Figure 4.12: Distribution de $\Delta z$ (figure de gauche), de son erreur (figure du milieu) et du résidu $\Delta z_{\text {géné }}-\Delta z_{\text {mes. }}$ (figure de droite) pour tous les événements (courbe jaune) et ceux mal reconstruits (courbe bleue).

Il est possible de corriger cette formule de conversion pour prendre en compte l'impulsion du $B_{\text {rec }}$ dans le centre de masse du $\Upsilon(4 S)$, puisque le $B_{\text {rec }}$ est reconstruit de façon exclusive. La formule devient alors [52]:

$$
\Delta z=\beta \gamma \gamma_{\mathrm{rec}}^{*} c \Delta t+\gamma \beta_{\mathrm{rec}}^{*} \gamma_{\mathrm{rec}}^{*} \cos \theta_{\mathrm{rec}}^{*} c\left(t_{\mathrm{rec}}+t_{\mathrm{sav}}\right),
$$

où $\theta_{\text {rec }}^{*}, \beta_{\text {rec }}^{*}$ et $\gamma_{\text {rec }}^{*}$ sont respectivement l'angle polaire du $B_{\text {rec }}$ par rapport à la direction des faisceaux, la vélocité et le facteur de boost du $B_{\text {rec }}$ dans le centre de masse du $\Upsilon(4 S)$. La précision de la mesure de $t_{\mathrm{rec}}+t_{\mathrm{sav}}$ est limitée par la grande taille de la zone d'interaction (cf. Eq. 4.5), et l'on remplace donc $t_{\text {rec }}+t_{\text {sav }}$ par sa valeur moyenne valant $\tau_{B}+|\Delta t|$.

Le terme rajouté dans l'eq. 4.8 par rapport à l'eq. 4.7 représente une différence négligeable par rapport à la résolution intrinsèque sur $\Delta t^{8}$. Par contre, ce terme introduit des corrélations dans le bruit de fond non- $B$ : en effet, $\gamma_{\text {rec }}^{*}$ est corrélé avec $m_{\mathrm{ES}}$, puisque $\gamma_{\mathrm{rec}}^{*}=E^{*} / m_{\mathrm{ES}}$. Des corrélations supplémentaires sont introduites par $\cos \theta_{\text {rec }}^{*}$ et $|\Delta t|$ (ce dernier terme génère une asymétrie en fonction du signe de $\Delta t$ ) [78]. L'analyse pour mesurer sin $2 \beta$, qui ne souffre pas d'un bruit de fond de continuum important, utilise l'eq. 4.8, mais puisque les analyses décrites dans cette thèse souffrent d'une grande contamination du bruit de fond continuum, nous choisissons d'utiliser l'eq. 4.7.

La figure 4.13 montre la distribution de $\Delta t$ et de son erreur dans le Monte Carlo $B^{0} \rightarrow$ $\rho^{ \pm} \pi^{\mp}$, ainsi que celles du résidu $\left(\Delta t_{\mathrm{rec}}-\Delta t_{\mathrm{vrai}}\right)$ et du pull $\left(\left(\Delta t_{\mathrm{rec}}-\Delta t_{\mathrm{vrai}}\right) / \sigma_{\Delta t}\right)$. La résolution sur $\Delta t$ est d'environ 1.1 ps.

La moyenne de la distribution du résidu est négative à cause de la présence de particules charmées ${ }^{9}$ dans le $B_{\text {sav }}$ qui biaisent la reconstruction de son vertex vers l'avant (vers des

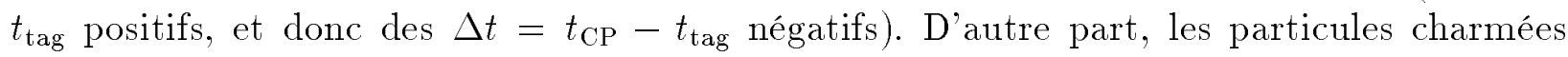
introduisent une corrélation entre l'erreur sur $\Delta t$ calculée pour chaque événement et la moyenne, ainsi que l'écart type, de la résolution sur $\Delta t$, comme montré sur la figure 4.14.

\subsubsection{Coupures sur $\Delta t$}

Pour éliminer les événements aberrants, on demande que $|\Delta t|$ soit plus petit que 20 ps et que l'erreur sur $\Delta t$ inférieure à 2.5 ps.

\footnotetext{
${ }^{8}$ Deux faits contribuent à cette conclusion: la distribution en $\cos \theta_{\text {rec }}^{*}$ est plus piquée à zéro qu'aux extrémités, et les valeurs positives et négatives du cosinus se compensent.

${ }^{9}$ Les $D^{0}$ et $D^{+}$possèdent par exemple une distance de désintégration $c \tau$ de respectivement 130 et $300 \mu \mathrm{m}$
} 

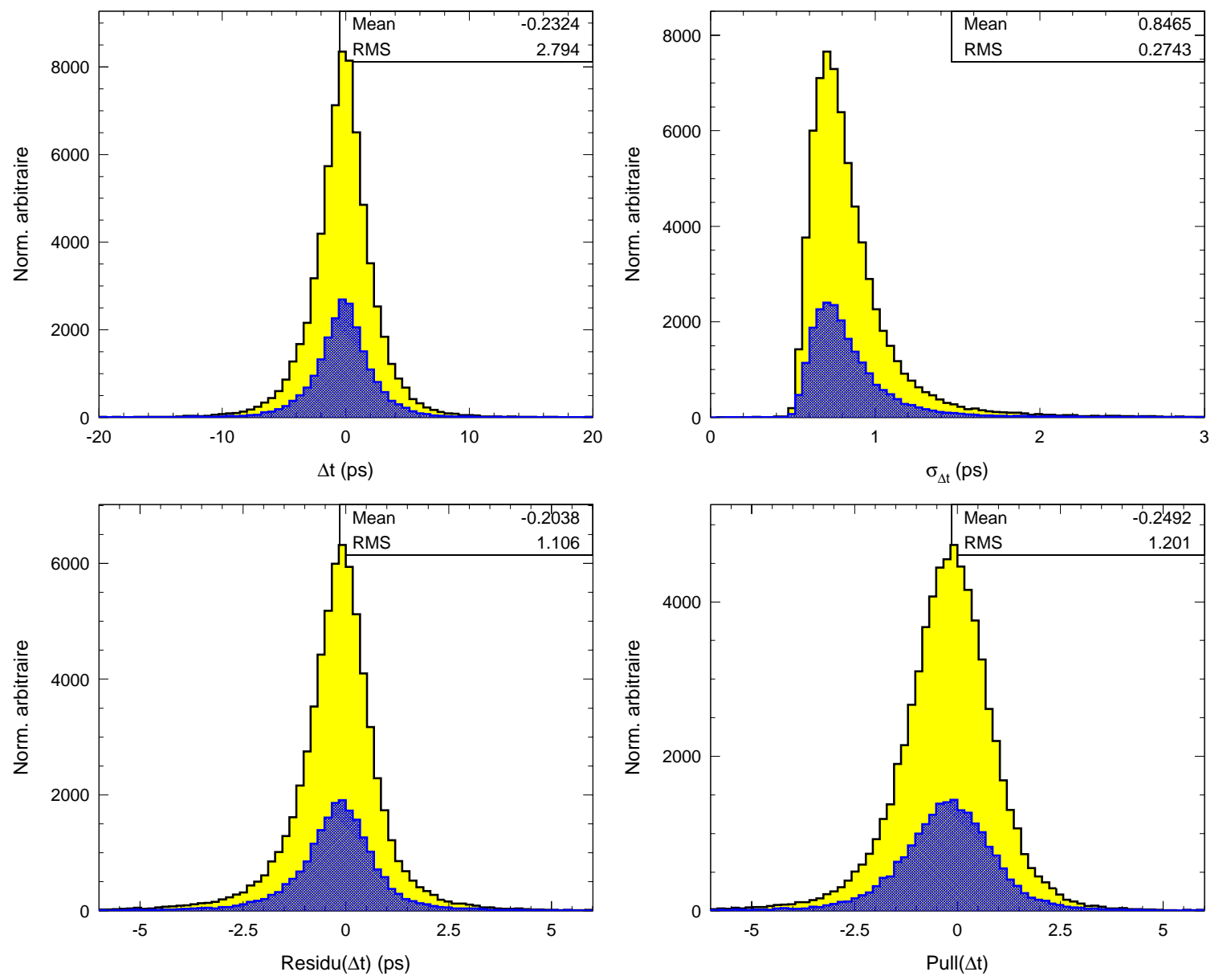

Figure 4.13: Distribution de $\Delta t$ (haut, gauche) et de son erreur (haut, droite), du résidu de $\Delta t\left(\Delta t_{\mathrm{rec}}-\Delta t_{\mathrm{vrai}}\right)$ (bas, gauche) et du pull $\left(\left(\Delta t_{\mathrm{rec}}-\Delta t_{\mathrm{vrai}}\right) / \sigma_{\Delta t}\right)$ (bas, droite), pour tous les événements (courbe jaune) et ceux mal reconstruits (courbe bleue).

\subsection{Lutte contre le bruit de fond $q \bar{q}$}

Le continuum $q \bar{q}$ représente le bruit de fond dominant dans les désintégrations non charmées analysées dans cette thèse. Comme introduit dans la section 4.2, la topologie et la cinématique des événements $B$ et du continuum sont très différentes. De nombreuses variables permettent d'exploiter ces différences. Nous combinons plusieurs d'entre elles (grâce à une analyse multidimensionnelle: ici, un réseau de neurones) pour former une unique variable optimale utilisée lors de l'ajustement final des données.

Trois configurations de variables ont été étudiées, et la meilleure configuration est retenue dans l'analyse finale (sur des critères définis dans la Sec. 4.6.5). Les variables retenues dans ces trois configurations sont décrites dans cette section. Celles qui n'ont pas été retenues, mais qui ont été considérées initialement sont décrites (ainsi que les raisons pour lesquelles elles ne n'ont pas été retenues) dans l'annexe B. Les distributions des variables les plus représentatives sont montrées sur les figures 4.15 à 4.17 .

Sauf mention contraire, les quantités discutées sont calculées dans le référentiel du centre de masse du $\Upsilon(4 S)$. 

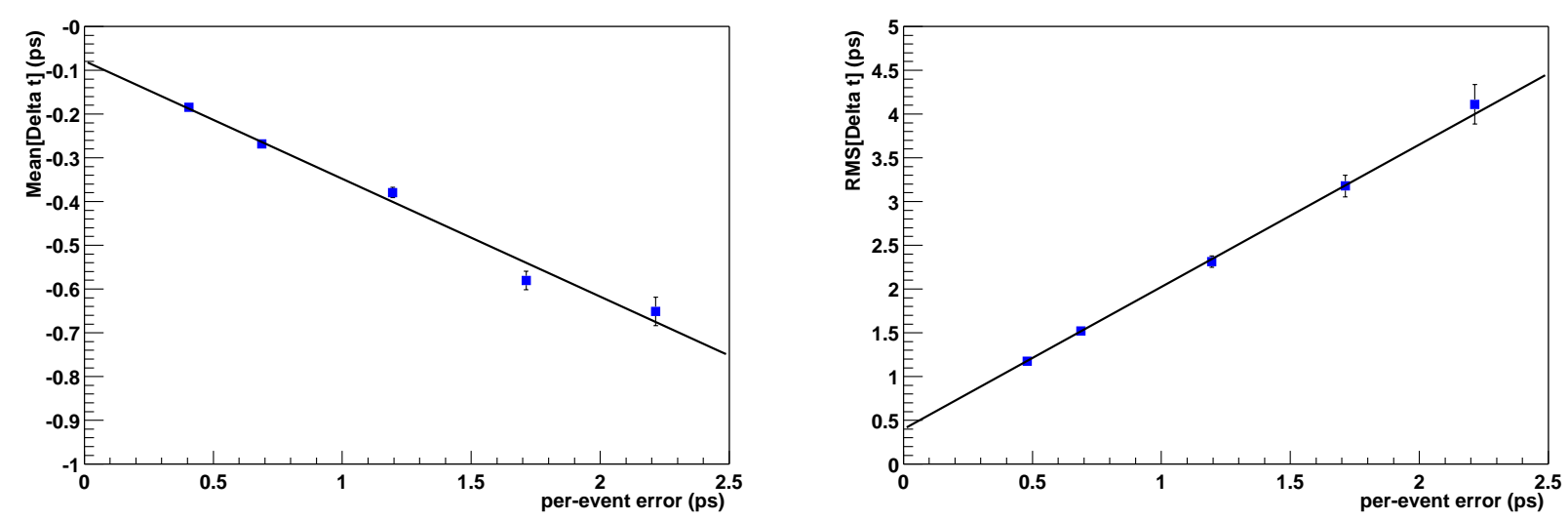

Figure 4.14: Corrélations entre la moyenne (gauche), ainsi que le RMS (droite), de la résolution sur $\Delta t$ et l'erreur sur $\Delta t$ pour chaque événement.

\subsubsection{Variables cinématiques}

Les variables cinématiques retenues sont les suivantes:

- $m\left(\rho^{ \pm}\right), m\left(\pi^{0}\right)$, les masses invariantes des combinaisons associées aux produits de désintégration du $\rho$ et $\pi^{0}$.

- $\cos \theta_{H}(\rho)$, défini dans la section 4.3.1.1.

- $\cos \theta_{H}\left(\pi^{0}\right)$, cosinus de l'angle polaire dans le centre de masse du $\pi^{0} \mathrm{~d} u \gamma$ par rapport à la direction de vol du $\pi^{0}$, qui suit une distribution uniforme (car le $\pi^{0}$ est scalaire).

- $\cos \theta(B, z)$, cosinus de l'angle entre la direction du $B$ et l'axe $z$. Comme expliqué dans la section 4.2, pour un $B$ de signal, la distribution est en $\sin ^{2} \theta$. Un candidat $B$ construit à partir du continuum possède quant à lui une distribution uniforme.

- $\cos \theta\left(T_{B}, z\right)$, cosinus de l'angle entre l'axe de poussée ${ }^{10}$ du candidat, et l'axe $z$. Cet angle permet de distinguer la structure en "jets" du continuum: on retrouve pour ce dernier la distribution en $1+\cos ^{2} \theta$ décrite dans la section 4.2. Le signal possède une distribution uniforme.

\subsubsection{Variables topologiques}

Parmi les variables topologiques, on distingue les trois catégories suivantes:

- Les Variables Globales (VG) sont définies pour l'événement entier sans distinguer le $B$ du ROE. Elles sont généralement corrélées avec les variables cinématiques comme par exemple $\Delta E$ et $m_{\mathrm{ES}}$.

\footnotetext{
${ }^{10} \mathrm{~L}$ 'axe de poussée est la direction $\vec{n}$ qui maximise la somme des impulsions longitudinales des traces considérées (ici, celles du candidat), appelée "poussée" ("thrust" en anglais, $T$ ):

$$
T=\operatorname{Max} \frac{\sum_{i}\left|\vec{n} \cdot \vec{p}_{i}\right|}{\sum_{i}\left|\vec{p}_{i}\right|} .
$$

Dans le cas d'une désintégration du $B$ en deux corps, l'axe de poussée est donné par la différence des vecteurs impulsion des deux traces.
} 

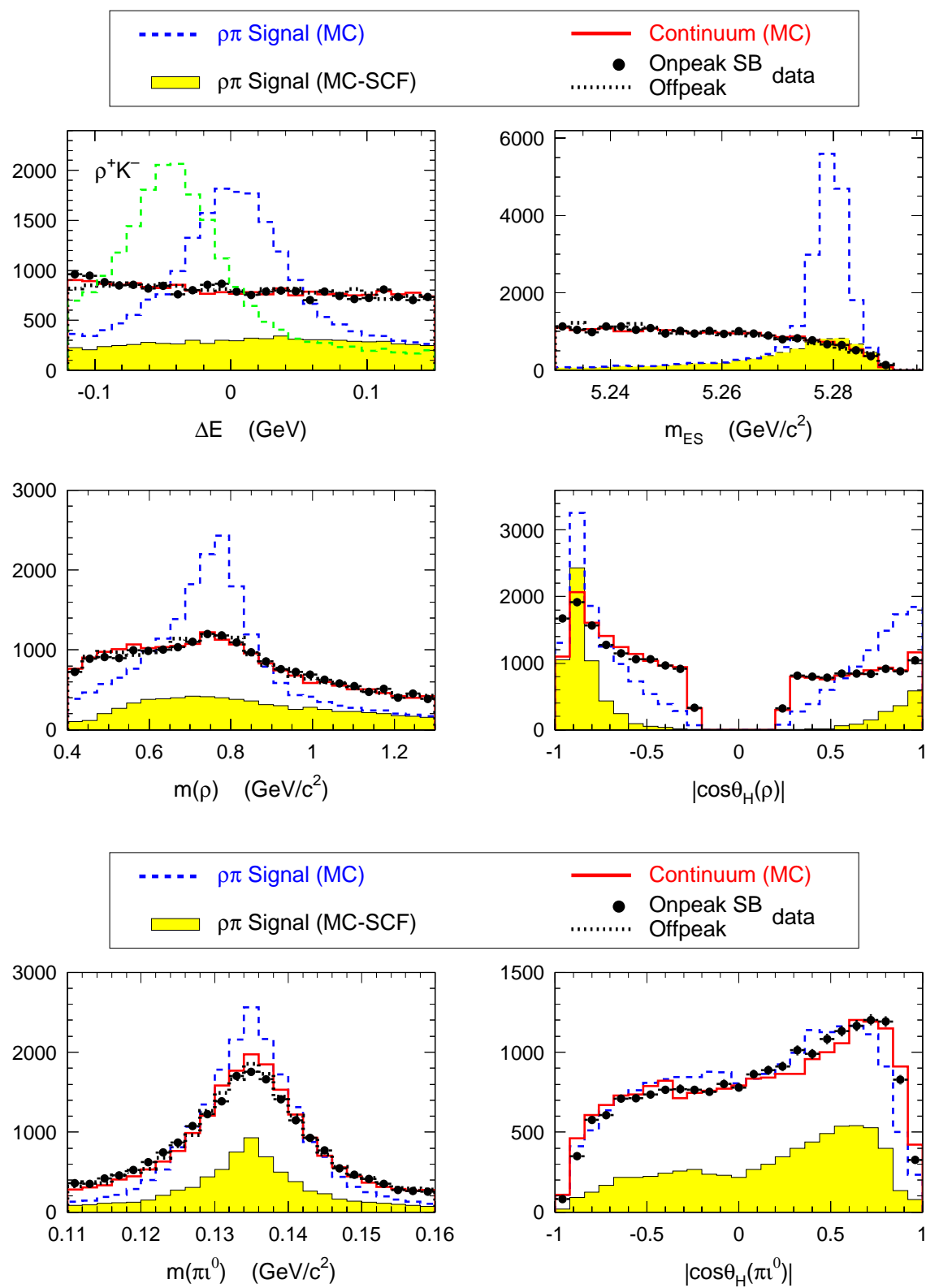

Onpeak SB data
$\ldots \ldots \ldots$, Offpeak
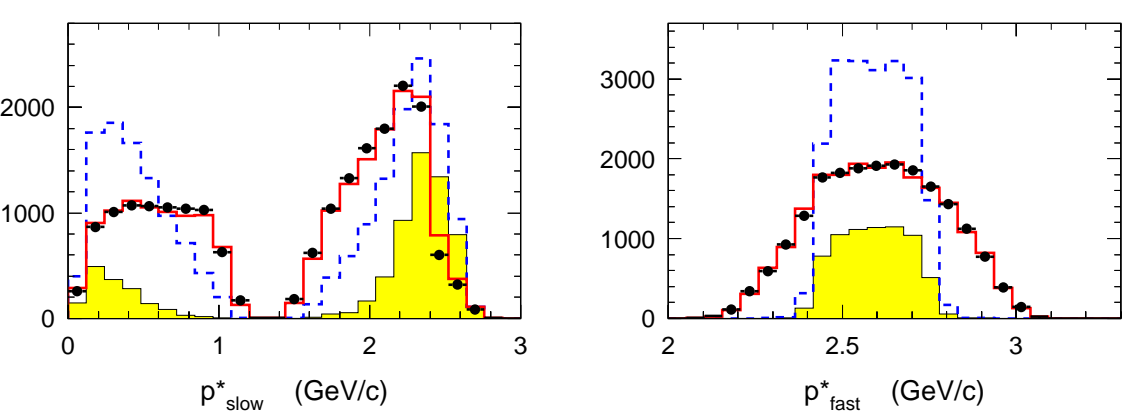

Figure 4.15: Distributions des variables cinématiques $\Delta E, m_{\mathrm{ES}}$ (utilisées dans l'ajustement final), $m\left(\rho^{ \pm}\right) \cos \theta_{H}(\rho), m\left(\pi^{0}\right), \cos \theta_{H}\left(\pi^{0}\right), p_{\text {slow }}^{*}$ et $p_{\text {fast }}^{*}$ (ces deux dernières variables sont définies dans l'annexe B.2). 

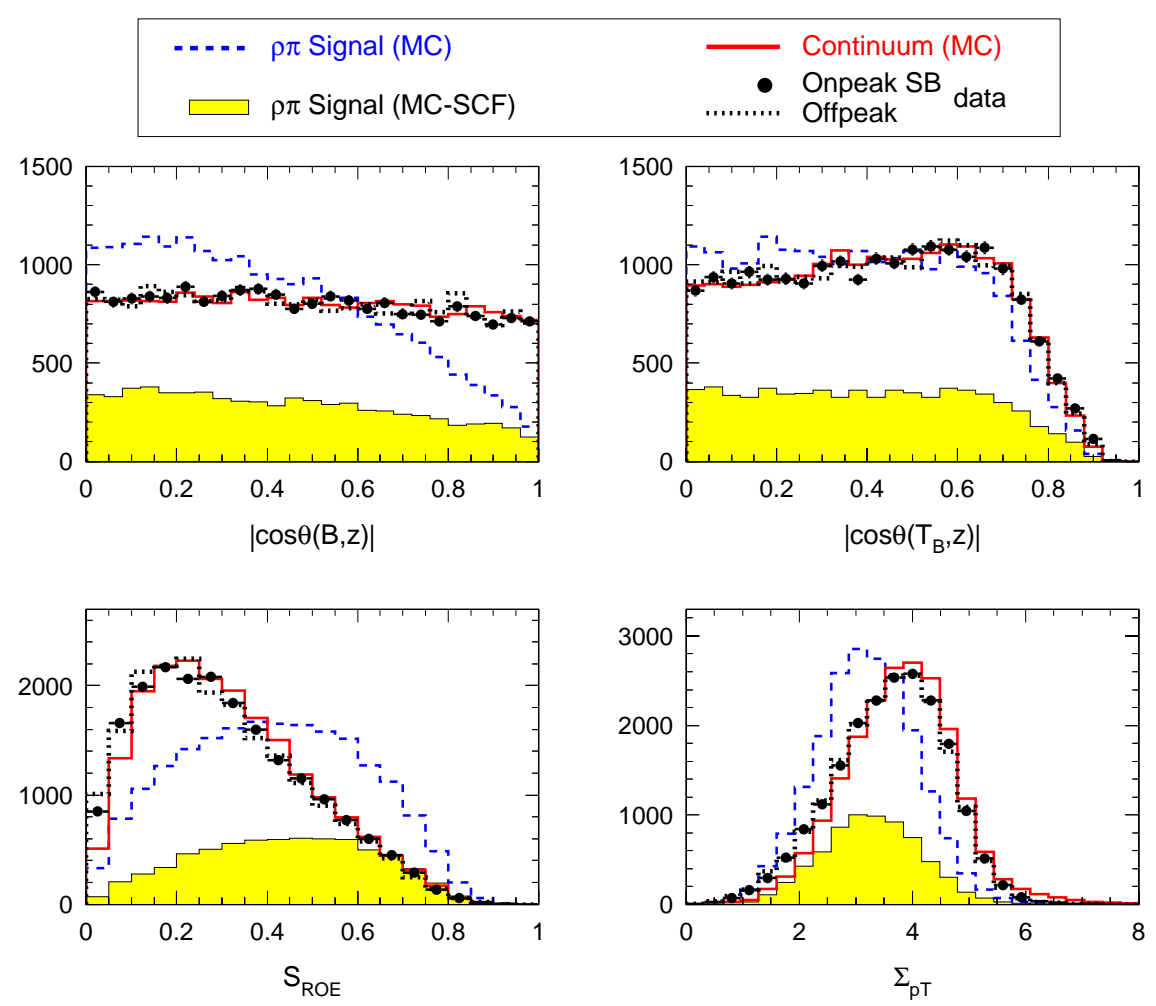

Figure 4.16: Distributions des variables cinématiques $\cos \theta(B, z), \cos \theta\left(T_{B}, z\right)$, et des variables topologiques $\hat{S}_{\mathrm{ROE}}, \Sigma_{\mathrm{pT}}$.

- Les Variables partiellement-Globales ( $\mathrm{VpG}$ ) distinguent explicitement le candidat $B$ du ROE, et dépendent de la cinématique particulière du mode $B$ considéré, car elles utilisent les traces des filles du candidat $B$. Elles sont peu corrélées avec les variables cinématiques.

- Variables non-Globales ( $\mathrm{VnG}$ ) distinguent explicitement le candidat $B$ du ROE, et ne dépendent pas de la cinématique particulière du mode $B$ considéré, car elles impliquent seulement la somme des impulsions des filles du candidat $B$. Elles ne sont pas corrélées avec les variables cinématiques (des exceptions sont possibles pour les candidats $B$ mal reconstruits créant ainsi des corrélations entre l'objet reconstruit et le ROE). Nous choisirons préférentiellement des variables appartenant à cette dernière catégorie, car nous pouvons valider ce type de variables grâce à d'autres modes $B$ produits de façon plus abondante.

Les variables topologiques considérées sont les suivantes:

- Les Monômes $L_{n}(\mathrm{VnG})$ définis par ${ }^{11}$ [79]:

$$
L_{n}=\sum_{i=\operatorname{ROE}} p_{i}\left|\cos \left(\theta_{T_{B}, i}\right)\right|^{n},
$$

où $\cos \left(\theta_{T_{B}, i}\right)$ est le cosinus entre l'axe de poussée du $B$ et la trace $i$ appartenant au reste de l'événement, ROE, et $p_{i}$ est l'impulsion de cette trace.

\footnotetext{
${ }^{11}$ Les monômes ont été utilisés pour la première fois dans l'analyse $B^{0} \rightarrow a_{0}^{ \pm} \pi^{\mp}$. Ils sont une généralisation des cônes de CLEO (voir Annexe B.2) communément utilisées dans BABAR avant d'être supplantés par les monômes.
} 

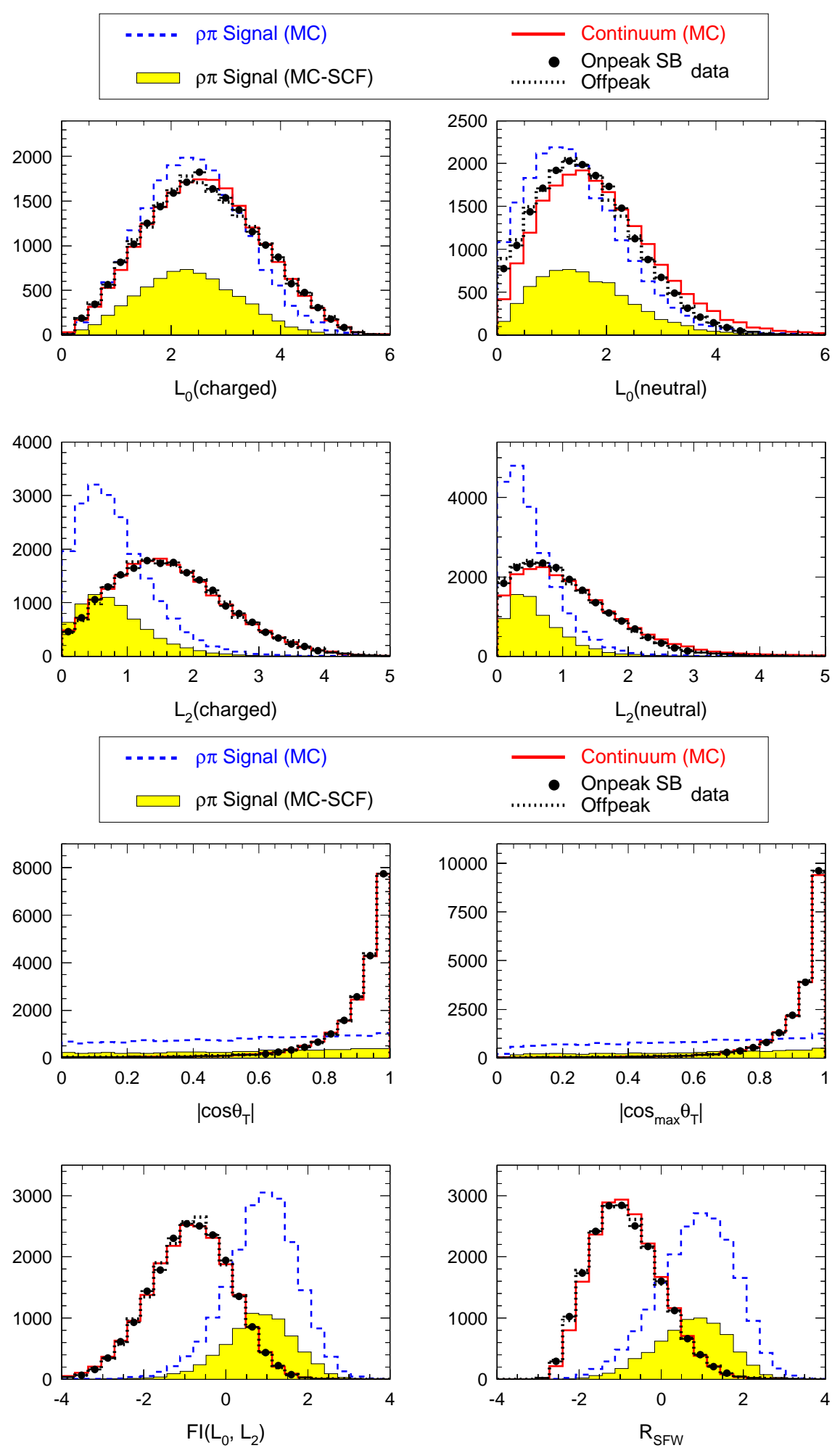

Figure 4.17: Figures du haut: distributions des monômes (4.10). On fait la distinction entre les monômes reconstruits avec les particules chargées ou neutres du ROE. Figures du bas: $\left|\cos \left(\theta_{T}\right)\right|,\left|\cos _{\max }\left(\tilde{\theta}_{T}\right)\right|$ (défini en annexe B.2), un discriminant de Fisher construit avec les monômes $L_{0}$ et $L_{2}$ (cf. Sec. 4.6.5), les supers moments de Fox-Wolfram (cf. Eq. B.1 en annexe B.2). 
- Les valeurs de poussée $T$ (Eq. 4.9) et de sphéricité $S^{12}: \hat{T}_{\text {Event }}, \hat{S}_{\text {Event }}(\mathrm{VG}), \hat{T}_{\mathrm{RoE}}, \hat{S}_{\mathrm{RoE}}$ $(\mathrm{VnG}), \hat{T}_{B}, \hat{S}_{B}(\mathrm{VpG})$ de l'événement, du ROE, et du candidat $B$ respectivement. Elles distinguent les structures "dos à dos" et isotropiques des événements.

- $\left|\cos \left(\theta_{T}\right)\right|(\mathrm{VnG})$, la valeur absolue du cosinus de l'angle entre l'axe de poussée du candidat et celui du ROE. Elle est uniformément distribuée pour le signal, et pique à 1 pour le continuum pour lesquelles les traces sont fortement corrélées à celles du ROE.

- La somme sur les impulsions transverses du ROE (par rapport à l'axe $z$ ) (VnG),

$$
\Sigma_{\mathrm{pT}}=\sum_{i=\mathrm{ROE}} p_{i} \sin \left(\theta_{i, z}\right)
$$

La valeur moyenne de $\Sigma_{\text {рт }}$ pour le continuum est environ supérieure de $0.8 \mathrm{GeV} / \mathrm{c}$ à celle des candidats $B$.

\subsubsection{Corrélations linéaires entre les variables}

Les modules des coefficients de corrélation linéaire entre des variables cinématiques et topologiques sont donnés dans les matrices 4.18 et 4.19 pour les événements ayant passé toutes les coupures décrites jusqu'ici. Il peut exister des corrélations non-linéaires entre certaines variables non-visibles dans ces matrices.

On remarque que:

- les corrélations sont plus importantes pour les événements de signal mal reconstruits que pour ceux correctement reconstruits.

- les variables topologiques montrées dans ces matrices sont corrélées entre elles, mais sont peu corrélées avec les variables cinématiques énoncées dans le point précédent.

Nous utilisons préférentiellement des variables non-corrélées dans l'ajustement final par maximum de fonction de vraisemblance, puisque la présence de corrélations nécessite l'introduction de distributions de probabilités multidimensionnelles modélisant ces corrélations, plus difficiles à maîtriser que les distributions unidimensionnelles.

\subsubsection{Outils d'analyse multidimensionnelle}

L'objectif de l'analyse multidimensionnelle décrite ici est de produire une variable optimale pour la lutte contre le bruit de fond continuum à partir d'un ensemble (dénoté par le vecteur $\mathbf{x}=\left\{x_{i}, i=1 . N\right\}$ par la suite) de $N$ variables cinématiques et/ou topologiques parmi celles décrites dans les sections 4.6.1 et 4.6.2. Parmi les types d'analyses multidimensionnelles existants, nous utiliserons les deux suivants:

\footnotetext{
${ }^{12}$ La sphéricité est donnée par la somme des carrés des impulsions transverses relative à la direction de l'événement: $S=3 / 2\left(\lambda_{2}+\lambda_{3}\right)$, où $\lambda_{i}$ sont les valeurs propres $\left(0<\lambda_{3}<\lambda_{2}<\lambda_{1}<1\right)$ du tenseur:

$$
S^{\alpha \beta}=\frac{\sum_{i} p_{i}^{\alpha} p_{i}^{\beta}}{\sum_{j}\left|p_{j}\right|^{2}}, \text { où } \alpha, \beta=x, y, z .
$$




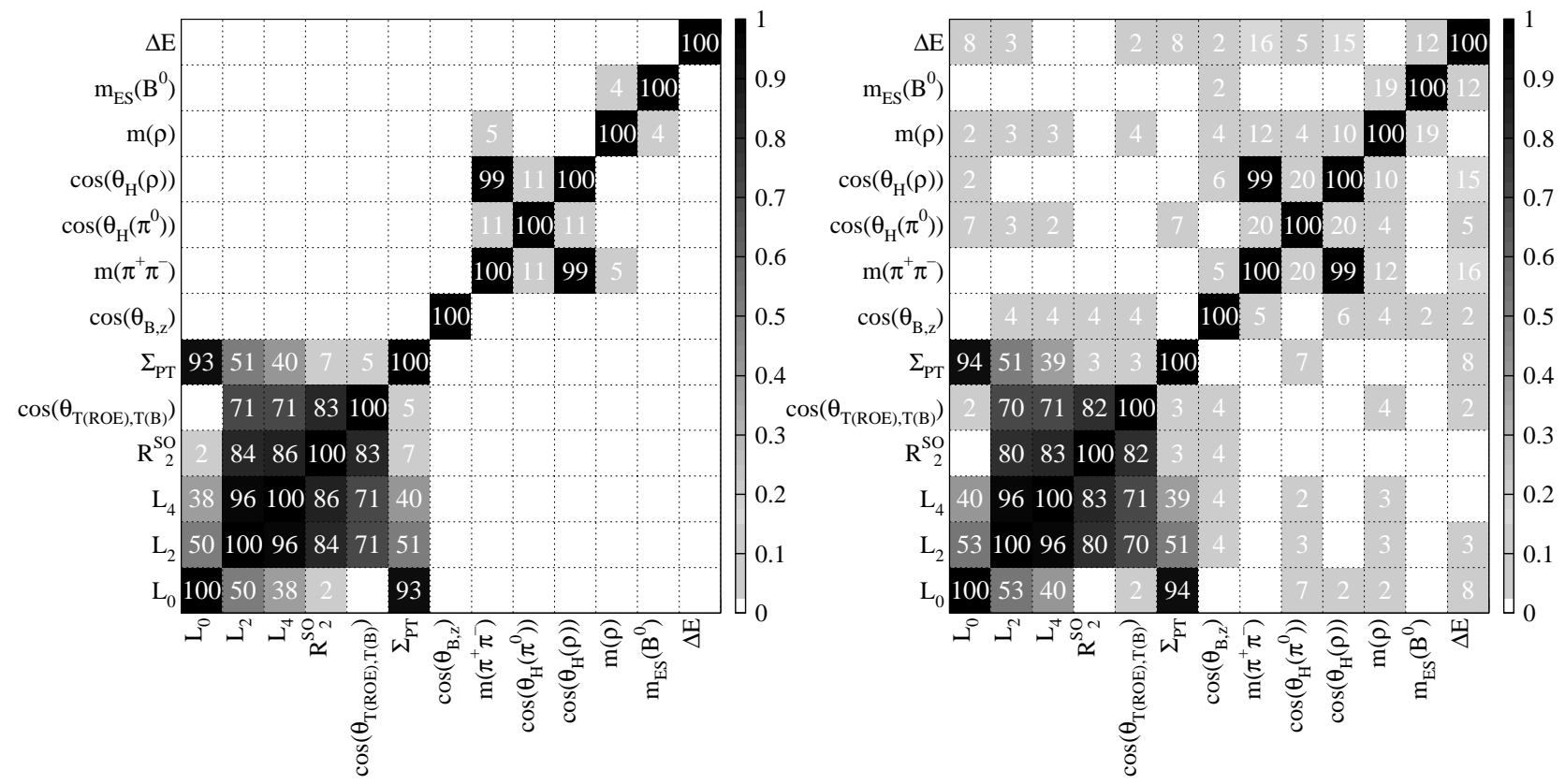

Figure 4.18: Modules des coefficients de corrélation linéaire entre les variables discriminantes pour les événements de signal correctement reconstruits (figure de gauche) et mal reconstruits (figure de droite), dans le Monte Carlo $B^{0} \rightarrow \rho^{ \pm} \pi^{\mp}$.
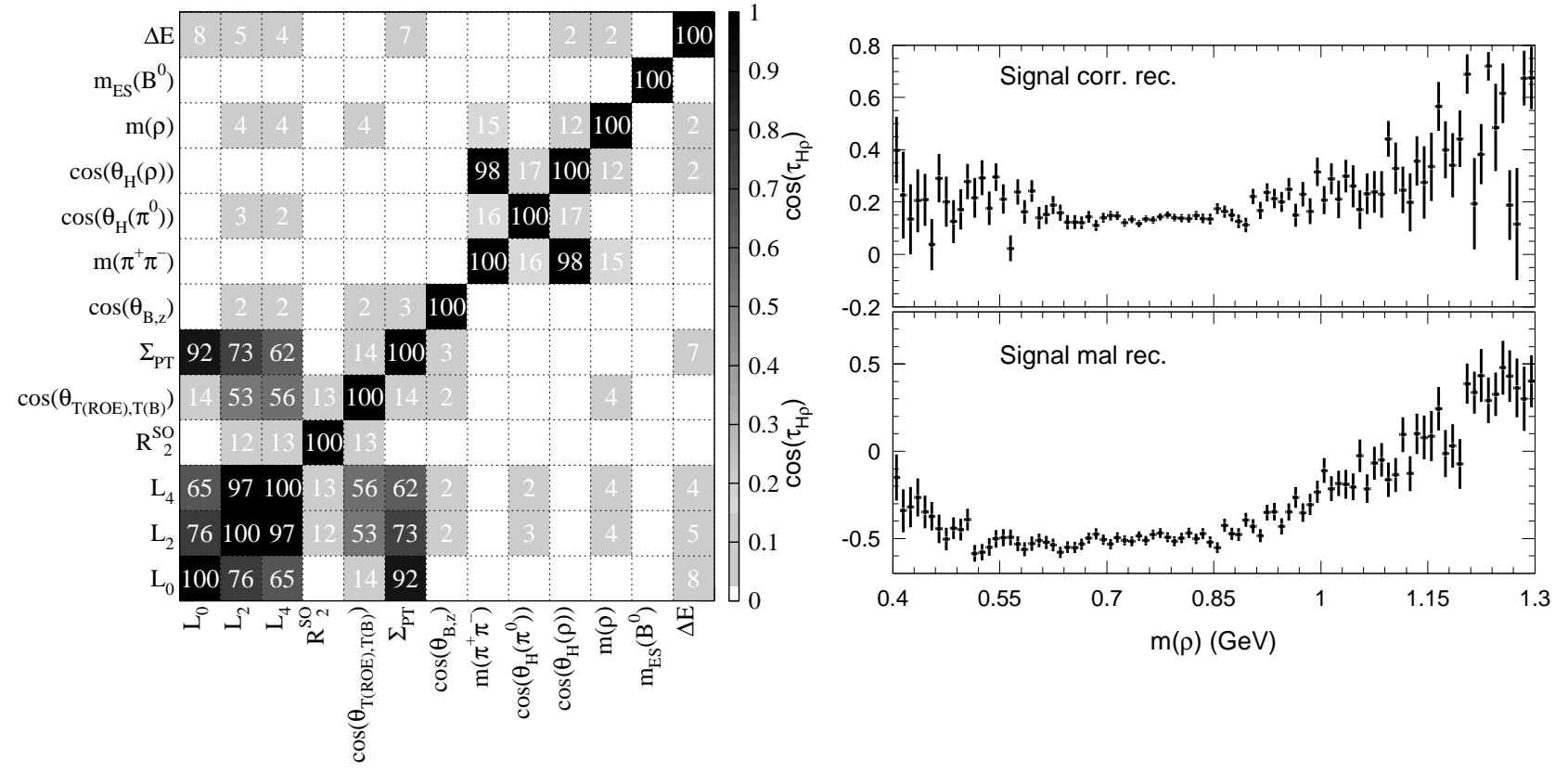

Figure 4.19: Figure de gauche: modules des coefficients de corrélation linéaire entre les variables discriminantes pour les événements on-peak (en dehors de la région de signal), dominés par le continuum. Figure de droite: corrélation entre $\left\langle\cos \theta_{H}(\rho)\right\rangle$ et $m\left(\rho^{ \pm}\right)$pour les événements de signal correctement (haut) et mal (bas) reconstruits. 


\subsubsection{Discriminant de Fisher}

Le discriminant de Fisher [80] est une combinaison linéaire des variables $x_{i}$ définie par:

$$
\mathcal{F}=c_{0}+\sum_{i=1}^{N} c_{i} x_{i}
$$

où les coefficients $c_{i}$ sont optimisés pour maximiser la séparation entre signal et bruit de fond (le coefficient $c_{0}$ sert à recentrer la distribution de sortie autour de 0 ). Plus précisément, on construit un axe dans l'espace N-dimensionnel des variables tel que les événements de signal et de bruit de fond soient le plus éloignés possible les uns des autres lorsque projetés sur cet axe. Le discriminant de Fisher traite les corrélations linéaires entre variables.

\subsubsection{Réseau de neurones}

Le réseau de neurones utilisé par la suite est un perceptron multicouche [81] consistant en plusieurs couches de neurones artificiels (dans notre cas, 2) entourées d'une couche de nœuds d'entrée $\mathbf{E}=\left\{E_{i}, i=1, N\right\}$ (autant de nouds d'entrée que de variables discriminantes utilisées, au nombre de $N$ ) et d'une couche de nouds de sortie $\mathbf{S}=\left\{S_{i}, i=1,2\right\}$ (une sortie pour chaque catégorie d'événements: signal et bruit de fond continuum). Nous plaçons $N-1$ neurones sur la première couche et $N-2$ sur la seconde ${ }^{13}$.

Le principe d'un réseau de neurones artificiel est inspiré des réseaux de neurones biologiques: un neurone reçoit par des synapses les signaux issus des neurones de la couche précédente, somme ces signaux, et si la somme est supérieure à un seuil, la transmet à travers une fonction d'activation $F$. Cette dernière est une fonction non-linéaire dérivable, une sigmoïde dans notre cas:

$$
F(x)=\frac{1}{1+e^{\alpha x}},
$$

où $\alpha$ est le paramètre de transfert, constant pour tous les neurones.

Les synapses transmettent les signaux entre neurones avec un certain poids. Ces poids sont déterminés lors de l'entraînement du réseau: on présente un lot d'événements, dits d'entraînement, pour chacun desquels on connaît la sortie désirée (signal ou bruit de fond), et on cherche les valeurs des poids de synapses qui minimisent la différence entre la sortie obtenue avec ces poids, et celle désirée.

Grâce à l'utilisation d'une fonction de transfert non-linéaire, ce discriminant permet d'exploiter les corrélations non-linéaires entre variables. Concernant les corrélations de type linéaire, les performances du réseau de neurones sont identiques à celles du discriminant de Fisher.

\subsubsection{Optimisation de la variable multidimensionnelle}

Parmi les variables cinématiques et topologiques (préférentiellement appartenant à la catégorie $\mathrm{VnG}$ ) à notre disposition, les trois configurations suivantes ont été retenues pour des critères de simplicité et de performance:

\footnotetext{
${ }^{13}$ Plusieurs configurations ont été expérimentées, et nous avons gardé celle-ci pour ses bonnes performances alliées à sa rapidité d'entraînement.
} 
- Base utilise les variables:

$$
m\left(\rho^{ \pm}\right), \cos \theta_{H}(\rho), L_{0}, L_{2} .
$$

- Base+ utilise les variables:

$$
m\left(\rho^{ \pm}\right), \cos \theta_{H}(\rho), L_{0}, L_{2}, \cos \theta(B, z), \cos \theta\left(T_{B}, z\right), \hat{S}_{\mathrm{ROE}} .
$$

- Base ++ utilise les variables:

$$
\begin{gathered}
\tilde{\chi}^{2}\left(m\left(\rho^{ \pm}\right), m\left(\pi^{0}\right)\right), \cos \theta_{H}(\rho), \cos \theta_{H}\left(\pi^{0}\right), \\
\mathcal{F}_{L}\left(L_{0}^{(c)}, L_{0}^{(n)}, L_{2}^{(c)}, L_{2}^{(n)}\right), \cos \theta(B, z), \cos \theta\left(T_{B}, z\right), \Sigma_{\mathrm{p}_{\mathrm{T}}},\left|\cos \left(\theta_{T}\right)\right|,
\end{gathered}
$$

où $\tilde{\chi}^{2}$ est défini par:

$$
\tilde{\chi}^{2} \equiv\left(\frac{m\left(\pi^{0}\right)-0.135}{0.0076}\right)^{2}+\left(\frac{m\left(\rho^{ \pm}\right)-0.769}{0.137}\right)^{2},
$$

et $\mathcal{F}_{L}$ est un discriminant de Fisher utilisant $L_{0}$ et $L_{2}$ calculés pour les traces neutres (n) et chargées (c). Les poids du Fisher sont calculés en utilisant des événements de signal Monte Carlo et des événements de bruit de fond continuum off-peak:

$$
\mathcal{F}_{L} \equiv 0.60+0.61 \cdot L_{0}^{(n)}+0.60 \cdot L_{0}^{(c)}-1.64 \cdot L_{2}^{(n)}-1.40 \cdot L_{2}^{(c)} .
$$

Les coefficients des monômes pour les traces chargées et neutres étant voisins, la séparation de ces deux composantes pourrait être évitée.

Les variables $m\left(\rho^{ \pm}\right)$et $\cos \theta_{H}(\rho)$ sont corrélées pour les événements mal reconstruits $(c f$. matrice 4.18 et figure 4.19): nous ne les utilisons donc pas dans l'ajustement final, mais dans l'analyse multidimensionnelle décrite ici qui permet de prendre en compte les corrélations entre ces variables.

Les performances des monômes $L_{0}$ et $L_{2}$ sont similaires à celles des moments de FoxWolfram ( $c f$. Eq. B.2 de l'annexe B), variables couramment utilisées pour la lutte contre le bruit de fond continuum. Les monômes sont néanmoins préférables aux moments de FoxWolfram en raison de leurs propriétés de variables non-globales ( $\mathrm{VnG})$.

Il existe des corrélations non-linéaires entre les variables de ces trois ensembles, et nous les traitons donc avec un réseau de neurones.

\subsubsection{Performances des trois configurations}

Afin de comparer les performances de ces trois configurations, nous procédons à un certain nombre de tests en entraînant les réseaux de neurones dans des conditions similaires:

- Nombre de cycles d'entrainement (= nombre de cycles d'optimisation des poids de synapses): 5000

- Nombre d'événements dans les lots entraînement: 10000 de Monte Carlo signal et 10000 de continuum off-peak

Les distributions de la sortie des réseaux de neurones $x_{\mathrm{NN}}$ pour chaque configuration de variables sont montrées sur la figure 4.20 .

Les critères pour le choix de la configuration finale sont les suivants: 


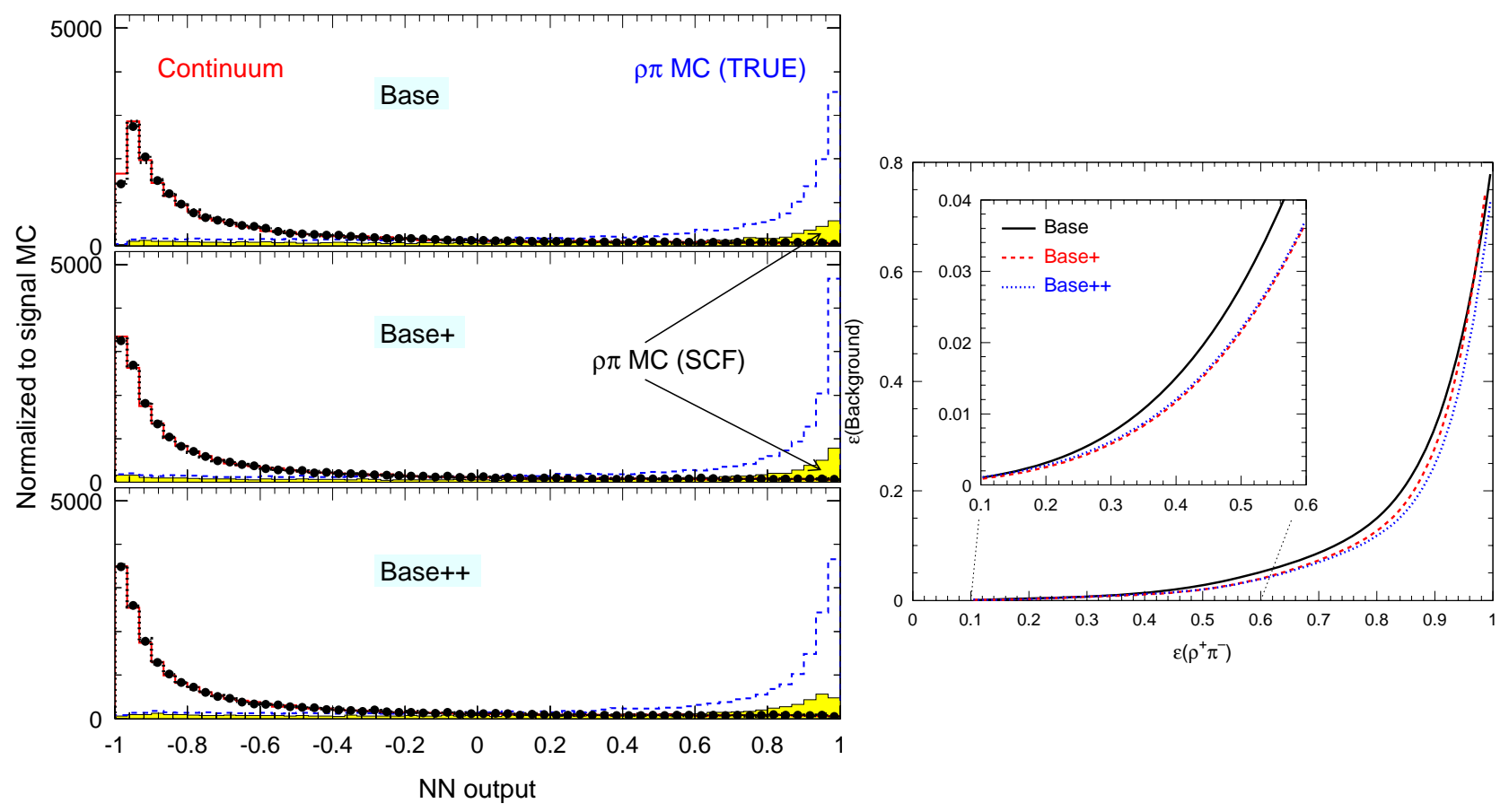

Figure 4.20: Figure de gauche: distributions de la sortie des réseaux de neurones $x_{\mathrm{NN}}$ pour chaque configuration Base (graphe du haut), Base+ (graphe du milieu) et Base++ (graphe du bas) pour des événements de signal correctement reconstruits (TRUE), mal reconstruits (SCF) du Monte Carlo $B^{0} \rightarrow \rho^{ \pm} \pi^{\mp}$, et des événements de bruit de fond on-peak (en dehors de la région de signal, points sur la figure) et off-peak (ligne pointillée). Figure de droite: comparaison de l'efficacité de sélection du signal en fonction de l'efficacité du bruit de fond pour une coupure sur $x_{\mathrm{NN}}$ pour les configurations Base (ligne pleine), Base+ (ligne en tirets) et Base++ (ligne pointillée).

- erreurs sur les paramètres $C P$ obtenues par des simulations rapides.

- simplicité du réseau et de la validation du réseau de neurones grâce à d'autres désintégrations $B$ abondantes (les variables non-globales sont préférées): cf. section 4.14.2.

- taille des corrélations entre $x_{\mathrm{NN}}$ et les variables utilisées dans l'ajustement final, $m_{\mathrm{ES}}$, $\Delta E$ et $\Delta t$ (on évite donc la présence de variables globales).

L'efficacité de sélection du signal en fonction de l'efficacité du bruit de fond pour une coupure sur $x_{\mathrm{NN}}$ est montrée sur la figure 4.20. On observe la hiérarchie $\epsilon_{\rho \pi}$ (Base) < $\epsilon_{\rho \pi}($ Base +$) \simeq \epsilon_{\rho \pi}($ Base ++$)$.

Des simulations rapides de l'analyse (dans sa configuration presque finale) faites avec les 3 configurations permettent de comparer les erreurs attendues sur les paramètres $C P$. On ne considère pas $\rho K$ dans ces analyses pour raison de simplicité. La sélection décrite dans les sections 4.3 et 4.5 est appliquée, ainsi qu'une coupure supplémentaire sur la sortie du réseau de neurones: $x_{\mathrm{NN}}($ Base $)>0, x_{\mathrm{NN}}($ Base +$)>0.1$ et $x_{\mathrm{NN}}($ Base ++$)>0.05$, menant à la même efficacité de sélection pour le signal de $29.9 \%$ (soit 517 événements de signal). En supposant une luminosité de $60 \mathrm{fb}^{-1}$ et un rapport d'embranchement pour $B^{0} \rightarrow \rho^{ \pm} \pi^{\mp}$ de $30 \times 10^{-6}$, on obtient les nombres d'événements pour signal et bruit de fond donnés dans le tableau 4.5 . 


\begin{tabular}{lccc}
\hline Configuration & $f_{\text {SCF }}$ & $w_{Q}$ & $N_{\rho \pi}^{B}$ \\
\hline Base & 0.305 & 0.136 & 29731 \\
Base + & 0.303 & 0.131 & 24577 \\
Base ++ & 0.305 & 0.128 & 25836 \\
\hline
\end{tabular}

Tableau 4.5: Fraction d'événements mal reconstruits $\left(f_{\mathrm{SCF}}\right)$, fraction de $\rho$ de mauvaise charge parmi les événements mal reconstruit $\left(w_{Q}\right)$ et nombre d'événements bruit de fond dans les simulations rapides.

L'ajustement par maximum de vraisemblance utilise les variables $m_{\mathrm{ES}}, \Delta E$ et $x_{\mathrm{NN}}$. Un total de 1000 expériences de simulation rapide sont générées et ajustées pour les trois configurations étudiées. Les valeurs moyennes des paramètres $C P$ et du nombre d'événements de signal, ainsi que les erreurs sur ces valeurs, sont montrées dans le tableau 4.6. Les résultats de ces simulations rapides confirment la hiérarchie observée sur la figure 4.20: Base + mène à une erreur en moyenne $3 \%$ plus petite que Base, et $2 \%$ plus petite que Base ++ .

\begin{tabular}{lcccccc}
\hline & $C^{-}$ & $C^{+}$ & $S^{-}$ & $S^{+}$ & $A_{\mathrm{CP}}^{\rho \pi}$ & $\mathcal{B}\left(\rho^{ \pm} \pi^{\mp}\right)$ \\
\hline Valeurs initiales & -0.59 & 0.59 & 0.37 & -0.30 & 0.05 & $30 \times 10^{-6}$ \\
\hline Configuration & $\sigma\left(C^{-}\right)$ & $\sigma\left(C^{+}\right)$ & $\sigma\left(S^{-}\right)$ & $\sigma\left(S^{+}\right)$ & $\sigma\left(A_{\mathrm{CP}}^{\rho \pi}\right)$ & $\sigma\left(N_{\rho \pi}\right) / \sqrt{N_{\rho \pi}}$ \\
\hline Base & 0.2502 & 0.2314 & 0.3579 & 0.33014 & 0.0754 & $\begin{array}{c}7.55 \% \\
\pm 0.01 \%\end{array}$ \\
Base+ & \pm 0.0010 & \pm 0.0007 & \pm 0.0015 & \pm 0.0011 & \pm 0.0001 & $\pm 0.01 \%$ \\
Base++ & $-3.0 \%$ & $-3.1 \%$ & $-2.8 \%$ & $-2.8 \%$ & $-3.3 \%$ & $-3.3 \%$ \\
& $-1.0 \%$ & $-1.5 \%$ & $-1.3 \%$ & $-1.0 \%$ & $-1.6 \%$ & $-0.9 \%$ \\
\hline
\end{tabular}

Tablea1 4.6: Résultats de simulations rapides (excluant $\rho K$ ) sur les 3 configurations de variables entrant dans le réseau de neurones: les valeurs générées pour les paramètres $C P$ et le rapport d'embranchement de $\rho \pi$ sont données dans la première partie du tableau. Les erreurs sur la configuration Base sont données plus bas, ainsi que la réduction relative des erreurs pour les deux autres configurations Base+ et Base+t.

\subsubsection{Corrélations}

La figure 4.21 montre les corrélations entre les sorties des réseaux de neurones pour les trois configurations et les deux variables $m_{\mathrm{ES}}, \Delta E$. On observe de petites corrélations, en particulier entre $x_{\mathrm{NN}}$ et $m_{\mathrm{ES}}$ pour le signal mal reconstruit, et entre $x_{\mathrm{NN}}$ et $\Delta E$. Cette dernière corrélation est due à l'utilisation de la variable $\cos \theta_{H}(\rho)$ dans le réseau de neurones, variable corrélée avec $\Delta E$ pour les événements de signal mal reconstruit, comme montré sur la figure 4.22. En effet, les événements avec un grand $\Delta E$ ont tendance à peupler la région $\cos \theta_{H}(\rho) \simeq 1$. 

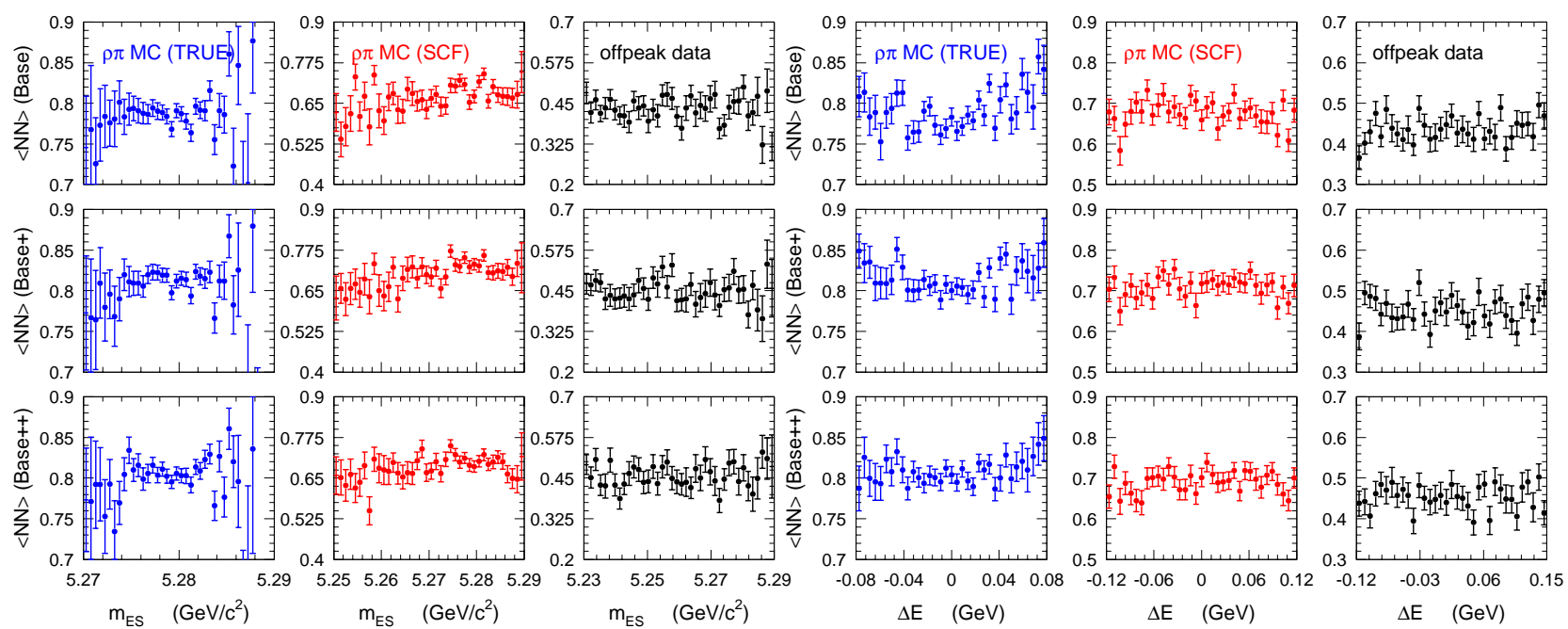

Figure 4.21: Corrélations entre $\left\langle x_{\mathrm{NN}}\right\rangle$ et $m_{\mathrm{ES}}$ (ensemble des 9 graphes de gauche), et entre $\left\langle x_{\mathrm{NN}}\right\rangle$ et $\Delta E$ (ensemble des 9 graphes de droite) pour chaque configuration Base (graphe du haut de l'ensemble), Base+ (graphe du milieu) et Baset+ (graphe du bas) pour des événements de signal correctement reconstruits (TRUE), mal reconstruits (SCF) du Monte Carlo $B^{0} \rightarrow \rho^{ \pm} \pi^{\mp}$, ainsi que des événements de bruit de fond continuum off-peak.

\subsubsection{Choix de la configuration finale}

Au vu du très faible gain en performance apporté par l'ajout de variables à la configuration Base et étant donnée la simplicité de cette dernière, nous gardons cette configuration pour l'ajustement final.

Afin de faciliter la paramétrisation des distributions de la sortie du réseau de neurones (en particulier pour le bruit de fond continuum), nous appliquons la transformation suivante sur $x_{\mathrm{NN}}$ :

$$
x_{\mathrm{NN}} \rightarrow 1-\operatorname{acos}\left(x_{\mathrm{NN}}+\text { constante }\right),
$$

où la constante vaut 0.003 . Pour ne pas garder un nombre trop important d'événements on-peak dans l'analyse (ce qui ralenti sensiblement les simulations rapides et autres études), nous coupons sur $x_{\mathrm{NN}}$ à zéro (après transformation de l'eq. 4.17). La distribution de $x_{\mathrm{NN}}$ pour le bruit de fond continuum devient quasiment linéaire, comme représenté sur le graphe de gauche de la figure 4.23. La distribution pour les événements de signal est montrée sur le graphe de droite.

\subsection{Sélection finale et choix du meilleur candidat}

\subsubsection{Efficacité finale de sélection}

Le tableau 4.7 résume les efficacités des coupures de la sélection finale. Les efficacités sont calculées dans le Monte Carlo relativement au nombre d'événements passant les coupures précédemment appliquées (après avoir appliqué les corrections dues aux $\pi^{0}$ : voir section 4.14.1). En pratique, une pré-sélection est appliquée ( $c f$. Ref. [39]) pour des raisons techniques: l'efficacité de la première coupure est donc relative au nombre d'événements issus de cette pre-sélection. 


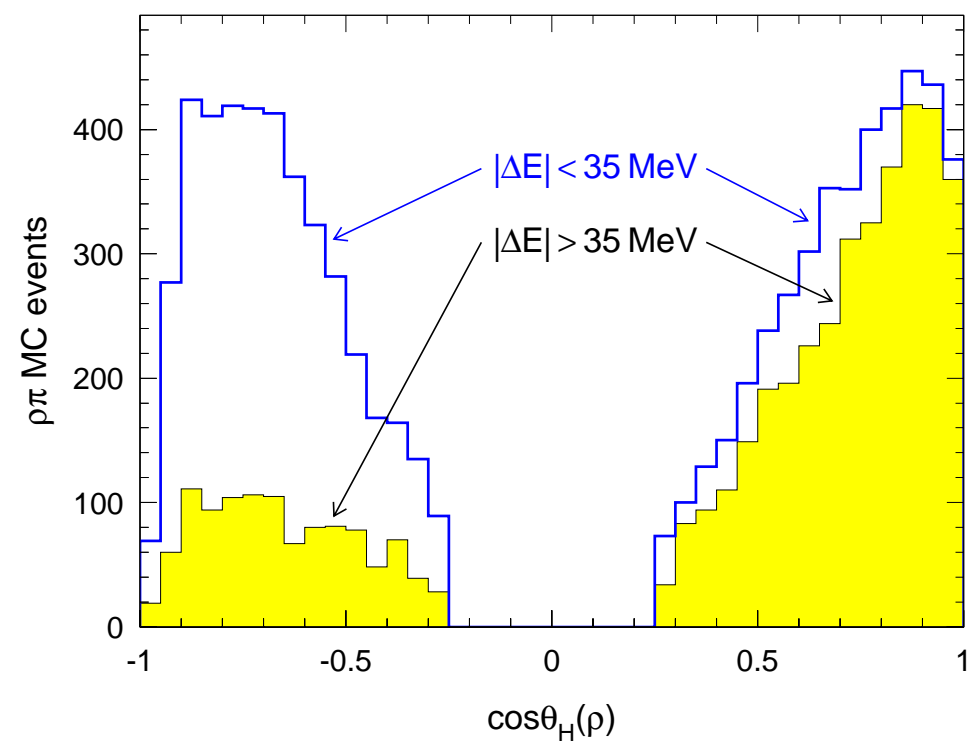

Figure 4.22: Distribution de $\cos \theta_{H}(\rho)$ pour deux différentes coupures sur $\Delta E$, démontrant la corrélation entre ces deux variables.
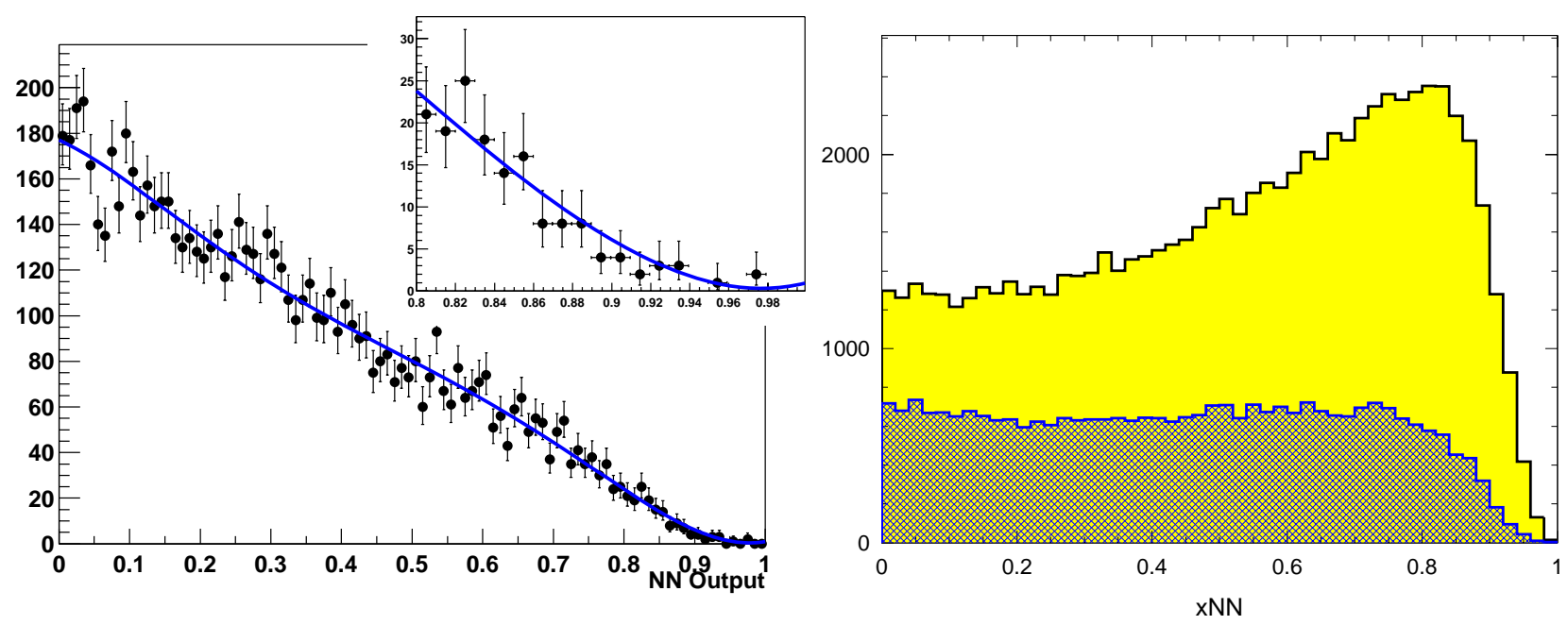

Figure 4.23: Distribution de $x_{\mathrm{NN}}$ après la transformation définie dans l'equation 4.17 pour les événements de bruit de fond continuum off-peak (graphe de gauche) et de signal Monte Carlo (graphe de droite). La courbe bleue hachurée montre les événements mal reconstruits. 


\begin{tabular}{|c|c|c|c|c|}
\hline Coupure & $\epsilon(\rho \pi)$ & $\epsilon(\rho K)$ & $\epsilon($ on - peak $)$ & $\epsilon($ off - peak $)$ \\
\hline Pré-sélection & 66.6 & $\overline{666.6}$ & 16.0 & 16.0 \\
\hline \multicolumn{5}{|l|}{ Traces chargées } \\
\hline Reconstruction & 97.4 & 95.5 & 99.6 & 99.6 \\
\hline Veto électrons & 98.7 & 99.0 & 94.1 & 94.1 \\
\hline Veto kaons & 98.7 & 95.7 & 88.2 & 88.1 \\
\hline Veto protons & 99.7 & 96.8 & 94.7 & 94.5 \\
\hline$\# \gamma$ Cherenkov $>5$ & 85.3 & 86.9 & 80.4 & 80.5 \\
\hline \multicolumn{5}{|l|}{ Traces neutres } \\
\hline$E>50 \mathrm{MeV}$ & 95.9 & 95.7 & 94.3 & 94.2 \\
\hline $0.01<\mathrm{LAT}<0.6$ & 96.7 & 96.5 & 93.9 & 94.2 \\
\hline $0.1<m\left(\pi^{0}\right)<0.16 \mathrm{GeV} / c^{2}$ & 98.2 & 98.2 & 96.2 & 96.2 \\
\hline \multicolumn{5}{|l|}{ Méson $\rho$} \\
\hline $0.4<m(\rho)<1.3 \mathrm{GeV} / \mathrm{c}^{2}$ & 94.8 & 94.5 & 65.0 & 64.5 \\
\hline Veto interférences & 96.2 & 96.1 & 98.3 & 98.3 \\
\hline$\left|\cos ^{2} \theta^{ \pm}\right|>0.25$ & 97.6 & 97.4 & 79.3 & 78.8 \\
\hline \multicolumn{5}{|l|}{ Candidat $B$} \\
\hline $5.23<m_{\mathrm{ES}}<5.29 \mathrm{GeV} / \mathrm{c}^{2}$ & 96.5 & 96.5 & 51.2 & 52.0 \\
\hline$-120<\Delta E<150 \mathrm{MeV}$ & 84.3 & 80.5 & 27.4 & 27.6 \\
\hline \multicolumn{5}{|l|}{ Temps } \\
\hline$|\Delta t|<20 \mathrm{ps}$ & 99.3 & 99.3 & 97.5 & 97.5 \\
\hline$\sigma_{\Delta t}<2.5 \mathrm{ps}$ & 96.5 & 96.6 & 93.2 & 93.4 \\
\hline$x_{\mathrm{NN}}$ & 63.1 & 62.7 & 5.6 & 5.3 \\
\hline 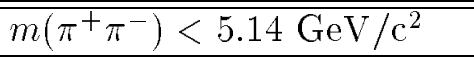 & 99.5 & 100. & 99.1 & 99.7 \\
\hline Total & 21.36 & 18.99 & $1.91 .10^{-3}$ & $1.85 .10^{-3}$ \\
\hline
\end{tabular}

Tableau 4.7: Efficacités de sélection (en \%) pour les signaux $B^{0} \rightarrow \rho^{ \pm} \pi^{\mp}, B^{0} \rightarrow \rho^{-} K^{+}$, et le bruit de fond continuum on- et off-peak. Les efficacités sont relatives aux coupures précédemment réalisées. Pour des raisons techniques, une pré-sélection est appliquée avant les coupures décrites dans cette section (cf. texte). 


\subsubsection{Choix du meilleur candidat}

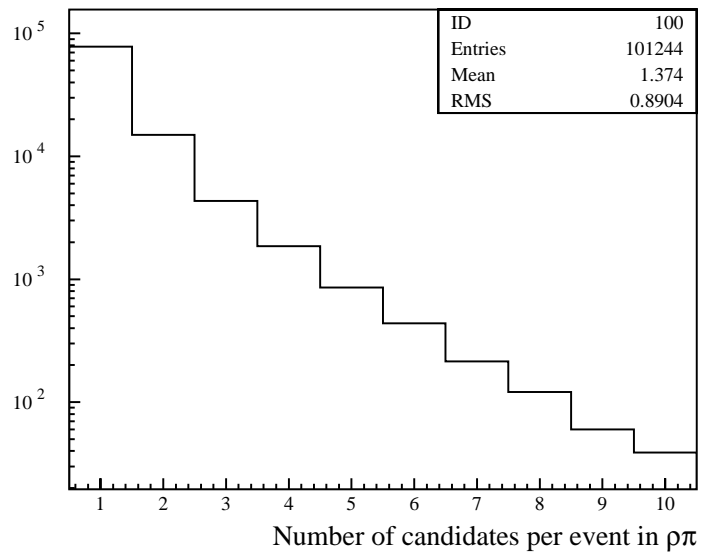

Figure 4.24: Distribution du nombre de candidats par événement après l'ensemble des coupures de sélection.

La figure 4.24 montre la distribution du nombre de candidats par événement pour le signal $B^{0} \rightarrow \rho^{ \pm} \pi^{\mp}$ après l'ensemble des coupures de sélection: $23 \%$ des événements ont plus d'un candidat, et le nombre moyen de candidats par événement est de 1.4 . Environ $20 \%$ des événements $\rho K$ ont plus d'un candidat. Ces candidats multiples sont dus majoritairement à la mauvaise reconstruction des $\pi^{0}$ ( $c f$. section 4.3.2). Nous choisissons donc le candidat pour lequel la masse invariante des deux photons est la plus proche de la masse nominale du $\pi^{0}$. Si deux candidats ont la même masse, nous gardons le premier des deux.

Le tableau 4.8 donne la fraction d'événements mal reconstruits $\left(f_{\mathrm{scf}}\right)$ et la fraction de $\rho$ de mauvaise charge parmi ces derniers $\left(w_{Q}\right)$, ainsi que les efficacités finales de sélection. La fraction $w_{Q}$ est plus élevée pour $B^{0} \rightarrow \rho^{ \pm} \pi^{\mp}$ que pour $B^{0} \rightarrow \rho^{-} K^{+}$, car dans ce dernier mode, la trace célibataire est un kaon qui ne peut être considérée comme issu d'un $\rho$ qu'en cas de mauvaise identification. Les fractions d'événements mal reconstruits varient par catégories d'étiquetage, et nous tenons compte de ceci lors de l'ajustement final.

\begin{tabular}{|c|c|c|c|c|c|}
\hline Mode & \multicolumn{2}{|l|}{$\epsilon$} & $f_{\mathrm{scf}}$ & \multicolumn{2}{|r|}{$w_{Q}$} \\
\hline$\rho^{ \pm} \pi^{\mp}$ & $21.36 \pm 0.01$ & \multicolumn{2}{|c|}{$31.30 \pm 0.1$} & \multirow{2}{*}{\multicolumn{2}{|c|}{$\begin{array}{c}16.46 \pm 0.2 \\
5.22 \pm 0.19\end{array}$}} \\
\hline$\rho^{ \pm} K^{\mp}$ & $18.99 \pm 0.01$ & \multicolumn{2}{|c|}{$29.70 \pm 0.22$} & & \\
\hline \multirow{2}{*}{ Mode } & \multicolumn{5}{|c|}{$f_{\mathrm{scf}}$} \\
\hline & Lepton & Kaon & NT1 & NT2 & NoTag \\
\hline$\rho^{ \pm} \pi^{\mp}$ & 21.12 & 31.85 & 28.41 & 32.91 & 34.11 \\
\hline$\rho^{ \pm} K^{\mp}$ & 19.04 & 29.95 & 26.96 & 31.41 & 32.89 \\
\hline
\end{tabular}

Tableau 4.8: Tableau du haut: effcacité de sélection ( $\epsilon)$, fraction d'événements mal reconstruits $\left(f_{\mathrm{scf}}\right)$ et taux de mauvaise charge $\left(w_{Q}\right)$ parmi les événements mal reconstruits (en \%). Ces chiffres sont obtenus dans les Monte Carlo $B^{0} \rightarrow \rho^{ \pm} \pi^{\mp}$ et $B^{0} \rightarrow \rho^{-} K^{+}$. Tableau du bas: fractions d'événements mal reconstruits par catégories d'étiquetage. 


\subsection{Bruit de fond provenant d'autres modes $B$}

En raison de la présence de la résonance large $\rho\left(\Gamma(\rho) \simeq 150 \mathrm{MeV} / \mathrm{c}^{2}\right.$ ) et d'un $\pi^{0}$ (pollué par le bruit de fond) dans l'état final considéré, les désintégrations $B^{0} \rightarrow \rho^{ \pm} \pi^{\mp}$ et $B^{0} \rightarrow \rho^{-} K^{+}$ souffrent de la contamination d'autres modes $B$, charmés ou non charmés.

Le traitement du bruit de fond $B$ est plus difficile que celui du bruit de fond continuum pour les raisons suivantes:

- les caractéristiques (topologiques et cinématiques) du bruit de fond $B$ sont proches de celles du signal: il est donc plus difficile de lutter contre ce bruit de fond que de lutter contre le continuum. D'autre part, il est difficile (du moins pour les modes $B$ non charmés) de trouver un échantillon de données permettant d'évaluer la contamination de ce bruit de fond (alors que pour le continuum, nous disposions des données off-peak, ainsi que des données on-peak en dehors de la région de signal).

- pour évaluer la contamination des modes de bruit de fond $B$, nous avons besoin de connaître leurs rapports d'embranchement. Or la plupart d'entre eux n'ont pas encore été mesurés: attribuer un rapport d'embranchement à chacun de ces modes représente la gageure principale pour un traitement correct du bruit de fond $B$.

- ce bruit de fond peut être affecté par la violation de $C P$, par des corrélations entre l'étiquette de saveur et la charge du $\rho$ reconstruit, ainsi que par des corrélations entre certaines variables de l'ajustement final.

Notre stratégie consiste à introduire un terme correctif dans la fonction de vraisemblance finale ( $c f$. section 4.10.1) pour chaque mode de bruit de fond $B$ (en fait, pour chaque classe de modes, puisque nous regroupons certaines modes entre eux afin de limiter le nombre de termes correctifs total).

La lutte contre le bruit $B$ s'avère infructueuse: pour diminuer significativement les incertitudes systématiques reliées au bruit de fond $B$, il faut sacrifier l'efficacité de sélection du signal (une analyse alternative utilisant un réseau de neurones de lutte contre le bruit de fond $B$ est résumée dans l'annexe C). Puisque l'on s'attend, avec la luminosité considérée dans cette analyse, à être limité par la statistique, nous n'adoptons pas cette stratégie.

Les modes $B$ non charmés sont traités exclusivement et sont détaillés dans la section 4.8.1. Les modes $B$ charmés sont quant à eux traités inclusivement et décrits dans la section 4.8.2.

\subsubsection{Modes $B$ non charmés}

Nous avons étudié une liste voulue exhaustive des modes $B$ non charmés et avons évalué la contamination de ces modes dans le signal $B^{0} \rightarrow \rho^{ \pm} \pi^{\mp}$ et $B^{0} \rightarrow \rho^{-} K^{+}$à l'aide de la simulation Monte Carlo. Il s'agit de désintégrations en 2-, 3- et 4(et plus)- corps, pour lesquelles la cinématique diffère:

- désintégrations en 2-corps: pour reconstruire notre état final à partir d'une désintégration en 2-corps, il faut rajouter une trace (chargée ou neutre) prise dans le ROE. Le $B$ ainsi reconstruit possède alors une énergie plus élevée que la moitié de l'énergie des faisceaux (dans le centre de masse), de sorte que ces événements peuplent la région $\Delta E>0$. Les rapports d'embranchement des désintégrations en 2-corps sont bien connus expérimentalement. 
- désintégrations en 3-corps: on peut reconstruire notre état final à partir d'une autre désintégration en 3-corps en échangeant des traces avec le ROE, ou en construisant un faux $\pi^{0}$ à partir des photons de bruit de fond. L'énergie du $B$ ainsi reconstruit reste de l'ordre de la moitié de l'énergie des faisceaux, et laisse donc $\Delta E \simeq 0$. La cinématique de ce bruit de fond étant très proche de celle du signal (en particulier celui mal reconstruit), il constitue un bruit de fond difficilement réductible. Les rapports d'embranchement des désintégrations en 3 -corps sont pour la plupart connus, mais souvent avec des erreurs statistiques encore importantes.

- désintégrations en 4-corps: en ne gardant que 3 traces d'une désintégration en 4-corps, on peut reconstruire notre état final. Dans ce cas, l'énergie du $B$ est plus petite que la moitié de l'énergie des faisceaux, et ces modes peuplent donc $\Delta E<0$. Les rapports d'embranchement des désintégrations en 4-corps sont mal connus expérimentalement.

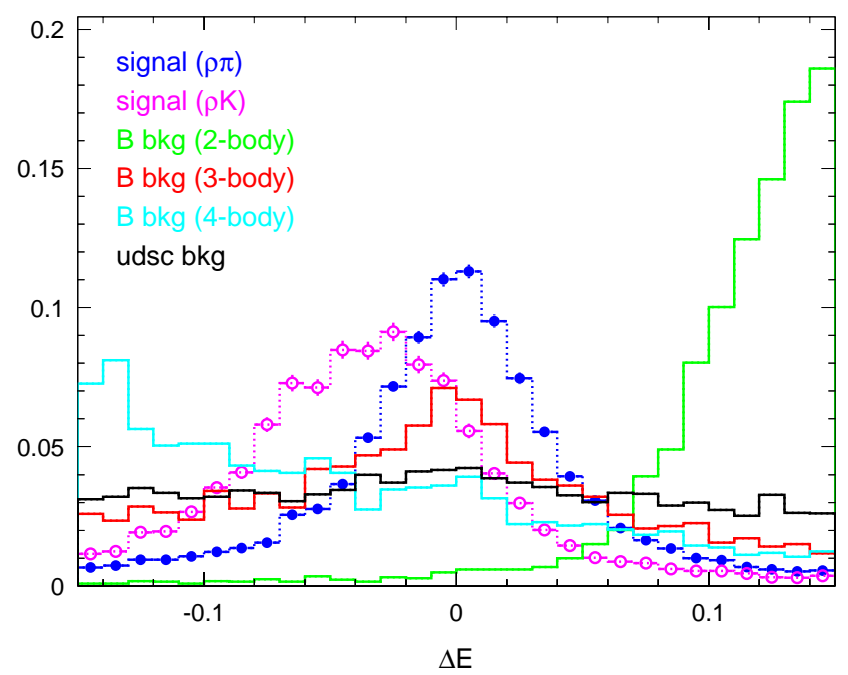

Figure 4.25: Distributions de $\Delta E$ pour le signal $B^{0} \rightarrow \rho^{ \pm} \pi^{\mp}$ (points bleus pleins) et $B^{0} \rightarrow$ $\rho^{-} K^{+}$(points blancs cerclés de mauve), et les bruits de fond continuum et $B$ en 2-, 3-, 4-corps (histogrammes de couleur).

Les distributions de $\Delta E$ pour le signal $B^{0} \rightarrow \rho^{ \pm} \pi^{\mp}$ et $B^{0} \rightarrow \rho^{-} K^{+}$, pour le bruit de fond continuum, et pour les bruits de fond $B$ en 2-, 3- et 4-corps sont montrées sur la figure 4.25.

Lors de l'attribution d'un rapport d'embranchement à chaque mode $B$ non charmé, plusieurs cas se présentent: lorsque le rapport d'embranchement a été mesuré, nous utilisons la moyenne mondiale des mesures effectuées. Dans le cas où seulement une limite supérieure a été placée, nous traduisons cette limite en rapport d'embranchement en utilisant l'information disponible dans les publications. Pour tous les autres modes encore non-mesurés, nous déduisons un intervalle de valeurs possibles à partir d'arguments théoriques. Par exemple, on peut relier certains modes non-mesurés à des modes mesurés en utilisant la symétrie d'isospin, ou des rapports de facteurs de forme. Si l'on ne peut relier un mode inconnu à un mode connu, nous donnons un intervalle très large basé sur des hypothèses ad-hoc.

Les discussions relatives aux rapports d'embranchement représentent un travail important (à la fois en quantité et en intérêt) pour la crédibilité du résultat de l'analyse de $B^{0} \rightarrow \rho^{ \pm} \pi^{\mp}$ et $B^{0} \rightarrow \rho^{-} K^{+}$. Il s'agit néanmoins d'un travail technique: nous le reportons donc dans l'annexe D. 
Les tableaux 4.9 à 4.11 résume les rapports d'embranchement (suivis d'un astérisque lorsqu'il s'agit d'une prédiction théorique), les efficacités de sélection et nombres d'événements attendus pour les catégories $B^{0} \rightarrow \rho^{ \pm} \pi^{\mp}$ et $B^{0} \rightarrow \rho^{-} K^{+}$, les numéros d'identification (détail technique), et les numéros de classe (CL, pour les modes gardés dans l'ajustement final) de tous les modes considérés dans l'analyse.

Les modes retenus dans l'analyse parmi cette liste sont décrits dans la section 4.8.3.

\subsubsection{Modes $B$ charmés}

La multiplicité moyenne des désintégrations charmées des $B$ est similaire à la multiplicité moyenne du bruit de fond continuum (cf. Fig. 4.1): reconstruit dans notre état final $B^{0} \rightarrow \rho h$, le bruit de fond $B$ charmé est donc cinématiquement plus proche du continuum que du bruit de fond $B$ non charmé. D'autre part, la plupart des rapports d'embranchement des modes charmés sont bien connus. Ce bruit de fond est donc moins dangereux que celui provenant des modes $B$ non charmés.

\subsubsection{Monte Carlo générique $b \rightarrow c$}

La figure 4.26 montre les distributions de $m_{\mathrm{ES}}, \Delta E$ et $x_{\mathrm{NN}}$ pour les événements charmés issus du Monte Carlo générique $B \bar{B}^{0}$ et $B^{+} B^{-}$, comparées aux distributions de bruit de fond continuum off-peak. Les distributions de $m_{\mathrm{ES}}$ sont quasiment identiques pour ces deux bruits de fond. La distribution de $\Delta E$ pour le bruit de fond charmé possède une pente négative supérieure à celle du continuum. Enfin, la distribution de $x_{\mathrm{NN}}$ pour le bruit de fond charmé pique à la fois sous le continuum et sous le signal.

A partir du Monte Carlo générique, nous attendons $164 \pm 36$ événements charmés provenant des $B$ chargés, et $102 \pm 23$ provenant des $B$ neutres.

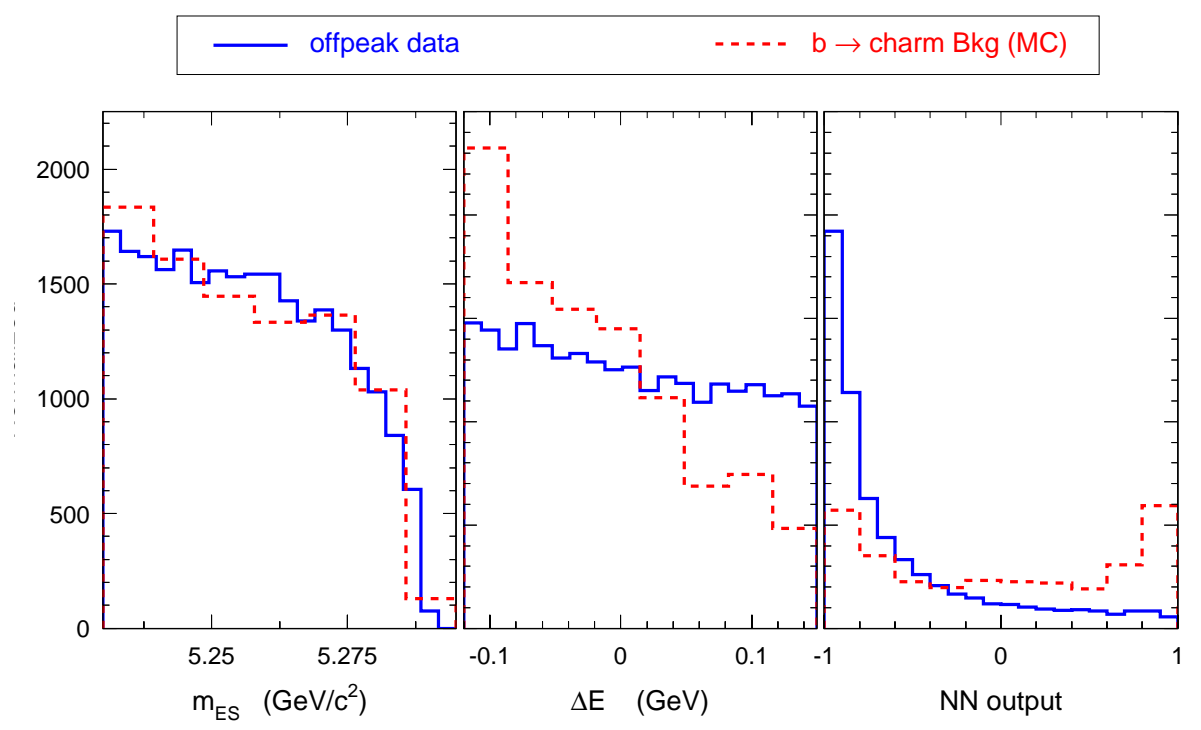

Figure 4.26: Distributions de $m_{\mathrm{ES}}, \Delta E$ et $x_{\mathrm{NN}}$ pour les données off-peak (ligne pleine) et Monte Carlo générique $b \rightarrow c$ (ligne pointillée). 


\begin{tabular}{|c|c|c|c|c|c|c|c|}
\hline \multirow{2}{*}{ Mode } & \multirow{2}{*}{$\begin{array}{c}\mathcal{B} \\
\left(\times 10^{-6}\right)\end{array}$} & \multicolumn{2}{|c|}{ Eff. (\%) } & \multicolumn{2}{|c|}{ Nombre attendu } & \multirow{2}{*}{$\mathrm{Id}$} & \multirow{2}{*}{$\mathrm{Cl}$. } \\
\hline & & $\rho \pi$ & $\rho K$ & & $K$ & & \\
\hline$B^{0} \rightarrow K^{\star 0} K^{\star 0}\left(\rightarrow K^{+} \pi^{-}\right)$[long] & $.0^{*}$ & 0.00 & 0.07 & $0.0 \pm 0.0$ & $0.0 \pm 0.2$ & 1 & - \\
\hline$B^{0} \rightarrow K^{\star 0} K^{\star 0}\left(\rightarrow K^{+} \pi^{-}\right)[$trans] & $0.0 \pm 3.0^{*}$ & 0.00 & 0.00 & $0.0 \pm 0.0$ & $0.0 \pm 0.0$ & 2 & - \\
\hline$B^{+} \rightarrow \rho^{+} K^{\star 0}\left(\rightarrow K^{+} \pi^{-}\right)[\mathrm{lo}$ & $12.0 \pm 12.0^{*}$ & 0.00 & 0.28 & $0.0 \pm 0.0$ & $2.9 \pm 2.9$ & 3 & 0 \\
\hline$B^{+} \rightarrow \rho^{+} K^{\star 0}\left(\rightarrow K^{+} \pi^{-}\right)[$trans] & $12.0 \pm 12.0^{*}$ & 0.00 & 0.01 & $0.0 \pm 0.0$ & $0.1 \pm 0.1$ & 4 & - \\
\hline$B^{+} \rightarrow \rho^{0} K^{\star+}\left(\rightarrow K^{+} \pi^{0}\right)[$ long] & $3.0 \pm 3.0^{*}$ & 0.06 & 0.25 & $0.2 \pm 0.2$ & $0.7 \pm 0.7$ & 5 & - \\
\hline$B^{+} \rightarrow \rho^{0} K^{\star+}\left(\rightarrow K^{+} \pi^{0}\right)$ [trans] & $3.0 \pm 3.0^{*}$ & 0.00 & 0.01 & $0.0 \pm 0.0$ & $0.0 \pm 0.0$ & 6 & - \\
\hline$B^{+} \rightarrow \rho^{0} K^{\star+}\left(\rightarrow K_{S}^{0} \pi^{+}\right)$[long] & $3.0 \pm 3.0^{*}$ & 0.02 & 0.00 & $0.0 \pm 0.0$ & $0.0 \pm 0.0$ & 7 & - \\
\hline$B^{+} \rightarrow \rho^{0} K^{\star+}\left(\rightarrow K_{S}^{0} \pi^{+}\right)$[trans] & $3.0 \pm 3.0^{*}$ & 0.00 & 0.00 & $0.0 \pm 0.0$ & $0.0 \pm 0.0$ & 8 & - \\
\hline$B^{0} \rightarrow \rho^{0} K^{\star 0}\left(\rightarrow K^{+} \pi^{-}\right)[$long] & $6.0 \pm 6.0^{*}$ & 0.04 & 0.19 & $0.2 \pm 0.2$ & $1.0 \pm 1.0$ & 91 & 3 \\
\hline$B^{0} \rightarrow \rho^{0} K^{\star 0}\left(\rightarrow K^{+} \pi^{-}\right)[$trans $]$ & $6.0 \pm 6.0^{*}$ & 0.00 & 0.00 & $0.0 \pm 0.0$ & $0.0 \pm 0.0$ & 92 & - \\
\hline$B^{0} \rightarrow \rho^{-} K^{\star+}\left(\rightarrow K^{+} \pi^{0}\right)$ [long] & $6.0 \pm 6.0^{*}$ & 0.09 & 0.51 & $0.5 \pm 0.5$ & $2.7 \pm 2.7$ & 9 & 3 \\
\hline$B^{0} \rightarrow \rho^{-} K^{\star+}\left(\rightarrow K^{+} \pi^{0}\right)$ [trans] & $6.0 \pm 6.0^{*}$ & 0.00 & 0.01 & $0.0 \pm 0.0$ & $0.1 \pm 0.1$ & 10 & - \\
\hline$B^{0} \rightarrow \rho^{-} K^{\star+}\left(\rightarrow K_{S}^{0} \pi^{+}\right)$[long] & $6.0 \pm 6.0^{*}$ & 0.01 & 0.00 & $0.0 \pm 0.0$ & $0.0 \pm 0.0$ & 11 & - \\
\hline$B^{0} \rightarrow \rho^{-} K^{\star+}\left(\rightarrow K_{S}^{0} \pi^{+}\right)[$trans $]$ & $6.0 \pm 6.0^{*}$ & 0.00 & 0.00 & $0.0 \pm 0.0$ & $0.0 \pm 0.0$ & 12 & - \\
\hline$B^{+} \rightarrow \rho^{+} \rho_{[\text {long] }}^{0}$ & $22.5_{-17}^{+27}$ & 1.02 & 0.00 & $20.1 \pm$ & $0.0 \pm 0.0$ & 13 & 0 \\
\hline$B^{+} \rightarrow \rho^{+} \rho_{[\text {trans] }}^{0}$ & $22.5_{-17.5}^{+27.5 *}$ & 0.02 & 0.00 & $0.4 \pm 0.4$ & $0.0 \pm 0.0$ & 14 & - \\
\hline$B^{0} \rightarrow \rho^{+} \rho_{[\text {long }]}^{-}$ & $40.0_{-35}^{+50 *}$ & 1.27 & 0.00 & $44.4 \pm 33.3$ & $0.0 \pm 0.0$ & 15 & 4 \\
\hline$B^{0} \rightarrow \rho^{+} \rho_{[\text {trans }]}^{-}$ & $40.0_{-35}^{+50 *}$ & 0.02 & 0.00 & $0.7 \pm 0.6$ & $0.0 \pm 0.0$ & 16 & - \\
\hline$B^{0} \rightarrow \rho^{0} \rho_{[\mathrm{long}]}^{0}$ & $3.5 \pm 3.5^{*}$ & 0.84 & 0.00 & $2.6 \pm 2.6$ & $0.0 \pm 0.0$ & 17 & 4 \\
\hline$B^{0} \rightarrow \rho^{0} \rho_{[\text {trans }]}^{0}$ & $3.5 \pm 3.5^{*}$ & 0.01 & 0.00 & $0.0 \pm 0.0$ & $0.0 \pm 0.0$ & 18 & - \\
\hline$B^{0} \rightarrow \eta^{\prime}\left(\rightarrow \rho^{0} \gamma\right) K^{\star 0}\left(\rightarrow K^{+} \pi^{-}\right)$ & $2.0 \pm 2.0^{*}$ & 0.00 & 0.02 & $0.0 \pm 0.0$ & $0.0 \pm$ & 20 & - \\
\hline$B^{+} \rightarrow \eta^{\prime}\left(\rightarrow \rho^{0} \gamma\right) K^{\star+}\left(\rightarrow K^{+} \pi^{0}\right)$ & $2.0 \pm 2.0^{*}$ & 0.00 & 0.02 & $0.0 \pm 0.0$ & $0.0 \pm 0.0$ & 21 & - \\
\hline$B^{+} \rightarrow \eta^{\prime}\left(\rightarrow \rho^{0} \gamma\right) \rho^{+}$ & $4.0 \pm 4.0^{*}$ & 0.04 & 0.00 & $0.0 \pm 0.0$ & $0.0 \pm 0.0$ & 22 & - \\
\hline$B^{+} \rightarrow \eta^{\prime}\left(\rightarrow \eta \pi^{+} \pi^{-}\right) \rho^{+}$ & $5.0 \pm 5.0^{*}$ & 0.00 & 0.00 & $0.0 \pm 0.0$ & $0.0 \pm 0.0$ & 23 & - \\
\hline$B^{0} \rightarrow \eta^{\prime}\left(\rightarrow \rho^{0} \gamma\right) \pi^{0}$ & $0.0 \pm 1.0^{*}$ & 0.17 & 0.00 & $0.0 \pm 0.2$ & $0.0 \pm 0.0$ & 41 & - \\
\hline$B^{+} \rightarrow \eta^{\prime}\left(\rightarrow \rho^{0} \gamma\right) \pi^{+}$ & $3.0 \pm 3.0^{*}$ & 0.64 & 0.00 & $1.7 \pm 1.7$ & $0.0 \pm 0.0$ & 43 & 0 \\
\hline$B^{+} \rightarrow \eta^{\prime}\left(\rightarrow \rho^{0} \gamma\right) K^{+}$ & $21.4 \pm 1.7$ & 0.01 & 0.41 & $0.1 \pm 0.1$ & $7.6 \pm 0.6$ & 42 & 0 \\
\hline$B^{+} \rightarrow K^{+} K^{-} K^{\star+}\left(\rightarrow K^{+} \pi^{0}\right)$ & )$^{*}$ & 0.00 & 0 & & & 24 & - \\
\hline$B^{+} \rightarrow K^{+} K^{+} K^{\star-}\left(\rightarrow K^{-} \pi^{0}\right)$ & $20.0 \pm 20.0^{*}$ & 0.00 & 0.00 & $0.0 \pm 0.0$ & $0.0 \pm 0.0$ & 25 & - \\
\hline$B^{+} \rightarrow \pi^{+} \pi^{0} K^{\star 0}\left(\rightarrow K^{+} \pi^{-}\right)$ & $0.0 \pm 10.0^{*}$ & 0.00 & 0.03 & $0.0 \pm 0.0$ & $0.0 \pm 0.1$ & 26 & - \\
\hline$B^{+} \rightarrow \pi^{+} \pi^{-} K^{\star+}\left(\rightarrow K^{+} \pi^{0}\right)$ [nonres] & $0.0 \pm 10.0^{*}$ & 0.00 & 0.00 & $0.0 \pm 0.0$ & $0.0 \pm 0.0$ & 27 & . \\
\hline$B^{+} \rightarrow \pi^{+} \pi^{-} K^{\star+}\left(\rightarrow K_{S}^{0} \pi^{+}\right)$[nonres] & $0.0 \pm 10.0^{*}$ & 0.00 & 0.00 & $0.0 \pm 0.0$ & $0.0 \pm 0.0$ & 28 & - \\
\hline$B^{0} \rightarrow \rho^{0} K^{+} \pi_{[\text {nonres] }}^{-}$ & $0.0 \pm 10.0^{*}$ & 0.00 & 0.00 & $0.0 \pm 0.0$ & $0.0 \pm 0.0$ & 29 & - \\
\hline$B^{0} \rightarrow \rho^{0} \pi^{+} \pi_{[\text {nonres }]}^{-}$ & $0.0 \pm 10.0^{*}$ & 0.05 & 0.00 & $0.0 \pm 0.3$ & $0.0 \pm 0.0$ & 30 & - \\
\hline
\end{tabular}

Tableau 4.9: Première partie de la liste des modes de bruit de fond $B$ considérés dans l'analyse. On donne le rapport d'embranchement $(\mathcal{B})$ (avec un astérisque dans le cas d'une estimation théorique), l'efficacité de sélection, le nombre d'événements attendu, le numéro d'identification (Id), et la classe des modes gardés dans l'ajustement final (CL). L'indice [long] et [trans] indique la composante longitudinale ou transversale des modes vecteur-vecteur. 


\begin{tabular}{|c|c|c|c|c|c|c|c|}
\hline \multirow{2}{*}{ Mode } & \multirow{2}{*}{$\begin{array}{c}\mathcal{B} \\
\left(\times 10^{-6}\right)\end{array}$} & \multicolumn{2}{|c|}{ Eff. (\%) } & \multicolumn{2}{|c|}{ Nombre attendu } & \multirow{2}{*}{ Id } & \multirow{2}{*}{$\mathrm{Cl}$. } \\
\hline & & $\rho \pi$ & $\rho K$ & $\rho \pi$ & $\rho K$ & & \\
\hline$B^{0} \rightarrow \rho^{-} \pi^{+} \pi_{[\text {nonres }]}^{0}$ & $0.0 \pm 10.0^{*}$ & 0.05 & 0.00 & $0.0 \pm 0.3$ & $0.0 \pm 0.0$ & 31 & - \\
\hline$B^{0} \rightarrow \rho^{-} K^{+} \pi_{[\text {nonres }]}^{0}$ & $0.0 \pm 10.0^{*}$ & 0.00 & 0.00 & $0.0 \pm 0.0$ & $0.0 \pm 0.0$ & 32 & - \\
\hline$B^{+} \rightarrow \rho^{0} K^{+} \pi_{[\text {nonres] }}^{0}$ & $0.0 \pm 10.0^{*}$ & 0.00 & 0.03 & $0.0 \pm 0.0$ & $0.0 \pm 0.1$ & 33 & - \\
\hline$B^{+} \rightarrow \rho^{0} \pi^{+} \pi_{[\text {nonres }]}^{0}$ & $0.0 \pm 10.0^{*}$ & 0.10 & 0.00 & $0.0 \pm 1.0$ & $0.0 \pm 0.0$ & 34 & - \\
\hline$B^{+} \rightarrow \rho^{+} K^{+} \pi_{[\text {nonres }]}^{-}$ & $0.0 \pm 10.0^{*}$ & 0.00 & 0.05 & $0.0 \pm 0.0$ & $0.0 \pm 0.1$ & 35 & - \\
\hline$B^{+} \rightarrow \rho^{+} \pi^{+} \pi_{[\text {nonres] }}^{-}$ & $0.0 \pm 10.0^{*}$ & 0.09 & 0.00 & $0.0 \pm 0.7$ & $0.0 \pm 0.0$ & 36 & - \\
\hline$B^{0} \rightarrow K^{+} \pi^{-} K_{S}^{0}$ [nonres] & $0.0 \pm 10.0^{*}$ & 0.03 & 0.05 & $0.0 \pm 0.3$ & $0.0 \pm 0.1$ & 37 & - \\
\hline$B^{0} \rightarrow \pi^{+} \pi^{-} K_{S}^{0}$ [nonres] & $0.0 \pm 10.0^{*}$ & 0.14 & 0.00 & $0.0 \pm 1.3$ & $0.0 \pm 0.0$ & 38 & - \\
\hline$B^{+} \rightarrow K^{+} K_{S}^{0} \pi_{[\text {nonres] }}^{0}$ & $0.0 \pm 10.0^{*}$ & 0.00 & 0.05 & $0.0 \pm 0.0$ & $0.0 \pm 0.1$ & 39 & - \\
\hline$B^{+} \rightarrow \pi^{+} K_{S}^{0} \pi_{[\text {nonres] }}^{0}$ & $0.0 \pm 10.0^{*}$ & 0.08 & 0.00 & $0.0 \pm 0.7$ & $0.0 \pm 0.0$ & 40 & - \\
\hline$B^{+} \rightarrow K^{+} K^{\star 0}\left(\rightarrow K_{S}^{0} \pi^{0}\right)$ & $0.0 \pm 3.0^{*}$ & 0.00 & 0.17 & $0.0 \pm 0.0$ & $0.0 \pm 0.4$ & 49 & - \\
\hline$B^{+} \rightarrow K^{+} K^{\star 0}\left(\rightarrow K^{+} \pi^{-}\right)$ & $0.0 \pm 3.0^{*}$ & 0.00 & 0.97 & $0.0 \pm 0.0$ & $0.0 \pm 2.5$ & 50 & - \\
\hline$B^{+} \rightarrow \pi^{0} \rho^{+}$ & $15.0_{-10}^{+15 *}$ & 1.29 & 0.00 & $17.0 \pm 11.3$ & $0.0 \pm 0.0$ & 51 & 1 \\
\hline$B^{+} \rightarrow \pi^{+} \rho^{0}$ & $9.5 \pm 2.0$ & 4.30 & 0.00 & $35.8 \pm 7.5$ & $0.0 \pm 0.0$ & 58 & 1 \\
\hline$B^{0} \rightarrow \pi^{0} \rho^{0}$ & $1.0 \pm 3.0^{*}$ & 2.81 & 0.00 & $2.5 \pm 7.4$ & $0.0 \pm 0.0$ & 76 & 6 \\
\hline$B^{0} \rightarrow \pi^{0} f_{X}(980,1370)$ & $0.0 \pm 3.0^{*}$ & $\sim 1.5$ & 0.00 & $0.0 \pm 4.0$ & $0.0 \pm 0.0$ & 77 & 13 \\
\hline$B^{0} \rightarrow \pi^{0} f(\sigma)_{0}(\sim 400)$ & $0.0 \pm 3.0^{*}$ & $\sim 2.9$ & 0.00 & $0.0 \pm 8.0$ & $0.0 \pm 0.0$ & $77^{\prime}$ & 13 \\
\hline$B^{0} \rightarrow \pi^{+} \pi^{-} \pi_{[\text {nonres }]}^{0}$ & $0.0 \pm 5.0^{*}$ & 2.89 & 0.00 & $0.0 \pm 12$ & $0.0 \pm 0.0$ & 104 & 13 \\
\hline$B^{+} \rightarrow K_{S}^{0}\left(\rightarrow \pi^{+} \pi^{-}\right) K^{+}$ & $0.0 \pm 0.0$ & 0.02 & 0.68 & $0.0 \pm 0.0$ & $0.0 \pm 0.0$ & 54 & - \\
\hline$B^{+} \rightarrow K_{S}^{0}\left(\rightarrow \pi^{+} \pi^{-}\right) \pi^{+}$ & $9.0 \pm 0.9$ & 1.00 & 0.00 & $7.9 \pm 0.8$ & $0.0 \pm 0.0$ & 55 & 1 \\
\hline$B^{0} \rightarrow\left(a_{1} \pi\right)^{0}$ & $35.0 \pm 25.0^{*}$ & 0.29 & 0.00 & $8.9 \pm 6.3$ & $0.0 \pm 0.0$ & 56 & 5 \\
\hline$B^{+} \rightarrow\left(a_{1} \pi\right)^{+}$ & $35.0 \pm 25.0^{*}$ & - & - & $10 \pm 10$ & $0.0 \pm 0.0$ & 56 & 0 \\
\hline$B^{0} \rightarrow K^{\star 0} \gamma$ & $40.2 \pm 2.7$ & 0.00 & 0.02 & $0.0 \pm 0.0$ & $0.7 \pm 0.1$ & 59 & - \\
\hline$B^{+} \rightarrow K^{\star+} \gamma$ & $40.8 \pm 3.8$ & - & - & $0.0 \pm 0.0$ & $1 \pm 1$ & 59 & - \\
\hline$B^{0} \rightarrow \eta(\rightarrow 2 \gamma) \rho^{0}$ & $1.0 \pm 1.5^{*}$ & 0.07 & 0.00 & $0.1 \pm 0.1$ & $0.0 \pm 0.0$ & 60 & - \\
\hline$B^{+} \rightarrow \eta(\rightarrow 2 \gamma) \rho^{+}$ & $2.0 \pm 2.0^{*}$ & 0.03 & 0.00 & $0.1 \pm 0.1$ & $0.0 \pm 0.0$ & 61 & - \\
\hline$B^{+} \rightarrow \pi^{+} \pi^{+} \pi_{[\text {nonres] }}^{-}$ & $0.0 \pm 3.0^{*}$ & 0.91 & 0.00 & $0.0 \pm 2.4$ & $0.0 \pm 0.0$ & 62 & - \\
\hline$B^{0} \rightarrow K^{+} K^{-} \pi_{[\text {nonres }]}^{0}$ & $0.0 \pm 3.0^{*}$ & 0.00 & 0.21 & $0.0 \pm 0.0$ & $0.0 \pm 0.6$ & 63 & - \\
\hline$B^{+} \rightarrow K^{+} K^{-} \pi_{\text {[nonres] }}^{+}$ & $0.0 \pm 3.0^{*}$ & 0.00 & 0.09 & $0.0 \pm 0.0$ & $0.0 \pm 0.2$ & 65 & - \\
\hline$B^{+} \rightarrow \pi^{0} K^{+} \pi_{[\text {nonres] }}^{0}$ & $0.0 \pm 3.0^{*}$ & 0.00 & 0.39 & $0.0 \pm 0.0$ & $0.0 \pm 1.0$ & 67 & - \\
\hline$B^{+} \rightarrow K^{+} K^{+} K^{-}$ & $34.7 \pm 2.7$ & 0.00 & 0.01 & $0.0 \pm 0.0$ & $0.3 \pm 0.0$ & 68 & - \\
\hline$B^{0} \rightarrow K^{+} \pi^{-}$ & $18.5 \pm 1.0$ & 0.08 & 0.10 & $1.4 \pm 0.1$ & $1.6 \pm 0.1$ & 69 & 6 \\
\hline$B^{0} \rightarrow \pi^{+} \pi^{-}$ & $4.8 \pm 0.5$ & 0.10 & 0.00 & $0.4 \pm 0.1$ & $0.0 \pm 0.0$ & 70 & - \\
\hline$B^{+} \rightarrow K^{+} \pi^{0}$ & $12.7 \pm 1.2$ & 0.00 & 1.63 & $0.0 \pm 0.0$ & $18.2 \pm 1.7$ & 71 & 2 \\
\hline$B^{+} \rightarrow \pi^{+} \pi^{0}$ & $5.8 \pm 1.0$ & 0.98 & 0.00 & $5.0 \pm 0.9$ & $0.0 \pm 0.0$ & 72 & 2 \\
\hline
\end{tabular}

Tableau 4.10: Deuxième partie de la liste des modes de bruit de fond $B$ considérés dans l'analyse (voir la légende du tableau 4.9). 


\begin{tabular}{|c|c|c|c|c|c|c|c|c|}
\hline \multirow{2}{*}{\multicolumn{2}{|c|}{ Mode }} & \multirow{2}{*}{$\begin{array}{c}\mathcal{B} \\
\left(\times 10^{-6}\right)\end{array}$} & \multicolumn{2}{|c|}{ Eff. (\%) } & \multicolumn{2}{|c|}{ Nombre attendu } & \multirow{2}{*}{ Id } & \multirow{2}{*}{$\mathrm{Cl}$. } \\
\hline & & & $\rho \pi$ & $\rho K$ & $\rho \pi$ & $\rho K$ & & \\
\hline \multirow{6}{*}{$B^{+} \rightarrow$} & $K^{+} \rho^{0}$ & $3.9 \pm 3.0$ & 0.26 & 2.99 & $0.9 \pm 0.7$ & $10.2 \pm 7.8$ & 53 & 1 \\
\hline & $\pi^{+} K^{\star 0}\left(\rightarrow K^{+} \pi^{-}\right)$ & $10.3 \pm 2.6$ & 0.04 & 0.00 & $0.4 \pm 0.1$ & $0.0 \pm 0.0$ & 47 & - \\
\hline & $K^{+} \pi^{+} \pi_{[\text {nonres }]}^{-}$ & $0.0 \pm 3.0$ & 0.10 & 0.19 & $0.0 \pm 0.3$ & $0.0 \pm 0.5$ & 66 & - \\
\hline & $\left(K_{Y}^{(* *)} \pi\right)^{+}$ & $15.0 \pm 8.0$ & 0.36 & 0.05 & $4.7 \pm 2.5$ & $0.7 \pm 0.4$ & - & 10 \\
\hline & $K^{+} f_{X}(1300)$ & $13.0 \pm 9.0$ & 0.31 & 1.20 & $3.5 \pm 2.4$ & $13.7 \pm 9.5$ & - & 1 \\
\hline & $K^{+} f_{0}(980)$ & $\frac{11.7 \pm 4.0}{53.9 \pm 15.0}$ & 0.16 & 1.40 & $1.6 \pm 0.6$ & $14.2 \pm 4.8$ & 57 & 1 \\
\hline \multicolumn{2}{|c|}{$B^{+} \rightarrow\left(K_{X}^{(* *)} \pi\right)^{+} \rightarrow$ autre que $K^{+} \pi^{-} \pi^{+}$} & $25 \pm 25^{*}$ & 0.21 & 0.03 & $4.7 \pm 4.7$ & $0.7 \pm 0.7$ & - & 10 \\
\hline \multicolumn{2}{|c|}{$B^{+} \rightarrow\left(K_{X}^{(* *)} \rho\right)^{+} \rightarrow K^{+} \pi^{-} \pi^{+} X$} & $15 \pm 15^{*}$ & 0.06 & 0.06 & $0.8 \pm 0.8$ & $0.8 \pm 0.8$ & 85 & 12 \\
\hline \multicolumn{2}{|c|}{$B^{+} \rightarrow K_{S}^{0} \rho^{+}$} & $4.0 \pm 3.0^{*}$ & 0.10 & 0.00 & $0.4 \pm 0.3$ & $0.0 \pm 0.0$ & 19 & - \\
\hline \multicolumn{2}{|c|}{$B^{+} \rightarrow K^{\star+}\left(\rightarrow K^{+} \pi^{0}\right) f_{0}(980)$} & $5.0 \pm 3.0^{*}$ & 0.04 & 0.07 & $0.2 \pm 0.1$ & $0.3 \pm 0.2$ & 93 & - \\
\hline \multicolumn{2}{|c|}{$B^{+} \rightarrow \pi^{+} K^{\star 0}\left(\rightarrow K_{S}^{0} \pi^{0}\right)$} & $4.4 \pm 2.5^{*}$ & 0.29 & 0.00 & $1.1 \pm 0.6$ & $0.0 \pm 0.0$ & 46 & 0 \\
\hline \multicolumn{2}{|c|}{$B^{+} \rightarrow \pi^{0} K^{\star+}\left(\rightarrow K^{+} \pi^{0}\right)$} & $4.4 \pm 2.5^{*}$ & 0.00 & 1.55 & $0.0 \pm 0.0$ & $6.0 \pm 3.4$ & 95 & 1 \\
\hline \multirow{4}{*}{$B^{0} \rightarrow$} & $\pi^{-} K^{\star+}\left(\rightarrow K^{+} \pi^{0}\right)$ & $8.7 \pm 5.0$ & 2.50 & 1.58 & $19.1 \pm 11.0$ & $12.1 \pm 6.9$ & 45 & 6 \\
\hline & $\pi^{0} K^{\star 0}\left(\rightarrow K^{+} \pi^{-}\right)$ & $8.7 \pm 5.0$ & 0.01 & 1.51 & $0.1 \pm 0.1$ & $11.5 \pm 6.6$ & 48 & 5 \\
\hline & $K^{+} \pi^{-} \pi_{[\text {nonres }]}^{0}$ & $0.0 \pm 3.0$ & 0.21 & 1.31 & $0.0 \pm 0.6$ & $0.0 \pm 3.4$ & 64 & - \\
\hline & $\left(K_{X}^{(* *)} \pi\right)^{0}$ & $\frac{20.0 \pm 15.0}{37.4 \pm 12.0}$ & 0.00 & 1.08 & $0.0 \pm 0.0$ & $19.0 \pm 14.3$ & - & 9 \\
\hline \multicolumn{2}{|c|}{$B^{0} \rightarrow\left(K_{X}^{(* *)} \pi\right)^{0} \rightarrow$ autre que $K^{+} \pi^{-} \pi^{0}$} & $52 \pm 52^{*}$ & 0.43 & 0.39 & $19.8 \pm 19.8$ & $17.6 \pm 17.6$ & - & 9 \\
\hline \multicolumn{2}{|c|}{$B^{0} \rightarrow\left(K_{X}^{(* *)} \rho\right)^{0} \rightarrow K^{+} \pi^{-} \pi^{0} X$} & $20.0 \pm 20.0^{*}$ & 0.02 & 0.17 & $0.4 \pm 0.4$ & $2.9 \pm 2.9$ & 90 & 11 \\
\hline \multicolumn{2}{|c|}{$B^{0} \rightarrow K^{\star 0}\left(\rightarrow K^{+} \pi^{-}\right) f_{0}(980)$} & $10.0 \pm 7.0^{*}$ & 0.02 & 0.06 & $0.2 \pm 0.1$ & $0.6 \pm 0.4$ & 94 & - \\
\hline \multicolumn{2}{|c|}{$B^{0} \rightarrow \pi^{-} K^{\star+}\left(\rightarrow K_{S}^{0} \pi^{+}\right)$} & $8.7 \pm 5.0$ & 0.35 & 0.00 & $2.6 \pm 1.5$ & $0.0 \pm 0.0$ & 44 & 3 \\
\hline
\end{tabular}

Tableau 4.11: Troisième partie de la liste des modes de bruit de fond $B$ considérés dans l'analyse (voir la légende du tableau 4.9). 


\subsubsection{Déterminer la contamination de $b \rightarrow c$ à partir des données}

Contrairement aux modes $B$ non charmés, les distributions de $\Delta E, m_{\mathrm{ES}}$ et $x_{\mathrm{NN}}$ des modes $B$ charmés sont suffisamment différentes de celles du signal pour ajuster les contributions charmées dans les données. On peut ainsi obtenir une validation du nombre d'événements prédits par le Monte Carlo générique. Néanmoins, l'efficacité de sélection des événements $b \rightarrow c$ étant très faible, et les événements charmés peuplant majoritairement la zone $\Delta E<0$, nous ouvrons la coupure nominale inférieure sur $\Delta E$ de -0.12 à -0.35 afin d'augmenter notre sensibilité relative du bruit de fond charmé par rapport au signal.

La figure 4.27 montre les distributions normalisées de $\Delta E$ sur l'intervalle élargi pour le signal Monte Carlo $B^{0} \rightarrow \rho^{ \pm} \pi^{\mp}$ et $B^{0} \rightarrow \rho^{-} K^{+}$, le bruit de fond continuum (off-peak), les principaux bruits de fond $B$ non charmés, et les bruits de fond charmés (Monte Carlo). D'après le Monte Carlo générique, avec cette nouvelle coupure, nous attendons 960 (261) événements de $B$ chargés et 511 (64) neutres dans la catégorie de signal $\rho \pi(\rho K)$.

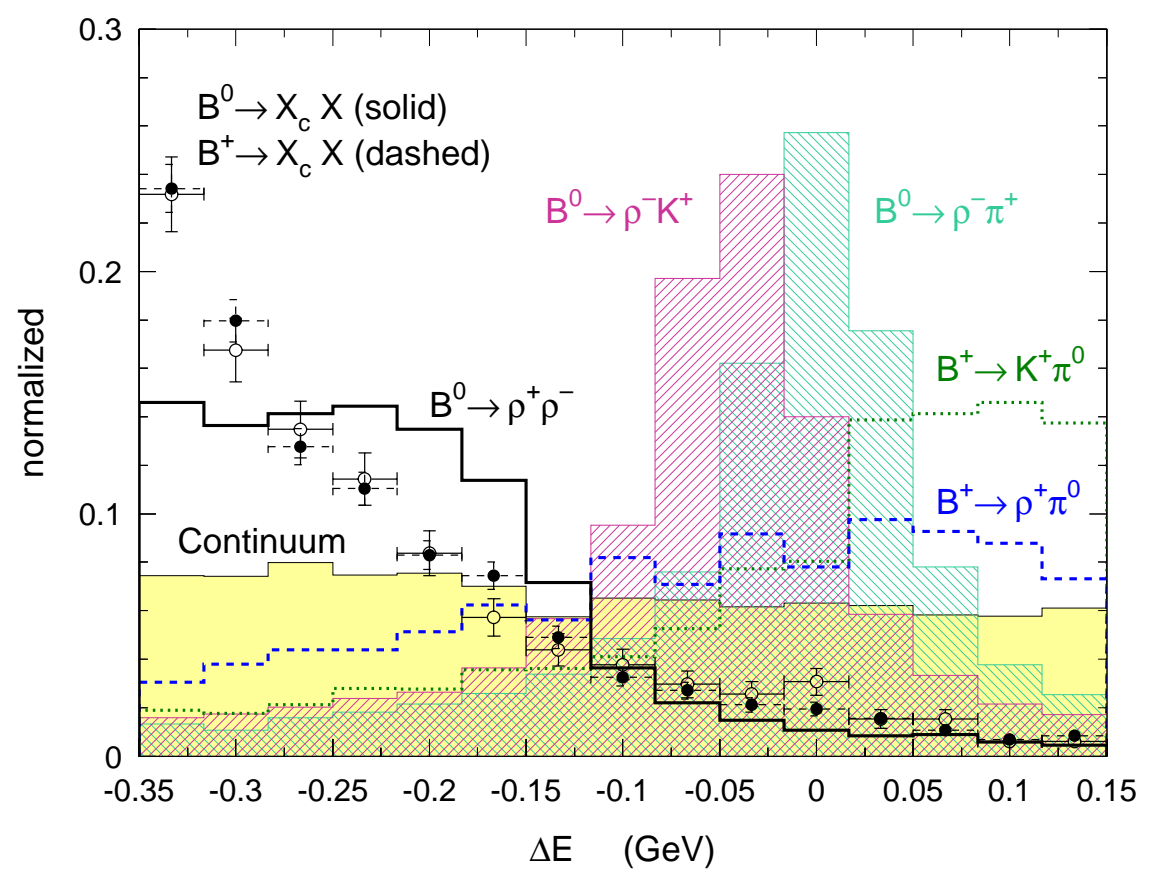

Figure 4.27: Distribution de la variable $\Delta E$ sur un intervalle élargi par rapport aux coupures nominales: le signal $B^{0} \rightarrow \rho^{ \pm} \pi^{\mp}$ correspond à l'histogramme avec des hachures descendant vers la droite, le signal $B^{0} \rightarrow \rho K$ à celui avec des hachures descendant vers la gauche. Les principaux modes de bruit de fond $B$ non charmés sont montrées par divers histogrammes: $B^{0} \rightarrow \rho^{+} \rho^{-}$avec une ligne pleine, $B^{+} \rightarrow K^{+} \pi^{0}$ avec une ligne pointillée, et $B^{+} \rightarrow \rho^{+} \pi^{0}$ avec une ligne en tirets. Le Monte Carlo générique pour les $B$ charmés neutres (cercles blancs avec barres d'erreur) et chargés (cercles noirs avec barres d'erreur) est superposé. Enfin, la contribution des données continuum off-peak est représenté par l'histogramme plein jaune.

Nous ajustons les contributions charmées dans l'échantillon ainsi sélectionné. Le résultat de l'ajustement est illustré sur la figure 4.28: on y voit les distributions de $\Delta E$ des données appartenant aux catégories $\rho \pi$ et $\rho K$ respectivement, sur lesquelles sont superposées les distributions de probabilités des différentes composantes: le signal, le continuum, les bruits de fond $B$ non charmés en 2-, 3- et 4-corps, et le bruit de fond $B$ charmé laissé libre lors de l'ajustement. 

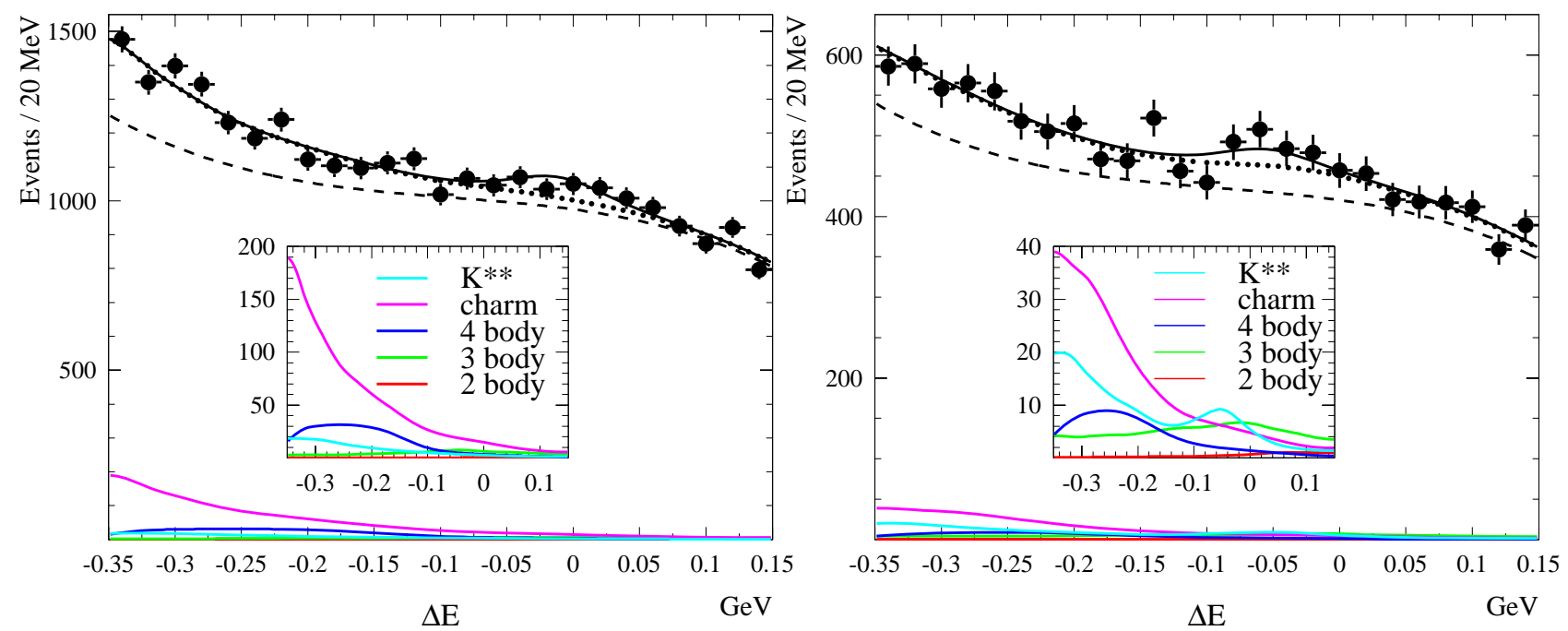

Figure 4.28: Distribution de $\Delta E$ des événements de la catégorie $B^{0} \rightarrow \rho^{ \pm} \pi^{\mp}$ (gauche), et $B^{0} \rightarrow \rho^{-} K^{+}$(droite). Les contributions individuelles des bruits de fond $B$ sont indiquées par les lignes de couleur. La contribution provenant de $b \rightarrow c$ est laissée libre dans l'ajustement. Les lignes en tirets, pointillés et pleines représentent les contributions continuum, continuum + bruit de fond $B$, et continuum + bruit de fond $B+$ signal, respectivement.

\begin{tabular}{|l|c|c|}
\hline Mode & Monte Carlo générique & Ajustement sur les données on-peak \\
\hline$B \rightarrow \operatorname{charme}\left(\rho^{ \pm} K^{\mp}\right)$ & 325 & $300 \pm 74$ \\
$B \rightarrow \operatorname{charme}\left(\rho^{ \pm} \pi^{\mp}\right)$ & 1471 & $1243 \pm 124$ \\
\hline
\end{tabular}

Tableau 4.12: Résultat de l'ajustement des contributions charmées. Les contributions des $B$ neutres et chargés sont sommées.

Les nombres d'événements obtenus lors de cet ajustement sont donnés dans le tableau 4.12 , et sont comparés aux nombres prédits par le Monte Carlo générique. La différence entre les deux résultats est prise comme erreur systématique sur le nombre d'événements attendus:

$$
\begin{array}{llll}
\Delta_{B^{0} \rightarrow \rho^{ \pm} \pi^{\mp}}(\text { ajust. }-M C) & =-25 \pm 74 & \text { soit } & -(8 \pm 23) \% \\
\Delta_{B^{0} \rightarrow \rho^{-} K^{+}}(\text {ajust. }-M C) & =-228 \pm 124 & \text { soit } & -(20 \pm 8) \%
\end{array}
$$

Après propagation de ces erreurs systématiques jusqu'au nombre d'événements de signal, on obtient une variation de 2.7 événements (sur 430 au total) pour $B^{0} \rightarrow \rho^{ \pm} \pi^{\mp}$ et 1 événement (sur 120 au total) pour $B^{0} \rightarrow \rho^{-} K^{+}$, soit une variation de $\simeq 0.5 \%$ et $\simeq 0.9 \%$ respectivement.

\subsubsection{Classification des modes $B$ charmés et non charmés}

Nous introduisons un terme correctif dans la fonction de vraisemblance finale pour les modes oì l'on attend au moins un événement dans l'échantillon sélectionné. Au total, 33 modes (dont 31 modes exclusifs non charmés, et 2 contributions charmées inclusives) passent ce critère, dont plus de la moitié possède des rapports d'embranchement pas encore mesurés.

Chacun de ces modes possède deux contributions: une au signal $B^{0} \rightarrow \rho^{ \pm} \pi^{\mp}$ et une au signal $B^{0} \rightarrow \rho^{-} K^{+}$. Pour simplifier la procédure, nous regroupons ces 33 modes en classes. Celles-ci sont formées de telle sorte que les modes qui y appartiennent:

- proviennent d'un $B$ de même charge (neutre ou chargé). 
- possèdent des distributions de $m_{\mathrm{ES}}, \Delta E$ et $x_{\mathrm{NN}}$ similaires.

Nous regroupons aussi ensembles les modes qui ne contribuent qu'à $B^{0} \rightarrow \rho^{ \pm} \pi^{\mp}$ ou qu'à $B^{0} \rightarrow \rho^{-} K^{+}$. La classification est donnée dans le tableau 4.13.

\begin{tabular}{|c|c|c|c|c|c|c|c|}
\hline $\mathrm{Cl}$. & Mode & $N_{\mathrm{exp}}^{\pi}$ & $N_{\mathrm{exp}}^{K}$ & & & & \\
\hline \multicolumn{4}{|c|}{$B$ chargés } & \multirow[b]{2}{*}{$\mathrm{Cl}$. } & \multirow[b]{2}{*}{ Mode } & \multirow[b]{2}{*}{$N_{\mathrm{exp}}^{\pi}$} & \multirow[b]{2}{*}{$N_{\mathrm{exp}}^{K}$} \\
\hline 0 & $\pi^{+} K^{* 0}\left(K_{S}^{0} \pi^{0}\right)$ & $1.1 \pm 0.6$ & $0.0 \pm 0.0$ & & & & \\
\hline \multirow{2}{*}{$\begin{array}{l}0 \\
0\end{array}$} & $\rho^{+} K^{* 0}\left(K^{+} \pi^{-}\right)_{1}$ & $0.0 \pm 0.0$ & $2.9 \pm 2.9$ & \\
\hline & $\rho^{+} \rho_{1}^{0}$ & $20.1 \pm 15.6$ & $0.0 \pm 0.0$ & 3 & $B$ neutres & $0.2 \pm 0.2$ & \multirow{2}{*}{$\begin{array}{l}1.0 \pm 1.0 \\
2.7+2.7\end{array}$} \\
\hline \multirow{2}{*}{$\begin{array}{l}0 \\
0\end{array}$} & $\left(a_{1} \pi\right)^{+}$ & $10.0 \pm 10.0$ & $0.0 \pm 0.0$ & \multirow{2}{*}{3} & $\begin{array}{l}\rho^{0} K^{* 0}\left(K^{+} \pi^{-}\right)_{1} \\
\rho^{-} K^{*+}\left(K^{+} \pi^{0}\right)_{1}\end{array}$ & $0.5 \pm 0.5$ & \\
\hline & $\eta^{\prime}\left(\rho^{0} \gamma\right) \pi^{+}$ & $1.7 \pm 1.7$ & $0.0 \pm 0.0$ & & $\pi^{-} K^{*+}\left(K_{S}^{0} \pi^{+}\right)$ & $2.6 \pm 1.5$ & $0.0 \pm 0.0$ \\
\hline 0 & $\eta^{\prime}\left(\rho^{0} \gamma\right) K^{+}$ & $0.1 \pm 0.1$ & $7.6 \pm 0.6$ & \multirow{2}{*}{$\begin{array}{l}4 \\
4\end{array}$} & $\rho^{+} \rho_{[\operatorname{lon} \mathrm{l}}^{-}$ & $44.4 \pm 33.3$ & \multirow{2}{*}{$\begin{array}{l}0.0 \pm 0.0 \\
0.0 \pm 0.0\end{array}$} \\
\hline \multirow{2}{*}{$\begin{array}{l}1 \\
1\end{array}$} & $\pi^{0} \rho^{+}$ & $17.0 \pm 11.3$ & $0.0 \pm 0.0$ & & $\rho^{0} \rho_{[\text {long }]}^{0}$ & $2.6 \pm 2.6$ & \\
\hline & $\begin{array}{l}\pi^{+} \rho^{\sigma} \\
K_{C}^{0}\left(\pi^{+} \pi^{-}\right) \pi^{+}\end{array}$ & $\begin{array}{c}35.8 \pm 7.5 \\
7.9 \pm 0.8\end{array}$ & $\begin{array}{l}0.0 \pm 0.0 \\
0.0 \pm 0.0\end{array}$ & & \multirow{2}{*}{$\begin{array}{l}\left(a_{1} \pi\right)^{0} \\
\pi^{0} K^{* 0}\left(K^{+} \pi^{-}\right)\end{array}$} & $8.9 \pm 6.3$ & $0.0 \pm 0.0$ \\
\hline 1 & $K^{+} \rho^{0}$ & $0.9 \pm 0.7$ & $10.2 \pm 7.8$ & $\begin{array}{l}5 \\
5\end{array}$ & & $0.1 \pm 0.1$ & $11.5 \pm 6.6$ \\
\hline 1 & $K^{+} f_{X}(1300)$ & $3.5 \pm 2.4$ & $13.7 \pm 9.5$ & 6 & $\pi^{0} \rho^{0}$ & $2.5 \pm 7.4$ & \multirow{2}{*}{$\begin{array}{l}0.0 \pm 0.0 \\
1.6 \pm 0.1\end{array}$} \\
\hline 1 & $K^{+} f_{0}(980)\left(\pi^{+} \pi^{-}\right)$ & $1.6 \pm 0.6$ & $14.2 \pm 4.8$ & \multirow{2}{*}{$\begin{array}{l}6 \\
6\end{array}$} & \multirow{2}{*}{$\begin{array}{l}K^{+} \pi^{-} \\
\pi^{-} K^{*+}\left(K^{+} \pi^{0}\right)\end{array}$} & $1.4 \pm 0.1$ & \\
\hline 1 & $\pi^{0} K^{*+}\left(K^{+} \pi^{0}\right)$ & $0.0 \pm 0.0$ & $6.0 \pm 3.4$ & & & $19.1 \pm 11.0$ & $\begin{array}{c}1.6 \pm 0.1 \\
12.1 \pm 6.9\end{array}$ \\
\hline 2 & $K^{+} \pi^{0}$ & $0.0 \pm 0.0$ & $18.2 \pm 1.7$ & \multirow[t]{2}{*}{9} & \multirow{2}{*}{$\begin{array}{l}\left(K_{X}^{(*)} \pi\right)^{0} \\
\quad \rightarrow K^{+} \pi^{-} \pi^{0}\end{array}$} & $0.0 \pm 0.0$ & $19.0 \pm 14.3$ \\
\hline 2 & $\pi^{+} \pi^{0}$ & $5.0 \pm 0.9$ & $0.0 \pm 0.0$ & & & & \\
\hline 10 & $\begin{array}{l}\left(K_{X}^{(* *)} \pi\right)^{+} \\
\quad \rightarrow K^{+} \pi^{-} \pi^{+}\end{array}$ & $4.7 \pm 2.5$ & $0.7 \pm 0.4$ & 9 & $\begin{array}{l}\left(K_{X}^{(* *)} \pi\right)^{0} \\
\quad \rightarrow \text { autre }\end{array}$ & $19.8 \pm 19.8$ & $17.6 \pm 17.6$ \\
\hline 10 & $\left(K_{X}^{(* *)} \pi\right)^{+}$ & $4.7 \pm 4.7$ & $0.7 \pm 0.7$ & 11 & $\left(K_{X}^{(* *)} \rho\right)^{0}$ & $0.4 \pm 0.4$ & $2.9 \pm 2.9$ \\
\hline & $\stackrel{A}{\rightarrow}$ autre & & & 8 & charme & $102 \pm 23$ & $13 \pm 4$ \\
\hline 12 & $\left(K_{X}^{(* *)} \rho\right)^{+}$ & $0.8 \pm 0.8$ & $0.8 \pm 0.8$ & & & & \\
\hline 7 & charme & $164 \pm 36$ & $41 \pm 10$ & & & & \\
\hline
\end{tabular}

Tableau 4.13: Classification des modes de bruit de fond provenant d'un B chargé (tableau de gauche) et neutre (tableau de droite) retenus dans l'analyse. On donne le nombre d'événements attendus quand la trace célibataire est un pion ou un kaon. L'indice "1" correspond à une contribution longitudinale pour le mode considéré. Les erreurs sur les nombres d'événements reflètent la statistique limitée du Monte Carlo à partir desquels ils sont estimés, ainsi que les erreurs sur les rapports d'embranchement. Dans le cas de la contribution charmée, l'erreur systématique sur le nombre d'événements attendu est prise en compte (voir la section 4.8.2).

\subsection{Distributions de référence}

Nous décrivons dans cette section les distributions de référence des variable utilisées lors de l'ajustement final: $\Delta E, m_{\mathrm{ES}}, x_{\mathrm{NN}}, \theta_{C}$ et $\Delta t$, pour les contributions du signal (correctement reconstruit, mal reconstruit de bonne charge, mal reconstruit de mavvaise charge), du continuum, et du bruit de fond $B$.

Ces distributions sont le plus souvent paramétrées par une fonction analytique, ce qui présente l'avantage qu'un (ou plusieurs) paramètre de la fonction peut être laissé libre lors de l'ajustement final, réduisant ainsi la dépendance de nos résultats en les prédictions du Monte Carlo. C'est un point particulièrement important pour le bruit de fond continuum qui, puisque dominant dans l'échantillon d'événements, doit être décrit très précisément. Lorsque 
la distribution est telle qu'elle est mal décrite par une fonction analytique (ou lorsqu'elle correspond à une contribution de faible importance), nous modélisons la distributions de façon non-paramétrique, en utilisant le programme KEYS [82] ("Kernel Estimating Your Shapes"). Il s'agit d'une modélisation plaçant un noyau ("kernel", en anglais, d'où le nom) de probabilité Gaussien à chaque événement (de largeur prédéfinie). La somme sur tous ces noyaux donne une modélisation "lisse" de la distribution.

\subsubsection{Signal et bruit de fond continuum}

\subsubsection{1 $m_{\mathrm{ES}}$}

Le signal correctement reconstruit est paramétré par une fonction de Crystal Ball [83] asymétrique, c'est à dire pour laquelle la largeur $\sigma_{L}$ à gauche de la valeur moyenne est différente de la largeur $\sigma_{R}$ à droite de la valeur moyenne:

$$
f_{\mathrm{CB}}\left(m_{\mathrm{ES}}, m_{\mathrm{ES}}^{0}, \sigma_{L}, \sigma_{R}, \alpha, n\right)=\frac{1}{N}\left\{\begin{array}{cc}
\frac{(n \alpha)^{n} \exp \left(\alpha^{2} / 2\right)}{\left(\left(m_{\mathrm{ES}}-m_{\mathrm{ES}}^{0}\right) / \sigma_{L}+n / \alpha-\alpha\right)^{n}} & m_{\mathrm{ES}} \leq m_{\mathrm{ES}}^{0}-\alpha \sigma_{L} \\
\exp \left(-\frac{\left(m_{\mathrm{ES}}-m_{\mathrm{ES}}^{0}\right)^{2}}{2 \sigma_{L}^{2}}\right) & m_{\mathrm{ES}}^{0}-\alpha \sigma_{L}<m_{\mathrm{ES}}<m_{\mathrm{ES}}^{0} \\
\exp \left(-\frac{\left(m_{\mathrm{ES}}-m_{\mathrm{ES}}^{0}\right)^{2}}{2 \sigma_{R}^{2}}\right) & m_{\mathrm{ES}}^{0}<m_{\mathrm{ES}}
\end{array}\right.
$$

où $m_{\mathrm{ES}}^{0}$ est la moyenne de la distribution et $N$ est la normalisation de la distribution. Le signal mal reconstruit (de bonne et mauvaise charge) est modélisé par les KEYS.

Le bruit de fond continuum est paramétré avec une fonction introduite par la collaboration ARGUS [84]:

$$
f_{A}\left(m_{\mathrm{ES}}, m_{\mathrm{ES}}^{0}, \xi\right)=\frac{1}{N} m_{\mathrm{ES}} \sqrt{1-\left(m_{\mathrm{ES}} / m_{\mathrm{ES}}^{0}\right)^{2}} \exp \left(\xi\left(1-\left(m_{\mathrm{ES}} / m_{\mathrm{ES}}^{0}\right)^{2}\right)\right),
$$

où $m_{\mathrm{ES}}^{0}$ est le point de chute de l'extrémité droite de la distribution, et $\xi$ un paramètre libre.

Les distributions de $m_{\mathrm{ES}}$ sont montrées sur les figures 4.29 et 4.30 dans le Monte Carlo signal et les données off-peak ${ }^{14}$.

\subsubsection{2 $\Delta E$}

Les événements de signal $B^{0} \rightarrow \rho^{ \pm} \pi^{\mp}$ correctement reconstruits sont décrits par des doubles Gaussiennes. Les paramétrisations pour le signal $B^{0} \rightarrow \rho^{-} K^{+}$sont obtenus à partir de celles de $B^{0} \rightarrow \rho^{ \pm} \pi^{\mp}$ en appliquant le décalage de l'eq. 4.3 .

Le bruit de fond continuum est paramétré par un polynôme du second ordre, avec des coefficients identiques pour les traces célibataires pions et kaons.

Les distributions de $\Delta E$ sont montrées sur la figure 4.31 .

\subsubsection{3 $x_{\mathrm{NN}}$}

Les distributions de $x_{\mathrm{NN}}$ pour le signal sont différentes selon la catégorie d'étiquetage. Nous modélisons chacune de ces distributions en utilisant les KEYS.

\footnotetext{
${ }^{14}$ Puisque l'énergie dans le centre de masse est décalée de $40 \mathrm{MeV}$ pour les données off-peak par rapport aux données on-peak, il faut décaler la distribution off-peak pour obtenir l'équivalent de on-peak.
} 

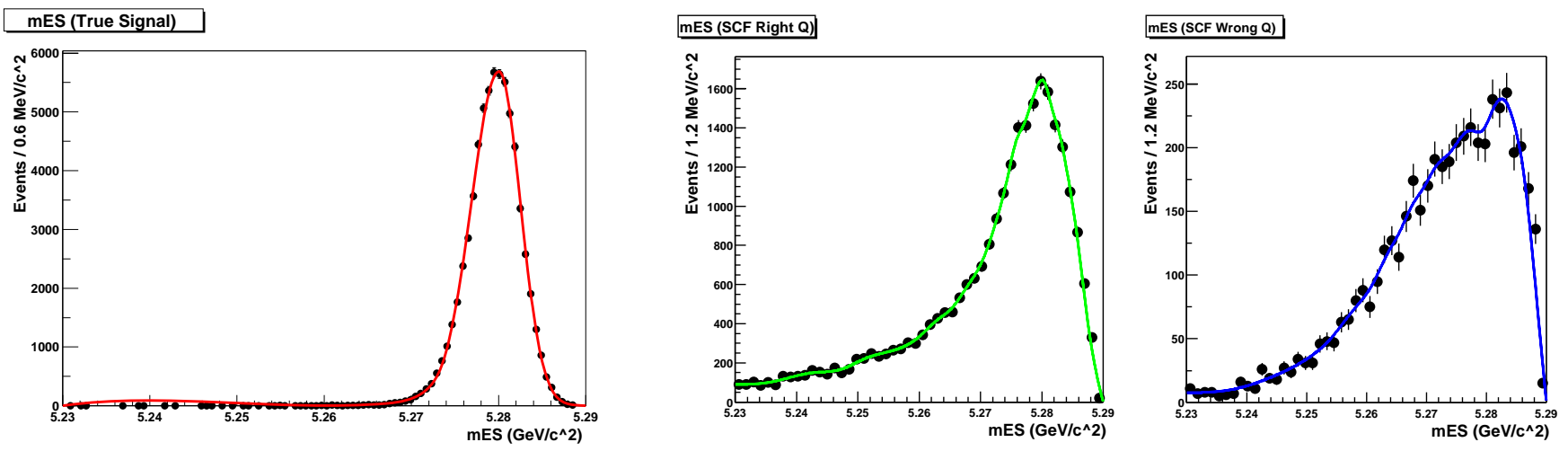

Figure 4.29: Distributions de $m_{\mathrm{ES}}$ du Monte Carlo $B^{0} \rightarrow \rho^{ \pm} \pi^{\mp}$ pour le signal correctement reconstruit (gauche), mal reconstruit de bonne charge (milieu), et de mauvaise charge (droite).

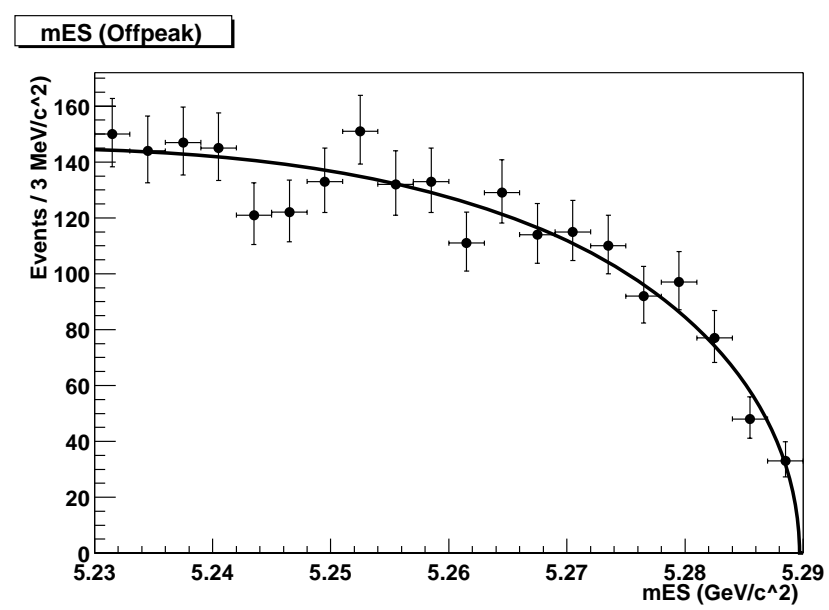

Figure 4.30: Distributions de $m_{\mathrm{ES}}$ pour le bruit de fond continuum off-peak.

Le bruit de fond continuum est paramétré par la fonction:

$$
f\left(x_{\mathrm{NN}} ; a_{1}, a_{2}, a_{3}, a_{4}\right)=\left(1-X_{\mathrm{RN}}\right)^{a_{1}}\left(a_{2} X_{\mathrm{RN}}^{2}+a_{3} X_{\mathrm{RN}}+a_{4}\right),
$$

où $X_{\mathrm{RN}}=\left(x_{\mathrm{NN}}-x_{\mathrm{NN}}^{\min }\right) /\left(x_{\mathrm{NN}}^{\max }-x_{\mathrm{NN}}^{\min }\right)$ pour $x_{\mathrm{NN}}^{\min }<x_{\mathrm{NN}}<x_{\mathrm{NN}}^{\max }$. Les coefficients $a_{i}, i=1,3$ sont laissés libres dans l'ajustement final, et $a_{4}$ est fixé à 1 .

Les distributions de $x_{\mathrm{NN}}$ sont montrées sur la figure 4.23 .

\subsubsection{4 $\theta_{C}$}

La distribution du pull de l'angle Cherenkov est déterminée pour 4 catégories d'événements (type $=\pi, K ;$ charge $= \pm 1$ ):

$$
\text { pull(type, charge })=\frac{\theta_{C}-\theta_{C}^{\text {attendu }}(\text { type }, \text { charge })+\operatorname{constante}(\theta)}{\sigma_{\theta_{C}}(\theta)},
$$

où $\theta_{C}^{\text {attendu }}$ est l'angle attendu pour une particule de type et de charge donnés, et d'impulsion donnée. La constante et la résolution $\sigma_{\theta_{C}}$ dépendent de l'angle polaire $\theta$ de la trace (ce qui permet de tenir compte des effets possibles de mauvais alignements entre le DIRC et la DCH, et de la dépendance en l'angle polaire du nombre de photons Cherenkov détectés). 


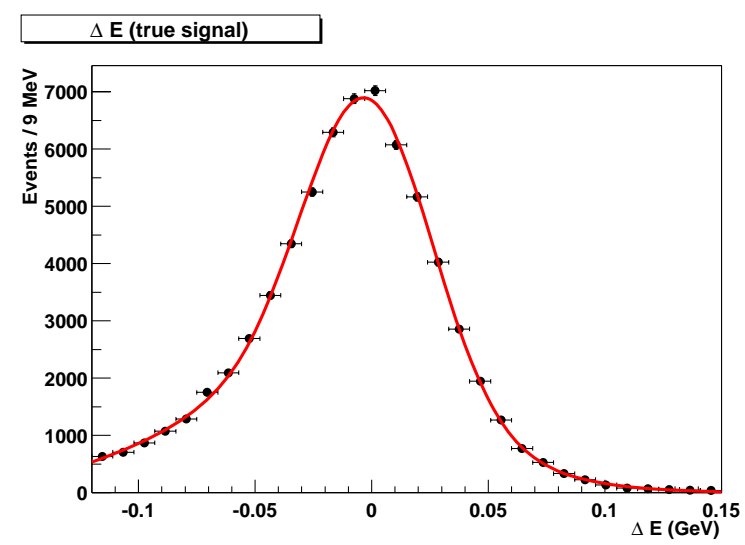

$\Delta \mathrm{E}$ (SCF wrong charge)

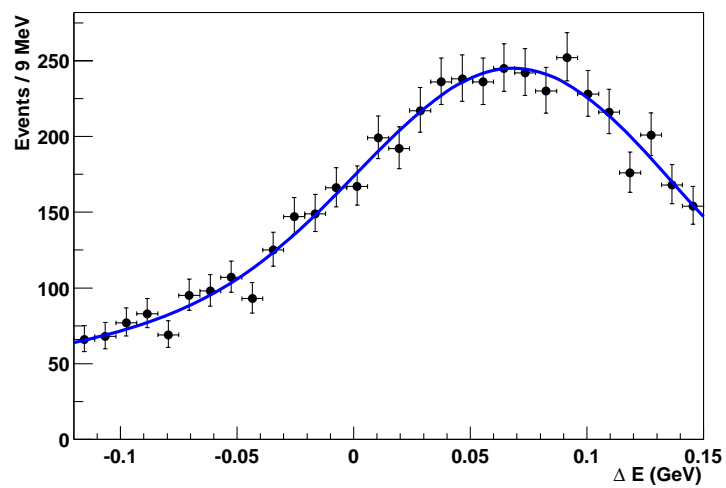

$\Delta \mathrm{E}$ (SCF right charge)

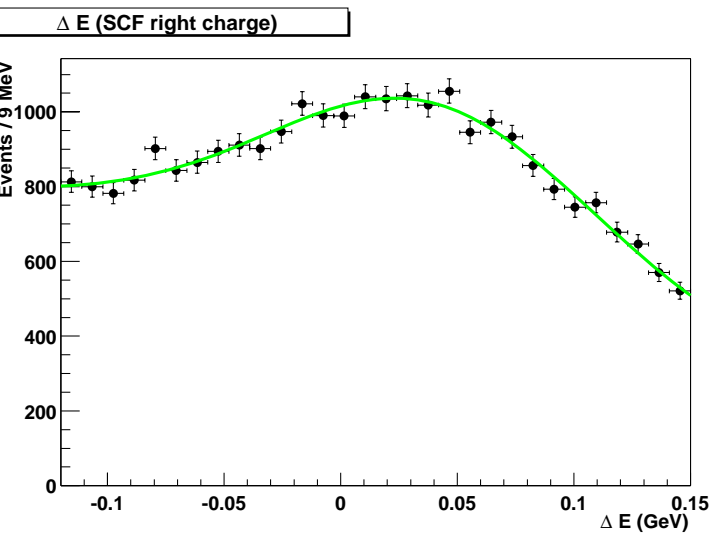

$\Delta \mathrm{E}$ (Offpeak)

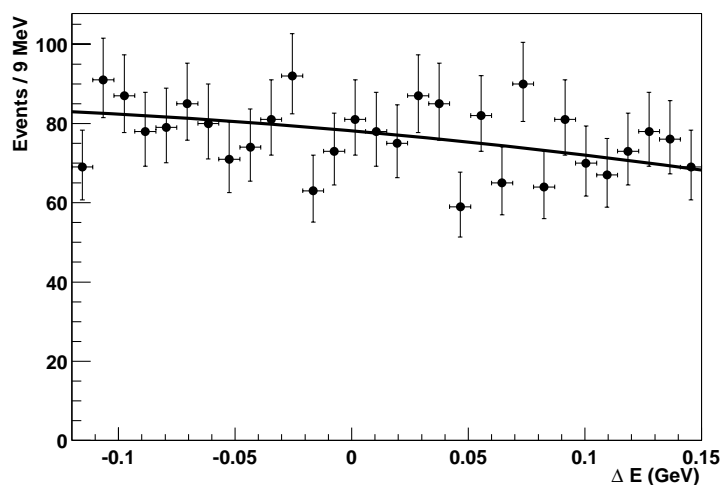

Figure 4.31: Distributions de $\Delta E$ du Monte Carlo $B^{0} \rightarrow \rho^{ \pm} \pi^{\mp}$ pour le signal correctement reconstruit (haut, gauche), mal reconstruit de bonne charge (haut, droite), de mauvaise charge (bas, gauche) et pour le bruit de fond continuum off-peak (bas, droite).

Les distributions de ces pulls sont déterminées sur un échantillon de grande statistique de $D^{*} \rightarrow D^{0}\left(K^{+} \pi^{-}\right) \pi$ [85] découpé en 20 intervalles d'angle polaire $\theta$, et sont paramétrées par une double Gaussienne. Pour chaque catégorie de type et charge, les 20 double Gaussiennes sont corrigées de façon à donner une distribution commune pour cette catégorie (paramétrée par deux Gaussiennes de moyenne nulle), qui est celle utilisée lors de l'ajustement final.

Les mêmes distributions sont utilisée pour le signal et le bruit de fond.

\subsubsection{5 $\Delta t$}

Distribution du signal: les taux de désintégration théoriques du signal en fonction du temps pour des événements étiquetés $B^{0}$ ou $\bar{B}^{0}$ sont donnés dans les eqs. 1.57 et 1.58. Les taux de désintégration mesurés sont obtenus en convoluant ces équations théoriques avec la fonction de résolution du détecteur, et en injectant les effets dus aux erreurs d'étiquetage. On obtient ainsi les expressions suivantes:

$$
\begin{aligned}
f_{B^{0} \text { tag }}^{\rho^{ \pm} x^{\mp}}\left(\Delta t, \sigma_{\Delta t}\right)=\frac{e^{-\left|\Delta t^{\prime}\right| / \tau}}{4 \tau} \cdot\left[1+\frac{\Delta D}{2}\right. & +\langle D\rangle\left(\left(S_{\rho x} \pm \Delta S_{\rho x}\right) \sin \left(\Delta m_{B} \Delta t^{\prime}\right)\right. \\
& \left.\left.-\left(C_{\rho x} \pm \Delta C_{\rho x}\right) \cos \left(\Delta m_{B} \Delta t^{\prime}\right)\right)\right] \otimes \mathcal{R}_{\mathrm{sig}}\left(\Delta t^{\prime}-\Delta t, \sigma_{\Delta t}\right),
\end{aligned}
$$




\begin{tabular}{|l|c|c|}
\hline Paramètre & BReco & $\begin{array}{c}\mathrm{MC} \\
B^{0} \rightarrow \rho \pi\end{array}$ \\
\hline$s_{1}^{\sigma}$ & $1.115 \pm 0.049$ & 1.140 \\
$s_{1}^{b}$ (Lepton) & $0.007 \pm 0.061$ & -0.244 \\
$s_{1}^{b}$ (Kaon) & $-0.217 \pm 0.038$ & -0.037 \\
$s_{1}^{b}$ (NT1) & $-0.232 \pm 0.074$ & -0.091 \\
$s_{1}^{b}$ (NT2) & $-0.171 \pm 0.055$ & -0.150 \\
$s_{1}^{b}$ (No Tag) & $-0.191 \pm 0.041$ & -0.189 \\
$s_{2}^{\sigma}$ & 3.0 (const) & 1.768 \\
$s_{2}^{b}$ & $-1.282 \pm 0.371$ & -1.115 \\
$f_{1}$ & $0.103 \pm 0.022$ & 0.135 \\
$s_{3}^{\sigma}$ & 8.0 (const) & 8.0 \\
$s_{3}^{b}$ & 0.0 (const) & 0.0 \\
$f_{3}$ & $0.0148 \pm 0.0019$ & 0.03 \\
\hline
\end{tabular}

Tablea1 4.14: Paramètres de la fonction de résolution de $\Delta t$ (cf. Eq. 4.25) pour l'échantillon Breco et le Monte Carlo signal $B^{0} \rightarrow \rho^{ \pm} \pi^{\mp}$. La moyenne de la Gaussienne principale est différente pour chaque catégorie d'étiquetage. Les valeurs marquées "const" sont fixées lors de leur ajustement dans l'échantillon Breco.

$$
\begin{aligned}
f_{\bar{B}^{0} x_{\text {tag }}^{\mp}}^{\rho^{ \pm}}\left(\Delta t, \sigma_{\Delta t}\right)=\frac{e^{-\left|\Delta t^{\prime}\right| / \tau}}{4 \tau} \cdot\left[1-\frac{\Delta D}{2}\right. & -\langle D\rangle\left(\left(S_{\rho x} \pm \Delta S_{\rho x}\right) \sin \left(\Delta m_{B} \Delta t^{\prime}\right)\right. \\
& \left.\left.-\left(C_{\rho x} \pm \Delta C_{\rho x}\right) \cos \left(\Delta m_{B} \Delta t^{\prime}\right)\right)\right] \otimes \mathcal{R}_{\mathrm{sig}}\left(\Delta t^{\prime}-\Delta t, \sigma_{\Delta t}\right)
\end{aligned}
$$

où les moyenne et différence des dilutions d'étiquetage, $\langle D\rangle$ et $\Delta D$, sont définis dans la section 4.4 .

La fonction de résolution $\mathcal{R}_{\mathrm{sig}}\left(\Delta t^{\prime}-\Delta t, \sigma_{\Delta t}\right)$ tient compte de la résolution sur la mesure de $\Delta t$, dominée par le $B_{\mathrm{sav}}$ : la fonction de résolution est donc similaire pour tous les modes étudiés à $B A B A R$. Elle est obtenue à partir du résidu de $\Delta t,\left(\Delta t_{\mathrm{rec}}-\Delta t_{\mathrm{vrai}}\right)$, montré sur la figure 4.13 .

Pour tenir compte des corrélations entre l'erreur sur $\Delta t$ et la moyenne ainsi que le RMS ( $c f$. figure 4.14), la fonction de résolution est paramétrée par la somme de trois Gaussiennes $G$ :

$$
\begin{aligned}
\mathcal{R}_{\mathrm{sig}}\left(\Delta t, \sigma_{\Delta t}\right)= & \left(1-f_{2}-f_{3}\right) G\left(\Delta t, s_{1}^{b} \sigma_{\Delta t}, s_{1}^{\sigma} \sigma_{\Delta t}\right) \\
& +f_{2} G\left(\Delta t, s_{2}^{b} \sigma_{\Delta t}, s_{2}^{\sigma} \sigma_{\Delta t}\right)+f_{3} G\left(\Delta t, s_{3}^{b}, \sigma_{2}\right)
\end{aligned}
$$

où $s_{i}^{b}$ et $s_{i}^{\sigma}$ sont des facteurs dans le biais et l'écart type des deux principales Gaussiennes multipliant l'erreur sur $\Delta t\left(\sigma_{\Delta t}\right)$.

Les valeurs des paramètres de cette fonction sont données dans le tableau 4.14 pour l'échantillon Breco et le Monte Carlo $B^{0} \rightarrow \rho \pi$.

Distribution du continuum: pour le continuum, les corrélations entre écart type de la résolution de $\Delta t$ et $\sigma_{\Delta t}$ sont similaires, mais celles entre la moyenne de la résolution de $\Delta t$ et $\sigma_{\Delta t}$ sont diluées par le fait qu'un candidat $B$ est construit à partir de traces appartenant aux deux "jets" ( $c f$. figure 4.32). 

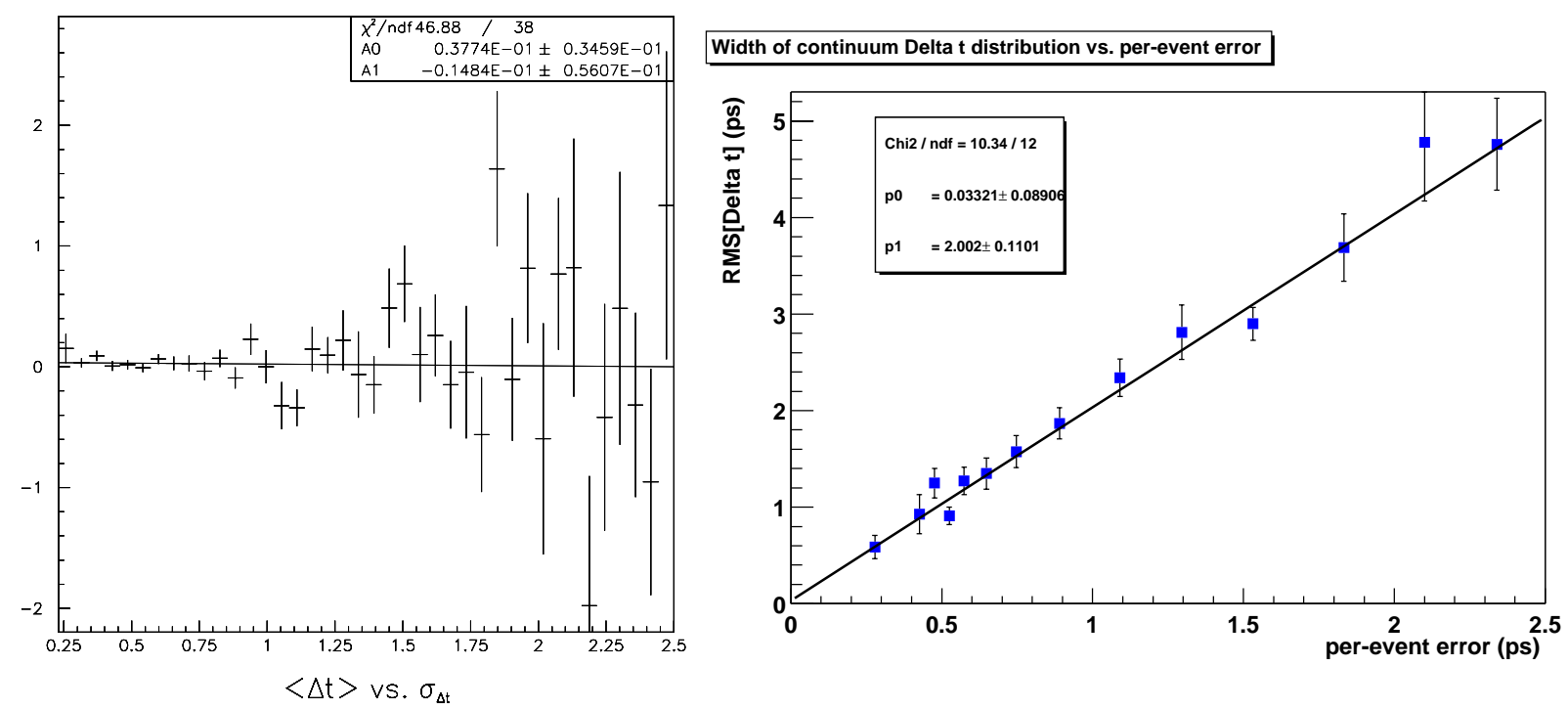

Figure 4.32: Moyenne (gauche) et écart-type (droite) de la distribution de $\Delta t$ des événements de continuum on-peak (en dehors de la région de signal) en fonction de l'erreur $\sigma_{\Delta t}$.

Ces observations motivent la paramétrisation suivante pour la distribution de $\Delta t$ du bruit de fond continuum:

$$
\begin{aligned}
f\left(\Delta t, \sigma_{\Delta t}\right)= & \left(1-f_{2}-f_{3}\right) \cdot G\left(\Delta t, \mu, s_{1} \sigma_{\Delta t}\right) \\
& +f_{2} \cdot G\left(\Delta t, \mu, s_{2} \sigma_{\Delta t}\right) \\
& +f_{3} \cdot G\left(\Delta t, \mu, s_{3} \sigma_{\Delta t}\right)
\end{aligned}
$$

où les paramètres $s_{i}$ multiplient $\sigma_{\Delta t}$. La moyenne $\mu$ est commune aux trois Gaussiennes. Les valeurs de ces paramètres sont obtenues dans un ajustement des événements on-peak (en dehors de la région de signal) et sont données dans la figure 4.33

Corrélations étiquette-charge dans le continuum: la charge $Q$ du $\rho$ du "candidat

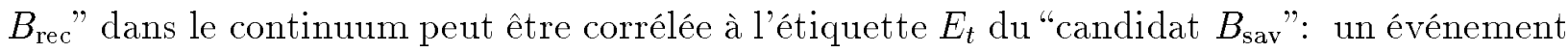
de continuum $s \bar{s}$ dont le quark $\bar{s}$ participe à la construction d'un candidat $\rho^{-} K^{+}$est corrélé à une étiquette $\bar{B}^{0}$ (car le $s$ produira un $K^{-}$, qui mène à une étiquette $\bar{B}^{0}$ ). On introduit donc un terme d'asymétrie étiquette-charge pour le continuum $A_{\text {mix }}^{q \rho h}$ pour chaque catégorie d'étiquetage:

$$
A_{\mathrm{mix}}^{q \rho h}=\frac{\left(N\left(B_{\mathrm{sav}}^{0}, \rho^{+} h^{-}\right)+N\left(\bar{B}_{\mathrm{sav}}^{0}, \rho^{-} h^{+}\right)\right)-\left(N\left(B_{\mathrm{sav}}^{0}, \rho^{-} h^{+}\right)+N\left(\bar{B}_{\mathrm{sav}}^{0}, \rho^{+} h^{-}\right)\right)}{N\left(B_{\mathrm{sav}}^{0}, \rho^{+} h^{-}\right)+N\left(\bar{B}_{\mathrm{sav}}^{0}, \rho^{-} h^{+}\right)+N\left(B_{\mathrm{sav}}^{0}, \rho^{-} h^{+}\right)+N\left(\bar{B}_{\mathrm{sav}}^{0}, \rho^{+} h^{-}\right)} .
$$

où $N\left(\mathrm{E}_{\mathrm{t}}, \mathrm{Q}_{\rho}\right)$ est le nombre d'événements d'étiquette $E_{t}$ et de charge $Q_{\rho}$ données. Les valeurs de ces asymétries sont extraites d'un ajustement global des données on-peak (dans la configuration finale telle que décrite dans la section 4.12, avec les paramètres $A_{\text {mix }}^{q \rho h}$ laissés libres) et sont fixées dans l'ajustement final. Le tableau 4.15 résume les valeurs obtenues. 


\section{Continuum Delta t distribution}

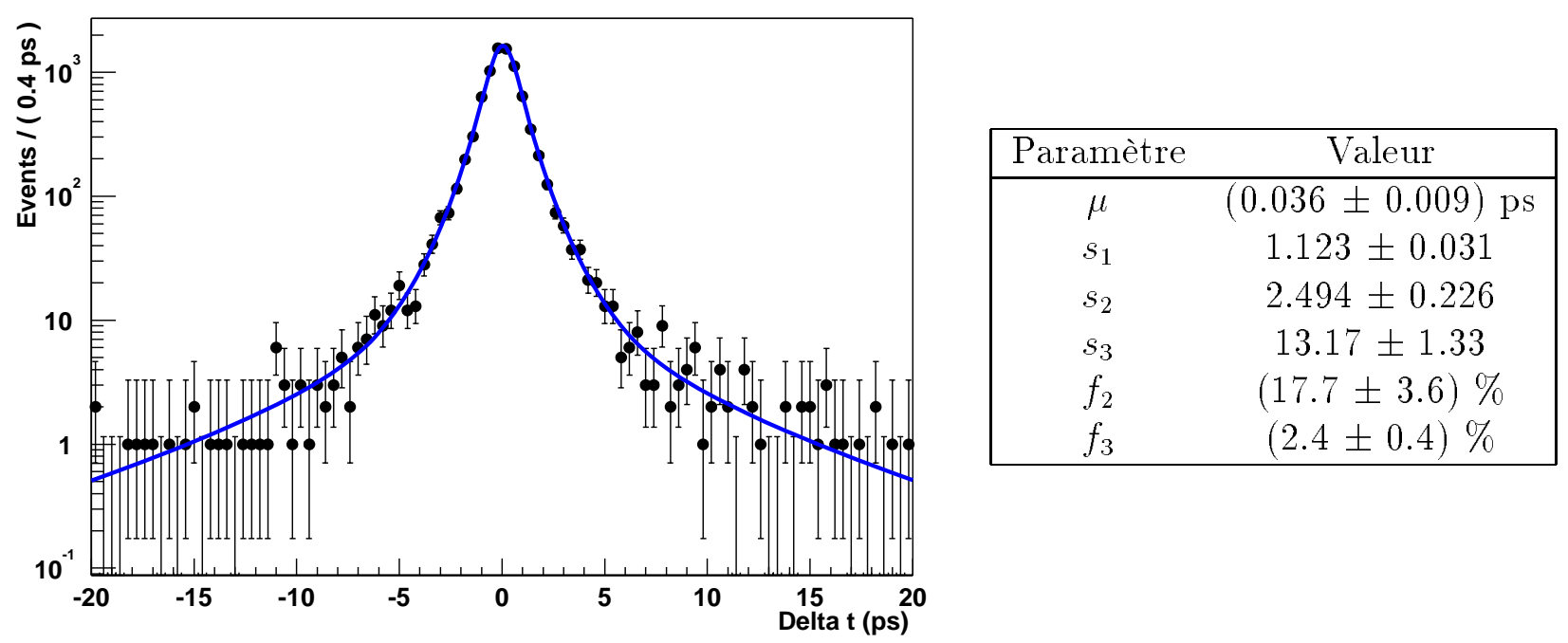

Figure 4.33: Figure de gauche: distribution de $\Delta t$ pour les événements continuum onpeak (hors de la région de signal). La paramétrisation par la fonction 4.26 est superposée. Tableau de droite: résultat d'un ajustement de vraisemblance du modèle décrit dans l'eq. 4.26 sur les données on-peak (en dehors de la région du signal)

\begin{tabular}{l|c|c} 
Categ. Etiq. & $\rho \pi$ & $\rho K$ \\
\hline Lepton & $-0.151 \pm 0.178$ & $-0.277 \pm 0.231$ \\
Kaon & $-0.020 \pm 0.019$ & $-0.530 \pm 0.023$ \\
NT1 & $-0.061 \pm 0.045$ & $-0.159 \pm 0.073$ \\
NT2 & $-0.011 \pm 0.023$ & $-0.101 \pm 0.037$
\end{tabular}

Tableau 4.15: Valeurs des asymétries étiquette-charge $A_{m i x_{c}}^{q \rho h} d u$ continuum pour les différentes catégories d'étiquetage.

\subsubsection{Bruits de fond $B$}

\subsubsection{1 $m_{\mathrm{ES}}, \Delta E$ et $x_{\mathrm{NN}}$}

La figure 4.34 montre les distributions de $m_{\mathrm{ES}}, \Delta E$ et $x_{\mathrm{NN}}$ pour les événements Monte Carlo du signal $\rho \pi$, des modes exclusifs $B^{0} \rightarrow K^{\star+} \pi^{-}, \mathrm{B}^{+} \rightarrow \rho^{0} \pi^{+}$et $B^{0} \rightarrow \rho^{+} \rho^{-}$, ainsi que le bruit de fond générique $b \rightarrow c$. Pour toutes les classes de bruits de fond $B$, les distributions sont paramétrées par des KEYS.

\subsubsection{Corrélations entre $m_{\mathrm{ES}}$ et $\Delta E$ dans le bruit de fond $B$}

La figure 4.35 montre les corrélations entre $m_{\mathrm{ES}}$ et $\Delta E$ pour des bruits de fond non charmés représentatifs de $B$ en 2-, 3-, 4-corps, et pour les bruits de fond charmés. 


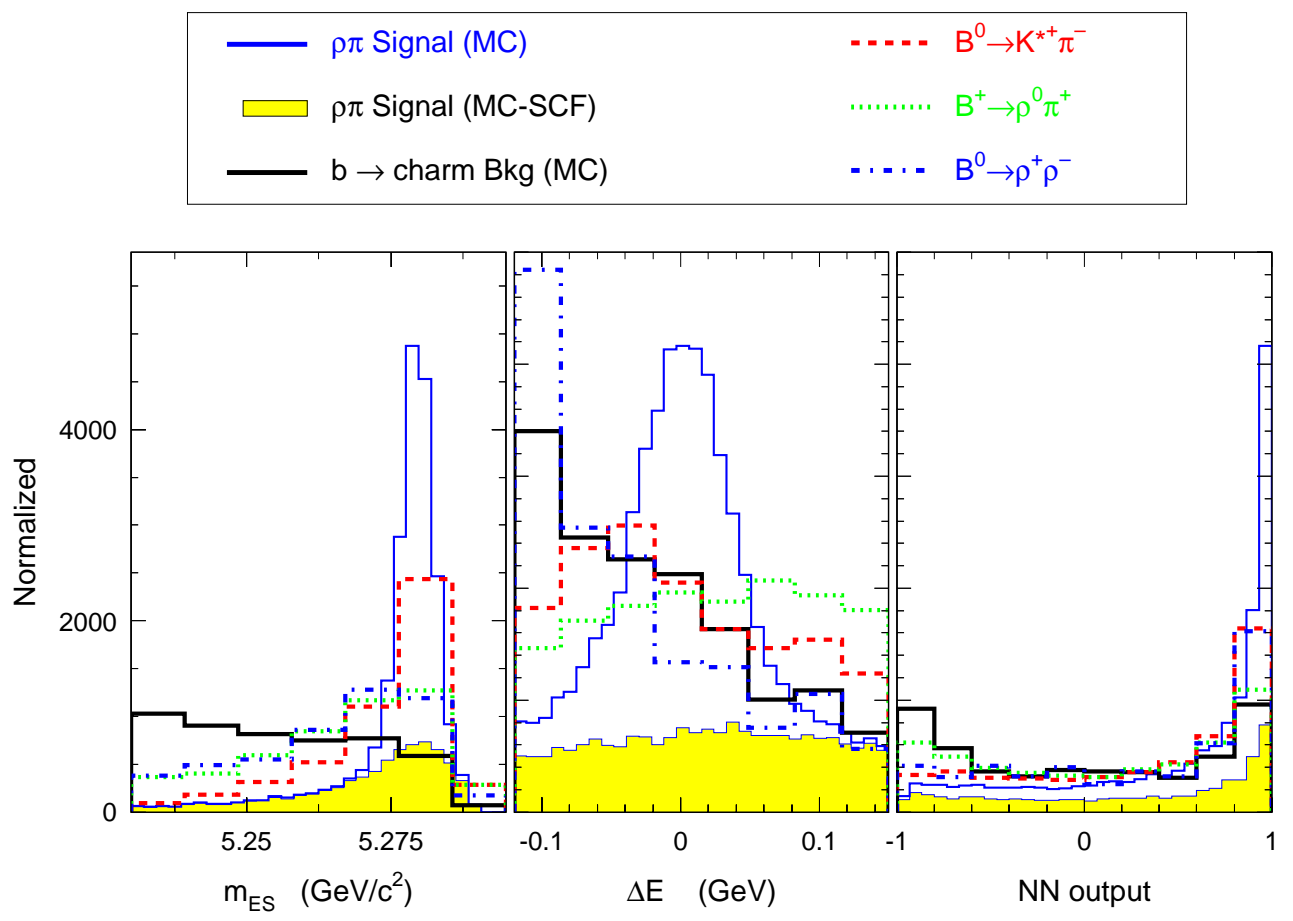

Figure 4.34: Distributions de $m_{\mathrm{ES}}, \Delta E$ et $x_{\mathrm{NN}}$ pour les événements Monte Carlo du signal $\rho \pi$ (les événements mal reconstruits sont indiqués par la zone jaune), et des modes exclusifs $B^{0} \rightarrow K^{\star+} \pi^{-}$(tirets), $B^{+} \rightarrow \rho^{0} \pi^{+}$(pointillés) et $B^{0} \rightarrow \rho^{+} \rho^{-}$(tirets-pointillés), ainsi que le bruit de fond générique $b \rightarrow c$ (ligne pleine).

Les coefficients de corrélation linéaire sont les suivants:

$\begin{array}{ccccr} & B^{+} \rightarrow K^{+} \pi^{0} & B^{+} \rightarrow \pi^{0} \rho^{+} & B^{0} \rightarrow \rho^{+} \rho^{-} & b \rightarrow c \\ \Delta E \leftrightarrow m_{\mathrm{ES}} & 0.05 & 0.02 & -0.24 & -0.15 \\ \Delta E \leftrightarrow \mathrm{NN} & 0.03 & 0.02 & -0.10 & 0.02 \\ m_{\mathrm{ES}} \leftrightarrow \mathrm{NN} & 0.09 & 0.02 & 0.05 & 0.01\end{array}$

On observe des anti-corrélations significatives entre $\Delta E$ et $m_{\mathrm{ES}}$ pour les modes $B$ en 4-corps, qui sont d'autant plus grandes qu'il y a un grand nombre de $\pi^{0}$ dans l'état final. On tient compte de ces corrélations dans l'ajustement final en introduisant des distributions en 2 dimensions (modélisées par des KEYS) pour les classes 0, 3, 4 et 5 de modes $B$ en 4-corps.

\subsubsection{Distributions de $\Delta t$ et corrêlations charge-étiquette}

La distribution de $\Delta t$ du bruit de fond $B$ (notée $P(\Delta t)$ ) diffère selon qu'il s'agit d'un $B$ chargé ou neutre:

- Pour les modes provenant d'un $B^{ \pm}: P(\Delta t)$ est une exponentielle tenant compte de la durée de vie des $B$ chargés. Il peut exister une corrélation entre la charge du $\rho$ et l'étiquette du $B_{\mathrm{sav}}$ : par exemple, pour le mode $B^{+} \rightarrow \rho^{+} \rho^{0}$, la charge du $\rho^{+}$est corrélée à l'étiquette $B_{\mathrm{sav}}^{-}$, soit $\bar{B}^{0}$. Nous introduisons donc un coefficient $A_{h}$ tenant compte de cette corrélation similaire à celui introduit pour le continuum dans l'eq. 4.27, mais où $N\left(\mathrm{E}_{\mathrm{t}}, \mathrm{Q}_{\rho}\right)$ correspond aux nombres d'événements d'étiquette et de charge données au 


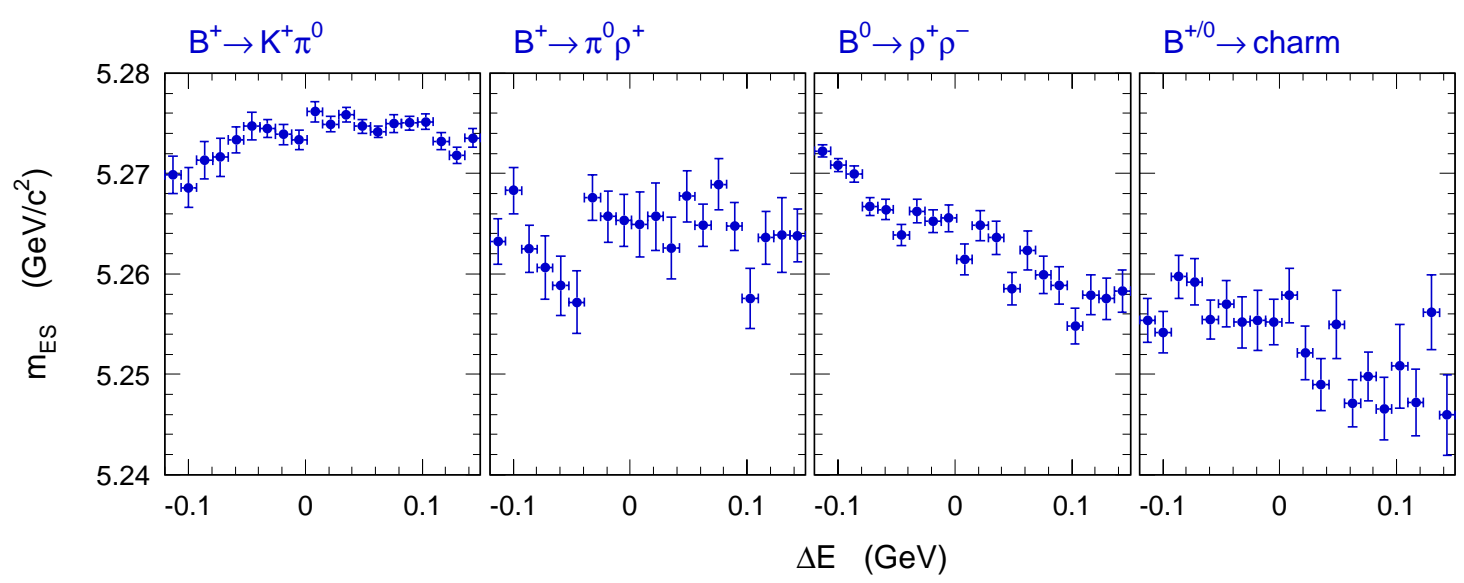

Figure 4.35: Corrélations entre $m_{\mathrm{ES}}$ et $\Delta E$ pour des bruits de fond non charmés représentatifs de $B$ en 2-, 3-, 4-corps, et pour les bruits de fond charmés.

niveau de la génération, $c-\grave{a}-d$ ne tenant pas compte du taux de mauvais étiquetage. Ce dernier est pris en compte dans le paramètre $\omega_{\text {cat }}$ calculé sur les événements $B$ chargés de l'échantillon Breco. L'asymétrie $A_{h}$ est calculé à partir du Monte Carlo. La distribution en temps des bruits de fond $B^{ \pm}$est donnée par:

$$
\begin{array}{r}
P_{\mathrm{cat}}\left(\Delta t, E_{t}= \pm 1, Q_{\rho}= \pm 1\right)=\frac{1}{4}\left(1+\mathrm{E}_{\mathrm{t}} \times \mathrm{Q}_{\rho} \times A_{h} \times\left(1-2 w_{\mathrm{cat}}\right)\right) \frac{e^{-\left|\Delta t^{\prime}\right| / \tau_{B^{+}}}}{2 \tau_{B^{+}}} \\
\otimes \mathcal{R}_{\mathrm{sig}}\left(\Delta t^{\prime}-\Delta t, \sigma_{\Delta t}\right)
\end{array}
$$

- Pour les modes provenant d'un $B^{0} / \bar{B}^{0}$ : la même distribution en temps que pour le signal est utilisée ( $c f$. Eq. 4.25), où $C_{h}, S_{h}, \Delta S_{h}$ sont supposés nuls pour $h=\pi / K$, et $\Delta C_{h}$ tient compte de la corrélation entre étiquette et charge (calculé à partir du Monte Carlo). Les efficacités d'étiquetage sont obtenues dans le Monte Carlo.

La fonction de résolution $\mathcal{R}_{\text {sig }}$ du bruit de fond $B$ est la même que pour le signal ( $c f$. Eq. 4.25 et tableau 4.14).

Le tableau 4.16 donne les valeurs des coefficients de corrélations étiquette-charge $A_{h}$ et $\Delta C_{h}$.

\subsection{Fonction de vraisemblance}

Les variables $m_{\mathrm{ES}}, \Delta E, x_{\mathrm{NN}}$ et $\Delta t$ sont utilisées pour distinguer le signal des bruits de fond continuum et $B$. L'angle Cherenkov $\theta_{C}$, et dans une moindre mesure $\Delta E$, déterminent la quantité relative d'événements avec une trace célibataire pion et kaon (pour le signal $B^{0} \rightarrow \rho^{ \pm} \pi^{\mp}$ ou $B^{0} \rightarrow \rho^{-} K^{+}$, mais aussi pour les bruits de fond respectifs). La variable $\Delta t$ permet de réaliser la mesure des paramètres $C P$ et de dilution entrant dans la dépendance en temps du taux de désintégration du signal (voir Eq. 4.25).

\subsubsection{Composition de la fonction de vraisemblance}

La densité de probabilité $\mathcal{P}_{i, c}$ pour un événement $i$, appartenant à la catégorie d'étiquetage $c_{i}$, est la somme des densités de probabilités sur les composantes de signal et de bruit de 


\begin{tabular}{|c|c|c|c|c|c|c|c|}
\hline $\mathrm{Cl}$ & Mode $\left(B^{+}\right)$ & $A_{\pi}$ & $\overline{A_{K}}$ & & & & \\
\hline 0 & $\pi^{+} K^{* 0}\left(K_{S}^{0} \pi^{0}\right)$ & -1 & - & & & & \\
\hline 0 & $\rho^{+} K^{* 0}\left(K^{+} \pi^{-}\right)$ & - & -1 & $\mathrm{Cl}$ & Mode $\left(B^{0}\right)$ & $\Delta C_{\pi}$ & $\Delta C_{K}$ \\
\hline 0 & $\rho^{+} \rho^{0}$ & $+0.10 \pm 0.05$ & - & 3 & $\rho^{0} K^{* 0}\left(K^{+} \pi^{-}\right)$ & $-0.77 \pm 0.66$ & $\frac{n}{-1}$ \\
\hline 0 & $\left(a_{1} \pi\right)^{+}$ & $\begin{array}{c}0 \pm 0.2 \\
-081+003\end{array}$ & - & 3 & $\rho^{-} K^{*+}\left(K^{+} \pi^{0}\right)$ & $+0.50 \pm 0.43$ & $-0.80 \pm 0.17$ \\
\hline $\begin{array}{l}0 \\
0\end{array}$ & $\begin{array}{l}\eta^{\prime}\left(\rho^{0} \gamma\right) \pi^{+} \\
\eta^{\prime}\left(\rho^{0} \gamma\right) K^{+}\end{array}$ & $\begin{array}{c}-0.81 \pm 0.03 \\
+1\end{array}$ & $\begin{array}{c}- \\
-1\end{array}$ & 3 & $\pi^{-} K^{*+}\left(K_{S}^{0} \pi^{+}\right)$ & +1 & - \\
\hline 1 & $\pi^{0} \rho^{+}$ & $\frac{T+1}{-1}$ & -1 & 4 & $\rho_{0}^{+} \rho^{-}$ & $+0.11 \pm 0.04$ & - \\
\hline 1 & $\pi^{+} \rho^{0}$ & $-0.46 \pm 0.02$ & - & $\frac{4}{5}$ & $\frac{\rho^{0} \rho^{0}}{\left(\rho^{0}\right.}$ & $-0.46 \pm 0.15$ & - \\
\hline 1 & $K_{S}^{0}\left(\pi^{+} \pi^{-}\right) \pi^{+}$ & $-0.75 \pm 0.04$ & - & 5 & $\begin{array}{l}\left(a_{1} \pi\right)^{0} \\
\tau^{0} H^{\prime} * 0\left(L^{+}-\tau^{-}\right.\end{array}$ & $+0.06 \pm 0.27$ & - \\
\hline 1 & $K^{+} \rho^{0}$ & +1 & -1 & 5 & & $0 \pm 1$ & $-0.55 \pm 0.10$ \\
\hline 1 & $K^{+} f_{X}(1300)$ & +1 & -1 & 6 & $\begin{array}{l}\pi^{0} \rho^{0} \\
U^{+}-\tau^{-}\end{array}$ & $\begin{array}{c}0 \\
\end{array}$ & $\stackrel{-}{-}$ \\
\hline 1 & $K^{+} f_{0}\left(\pi^{+} \pi^{-}\right)$ & +1 & -1 & $\begin{array}{l}6 \\
6\end{array}$ & $\begin{array}{l}K^{+} \pi^{-} \\
\pi^{-} K^{*+}\left(K^{+} \pi^{0}\right)\end{array}$ & $\begin{array}{l}+0.33 \pm 0.10 \\
+0.88 \pm 0.06\end{array}$ & $\begin{array}{c}-0.23 \pm 0.15 \\
-1\end{array}$ \\
\hline$\frac{1}{9}$ & $\frac{\pi^{0} K^{*+}\left(K^{+} \pi^{0}\right)}{K^{+} \pi^{0}}$ & - & $\frac{-1}{-1}$ & 9 & $\left(K_{X}^{(* *)} \pi\right)^{0}$ & +0.25 & -1 \\
\hline 2 & $\pi^{+} \pi^{0}$ & -1 & - & 11 & $\left(K_{X}^{(* *)} \rho\right)^{0}$ & $0 \pm 1$ & -1 \\
\hline 10 & $\left(K_{X}^{(* *)} \pi\right)^{+}$ & -0.63 & $\overline{-1}$ & 8 & charm & 0 & 0 \\
\hline 12 & $\left(K_{X}^{(* *)} \rho\right)^{+}$ & $+0.19 \pm 0.18$ & -1 & & & & \\
\hline 7 & charm & $-0.20 \pm 0.05$ & $-0.75 \pm 0.08$ & & & & \\
\hline
\end{tabular}

Tableau 4.16: Coefficients de corrélation entre étiquette et charge du $\rho$ reconstruits pour les modes de bruit de fond des $B$ chargés (tableau de gauche) et des $B$ neutres (tableau de droite). Les erreurs sur les coefficients sont dues à la statistique limitée du Monte Carlo à partir desquels ils sont estimés.

fond:

$$
\begin{aligned}
\mathcal{P}_{i, c}=\sum_{h=\pi, K} & {\left[N _ { \rho h } f _ { c } ^ { \rho h } \frac { 1 } { 2 } \left(\left(1+Q_{\rho} A_{C P}^{\rho h}\right)\left[\left(1-f_{\mathrm{scf}, c}^{\rho h}\right) \mathcal{P}_{i, c, \mathrm{tru}}^{\rho h}+f_{\mathrm{scf}, c}^{\rho h}\left(1-w_{Q}^{\rho h}\right) \mathcal{P}_{i, c, \mathrm{scf}\left[Q_{\rho}\right]}^{\rho h}\right]\right.\right.} \\
& \left.+\left(1-Q_{\rho} A_{C P}^{\rho h}\right) f_{\mathrm{scf}}^{\rho h} w_{Q}^{\rho h} \mathcal{P}_{i, c, \mathrm{scf}\left[-Q_{\rho}\right]}^{\rho h}\right) \\
& +N_{q \rho h}^{c} \frac{1}{4}\left(1+Q_{\rho_{i}} A_{C P}^{q \rho h}\right)\left(1+\mathrm{E}_{\mathrm{t}} \mathrm{Q}_{\rho_{\mathrm{i}}} \mathrm{A}_{\mathrm{mix}, \mathrm{q}, \mathrm{c}}^{\mathrm{q} \rho \mathrm{h}} \mathcal{P}_{\mathrm{i}, \mathrm{q}}^{\mathrm{q} \rho \mathrm{h}}\right. \\
& \left.+\sum_{b=1, \ldots N_{B}} \mathcal{L}_{i, b, c}^{\rho h}\right]
\end{aligned}
$$

où:

- $N_{\rho h}$ est le nombre d'événements de signal de type $\rho h(h=\pi, K)$.

- $f_{c}^{\rho h}$ est la fraction d'événements de signal de type $\rho h$ dans la catégorie d'étiquetage $c$.

- $f_{\text {scf,c }}^{\rho h}$ est la fraction d'événements mal reconstruits de signal $\rho h$ dans la catégorie d'étiquetage $c$.

- $w_{Q}^{\rho h}$ est la fraction d'événements de mauvaise charge parmi les événements mal reconstruits du signal $\rho h$.

- $N_{q \rho h}^{c}$ est le nombre d'événements de bruit de fond de continuum avec une trace célibataire de type $h$, dans la catégorie d'étiquetage $c$.

- $Q_{\rho_{i}}$ est la charge du candidat $\rho$. 
- $\mathrm{E}_{\mathrm{t}}=-1 /+1$ pour $B^{0} / \overline{B^{0}}$ est l'étiquette de saveur du $B_{\mathrm{sav}}$.

- $A_{C P}^{\rho h}=\left(N_{\rho^{+} h^{-}}-N_{\rho^{-} h^{+}}\right) /\left(N_{\rho^{+} h^{-}}+N_{\rho^{-h^{+}}}\right)$est l'asymétrie $C P$ directe intégrée sur le temps et la saveur.

- $A_{m i x, c}^{\rho h}$ est le coefficient d'asymétrie étiquette-charge du continuum défini dans l'eq. 4.27.

- $\mathcal{P}_{c}^{(q) \rho h}$ est le produit des densités de probabilités pour le signal $\rho h$ et le bruit de fond continuum $q \rho h$ des variables entrant dans l'ajustement: $\mathcal{P}^{(q) \rho h}\left(m_{\mathrm{ES}}\right) \cdot \mathcal{P}^{(q) \rho h}\left(\Delta E \mid p_{\text {célib }}\right) \cdot$ $\mathcal{P}^{(q) \rho h}\left(x_{\mathrm{NN}}\right) \cdot \mathcal{P}^{(q) \rho h}\left(\theta_{c} \mid p_{\text {célib }}^{\mathrm{D}}, \theta\right) \cdot \mathcal{P}_{c}^{(q) \rho h}(\Delta t)$, où $p_{\text {célib }}$ et $\theta$ dénotent respectivement l'impulsion et l'angle polaire (dans le référentiel du laboratoire) de la trace célibataire, et

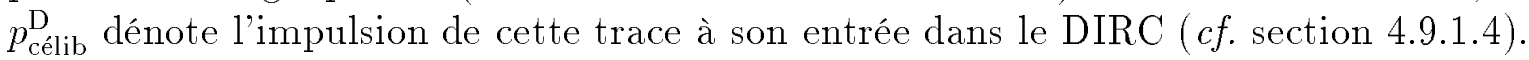
Les variables contenues dans $\mathcal{P}_{c}^{(q) \rho h}$ sont supposées non-corrélées, et leurs distributions de probabilité sont donc unidimensionnelles. Les corrélations résiduelles existant entre ces variables ( $c f$. matrices 4.18 et 4.19 ) sont traitées comme erreurs systématiques.

- $\sum_{b=1, \ldots N_{B}} \mathcal{L}_{i, b, c}^{\rho h}$ est la somme des termes correctifs pour les $N_{B}$ classes de bruits de fond $B$ ayant une trace célibataire de type $h$ ( $c f$. tableau 4.13 ).

La fonction de vraisemblance étendue sur toutes les catégories d'étiquetage est donnée par:

$$
\mathcal{L}=\prod_{c=1}^{5} e^{-N_{c}^{\prime}} \prod_{i}^{N_{c}} \mathcal{P}_{i, c}
$$

où $N_{c}^{\prime}$ est le nombre d'événements attendus (signal et bruit de fond) dans la catégorie $c$, c'est à dire la somme des nombres d'événements de signal $N_{\rho h} f_{c}^{\rho h}$ et de bruit de fond $N_{q \rho h}^{c}$ ajustés dans chaque catégorie $c$. Ajuster ces nombres d'événements, plutôt que des fractions de signal et bruit de fond par rapport au nombre total d'événements dans une catégorie $N_{c}$, mène à l'introduction du terme exponentiel $e^{-N_{c}^{\prime}}$ dans la formule ci-dessus ${ }^{15}$.

\subsubsection{Paramètres libres de l'ajustement final}

Parmi tous les paramètres de la fonction de vraisemblance décrite précédemment, 34 sont laissés libres dans l'ajustement final:

- Paramètres $C P$ et de dilution:

$A_{C P}^{\rho \pi}$ : asymétrie de charge dans $B^{0} \rightarrow \rho \pi$.

$A_{C P}^{\rho K}$ : asymétrie de charge dans $B^{0} \rightarrow \rho K$.

$C_{\rho \pi}$ : moyenne des coefficients du cosinus pour $B^{0} \rightarrow \rho^{+} \pi^{-}$et $B^{0} \rightarrow \rho^{-} \pi^{+}$.

$\Delta C_{\rho \pi}$ : demi-différence des coefficients du cosinus pour $B^{0} \rightarrow \rho^{+} \pi^{-}$et $B^{0} \rightarrow \rho^{-} \pi^{+}$.

$S_{\rho \pi}$ : moyenne des coefficients du sinus pour $B^{0} \rightarrow \rho^{+} \pi^{-}$et $B^{0} \rightarrow \rho^{-} \pi^{+}$.

$\Delta S_{\rho \pi}$ : demi-différence des coefficients du sinus pour $B^{0} \rightarrow \rho^{+} \pi^{-}$et $B^{0} \rightarrow \rho^{-} \pi^{+}$.

\footnotetext{
${ }^{15}$ La fonction de vraisemblance non-étendue s'écrit $\mathcal{L}=\Pi_{i=1}^{N} f \mathcal{P}^{S}+(1-f) \mathcal{P}^{B}$, où $f$ est la fraction de signal dans le lot de $N$ événements, et $\mathcal{P}^{S(B)}$ les distributions de probabilités du signal (bruit de fond). La fonction de vraisemblance étendue s’écrit

$$
\begin{aligned}
\mathcal{L}_{\text {étendue }} & =\left(n_{S}+n_{B}\right)^{N} / N ! e^{-\left(n_{S}+n_{B}\right)} \mathcal{L}, \\
& =e^{-\left(n_{S}+n_{B}\right)} \Pi_{i=1}^{N} n_{S} \mathcal{P}^{S}+n_{B} \mathcal{P}^{B}
\end{aligned}
$$
}

où $n_{S}\left(n_{B}\right)$ est le nombre d'événements de signal (bruit de fond) et où le terme $N$ ! a été retiré. 
- Asymétries de charge du continuum:

$A_{C P}^{\rho \pi, c o n t}$ : asymétrie de charge du continuum pour la catégorie $\rho \pi$.

$A_{C P}^{\rho K, \text { cont }}$ : asymétrie de charge du continuum pour la catégorie $\rho K$.

- Nombre d'événements de signal:

$N_{\rho \pi}:$ pour $B^{0} \rightarrow \rho \pi$.

$N_{\rho K}:$ pour $B^{0} \rightarrow \rho K$.

- Nombre d'événements du continuum par catégorie d'étiquetage:

$N_{B \rho \pi}^{\mathrm{Lepton}}, N_{B \rho \pi}^{\mathrm{Kaon}}, N_{B \rho \pi}^{\mathrm{NT} 1}, N_{B \rho \pi}^{\mathrm{NT} 2}, N_{B \rho \pi}^{\mathrm{NoTag}}:$ pour le continuum $\rho \pi$

$N_{B \rho K}^{\mathrm{Lepton}}, N_{B \rho K}^{\mathrm{Kaon}}, N_{B \rho K}^{\mathrm{NT} 1}, N_{B \rho K}^{\mathrm{NT} 2}, N_{B \rho K}^{\mathrm{NoTag}}:$ pour le continuum $\rho K$

- Paramétrisation du signal :

$\mu(\Delta E)$ : moyenne de la principale Gaussienne de $\Delta E$ (événements correctement reconstruits)

$\mu\left(m_{\mathrm{ES}}\right)$ : moyenne de la fonction Crystal Ball de $m_{\mathrm{ES}}$ (événements correctement reconstruits)

- Paramétrisation du continuum :

$\xi$ : paramètre de la fonction d'Argus pour $m_{\mathrm{ES}}$

$\Delta E_{p 1}, \Delta E_{p 2}$ : coefficients linéaire et quadratique de la fonction polynomiale pour $\Delta E$ $a_{1}, a_{2}, a_{3}$ : coefficients de la fonction polynomiale pour $x_{\mathrm{NN}}$

$\mu(\Delta t)_{1}$ : moyenne de la 1-ère Gaussienne pour la distribution de $\Delta t$

$\sigma(\Delta t)_{1}, \sigma(\Delta t)_{2}, \sigma(\Delta t)_{3}$ : écart-type des trois Gaussiennes pour la distribution de $\Delta t$

$f(\Delta t)_{1}, f(\Delta t)_{2}$ : fractions des deux principales Gaussiennes pour la distribution de $\Delta t$

\subsection{Validation de l'analyse en aveugle}

L'analyse, dont l'élaboration est développée dans les sections précédentes, est validée par divers ajustements sur des simulations rapides, des événements de Monte Carlo, et des données off-peak.

\subsubsection{Validation par des simulations rapides}

Pour valider l'outil d'ajustement, environ 1800 expériences de simulations rapides dans la configuration nominale ( $c f$. Sec. 4.12) ont été réalisées. Les pulls des distributions des variables de l'ajustement doivent être des Gaussiennes centrés à zéro et écart type égal à l'unité. Les résultats pour les paramètres $C P$ et de dilution, ainsi que pour les nombres d'événements de signal, sont résumés dans le tableau 4.17. Des biais sont observés, dont on tient compte dans les erreurs systématiques.

\subsubsection{Ajustements sur des échantillons connus (MC, simulation rapide, off-peak)}

Les résultats des ajustements décrits dans cette section sont détaillés dans la référence [39]. Seules les conclusions de ces études sont reportées ici. 


\begin{tabular}{ccr}
\hline \hline & \multicolumn{2}{c}{ Valeurs nominales } \\
& Moyenne & Largeur \\
\hline$C$ & $-0.060 \pm 0.024$ & $0.998 \pm 0.018$ \\
$\Delta C$ & $-0.052 \pm 0.023$ & $0.969 \pm 0.017$ \\
$S$ & $-0.001 \pm 0.024$ & $1.030 \pm 0.019$ \\
$\Delta S$ & $-0.021 \pm 0.021$ & $1.015 \pm 0.018$ \\
$A_{C P}$ & $0.055 \pm 0.025$ & $1.012 \pm 0.017$ \\
$A_{C P}^{K}$ & $-0.004 \pm 0.024$ & $1.003 \pm 0.018$ \\
$N_{\rho \pi}$ & $0.046 \pm 0.023$ & $0.981 \pm 0.017$ \\
$N_{\rho K}$ & $-0.097 \pm 0.024$ & $0.973 \pm 0.019$ \\
\hline \hline
\end{tabular}

Tablea1 4.17: Moyennes et largeurs des pulls des paramètres $C P$ et de dilution, ainsi que des nombres d'événements de signal, issus des simulations rapides.

\subsubsection{Monte Carlo $\rho \pi$ et $\rho K$ (grande statistique)}

Nous avons réalisé des ajustements d'échantillons de grande statistique de signal Monte Carlo, afin de tester la qualité des paramétrisations des différentes variables, et de vérifier la présence de corrélations résiduelles entre ces variables. Les événements de signal du Monte Carlo sont pondérés pour mener aux valeurs suivantes:

$$
\begin{aligned}
A_{C P}^{\rho \pi}=-0.17, & A_{C P}^{\rho K}=0 \\
C_{\rho \pi}=0.36, & \Delta C_{\rho \pi}=0.27, \\
S_{\rho \pi}=0.19, & \Delta S_{\rho \pi}=0.14
\end{aligned}
$$

Pour des raisons techniques, nous mélangeons aux événements de signal 1000 événements de continuum générés par la simulation rapide.

Nous observons que:

- $0.1 \%$ des événements de $\rho \pi$ contaminent $\rho K$, et $0.4 \%$ contaminent le continuum.

- $0.5 \%$ des événements de $\rho K$ contaminent $\rho \pi$, et $0.7 \%$ contaminent le continuum.

- il n'y a aucun biais significatif dans les valeurs ajustées.

\subsubsection{Monte Carlo Continuum et données off-peak (grande statistique)}

Nous réalisons un ajustement de tous les paramètres reliés au bruit de fond continuum sur un échantillon de 2291 événements de données off-peak, mélangés à des événements de Monte Carlo continuum. Les nombres d'événements de signal, et l'asymétrie de charge dans le continuum, sont compatibles avec zéro. Les paramètres pour $\Delta t$ et $x_{\mathrm{NN}}$ sont en bon accord avec ceux utilisés dans l'ajustement nominal.

\subsubsection{Cocktails de MC signal et bruit de fond $B$, et de continuum de la simulation rapide (échantillons de taille réaliste)}

Des échantillons contenant un nombre réaliste (proche du nombre attendu dans les données) d'événements de signal (430 pour $B^{0} \rightarrow \rho^{ \pm} \pi^{\mp}$ et 120 pour $B^{0} \rightarrow \rho^{-} K^{+}$, pondérés pour mener aux valeurs de l'eq. 4.31), de bruit de fond continuum et de bruits de fond $B$ (suivant 
les nombres d'événements donnés dans le tableau 4.13) sont formés. Lorsque la statistique du Monte Carlo le permet, les événements sont issus du Monte Carlo (c'est le cas du signal et des bruits de fond $B$ non charmés). Le cas échéant, les événements sont issus de la simulation rapide (continuum et bruits de fond charmés).

Les conclusions de ces ajustements sont énoncées ci-après:

- Ajustements de 73 échantillons de signal $B^{0} \rightarrow \rho^{ \pm} \pi^{\mp}$ et $B^{0} \rightarrow \rho^{-} K^{+}$: les paramètres $C P$ et de dilution sont en bon accord avec ceux générés, alors que les nombres d'événements sont biaisés de $-0.4 \pm 0.1 \%$ pour $B^{0} \rightarrow \rho^{ \pm} \pi^{\mp}$ et $-3.6 \pm 0.4 \%$ pour $B^{0} \rightarrow \rho^{-} K^{+}$.

- Ajustement de 73 échantillons de continuum: la contamination du continuum vers le signal est négligeable.

- Ajustements de 73 échantillons de signal et de continuum: nous n'observons aucun biais sur les paramètres $C P$ et de dilution, ni sur le nombre d'événements de $B^{0} \rightarrow \rho^{-} K^{+}$(ce qui signifie qu'un nouveau biais a compensé le biais observé dans l'ajustement $d u$ signal seulement), mais une contamination significative $d u$ continuum vers le signal $B^{0} \rightarrow \rho^{ \pm} \pi^{\mp}$ de $+2.8 \pm 0.7 \%$.

- Ajustements de 19 échantillons complets (signal, continuum, bruit de fond $B)$ : les paramètres $C P$ et de dilution ne montrent aucun biais, alors que les nombres d'événements de $B^{0} \rightarrow \rho^{ \pm} \pi^{\mp}$ et $B^{0} \rightarrow \rho^{-} K^{+}$montrent des biais de respectivement $+5.1 \pm 1.3 \%$ et $+6.6 \pm 3.1 \%$.

\subsubsection{Discussion des résultats}

Les ajustements d'échantillons Monte Carlo révèlent des biais significatifs sur les nombres d'événements de signal $B^{0} \rightarrow \rho^{ \pm} \pi^{\mp}$ et $B^{0} \rightarrow \rho^{-} K^{+}$, mais aucun biais sur les paramètres $C P$ et de dilution. Des études plus poussées ne pointent pas vers un problème concernant une des variables discriminantes en particulier, ni les divers paramètres utilisés dans l'ajustement (fractions de mauvais étiquetage, efficacités d'étiquetage, etc.).

Ces biais pourraient être crées par la présence de corrélations résiduelles entre les variables de l'ajustement final. Nous pouvons détecter la présence de telles corrélations en comparant la composante de signal $B^{0} \rightarrow \rho \pi(\mathcal{L}(\rho \pi))$ de la fonction de vraisemblance (cf. Eq. 4.10.1) dans les données ou le Monte Carlo (possédant éventuellement des corrélations) et les événements issus de la simulation rapide (n'en possédant pas). Pour obtenir une distribution bornée (entre 0 et 1 ), nous normalisons la composante de signal par la fonction de vraisemblance totale $\mathcal{L}$ (tot). L'effet des corrélations positives est un peuplement excessif des extrémités de la distribution du Monte Carlo.

Les distributions de $\mathcal{L}(\rho \pi) / \mathcal{L}($ tot $)$ pour le signal $B^{0} \rightarrow \rho^{ \pm} \pi^{\mp}$ (correctement et mal reconstruit) et le continuum sont montrées sur la figure 4.36. Nous observons la signature de corrélations pour les événements de signal ( $c$-àd-d, des excès d'événements Monte Carlo aux extrémités des distributions - noter l'échelle logarithmique: il s'agit d'excès significatifs). Nous n'observons pas de corrélations dans le continuum.

Ces corrélations peuvent expliquer qualitativement les biais observés dans les ajustements sur cocktails Monte Carlo ( $c f$. section 4.11.2.3). Une preuve plus quantitative nécessite des études supplémentaires. 

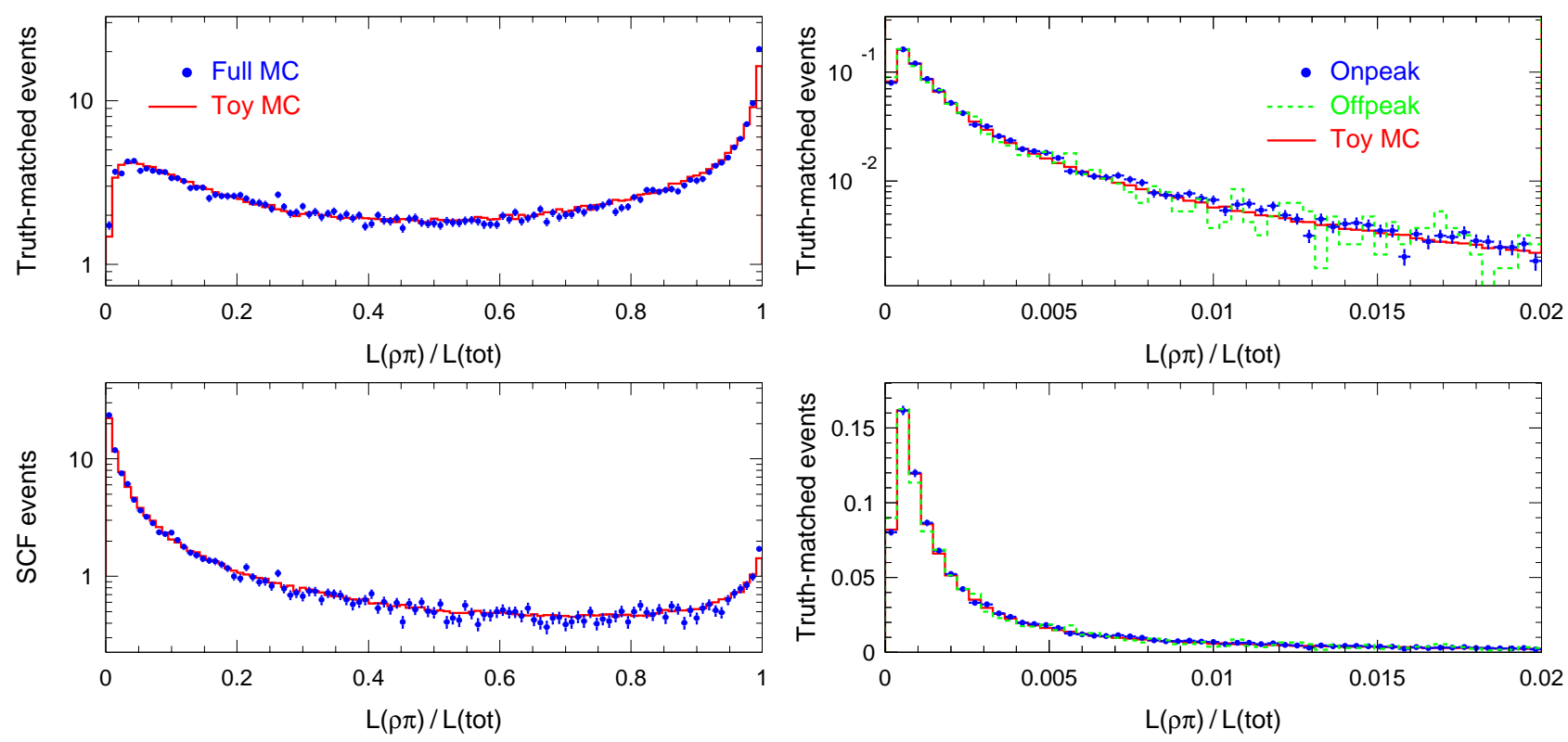

Figure 4.36: Distributions des projections de vraisemblance (cf. texte et Sec. 4.12.3) pour les événements de signal correctement reconstruits (graphe en haut à gauche), et mal reconstruits (graphe en bas à gauche), ainsi que les données (graphes de droite) on-peak (points avec barres d'erreur) et off-peak (pointillés), comparées aux distributions obtenues par des simulations rapides (ligne pleine).

\subsection{Ajustement final des données (dévoilement du ré- sultat)}

Nous procédons dans cette section au dévoilement du résultat final sur les 20497 événements on-peak sélectionnés.

\subsubsection{Résultat de l'ajustement final sur les données on-peak}

Le résultat de l'ajustement final est donné dans le tableau 4.18.

Le tableau 4.19 montre la matrice de corrélation entre les paramètres physiques de l'ajustement. Les corrélations entre les paramètres $\Delta C, \Delta S, C, S, A_{C P}$, et $N_{\rho \pi}$ sont inférieures à $23 \%$, et celles entre les paramètres $A_{C P}^{K}$, et $N_{\rho K}$ à $9 \%$.

Les figures 4.37 et 4.38 montrent les distributions de $m_{\mathrm{ES}}, \Delta E$ et $x_{\mathrm{NN}}$ pour des échantillons enrichis en signal $B^{0} \rightarrow \rho^{ \pm} \pi^{\mp}$ et $B^{0} \rightarrow \rho^{-} K^{+}$grâce à une coupure sur le rapport des fonctions de vraisemblance:

$$
\mathcal{R}^{\rho h}=\frac{\mathcal{P}_{i, c}^{\rho h[\text { tot }]}}{\mathcal{P}_{i, c}^{\text {continuum }}},
$$

où $\mathcal{P}$ est le produit des distributions de probabilités des variables (celle montrée sur le graphe étant exclue) incluses dans l'ajustement ( $c f$. Sec. 4.10.1). Les fonctions de vraisemblance de la variable représentée pour le signal, le continuum et le bruit de fond $B$ sont superposées à la distribution des événements (en ayant prit soin de les corriger de l'efficacité de la coupure sur le rapport $\left.\mathcal{R}^{\rho h}\right)^{16}$. La figure 4.38 représente la projection de la fonction de vraisemblance

\footnotetext{
${ }^{16}$ Dans la jargon de $B A B A R$, on appelle ce type de graphe une "projection de la fonction de vraisemblance",
} 


\begin{tabular}{|c|c|c|}
\hline Paramètre & Description & Valeur \\
\hline$N_{\rho \pi}$ & Nombre d'événements de $B^{0} \rightarrow \rho \pi$ & $428.0 \pm 33.9$ \\
\hline$N_{\rho K}$ & Nombre d'événements de $B^{0} \rightarrow \rho K$ & $120.4 \pm 20.7$ \\
\hline$A_{C P}^{\rho \pi}$ & Asymétrie de charge dans $B^{0} \rightarrow \rho \pi$ & $-0.178 \pm 0.079$ \\
\hline$A_{C P}^{\rho K}$ & Asymétrie de charge dans $B^{0} \rightarrow \rho K$ & $0.284 \pm 0.17$ \\
\hline$C_{\rho \pi}$ & Moyenne des coef. du cos dans $B^{0} \rightarrow \rho^{+} \pi^{-}$et $B^{0} \rightarrow \rho^{-} \pi^{+}$ & $0.36 \pm 0.18$ \\
\hline$\Delta C_{\rho \pi}$ & Demi-différence des coef. du cos dans $B^{0} \rightarrow \rho^{+} \pi^{-}$et $B^{0} \rightarrow \rho^{-} \pi^{+}$ & $0.28 \pm 0.18$ \\
\hline$S_{\rho \pi}$ & Moyenne des coef. du sin dans $B^{0} \rightarrow \rho^{+} \pi^{-}$et $B^{0} \rightarrow \rho^{-} \pi^{+}$ & $0.19 \pm 0.24$ \\
\hline$\Delta S_{\rho \pi}$ & Demi-différence des coef. du sin dans $B^{0} \rightarrow \rho^{+} \pi^{-}$et $B^{0} \rightarrow \rho^{-} \pi^{+}$ & $0.15 \pm 0.25$ \\
\hline$\mu(\Delta E)$ & Moyenne de la principale Gaussienne pour $\Delta E$ signal & $-0.009 \pm 0.004$ \\
\hline$\mu\left(m_{\mathrm{ES}}\right)$ & Moyenne de la fonction de Crystal Ball pour $m_{\mathrm{ES}}$ signal & $5.2812 \pm 0.0002$ \\
\hline$N_{B \rho \pi}^{\text {Lepton }}$ & Nombre d'événements de continuum $\rho \pi$ dans la catég. Lepton & $50.1 \pm 8.9$ \\
\hline$N_{B \rho \pi}^{\mathrm{Kaon}}$ & Nombre d'événements de continuum $\rho \pi$ dans la catég. Kaon & $3220 \pm 60$ \\
\hline$N_{B \rho \pi}^{N T 1}$ & Nombre d'événements de continuum $\rho \pi$ dans la catég. NT1 & $561.3 \pm 25.0$ \\
\hline$N_{B \rho \pi}^{\mathrm{NT} 2}$ & Nombre d'événements de continuum $\rho \pi$ dans la catég. NT2 & $2087 \pm 47.7$ \\
\hline$N_{B \rho \pi}^{\text {NoTag }}$ & Nombre d'événements de continu1um $\rho \pi$ dans la catég. No Tag & $7633 \pm 91$ \\
\hline$N_{B \rho K}^{\text {Lepton }}$ & Nombre d'événements de continu1um $\rho K$ dans la catég. Lepton & $26.5 \pm 6.4$ \\
\hline$N_{B \rho K}^{\mathrm{Kaon}}$ & Nombre d'événements de continuum $\rho K$ dans la catég. Kaon & $1598 \pm 42.9$ \\
\hline$N_{B \rho K}^{\mathrm{NT1}}$ & Nombre d'événements de continuum $\rho K$ dans la catég. NT1 & $218.9 \pm 16.2$ \\
\hline$N_{B \rho K}^{\mathrm{NT} 2}$ & Nombre d'événements de continuum $\rho K$ dans la catég. NT2 & $827.2 \pm 30.8$ \\
\hline$N_{B \rho K}^{\text {NóTag }}$ & Nombre d'événements de continuum $\rho K$ dans la catég. No Tag & $3055 \pm 59.1$ \\
\hline$a_{1}$ & Coefficient de la distribution de $x_{\mathrm{NN}}$ & $3.774 \pm 0.669$ \\
\hline$a_{2}$ & Coefficient de la distribution de $x_{\mathrm{NN}}$ & $1.330 \pm 0.131$ \\
\hline$a_{3}$ & Coefficient de la distribution de $x_{\mathrm{NN}}$ & $1.628 \pm 0.019$ \\
\hline$\xi$ & Paramètre de la fonction d'ARGUS & $-19.2 \pm 1.3$ \\
\hline$\Delta E_{p 1}$ & Coefficient linéaire du polynôme pour $\Delta E$ continuum & $-0.66 \pm 0.10$ \\
\hline$\Delta E_{p 2}$ & Coefficient quadratique du polynôme pour $\Delta E$ continuum & $-1.2 \pm 1.3$ \\
\hline$\mu(\Delta t)_{1}$ & Moyenne de la distribution de $\Delta t$ continuum & $0.025 \pm x \circ 0.006$ \\
\hline$\sigma(\Delta t)_{1}$ & Ecart-type de la $1^{\text {re }}$ Gaussienne de $\Delta t$ continuum & $1.094 \pm 0.021$ \\
\hline$\sigma(\Delta t)_{2}$ & Ecart-type de la $2^{e}$ Gaussienne de $\Delta t$ continuum & $2.410 \pm 0.188$ \\
\hline$\sigma(\Delta t)_{3}$ & Ecart-type de la $3^{e}$ Gaussienne de $\Delta t$ continuum & $12.03 \pm 1.04$ \\
\hline$f(\Delta t)_{1}$ & Fraction de la $1^{\text {re }}$ Gaussienne de $\Delta t$ continuum & $0.161 \pm 0.027$ \\
\hline$f(\Delta t)_{2}$ & Fraction de la $2^{e}$ Gaussienne de $\Delta t$ continuum & $0.022 \pm 0.003$ \\
\hline$A_{C P}^{\rho \pi, \text { cont }}$ & Asymétrie de charge pour le continu1um $\rho \pi$ & $-0.009 \pm 0.009$ \\
\hline$A_{C P}^{\rho K, \text { cont }}$ & Asymétrie de charge pour le continuum $\rho K$ & $0.001 \pm 0.014$ \\
\hline
\end{tabular}

Tableau 4.18: Résultat de l'ajustement final sur les données on-peak. 


\begin{tabular}{c|cccccccc}
\hline \hline & $N_{\rho \pi}$ & $N_{\rho K}$ & $A_{C P}^{\rho \pi}$ & $A_{C P}^{\rho K}$ & $C_{\rho \pi}$ & $\Delta C_{\rho \pi}$ & $S_{\rho \pi}$ & $\Delta S_{\rho \pi}$ \\
\hline$N_{\rho \pi}$ & 100 & -1.0 & 6.1 & 0.6 & -4.7 & -6.7 & 0.3 & -0.2 \\
$N_{\rho K}$ & & 100 & -0.4 & -8.3 & 0.6 & 0.5 & 0.9 & -0.5 \\
$A_{C P}^{\rho \pi}$ & & & 100 & -3.7 & -8.0 & -5.9 & -0.4 & 0.8 \\
$A_{C P}^{\rho K}$ & & & & 100 & 0.7 & 0.5 & -0.9 & 1.0 \\
$C_{\rho \pi}$ & & & & & 100 & 17.6 & -10.0 & -8.7 \\
$\Delta C_{\rho \pi}$ & & & & & & 100 & -8.7 & -10.2 \\
$S_{\rho \pi}$ & & & & & & & 100 & 23.0 \\
$\Delta S_{\rho \pi}$ & & & & & & & & 100 \\
\hline \hline
\end{tabular}

Tableau 4.19: Matrice de corrélations (en \%) entre les principaux paramètres de l'ajustement final.

de l'angle Cherenkov pour les traces célibataires $\pi$ et $K$.

Sur la figure 4.39 représentant $m_{\mathrm{ES}}$ séparément pour $\rho^{+} \pi^{-}$et $\rho^{-} \pi^{+}$, on peut directement observer l'asymétrie de charge $A_{C P}^{\rho \pi}=-0.18 \pm 0.08$. La figure 4.40 montre l'asymétrie $A_{B^{0}} / \bar{B}^{0}$ entre les étiquettes $B^{0}$ et $\bar{B}^{0}$ pour les événements $\rho \pi$ dans les catégories d'étiquetage Lepton et Kaon, ainsi que l'asymétrie $A_{\rho^{+} \pi^{-} / \rho^{-} \pi^{+}}$pour toutes les catégories d'étiquetage.

\subsubsection{Niveau de confiance des résultats}

Nous estimons le niveau de confiance d'obtenir en l'absence de violation de $C P$ des valeurs pour les asymétries $C P$ égales à celles obtenues lors de l'ajustement nominal. Le niveau de confiance est obtenu en calculant la différence entre la valeur de $-2 \ln (\mathcal{L})$ obtenue dans l'ajustement nominal, et celle obtenue dans un ajustement où certains paramètres $C P$ sont fixés à zéro. Nous obtenons $\mathrm{CL}=1.5 \cdot 10^{-2}$ (ce qui correspond à $2.4 \sigma$ ) lorsque l'on demande $A_{C P}^{\rho \pi}=C=0$ (pas de violation de $C P$ directe), et $\mathrm{CL}=2.4 \cdot 10^{-2}(2.3 \sigma)$ en demandant $A_{C P}^{\rho \pi}=C=S=0$ (aucune violation de $C P$ ). Ces résultats concernent les erreurs statistiques seulement.

De façon similaire, nous calculons la signification statistique pour les nombres d'événements de signal: fixer $N_{\rho \pi}=0$ dans l'ajustement résulte en $|\Delta \ln (\mathcal{L})|=205.1$, soit $20.3 \sigma$. Fixer $N_{\rho K}=0$ résulte en $|\Delta \ln (\mathcal{L})|=31.8$, soit $8.0 \sigma$.

\subsubsection{Qualité de l'ajustement}

Une mesure qualitative de la qualité de l'ajustement des données peut se faire grâce au rapport des fonctions de vraisemblance ${ }^{17}$ :

$$
r_{i, c}^{\rho h[\mathrm{tot}]}=\frac{\mathcal{P}_{i, c}^{\rho h[\mathrm{tot}]}}{\mathcal{P}_{i, c}},
$$

défini pour chaque événement $i$ appartenant à la catégorie d'étiquetage $c$. Les données sont comparées aux fonctions de vraisemblance du signal et des bruits de fond correctement normalisées au résultat de l'ajustement par intégration Monte Carlo. Les résultats sont montrés

terme qui sera parfois utilisé dans la suite.

${ }^{17} \mathrm{Ce}$ rapport est similaire à celui défini dans l'eq. 4.32 , mais borné entre 0 et 1 . Nous l'avons déjà utilisé dans la section 4.11.2.4 pour visualiser la présence de corrélations dans les données. 

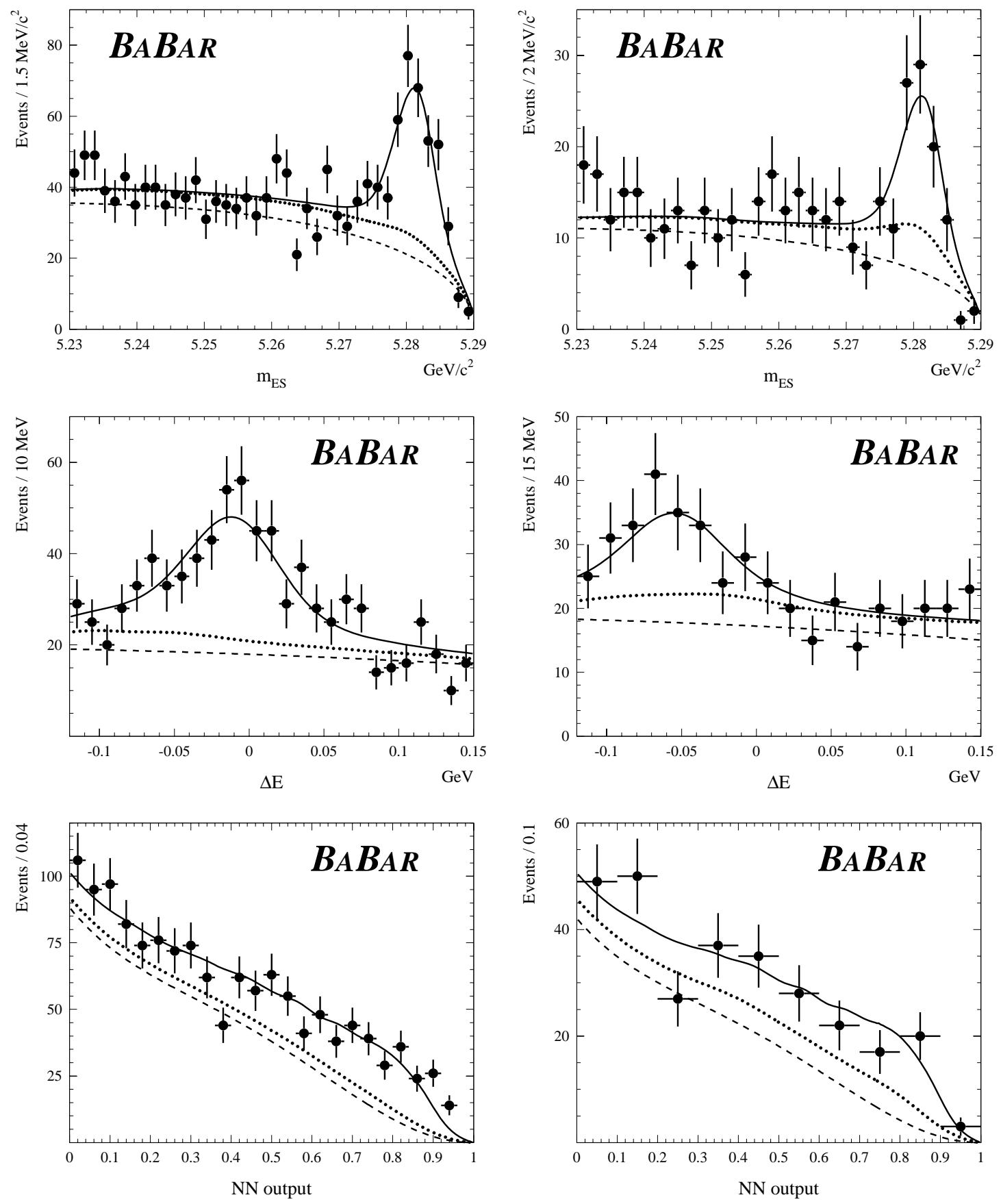

Figure 4.37: Distributions de $m_{\mathrm{ES}}$ (haut), $\Delta E$ (milieu), et $x_{\mathrm{NN}}$ (bas) pour des échantillons enrichis en signal $B^{0} \rightarrow \rho^{ \pm} \pi^{\mp}$ (gauche) et $B^{0} \rightarrow \rho^{-} K^{+}$(droite) grâce à une coupure sur des rapports de fonctions de vraisemblance $\mathcal{R}^{\rho h}$ (cf. Eq. 4.32). La courbe en trait plein montre la projection du résultat de l'ajustement du maximum de la fonction de vraisemblance. La courbe en tirets montre la contribution des événements de continuum (à la fois pour une trace célibataire $\pi$ et $K$ ), et la courbe pointillée montre les contributions du continuum et des bruits de fond $B$. 


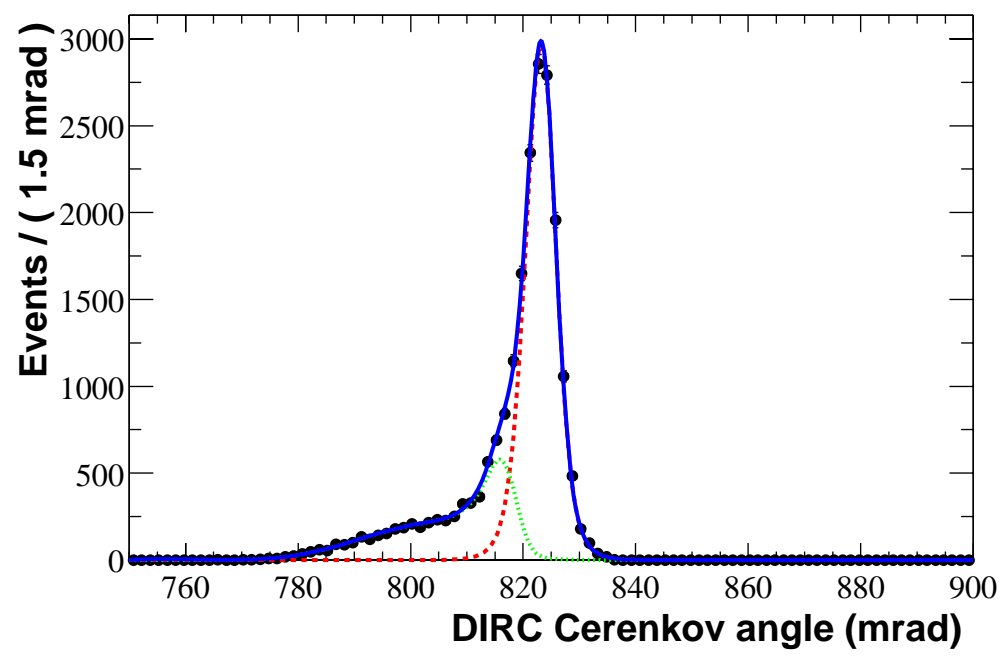

Figure 4.38: Distribution de l'angle Cherenkov de la trace célibataire des candidats ph de l'échantillon. La courbe en tirets montre la contribution des événements ayant une trace célibataire $\pi$, et la courbe pointillée celle correspondant aux $K$.
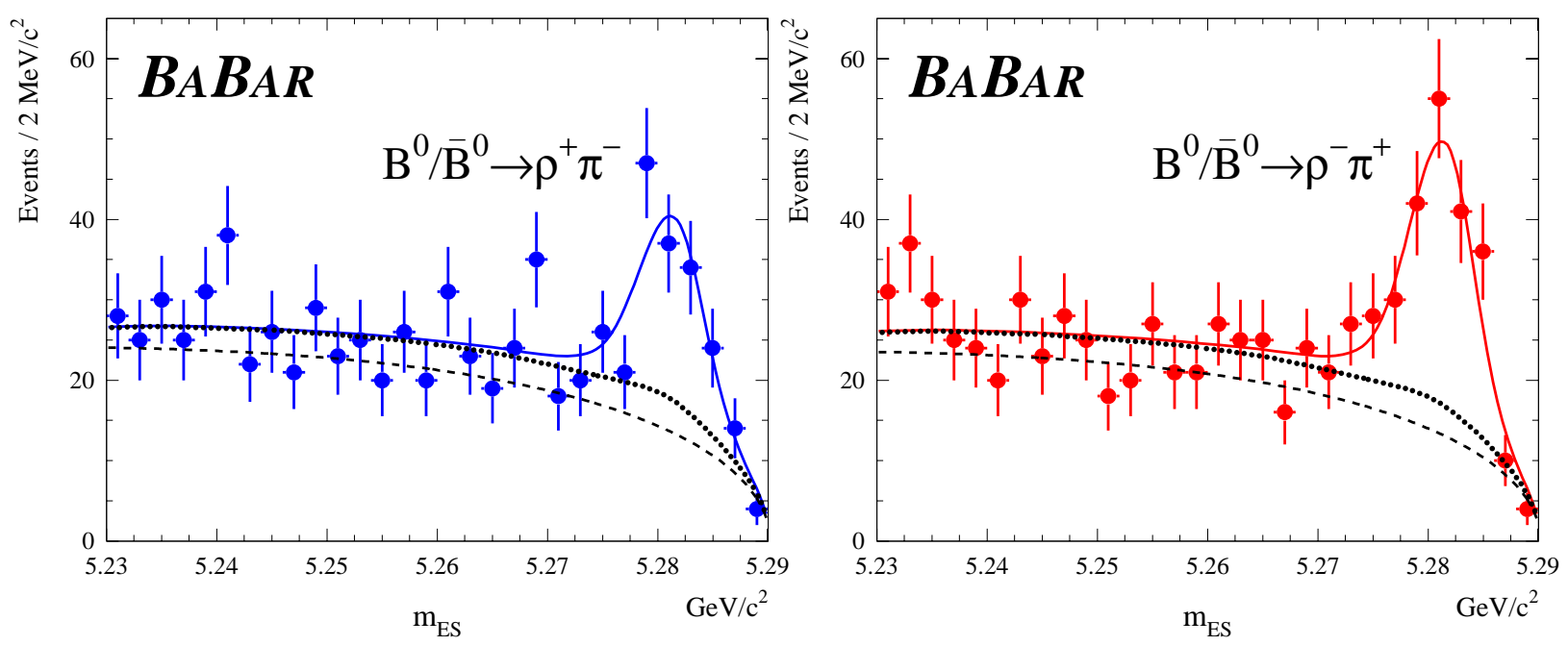

Figure 4.39: Distributions de $m_{\mathrm{ES}}$ pour des échantillons enrichis en signal $\rho^{+} \pi^{-}$(gauche) et $\rho^{-} \pi^{+}$(droite) grâce à une coupure sur des rapports de fonctions de vraisemblance. La courbe en trait plein montre la projection du résultat de l'ajustement du maximum de la fonction de vraisemblance. La courbe en tirets montre la contribution des événements de continuum (à la fois pour une trace célibataire $\pi$ et $K$ ), et la courbe pointillée montre les contributions du continuum et des bruits de fond $B$, incluant $\rho K$. 

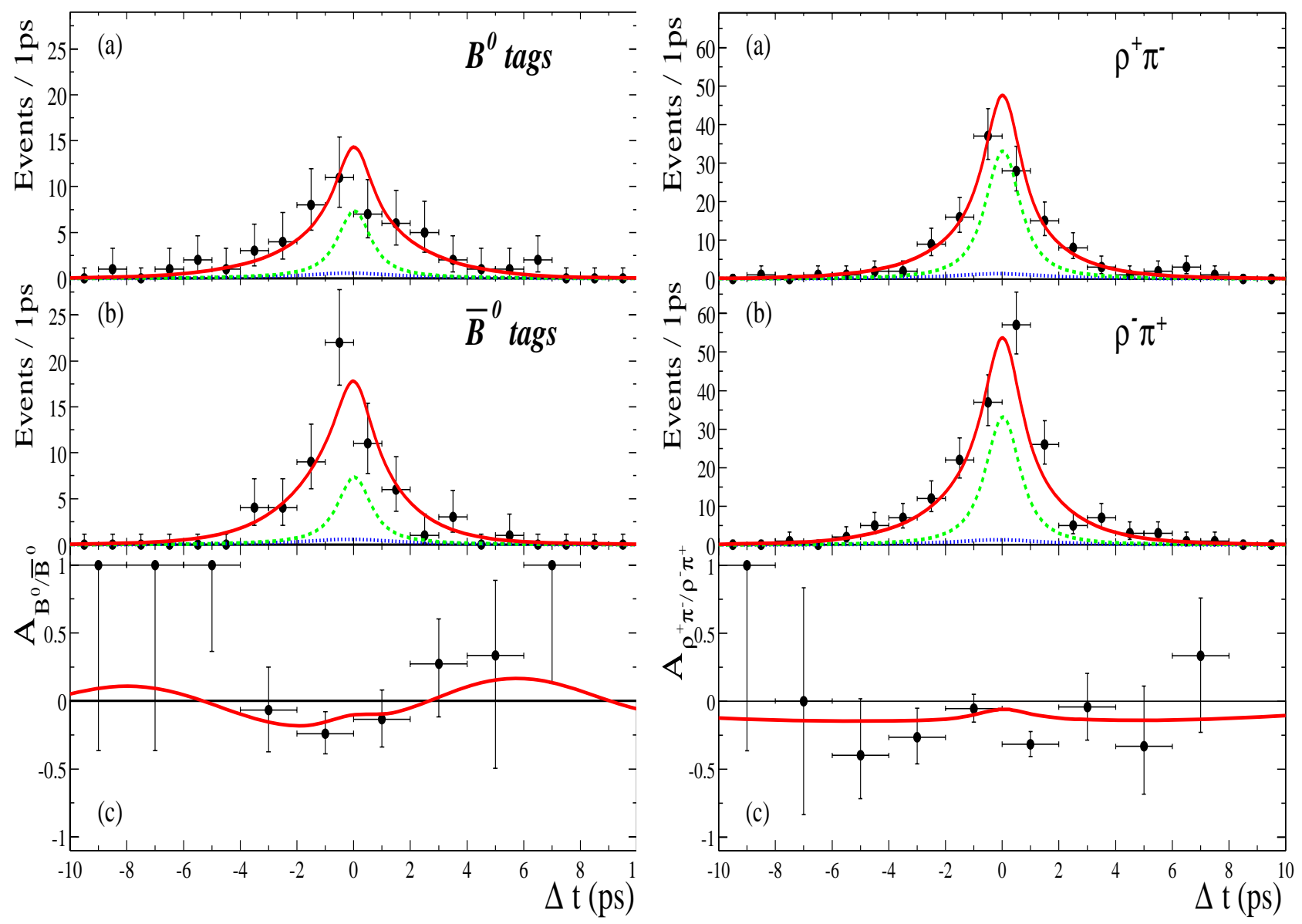

Figure 4.40: Figure de gauche: Distribution de $\Delta t$ et de l'asymétrie $A_{B^{0}} \bar{B}^{\circ}$ dans les catégories d'étiquetage Lepton et Kaon. Figure de droite: Distribution de $\Delta t$ et de l'asymétrie $A_{\rho^{+} \pi^{-} / \rho^{-} \pi^{+}}$entre $\rho^{+} \pi^{-}$et $\rho^{-} \pi^{+}$sur l'ensemble des catégories d'étiquetage. L'échantillon enrichi en signal $\rho \pi$ grâce $\grave{a}$ une coupure sur des rapports de fonctions de vraisemblance. La courbe en trait plein montre la projection du résultat de l'ajustement du maximum de la fonction de vraisemblance. La courbe pointillée montre la contribution des bruits de fond $B$, et la courbe en tirets montre les contributions du continuum et des bruits de fond $B$. La dépression aux alentours de 0 est due à la fraction de continuum plus large dans cette région de $\Delta t$. La valeur non-nulle de $S_{\rho \pi}$ introduit une asymétrie impaire dans la courbe de $A_{B^{0}} / \bar{B}^{0}$. En l'absence de bruit de fond continuum, la courbe $A_{\rho^{+} \pi^{-} / \rho^{-} \pi^{+}}$serait plate et égale à $A_{\mathrm{CP}}^{\rho \pi}$. 
sur la figure 4.41: on observe un bon accord qualitatif entre les données et la somme des fonctions de vraisemblance.
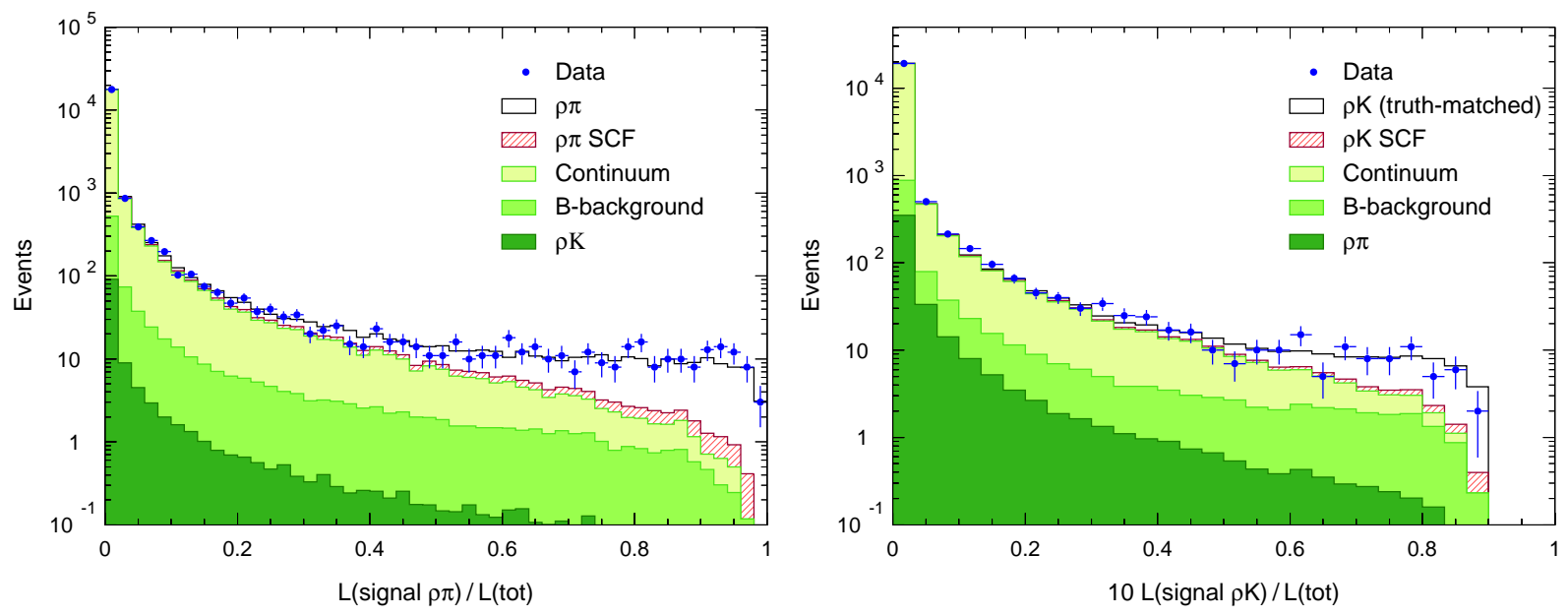

Figure 4.41: Distributions des projections de vraisemblance (Eq. 4.33) de $B^{0} \rightarrow \rho^{ \pm} \pi^{\mp}$ (gauche) et $B^{0} \rightarrow \rho^{-} K^{+}$(droite) pour les données, et les contributions obtenues par simulation rapide.

On peut mesurer quantitativement la qualité de l'ajustement de la façon suivante: on obtient une distribution de $-\ln \mathcal{L}$ à partir de simulations rapides que l'on compare à la valeur obtenue dans l'ajustement nominal sur les données. La distribution issue des simulations rapides est montrée sur la figure 4.42, et une flèche indique la valeur de la fonction de vraisemblance de l'ajustement nominal.

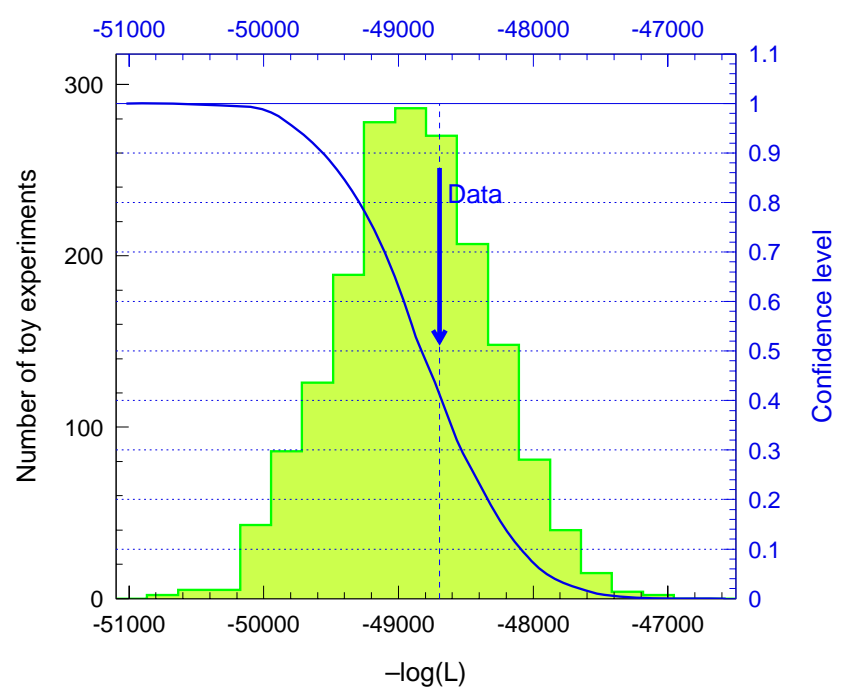

Figure 4.42: Distribution de $-\ln \mathcal{L}$ pour des expériences de simulations rapides. La flèche indique la valeur de la fonction de vraisemblance de l'ajustement nominal.

Le niveau de confiance de l'ajustement est ensuite calculé en intégrant la distribution de $-\ln \mathcal{L}$ (par la droite) jusqu'à la valeur nominale: le niveau de confiance ainsi obtenu est de $42 \%$.

Néanmoins, puisque l'échantillon contient essentiellement des événements de continuum, ce niveau de confiance concerne surtout la qualité de l'ajustement du continuum. Pour tester la qualité du signal, nous sélectionnons un échantillon des données enrichi en signal en 


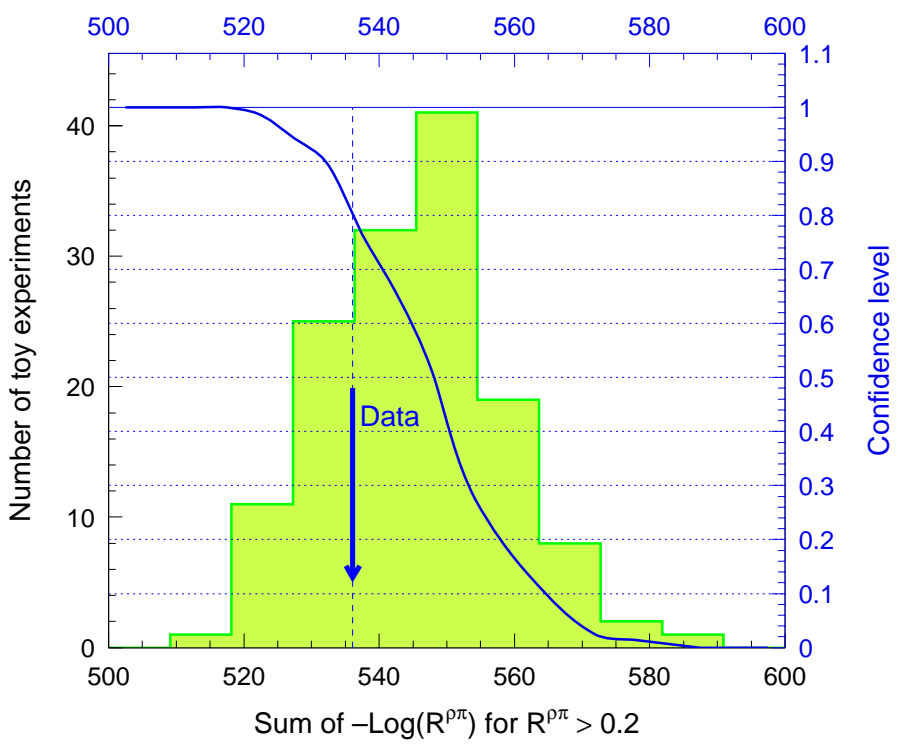

Figure 4.43: Distribution de $-\ln \left(r^{\rho h[\mathrm{tot}]}\right)$ pour des expériences de simulation rapide dans un échantillon de données enrichi en signal. La courbe en trait plein indique l'intégration de la distribution menant au niveau de confiance. La flèche montre la valeur obtenue lors de l'ajustement nominal sur les données on-peak.

appliquant une coupure sur la fonction de vraisemblance $\left(r_{i, c}^{\rho h \text { [tot] }}>0.2\right)$. Cette coupure possède une efficacité de $62 \%$ pour le signal $B^{0} \rightarrow \rho^{ \pm} \pi^{\mp}$ : il reste ainsi 649 événements dans l'échantillon obtenu, dont 271 événements de signal $B^{0} \rightarrow \rho^{ \pm} \pi^{\mp}$.

La figure 4.43 montre la distribution de $-\ln \mathcal{L}$ pour des expériences de simulation rapide après coupure. On obtient alors un niveau de confiance de $80 \%$.

\subsubsection{Nombre d'événements $\rho \pi$ pour une charge de $\rho$ et une saveur de $B^{0}$ données}

Nous définissons un "nombre d'événements" pour une charge de $\rho$ et une saveur de $B^{0}$ données $\operatorname{par}^{18}$ :

$$
\begin{array}{lll}
N^{+-}=N\left(B^{0} \rightarrow \rho^{+} \pi^{-}\right) & \propto\left|A\left(B^{0} \rightarrow \rho^{+} \pi^{-}\right)\right|^{2}, \\
N^{-+}=N\left(B^{0} \rightarrow \rho^{-} \pi^{+}\right) & \propto\left|A\left(B^{0} \rightarrow \rho^{-} \pi^{+}\right)\right|^{2}, \\
\bar{N}^{+-}=|q / p| N\left(\bar{B}^{0} \rightarrow \rho^{+} \pi^{-}\right) & \propto|q / p|\left|A\left(\bar{B}^{0} \rightarrow \rho^{+} \pi^{-}\right)\right|^{2}, \\
\bar{N}^{-+} & =|q / p| N\left(\bar{B}^{0} \rightarrow \rho^{-} \pi^{+}\right) & \propto|q / p|\left|A\left(\bar{B}^{0} \rightarrow \rho^{-} \pi^{+}\right)\right|^{2},
\end{array}
$$

où $A$ est l'amplitude de la désintégration considérée.

Pour chaque catégorie de saveur $f$ et de charge $Q$, le nombre d'événements $N(f, Q)$ est donné par:

$$
N(f, Q)=\frac{1}{4}\left(1+Q A_{C P}\right)(1+f(C+Q \Delta C)) N_{\rho \pi},
$$

où $f\left(B^{0}\right)=1$ et $f\left(\bar{B}^{0}\right)=-1$. On suppose $|q / p|=1$. En prenant en compte les corrélations entre les différents paramètres $\left(-61 \%\right.$ entre $N^{+-}$et $\bar{N}^{+-}$, ainsi qu'entre $N^{-+}$et $\left.\bar{N}^{-+}\right)$, on

\footnotetext{
${ }^{18}$ La notion de nombre d'événements n'est pas rigoureusement définie à cause de l'interférence entre les $B^{0}$ et $\bar{B}^{0}$.
} 
obtient:

$$
\begin{aligned}
& N^{+-}=144_{-27}^{+30} \pm 11, \\
& N^{-+}=137_{-32}^{+34} \pm 11, \\
& \bar{N}^{+-}=32_{-25}^{+26} \pm 6, \\
& \bar{N}^{-+}=116_{-31}^{+33} \pm 10,
\end{aligned}
$$

oì les premières erreurs sont statistiques, et les secondes systématiques. Ces dernières sont discutées dans la section 4.14. Les valeurs des paramètres $C P$ et de dilution obtenues se traduisent donc en un déficit du nombre d'événements $\bar{B}^{0} \rightarrow \rho^{+} \pi^{-}$.

\subsubsection{Paramétrisation physique de la violation de $C P$ directe}

Dans la paramétrisation des taux de désintégration dépendant du temps de $B^{0} \rightarrow \rho^{ \pm} \pi^{\mp}$ utilisée jusque là, la violation de $C P$ directe correspond aux paramètres $C$ et $A_{C P}$. Pratiques d'un point de vue expérimental ( $C$ est un coefficient du cosinus, et $A_{C P}$ une normalisation relative entre $\rho^{+} \pi^{-}$et $\left.\rho-\pi^{+}\right)$, ces paramètres sont plus difficiles à interpréter au niveau de la physique.

Nous définissons les deux paramètres $A_{\rho \pi}^{+-}$et $A_{\rho \pi}^{-+}[24]$ à partir des paramètres $C, \Delta C$ et $A_{C P}$ comme (on suppose $|q / p|=1$ ):

$$
\begin{aligned}
& A_{\rho \pi}^{-+}=\frac{N\left(\bar{B}^{0} \rightarrow \rho^{+} \pi^{-}\right)-N\left(B^{0} \rightarrow \rho^{-} \pi^{+}\right)}{N\left(\bar{B}^{0} \rightarrow \rho^{+} \pi^{-}\right)+N\left(B^{0} \rightarrow \rho^{-} \pi^{+}\right)}=\frac{A_{\mathrm{CP}}^{\rho \pi}-C-A_{\mathrm{CP}}^{\rho \pi} \cdot \Delta C}{1-\Delta C-A_{\mathrm{CP}}^{\rho \pi} \cdot C}, \\
& A_{\rho \pi}^{+-}=\frac{N\left(\bar{B}^{0} \rightarrow \rho^{-} \pi^{+}\right)-N\left(B^{0} \rightarrow \rho^{+} \pi^{-}\right)}{N\left(\bar{B}^{0} \rightarrow \rho^{-} \pi^{+}\right)+N\left(B^{0} \rightarrow \rho^{+} \pi^{-}\right)}=-\frac{A_{\mathrm{CP}}^{\rho \pi}+C+A_{\mathrm{CP}}^{\rho \pi} \cdot \Delta C}{1+\Delta C+A_{\mathrm{CP}}^{\rho \pi} \cdot C} .
\end{aligned}
$$

Ces asymétries comparent les taux des désintégrations conjuguées de $C P$, et sont similaires aux asymétries habituellement utilisées pour la violation de $C P$ directe dans les désintégrations des $B$ chargés. Si l'un de ces paramètres est non-nul, alors nous sommes en présence de violation de $C P$ directe.

D'autre part, ces deux asymétries correspondent à deux topologies de diagrammes différentes: $A_{\rho \pi}^{-+}$correspond aux diagrammes où le $\rho$ est formé à partir du quark spectateur du $B$, alors que $A_{\rho \pi}^{+-}$correspond aux diagrammes où le $\rho$ est issu du $W$.

A partir des valeurs nominales $C, \Delta C$ et $A_{C P}$ du tableau 4.18, nous obtenons:

$$
A_{\rho \pi}^{-+}=-0.62_{-0.28}^{+0.24} \pm 0.06, \quad A_{\rho \pi}^{+-}=-0.11_{-0.17}^{+0.16} \pm 0.04
$$

avec une corrélation linéaire de $51 \%$ entre ces deux variables. Les erreurs systématiques associées à ces paramètres sont discutées dans la section 4.14 .

La figure 4.44 montre les niveaux de confiance des paramètres $A_{\rho \pi}^{+-}$et $A_{\rho \pi}^{-+}$(utilisant les erreurs statistiques seulement).

\subsection{Validations a posteriori}

Une fois le résultat final dévoilé, nous réalisons plusieurs tests de validation supplémentaires. 


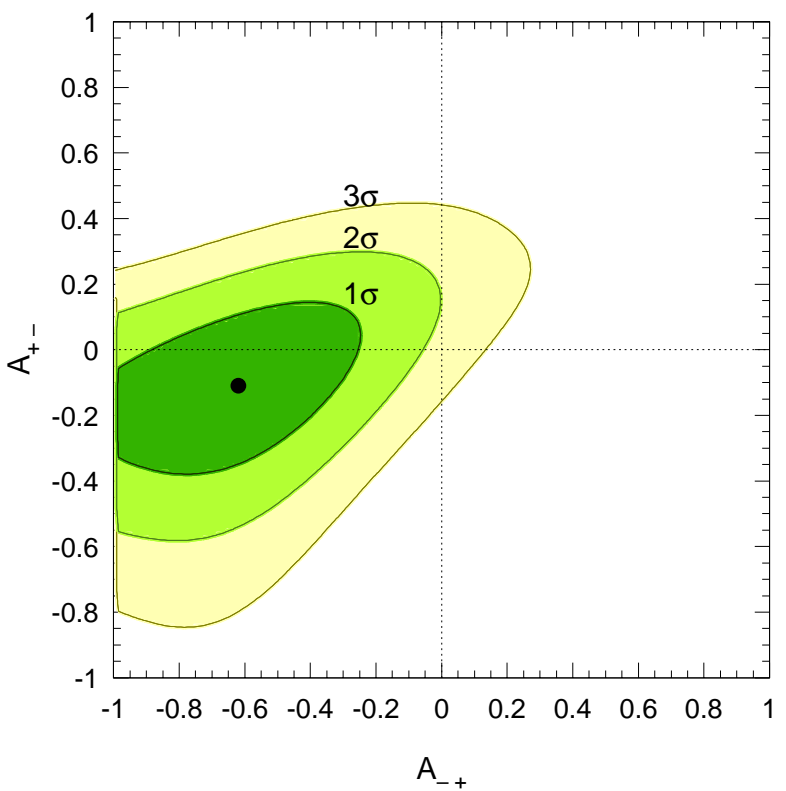

Figure 4.44: Niveaux de confiance des paramètres $A_{\rho \pi}^{+-}$et $A_{\rho \pi}^{-+}$(pour les erreurs statistiques seulement).

\subsubsection{Différentes configurations d'ajustements}

Plusieurs ajustements dans différentes configurations sont réalisés soit pour vérifier la stabilité des résultats, soit pour quantifier les contributions des bruits de fond (en particulier, du bruit de fond $B$ qui soustrait 68 événements à $B^{0} \rightarrow \rho^{ \pm} \pi^{\mp}$ et 52 à $\left.B^{0} \rightarrow \rho^{-} K^{+}\right)$. Lorsque $\tau$ et $\Delta m_{d}$ sont laissés libres dans l'ajustement, les résultats obtenus pour ces deux paramètres sont compatibles avec ceux du PDG [44].

Aucune anomalie n'est à noter dans ces ajustements de validation.

\subsubsection{Vérifications sur $\Delta C_{K}, \Delta S_{K}$ et $C_{K}$}

Les paramètres $\Delta S_{K}$ et $C_{K}$ sont fixés à 0 dans l'ajustement nominal, et le paramètres $\Delta C_{K}$ à -1 , comme dicté par le caractère auto-étiquetant de ce mode ( $c f$. section 1.3.4). Nous réalisons un ajustement avec ces paramètres libres. Les résultats sont donnés par le tableau 4.21, et correspondent aux valeurs attendues, dans la mesure de la (faible) sensibilité que nous possédons sur ces paramètres.

\subsubsection{Information contenue dans $m(\rho)$ et $\cos \theta_{H}(\rho)$}

Nous vérifions la compatibilité des événements de signal obtenus avec la présence d'un $\rho$.

Une évaluation qualitative de cette compatibilité consiste à projeter les fonctions de vraisemblance des variables $m(\rho)$ et $\cos \theta_{H}(\rho)$ des données comme nous l'avons fait pour les autres variables ( $c f$. figure 4.37 ). Néanmoins, dans l'ajustement nominal, ces deux variables sont utilisées dans le réseau de neurones sur lequel nous avons appliqué une coupure: les distributions obtenues sont donc biaisées (le continuum pique à la masse du $\rho$ ).

Nous réalisons une nouvelle analyse sans utiliser les deux variables $m(\rho)$ et $\cos \theta_{H}(\rho)$ dans le réseau de neurones (utilisant alors uniquement $L_{0}$ et $L_{2}$ ). Les détails relatifs à cette analyse sont donnés dans la référence [39]. 


\begin{tabular}{|c|c|c|c|c|c|c|c|c|c|c|}
\hline & $\overline{N_{\rho \pi}}$ & $\overline{\overline{N_{\rho K}}}$ & $\overline{A_{C P}^{\rho \pi}}$ & $\overline{A_{C P}^{\rho K}}$ & $\bar{C}$ & $\overline{\Delta C}$ & $\bar{S}$ & $\overline{\Delta S}$ & $\bar{\tau}$ & $\overline{\Delta \Delta m}$ \\
\hline $\begin{array}{l}\text { Ajustement } \\
\text { nominal }\end{array}$ & $\begin{array}{l}428.0 \\
\pm 34.0\end{array}$ & $\begin{array}{l}120.4 \\
\pm 20.7\end{array}$ & $\begin{array}{l}-0.18 \\
\pm 0.08\end{array}$ & $\begin{array}{c}0.28 \\
\pm 0.17\end{array}$ & $\begin{array}{c}0.36 \\
\pm 0.18\end{array}$ & $\begin{array}{c}0.28 \\
\pm 0.18\end{array}$ & $\begin{array}{c}0.19 \\
\pm 0.24\end{array}$ & $\begin{array}{c}0.15 \\
\pm 0.25\end{array}$ & 1.542 & 0.489 \\
\hline $\begin{array}{c}\text { Ajustement } \\
\text { nominal MINOS }\end{array}$ & $\begin{array}{l}428.0 \\
+33.9 \\
-33.3 \\
\end{array}$ & $\begin{array}{l}120.4 \\
+20.7 \\
-20.1 \\
\end{array}$ & $\begin{array}{l}-0.18 \\
+0.08 \\
-0.08\end{array}$ & $\begin{array}{l}0.28 \\
+0.17 \\
-0.17 \\
\end{array}$ & $\begin{array}{r}0.36 \\
+0.18 \\
-0.18 \\
\end{array}$ & $\begin{array}{l}0.28 \\
+0.18 \\
-0.19 \\
\end{array}$ & $\begin{array}{l}0.19 \\
+0.24 \\
-0.24\end{array}$ & $\begin{array}{l}0.15 \\
+0.25 \\
-0.25 \\
\end{array}$ & 1.542 & 0.489 \\
\hline $\begin{array}{c}\text { Sans modes } \\
K^{* *}\end{array}$ & $\begin{array}{l}431.1 \\
\pm 33.9\end{array}$ & $\begin{array}{l}142.9 \\
\pm 21.1\end{array}$ & $\begin{array}{l}-0.18 \\
\pm 0.08\end{array}$ & $\begin{array}{c}0.24 \\
\pm 0.14\end{array}$ & $\begin{array}{c}0.36 \\
\pm 0.18\end{array}$ & $\begin{array}{c}0.27 \\
\pm 0.18\end{array}$ & $\begin{array}{c}0.19 \\
\pm 0.24\end{array}$ & $\begin{array}{c}0.15 \\
\pm 0.25\end{array}$ & 1.542 & 0.489 \\
\hline $\begin{array}{l}\text { Sans modes } K^{* *} \text {, } \\
\text { et charme }\end{array}$ & $\begin{array}{l}441.9 \\
\pm 34.2\end{array}$ & $\begin{array}{l}145.2 \\
\pm 21.1\end{array}$ & $\begin{array}{l}-0.17 \\
\pm 0.08\end{array}$ & $\begin{array}{c}0.23 \\
\pm 0.14\end{array}$ & $\begin{array}{c}0.36 \\
\pm 0.18\end{array}$ & $\begin{array}{c}0.25 \\
\pm 0.18\end{array}$ & $\begin{array}{c}0.20 \\
\pm 0.23\end{array}$ & $\begin{array}{c}0.15 \\
\pm 0.24\end{array}$ & 1.542 & 0.489 \\
\hline $\begin{array}{l}\text { Sans bruit de } \\
\text { fond } B\end{array}$ & $\begin{array}{l}495.8 \\
\pm 35.0\end{array}$ & $\begin{array}{l}171.8 \\
\pm 21.8\end{array}$ & $\begin{array}{l}-0.15 \\
\pm 0.07\end{array}$ & $\begin{array}{c}0.20 \\
\pm 0.12\end{array}$ & $\begin{array}{c}0.30 \\
\pm 0.16\end{array}$ & $\begin{array}{c}0.21 \\
\pm 0.17\end{array}$ & $\begin{array}{c}0.23 \\
\pm 0.20\end{array}$ & $\begin{array}{c}0.13 \\
\pm 0.21\end{array}$ & 1.542 & 0.489 \\
\hline $\begin{array}{l}\tau \text { laissé } \\
\text { libre }\end{array}$ & $\begin{array}{l}423.0 \\
\pm 34.3\end{array}$ & $\begin{array}{l}118.8 \\
\pm 20.6\end{array}$ & $\begin{array}{l}-0.18 \\
\pm 0.08\end{array}$ & $\begin{array}{c}0.28 \\
\pm 0.17\end{array}$ & $\begin{array}{c}0.37 \\
\pm 0.18\end{array}$ & $\begin{array}{c}0.28 \\
\pm 0.19\end{array}$ & $\begin{array}{c}0.18 \\
\pm 0.24\end{array}$ & $\begin{array}{c}0.14 \\
\pm 0.25\end{array}$ & $\begin{array}{l}1.64 \\
\pm 0.13\end{array}$ & 0.489 \\
\hline $\begin{array}{c}\Delta m \text { laissé } \\
\text { libre }\end{array}$ & $\begin{array}{l}427.9 \\
\pm 33.9\end{array}$ & $\begin{array}{l}120.2 \\
\pm 20.7\end{array}$ & $\begin{array}{l}-0.18 \\
\pm 0.08\end{array}$ & $\begin{array}{c}0.28 \\
\pm 0.17\end{array}$ & $\begin{array}{c}0.37 \\
\pm 0.19\end{array}$ & $\begin{array}{c}0.29 \\
\pm 0.19\end{array}$ & $\begin{array}{c}0.19 \\
\pm 0.24\end{array}$ & $\begin{array}{c}0.15 \\
\pm 0.25\end{array}$ & 1.542 & $\begin{array}{c}0.517 \\
\pm 0.115\end{array}$ \\
\hline Sans $\Delta t$ & $\begin{array}{l}439.2 \\
\pm 40.7\end{array}$ & $\begin{array}{l}132.6 \\
\pm 24.3\end{array}$ & - & - & - & - & - & - & - & - \\
\hline Sans $x_{\mathrm{NN}}$ & $\begin{array}{l}430.1 \\
\pm 38.4\end{array}$ & $\begin{array}{l}124.9 \\
\pm 23.4\end{array}$ & $\begin{array}{l}-0.13 \\
\pm 0.09\end{array}$ & $\begin{array}{c}0.41 \\
\pm 0.18\end{array}$ & $\begin{array}{c}0.29 \\
\pm 0.20\end{array}$ & $\begin{array}{c}0.15 \\
\pm 0.20\end{array}$ & $\begin{array}{c}0.21 \\
\pm 0.25\end{array}$ & $\begin{array}{c}0.23 \\
\pm 0.26\end{array}$ & 1.542 & 0.489 \\
\hline Sans $\Delta E$ & $\begin{array}{l}419.2 \\
\pm 36.2\end{array}$ & $\begin{array}{l}125.9 \\
\pm 23.2\end{array}$ & $\begin{array}{l}-0.19 \\
\pm 0.09\end{array}$ & $\begin{array}{c}0.34 \\
\pm 0.18\end{array}$ & $\begin{array}{c}0.32 \\
\pm 0.20\end{array}$ & $\begin{array}{c}0.25 \\
\pm 0.21\end{array}$ & $\begin{array}{c}0.17 \\
\pm 0.26\end{array}$ & $\begin{array}{c}0.12 \\
\pm 0.27\end{array}$ & 1.542 & 0.489 \\
\hline
\end{tabular}

Tableau 4.20: Ajustements dans différentes configurations.

\begin{tabular}{|c|c|c|c|c|c|c|c|c|c|c|}
\hline \hline & $A_{C P}^{\rho \pi}$ & $A_{C P}^{\rho K}$ & $C$ & $\Delta C$ & $S$ & $\Delta S$ & $\Delta C_{\rho K}$ & $S_{\rho K}$ & $\Delta S_{\rho K}$ & $\ln (\mathcal{L})$ \\
\hline Ajustement & -0.18 & 0.28 & 0.36 & 0.28 & 0.19 & 0.15 & -1 & 0 & 0 & 48695.5 \\
nominal & \pm 0.08 & \pm 0.17 & \pm 0.18 & \pm 0.18 & \pm 0.24 & \pm 0.25 & & & & \\
\hline$\Delta C_{\rho K}$ & -0.18 & 0.29 & 0.36 & 0.28 & 0.19 & 0.15 & -1.04 & 0 & 0 & 48695.5 \\
libre & \pm 0.08 & \pm 0.17 & \pm 0.18 & \pm 0.18 & \pm 0.24 & \pm 0.25 & \pm 0.39 & & & \\
\hline$S_{\rho K}$ & -0.18 & 0.30 & 0.36 & 0.28 & 0.21 & 0.15 & -1 & -0.80 & 0 & 48696.4 \\
libre & \pm 0.08 & \pm 0.17 & \pm 0.18 & \pm 0.18 & \pm 0.24 & \pm 0.25 & & \pm 0.90 & & \\
\hline$\Delta S_{\rho K}$ & $-\mathbf{0 . 1 8}$ & 0.32 & 0.36 & 0.28 & 0.19 & $\mathbf{0 . 1 7}$ & -1 & $\mathbf{0}$ & $-\mathbf{0 . 8 0}$ & 48696.5 \\
libre & $\pm \mathbf{0 . 0 8}$ & $\pm \mathbf{0 . 1 7}$ & \pm 0.18 & \pm 0.18 & \pm 0.24 & \pm 0.25 & & & \pm 0.98 & \\
\hline \hline
\end{tabular}

Tableau 4.21: Ajustements avec $\Delta C_{k}, \Delta S_{k}$ et $C_{k}$ libres.

\subsubsection{Projections de $m\left(\rho^{ \pm}\right)$et $\cos \theta_{H}(\rho)$}

La figure 4.45 montre la projection des fonctions de vraisemblance du signal et bruits de fond continuum et $B$ dans un échantillon de données enrichi en signal obtenue dans l'analyse décrite au paragraphe précédent.

De vrais $\rho$ sont produits dans les bruit de fond continuum et $B$, et ces derniers piquent donc légèrement à la masse $d u \rho$. Par contre, le fait que $\cos \theta_{H}(\rho)$ pique à \pm 1 pour le continuum n'est pas dû̀ à la polarisation des $\rho$, car celle-ci est nulle. Les événements à $\cos \theta_{H}(\rho)= \pm 1$ sont ceux possédant un $\pi^{0}$ mou ou une trace molle. Ces dernières sont présentes en abondance dans le bruit de fond continuum, et donc plus d'événements du continuum passent les coupures de sélection dans cette région de l'espace de phase. 


\section{A projection plot for $\rho$ mass}

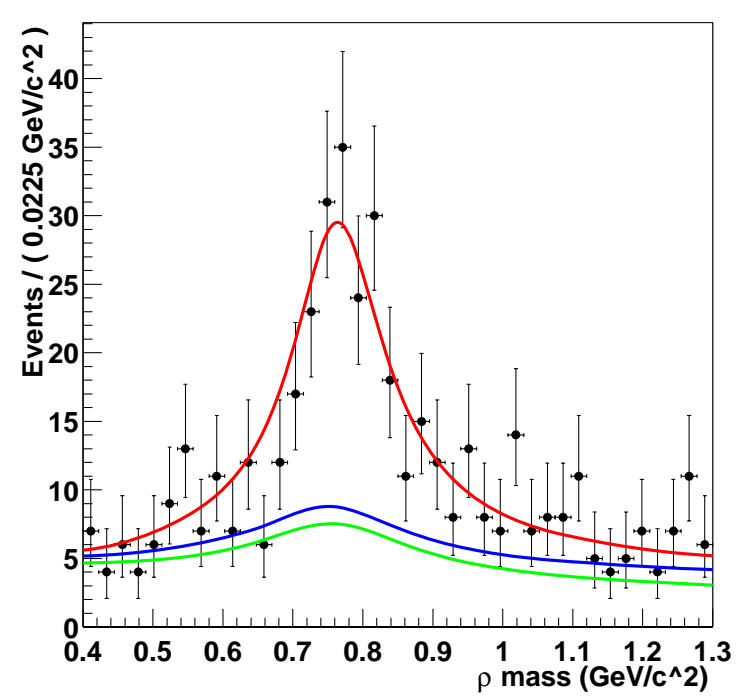

\section{A projection plot for $\rho$ mass}

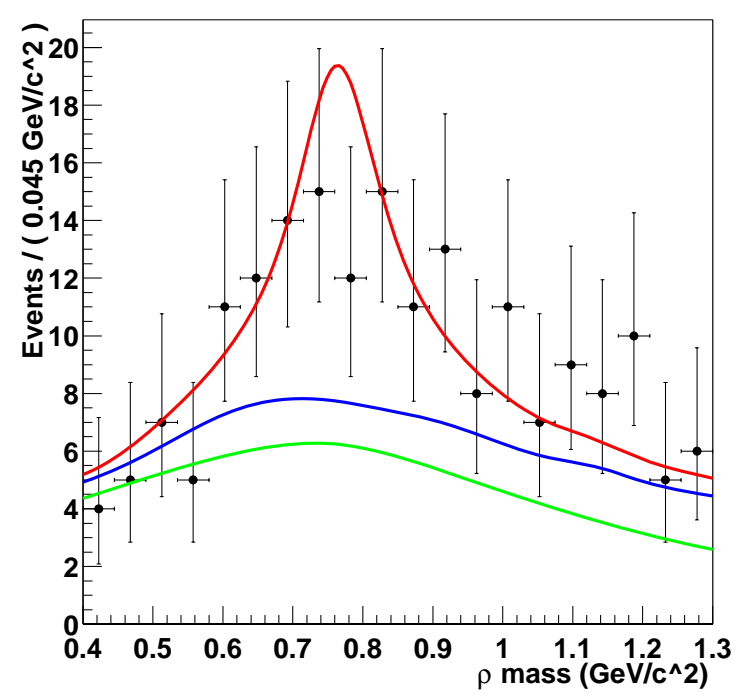

\section{A projection plot for $\cos (\rho$ helicity}

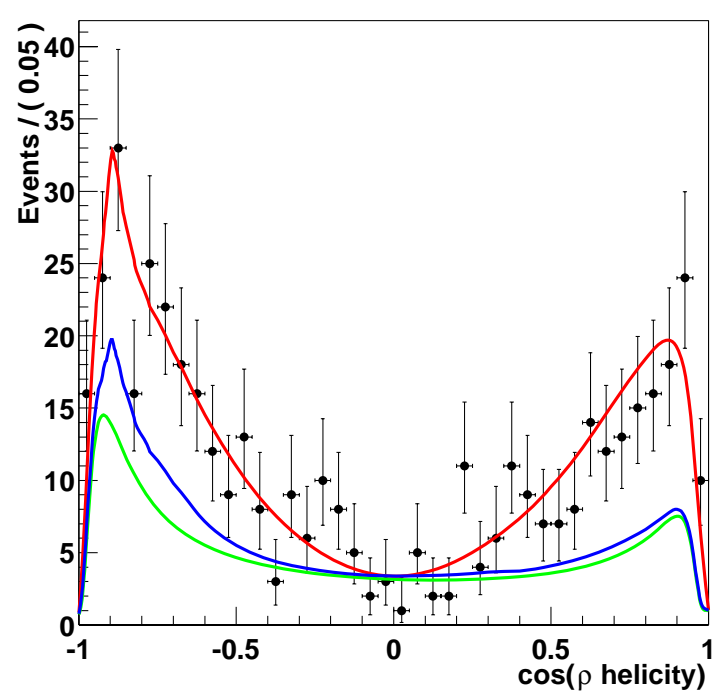

A projection plot for $\cos (\rho$ helicity $)$

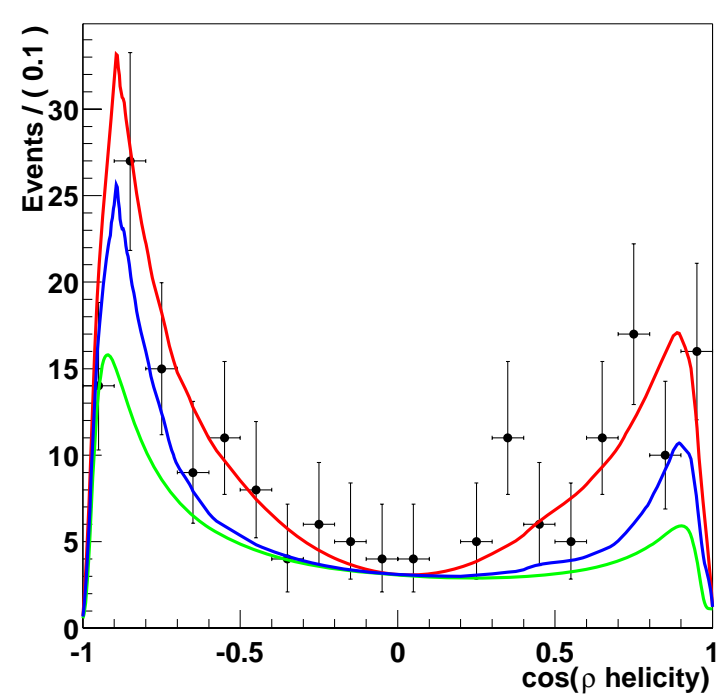

Figure 4.45: Distributions de $m(\rho)$ (figure de gauche) et $\cos \theta_{H}(\rho)$ (figure de droite) pour une trace célibataire $\pi$ (haut) et $K$ (bas) dans les données on-peak (points avec barres d'erreurs) après coupure sur le rapport des fonctions de vraisemblance. La courbe verte (resp. bleue et rouge) montre la fonction de vraisemblance du bruit de fond continuum (resp. du bruit de fond $B$ et du signal). 


\subsubsection{Ajustements avec et sans $m\left(\rho^{ \pm}\right)$}

Une estimation quantitative de la compatibilité du signal avec la présence d'un $\rho$ consiste à comparer les résultats obtenus dans la configuration exposée ci-dessus $(c-\grave{a}-d$, avec le réseau de neurones $L_{0}, L_{2}$ uniquement) sans et avec utilisation de la variable $m(\rho)$ lors de l'ajustement: la présence d'autres types d'événements de signal (e.g., $B^{0} \rightarrow \pi^{+} \pi^{-} \pi^{0}$ nonrésonnant) se traduirait dans des nombres d'événements inférieurs dans l'ajustement avec $m\left(\rho^{ \pm}\right)$à comparé de celui sans $m\left(\rho^{ \pm}\right)$.

Nous obtenons une différence entre les ajustements sans $m\left(\rho^{ \pm}\right)$et avec $m\left(\rho^{ \pm}\right)$de -0.4 événements de $B^{0} \rightarrow \rho^{ \pm} \pi^{\mp}$ et de -7.1 événements pour $B^{0} \rightarrow \rho^{-} K^{+}$. Nous évaluons le niveau de confiance des fluctuations statistiques entre les deux résultats obtenus grâce à des simulations rapides (où les ajustements sans et avec $m(\rho)$ se font sur les mêmes échantillons générés): nous obtenons $(97 \pm 1) \%$ pour $B^{0} \rightarrow \rho^{ \pm} \pi^{\mp}$ et $(42 \pm 3) \%$ pour $B^{0} \rightarrow \rho^{-} K^{+}$. Les résultats sont donc parfaitement compatibles et confirment la domination du $\rho$ dans les événements de signal ajustés.

\subsection{Etudes des erreurs systématiques}

Avec la statistique actuelle, on s'attend à ce que l'erreur statistique des paramètres mesurés domine l'erreur systématique. Les principales sources d'incertitudes systématiques sont énumérées ci-dessous.

\subsubsection{Reconstruction des $\pi^{0}$}

Des corrections doivent être appliquées aux Monte Carlo pour tenir compte des différences dans la reconstruction des traces neutres $\left(\gamma\right.$ et $\left.\pi^{0}\right)$ entre le Monte Carlo et les données. Pour les $\pi^{0}$, ces corrections sont de trois types [86]:

- Efficacité de reconstruction des $\pi^{0}$ : des événements $e^{+} e^{-} \rightarrow \tau^{+} \tau^{-}$sont utilisés pour mesurer le rapport des efficacités $\epsilon\left(2 \pi^{0}\right) / \epsilon\left(1 \pi^{0}\right)$ en fonction de l'énergie des $\pi^{0}$. Cette étude conclue que l'efficacité de reconstruction des $\pi^{0}$ est sur-évaluée par environ 3\% dans le Monte Carlo. La différence entre données et Monte Carlo est appliquée comme correction au Monte Carlo par une méthode de "killing" ( $c$-à- $d$ certains événements du Monte Carlo sont supprimés afin que l'efficacité reproduise celle des données).

- Résolution sur la masse des $\pi^{0}$ : les événements $e^{+} e^{-} \rightarrow \tau^{+} \tau^{-}$sont aussi utilisés pour mesurer la différence de résolution sur $m\left(\pi^{0}\right)$ entre les données et le Monte Carlo en fonction de l'énergie. Cette différence varie entre 0 et $3 \%$.

- Décalage sur l'énergie des neutres: il existe un décalage sur l'énergie reconstruite pour certains cristaux de l'EMC. Des corrections sont appliquées sur le Monte Carlo pour tenir compte de cet effet.

L'erreur systématique associée à ces corrections est constituée de deux sources [86]: une somme en quadrature d'erreurs corrélées dues au mécanisme d'évaluation des corrections (comme les incertitudes sur les rapports d'embranchement des désintégrations des $\tau$, les incertitudes lors des ajustements des données, etc.), d'un total de $5 \%$, et une erreur non corrélée de $1 \%$ associée à la méthode de correction de l'efficacité elle-même. 
La figure 4.46 montre la comparaison des distributions de $\Delta E, m_{\mathrm{ES}}$ et $x_{\mathrm{NN}}$ pour le Monte Carlo $B^{0} \rightarrow \rho^{ \pm} \pi^{\mp}$ corrigé ("smeared") pour la reconstruction des $\pi^{0}$, et non-corrigé ("not smeared"). La distribution la plus affectée est $\Delta E$ (par le décalage de l'énergie des neutres).
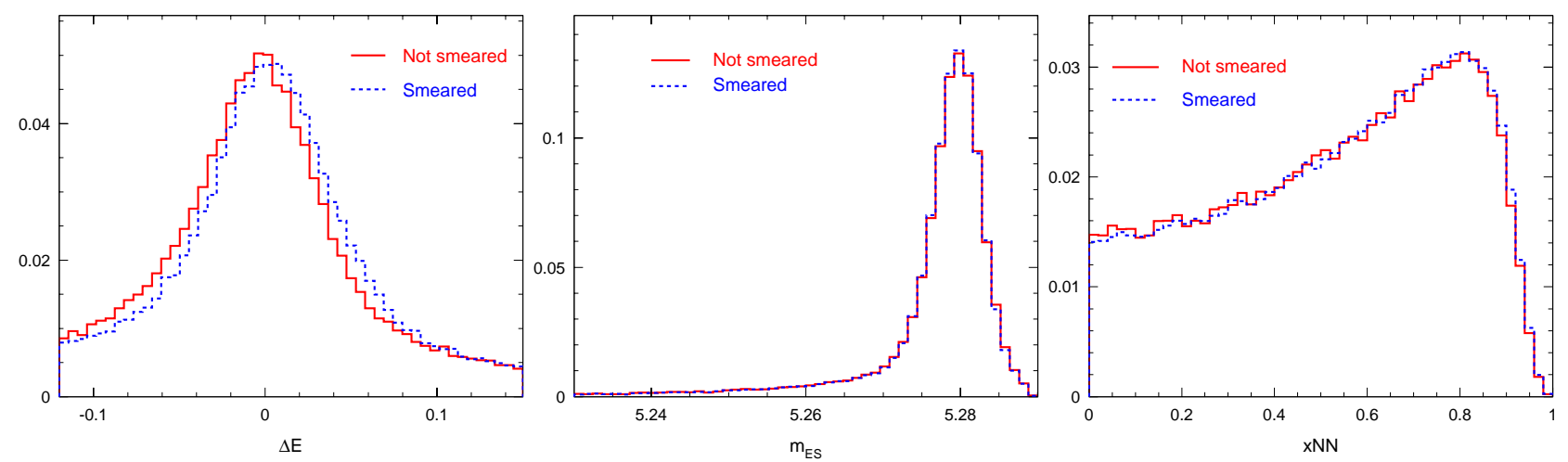

Figure 4.46: Comparaison des distributions de $\Delta E, m_{\mathrm{ES}}$ et $x_{\mathrm{NN}}$ pour le Monte Carlo $B^{0} \rightarrow$ $\rho^{ \pm} \pi^{\mp}$ corrigé ("smeared") pour la reconstruction des $\pi^{0}$ (courbe bleue), et non-corrigée ("not smeared", courbe rouge).

\subsubsection{Validations du signal en utilisant le mode $B^{0} \rightarrow D^{-} \rho^{+}$}

Les propriétés du mode $B^{0} \rightarrow D^{-} \rho^{+}$sont similaires à celles de $B^{0} \rightarrow \rho h$ : il s'agit d'une désintégration en deux corps avec un $\rho$ chargé dans l'état final. Ce mode n'étant pas supprimé de Cabibbo $\left(\mathcal{B}\left(B^{0} \rightarrow D^{-} \rho^{+}\right)=(7.8 \pm 1.4) \times 10^{-3}[44]\right)$, nous bénéficions d'un grand échantillon d'événements que nous pouvons utiliser pour étudier les différences entre Monte Carlo et données.

Pour la reconstruction de ce mode, nous appliquons une sélection similaire à celle de l'analyse courante. Après soustraction du bruit de fond continuum et $B$, nous disposons de 8015 événements dans les données, et 9271 dans le Monte Carlo.

Les détails relatifs à cette analyse sont données dans la ref. [39]. Les conclusions sont résumées ici.

\subsubsection{Distribution de $x_{\mathrm{NN}}$}

La figure 4.47 montre la distribution de $x_{\mathrm{NN}}$ pour les données et le Monte Carlo $B^{0} \rightarrow D^{-} \rho^{+}$ (avec et sans les effets de détecteur). La figure montre aussi le rapport de la distribution des données sur celle du Monte Carlo, ajusté avec une droite dont la pente est compatible avec zéro à $2 \sigma$.

\subsubsection{Distributions de $\Delta E$ et $m_{\mathrm{ES}}$}

Les valeurs moyennes des distributions de signal correctement reconstruit de $\Delta E$ et $m_{\mathrm{ES}}$ sont laissées libres lors de l'ajustement final, et il n'y a donc pas d'erreurs systématiques associées à ces paramètres. Nous utilisons par contre l'échantillon $B^{0} \rightarrow D^{-} \rho^{+}$pour évaluer la systématique associée aux largeurs de ces distributions. Le rapport de ces largeurs obtenues dans le Monte Carlo et les données $B^{0} \rightarrow D^{-} \rho^{+}$est de 1.036 pour $\Delta E$ et 1.076 pour $m_{\mathrm{Es}}$. 

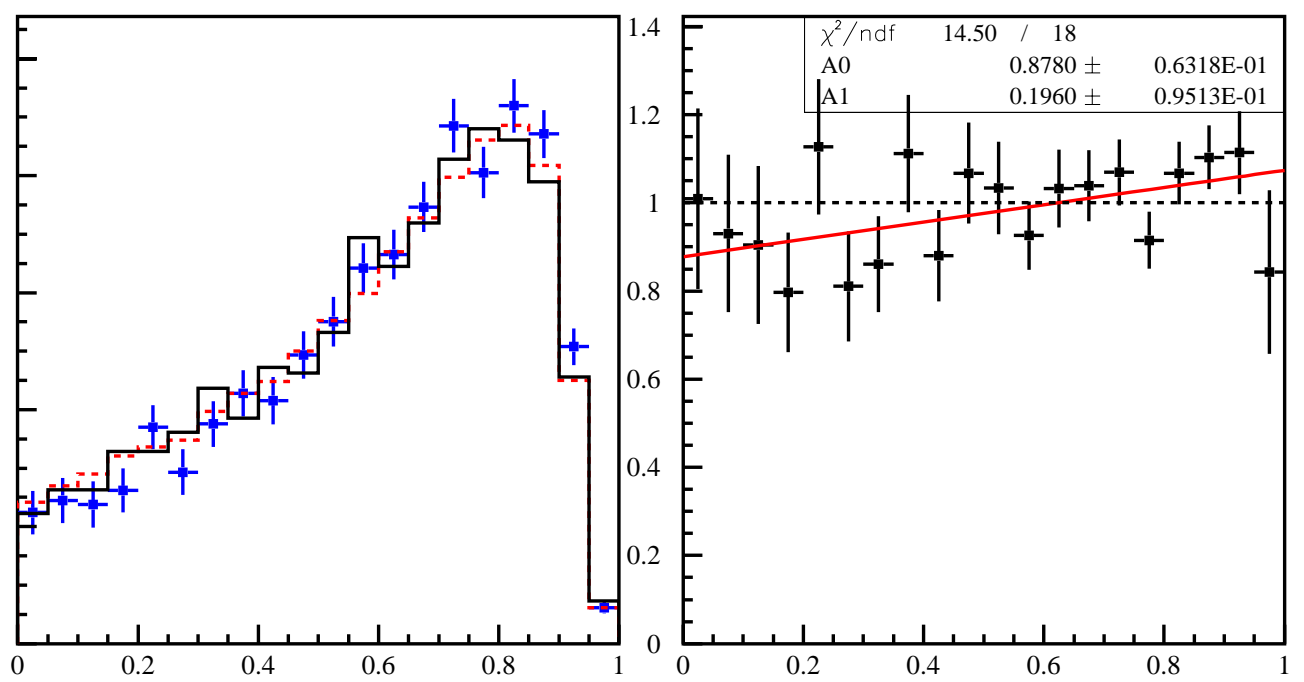

Figure 4.47: Figure de gauche: distribution de $x_{\mathrm{NN}}$ pour les données $B^{0} \rightarrow D^{-} \rho^{+}$(points avec barres d'erreurs), le Monte Carlo avec effets du détecteur (trait plein) et au niveau de la génération (pointillés). Figure de droite: rapport des données et du Monte Carlo $B^{0} \rightarrow$ $D^{-} \rho^{+}$, ajusté avec une droite.

\subsubsection{Fraction de signal mal reconstruit}

Nous voulons valider la fraction de signal mal reconstruit dans les événements $B^{0} \rightarrow D^{-} \rho^{+}$. Comme pour $B^{0} \rightarrow \rho h$, la composante de signal mal reconstruit est due aux $\pi^{0}$ de basse énergie. Le spectre d'impulsion des $\pi^{0}$ est différent dans $B^{0} \rightarrow D^{-} \rho^{+}$et $B^{0} \rightarrow \rho^{ \pm} \pi^{\mp}$, comme indiqué sur la figure 4.48 (puisque $m_{D} \gg m_{\pi}$ ). Nous corrigeons la distribution de $B^{0} \rightarrow D^{-} \rho^{+}$par une méthode de réjection pour obtenir la même distribution que le signal $B^{0} \rightarrow \rho^{ \pm} \pi^{\mp}$.

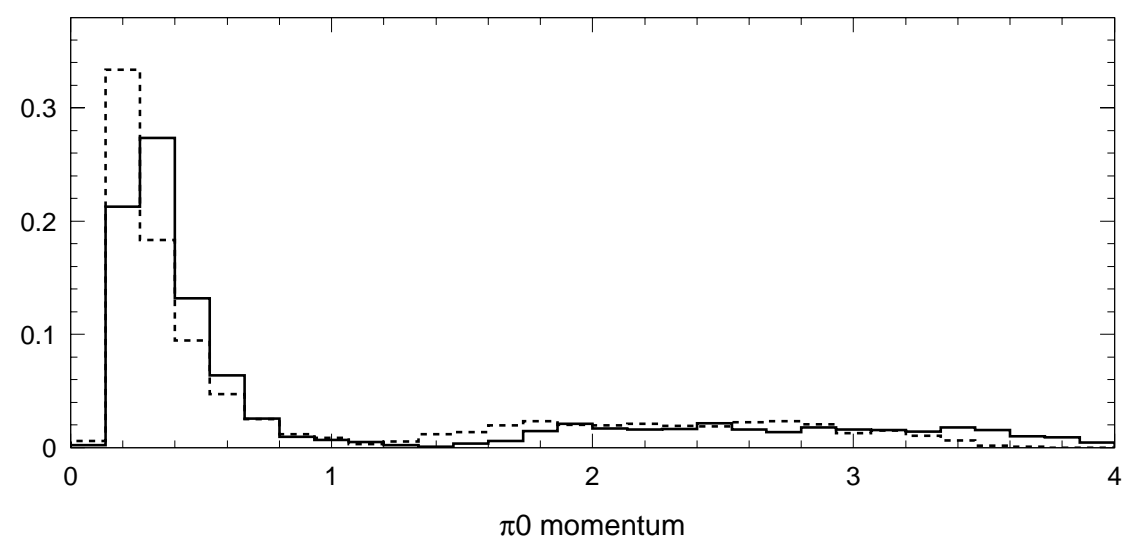

Figure 4.48: Distribution de l'impulsion du $\pi^{0}$ dans $B^{0} \rightarrow D^{-} \rho^{+}$(pointillés) et $B^{0} \rightarrow \rho^{ \pm} \pi^{\mp}$ (trait plein).

Nous mettons en place une procédure pour mesurer la fraction d'événements mal reconstruits dans les données $B^{0} \rightarrow D^{-} \rho^{+}$. Pour ce faire, nous séparons les différentes composantes de l'échantillon de données: signal correctement reconstruit, celui mal reconstruit, bruit de fond $B$, et bruit de fond continuum par une méthode que nous avons dénommée "pelure d'oignons". 
Un réseau de neurones comprenant les variables

$$
\left\{L_{0}, L_{2}, m_{\mathrm{ES}}, \Delta E, m(\rho), \cos \theta_{H}(\rho), p_{\mathrm{CMS}}\left(\pi^{0}\right), \mathrm{nCand}, \cos \theta(B, z), \cos \theta\left(T_{B}, z\right)\right\},
$$

où nCand est le nombre de candidats par événement, est entraîné successivement dans les configurations indiquées dans le tableau 4.22, permettant de séparer les composantes une par une en fixant les composantes déterminées à l'étape précédente. Ainsi, lors de la première étape, nous entraînons un réseau de neurones pour discriminer le continuum des autres composantes. Nous obtenons ainsi la contribution du continuum, que nous fixons dans la suite. Lors de la seconde étape, le réseau de neurones est entraîné pour discriminer le bruit de fond $B$ du signal mal reconstruit. Nous ajustons ainsi le bruit de fond $B$ en ayant fixé le continuum au résultat de la première étape. Enfin, la troisième étape distingue le signal correctement reconstruit de celui mal reconstruit.

\begin{tabular}{|c|l|l|l|l|}
\hline \multirow{2}{*}{ Etape } & \multicolumn{2}{|c|}{ Entraînement } & $\begin{array}{l}\text { Composante } \\
\text { ajustée }\end{array}$ & $\begin{array}{l}\text { Composantes } \\
\text { fixées }\end{array}$ \\
\cline { 2 - 3 } & Catégorie 1 & Catégorie 2 & continuum & aucune \\
\hline \hline 1 & continuum & le reste & contin de & continuum \\
\hline 2 & $\begin{array}{l}\text { bruit de } \\
\text { fond } B\end{array}$ & $\begin{array}{l}\text { signal mal } \\
\text { reconstruit }\end{array}$ & $\begin{array}{l}\text { bruit de } \\
\text { fond } B\end{array}$ & \\
\hline 3 & $\begin{array}{l}\text { signal corr. } \\
\text { reconstruit }\end{array}$ & $\begin{array}{l}\text { signal mal } \\
\text { reconstruit }\end{array}$ & $\begin{array}{l}\text { bruits de fond } \\
\text { cont. et } B\end{array}$ & continuum, $B$ \\
\hline
\end{tabular}

Tableau 4.22: Configurations des différentes étapes de la méthode de "pelure d'oignons". Pour chaque étape, le réseau de neurones est entraîné sur les catégories 1 et 2 . On ajuste ensuite la composante de la colonne "composante ajustée" alors que les composantes de la dernière colonne sont fixées.

La figure 4.49 montre les distributions de la sortie des réseaux de neurones lors de ces 3 étapes pour les différentes composantes en jeu.

Nous calculons la fraction d'événements mal reconstruits dans le Monte Carlo par la méthode précédente, ainsi que par une méthode de comptage standard, afin de vérifier la validité de la méthode appliquée sur les données. Nous obtenons des résultats en parfais accord à l'intérieur des erreurs statistiques de l'échantillon Monte Carlo $(c-\grave{a}-d, 3 \%)$.

Le rapport entre la fraction d'événements mal reconstruits dans les données et dans le Monte Carlo vaut:

$$
R=\frac{f_{\text {scf }}(\text { données })}{f_{\text {scf }}(\text { Monte Carlo })}=1.04 \pm 0.05
$$

L'erreur statistique sur la fraction d'événements mal reconstruits dans les données est la somme en quadrature de l'erreur obtenue dans l'ajustement et des erreurs des nombres de bruits de fond continuum et $B$.

Nous assignons une erreur systématique de $5 \%$ à la fraction d'événements mal reconstruits. Nous utilisons la même incertitude systématique pour la fraction d'événements de mauvaise charge.

\subsubsection{Fonction de résolution et étiquetage}

- Incertitudes sur $\Delta m_{d}$ et $\tau_{B}$ : l'erreur systématique due aux incertitudes sur $\Delta m_{d}$ et $\tau_{B}$ est obtenue en variant ces deux paramètres à l'intérieur de leur erreur statistique [44]. 


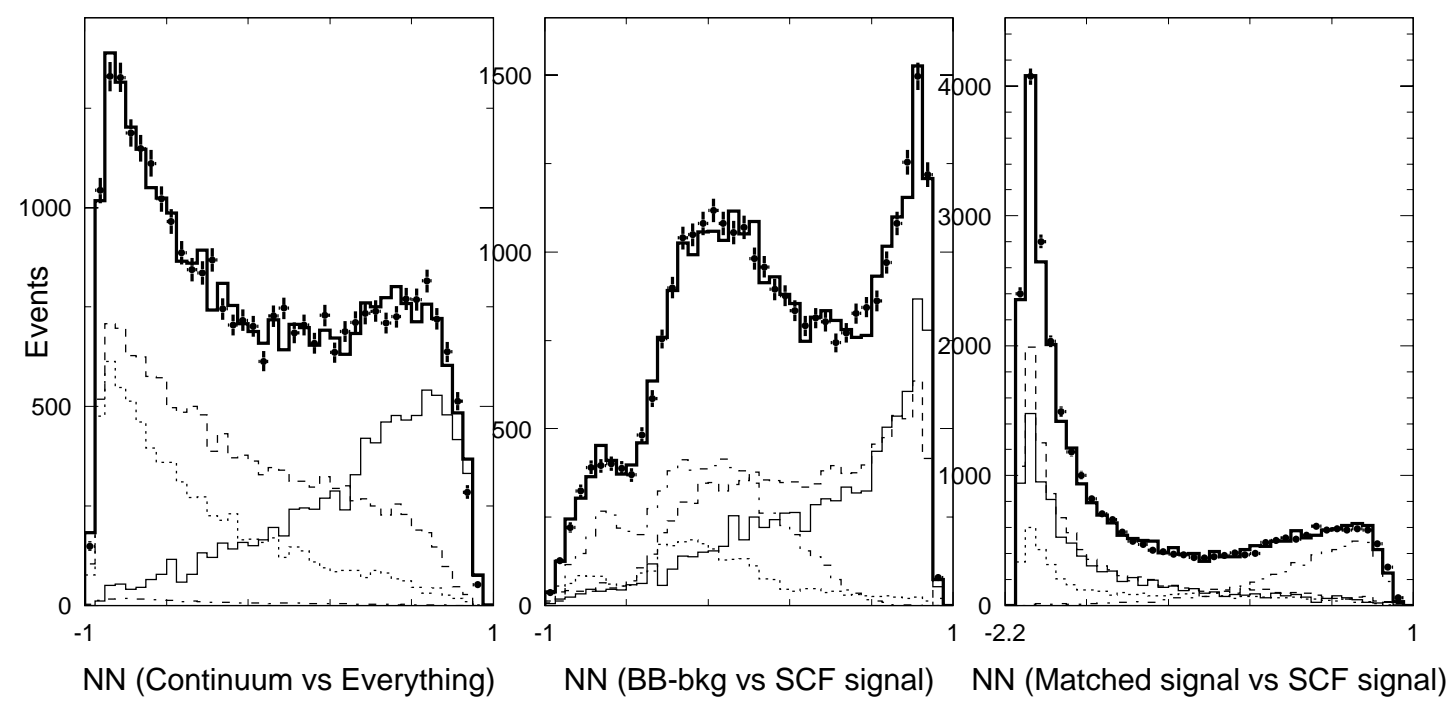

Figure 4.49: Distributions aux étapes 1 (gauche), 2 (milieu) et 3 (droite) de la sortie du réseau de neurones pour les composantes de signal correctement reconstruit (tirets-points), mal reconstruit (pointillés), bruit de fond $B$ (tirets) et continuum (trait plein), comparées aux événements on-peak (points avec barres d'erreurs).

- Fonction de résolution de $\Delta t$ : les 6 paramètres décrivant la fonction de résolution du bruit de fond continuum ( $c f$. Eq. 4.26) sont laissés libres lors de l'ajustement final, et il n'y a donc pas d'incertitude systématique supplémentaire à prendre en compte.

Les paramètres décrivant la fonction de résolution du signal ( $c f$. Eq. 4.25) sont obtenus grâce à un ajustement sur les événements Breco: nous varions ces paramètres à l'intérieur de leur incertitude statistique.

- Paramètres d'étiquetage: ils sont déterminés soit dans Breco (signal correctement reconstruit), soit dans le Monte Carlo (signal mal reconstruit): nous varions tous ces paramètres à l'intérieur de leur erreurs statistiques.

\subsubsection{Désintégrations doublement supprimées de Cabibbo}

Pour déterminer la saveur de $B_{\text {sav }}$, nous exploitons le caractère étiquetant d'un certain nombre de désintégrations, comme, par exemple, le signe du $D$ dans $B^{0} \rightarrow D^{*-} \rho^{+}$. Néanmoins, certaines d'entre elles ne sont pas à strictement parler des états propres de saveur à cause de la présence de désintégrations doublement supprimées de Cabibbo ("Doubly Cabibbo Suppressed Decays" en anglais, DCSD). Dans notre exemple précédent, il s'agit du mode $B^{0} \rightarrow D^{*+} \rho^{-}$. Les DCSD entraînent une modification des distributions temporelles de $B_{\text {sav }}$ et $B_{\text {rec }}$ [87] et donc une systématique sur sur les paramètres $C P$ et de dilution.

Nous estimons cette systématique par des simulations rapides dont les détails sont décrits dans la référence [39]. Les biais obtenus sont donnés dans le tableau 4.23. Les simulations ne tiennent pas compte de la fraction d'événements semi-leptoniques utilisés lors de l'étiquetage: or ces derniers ne souffrent pas de la contamination des DCSD. Les biais sont donc corrigés par un facteur de 1.6. Nous prenons la demi-largeur des distributions de biais (interprétation fréquentiste du résultat) comme erreur systématique. 


\begin{tabular}{cccccc}
\hline \hline Paramètre & généré & minimum & maximum & demi-largeur & RMS \\
\hline$C$ & 0.36 & 0.325 & 0.395 & 0.035 & 0.021 \\
$\Delta C$ & 0.27 & 0.235 & 0.304 & 0.035 & 0.011 \\
$S$ & 0.19 & 0.175 & 0.205 & 0.013 & 0.005 \\
$\Delta S$ & 0.14 & 0.128 & 0.151 & 0.012 & 0.003 \\
\hline \hline
\end{tabular}

Tableau 4.23: Résultats de l'évaluation des biais introduits par les DCSD par des simulations rapides. L intervalle donné par la demi-largeur correspond à une interprétation fréquentiste de l'erreur associée, alors que le RMS à une interprétation Bayesienne.

\subsubsection{Méthode d'ajustement}

Les biais observés lors de la validation de l'analyse par des ajustements de divers échantillons (voir section 4.11) sont répercutés comme incertitudes systématiques:

- les biais observés dans les ajustements sans bruits de fond $B$ décrits dans la section 4.11.2.3 sont corrigés dans les nombres d'événements de signal, auxquels on assigne une erreur systématique égale à ces biais.

- les biais observés dans les ajustements avec bruit de fond $B$ n'entraînent pas de corrections sur les nombres d'événements (car l'origine des biais n'est pas comprise, et ces biais sont moins significatifs du fait du nombre plus limité d'échantillons). Nous attribuons néanmoins une erreur systématique sur les nombres d'événements de signal égale à ces biais.

\subsubsection{Identification de la trace célibataire}

La distribution de référence de l'angle Cherenkov $\theta_{C}$ est décrite dans la section 4.9.1.4. On propage sur les paramètres mesurés les incertitudes statistiques des résolutions des deux Gaussiennes et de la fraction de la seconde Gaussienne (tout en gardant les moyennes à leur valeur nominale, 0 ) pour chacune des 4 catégories (type $=\pi, K ;$ charge $= \pm 1$ ).

D'autre part, nous réalisons un ajustement où les moyennes des deux Gaussiennes sont laissées libres, et nous prenons la différence obtenue sur les paramètres mesurés comme incertitude systématique supplémentaire.

\subsubsection{Bruit de fond $B$}

Les systématiques dues au bruit de fond $B$ (résumées dans le tableau 4.26) sont dues aux incertitudes sur les rapports d'embranchement, et sur les paramètres $C P$ et de dilution. Elles constituent les incertitudes systématiques principales pour l'analyse $B^{0} \rightarrow \rho h$.

\subsubsection{Incertitudes dues aux rapports d'embranchement}

L'incertitude due aux erreurs sur les rapports d'embranchement des modes $B$ est obtenue de la façon suivante:

- si le rapport d'embranchement est issu d'une mesure, nous varions ce rapport à l'intérieur de son incertitude statistique. 
- si le rapport d'embranchement est issu d'arguments théoriques, nous varions le rapport à l'intérieur de son intervalle. Notre approche Bayesienne pour l'évaluation de ces incertitudes nous amène à diviser l'erreur obtenue par $\sqrt{3}$ (qui est le facteur existant entre le RMS et la demi-largeur d'un intervalle).

- dans le cas des modes charmés, l'erreur systématique correspond à la différence entre le nombre d'événements extrapolé à partir du Monte Carlo générique, et celui obtenu par un ajustement direct des données sur l'intervalle $-0.35<\Delta E<0.15$ (comme décrit dans la section 4.8.2.2).

Les erreurs systématiques associées à chaque mode sont sommées en quadrature. Leur impact principal se retrouve sur les nombres d'événements de $B^{0} \rightarrow \rho^{ \pm} \pi^{\mp}$ (dont la systématique est dominée par $\left.B^{0} \rightarrow \rho^{+} \rho^{-}\right)$et de $B^{0} \rightarrow \rho^{-} K^{+}\left(\right.$dominé par $\left.B^{0} \rightarrow K_{X}^{(* *)} \pi\right)$.

\subsubsection{Incertitudes reliées aux paramètres $C P$ et de dilution}

Les incertitudes systématiques reliées aux paramètres $C P$ et de dilution du bruit de fond $B$ sont étudiées pour les modes dominants, tel que décrit dans le tableau 4.24. Ces paramètres sont variés à l'intérieur d'intervalles choisis selon les informations obtenues du Monte Carlo. Les incertitudes obtenues sont données dans le tableau 4.25.

\begin{tabular}{|c|c|c|c|c|c|c|c|}
\hline Param. & $\rho^{+} \rho^{-}$ & $\begin{array}{c}K^{*+} \pi^{-} \\
\text {et }\left(K^{* *} \pi\right)^{0}\end{array}$ & $b \rightarrow c$ & & & & \\
\hline 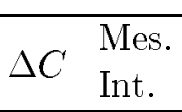 & $\begin{array}{c}0.11 \pm 0.04 \\
\pm 0.3\end{array}$ & $\begin{array}{l}\text { Tab. } 4.16 \\
\Delta C \pm 0.5\end{array}$ & $\begin{array}{c}- \\
\pm 0.5\end{array}$ & Param. & $\begin{array}{c}\rho^{+} \pi^{0} \\
\text { et } \rho^{0} \pi^{+}\end{array}$ & $\begin{array}{c}\rho^{0} K^{+} \\
\text {et } \pi^{0} K^{+}\end{array}$ & $b \rightarrow c$ \\
\hline \begin{tabular}{ll}
\multirow{2}{*}{$\Delta S$} & $\begin{array}{l}\text { Mes. } \\
\text { Int. }\end{array}$ \\
\end{tabular} & $\begin{array}{c}0.12 \pm 0.07 \\
\pm 0.3\end{array}$ & - & $\begin{array}{r}- \\
\pm 0.5 \\
\end{array}$ & \begin{tabular}{ll}
\multirow{2}{*}{$A_{\pi}$} & Mes. \\
& Int.
\end{tabular} & $\begin{array}{l}\text { Tab. } 4.16 \\
A_{\pi} \pm 0.5\end{array}$ & $\begin{array}{l}\text { Tab. } 4.16 \\
A_{\pi} \pm 0.5\end{array}$ & $\begin{array}{c}- \\
\pm 0.5\end{array}$ \\
\hline \begin{tabular}{ll}
\multirow{2}{*}{$C$} & Mes. \\
& Int. \\
\end{tabular} & $\begin{array}{c} \pm 0.71 \pm 0.06 \\
\pm 0.7\end{array}$ & - & $\begin{array}{c}- \\
\pm 0.5 \\
\end{array}$ & $\begin{array}{ll}A_{K} & \text { Mes. } \\
& \text { Int. }\end{array}$ & - & $\begin{array}{l}\text { Tab. } 4.16 \\
A_{K} \pm 0.5\end{array}$ & $\begin{array}{c}- \\
\pm 0.5 \\
\end{array}$ \\
\hline $\begin{array}{ll}S & \text { Mes. } \\
& \text { Int. }\end{array}$ & $\begin{array}{c}-0.28 \pm 0.07 \\
\pm 0.7\end{array}$ & - & $\begin{array}{r}- \\
\pm 0.5 \\
\end{array}$ & \begin{tabular}{ll}
\multirow{2}{*}{$A_{C P}^{\rho}$} & Mes. \\
& Int. \\
\end{tabular} & $\begin{array}{c} \pm 0.47 \pm 0.02 \\
\pm 0.5\end{array}$ & $\begin{array}{c}- \\
\pm 1 \\
\end{array}$ & $\begin{array}{r}- \\
\pm 0.5 \\
\end{array}$ \\
\hline $\begin{array}{ll}A_{C P}^{\rho} & \text { Mes. } \\
& \text { Int. } \\
\end{array}$ & $\begin{array}{l}- \\
-\end{array}$ & $\begin{array}{c}- \\
\pm 1 \\
\end{array}$ & $\begin{array}{c}- \\
\pm 0.5 \\
\end{array}$ & \begin{tabular}{ll}
\multirow{2}{*}{$A_{C P}^{K}$} & Mes. \\
& Int. \\
\end{tabular} & - & $\begin{array}{c}- \\
\pm 1 \\
\end{array}$ & $\begin{array}{c}- \\
\pm 0.5 \\
\end{array}$ \\
\hline $\begin{array}{ll}A_{C P}^{K} & \text { Mes. } \\
& \text { Int. } \\
\end{array}$ & $\begin{array}{l}- \\
- \\
\end{array}$ & $\begin{array}{c}- \\
\pm 1 \\
\end{array}$ & $\begin{array}{c}- \\
\pm 0.5 \\
\end{array}$ & & & & \\
\hline
\end{tabular}

Tablea1 4.24: Valeurs des paramètres $C P$ et de dilution pour les modes $B$ neutres (tableau de gauche) et des $B$ chargés (tableau de droite) mesurées ("Mes.") dans les Monte Carlo, à partir desquelles sont inférés des intervalles ("Int.") utilisés dans le calcul des incertitudes systématiques. Les intervalles sont centrée en zéro (syntaxe " $\pm 0.5 "$ ), ou autour de la valeur issue du Monte Carlo (syntaxe " $X \pm 0.5$ ").

\subsubsection{Incertitudes reliées aux autres hypothèses}

- $B^{0} \rightarrow \pi^{+} \pi^{-} \pi^{0}$ non-résonnant et contributions scalaires: nous avons supposé leur contribution nulle ( $c f$. tableau 4.10 et annexe D). Nous attribuons une erreur systématique liée à cette hypothèse en nous servant de la limite supérieure à $68 \% \mathrm{CL}$ 


\begin{tabular}{|c|c|c|c|c|c|c|c|c|}
\hline Mode & $N_{\rho K}$ & $N_{\rho \pi}$ & $A_{K}$ & $A_{\pi}$ & $C$ & $\Delta C$ & $S$ & $\Delta S$ \\
\hline$\rho^{+} \rho^{-}$long & 0.03 & 0.16 & 0.000 & 0.000 & 0.012 & 0.005 & 0.013 & 0.006 \\
$\rho^{+} \rho^{0}$ long & 0.09 & 0.10 & 0.004 & 0.003 & 0.000 & 0.004 & 0.001 & 0.000 \\
\hline$\rho^{0} \pi^{+}+\rho^{-} \pi^{0}$ & 0.26 & 0.10 & 0.025 & 0.011 & 0.011 & 0.015 & 0.002 & 0.003 \\
\hline$K^{*+}\left(K^{+} \pi^{0}\right) \pi^{-}$ & 0.14 & 0.30 & 0.016 & 0.019 & 0.008 & 0.010 & 0.005 & 0.001 \\
$\left(K^{* *} \pi\right)^{0}$ & 0.80 & 0.21 & 0.003 & 0.000 & 0.007 & 0.003 & 0.001 & 0.000 \\
\hline$\rho^{0} K^{+}$ & 0.26 & 0.09 & 0.025 & 0.000 & 0.000 & 0.001 & 0.000 & 0.000 \\
$\pi^{0} K^{+}$ & 0.15 & 0.06 & 0.011 & 0.000 & 0.000 & 0.000 & 0.000 & 0.000 \\
\hline Charme (chargé) & 0.08 & 0.30 & 0.014 & 0.008 & 0.007 & 0.013 & 0.001 & 0.001 \\
Charme (neutre) & 0.02 & 0.18 & 0.006 & 0.012 & 0.012 & 0.012 & 0.011 & 0.009 \\
\hline Total & 0.91 & 0.57 & 0.044 & 0.027 & 0.024 & 0.026 & 0.017 & 0.011 \\
\hline
\end{tabular}

Tableau 4.25: Incertitudes systématiques reliées aux paramètres $C P$ et de dilution.

sur la contribution non résonnante $\mathcal{B}\left(B^{0} \rightarrow \pi^{+} \pi^{-} \pi^{0}\right)<5 \cdot 10^{-6}$ issue des références [88, 89]. Ceci entraîne une diminution du nombre d'événements de $B^{0} \rightarrow \rho^{ \pm} \pi^{\mp}$ de 8.9 événements. Les paramètres $C P$ et de dilution de la contribution non résonnante sont variés entre \pm 1 .

- Efficacités d'étiquetage: les efficacités d'étiquetage sont calculées dans le Monte Carlo des bruits de fond $B$. Les erreurs statistiques de ces efficacités sont propagées comme erreur systématique sur les quantités mesurées. D'autre part, nous propageons aussi la différence entre les efficacités d'étiquetage calculées dans les Monte Carlo et celles de Breco.

- Distribution de $\Delta t$ : nous avons fait l'hypothèse que la fonction de résolution de $\Delta t$ pour les bruits de fond $B$ est la même que celle du signal. Néanmoins, dans les cas où une trace est prise du ROE pour former le candidat $B$, la fonction de résolution de $\Delta t$ peut être modifiée. C'est le cas par exemple du mode $B^{+} \rightarrow \rho^{+} \pi^{0}$. Cet effet peut être pris en compte en modifiant la durée de vie de la distribution en temps: nous évaluons que la différence observée correspond en moyenne à un décalage de 0.1 ps que nous propageons sur les quantités mesurées.

- Distributions de $\Delta E, m_{\mathrm{ES}}$ et $x_{\mathrm{NN}}$ : les mêmes corrections que pour le signal sont appliquées sur les distributions des bruits de fond $B$ prises des Monte Carlo (voir Sec. 4.14.5).

\subsubsection{Résumé des incertitudes systématiques liées aux bruits de fond $B$}

Le tableau 4.26 résume l'ensemble des incertitudes systématiques reliées au bruit de fond $B$. L'incertitude dominante pour les nombres d'événements de signal est liée aux incertitudes sur les rapports d'embranchement des bruits de fond $B$. L'incertitude dominante sur les paramètres $C P$ et de dilution est liée à ces mêmes paramètres dans les bruits de fond $B$. La contribution non-résonnante $\pi^{+} \pi^{-} \pi^{0}$ contribue significativement aux erreurs des paramètres reliés à $B^{0} \rightarrow \rho^{ \pm} \pi^{\mp}$.

\subsubsection{Résumé des incertitudes systématiques}

Le tableau 4.27 résume les incertitudes systématiques sur les paramètres mesurés. L'incertitude systématique dominante sur tous les paramètres provient du bruit de fond $B$, mis à part 


\begin{tabular}{l|c|c|c|c|c|c|c|c}
\hline \hline Source & $N_{\rho K}$ & $N_{\rho \pi}$ & $A_{K}$ & $A_{\pi}$ & $C$ & $\Delta C$ & $S$ & $\Delta S$ \\
\hline Rapports d'embranchement & 15.90 & 10.41 & 0.032 & 0.005 & 0.013 & 0.015 & 0.004 & 0.006 \\
Paramètres $C P$ et de dilution & 0.50 & 0.53 & 0.072 & 0.027 & 0.023 & 0.026 & 0.017 & 0.011 \\
$B^{0} \rightarrow \pi^{+} \pi^{-} \pi^{0}$ non résonant & 0.94 & 8.86 & 0.000 & 0.003 & 0.012 & 0.012 & 0.011 & 0.011 \\
Efficacité d'étiquetage & 0.27 & 0.22 & 0.002 & 0.002 & 0.004 & 0.013 & 0.002 & 0.003 \\
Distribution de $\Delta t$ & 0.60 & 1.40 & 0.002 & 0.001 & 0.001 & 0.000 & 0.002 & 0.002 \\
$\Delta E, m_{\mathrm{ES}}, x_{\mathrm{NN}}$ & 1.90 & 3.33 & 0.006 & 0.001 & 0.008 & 0.004 & 0.001 & 0.005 \\
\hline Total & 16.03 & 14.15 & 0.079 & 0.028 & 0.030 & 0.035 & 0.021 & 0.018 \\
\hline \hline
\end{tabular}

Tableau 4.26: Résumé des incertitudes systématiques provenant du bruit de fond $B$.

pour le nombre d'événements de signal pour lequel la systématique due aux biais observés lors des tests de validation sur Monte Carlo est du même ordre de grandeur.

\begin{tabular}{l|c|c|c|c|c|c|c|c}
\hline \hline Type & $N_{\rho K}$ & $N_{\rho \pi}$ & $A_{\mathrm{CP}}^{\rho K}$ & $A_{\mathrm{CP}}^{\rho \pi}$ & $C$ & $\Delta C$ & $S$ & $\Delta S$ \\
\hline$\Delta m \pm 0.008 \mathrm{ps}^{-1}$ & 0.1 & 0.1 & 0.000 & 0.000 & 0.004 & 0.004 & 0.002 & 0.001 \\
\hline$\tau \pm 0.016 p s$ & 0.1 & 0.1 & 0.000 & 0.000 & 0.000 & 0.000 & 0.000 & 0.000 \\
\hline Résolution de $\Delta t$ & 0.6 & 1.3 & 0.002 & 0.001 & 0.006 & 0.002 & 0.009 & 0.006 \\
\hline Etiquetage & 0.7 & 1.3 & 0.003 & 0.000 & 0.011 & 0.005 & 0.011 & 0.010 \\
\hline Frac. de mauvaise charge & 0.0 & 0.1 & 0.000 & 0.000 & 0.000 & 0.001 & 0.000 & 0.000 \\
\hline Identif. de la trace du $\rho$ & 0.2 & 0.1 & 0.000 & 0.000 & 0.000 & 0.000 & 0.000 & 0.000 \\
\hline Fraction de signal mal rec. & 1.9 & 5.4 & 0.000 & 0.000 & 0.002 & 0.001 & 0.002 & 0.001 \\
\hline Distr. du signal mal rec. & 0.0 & 0.2 & 0.000 & 0.000 & 0.000 & 0.000 & 0.000 & 0.001 \\
\hline Distributions du DIRC & 0.6 & 0.6 & 0.005 & 0.002 & 0.001 & 0.001 & 0.001 & 0.001 \\
\hline Distribution de $\Delta E$ & 1.0 & 1.4 & 0.002 & 0.001 & 0.004 & 0.002 & 0.002 & 0.003 \\
\hline Distribution de $m_{\mathrm{ES}}$ & 2.0 & 9.6 & 0.010 & 0.007 & 0.001 & 0.002 & 0.014 & 0.008 \\
\hline Distribution de $x_{\text {NN }}$ & 2.7 & 7.0 & 0.007 & 0.003 & 0.005 & 0.007 & 0.001 & 0.003 \\
\hline$A_{\text {mix }}^{\rho h}$ continuum & 0.8 & 0.5 & 0.001 & 0.002 & 0.008 & 0.006 & 0.006 & 0.006 \\
\hline Bruit de fond $B$ & $\mathbf{1 6 . 0}$ & $\mathbf{1 4 . 2}$ & $\mathbf{0 . 0 7 9}$ & $\mathbf{0 . 0 2 8}$ & $\mathbf{0 . 0 3 0}$ & $\mathbf{0 . 0 3 5}$ & $\mathbf{0 . 0 2 1}$ & $\mathbf{0 . 0 1 8}$ \\
\hline Méthode (simul. rapide) & 0.5 & 0.8 & 0.004 & 0.002 & 0.004 & 0.004 & 0.004 & 0.003 \\
\hline Méthode (Monte Carlo $)$ & $\mathbf{8 . 0}$ & $\mathbf{1 5 . 7}$ & 0.000 & 0.000 & 0.000 & 0.000 & 0.000 & 0.000 \\
\hline DCSD & 0.0 & 0.3 & 0.000 & 0.001 & 0.022 & 0.022 & 0.008 & 0.007 \\
\hline \hline Total & 18.5 & 25.0 & 0.080 & 0.029 & 0.041 & 0.043 & 0.031 & 0.025 \\
\hline \hline
\end{tabular}

Tableau 4.27: Résumé des incertitudes systématiques sur les observables mesurées. Les incertitudes dominantes sont indiquées en gras.

\subsection{Rapports d'embranchement de $B^{0} \rightarrow \rho^{ \pm} \pi^{\mp}$ et $B^{0} \rightarrow$ $\rho^{-} K^{+}$}

Les rapports d'embranchement de $B^{0} \rightarrow \rho^{ \pm} \pi^{\mp}$ et $B^{0} \rightarrow \rho^{-} K^{+}$sont donnés par:

$$
\mathcal{B}\left(B^{0} \rightarrow \rho h\right)=\frac{N_{\rho h}}{\left(\epsilon_{M C}^{\rho h} \times N_{B^{0}}\right)}
$$


où $N_{\rho h}$ sont les nombres d'événements de $B^{0} \rightarrow \rho h$ obtenus dans l'ajustement final (voir tableau 4.18), $\epsilon_{M C}^{\rho h}$ est l'efficacité de sélection de $B^{0} \rightarrow \rho h$ calculée dans le Monte Carlo (l'efficacité brute est donnée dans le tableau 4.8), et $N_{B^{0}}$ le nombre de $B^{0}$ produits.

L'efficacité de sélection doit être corrigée de certains effets systématiques:

- nous appliquons événement par événement des corrections sur l'efficacité de reconstruction des traces(distinctes selon le signe de la trace et la haute-tension appliquée à la DCH, $c f$. section 3.2.2). En moyenne, l'efficacité est corrigée par un facteur 0.982 .

- la coupure dans le Monte Carlo sur le nombre de photons détectés dans le DIRC ( $c f$. section 3.2.3) entraîne une sur-estimation de l'efficacité de sélection par rapport aux données. Tel que suggéré par la référence [90], nous appliquons un facteur correctif de 0.99 , avec une erreur systématique de $1 \%$.

- les corrections dues à la reconstruction des $\pi^{0}$ ( $c f$. section 4.14.1) sont déjà comptées dans le calcul de l'efficacité, puisque cette dernière est calculée sur des échantillons Monte Carlo prenant en compte ces corrections.

Les efficacités corrigées des effets précédemment énoncés valent $(20.77 \pm 0.06) \%$ pour $B^{0} \rightarrow \rho^{ \pm} \pi^{\mp}$ et $(18.46 \pm 0.08) \%$ pour $B^{0} \rightarrow \rho^{-} K^{+}$.

Le nombre de $B^{0} / \bar{B}^{0}$ est $N_{B^{0}}=(88.9 \pm 0.2) \cdot 10^{6}$, et nous obtenons donc les rapports d'embranchement suivants:

$$
\begin{aligned}
\mathcal{B}\left(B^{0} \rightarrow \rho^{ \pm} \pi^{\mp}\right) & =\left(22.5_{-1.8}^{+1.8}\right) \pm 2.2 \times 10^{-6}, \\
\mathcal{B}\left(B^{0} \rightarrow \rho^{-} K^{+}\right) & =\left(7.3_{-1.2}^{+1.3}\right) \pm 1.3 \times 10^{-6} .
\end{aligned}
$$

Les incertitudes systématiques sur les rapports d'embranchement sont listées dans le tableau 4.28. Les incertitudes systématiques dominantes pour $B^{0} \rightarrow \rho^{ \pm} \pi^{\mp}$ sont dues à la reconstruction des $\pi^{0}$, à la validation du réseau de neurones dans Breco, des biais observés sur les ajustements de Monte Carlo, et du bruit de fond $B$. Pour $B^{0} \rightarrow \rho^{-} K^{+}$, il s'agit principalement du bruit de fond $B$.

Les erreurs statistiques sur les rapports d'embranchement mesurés sont améliorées de $70 \%$ pour $B^{0} \rightarrow \rho^{ \pm} \pi^{\mp}$ et $80 \%$ pour $B^{0} \rightarrow \rho^{-} K^{+}$par rapport aux mesures précédentes les plus précises ( $c f$. tableau 2.3). Les erreurs systématiques sont aussi significativement inférieures à celles des précédentes mesures. 


\begin{tabular}{|c|c|c|c|}
\hline & $B^{0} \rightarrow \rho^{ \pm} \pi^{\mp}$ & $B^{0} \rightarrow \rho^{ \pm} K^{\mp}$ & Commentaires \\
\hline \multicolumn{4}{|c|}{ Nombres d'événements et efficacité } \\
\hline $\begin{array}{l}N_{\text {signal }} \\
N_{\text {signal }}-N_{\text {biais }} \\
\epsilon_{\text {signal }}^{0} \\
\epsilon_{\text {signal }} \\
\epsilon_{\text {signal }} \\
\text { Erreurs statistique } \\
\end{array}$ & $\begin{array}{c}428 \\
416 \\
(21.36 \pm 0.06) \% \\
(20.98 \pm 0.06) \% \\
(20.77 \pm 0.06) \% \\
+7.9 \% \\
{ }_{-7.8} \% \\
\end{array}$ & $\begin{array}{c}120 \\
120 \\
(18.99 \pm 0.08) \% \\
(18.65 \pm 0.08) \% \\
(18.46 \pm 0.08) \% \\
+17.2 \% \\
{ }_{-16.7} \% \\
\end{array}$ & $\begin{array}{l}\text { ajustement (Tab. 4.18) } \\
\text { biais (Sec. 4.14.5) } \\
\text { MC corrigés pour } \pi^{0} \\
\text { correction traces } \\
\text { correction DIRC } \\
\text { erreurs MINOS } \\
\end{array}$ \\
\hline \multicolumn{4}{|c|}{ Systématiques sur l'efficacité[\%] } \\
\hline $\begin{array}{l}\text { Rec. des traces } \\
\text { Erreurs corrélées } \pi^{0} \\
\text { Erreurs dé-corrélées } \pi^{0} \\
\text { Coupure sur } \Delta E \\
\text { Coupure sur } m_{\mathrm{ES}} \\
\text { Coupure sur NN } \\
\text { Coupure sur } m\left(\rho^{ \pm}\right) \\
\text {Coupure sur } \cos \theta_{H}(\rho) \\
\text { Coupure sur } N_{\gamma}^{\mathrm{DIRC}}>5 \\
\mathcal{B}\left(\rho \rightarrow \pi^{+} \pi^{-}\right) \\
\text {Statistiques du MC } \\
N_{B^{0}}\end{array}$ & $\begin{array}{l}1.2 \\
5.0 \\
2.0 \\
0.4 \\
0.0 \\
4.3 \\
0.5 \\
0.2 \\
1.0 \\
1.6 \\
0.3 \\
2.2 \\
\end{array}$ & $\begin{array}{l}1.2 \\
5.0 \\
2.0 \\
1.0 \\
0.1 \\
4.3 \\
0.6 \\
0.3 \\
1.0 \\
1.6 \\
0.4 \\
2.2\end{array}$ & $\begin{array}{l}\text { corrections par trace } \\
\text { correction } \pi^{0} \\
\text { correction } \pi^{0} \\
\text { Breco } \\
\text { Breco } \\
\text { Breco } \\
\text { Breco } \\
\text { Breco } \\
\text { correction DIRC } \\
\Gamma\left(\pi^{+} \pi^{-}\right) / \Gamma(T o t a l) \\
\text { taille de l'échantillon } \\
\text { comptage des } B\end{array}$ \\
\hline Sous total: & 7.7 & 7.7 & \\
\hline \multicolumn{4}{|c|}{ Systématiques dues à l'ajustement [\%] } \\
\hline $\begin{array}{l}\Delta m_{d} \pm 0.008 \mathrm{ps}^{-1} \\
\tau_{B^{0}} \pm 0.016 \mathrm{ps} \\
\text { Résolution de } \Delta t \\
\text { Etiquetage } \\
\rho \text { de mauvaise charge } \\
\text { Ident. trace molle } \\
\text { Frac. de signal mal rec. } \\
\text { Distr. de signal mal rec. } \\
\text { Distr. } \theta_{C} \\
\text { Distr. de } \Delta E \\
\text { Distr. de } m_{\mathrm{ES}} \\
\text { Distr. de } x_{\mathrm{NN}} \\
A_{m i x_{c}}^{\rho h} \text { continuum } \\
\text { bruit de fond } B \\
\text { Méthodes (simul rapide) } \\
\text { Méthodes (MC) }\end{array}$ & $\begin{array}{c}0.00 \\
0.03 \\
0.29 \\
0.32 \\
0.02 \\
0.03 \\
1.25 \\
0.05 \\
0.15 \\
0.33 \\
2.22 \\
1.61 \\
0.11 \\
3.10 \\
0.20 \\
3.7\end{array}$ & $\begin{array}{c}0.00 \\
0.09 \\
0.53 \\
0.54 \\
0.00 \\
0.13 \\
1.56 \\
0.01 \\
0.51 \\
0.86 \\
1.67 \\
2.28 \\
0.65 \\
\mathbf{1 3 . 4} \\
0.45 \\
6.7\end{array}$ & $\begin{array}{l}\text { variation du paramètre } \\
\text { variation du paramètre } \\
\text { variation distribution } \Delta t \\
\text { Breco } \\
\text { variation du paramètre } \\
\text { erreur sur bruit de fond } B \\
\text { variation du paramètre } \\
\text { BReco } \\
\text { distr. pour le DIRC } \\
\text { BReco } \\
\text { BReco } \\
\text { BReco } \\
\text { variation du paramètre } \\
\text { bruit de fond } B \\
\text { simulations rapides } \\
\text { ajustements sur MC }\end{array}$ \\
\hline Sous-total: & 5.8 & 15.4 & \\
\hline Erreur systématique totale & 9.6 & 17.2 & \\
\hline $\mathcal{B}\left(\times 10^{-6}\right)$ & $22.5 \pm 1.8 \pm 2.2$ & $7.3_{-1.2}^{+1.3} \pm 1.3$ & \\
\hline
\end{tabular}

Tableau 4.28: Erreurs systématiques sur les rapports d'embranchement de $B^{0} \rightarrow \rho^{ \pm} \pi^{\mp}$ et $B^{0} \rightarrow \rho^{-} K^{+}$. L'erreur dominante est indiquée en gras. 


\subsection{Conclusion}

Nous avons réalisé une analyse temporelle des modes $B^{0} \rightarrow \rho^{ \pm} \pi^{\mp}$ et $B^{0} \rightarrow \rho^{-} K^{+}$sur une luminosité intégrée de $81.9 \mathrm{fb}^{-1}$. Grâce à un ajustement par maximum de vraisemblance, nous avons mesuré les rapports d'embranchement de $B^{0} \rightarrow \rho^{ \pm} \pi^{\mp}$ et $B^{0} \rightarrow \rho^{-} K^{+}$suivants:

$$
\begin{aligned}
\mathcal{B}\left(B^{0} \rightarrow \rho^{ \pm} \pi^{\mp}\right) & =(22.5 \pm 1.8 \pm 2.2) \times 10^{-6} \\
\mathcal{B}\left(B^{0} \rightarrow \rho^{ \pm} K^{\mp}\right) & =(7.3 \pm 1.3 \pm 1.3) \times 10^{-6}
\end{aligned}
$$

ainsi que les paramètres $C P$ :

$$
\begin{aligned}
A_{\mathrm{CP}}^{\rho K} & =0.28 \pm 0.17 \pm 0.080, \\
A_{\mathrm{CP}}^{\rho \pi} & =-0.18 \pm 0.08 \pm 0.029, \\
C & =0.36 \pm 0.18 \pm 0.041, \\
S & =0.19 \pm 0.24 \pm 0.031
\end{aligned}
$$

et les paramètres de dilution:

$$
\begin{aligned}
\Delta C & =0.28 \pm 0.19 \pm 0.043 \\
\Delta S & =0.15 \pm 0.25 \pm 0.025
\end{aligned}
$$

où la première erreur est statistique, et la seconde systématique. Nous n'observons pas de signature significative de violation de $C P$.

Les incertitudes sur les rapports d'embranchement sont dominées par les erreurs systématiques sur la reconstruction des $\pi^{0}$, le bruit de fond $B$, et celles des paramètres $C P$ et de dilution par le bruit de fond $B$. Les erreurs liées à ce dernier devrait diminuer dans un proche futur grâce à la mesure des rapports d'embranchement des modes contaminant notre signal. 


\section{Chapitre 5}

\section{Mesure du rapport d'embranchement de $B^{0} \rightarrow \rho^{0} \pi^{0}$}

\subsection{Stratégie de l'analyse et différences avec $B^{0} \rightarrow \rho^{ \pm} \pi^{\mp}$}

Le mode $B^{0} \rightarrow \rho^{0} \pi^{0}$, état propre de $C P$, est un ingrédient essentiel de l'analyse Dalitz de $B^{0} \rightarrow \pi^{+} \pi^{-} \pi^{0}$. Il s'agit d'une désintégration supprimée de couleur et d'isospin: on attend donc son rapport d'embranchement réduit d'un facteur compris entre 3.5 et 70 [91] par rapport à celui du mode $B^{0} \rightarrow \rho^{ \pm} \pi^{\mp}\left(\operatorname{soit} \mathcal{B}\left(B^{0} \rightarrow \rho^{0} \pi^{0}\right) \simeq(0.3-5.7) \times 10^{-6}\right)$. Le rapport signal sur bruit de fond continuum est donc beaucoup plus faible pour $B^{0} \rightarrow \rho^{0} \pi^{0}$ que pour $B^{0} \rightarrow \rho^{ \pm} \pi^{\mp}$.

A l'opposé de $B^{0} \rightarrow \rho^{ \pm} \pi^{\mp}$, les deux traces chargées sont issues du $\rho$, et le $\pi^{0}$ correspond à la trace célibataire: il s'agit donc d'un $\pi^{0}$ dur ( $c f$. figure 4.2) menant à un plus faible taux d'événements mal reconstruits ( $c f$. Sec. 4.3.2) et à une plus petite contamination de bruit de fond $B$ par rapport à $B^{0} \rightarrow \rho^{ \pm} \pi^{\mp}$. Enfin, puisque le $\rho$ est neutre, il n'y a pas d'événements de mauvaise charge.

Le signal $B^{0} \rightarrow \rho^{0} \pi^{0}$ étant attendu faible, les effets des interférences avec d'autres résonances ou des contributions non résonantes sont relativement plus importants que pour $B^{0} \rightarrow \rho^{ \pm} \pi^{\mp}$. Idéalement, l'utilisation de ces interférences devrait permettre de mettre en évidence $B^{0} \rightarrow \rho^{0} \pi^{0}$ (puisque l'amplitude de ce dernier entre linéairement dans les termes d'interférences). Néanmoins, ce type d'analyse implique la compréhension détaillée du diagramme de Dalitz. Une analyse plus simple consiste à couper les zones d'interférences (perdant ce faisant l'accès à la partie linéaire de l'amplitude) pour tenter de mettre en évidence les amplitudes au carré. C'est la stratégie suivie ici: elle n'est sensible qu'à des valeurs élevées du rapport d'embranchement de $B^{0} \rightarrow \rho^{0} \pi^{0}$.

Nous excluons donc les coins du diagramme de Dalitz où les interférences entre le $\rho^{0}$ et les $\rho^{ \pm}$sont les plus importantes (l'effet de la polarisation du $\rho$ est alors néfaste pour cette stratégie d'analyse, puisque $B^{0} \rightarrow \rho^{0} \pi^{0}$ peuple principalement les coins du Dalitz), et reconstruisons le $\rho^{0}$ dans une fenêtre plus resserrée que dans le cas de $\rho^{ \pm}$pour limiter la contamination du $f^{0}(980)$.

Comme ce signal n'a encore jamais été observé ( $c f$. tableau 2.3), dans un premier temps, nous nous limitons à la mesure du rapport d'embranchement sans réaliser d'analyse $C P$. Si le signal s'avère statistiquement significatif, nous réaliserons alors ultérieurement une analyse $C P$ dépendante du temps. Le mode $B^{0} \rightarrow \rho^{0} \pi^{0}$ étant un état propre de $C P$, sa dépendance en temps est similaire à celle du mode $B^{0} \rightarrow \pi^{+} \pi^{-}$décrite dans la section 1.5.2, menant 
à un total de trois observables: $\mathcal{B}^{\prime \prime}, C^{00}$ et $S^{00}$. La variable $\Delta t$, ainsi que les catégories d'étiquetage sont utilisées dans la mesure du rapport d'embranchement, car elles permettent de rejeter du bruit de fond continuum.

A ce jour, l'analyse $B^{0} \rightarrow \rho^{0} \pi^{0}$ n'est pas encore complètement finalisée: le résultat est encore voilé au sein de la collaboration $B A B A R$, et certaines erreurs systématiques sont encore préliminaires. La finalisation de l'analyse étant néanmoins proche, je dévoile de façon préliminaire les résultats dans ce document.

\section{$5.2 \quad$ Sélection de $B^{0} \rightarrow \rho^{0} \pi^{0}$}

\subsubsection{Reconstruction et sélection}

La reconstruction de $B^{0} \rightarrow \rho^{0} \pi^{0}$ est similaire à celle de $B^{0} \rightarrow \rho^{ \pm} \pi^{\mp}$. La liste des coupures de sélection est donnée ci-dessous:

- Reconstruction des traces: identique à $B^{0} \rightarrow \rho^{ \pm} \pi^{\mp}$ (cf. section 4.3.1.1).

- Identification des traces: les deux traces ne doivent pas être identifiées comme électrons, protons ou kaons ( $c f$. section 4.3.1.2).

- Reconstruction du $\pi^{0}$ : identique à $B^{0} \rightarrow \rho^{ \pm} \pi^{\mp}(c f$. section 4.3.2).

- Reconstruction du $\rho^{0}$ :

- $0.4<m\left(\pi^{+} \pi^{-}\right)<0.9 \mathrm{GeV} / c^{2}$ : la coupure supérieure sur $m\left(\pi^{+} \pi^{-}\right)$est plus resserrée que celle sur $m\left(\pi^{ \pm} \pi^{0}\right)$ pour $B^{0} \rightarrow \rho^{ \pm} \pi^{\mp}$, afin de limiter la contamination par le $f_{0}$.

- coupure des zones d'interférences principales avec les $\rho^{ \pm}$: on exclut les zones du plan Dalitz où $0.4<m\left(\pi^{ \pm} \pi^{0}\right)<1.3 \mathrm{GeV} / \mathrm{c}^{2}$.

- Hélicité du $\rho$ : coupure identique à $B^{0} \rightarrow \rho^{ \pm} \pi^{\mp}\left(\left|\cos \theta_{H}(\rho)\right|>0.25\right)$.

- Bruit de fond $B$ en 2 corps: $m\left(\pi^{ \pm} \pi^{0}\right)<5.14 \mathrm{GeV} / c^{2}$.

- Reconstruction du $B$ :

- $5.23<m_{\mathrm{ES}}<5.2875 \mathrm{GeV} / \mathrm{c}^{2}$ : la coupure supérieure sur $m_{\mathrm{ES}}$ est différente de celle pour $B^{0} \rightarrow \rho^{ \pm} \pi^{\mp}$ pour des raisons techniques. Cela a un effet négligeable sur l'efficacité de sélection.

- $-0.15<\Delta E<0.10 \mathrm{GeV}$ : la coupure est différente de celle de $B^{0} \rightarrow \rho^{ \pm} \pi^{\mp}$ à cause de la présence du $\pi^{0}$ dur (la correction du Monte Carlo pour la reconstruction du $\pi^{0}$ entraîne un décalage vers la gauche de $20 \mathrm{MeV}$ de la distribution de $\Delta E$ ).

- Reconstruction de $\Delta t$ : identique à $B^{0} \rightarrow \rho^{ \pm} \pi^{\mp}$.

- Lutte contre le bruit de fond continuum: cf. section 5.4.

Le tableau 5.1 donne les efficacités relatives de sélection des coupures décrites ci-dessus pour le signal Monte Carlo, ainsi que les données off-peak, on-peak et Monte Carlo continuum. 


\begin{tabular}{c|ccccc}
\hline Coupure & \multicolumn{4}{|c}{ Efficacité $(\%)$} \\
& $\epsilon_{\rho^{0} \pi^{0}}^{\mathrm{MC}-\pi^{0}}$ & $\epsilon_{\rho^{0} \pi^{0}}^{\mathrm{MC}}$ & $\epsilon_{\mathrm{off}}^{\text {donnés }}$ & $\epsilon_{\text {on(SB) }}^{\text {données }}$ & $\epsilon_{\mathrm{udsc}}^{\mathrm{MC}}$ \\
\hline Reconstruction traces & $99.8 \pm 0.02$ & $99.8 \pm 0.01$ & $99.7 \pm 0.01$ & $99.7 \pm 0.01$ & $99.7 \pm 0.01$ \\
$0.01<\mathrm{LAT}(\gamma)<0.6$ & $97.2 \pm 0.07$ & $97.7 \pm 0.06$ & $92.3 \pm 0.03$ & $92.1 \pm 0.05$ & $92.4 \pm 0.07$ \\
$E_{\gamma}>0.05$ & $98.4 \pm 0.05$ & $98.7 \pm 0.04$ & $95.7 \pm 0.03$ & $96.2 \pm 0.04$ & $96.0 \pm 0.05$ \\
$0.11<m\left(\pi^{0}\right)<0.16$ & $97.9 \pm 0.06$ & $98.1 \pm 0.05$ & $96.1 \pm 0.03$ & $96.4 \pm 0.04$ & $96.3 \pm 0.05$ \\
$0.4<m\left(\rho^{0}\right)<0.9$ & $73.6 \pm 0.19$ & $73.9 \pm 0.17$ & $21.6 \pm 0.06$ & $22.1 \pm 0.08$ & $22.1 \pm 0.11$ \\
$0.25<\left|\cos \left(\theta_{H\left(\rho^{0}\right)}\right)\right|$ & $98.4 \pm 0.06$ & $98.4 \pm 0.06$ & $80.9 \pm 0.12$ & $81.4 \pm 0.16$ & $82.1 \pm 0.22$ \\
$-0.15<\Delta E<0.1$ & $81.1 \pm 0.20$ & $82.3 \pm 0.17$ & $23.3 \pm 0.14$ & $48.0 \pm 0.23$ & $23.1 \pm 0.28$ \\
$5.23<m_{E S}<5.2895$ & $99.4 \pm 0.04$ & $99.4 \pm 0.04$ & $44.3 \pm 0.34$ & $60.3 \pm 0.33$ & $42.4 \pm 0.66$ \\
$|\Delta t|<20 \mathrm{ps}$ & $98.9 \pm 0.06$ & $98.9 \pm 0.05$ & $94.1 \pm 0.24$ & $93.0 \pm 0.22$ & $93.5 \pm 0.50$ \\
$\sigma(\Delta t)<2.5 \mathrm{ps}$ & $95.3 \pm 0.12$ & $95.3 \pm 0.11$ & $92.1 \pm 0.29$ & $90.6 \pm 0.26$ & $91.4 \pm 0.59$ \\
veto $\rho^{ \pm}$ & $96.6 \pm 0.10$ & $96.6 \pm 0.09$ & $98.3 \pm 0.14$ & $97.6 \pm 0.14$ & $98.5 \pm 0.27$ \\
veto $B 2-\operatorname{corps}$ & $99.9 \pm 0.01$ & $99.9 \pm 0.02$ & $100 \pm 0.00$ & $97.1 \pm 0.16$ & $100.0 \pm 0.00$ \\
veto électron & $98.4 \pm 0.07$ & $98.4 \pm 0.07$ & $92.6 \pm 0.29$ & $93.1 \pm 0.24$ & $94.5 \pm 0.49$ \\
veto kaon & $97.1 \pm 0.10$ & $97.2 \pm 0.09$ & $76.5 \pm 0.49$ & $75.1 \pm 0.43$ & $69.6 \pm 0.99$ \\
veto proton & $99.5 \pm 0.04$ & $99.5 \pm 0.04$ & $94.1 \pm 0.31$ & $94.6 \pm 0.26$ & $92.0 \pm 0.74$ \\
$x_{\text {NN }}$ & $81.1 \pm 0.23$ & $81.2 \pm 0.21$ & $6.97 \pm 0.31$ & $7.61 \pm 0.31$ & $6.89 \pm 0.62$ \\
\hline \hline
\end{tabular}

Tableau 5.1: Efficacités de sélection relatives aux coupures précédemment pour le signal (après (noté " $M C-\pi^{0}$ ") et avant corrections sur le Monte Carlo dues aux $\pi^{0}$ ), les données off-peak, on-peak (en dehors de la région de signal), et Monte Carlo continuum.

\subsubsection{Choix du meilleur candidat}

Grâce à la présence du $\pi^{0}$ dur, la fraction d'événements ayant plus d'un candidat est seulement de $8 \%$ pour $B^{0} \rightarrow \rho^{0} \pi^{0}$, à comparer aux $23 \%$ pour $B^{0} \rightarrow \rho^{ \pm} \pi^{\mp}$ ( $c f$. sections 4.3 .2 et 4.7.2). Le nombre moyen de candidats par événement est 1.1. Les candidats multiples sont dus majoritairement à la mauvaise reconstruction d'une trace chargée de faible impulsion issue du $\rho$. Nous choisissons le candidat dont la paire de pions chargés possède la masse invariante la plus proche de la masse nominale du $\rho$ [44]. Ceci mène à une fraction de mauvais événements de $9.4 \%$.

\subsubsection{Statistiques finales de la sélection}

Le tableau 5.2 donne l'efficacité $\epsilon$ de sélection et la fraction d'événements mal reconstruits $f_{\text {scf }}$ obtenues dans le Monte Carlo $B^{0} \rightarrow \rho^{0} \pi^{0}$, ainsi que le nombre d'événements sélectionnés dans les données on-peak et off-peak (normalisé à une luminosité de $81.9 \mathrm{fb}^{-1}$ ). L'efficacité de sélection est similaire à celle de $B^{0} \rightarrow \rho^{ \pm} \pi^{\mp}$ ( $c f$. tableau 4.8): la coupure des zones d'interférence est plus sévère que pour $B^{0} \rightarrow \rho^{ \pm} \pi^{\mp}$ (puisqu'il existe deux zones à la place d'une seule auparavant), mais on ne se restreint plus à l'acceptance du DIRC pour l'identification de la trace célibataire. Au total, l'efficacité reste donc stable. 


\begin{tabular}{l|cc}
\hline Mode & $\epsilon(\%)$ & $f_{\text {SCF }}(\%)$ \\
\hline$B^{0} \rightarrow \rho^{0} \pi^{0}$ & $20.2 \pm 0.1$ & $9.4 \pm 0.2$ \\
\hline \hline Mode & \multicolumn{2}{|c}{$N_{\text {sel }}$} \\
\hline off-peak & 6397 (normalisé à $\left.81.9 \mathrm{fb}^{-1}\right)$ \\
on-peak & \multicolumn{2}{|c}{6648} \\
\hline
\end{tabular}

Tablea1 5.2: La partie supérieure du tableau donne l'efficacité de sélection ( $\epsilon$ ) et la fraction d'événements mal reconstruits $\left(f_{\mathrm{scf}}\right)$ obtenues dans le Monte Carlo $B^{0} \rightarrow \rho^{0} \pi^{0}$. La partie inférieure du tableau donne le nombre d'événements sélectionnés dans les données on-peak et off-peak (normalisé à une luminosité de $81.9 \mathrm{fb}^{-1}$ ).

\subsection{Catégories d'étiquetage de la saveur}

Nous utilisons les catégories d'étiquetage de la saveur dans la mesure du rapport d'embranchement de $B^{0} \rightarrow \rho^{0} \pi^{0}$ puisque les niveaux de bruits de fond dans chaque catégorie sont différents, ce qui aide à la séparation bruit de fond - signal. L'algorithme d'étiquetage de la saveur est décrit dans la section 4.4 .

Le tableau 5.3 montre les fractions de signal et off-peak dans les différentes catégories d'étiquetage. Nous utilisons des fractions différentes pour le signal correctement et mal reconstruit, puisqu'elles sont différentes pour ces deux classes de signal.

\begin{tabular}{|l|ccccc|}
\hline Mode & $\varepsilon_{\text {Lepton }}(\%)$ & $\varepsilon_{\text {Kaon }}(\%)$ & $\varepsilon_{\text {IIT } 1}(\%)$ & $\varepsilon_{\text {IT2 }}(\%)$ & $\varepsilon_{\text {IoTag }}(\%)$ \\
\hline \hline Signal corr. rec. & $12.6 \pm 0.2$ & $35.0 \pm 0.3$ & $7.4 \pm 0.2$ & $14.2 \pm 0.2$ & $30.8 \pm 0.3$ \\
Signal mal rec. & $6.1 \pm 0.5$ & $37.6 \pm 1.1$ & $5.4 \pm 0.5$ & $15.3 \pm 0.8$ & $35.6 \pm 1.0$ \\
Signal total & $12.0 \pm 0.2$ & $35.2 \pm 0.3$ & $7.2 \pm 0.2$ & $14.3 \pm 0.2$ & $31.3 \pm 0.3$ \\
\hline Off-peak & $0.8 \pm 0.5$ & $22.6 \pm 2.2$ & $4.9 \pm 1.1$ & $15.1 \pm 1.9$ & $56.6 \pm 2.6$ \\
\hline
\end{tabular}

Tableau 5.3: Fractions de signal et off-peak dans les différentes catégories d'étiquetage.

\subsection{Lutte contre le bruit de fond $q \bar{q}$}

\subsubsection{Configurations de variables}

La lutte contre le bruit de fond $q \bar{q}$ pour $B^{0} \rightarrow \rho^{0} \pi^{0}$ est similaire à celle réalisée pour le mode $B^{0} \rightarrow \rho^{ \pm} \pi^{\mp}$ (cf. section 4.6). Nous avons étudié cinq configurations de variables:

- NN à 2 variables: $L_{0}, L_{2}$,

- NN à 4 variables: $N N$ à 2 variables $+m\left(\rho^{ \pm}\right),\left|\cos \theta_{H}(\rho)\right|$,

- NN à 6 variables: NN à 4 variables $+\cos \theta(B, z)+\cos \theta\left(T_{B}, z\right)$,

- $\mathrm{NN}$ à 7 variables: $\mathrm{NN}$ à 6 variables $+\Delta E$,

- NN à 8 variables: $\mathrm{NN}$ à 6 variables $+\hat{S}_{\mathrm{ROE}}+\cos \theta\left(A p_{B}, T_{R O E}\right)^{1}$.

\footnotetext{
${ }^{1}$ La variable $\cos \theta\left(A p_{B}, T_{R O E}\right)$ est le cosinus de l'angle entre la direction transverse au plan de la désintégration du $B$ (dénommée “aplanarité") et la direction de l'axe de poussée du ROE.
} 
Nous entraînons un réseau de neurones dans chacune de ces configurations sur environ 30000 événements de signal $B^{0} \rightarrow \rho^{0} \pi^{0}$ Monte Carlo, et 15000 événements de données offpeak ${ }^{2}$.

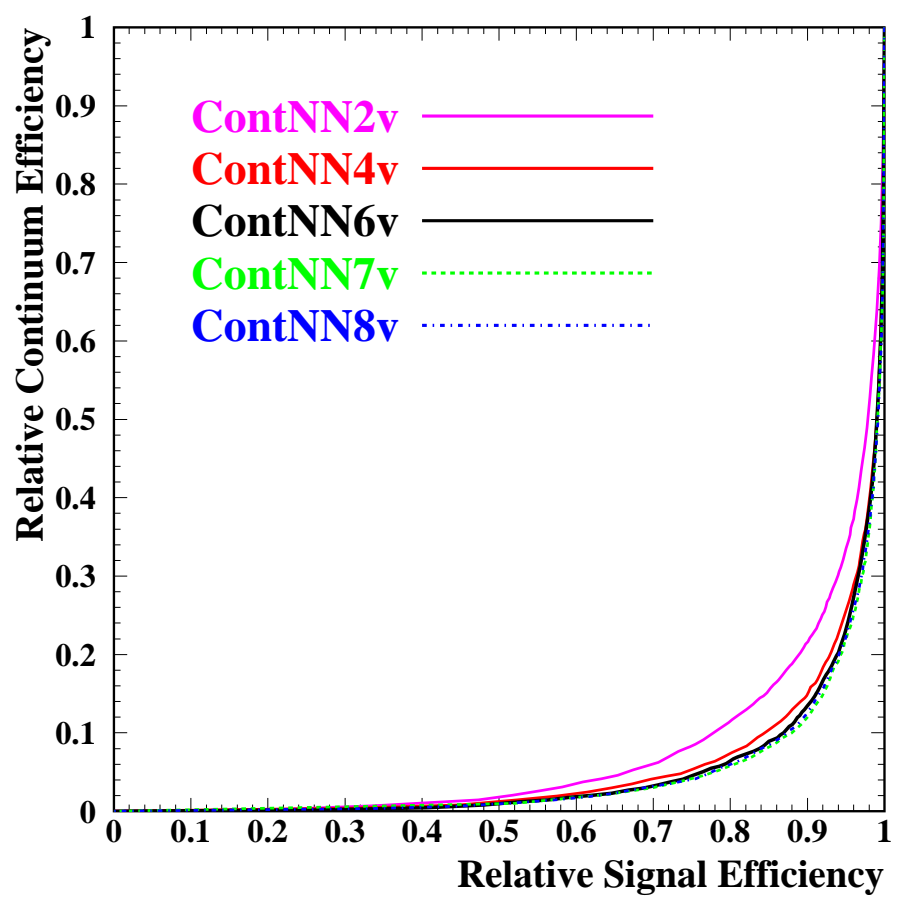

Figure 5.1: Efficacité du bruit de fond en fonction de l'effcacité du signal pour une coupure sur la sortie du réseau de neurones pour les différentes configurations de variables.

La figure 5.1 montre l'efficacité du bruit de fond en fonction de l'efficacité du signal pour une coupure sur la sortie du réseau de neurones pour les différentes configurations de variables. Les configurations à 6,7 et 8 variables montrent de meilleures performances que celle à 2 variables (et marginalement, que celle à 4 variables).

Nous choisissons de garder la configuration à 6 variables, puisque l'ajout de variables supplémentaires n'augmente pas significativement les performances du réseau de neurones.

\subsubsection{Corrélations}

La figure 5.2 montre les corrélations entre la moyenne de la sortie du réseau de neurones à 6 variables et $m_{\mathrm{ES}}$, ainsi que $\Delta E$, pour les événements de signal correctement reconstruits, mal reconstruits, et les données off-peak. De petites corrélations existent pour le signal, en particulier les événements mal reconstruits, et sont inexistantes pour le bruit de fond.

\subsection{Bruit de fond provenant d'autres modes $B$}

La stratégie utilisée pour $B^{0} \rightarrow \rho^{ \pm} \pi^{\mp}\left(c f\right.$. section 4.8) est appliquée à $B^{0} \rightarrow \rho^{0} \pi^{0}$ : nous isolons parmi plus de 100 modes de désintégrations non charmées ceux qui contribuent avec au moins un événement dans l'échantillon sélectionné. Nous incluons aussi les contributions inclusives des désintégrations charmées. Un terme correctif est ensuite ajouté à la fonction

\footnotetext{
${ }^{2}$ Le réseau de neurones est entraîné dans la même configuration que pour $B^{0} \rightarrow \rho^{ \pm} \pi^{\mp}$ : $c f$. section 4.6.4.2
} 

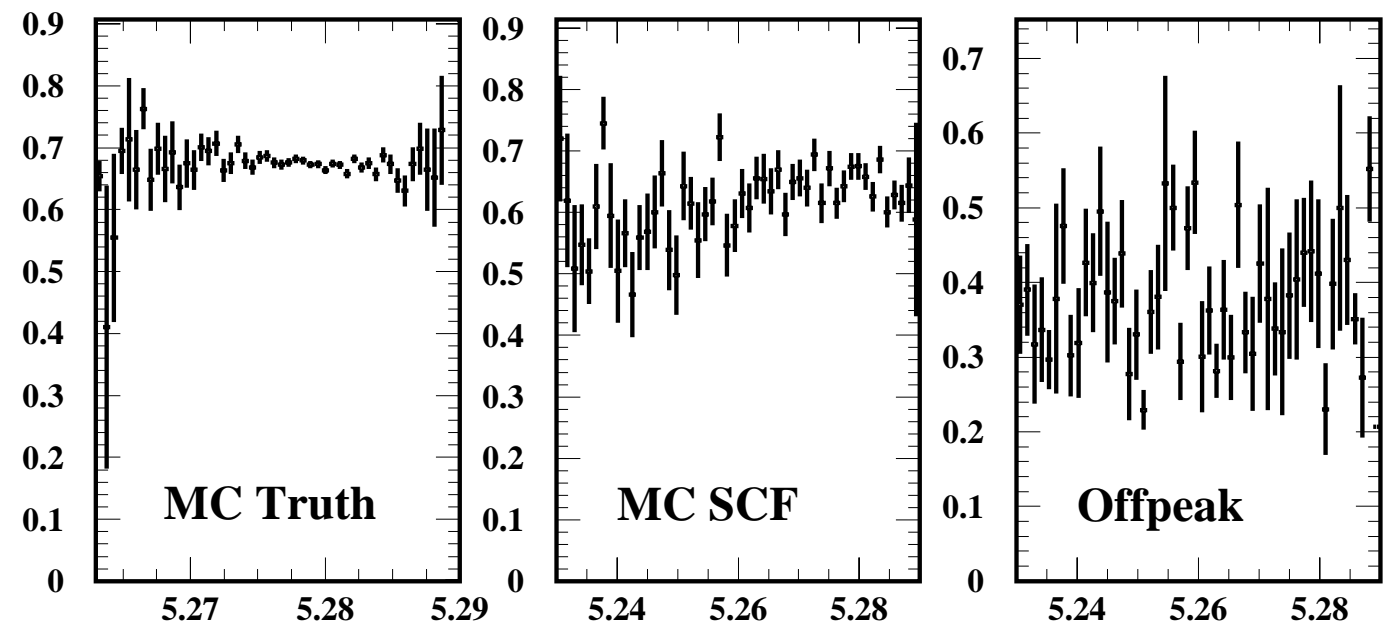

ContNN vs. mES
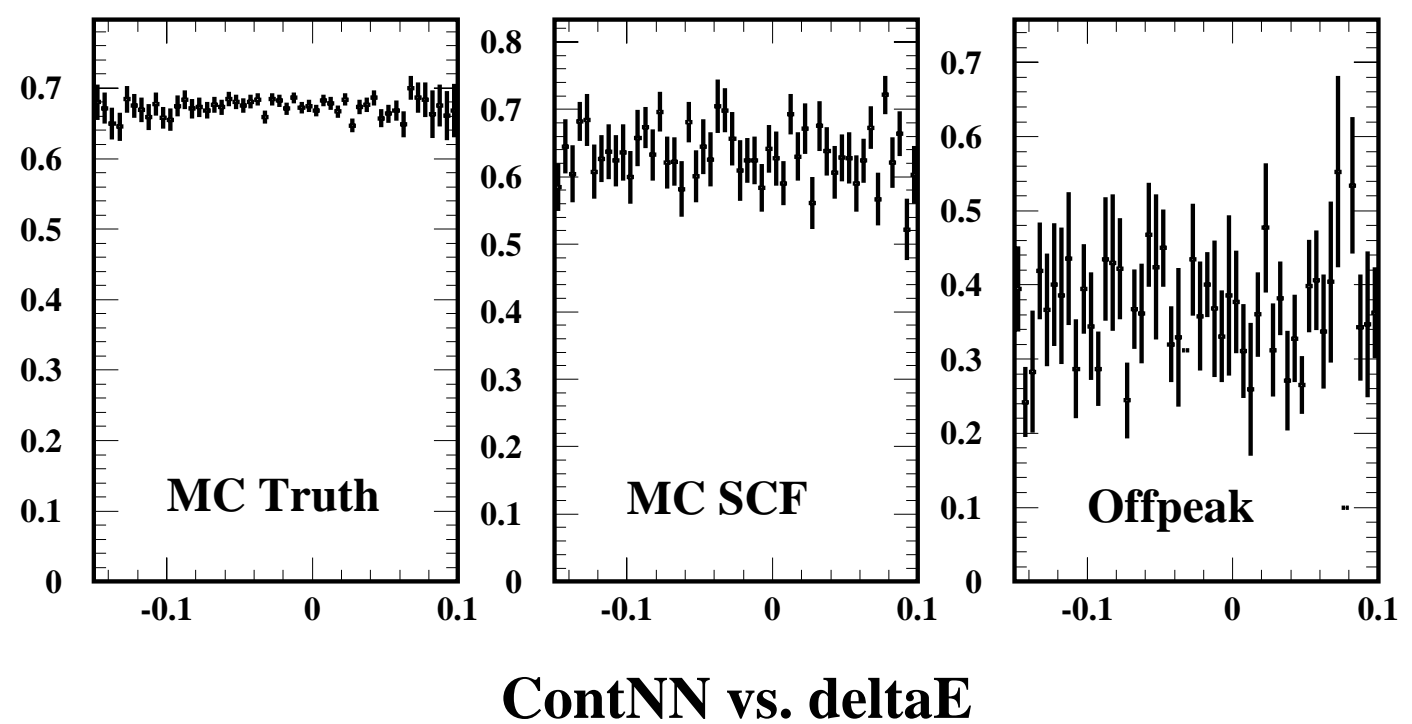

Figure 5.2: Corrélations entre la moyenne de la sortie du réseau de neurones à 6 variables et $m_{\mathrm{ES}}$ (haut), ainsi que $\Delta E$ (bas) pour les événements de signal correctement reconstruits (gauche, "Truth"), mal reconstruits (milieu, "SCF"), et les données off-peak (droite).

de vraisemblance ( $c f$. section 5.6.1) pour chaque mode retenu (nous ne recourons pas aux classes comme dans le cas de $B^{0} \rightarrow \rho^{ \pm} \pi^{\mp}$, puisque le nombre de modes retenus est plus réduit).

La liste détaillée des modes non charmés étudiés (similaire à celle donnée dans les tableaux 4.9 à 4.11) se trouve dans la référence [40]. L'évaluation des rapports d'embranchement est décrite dans l'annexe D. La liste des modes retenus est donnée dans le tablean 5.4: la plupart de ces modes possèdent un $\pi^{0}($ ou $\gamma)$ dur dans l'état final. 


\begin{tabular}{lll}
\hline Mode & $\mathcal{B}\left(\times 10^{-6}\right)$ & $\mathrm{N}_{\text {exp }}$ \\
\hline \hline$B^{+} \rightarrow \rho^{+} \rho_{\text {long }}^{0}$ & $22.5 \pm 17.5^{*}$ & $11.9 \pm 9.3$ \\
$B^{0} \rightarrow \rho^{+} \rho_{\text {long }}^{-}$ & $40.0 \pm 30.0^{*}$ & $10.9 \pm 8.2$ \\
$B^{0} \rightarrow \eta^{\prime}\left(\rho^{0} \gamma\right) \pi^{0}$ & $1.5 \pm 1.5^{*}$ & $3.1 \pm 3.1$ \\
$B^{0} \rightarrow \pi^{0} K^{* 0}\left(K^{+} \pi^{-}\right)$ & $8.7 \pm 5.0^{*}$ & $13.6 \pm 7.8$ \\
$B^{+} \rightarrow \rho^{+} \pi^{0}$ & $15.0 \pm 8.0^{*}$ & $15.9 \pm 8.5$ \\
$B^{0} \rightarrow K^{* 0}\left(\rightarrow(K \pi)^{0}\right) \gamma$ & $40.2 \pm 2.7$ & $1.7 \pm 0.1$ \\
$B^{0} \rightarrow \rho^{ \pm} \pi^{\mp}$ & $11.6 \pm 1.4$ & $8.0 \pm 1.0$ \\
$B^{0} \rightarrow \rho^{-} K^{+}$ & $7.3 \pm 1.8$ & $0.7 \pm 0.1$ \\
$B^{+} \rightarrow K^{+} \pi^{0}$ & $12.7 \pm 1.2$ & $0.85 \pm 0.08$ \\
$B^{+} \rightarrow \pi^{+} \pi^{0}$ & $5.8 \pm 1.0$ & $1.4 \pm 0.24$ \\
$B^{+} \rightarrow\left(K^{* *} \pi\right)^{+}$ & $40 \pm 26^{*}$ & $2.3 \pm 1.5$ \\
$B^{0} \rightarrow\left(K^{* *} \pi\right)^{0}$ & $72 \pm 54^{*}$ & $3.2 \pm 2.4$ \\
$B^{+} \rightarrow a_{1}^{+}\left(\rightarrow(\rho \pi)^{+}\right) \pi^{0}$ & $35.0 \pm 25.0^{*}$ & $5.2 \pm 3.7$ \\
$B^{0} \rightarrow f^{0} \pi^{0}$ & $1.0 \pm 1.0^{*}$ & $1.0 \pm 1.0$ \\
$B^{0} \rightarrow \pi^{+} \pi^{-} \pi^{0}($ non - res $)$ & $0.0 \pm 3.0^{*}$ & $0.0 \pm 2.5$ \\
\hline Sous-total $B \rightarrow$ non charmé & - & 83.4 \\
\hline$B^{0} \rightarrow$ charme & - & $23.57 \pm 7.1$ \\
$B^{ \pm} \rightarrow$ charme & - & $34.84 \pm 10.4$ \\
\hline Total & & 141.8 \\
\hline \hline
\end{tabular}

Tableau 5.4: Modes de bruit de fond $B$ retenus dans l'analyse $B^{0} \rightarrow \rho^{0} \pi^{0}$. Un astérisque indique que le rapport d'embranchement a été obtenu à partir d'arguments théoriques.

\subsection{Fonction de vraisemblance}

\subsubsection{Composition de la fonction de vraisemblance}

La fonction de vraisemblance pour l'analyse de $B^{0} \rightarrow \rho^{0} \pi^{0}$ est simplifiée par rapport à celle de $B^{0} \rightarrow \rho^{ \pm} \pi^{\mp}$ ( $c f$. Eq. 4.29), puisque tous les paramètres reliés à la charge du $\rho^{ \pm}$(taux de mauvaise charge $w_{Q}$, asymétrie de charge $A_{C P}$, corrélations étiquette-charge $\left.A_{\text {mix }}\right)$ n'existent plus pour le $\rho^{0}$ et son bruit de fond.

La densité de probabilité $\mathcal{P}_{i, c}$ pour un événement $i$, appartenant à la catégorie d'étiquetage $c_{i}$, est donc donnée par:

$$
\begin{aligned}
\mathcal{P}_{i, c} & =N_{\rho^{0} \pi^{0}} f_{c}^{\rho^{0} \pi^{0}}\left(1-f_{\mathrm{scf}, c}^{\rho^{0} \pi^{0}}\right) \mathcal{P}_{i, c, \text { tru }}^{\rho^{0} \pi^{0}}+f_{\mathrm{scf}, c}^{\rho^{0} \pi^{0}} \mathcal{P}_{i, c, \mathrm{scf}}^{\rho^{0} \pi^{0}} \\
& +N_{q \rho^{0} \pi^{0}}^{c} \mathcal{P}_{i, c}^{q \rho^{0} \pi^{0}} \\
& +\sum_{b=1, \ldots N_{B}} \mathcal{L}_{i, b, c}^{\rho^{0} \pi^{0}}
\end{aligned}
$$

ò̀:

- $N_{\rho^{0} \pi^{0}}$ est le nombre d'événements de signal $B^{0} \rightarrow \rho^{0} \pi^{0}$.

- $f_{c}^{\rho^{0}} \pi^{0}$ est la fraction d'événements de signal dans la catégorie d'étiquetage $c$.

- $f_{\mathrm{scf}, c}^{\rho^{0} \pi^{0}}$ est la fraction d'événements de signal mal reconstruits dans la catégorie d'étiquetage $c$. 
- $N_{q \rho^{0} \pi^{0}}$ est le nombre d'événements de bruit de fond de continuum dans la catégorie d'étiquetage $c$.

- $\mathcal{P}_{c}^{(q) \rho^{0} \pi^{0}}$ est le produit des densités de probabilités pour le signal et le bruit de fond continuum des variables entrant dans l'ajustement: $\mathcal{P}^{(q) \rho^{0} \pi^{0}}\left(m_{\mathrm{ES}}\right) \cdot \mathcal{P}^{(q) \rho^{0} \pi^{0}}(\Delta E)$. $\mathcal{P}^{(q) \rho^{0} \pi^{0}}\left(x_{\mathrm{NN}}\right) \cdot \mathcal{P}_{c}^{(q) \rho^{0} \pi^{0}}(\Delta t)$

La fonction de vraisemblance étendue sur toutes les catégories d'étiquetage est donnée par l'eq. 4.30 .

\subsubsection{Corrélations entre les variables entrant la fonction de vrai- semblance}

La figure 5.3 montre les coefficients de corrélation linéaire entre les variables discriminantes pour les événements de signal correctement reconstruits et mal reconstruits, ainsi que pour les données off-peak.

Les corrélations linéaires entre les variables utilisées dans l'ajustement de vraisemblance final sont faibles.

\subsection{Validation de l'analyse en aveugle}

\subsubsection{Validation par des simulations rapides}

\subsubsection{Erreurs sur $N_{\rho^{0} \pi^{0}}$ pour différentes configurations}

Nous avons réalisé 1000 expériences de simulation rapide pour différents rapports d'embranchement de $B^{0} \rightarrow \rho^{0} \pi^{0}$ et différentes configurations d'ajustement (nous générons 6397 événements de continuum, tel qu'évalué sur les données off-peak). Les erreurs sur $N_{\rho^{0} \pi^{0}}$ obtenues pour chaque configuration sont résumées dans le tableau 5.5.

La dernière configuration dans ce tableau consiste en l'ajustement d'un facteur $c_{\text {fact }}$ devant

le terme $\mathcal{L}_{i, b, c}^{\rho^{0} \pi^{0}}$ de bruit de fond $B$ de la fonction de vraisemblance ( $c f$. Eq. 5.1). Ce facteur est consistant avec 1, montrant que le terme correctif du bruit de fond $B$ est du bon ordre de grandeur.

Les variations des valeurs centrales entre les différentes configurations où une variable est retirée de l'ajustement sont données dans le même tableau. Ces variations sont obtenues à partir d'ajustements sur des échantillons communs de simulation rapide afin de ne pas être sensible aux fluctuations statistiques. Les variations sont compatibles avec zéro.

\subsubsection{Pulls des variables libres dans l'ajustement final}

Le tableau 5.6 donne le biais et l'écart-type des pulls pour les nombres d'événements du signal et du bruit de fond continuum obtenus par simulations rapides. Nous avons aussi vérifié les pulls de tous les autres paramètres libres de l'ajustement. Aucun biais significatif n'est observé. 


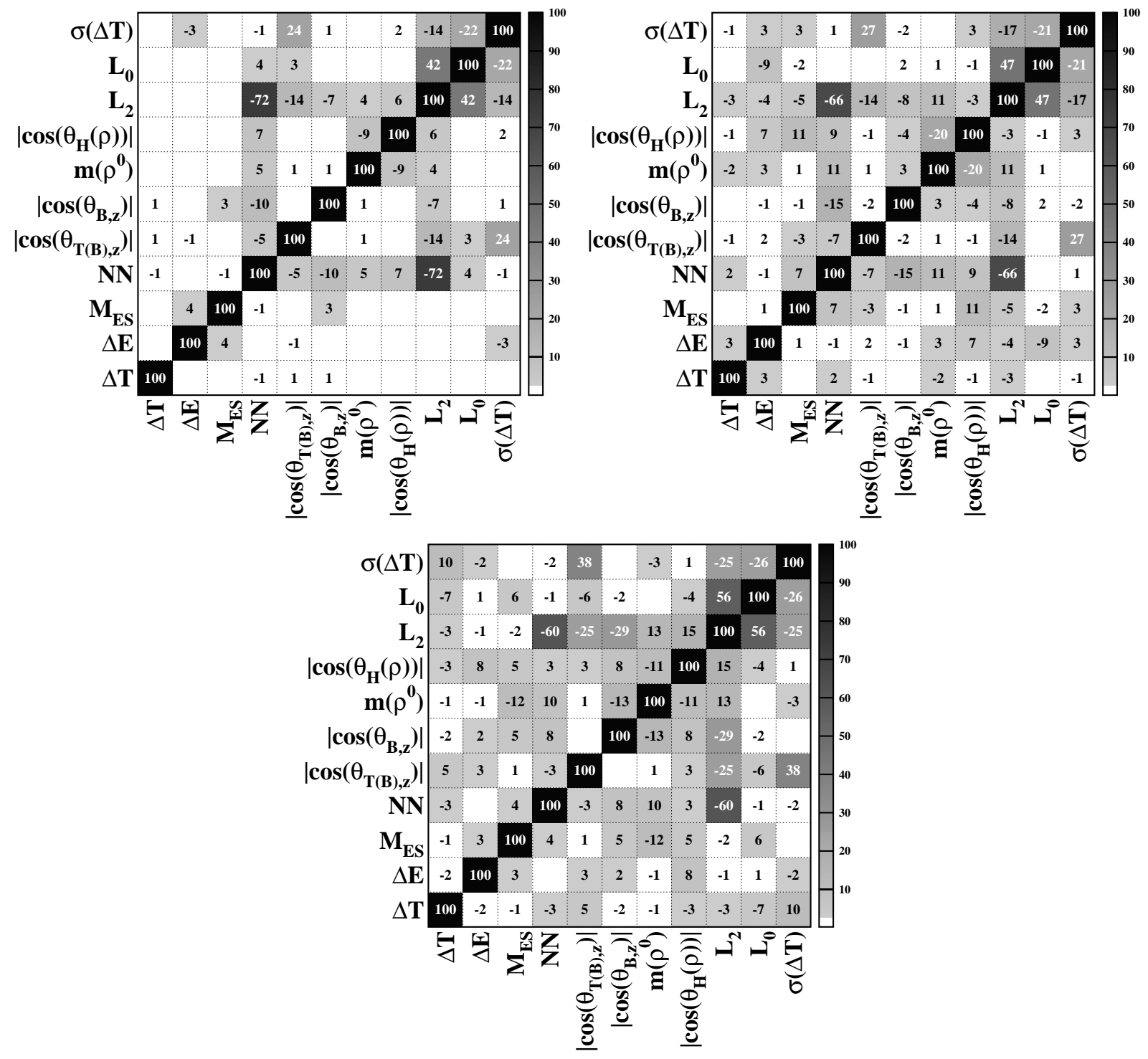

Figure 5.3: Coefficients de corrélation linéaire entre les variables discriminantes pour les événements de signal correctement reconstruits (haut, gauche), mal reconstruits (haut, droite) et les données off-peak (bas).

\subsubsection{Qualitê de l'ajustement (en aveugle)}

La qualité de l'ajustement a été évaluée en aveugle sur les données on-peak. La procédure est similaire à celle décrite dans la section 4.12 .3 pour l'analyse $B^{0} \rightarrow \rho^{ \pm} \pi^{\mp}$, mis à part la génération des paramètres des simulations rapides qui est faite à partir de valeurs obtenues par un ajustement en aveugle sur les données on-peak.

Les distributions de $-\ln \mathcal{L}$ et de $\sigma\left(N_{\rho^{0}} \pi^{0}\right)$ obtenues par ces simulations rapides sont montrées sur la figure 5.4. Les valeurs de $-\ln \mathcal{L}$ et de $\sigma\left(N_{\rho^{0} \pi^{0}}\right)$ obtenues lors de l'ajustement des données on-peak sont représentées par une flèche: on voit que les valeurs obtenues dans l'ajustement nominal sont en parfait accord avec celles des distributions obtenues par simulations rapides. 


\begin{tabular}{|c|c|c|c|}
\hline Configuration & $\sigma\left(N_{\rho^{0} \pi^{0}}\right)$ & & \\
\hline \multicolumn{2}{|l|}{ Nominale (pour différents $\mathcal{B}$ ) } & & \\
\hline $\mathcal{B}=0.5 \times 10^{-6}$ & $10.8 \pm 1.7$ & & \\
\hline $\mathcal{B}=1.0 \times 10^{-6}$ & $12.0 \pm 1.6$ & \multirow{8}{*}{$\begin{array}{c}\text { Différences de } N_{\rho^{0}} \pi^{0} \\
\text { dans } \neq \text { configurations } \\
\left\langle N_{\text {no } \Delta \mathrm{t}}-N_{\text {nominal }}\right\rangle \\
\left\langle N_{\text {no } \Delta \mathrm{E}}-N_{\text {nominal }}\right\rangle \\
\left\langle N_{\text {noNN }}-N_{\text {nominal }}\right\rangle \\
\left\langle N_{\text {no } \Delta \mathrm{t}}-N_{\text {no } \Delta \mathrm{E}}\right\rangle \\
\left\langle N_{\mathrm{no} \Delta \mathrm{t}}-N_{\mathrm{noNN}}\right\rangle \\
\left\langle N_{\mathrm{no} \Delta \mathrm{E}}-N_{\mathrm{noNN}}\right\rangle \\
\end{array}$} & \multirow{2}{*}{$\begin{array}{l}\text { Moyenne \& RMS } \\
\text { de la différence }\end{array}$} \\
\hline $\mathcal{B}=2.0 \times 10^{-6}$ & $13.6 \pm 1.4$ & & \\
\hline $\mathcal{B}=4.0 \times 10^{-6}$ & $16.2 \pm 1.1$ & & $-0.56 \pm 10.87$ \\
\hline \multicolumn{2}{|l|}{ Variations $\left(\mathcal{B}=2.0 \times 10^{-6}\right)$} & & $+0.21 \pm 6.07$ \\
\hline Pas de bruit de fond $B$ & $11.6 \pm 1.3$ & & $+0.14 \pm 11.34$ \\
\hline Pas de bruit de fond conti. & $9.1 \pm 0.7$ & & $-0.81 \pm 12.60$ \\
\hline Pas de bruits de fond & $6.0 \pm 0.5$ & & $-0.77 \pm 18.47$ \\
\hline Sans $\Delta E$ & $15.1 \pm 1.4$ & & $+0.02 \pm 13.29$ \\
\hline Sans $\Delta t$ & $\begin{array}{l}17.0 \pm 1.4 \\
18.5+1.7\end{array}$ & & \\
\hline Sans $m_{\mathrm{ES}}$ & $24.6 \pm 1.4$ & & \\
\hline Sans étiquetage & $14.8 \pm 1.3$ & & \\
\hline Facteur $c_{\text {fact }}(c f$. texte) & $15.8 \pm 1.5$ & & \\
\hline
\end{tabular}

Tableau 5.5: Gauche: erreur sur $N_{\rho^{0}} \pi^{0}$ obtenue dans diverses configurations de simulations rapides. Droite: différences entre les nombres d'événements de signal obtenus dans diverses configurations.

\subsubsection{Ajustements sur des échantillons connus}

Les détails relatifs aux ajustements de validation sur des échantillons connus sont donnés dans la référence [40], et leurs conclusions sont énoncées ci-dessous.

\subsubsection{Monte Carlo $B^{0} \rightarrow \rho^{0} \pi^{0}$ et données off-peak (grande statistique)}

Un ajustement de 22640 événements issus du Monte Carlo $B^{0} \rightarrow \rho^{0} \pi^{0}$ est réalisé: nous obtenons $N_{\rho^{0} \pi^{0}}=22588.7 \pm 151.5$. Similairement, un ajustement est réalisé sur 371 événements de données off-peak, et nous obtenons $N_{\rho^{0} \pi^{0}}=0.0 \pm 1.4$ événements de signal: la contamination entre continuum et signal est donc négligeable.

\subsubsection{Cocktails de MC signal et bruits de fond $B$, et de continuum issu de la simulation rapide}

Nous formons des échantillons contenant un nombre réaliste d'événements de signal (36 événements du Monte Carlo), de bruits de fond $B$ ( $c f$. tableau 5.4), et de bruit de fond continuum de la simulation rapide.

Le tableau 5.7 résume les configurations et résultats de ces ajustements. Aucun biais de plus de $3 \sigma$ n'est observé, mais plusieurs biais au-delà de $2 \sigma$ sont présents. Ces derniers sont pris en compte dans l'erreur systématique ( $c f$. section 5.9).

Comme pour l'analyse $B^{0} \rightarrow \rho^{ \pm} h^{\mp}$, ces biais trouvent probablement leur origine dans la présence de corrélations résiduelles non linéaires entre les variables de l'ajustement final. 


\begin{tabular}{|c|c|c|}
\hline Paramètre & Biais & Ecart type \\
\hline$N_{\rho^{0} \pi^{0}}$ & $-0.087 \pm 0.032$ & $0.95 \pm 0.02$ \\
\hline$N_{q \rho^{0} \pi^{0}}^{\text {Lepton }}$ & $-0.147 \pm 0.035$ & $0.94 \pm 0.02$ \\
\hline$N_{q \rho^{0} \pi^{0}}^{\text {Kaon }}$ & $+0.022 \pm 0.033$ & $0.96 \pm 0.03$ \\
\hline$N_{q \rho^{0} \pi^{0}}^{\mathrm{NT} 1}$ & $+0.011 \pm 0.033$ & $0.99 \pm 0.03$ \\
\hline$N_{q \rho^{0} \pi^{0}}^{\mathrm{ITT}}$ & $-0.011 \pm 0.033$ & $0.99 \pm 0.03$ \\
\hline$N_{q \rho^{0} \pi^{0}}^{\mathrm{NOTag}}$ & $+0.055 \pm 0.032$ & $0.97 \pm 0.02$ \\
\hline
\end{tabular}

Tableau 5.6: Biais et écart-type des pulls des nombres d'événements de signal et de bruit de fond continuum.

\begin{tabular}{|c|c|c|c|}
\hline $\begin{array}{c}\begin{array}{c}\text { Valeur ajustée } \\
\text { moyenne }\end{array} \\
\end{array}$ & $\begin{array}{c}\text { Nombre } \\
\text { d'échantillons } \\
\end{array}$ & $\begin{array}{c}\text { Ecart type moyen } \\
\sqrt{\mathrm{N} . \mathrm{d}^{\prime} \text { échant. }}\end{array}$ & $\begin{array}{l}\text { Biais } \\
(\# \sigma)\end{array}$ \\
\hline \multicolumn{4}{|c|}{ Signal seulement } \\
\hline 35.34 & 572 & 0.24 & 2.75 \\
\hline \multicolumn{4}{|c|}{ Signal + BBkg } \\
\hline 38.9 & 50 & 1.3 & 2.2 \\
\hline \multicolumn{4}{|c|}{ Signal + Continuum } \\
\hline 37.1 & 88 & 1.3 & 0.8 \\
\hline \multicolumn{4}{|c|}{ Signal + BBkg + Continuum } \\
\hline 41.1 & 50 & 2.2 & 2.3 \\
\hline
\end{tabular}

Tableau 5.7: Valeurs de $N_{\rho^{0}} \pi^{0}$ obtenues par des ajustements sur des cocktails de Monte Carlo et simulations rapides (cf. texte). Le nombre d'événements générés est 36.

\subsection{Ajustement final des données}

\subsubsection{Dévoilement du résultat}

Le tableau 5.8 donne le résultat dévoilé de l'ajustement nominal sur les données on-peak dans lequel 18 paramètres sont laissés libres. Nous trouvons $25.6 \pm 11.9$ événements de signal $B^{0} \rightarrow \rho^{0} \pi^{0}$.

\subsubsection{Signification statistique du résultat}

La signification statistique du nombre d'événements de signal $B^{0} \rightarrow \rho^{0} \pi^{0}$ est calculée à partir de la différence entre la valeur de la fonction de vraisemblance de l'ajustement nominal, et celle pour un ajustement où $N_{\rho^{0} \pi^{0}}=0$. On obtient $|\Delta \ln (\mathcal{L})|=7.2$, ce qui correspond à une signification statistique $2.7 \sigma$. Le résultat n'étant pas statistiquement significatif, nous calculons donc une limite supérieur sur le rapport d'embranchement de $B^{0} \rightarrow \rho^{0} \pi^{0}$. 

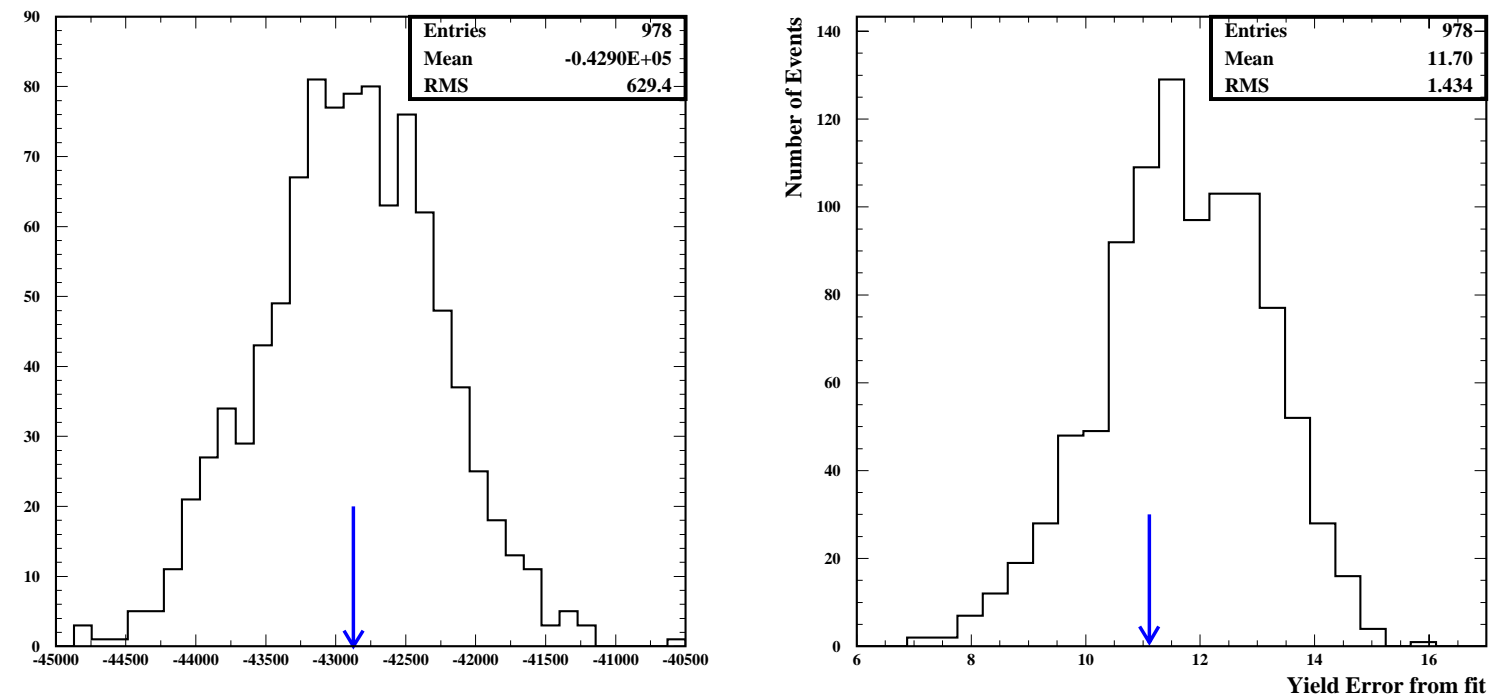

Figure 5.4: Gauche: distribution de $-\ln \mathcal{L}$ pour des expériences de simulations rapides. La flèche indique la valeur de la fonction de vraisemblance de l'ajustement nominal sur les données on-peak. Droite: distribution de $\sigma\left(N_{\rho^{0}} \pi^{0}\right)$ obtenue dans des expériences de simulations rapides. La flèche indique la valeur obtenu dans l'ajustement nominal.

\begin{tabular}{clc}
\hline Paramètre & Description & Valeur \\
\hline$N_{\rho \pi}$ & Nombre d'événements de $B^{0} \rightarrow \rho^{0} \pi^{0}$ & $25.6 \pm 11.9$ \\
$N_{B \rho \pi}^{\mathrm{Lepton}}$ & Nombre d'événements de continuum dans cat. Leptons & $23.5 \pm 5.4$ \\
$N_{B \rho \pi}^{\mathrm{Kaon}}$ & Nombre d'événements de continuum dans cat. Kaons & $1507.2 \pm 39.5$ \\
$N_{B \rho \pi}^{\mathrm{NT}}$ & Nombre d'événements de continuum dans cat. NT1 & $300.7 \pm 17.5$ \\
$N_{B \rho \pi}^{\mathrm{NT} 2}$ & Nombre d'événements de continuum dans cat. NT2 & $1042.0 \pm 32.6$ \\
$N_{B \rho \pi}^{\mathrm{NoT}}$ & Nombre d'événements de continuum dans cat. NoTag & $3605.5 \pm 60.6$ \\
$\xi$ & Paramètre de la fonction d'ARGUS & $-23.5 \pm 2.2$ \\
$\Delta E_{p 1}^{\pi}$ & Coefficient linéaire de la distr. de $\Delta E$ & $-0.68 \pm 0.18$ \\
$a_{2}$ & Coefficient de $x_{\mathrm{NN}}$ & $-0.020 \pm 0.024$ \\
$a_{3}$ & Coefficient de $x_{\mathrm{NN}}$ & $0.060 \pm 0.022$ \\
$a_{4}$ & Coefficient de $x_{\mathrm{NN}}$ & $-0.111 \pm 0.021$ \\
$a_{5}$ & Coefficient de $x_{\mathrm{NN}}$ & $0.090 \pm 0.014$ \\
$\mu(\Delta t)_{1}$ & Moyenne de la $1^{\text {re }}$ Gaussienne pour $\Delta t$ & $0.045 \pm 0.011$ \\
$\sigma(\Delta t)_{1}$ & Ecart-type de la $1^{e}$ Gaussienne pour $\Delta t$ & $1.079 \pm 0.035$ \\
$\sigma(\Delta t)_{2}$ & Ecart-type de la $2^{e}$ Gaussienne pour $\Delta t$ & $2.256 \pm 0.264$ \\
$\sigma(\Delta t)_{3}$ & Ecart-type de la $3^{e}$ Gaussienne pour $\Delta t$ & $11.23 \pm 1.137$ \\
$f(\Delta t)_{1}$ & Fraction de la $1^{r e}$ Gaussienne pour $\Delta t$ & $0.168 \pm 0.047$ \\
$f(\Delta t)_{2}$ & Fraction de la $2^{e}$ Gaussienne pour $\Delta t$ & $0.034 \pm 0.005$ \\
\hline & &
\end{tabular}

Tableau 5.8: Résultats de l'ajustement final. 


\subsubsection{Limite supérieure à $90 \%$ de niveau de confiance sur le nombre d'événements de signal}

Nous suivons la méthode fréquentiste donnée dans la référence [92] pour calculer une limite supérieure sur le rapport d'embranchement de $B^{0} \rightarrow \rho^{0} \pi^{0}$ :

- Nous générons des simulations rapides pour une hypothèse de signal donnée $\mu_{s}^{\text {hyp. }}$. L'ajustement de ces échantillons donne $\mu_{s}^{\prime}$ événements de signal. Nous disposons alors d'une distribution $D_{s+b}\left(\mu_{s}^{\prime}, \mu_{s}^{h y p .}\right)$ pour une hypothèse de signal donnée.

- Nous obtenons la distribution $D_{b}\left(\mu_{s}^{\prime}, 0\right)$ pour une hypothèse de signal nul de la même manière en générant des échantillons avec zéro événement de signal.

- Nous calculons le niveau de confiance pour un résultat $\left(\mu_{s}^{r e s .}\right)$ obtenu sur un échantillon particulier par:

$$
\mathrm{CL}\left(\mu_{\mathrm{s}}^{\mathrm{res}}, \mu_{\mathrm{s}}^{\mathrm{hyp} .}\right) \equiv \frac{D_{s+b}\left(\mu_{s}^{\prime} \leq \mu_{s}^{\mathrm{res} .} ; \mu_{s}^{\mathrm{hyp} .}\right)}{D_{b}\left(\mu_{s}^{\prime} \leq \mu_{s}^{\mathrm{res}}, 0\right)}
$$

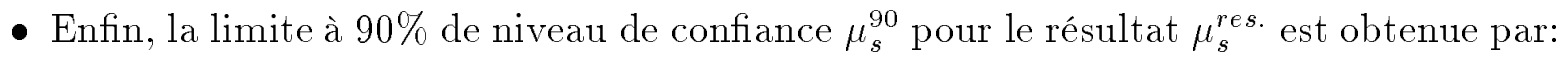

$$
\mathrm{CL}\left(\mu_{\mathrm{s}}^{\mathrm{res}}, \mu_{\mathrm{s}}^{90}\right)=1-0.90
$$

La relation entre $\mu_{s}^{90}$ et $\mu_{s}^{\text {res. }}$ est montrée sur la figure 5.5. Cette courbe est obtenue à partir de 10000 expériences de simulation rapide.

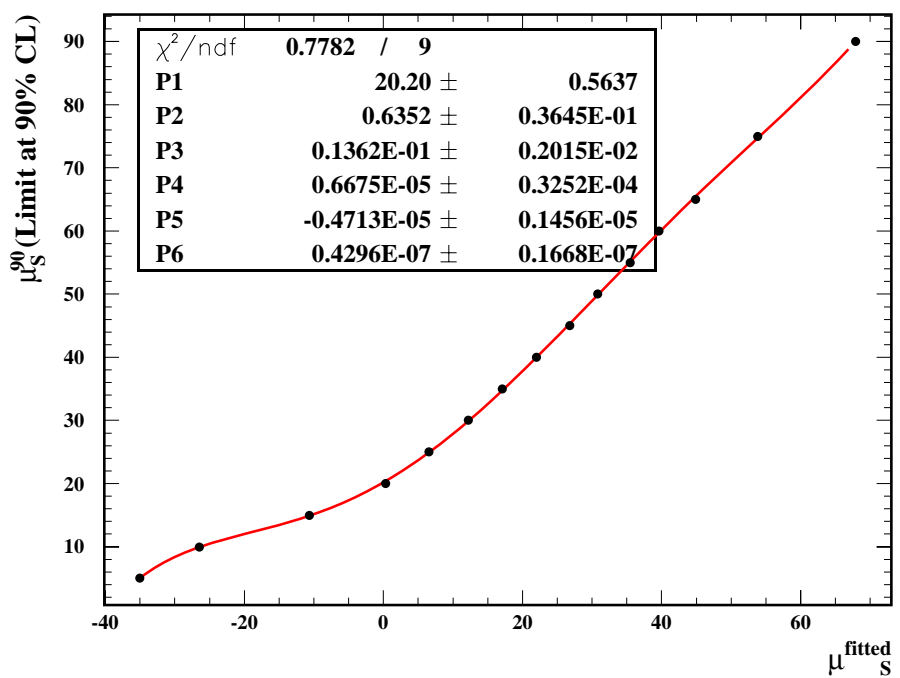

Figure 5.5: Relation entre le nombre d'événements $\mu_{s}^{\text {res. }}$ obtenu dans un échantillon particulier, et la limite supérieure à $90 \%$ de niveau de confiance $\mu_{s}^{90}$ correspondante à ce nombre d'événements. La courbe superposée est un polynôme du $5^{e}$ ordre. 


\subsection{Etudes des erreurs systématiques}

L'étude des erreurs systématiques n'est pas encore complète à ce jour: certaines erreurs présentées ici sont encore préliminaires. Le tableau 5.9 résume ces incertitudes systématiques. Lorsque nécessaire, les détails relatifs au calcul de ces incertitudes sont exposés dans les sections suivantes.

L'erreur systématique est dominée par les biais observés dans les ajustements sur cocktails de Monte Carlo ( $c f$. section 5.7.2.2). L'origine de ces biais est en cours d'investigation. Les erreurs systématiques les plus importantes mis à part ces biais sont dues à l'identification des particules, la reconstruction du $\pi^{0}$ et le bruit de fond $B$.

\subsubsection{Bruits de fond $B$}

Comme dans l'analyse $B^{0} \rightarrow \rho h$, nous varions les rapports d'embranchement des modes de bruit de fond $B$ à l'intérieur de leur incertitude, et obtenons ainsi la systématique due au bruit de fond $B$ sur le nombre d'événements de signal $B^{0} \rightarrow \rho^{0} \pi^{0}$. Les résultats sont donnés dans le tableau 5.10. L'erreur systématique due au bruit de fond $B$ est dominée par le mode $B^{0} \rightarrow K^{* 0}\left(K^{+} \pi^{-}\right) \pi^{0}$.

\subsubsection{Validations des distributions du signal en utilisant le mode $B^{0} \rightarrow D^{-} \rho^{+}$}

Comme pour l'analyse $B^{0} \rightarrow \rho^{ \pm} \pi^{\mp}$, nous utilisons le mode $B^{0} \rightarrow D^{-} \rho^{+}$pour valider les distributions du signal. Ce mode est moins approprié pour $B^{0} \rightarrow \rho^{0} \pi^{0}$ que pour $B^{0} \rightarrow \rho^{ \pm} \pi^{\mp}$, puisqu'il contient un $\rho$ chargé à la place d'un $\rho$ neutre, et ne contient pas de $\pi^{0}$ célibataire. Néanmoins, il s'agit du mode de grande statistique le plus proche de notre signal (les modes $B^{0} \rightarrow D^{0} \rho^{0}, B^{0} \rightarrow D^{0} \pi^{0}$ étant supprimés de couleur). La méthode utilisée ici est similaire à celle décrite dans la section 4.14.2.

\subsubsection{Distribution de $\Delta E$ et $m_{\mathrm{ES}}$}

Les distributions de $\Delta E$ et $m_{\mathrm{ES}}$ sont ajustées avec une fonction de Crystal-Ball dans les données et Monte Carlo $B^{0} \rightarrow D^{-} \rho^{+}$. La différence entre les paramètres des fonctions entre données et Monte Carlo est utilisée pour évaluer la systématique due à ces distributions. Les résultats sont résumés dans le tableau 5.11 .

\subsubsection{Distribution de $x_{\mathrm{NN}}$}

Une méthode similaire à celle utilisée dans l'analyse $B^{0} \rightarrow \rho h$ ( $c f$. section 4.14.2.1) mène à une erreur systématique sur $N_{\rho^{0}} \pi^{0}$ de 1.8 événements. Cette erreur est néanmoins encore préliminaire (la procédure et les résultats ne sont donc pas détaillés ici en détail). Nous utilisons cependant cette erreur préliminaire pour le résultat dévoilé ici.

\subsubsection{Interférences résiduelles $\rho^{0}-f^{0}$}

La figure 5.6 montre les effets des interférences résiduelles entre le $\rho^{0}$ et le $f^{0}$ pour une valeur d'hélicité du $\rho^{0}$ donnée (sur la figure, $\cos \theta_{H}(\rho)=1$ ). Néanmoins, l'effet intégré dans le diagramme de Dalitz ( $c$ - ̀̀- $d$ pour toutes les valeurs d'helicité du $\rho^{0}$ ) est nul, puisque 


\begin{tabular}{|c|c|c|}
\hline Incertitudes & Valeur & Commentaires \\
\hline \multicolumn{3}{|c|}{ Nombre d'événements et efficacités } \\
\hline$N_{\rho^{0} \pi^{0}}$ & $25.6 \pm 11.9$ & ajustement nominal \\
\hline$N_{\rho^{0} \pi^{0}}-N_{\text {biais }}$ & $22.0 \pm 10.2$ & biais ( $c f$. section 5.7.2.2) \\
\hline$N_{\rho^{0} \pi^{0}}$ à $90 \% \mathrm{CL}$ & 40 & limite supérieur à $90 \% \mathrm{CL}$ \\
\hline$\epsilon_{\text {signal }}^{\text {init. }}$ & $20.2 \pm 0.1 \%$ & calculée sur MC corrigé pour $\pi^{0}$ \\
\hline$\epsilon_{\text {signal }}$ & $20.0 \pm 0.1 \%$ & correction reconstruction traces \\
\hline \multicolumn{3}{|c|}{ "Systématiques sur l'efficacité de sélection (en \%) } \\
\hline Rec. traces & 1.6 & correction pour chaque trace \\
\hline Ident. des particules & 4.0 & cf. ref. $[93]$ \\
\hline Erreur corr. sur $\pi^{0}$ & 5.0 & cf. section 4.14 .1 \\
\hline Erreur décorr. sur $\pi^{0}$ & 0.8 & cf. section 4.14 .1 \\
\hline Coupure sur $\Delta E$ & 0.1 & $B \rightarrow D \rho(c f$. section 5.9 .2$)$ \\
\hline Coupure sur $M_{E S}$ & 0.0 & $B \rightarrow D \rho$ \\
\hline $\mathcal{B}\left(\rho \rightarrow \pi^{+} \pi^{-}\right)$ & 1.6 & Erreur sur $\Gamma\left(\pi^{+} \pi^{-}\right) / \Gamma$ (Total) [44] \\
\hline$N(B \bar{B})$ & 1.1 & Comptage des $B$ \\
\hline Sous-total: & 6.9 & \\
\hline \multicolumn{3}{|c|}{ "Autres systématiques (en nombre d'événements) } \\
\hline$\Delta m \pm 0.008 \mathrm{ps}^{-1}$ & 0.09 & PDG \\
\hline$\tau \pm 0.016 \mathrm{ps}$ & 0.12 & PDG \\
\hline$\Delta t$ Résolution & 1.05 & Breco (cf. section 4.14.3) \\
\hline Etiquetage des $B$ & 0.20 & Breco (cf. section 4.14.3) \\
\hline Fract. signal mal rec. & 0.81 & $( \pm 100 \%)$ \\
\hline Distribution $\Delta E$ & 0.53 & $B \rightarrow D \rho$ \\
\hline Distribution $m_{\mathrm{ES}}$ & 2.00 & $B \rightarrow D \rho$ \\
\hline Distribution $x_{\mathrm{NN}}$ & 1.8 & $B \rightarrow D \rho$ \\
\hline Bruit de fond $B$ & 4.9 & cf. section 5.9 .1 \\
\hline Méthode d'ajust. & 3.6 & cf. section 5.7.2.2 \\
\hline Sous-total: & 6.8 & événements \\
\hline Totale systématiques: & 7.4 & événements \\
\hline \multicolumn{3}{|c|}{ 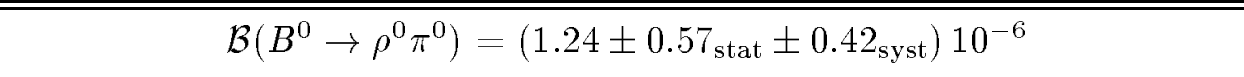 } \\
\hline Limite à $90 \% \mathrm{CL}$ & $2.7 \cdot 10^{-6}$ & statistique et systématiques incluses \\
\hline
\end{tabular}

Tableau 5.9: Incertitudes systématiques sur le rapport d'embranchement de $B^{0} \rightarrow \rho^{0} \pi^{0}$ : les systématiques sur l'efficacité de sélection sont données relativement au nombre d'événements $B^{0} \rightarrow \rho^{0} \pi^{0}$. Elles sont additionnées en quadrature aux incertitudes systématiques dues à l'ajustement (données en nombre d'événements). Les deux dernières lignes donnent le rapport d'embranchement de $B^{0} \rightarrow \rho^{0} \pi^{0}$ et sa limite supérieure incluant les incertitudes statistique et systématiques. 


\begin{tabular}{l|c}
\hline \hline Mode & $\sigma_{\text {syst }}^{\rho^{0} \pi^{0}}$ \\
\hline$B^{+} \rightarrow \pi^{+} \pi^{0}$ & 0.03 \\
$B^{+} \rightarrow K^{+} \pi^{0}$ & 0.01 \\
$B^{+} \rightarrow \rho^{+} \pi^{0}$ & 1.57 \\
$B^{0} \rightarrow \rho^{+} \pi^{-}$ & 0.10 \\
$B^{0} \rightarrow \rho^{-} \pi^{+}$ & 0.11 \\
$B^{0} \rightarrow \rho^{-} K^{+}$ & 0.02 \\
$B^{0} \rightarrow K^{* 0}\left(K^{+} \pi^{-}\right) \pi^{0}$ & 3.86 \\
$B^{0} \rightarrow K^{* 0}\left(K^{+} \pi^{-}\right) \gamma$ & 0.61 \\
$B^{0} \rightarrow\left(K^{* *} \pi\right)^{0}$ & 0.17 \\
$B^{+} \rightarrow\left(K^{* *} \pi\right)^{+}$ & 0.08 \\
$B^{0} \rightarrow \eta^{\prime}\left(\rho^{0} \gamma\right) \pi^{0}$ & 0.66 \\
$B^{0} \rightarrow f^{0}(980) \pi^{0}$ & 0.18 \\
$B^{0} \rightarrow \pi^{+} \pi^{-} \pi^{0}($ non-res $)$ & 1.88 \\
$B^{0} \rightarrow \rho^{+} \rho_{\text {long }}^{-}$ & 0.66 \\
$B^{+} \rightarrow \rho^{+} \rho_{\text {long }}^{0}$ & 1.09 \\
$B^{+} \rightarrow a_{1}^{+} \pi^{0}$ & 0.39 \\
$B^{0} \rightarrow$ charme & 0.64 \\
$B^{ \pm} \rightarrow$ charme & 0.24 \\
\hline Total & 4.9 \\
\hline \hline
\end{tabular}

Tablea1 5.10: Contributions des incertitudes sur les rapports d'embranchement des modes de bruits de fond $B$ à l'erreur systématique sur $N_{\rho^{0}} \pi^{0}$.

l'intégrale de $\cos \theta_{H}(\rho)$ entre -1 et +1 est nulle. L'effet des interférences est donc inexistant pour la mesure du rapport d'embranchement de $B^{0} \rightarrow \rho^{0} \pi^{0}$.

\subsubsection{Interférences résiduelles $\rho^{0}-\rho^{ \pm}$et avec $\pi^{+} \pi^{-} \pi^{0}$ non résonant}

L'incertitude systématique due aux interférences résiduelles entre le $\rho^{0}$ et le $\rho^{ \pm}$est encore en cours d'évaluation, mais est attendue plus faible que l'incertitude due au $f^{0}$, car les interférences avec les $\rho^{ \pm}$ne se produisent que dans les coins du diagramme de Dalitz, et non pas tout le long de la bande du $\rho^{0}$ comme c'est le cas avec le $f^{0}$. D'autre part, la coupure de réjection des $\rho^{ \pm}$est très sévère.

L'incertitude due aux interférences avec le $\pi^{+} \pi^{-} \pi^{0}$ non-résonant est aussi en cours d'évaluation.

\subsection{Limite supérieure à $90 \% \mathrm{CL}$ sur $\mathcal{B}\left(B^{0} \rightarrow \rho^{0} \pi^{0}\right)$ in- cluant les incertitudes systématiques}

Suivant une approche fréquentiste, nous additionnons linéairement l'erreur systématique totale (11.8) à la limite supérieure à $90 \%$ CL précédemment calculée sur la base du nombre d'événements obtenus dans l'ajustement (corrigé du biais observé dans la méthode d'ajustement). La limite statistique valait 40 événements, et la nouvelle limite incluant aussi les incertitudes systématiques vaut 47.4 événements. 


\begin{tabular}{ccc}
\hline Paramètre & Données & Monte Carlo \\
\hline Moyenne de $\Delta E(\mathrm{MeV})$ & -5.4 & 1.2 \\
Largeur de $\Delta E(\mathrm{MeV})$ & 24.7 & 27.1 \\
Moyenne de $m_{\mathrm{ES}}\left(\mathrm{MeV} / \mathrm{c}^{2}\right)$ & 5.2797 & 5.2803 \\
Largeur de $m_{\mathrm{ES}}\left(\mathrm{MeV} / \mathrm{c}^{2}\right)$ & 2.7 & 3.2 \\
\hline
\end{tabular}

Tableau 5.11: Paramètres de la fonction de Crystal-Ball paramétrant $\Delta E$ et $m_{\mathrm{ES}}$ dans les données et le Monte Carlo $B^{0} \rightarrow \rho^{0} \pi^{0}$.

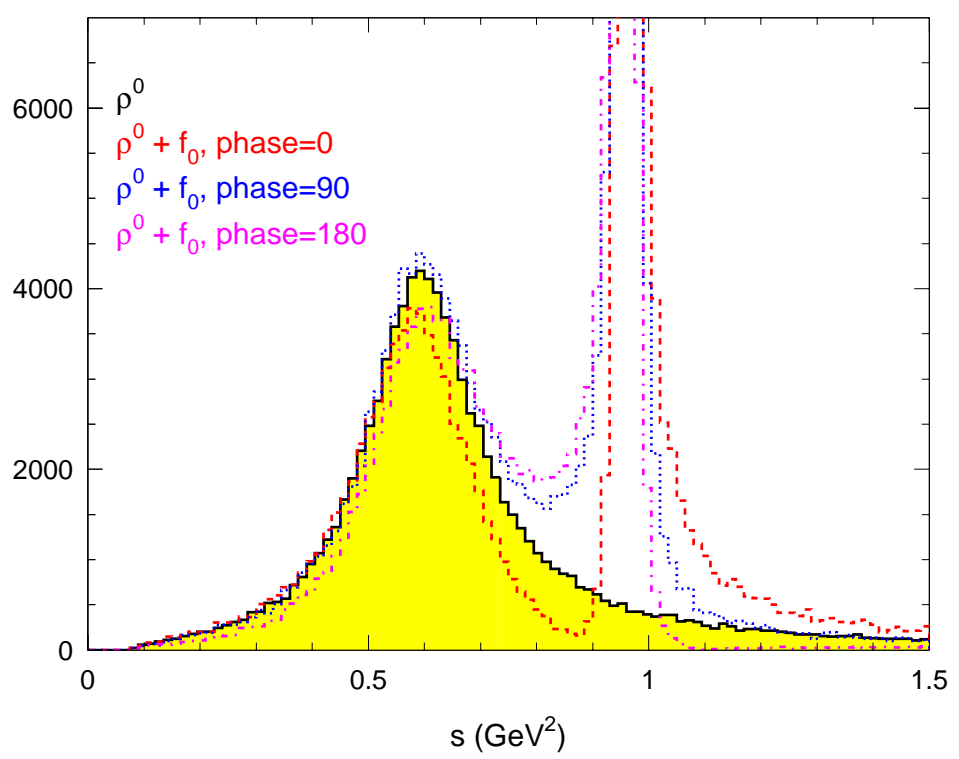

Figure 5.6: Distributions des Breit-Wigner de $\pi^{+} \pi^{-}$pour le $\rho^{0}$ seul (histogramme plein jaune), et pour $\rho^{0}+f^{0}$ (de telle sorte que les quantités de $\rho^{0}$ et $f^{0}$ soient égales en l'absence d'interférences, soit $\eta=1$ - cf. Eq. ??) avec diverses phases fortes relatives (histogrammes de diverses couleurs). Les histogrammes sont normalisés à luminosité constante.

Utilisant l'efficacité de sélection corrigée du tableau $5.9(20.0 \pm 0.1 \%)$, nous obtenons la limite suivante sur le rapport d'embranchement de $B^{0} \rightarrow \rho^{0} \pi^{0}$ :

$$
\mathcal{B}\left(B^{0} \rightarrow \rho^{0} \pi^{0}\right)<2.7 \times 10^{-6} \quad \text { à } 90 \% \mathrm{CL} .
$$

\subsection{Conclusion}

Nous avons réalisé une analyse pour mesurer le rapport d'embranchement de $B^{0} \rightarrow \rho^{0} \pi^{0}$ utilisant une luminosité intégrée de $81.9 \mathrm{fb}^{-1}$. Le nombre d'événements de signal obtenus par l'ajustement du maximum de vraisemblance possède une signification de $2.7 \sigma$. Nous sommes ainsi à la limite de l'observation d'un signal, mais avec une signification statistique faible.

Le rapport d'embranchement obtenu est le suivant:

$$
\mathcal{B}\left(B^{0} \rightarrow \rho^{0} \pi^{0}\right)=\left(1.24 \pm 0.57_{\text {stat }} \pm 0.42_{\mathrm{syst}}\right) \times 10^{-6}
$$

où la première erreur est statistique et la seconde correspond aux incertitudes systématiques expérimentales. 
Nous posons la limite supérieure à $90 \%$ de niveau de confiance sur le rapport d'embranchement de $B^{0} \rightarrow \rho^{0} \pi^{0}$ suivante:

$$
\mathcal{B}\left(B^{0} \rightarrow \rho^{0} \pi^{0}\right)<2.7 \times 10^{-6} \text { à } 90 \% \mathrm{CL} .
$$

Cette limite est à comparer aux meilleures limites supérieures précédentes mesurées par CLEO sur $9.7 \cdot 10^{6}$ paires $B \bar{B}, \mathcal{B}\left(B^{0} \rightarrow \rho^{0} \pi^{0}\right)<5.1 \times 10^{-6}$, et Belle sur $31.9 \cdot 10^{6}$ paires $B \bar{B}$, $\mathcal{B}\left(B^{0} \rightarrow \rho^{0} \pi^{0}\right)<5.3 \times 10^{-6}$, qui n'incluent que les incertitudes statistiques. La limite mesurée ici n'a pas évoluée aussi rapidement que l'augmentation de données pourrait le permettre, parce que la signification statistique de notre signal est plus élevée que pour les résultats précédents (nous observons quasiment un signal). D'autre part, notre limite tient compte des incertitudes systématiques, ce qui n'est pas le cas pour les limites précédentes. Finalement, comme pour la mesure des rapports d'embranchement de $B^{0} \rightarrow \rho^{ \pm} \pi^{\mp}$ et $B^{0} \rightarrow \rho^{-} K^{+}$, le traitement du bruit de fond $B$ est plus correct que dans les analyses précédemment réalisées. 


\section{Chapitre 6}

\section{Premiers pas dans l'analyse Dalitz de $B^{0} \rightarrow \pi^{+} \pi^{-} \pi^{0}$}

\subsection{Introduction}

Les chapitres 4 et 5 décrivent l'analyse en deux corps des modes $B^{0} \rightarrow \rho^{ \pm} h^{\mp}$ et $B^{0} \rightarrow \rho^{0} \pi^{0}$ : pour réaliser ces analyses, nous avons isolé dans le diagramme de Dalitz les bandes dominées par le $\rho(770)$, et avons exclu les régions où les interférences entre les $\rho$ de différentes charges sont les plus importantes ( $c$ - $\grave{a}-d$ les coins du diagramme).

Les observables ainsi mesurées peuvent être traduites en contraintes sur l'angle $\alpha$ en utilisant des ingrédients supplémentaires tels que les désintégrations des $B$ chargés, ou les modes $\rho K$ et $K^{*} \pi$ ( $c f$. chapitre 2). Les contraintes obtenues sont détaillées dans le chapitre 9 .

Mais le réel potentiel de l'état final $\pi^{+} \pi^{-} \pi^{0}$ réside dans l'analyse de la distribution des événements dans le diagramme de Dalitz tenant compte des interférences entre les diverses résonances menant à cet état final ( $c f$. section 2.2.4).

Ce chapitre expose les premiers pas expérimentaux dans cette analyse Dalitz complète. La transition entre les analyses en deux corps exposées dans les chapitres 4 et 5 et l'analyse en trois corps de $B^{0} \rightarrow \pi^{+} \pi^{-} \pi^{0}$ comporte les étapes suivantes:

- Nous considérons désormais la totalité du diagramme Dalitz: nous retirons donc les coupures sur les masses $\pi^{ \pm} \pi^{0}$, l'angle d'hélicité du $\rho$, et retirons ces variables du réseau de neurones de lutte contre le bruit de fond continuum

- Nous devons modéliser l'amplitude ( $c-\grave{a}-d$, à la fois leur module et leur phase forte) des différentes résonances intermédiaires menant à l'état final $\pi^{+} \pi^{-} \pi^{0}$.

- A cause des interférences, la notion de "charge" du $\rho$ tel qu'il en était fait usage dans l'analyse en deux corps de $B^{0} \rightarrow \rho^{ \pm} h^{\mp}$ n'a plus le même sens: on définit des événements reconstruits de "bonne charge" (respectivement "mauvaise charge") comme étant situés du même (respectivement opposé) coté de la diagonale $m^{2}\left(\pi^{+} \pi^{0}\right)=m^{2}\left(\pi^{-} \pi^{0}\right)$ du diagramme de Dalitz que lors de leur génération ( $c-\grave{a}-d$, selon que leur reconstruction a engendré ou pas un "saut" de l'autre coté de la diagonale du diagramme Dalitz).

On garde donc dans la suite les trois catégories de signal: correctement reconstruit, mal reconstruit de "bonne charge", et mal reconstruit de "mauvaise charge", au sens où nous venons de les définir. 
- Nous ne considérons plus $B^{0} \rightarrow \rho^{-} K^{+}$comme un signal, mais comme un bruit de fond à $B^{0} \rightarrow \pi^{+} \pi^{-} \pi^{0}$.

La figure 6.1 montre les distributions dans le diagramme de Dalitz du Monte Carlo noninterférant de $B^{0} \rightarrow \rho^{ \pm} \pi^{\mp}, B^{0} \rightarrow \rho^{0} \pi^{0}$ (pour les événements correctement et mal reconstruits) et pour les données offpeak. On observe la distribution angulaire en $\cos ^{2} \theta$ pour le signal, alors que celle du bruit de fond continuum est modelée par les coupures de sélection.
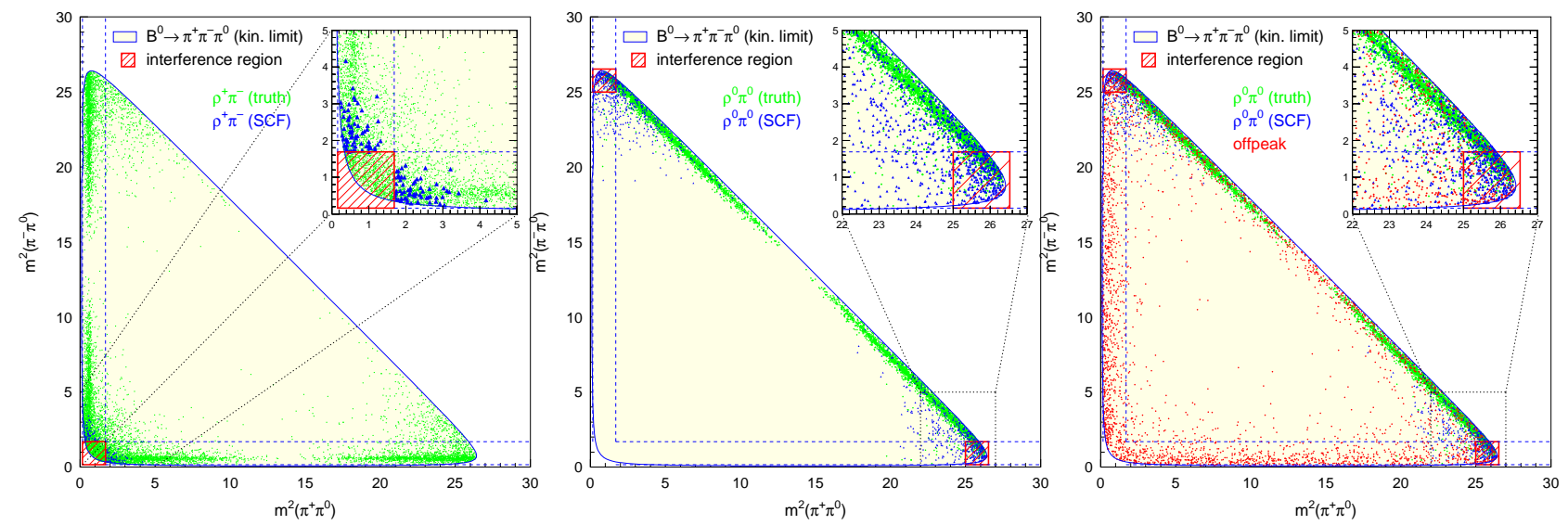

Figure 6.1: Distributions dans le diagramme de Dalitz du Monte Carlo non-interférant de $B^{0} \rightarrow \rho^{ \pm} \pi^{\mp}$ (gauche), $B^{0} \rightarrow \rho^{0} \pi^{0}$ (milieu), et données offpeak (droite, points en rouge). Les points triangulaires montrent les événements de mauvaise charge du signal $B^{0} \rightarrow \rho^{ \pm} \pi^{\mp}$.

On peut trouver dans la référence [33] une étude Monte Carlo (antérieure au démarrage de $B A B A R$ ) de l'analyse Dalitz $B^{0} \rightarrow \pi^{+} \pi^{-} \pi^{0}$, analysant entre autres les effets expérimentaux et les systématiques théoriques associées à la mesure de l'angle $\alpha$. Cette étude concluait à la nécessité d'une statistique importante pour mener à bien l'analyse, et soulignait l'importance de considérer les résonances $\rho$ excitées $\left(\rho(1450), \rho(1700)\right.$ et $\left.\rho_{3}(1690)\right)$.

L'objectif de l'étude exposée ici est de mettre en oeuvre l'analyse Dalitz dans un contexte expérimental plus réaliste ( $c$ - $\grave{a}-d$ utilisant tout ce que les premières années de $B A B A R$ nous ont appris, et en particulier les analyses de $B^{0} \rightarrow \rho^{ \pm} \pi^{\mp}$ et $B^{0} \rightarrow \rho^{0} \pi^{0}$ présentées dans les chapitres 4 et 5).

Nous exposons la sélection, lutte contre le bruit de fond continuum et traitement du bruit de fond $B$ dans les sections 6.2 à 6.4 . La section 6.5 détaille les ingrédients nécessaires à l'élaboration de la distribution des événements de signal dans le diagramme de Dalitz: les facteurs de forme du $\pi$, les effets dus au détecteur, et les effets dus à la sélection. Après avoir donné la fonction de vraisemblance (section 6.6), nous présentons les résultats des premières simulations réalisées dans ce contexte d'analyse dans la section 6.7 .

\subsection{Reconstruction de $B^{0} \rightarrow \pi^{+} \pi^{-} \pi^{0}$}

La reconstruction de l'état final $B^{0} \rightarrow \pi^{+} \pi^{-} \pi^{0}$ est similaire à celle de l'état final $B^{0} \rightarrow \rho^{ \pm} \pi^{\mp}$ (voir Sec. 4.3):

- Traces chargées: les traces chargées sont reconstruites de la même manière que pour $B^{0} \rightarrow \rho^{ \pm} \pi^{\mp}$ (cf. section 4.3.1.1). Puisque $B^{0} \rightarrow \rho^{-} K^{+}$est désormais considéré comme du bruit de fond, nous rejetons les événements pour lesquels une de deux traces chargées 
est compatible avec l'hypothèse d'un kaon (alors que dans l'analyse $B^{0} \rightarrow \rho^{ \pm} \pi^{\mp}$, nous imposions cette condition sur la trace issue du $\rho$ seulement).

- Traces neutres: la sélection est identique à celle effectuée pour $B^{0} \rightarrow \rho^{ \pm} h^{\mp}, c$ - ̀-a $d$, $E(\gamma)>50 \mathrm{MeV}, 0.01<\operatorname{LAT}(\gamma)<0.6,0.1<m\left(\pi^{0}\right)<0.16 \mathrm{GeV} / c^{2}$

- Candidat $\rho$ : à la différence de $B^{0} \rightarrow \rho^{ \pm} h^{\mp}$, nous gardons l'ensemble du diagramme de Dalitz. Nous ne coupons donc pas sur la masse et l'angle d'hélicité du $\rho$, ni n'excluons les zones d'interférence. Néanmoins, nous appliquons la coupure contre le bruit de fond $B^{0} \rightarrow \pi^{+} \pi^{-}: m\left(\pi^{+} \pi^{-}\right)<5.14 \mathrm{GeV} / \mathrm{c}^{2}$.

- Candidat $B:$ puisque nous avons relâché les coupures sur le candidat $\rho$, nous appliquons des coupures plus dures sur $m_{\mathrm{ES}}$ et $\Delta E$ que dans $B^{0} \rightarrow \rho^{ \pm} h^{\mp}$, afin de limiter le nombre d'événements onpeak sélectionnés ${ }^{1}$. D'autre part, $B^{0} \rightarrow \rho^{-} K^{+}$n'étant plus considéré comme du signal, on peut appliquer une coupure inférieure sur $\Delta E$ plus élevée. Nous appliquons ainsi les coupures suivantes: $5.24<m_{\mathrm{ES}}<5.2895 \mathrm{GeV} / \mathrm{c}^{2}$ et $-0.10<\Delta E<0.09 \mathrm{GeV}$.

- $\Delta t$ et $\sigma_{\Delta t}$ : les mêmes coupures que pour $B^{0} \rightarrow \rho^{ \pm} h^{\mp}$ sont appliquées: $|\Delta t|<20$ ps, $\sigma_{\Delta t}<2.5 \mathrm{ps}$.

- Réseau de neurones: la section 6.3 donne les détails relatifs à la réalisation du réseau de neurones pour la lutte contre le bruit de fond continuum dans l'analyse Dalitz. Le réseau contient les variables $L_{0}, L_{2}, \cos \theta(B, z), \cos \theta\left(T_{B}, z\right)$, et nous coupons sur $x_{\mathrm{NN}}$ à zéro.

L'efficacité finale ainsi que la fraction d'événements mal-reconstruits sont données dans le tableau 6.1. Cette dernière est plus élevée que pour $B^{0} \rightarrow \rho^{ \pm} \pi^{\mp}$, puisque les événements mal reconstruits s'accumulent dans les coins du diagramme de Dalitz précédemment rejetés mais désormais retenus dans la sélection.

\begin{tabular}{c|cc}
\hline Monte Carlo & $\epsilon(\%)$ & $f_{\mathrm{SCF}}(\%)$ \\
\hline$B^{0} \rightarrow \rho^{ \pm} \pi^{\mp}$ & $23.53 \pm 0.06$ & 37.70 \\
\hline
\end{tabular}

Tableau 6.1: Efficacité finale de sélection et fraction d'événements mal-reconstruits dans le Monte Carlo $B^{0} \rightarrow \rho^{ \pm} \pi^{\mp}$.

\subsection{Lutte contre le bruit de fond $q \bar{q}$}

La lutte contre le bruit de fond continuum dans l'analyse $B^{0} \rightarrow \pi^{+} \pi^{-} \pi^{0}$ est similaire à celle de l'analyse de $B^{0} \rightarrow \rho h$ ( $c f$. section 4.6), mis à part que les variables $m(\rho)$ et $\cos \theta_{H}(\rho)$ sont retirées du réseau de neurones.

La configuration de variables retenue pour l'analyse Dalitz est la suivante:

$$
L_{0}, L_{2}, \cos \theta(B, z), \cos \theta\left(T_{B}, z\right)
$$

La configuration d'entraînement du réseau de neurones est identique à celle de l'analyse $B^{0} \rightarrow \rho h$. Nous appliquons une coupure sur la sortie du réseau de neurones à $x_{\mathrm{NN}} \geq 0$.

\footnotetext{
${ }^{1}$ L'intérêt principal est de limiter le coût en CPU de l'ajustement final.
} 
Le graphe de gauche sur la figure 6.2 montre l'efficacité de sélection du signal en fonction de l'efficacité du bruit de fond pour une coupure sur $x_{\mathrm{NN}}$, et sur un discriminant de Fisher entraîné sur les mêmes variables pour comparaison. Le graphe de droite montre la distribution de $x_{\mathrm{NN}}$ pour le signal (correctement et mal reconstruit), et le bruit de fond continuum.
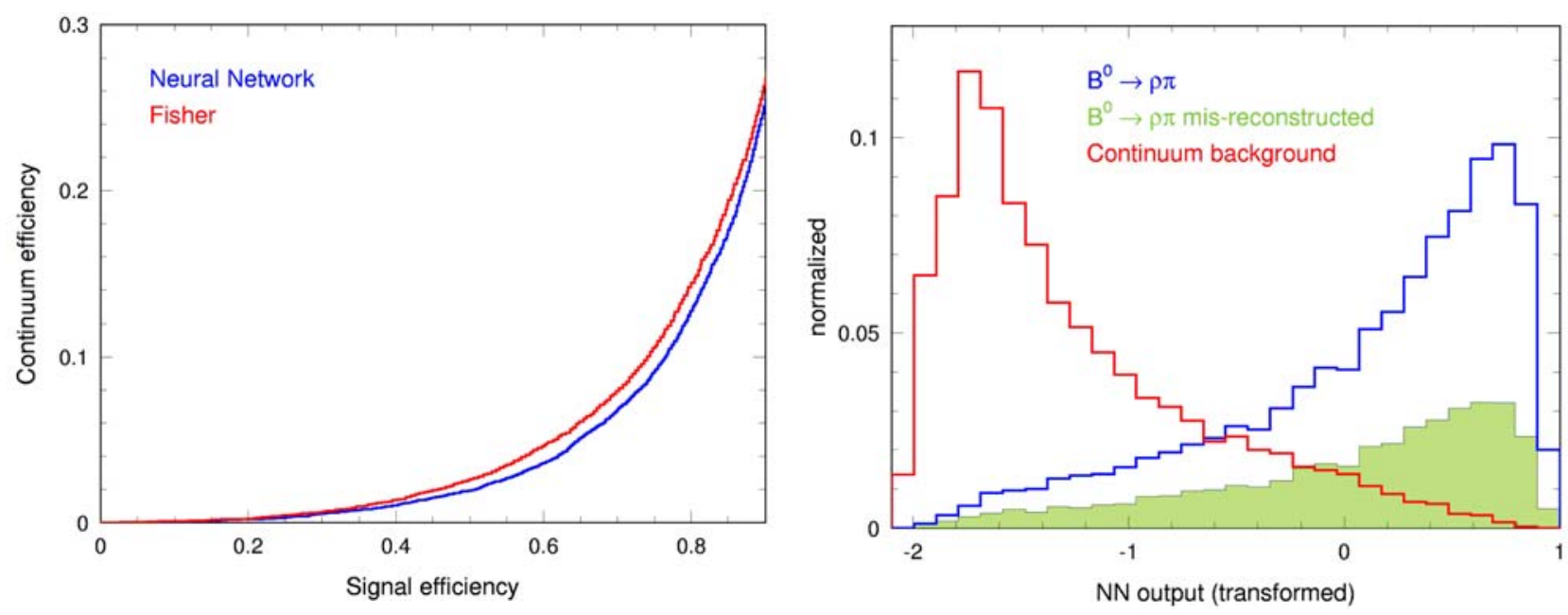

Figure 6.2: Gauche: efficacité de sélection du signal en fonction de l'efficacité du bruit de fond pour une coupure sur $x_{\mathrm{NN}}$, comparé à celle pour une coupure sur un discriminant de Fisher entraîné sur les mêmes variables. Droite: distribution de $x_{\mathrm{NN}}$ pour le signal (correctement et mal reconstruit), et le bruit de fond continuum.

\subsection{Bruit de fond provenant d'autres modes $B$}

Nous avons considéré, comme dans l'analyse $B^{0} \rightarrow \rho^{ \pm} h^{\mp}$, plus de 100 modes non-charmés (voir les tableaux 4.9 à 4.11 montrant l'ensemble de ces modes). Nous ne répétons pas ici ces tableaux complets, puisque les résultats sont assez similaires à ceux obtenus pour $\left.B^{0} \rightarrow \rho^{ \pm} h^{\mp}\right)$. Les rapports d'embranchement utilisés dans l'évaluation du nombre attendu d'événements sont les mêmes que dans la section 4.8.1.

Le tableau 6.2 donne la liste des modes non-charmés retenus dans l'analyse Dalitz. Les coupures étant plus lâches que pour l'analyse $B^{0} \rightarrow \rho^{ \pm} \pi^{\mp}$, le nombre attendu d'événements de bruits de fond est légèrement plus élevé que pour $B^{0} \rightarrow \rho^{ \pm} \pi^{\mp}$.

Les contributions charmées sont évaluées à partir du Monte Carlo générique, comme pour $B^{0} \rightarrow \rho^{ \pm} \pi^{\mp}$ ( $c f$. section 4.8.2). Nous attendons ainsi 1033 événements charmés des $B$ chargés, et 524 événements des $B$ neutres, soit environ 5 fois plus que dans le cas de $B^{0} \rightarrow \rho^{ \pm} \pi^{\mp}$, dû au relâchement des coupures sur la masse du $\rho$.

L'ensemble des modes retenus sont regroupés en 13 classes, décrites dans le tableau 6.3.

\subsection{Distributions de $B^{0} \rightarrow \pi^{+} \pi^{-} \pi^{0}$ dans le diagramme de Dalitz}

Les distributions de $m^{2}\left(\pi^{+} \pi^{0}\right)$ et $m^{2}\left(\pi^{-} \pi^{0}\right)$ sont l'ingrédient essentiel de l'analyse Dalitz. On aurait néanmoins pu choisir n'importe quelle paire de variables parmi:

$$
\left(m^{2}\left(\pi^{+} \pi^{0}\right), m^{2}\left(\pi^{-} \pi^{0}\right), m^{2}\left(\pi^{+} \pi^{-}\right), \cos \theta_{H}(\rho)^{+0}, \cos \theta_{H}(\rho)^{-0}, \cos \theta_{H}(\rho)^{+-}\right),
$$




\begin{tabular}{|c|c|c|c|}
\hline Mode & $\mathcal{B}$ & $\epsilon(\%)$ & $N_{\text {exp }}$ \\
\hline$B^{+} \rightarrow \rho^{+} K^{* 0} \rightarrow K^{+} \pi_{\text {long }}^{-}$ & $12 \pm 12$ & 0.038 & $0.40 \pm 0.40$ \\
\hline$B^{+} \rightarrow \rho^{0} K^{*+} \rightarrow K^{+} \pi_{\mathrm{long}}^{0}$ & $3 \pm 3$ & 0.105 & $0.28 \pm 0.28$ \\
\hline$B^{+} \rightarrow \rho^{+} \rho_{\text {long }}^{0}$ & $22.5 \pm 17.5$ & 1.0 & $36.95 \pm 28.74$ \\
\hline$B^{+} \rightarrow \eta^{\prime} K^{+} \rightarrow \rho^{0} \gamma$ & $22.1 \pm 2.1$ & 0.05 & $0.97 \pm 0.09$ \\
\hline$B^{+} \rightarrow \eta^{\prime} \pi^{+} \rightarrow \rho^{0} \gamma$ & $3 \pm 2$ & 0.895 & $2.35 \pm 1.57$ \\
\hline$B^{+} \rightarrow K^{* 0} \pi^{+} \rightarrow K_{S} \pi^{0}$ & $2.2 \pm 5$. & 0.003 & $0.65 \pm 0.15$ \\
\hline$B^{-} \rightarrow K^{* 0} \pi^{-} \rightarrow K^{+} \pi^{-}$ & $8.9 \pm 1.9$ & 0.090 & $0.70 \pm 0.15$ \\
\hline$B^{+} \rightarrow \pi^{0} \rho^{+}$ & $15 \pm 8$ & 2.70 & $35.58 \pm 18.97$ \\
\hline$B^{-} \rightarrow K^{-} \rho^{0}$ & $3.9 \pm 3$ & 0.547 & $1.87 \pm 1.44$ \\
\hline$B^{-} \rightarrow K_{S} \pi^{-} \rightarrow \pi^{+} \pi^{-}$ & $9 \pm 09$ & 0.014 & $10.89 \pm$ \\
\hline$B^{+} \rightarrow K^{+} f 0 \rightarrow \pi^{+} \pi^{-}$ & $11.7 \pm 4$ & 0.270 & $2.78 \pm$ \\
\hline$B^{+} \rightarrow \pi^{+} \rho^{0}$ & $8 \pm 2.3$ & 6.08 & $42.61 \pm$ \\
\hline$B^{+} \rightarrow K^{+} \pi^{0}$ & $12.7 \pm 1.2$ & 0.095 & $1.05 \pm$ \\
\hline$B^{-} \rightarrow \pi^{-} \pi^{0}$ & $5.8 \pm 1$ & 0.861 & $4.38 \pm$ \\
\hline$B^{+} \rightarrow K_{1}^{*+}(1270) \pi^{0}, K_{1}^{* 0}(12$ & $20 \pm 20$ & 0.012 & $0.21 \pm$ \\
\hline$B^{+} \rightarrow K_{1}^{*+}(1400) \pi^{0}, K_{1}^{* 0}(1400) \pi^{+}$ & $20 \pm 20$ & 0.03 & $0.53 \pm$ \\
\hline$B^{+} \rightarrow K_{2}^{*+}(1430) \pi^{0}, K_{2}^{* 0}(1430) \pi^{+}$ & $20 \pm 20$ & 0.698 & $12.22 \pm 12.22$ \\
\hline$B^{+} \rightarrow K^{*+}(1680) \pi^{0}, K^{* 0}(1680) \pi^{+}$ & $20 \pm 20$ & 0.420 & $7.37 \pm$ \\
\hline$B^{+} \rightarrow K^{*+}(1680) \rho^{0}, K^{* 0}(16$ & $20 \pm 20$ & 0.3 & $5.26 \pm$ \\
\hline$B^{+} \rightarrow K^{*+} \pi^{0}, K^{+} \pi^{0}$ & $4.4 \pm 2.5$ & 0.062 & $0.24 \pm$ \\
\hline$B^{0} \rightarrow \rho^{-} K^{*+} \rightarrow K^{+} \pi_{\mathrm{long}}^{0}$ & $6 \pm 6$ & 0.136 & $0.72 \pm$ \\
\hline$B^{0} \rightarrow \rho^{+} \rho_{\text {long }}^{-}$ & $40 \pm 30$ & 2.12 & $74.49 \pm 55.87$ \\
\hline$B^{0} \rightarrow \rho^{0} \rho_{\text {long }}^{0}$ & $3.5 \pm 3.5$ & 1.53 & $4.70 \pm 4.70$ \\
\hline$B^{0} \rightarrow K^{*+} \pi^{-} \rightarrow K_{S} \pi^{+}$ & $17.4 \pm 10$ & 0.482 & $7.36 \pm$ \\
\hline$B^{0} \rightarrow K^{*+} \pi^{-} \rightarrow K^{+} \pi^{0}$ & $8.7 \pm 5$ & 2.69 & $20.52 \pm 11.79$ \\
\hline$B^{0} \rightarrow K^{* 0} \pi^{0} \rightarrow K^{+} \pi^{-}$ & $8.7 \pm 5$ & 0.795 & $6.06 \pm 3.48$ \\
\hline$B^{0} \rightarrow a_{1}^{-} \pi^{+}$ & $30 \pm 20$ & 0.460 & $12.10 \pm 8.06$ \\
\hline$B^{0} \rightarrow K^{+} \pi^{-}$ & $18.5 \pm 1$ & 0.201 & $3.27 \pm 0.18$ \\
\hline$B^{0} \rightarrow K_{1}^{*+}(1127) \pi^{-}, K_{1}^{*}$ & $20 \pm 20$ & 0.032 & $0.56 \pm 0.56$ \\
\hline$B^{0} \rightarrow K^{*+}(1400) \pi^{-}, K^{* 0}(14$ & $20 \pm 20$ & 0.046 & $0.81 \pm 0.82$ \\
\hline$B^{0} \rightarrow K_{2}^{*+}(1430) \pi^{-}, K_{2}^{* 0}(1430) \pi^{0}$ & $20 \pm 20$ & 0.964 & $16.8 \pm 16.8$ \\
\hline$B^{0} \rightarrow K^{*+}(1680) \pi^{-}, K^{* 0}(1680) \pi^{0}$ & $20 \pm 20$ & 0.712 & $12.47 \pm 12.47$ \\
\hline$B^{0} \rightarrow K^{*+}(1680) \rho^{-}, K^{* 0}(1680) \rho^{0}$ & $20 \pm 20$ & 0.14 & $2.52 \pm 2.53$ \\
\hline$B^{0} \rightarrow \rho^{0} K^{* 0} \rightarrow K^{+} \pi_{\text {long }}^{-}$ & $6 \pm 6$ & 0.075 & $0.39 \pm 0.39$ \\
\hline
\end{tabular}

Tableau 6.2: Liste des modes $B$ non-charmés considérés dans l'analyse Dalitz. On donne le rapport d'embranchement $(\mathcal{B})$, l'efficacité de sélection, et le nombre d'événements attendus. 


\begin{tabular}{c|c|c}
\hline Classe & Mode & $N_{\text {exp }}$ \\
\hline 0 & $B^{+} \rightarrow 4$-corps & 57.61 \\
1 & $B^{+} \rightarrow 3$-corps & 106.41 \\
2 & $B^{+} \rightarrow 2$-corsp & 5.43 \\
3 & $B^{0} \rightarrow 4$-corps $\left(K^{*}\right)$ & 3.68 \\
4 & $B^{0} \rightarrow \rho^{+} \rho^{-}$ & 79.20 \\
5 & $B^{0} \rightarrow 4$-corps & 20.18 \\
6 & $B^{0} \rightarrow K^{*} \pi$ & 23.79 \\
7 & $B^{+} \rightarrow \operatorname{charme}$ & 1033.00 \\
8 & $B^{0} \rightarrow \operatorname{charme}$ & 524.00 \\
9 & $B^{0} \rightarrow 3 / 4$-corps $\left(K^{* *} \pi\right)$ & 62.73 \\
10 & $B^{+} \rightarrow 3 / 4$-corps $\left(K^{* *} \pi\right)$ & 19.25 \\
11 & $B^{0} \rightarrow 4$-corps $\left(K^{* *} \rho\right)$ & 2.53 \\
12 & $B^{+} \rightarrow 4$-corps $\left(K^{* *} \rho\right)$ & 3.96 \\
13 & $B^{0} \rightarrow \rho^{-} K^{+}$ & 9.57 \\
\hline
\end{tabular}

Tablean 6.3: Liste des 13 classes regroupant les modes charmés et non-charmés contaminant $B^{0} \rightarrow \pi^{+} \pi^{-} \pi^{0}$.

puis calculer ensuite le reste des variables grâce aux relations suivantes:

$$
\begin{aligned}
m_{B}^{2} & =m^{2}\left(\pi^{+} \pi^{0}\right)+m^{2}\left(\pi^{-} \pi^{0}\right)+m^{2}\left(\pi^{+} \pi^{-}\right)-2 m_{\pi^{ \pm}}^{2}-m_{\pi^{0}}^{2}, \\
\cos \theta_{H}(\rho)^{i j} & \simeq-1+2 \frac{m^{2}\left(\pi^{j} \pi^{k}\right)}{m_{B}^{2}-m^{2}\left(\pi^{i} \pi^{k}\right)} .
\end{aligned}
$$

Comme pour $\Delta t$, les distributions en $m^{2}\left(\pi^{+} \pi^{0}\right)$ et $m^{2}\left(\pi^{-} \pi^{0}\right)$ des données sont la convolution de la distribution physique avec la fonction de résolution du détecteur. D'autres effets entrent aussi en jeu, comme la variation de l'acceptance à travers le diagramme de Dalitz, ainsi que de l'efficacité de sélection, et de la fraction d'événements mal reconstruits.

Dans cette section, nous passons en revue chacun des ingrédients énoncés ci-dessus permettant l'élaboration des distributions de $m^{2}\left(\pi^{+} \pi^{0}\right)$ et $m^{2}\left(\pi^{-} \pi^{0}\right)$ de $B^{0} \rightarrow \pi^{+} \pi^{-} \pi^{0}$.

\subsubsection{Facteur de forme du $\pi$}

L'amplitude de la désintégration $B^{0} \rightarrow \pi^{+} \pi^{-} \pi^{0}$ est donnée par (voir Eq. 2.12) ${ }^{2}$ :

$$
\begin{aligned}
& A_{3 \pi} \equiv f_{+} A^{+-}+f_{-} A^{-+}+f_{0} A^{00} \\
& \bar{A}_{3 \pi} \equiv f_{+} \bar{A}^{+-}+f_{-} \bar{A}^{-+}+f_{0} \bar{A}^{00},
\end{aligned}
$$

où les $f_{i}$ sont les facteurs de forme du pion.

La principale difficulté de l'analyse Dalitz réside dans la modélisation de ces facteurs de forme. Nous devons en effet déterminer toutes les contributions à l'amplitude de $B^{0} \rightarrow$ $\pi^{+} \pi^{-} \pi^{0}$, et évaluer leurs poids et phases fortes relatives.

Plusieurs voies sont à explorer pour y répondre:

- Les prédictions théoriques: puisque nous nous situons dans le régime non-perturbatif de QCD, la théorie reste peu prédictive.

\footnotetext{
${ }^{2}$ Cette équation ne considère que les contributions résonnantes.
} 
- Les précédentes études dans d'autres systèmes que les mésons $B$ : les facteurs de forme du $\pi$ ont déjà été précisemment étudiés dans les données $e^{+} e^{-} \rightarrow \pi^{+} \pi^{-}$et les désintégrations des $\tau[96,97]$. Les résultats de ces études servent de base aux études menées dans ce chapitre. D'autre part, l'analyse Dalitz de $D^{+} \rightarrow \pi^{+} \pi^{-} \pi^{+}$a produit des résultats intéressants. Néanmoins, tous ces résultats ne sont pas directement transposables à la physique du $B$ (nous y reviendrons).

- Bien entendu, la paramétrisation doit être vérifiée sur nos données. Mais puisque nous disposons de peu d'événements, et que nous sommes dans un environnement moins idéal (notamment dû à la présence d'importants bruits de fond), il n'est pas garanti que nous puissions déterminer toutes les inconnues significatives reliées à ces facteurs de forme sur nos données. L'étude du diagramme Dalitz des désintégrations des $B$ chargés $B^{+} \rightarrow \pi^{+} \pi^{-} \pi^{+}$peut aussi aider à déterminer les facteurs de forme $\mathrm{d} u \pi$.

Dans cette section, nous commençons par rappeler la liste des résonances se désintégrant en $\pi \pi$. Nous résumons ensuite les résultats précédemment obtenus sur les données $e^{+} e^{-} \rightarrow$ $\pi^{+} \pi^{-}$et les désintégrations des $\tau[96,97]$. Dans un deuxième temps, nous proposons une paramétrisation de base pour les études de faisabilité réalisées par la suite.

\subsubsection{Les rêsonances qui se dêsintègrent en $\pi \pi$}

Le tableau 6.4 donne la liste des résonances [44] se désintégrant en $\pi \pi$, et donc susceptibles de se trouver dans le diagramme de Dalitz $B^{0} \rightarrow \pi^{+} \pi^{-} \pi^{0}$. Il faut ajouter à cela une possible contribution non résonnante $B^{0} \rightarrow \pi^{+} \pi^{-} \pi^{0}$. Selon le spin de la résonance, la distribution angulaire des produits de désintégration ( $c$-à- $d$ de l'angle d'hélicité $\theta$ ) est différente (les dépendances ci-dessous sont au niveau de l'amplitude de désintégration, pas du rapport d'embranchement):

- spin 0 et non-résonnant: distribution angulaire uniforme

- $\operatorname{spin} 1: \sqrt{\frac{3}{4 \pi}} \cos \theta$

- $\operatorname{spin} 2: \sqrt{\frac{5}{4 \pi}}\left(\frac{3}{2} \cos ^{2} \theta-\frac{1}{2}\right)$

- $\operatorname{spin} 3: \sqrt{\frac{7}{4 \pi}}\left(\frac{5}{2} \cos ^{3} \theta-\frac{3}{2} \cos \theta\right)$

Les résonances $\sigma, \rho(770), f_{0}(980), f_{2}(1270)$ (et marginalement, $\rho_{3}(1690)$ et $f_{4}(2050)$ ) se désintègrent majoritairement en $\pi \pi^{3}$. Les autres ont un rapport d'embranchement en $\pi \pi$ faible ou inconnu. Les rapports d'embranchement du $B^{0}$ en résonances non-charmées et un pion sont tous inconnus, sauf pour $B^{0} \rightarrow \rho^{ \pm} \pi^{\mp}$ (la valeur moyenne mondiale actuelle vaut $(25.9 \pm 4.3) \times 10^{-6}$ - voir Tab. 2.3$)$. Les rapports d'embranchement des désintégrations des $B$ en modes charmés sont tous connus, sauf pour celles supprimées de couleur.

\footnotetext{
${ }^{3}$ Les résonnances de spin pair peuvent se désintégrer à la fois en $\pi^{+} \pi^{-}$et $\pi^{0} \pi^{0}$, alors que celles de spin impair ne peuvent se désintégrer qu'en $\pi^{+} \pi^{-}$.
} 


\begin{tabular}{|c|c|c|c|c|}
\hline Résonance & $J^{P C}$ & Masse $(\mathrm{MeV})$ & Largeur (MeV) & $\operatorname{Br}(\rightarrow \pi \pi)(\%)$ \\
\hline \multicolumn{5}{|c|}{ Non-charmé } \\
\hline$f_{0}^{0}(600)($ ou $\sigma)$ & $0^{++}$ & $400-1200$ & $600-1000$ & dominant \\
\hline$\rho^{ \pm, 0}(770)$ & $1^{--}$ & $771 \pm 0.9$ & $149.2 \pm 0.7$ & $\simeq 100$ \\
\hline$\omega^{0}(782)$ & $1^{--}$ & $782.57 \pm 0.12$ & $8.44 \pm 0.9$ & $1.70 \pm 0.28$ \\
\hline$f_{0}^{0}(980)$ & $0^{++}$ & $980 \pm 10$ & $40-100$ & dominant \\
\hline$f_{2}^{0}(1270)$ & $2^{++}$ & $1275.4 \pm 1.2$ & $185.1 \pm 3.4$ & $84.7 \pm 2.4$ \\
\hline$f_{0}^{0}(1370)$ & $0^{++}$ & $1200-1500$ & $200-500$ & v11 \\
\hline$\rho^{ \pm, 0}(1450)$ & $1^{--}$ & $1465 \pm 25$ & $310 \pm 60$ & V11 \\
\hline$f_{0}^{0}(1500)$ & $0^{++}$ & $1507 \pm 5$ & $109 \pm 7$ & v1 \\
\hline$f_{2}^{\prime 0}(1525)$ & $2^{++}$ & $1525 \pm 5$ & $76 \pm 10$ & $(8.2 \pm 1.5) \cdot 10^{-3}$ \\
\hline$\rho_{3}^{ \pm, 0}(1690)$ & $3^{--}$ & $1691 \pm 5$ & $161 \pm 10$ & $23.6 \pm 1.3$ \\
\hline$\rho^{ \pm, 0}(1700)$ & $1^{--}$ & $1700 \pm 20$ & $240 \pm 60$ & v11 \\
\hline$f_{0}^{0}(1710)$ & $0^{++}$ & $1713 \pm 6$ & $125 \pm 10$ & V11 \\
\hline$f_{4}^{0}(2050)$ & $4^{++}$ & $2025 \pm 8$ & $194 \pm 13$ & $17.0 \pm 1.5$ \\
\hline \multicolumn{5}{|c|}{ Charmé } \\
\hline$D^{0}$ & $0^{-}$ & $1864.5 \pm 0.5$ & negl. & $(1.43 \pm 0.07) \cdot 10^{-3}$ \\
\hline$D^{+}$ & $0^{-}$ & $1869.3 \pm 0.5$ & negl. & $(2.5 \pm 0.7) \cdot 10^{-3}$ \\
\hline$J / \psi(1 S)$ & $1^{--}$ & $3096.87 \pm 0.04$ & $(87 \pm 5) \cdot 10^{-3}$ & $(1.47 \pm 0.23) \cdot 10^{-4}$ \\
\hline$\chi_{c 0}(1 P)$ & $0^{++}$ & $3415.1 \pm 0.8$ & $16.2 \pm 2.3$ & $(5.0 \pm 0.7) \cdot 10^{-3}$ \\
\hline$\chi_{c 2}(1 P)$ & $2^{++}$ & $3556.18 \pm 0.13$ & $2.08 \pm 0.17$ & $(1.52 \pm 0.25) \cdot 10^{-3}$ \\
\hline$\psi(2 S)$ & $1^{--}$ & $3685.96 \pm 0.09$ & $2.19 \pm 0.15$ & $(8 \pm 5) \cdot 10^{-5}$ \\
\hline
\end{tabular}

Tableau 6.4: Résonances se désintégrant en $\pi \pi$, et donc susceptibles de se trouver dans le diagramme de Dalitz $B^{0} \rightarrow \pi^{+} \pi^{-} \pi^{0}$. On donne leurs nombres quantiques $J, P, C$, leur masse et largeur, ainsi que leur rapport d'embranchement en $\pi \pi$.

\subsubsection{Les données $e^{+} e^{-} \rightarrow \pi^{+} \pi^{-}$et les désintégrations des $\tau^{ \pm}$}

Le facteur de forme du $\pi$ a été étudié dans les données $e^{+} e^{-} \rightarrow \pi^{+} \pi^{-}$collectées auprès des collisionneurs de Novosibirsk et Orsay, et dans les désintégrations des $\tau$ dans les expériences ALEPH, CLEO et OPAL du LEP ( $c f$. ref. [97] pour un compendium des expériences et la description des données). Ces deux systèmes permettent de produire des résonances vecteur (car le photon est un vecteur, et le $W$ un vecteur) uniquement, c'est à dire les résonances $\rho$ et $\omega$.

Les données $\tau^{ \pm}$couvre un spectre jusqu'à $s=m^{2}(\tau)$ ', et produisent une combinaison de $\pi$ chargés $\left(\rho^{ \pm}\right)$, alors que les données $e^{+} e^{-} \rightarrow \pi^{+} \pi^{-}$produisent des combinaisons neutres $\left(\rho^{0}\right.$ et $\left.\omega\right)$. Pour comparer les distributions obtenues des deux types de données, il faut tenir compte des corrections d'isospin.

La figure 6.3 montre les fonctions spectrales $\pi^{+} \pi^{-}$des données $e^{+} e^{-}$et celles des désintégrations des $\tau$ corrigées pour la brisure d'isospin et l'interférence $\rho-\omega$. La cassure dans la distribution montrée sur le zoom en bas à droite de la figure montre l'effet de cette interférence.

La figure 6.4 montre la fonction spectrale pour les données $e^{+} e^{-}$uniquement, jusqu'à $s=4 \mathrm{Gev}^{2}$. En plus du $\rho(770)$ et du $\omega(782)$, on voit apparaître les résonances $\rho(1450)$ et $\rho(1700)$, ainsi que l'effet de leurs interférences. La paramétrisation de Gounaris-Sakurai donnée dans la section 6.5.1.3 est superposée aux données. 

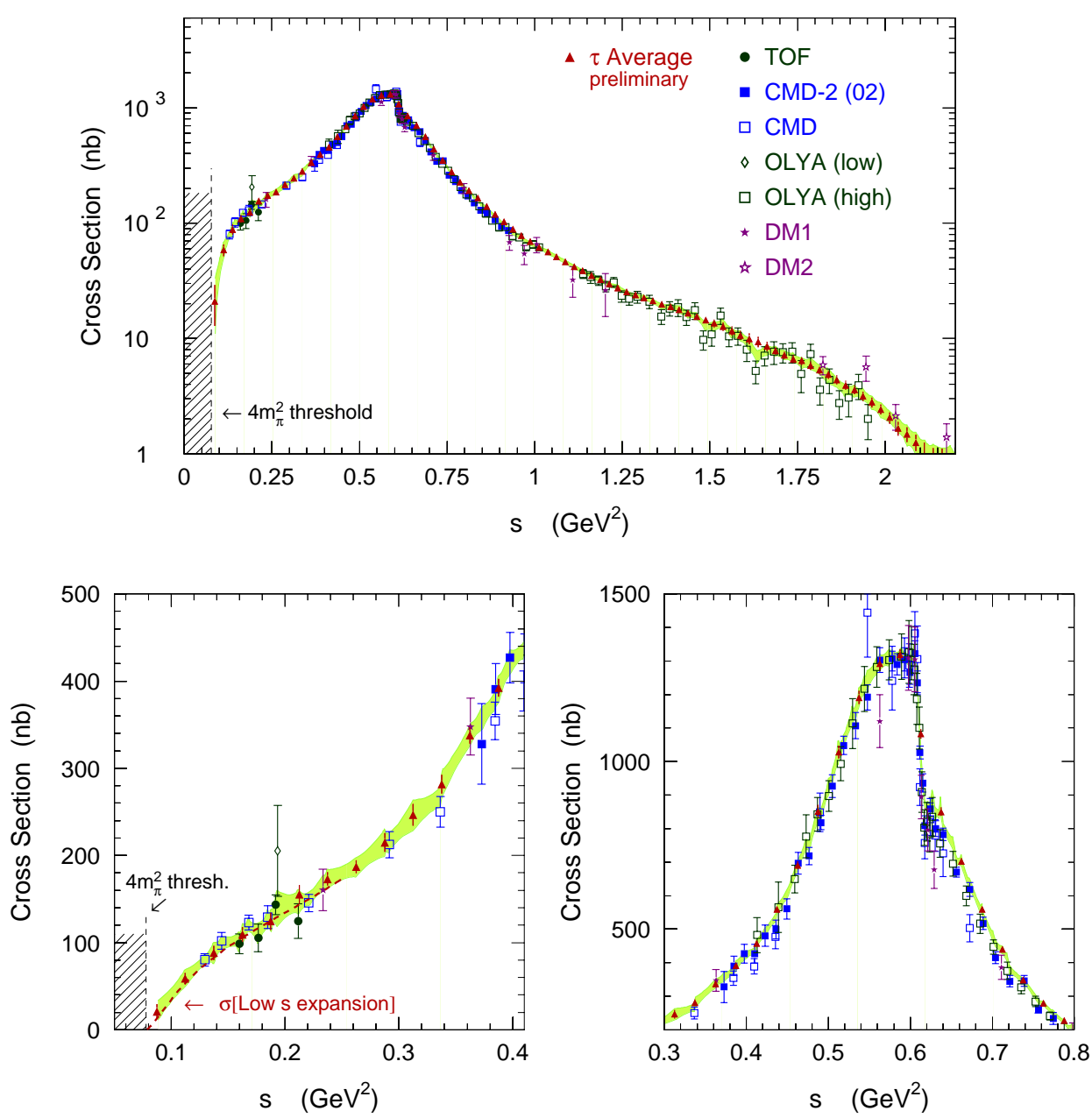

Figure 6.3: Fonctions spectrales $\pi^{+} \pi^{-}$des données $e^{+} e^{-}$et les données des désintégrations des $\tau$ corrigées pour la brisure d'isospin [97]. La bande verte correspond à une déviation d'un écart type des résultats combinés $e^{+} e^{-}$et $\tau$. La figure en bas à droite est un zoom sur l'interférence $\rho^{0}-\omega$.

\subsubsection{Paramétrisation de Gounaris-Sakurai du facteur de forme}

Il existe plusieurs paramétrisations du facteur de forme du $\pi[98,99,100,101]$. Par la suite, nous utilisons la paramétrisation de Gounaris-Sakurai [99] (au niveau de précision actuel, le choix de la paramétrisation n'a pas une grande importance).

Nous utilisons comme point de départ l'ajustement des données présentées dans la section précédente, tel que réalisé dans la référence [96]. Le facteur de forme total du $\pi$ est donné par:

$$
f(\pi)=\left(B W_{\rho(770)} \frac{1+\alpha B W_{\omega(782)}}{1+\alpha}+\beta B W_{\rho(1450)}+\gamma B W_{\rho(1700)}\right) \frac{1}{1+\beta+\gamma}
$$

où les propagateurs des Breit-Wigner $B W$ sont une version plus élaborée des simples BreitWigner relativistes, et sont donnés par:

$$
B W_{\rho, M_{\rho}}(s)=\frac{M_{\rho}^{2}\left(1+d \cdot \Gamma_{\rho} / M_{\rho}\right)}{M_{\rho}^{2}-s+f(s)-i \sqrt{s} \Gamma_{\rho}(s)},
$$




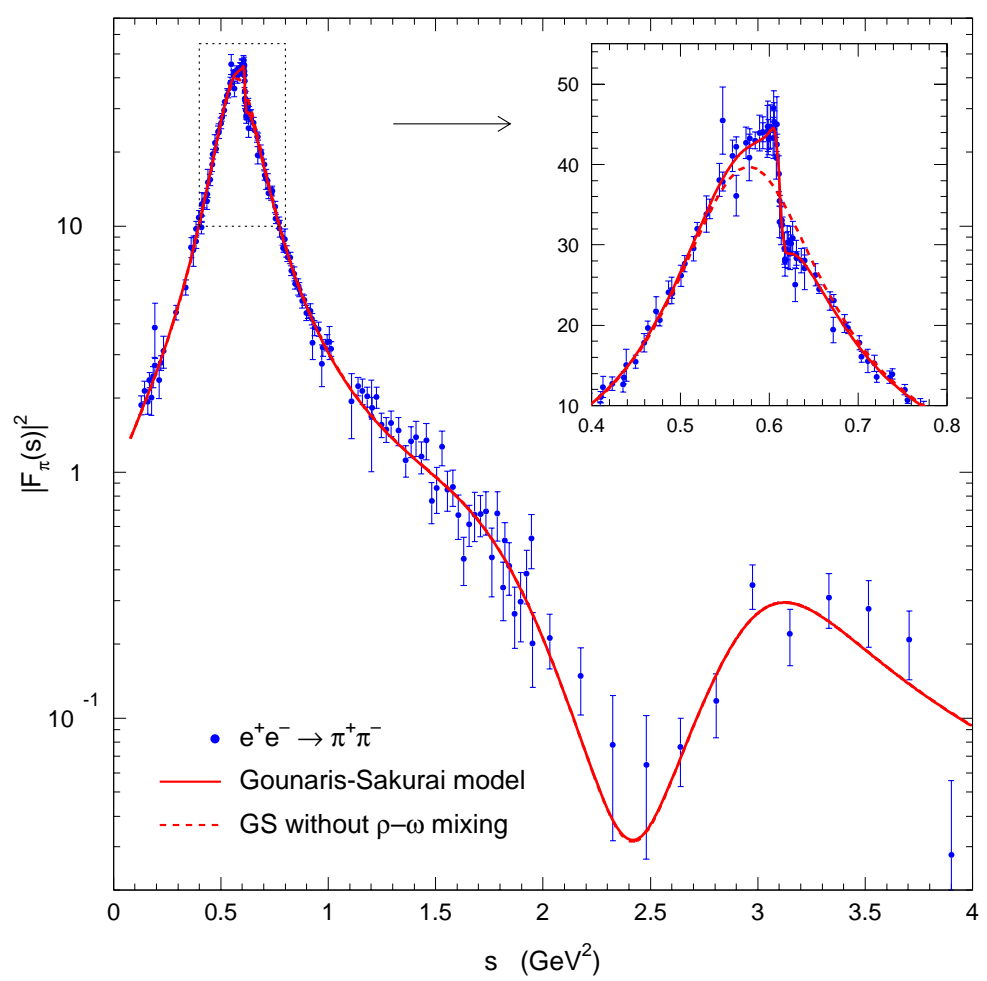

Figure 6.4: Fonction spectrale pour les données $e^{+} e^{-}$[97]: la structure à droite correspond aux résonances $\rho(1450)$ et $\rho(1700)$.

où

$$
f(s)=\Gamma_{\rho} \frac{M_{\rho}^{2}}{k^{3}\left(M_{\rho}^{2}\right)}\left[k^{2}(s)\left(h(s)-h\left(M_{\rho}^{2}\right)\right)+\left.\left(M_{\rho}^{2}-s\right) k^{2}\left(M_{\rho}^{2}\right) \frac{d h}{d s}\right|_{s=M_{\rho}^{2}}\right]
$$

et

$$
h(s)=\frac{2}{\pi} \frac{k(s)}{\sqrt{s}} \ln \frac{\sqrt{s}+2 k(s)}{2 m_{\pi}},
$$

où $k(s)=1 / 2 \sqrt{s} \beta_{\pi}(s)$ et $k\left(M_{\rho}^{2}\right)$ est l'impulsion du $\pi$ dans le référentiel du $\rho$. La largeur de la résonance est fonction de l'énergie $s$ :

$$
\Gamma_{\rho}(s)=\Gamma_{\rho}\left(M_{\rho}^{2}\right)\left(\frac{M_{\rho}^{2}}{s}\right)^{1 / 2}\left(\frac{k(s)}{k\left(M_{\rho}^{2}\right)}\right) .
$$

La normalisation de la Breit-Wigner est telle que $B W(0)=1$, ce qui fixe la valeur de $d$.

Les résultats de l'ajustement des données $e^{+} e^{-}$et $\tau$ se trouvent dans le tableau 6.5. Il existe des phases fortes relatives entre les différentes résonances si leur processus de production diffèrent entre eux. Les $\rho(1450)$ et $\rho(1700)$ étant des excitations radiales du $\rho(770)$, on peut penser qu'il n'existe pas de couplage entre ces excitations du $\rho$ et la production du $\rho$ par le $\gamma$ ou $W$, et donc pas de phase forte relative non-triviale $(c-\grave{a}-d \neq 0$ ou $\pi$ ) entre ces différentes résonances. Comme visible dans le tableau 6.5, il existe un signe relatif entre les paramètres $\beta, \gamma$ et $\delta$, soit une phase de $180^{\circ}$.

Les argument développés ici sur la valeur attendue de la phase relative des différentes résonances $\rho$ ne tiennent plus forcemment dans le système des $B$ : il n'est pas évident en effet

\footnotetext{
${ }^{4} \mathrm{Il}$ n'existe pas d'explication rigoureuse à cette observation. Cette phase de $180^{\circ}$ pourrait provenir de l'excitation radiale du $\rho$.
} 


\begin{tabular}{ll}
\hline Paramètres & Valeur \\
\hline$\delta$ & $(1.97 \pm 0.15) \times 10^{-3}$ \\
\hline$M_{\rho^{ \pm}}(770)$ & $783.8 \pm 3.0$ \\
$M_{\rho^{0}}(770)$ & $783.8 \pm 3.0$ \\
\hline$\Gamma_{\rho^{ \pm}}(770)$ & $162.0 \pm 5.3$ \\
$\Gamma_{\rho^{0}}(770)$ & $162.4 \pm 5.0$ \\
\hline$\beta$ & $-0.184 \pm 0.010$ \\
$M_{\rho}(1450)$ & $1490 \pm 23$ \\
$\Gamma_{\rho}(1450)$ & $591 \pm 53$ \\
\hline$\gamma$ & $0.074 \pm 0.010$ \\
$M_{\rho}(1700)$ & $1799 \pm 34$ \\
$\Gamma_{\rho}(1700)$ & $255 \pm 39$ \\
\hline
\end{tabular}

Tablea1 6.5: Valeurs des paramètres entrant dans la paramétrisation de Gounaris-Sakourai des données $e^{+} e^{-}$et $\tau$.

que l'excitation radiale des $\rho$ soit découplée de leur production par les diagrammes en arbre et pingouin ( $c f$. Fig. 2.1). Il faut donc considérer des déviations éventuelles au cas exposé ici. Les poids de chaque résonance peuvent aussi changer dans le système des $B$. Enfin, il peut y avoir d'autres résonances produites que celles des données $e^{+} e^{-}$et $\tau$. Ces dernières n'apportent donc qu'une information limitée pour le cas qui nous concerne.

Dans les études qui suivent, nous utilisons le modèle décrit dans cette section comme base de nos simulations.

\subsubsection{Résolution du détecteur}

La figure 6.5 montre les effets de la résolution du détecteur sur la reconstruction de $m^{2}\left(\pi^{+} \pi^{0}\right)$, $m^{2}\left(\pi^{-} \pi^{0}\right)^{5}$ :

- le résidu $\left(m^{2}\left(\pi^{ \pm} \pi^{0}\right)\right.$ géné. $-m^{2}\left(\pi^{ \pm} \pi^{0}\right)$ mes. $)$ des événements correctement reconstruits est porté sur le graphe de gauche. La distribution est ajustée avec une double Gaussienne: la largeur de la Gaussienne principale est de $8.5 \mathrm{MeV}$.

- sur le graphe de droite, on donne $m^{2}\left(\pi^{ \pm} \pi^{0}\right)_{\text {mes. }}$ en fonction de $m^{2}\left(\pi^{ \pm} \pi^{0}\right)$ géné. pour les événements de signal mal reconstruits. On voit que pour ces événements, le décalage entre la masse générée et reconstruite peut atteindre plusieurs centaines de $\mathrm{MeV}$. D'autre part, la masse reconstruite a tendance à être plus élevée que la masse générée, dû̀ à l'espace de phase.

La distribution de $\Delta t$ (voir Sec. 4.9.1.5)est une convolution d'une triple-Gaussienne avec la fonction physique de $\Delta t$ contenant une exponentielle, et des cosinus et sinus: cette convolution est calculable de façon quasi-analytique. Dans le cas de $m^{2}\left(\pi^{ \pm} \pi^{0}\right)$, la fonction de physique contient des propagateurs complexes de Breit-Wigner, et sa convolution avec la fonction de résolution n'est pas calculable analytiquement.

\footnotetext{
${ }^{5}$ Les effets de la résolution sur la masse ont été étudiés dans la réf. [33], concluant qu’il s'agit d'effets négligeables.
} 

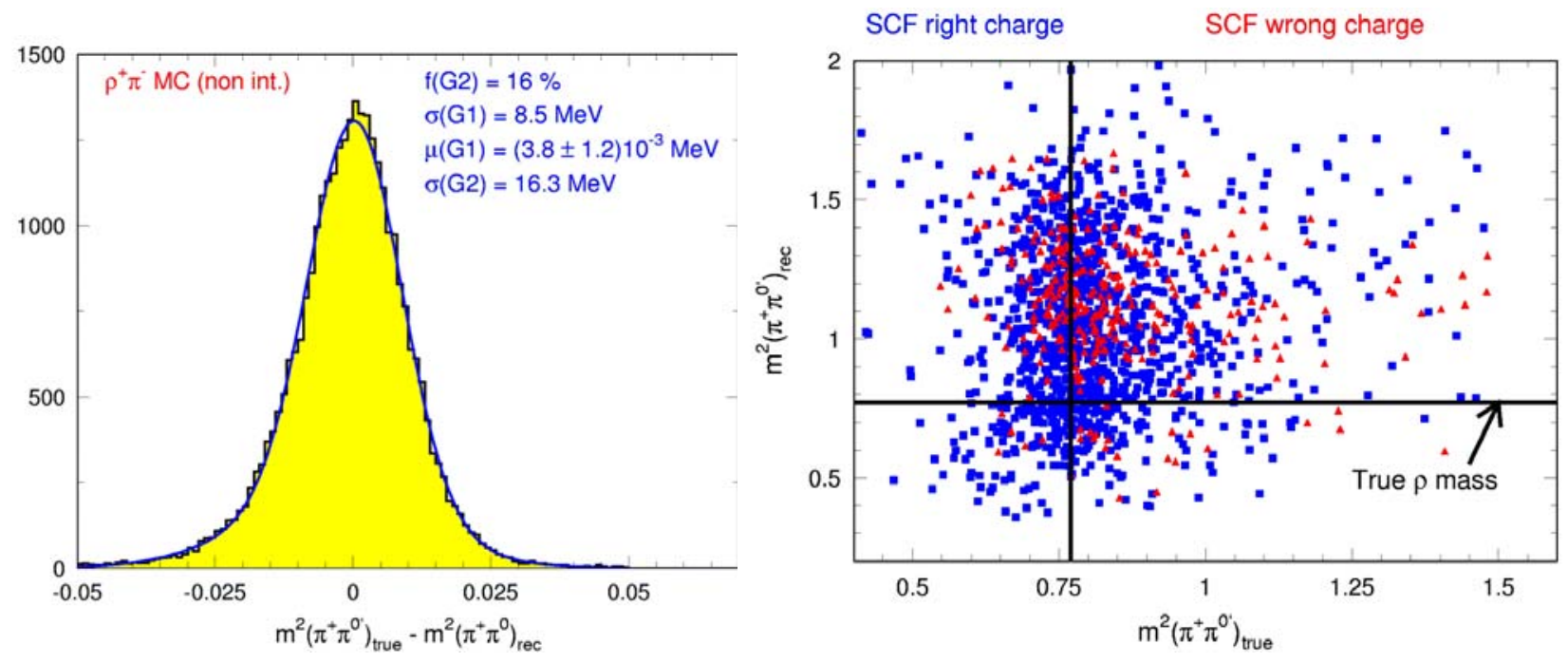

Figure 6.5: Résidu $\left(m^{2}\left(\pi^{ \pm} \pi^{0}\right)_{\text {géné. }}-m^{2}\left(\pi^{ \pm} \pi^{0}\right)_{\text {mes. }}\right)$ pour les événements de signal correctement reconstruit (gauche), et $m^{2}\left(\pi^{ \pm} \pi^{0}\right)$ mes. en fonction de $m^{2}\left(\pi^{ \pm} \pi^{0}\right)$ géné. pour les événements de signal mal reconstruits (droite).

La résolution sur la masse n'est pas encore implémentée dans le modèle utilisé ici, mais la méthode envisagée est de convoluer numériquement des matrices de résolution de dimension 4 permettant de passer de $\left(m^{2}\left(\pi^{+} \pi^{0}\right)_{\text {géné. }}, m^{2}\left(\pi^{-} \pi^{0}\right)_{\text {géné. }}\right)$ à $\left(m^{2}\left(\pi^{+} \pi^{0}\right)_{\text {mes. }}, m^{2}\left(\pi^{-} \pi^{0}\right)_{\text {mes. }}\right)$.

\subsubsection{Efficacité de sélection dans le diagramme de Dalitz}

L'efficacité de sélection dans le diagramme de Dalitz est montrée sur le graphe de gauche de la figure 6.6: elle est relativement uniforme en chaque point du diagramme, mis à part dans les régions où les traces chargées sont molles (dans les coins du diagramme de Dalitz touchant la diagonale $\left.\mathrm{d} u \rho^{0}\right)$, à cause du seuil de sélection de ces traces. Les autres inhomogénéités sont principalement d'origine statistique.

Les graphes de la figure 6.7 montrent les efficacités de sélection en fonction de 1'hélicité et de la masse carré des paires de pions $\pi^{ \pm} \pi^{0}$ et $\pi^{+} \pi^{-}$, confirmant les conclusions énoncées plus haut.

\subsubsection{Fraction d'événements mal reconstruits dans le diagramme de Dalitz}

Le graphe de droite de la figure 6.6 montre la fraction d'événements mal reconstruits dans le diagramme de Dalitz. Les variations sont violentes en fonction du point dans le Dalitz: la fraction est maximale dans les coins du diagramme (où une particule a une trace molle, augmentant ainsi le nombre d'événements mal reconstruits), et faible au centre (où toutes les particules de l'état final ont une impulsion élevée). 

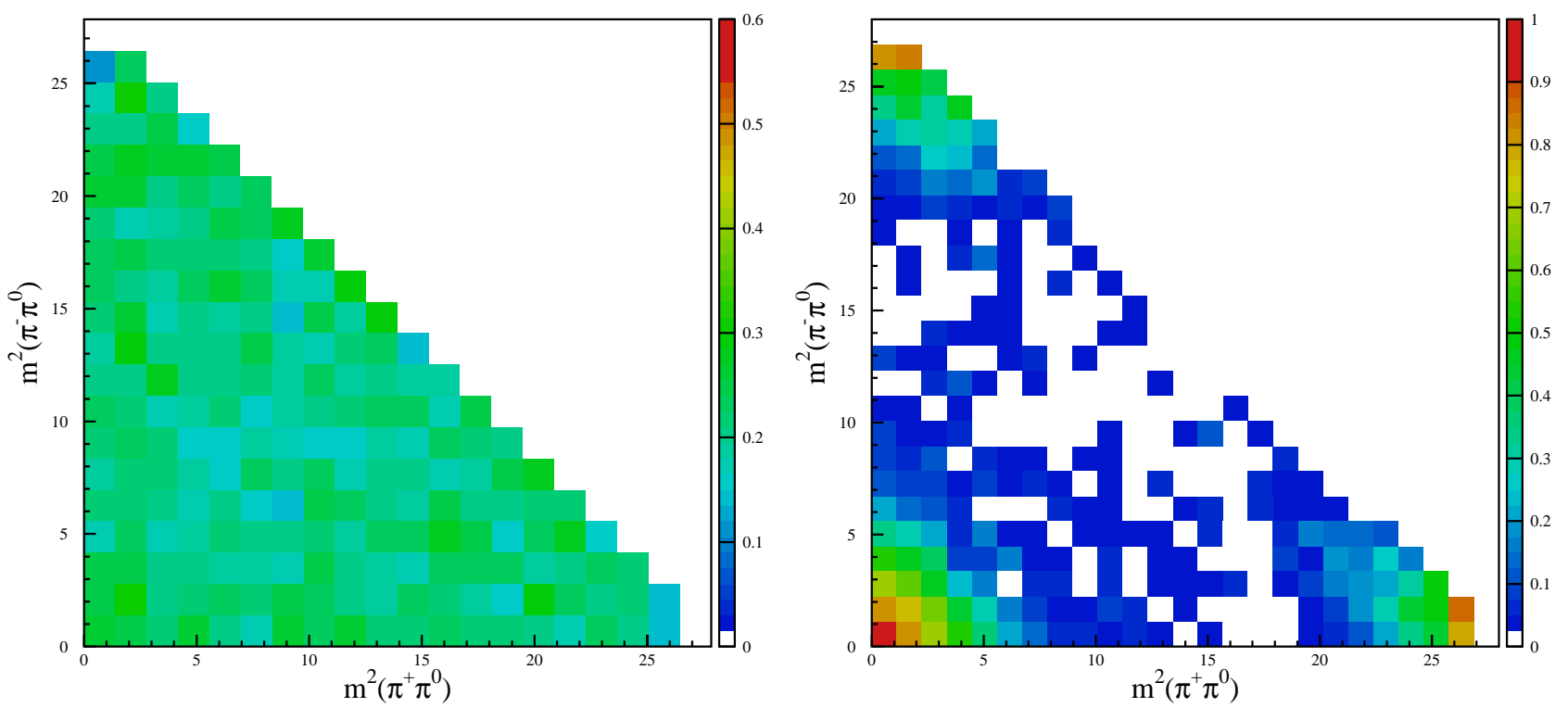

Figure 6.6: Figure de gauche: efficacité de sélection dans le diagramme de Dalitz. Figure de droite: fraction d'événements mal reconstruits dans le diagramme de Dalitz.

\subsection{Fonction de vraisemblance et paramétrisations}

\subsubsection{Fonction de vraisemblance}

Les variables entrant dans l'ajustement final des données sont $\Delta E, m_{\mathrm{ES}}, \Delta t, m^{2}\left(\pi^{+} \pi^{0}\right)$ et $m^{2}\left(\pi^{-} \pi^{0}\right)$. La probabilité $\mathcal{P}_{i, c}$ pour un événement $i$, appartenant à la catégorie d'étiquetage $c_{i}$, est la somme des probabilités sur les composantes de signal $B^{0} \rightarrow \pi^{+} \pi^{-} \pi^{0}$, de bruit de fond continuum et du bruit de fond $B$ :

$$
\mathcal{P}_{i, c}=N_{3 \pi} f_{c}^{3 \pi} \mathcal{P}_{i, c}^{3 \pi}+N_{q 3 \pi}^{c} \mathcal{P}_{i, c}^{q 3 \pi}+\Xi_{c}^{3 \pi}
$$

où

- $N_{3 \pi}$ est le nombre ajusté d'événement de signal $B^{0} \rightarrow \pi^{+} \pi^{-} \pi^{0}$.

- $f_{c}^{3 \pi}$ est la fraction d'événements de signal $B^{0} \rightarrow \pi^{+} \pi^{-} \pi^{0}$ dans la catégorie d'étiquetage $c$.

- $N_{q 3 \pi}^{c}$ est le nombre d'événements de bruit de fond de continuum dans la catégorie d'étiquetage $c$.

- $\mathcal{P}_{i, c}^{3 \pi}=\mathcal{P}^{3 \pi}\left(m_{\mathrm{ES}}\right) \mathcal{P}^{3 \pi}(\Delta E) \mathcal{P}^{3 \pi}\left(x_{\mathrm{NN}}\right) \mathcal{P}^{3 \pi}\left(\Delta t, m^{2}\left(\pi^{+} \pi^{0}\right), m^{2}\left(\pi^{-} \pi^{0}\right)\right)$ est le produit des probabilités sur les différentes variables.

- $\Xi_{c}^{3 \pi}$ est le terme correctif pour le bruit de fond $B$ (voir Sec. 6.4).

La contribution du signal est divisée entre les événements correctement reconstruits, et ceux mal reconstruits. Parmi ces derniers, comme expliqué en introduction de ce chapitre, 

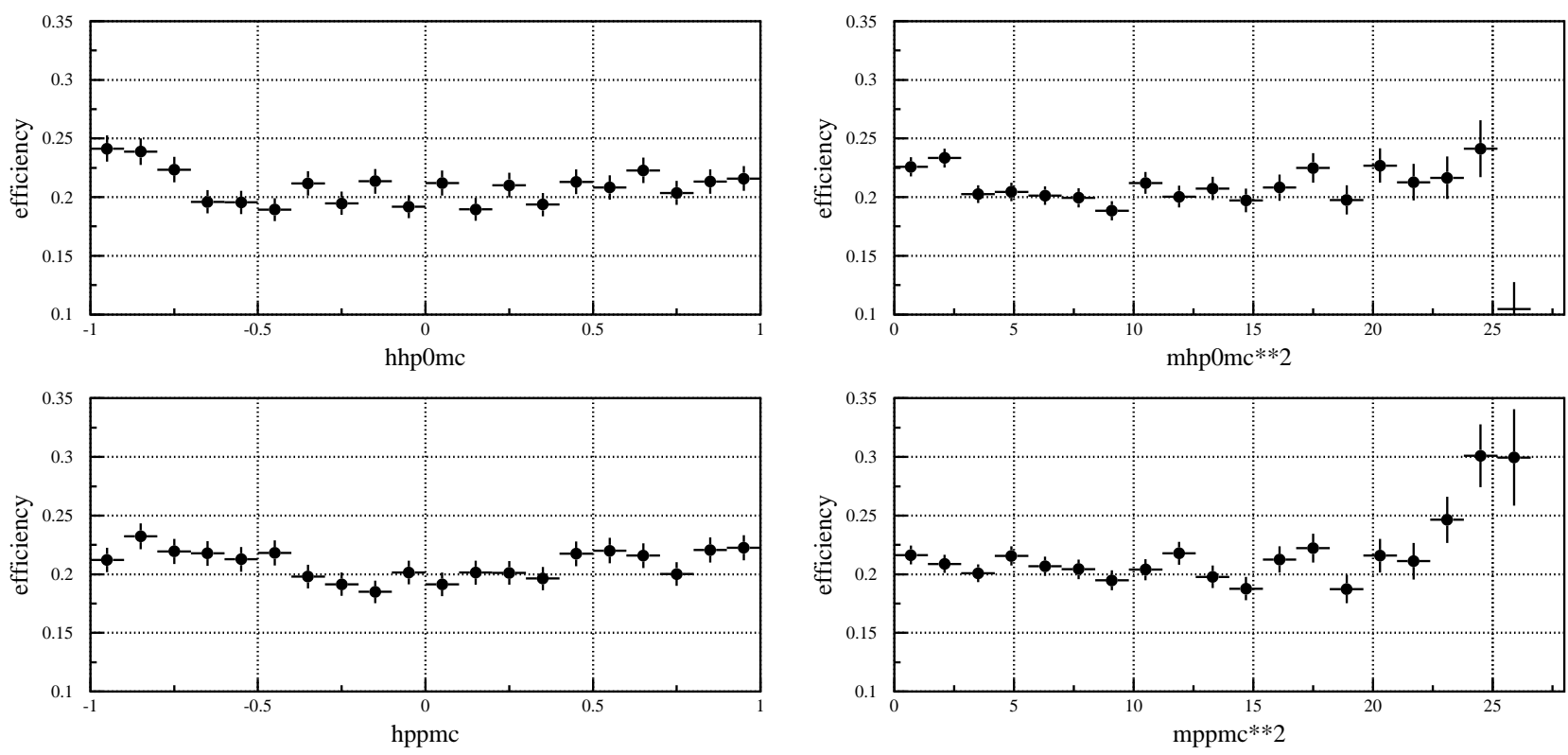

Figure 6.7: Distributions des efficacités de sélection en fonction de l'hélicité (gauche) et de la masse carré (droite) des paires de pions $\pi^{ \pm} \pi^{0}$ (haut) et $\pi^{+} \pi^{-}$(bas).

nous distinguons les événements de bonne charge ( $c$-à- $d$, du même coté de la diagonale $m^{2}\left(\pi^{+} \pi^{0}\right)=m^{2}\left(\pi^{-} \pi^{0}\right)$ que lors de leur génération) de ceux de mauvaise charge ( $c$ - $a-d, d u$ coté opposé):

$$
\mathcal{P}_{i, c}^{\rho x} \longrightarrow\left(1-f_{\mathrm{scf}}\right) \mathcal{P}_{i, c, \mathrm{tru}}^{3 \pi}+f_{\mathrm{scf}}\left(1-\omega_{Q}\right) \mathcal{P}_{i, c, \mathrm{scf}[Q]}^{3 \pi}+f_{\mathrm{scf}} \omega_{Q} \mathcal{P}_{i, c, \mathrm{scf}[-Q]}^{\rho x}
$$

\subsubsection{Paramétrisations de $m_{\mathrm{ES}}, \Delta E, x_{\mathrm{NN}}$}

Les distributions de référence pour les variables $m_{\mathrm{ES}}, \Delta E, x_{\mathrm{NN}}$ sont similaires à celles de l'analyse $B^{0} \rightarrow \rho^{ \pm} \pi^{\mp}$, et sont paramétrées de la même façon.

\subsubsection{Paramétrisations de $\mathcal{P}^{3 \pi}\left(\Delta t, m^{2}\left(\pi^{+} \pi^{0}\right), m^{2}\left(\pi^{-} \pi^{0}\right)\right)$}

\subsubsection{Signal}

La distribution de $\mathcal{P}^{3 \pi}\left(\Delta t, m^{2}\left(\pi^{+} \pi^{0}\right), m^{2}\left(\pi^{-} \pi^{0}\right)\right)$ pour le signal est la convolution de la fonction physique donnée dans l'eq. 2.13, et les fonctions de résolutions sur $\Delta t$ et $m^{2}\left(\pi^{ \pm} \pi^{0}\right)$.

Les termes contenant les intégrales de Breit-Wigner dans le facteur de normalisation de la fonction physique ( $c f$. Eq. 2.15) sont calculés à l'avance une fois pour toutes.

La fonction de résolution de $\Delta t$ est la même que dans l'analyse $B^{0} \rightarrow \rho h$ ( $c f$. section 4.9.1.5). La résolution sur la masse est différente pour les événements correctement et mal reconstruits, comme indiqué dans la section 6.5.2, et n'a pas encore été prise en compte dans le travail exposé ici. Enfin, à ce stade, nous ne prenons pas non plus en compte les variations de l'efficacité de sélection, et de la fraction d'événements mal reconstruits dans la paramétrisation de $\mathcal{P}^{3 \pi}\left(\Delta t, m^{2}\left(\pi^{+} \pi^{0}\right), m^{2}\left(\pi^{-} \pi^{0}\right)\right)$.

Parmi les différentes possibilités de paramètres à ajuster dans la fonction physique ( $c f$. section 2.2.4), nous choisissons par esprit de simplicité d'ajuster directement les amplitudes 
des diagrammes en arbre et pingouins, et $\alpha$.

\subsubsection{Bruit de fond continuum}

La distribution de $\mathcal{P}^{3 \pi}\left(\Delta t, m^{2}\left(\pi^{+} \pi^{0}\right), m^{2}\left(\pi^{-} \pi^{0}\right)\right)$ pour le bruit de fond continuum est factorisée entre les variables $\Delta t$ et $m^{2}\left(\pi^{ \pm} \pi^{0}\right)$ : la distribution pour $\Delta t$ est la même que celle utilisée dans l'analyse $B^{0} \rightarrow \rho^{ \pm} \pi^{\mp}$ (cf. section 4.9.1.5), et nous utilisons des KEYS bidimensionnelles pour paramétrer le diagramme $m^{2}\left(\pi^{+} \pi^{0}\right)-m^{2}\left(\pi^{-} \pi^{0}\right)$.

\subsubsection{Bruits de fond $B$}

Comme pour le bruit de fond continuum, la paramétrisation est factorisée entre les variables $\Delta t$ et $m^{2}\left(\pi^{ \pm} \pi^{0}\right)$. C'est un traitement correct pour les $B$ chargés, mais il s'agit d'une approximation pour les $B$ neutres. Néanmoins, pour le niveau de précision des études présentées ici, c'est une approximation valable $^{6}$ (d'autant plus que seuls des simulations Monte Carlo sont présentées, qui sont donc auto-cohérentes).

\subsection{Les premiers pas de l'analyse}

Nous donnons dans cette section quelques résultats sur des configurations simples basées sur ce qui a été décrit dans les sections précédentes. Nous considérons 500 événements de signal reconstruits, soit le nombre d'événements attendus avec la luminosité utilisée pour l'analyse $B^{0} \rightarrow \rho h\left(80.9 \mathrm{fb}^{-1}\right)$.

\subsubsection{Etude de sensibilité}

Nous nous plaçons pour commencer dans une configuration sans bruit de fond, où nous ne considérons que le signal correctement reconstruit, et nous ne tenons pas compte des effets de résolution sur la reconstruction de la masse. Nous étudions la sensibilité sur $\alpha$ pour différentes configurations de diagrammes arbre et pingouin: en effet, l'erreur sur alpha dépend de ces configurations. Les résultats obtenus sont donnés dans le tableau 6.6. Comme on s'y attend, la sensibilité est la moins bonne quand le mode $B^{0} \rightarrow \rho \pi$ est auto-étiquetant $\left(T^{-+}=0\right)$, et la meilleure quand la dilution ( $c$ - ̀̀- $d$, l'asymétrie entre $B^{0} \rightarrow \rho^{+} \pi^{-}$et $B^{0} \rightarrow \rho^{+} \pi^{-}$) est nulle $\left(T^{+-}=T^{-+}\right)$. L'erreur sur $\alpha$ s'améliore quand l'amplitude $A^{00}$ est plus grande ${ }^{7}$. Enfin, les pingouins dégradent l'erreur sur $\alpha$, en particulier en présence de phases fortes non nulles. La dernière ligne correspond au scénario de "large penguin" du BABAR book [59].

\subsubsection{Impact des phases fortes des résonances $\rho(1450)$ et $\rho(1700)$}

Dans la même configuration que précédemment (sec. 6.7.1) mais avec 10000 événements de signal, nous étudions l'effet de phases fortes relatives non-triviales entre le $\rho(770)$ et les $\rho(1450)$ et $\rho(1700)^{8}$. Les paramètres $\beta$ et $\gamma$ définis dans la section 6.5.1.3 donnent le poids relatif des $\rho$ excités par rapport au $\rho(770)$. Par défaut, la phase relative est de $180^{\circ}$ ( $c f$.

\footnotetext{
${ }^{6}$ La dilution due au mélange $B^{0} \bar{B}^{0}$ est d'environ $17 \%$

${ }^{7}$ Notons néanmoins que nous gardons dans cette étude le nombre d'événements total constant: ainsi, si on augmente la contribution de $B^{0} \rightarrow \rho^{0} \pi^{0}$, on diminue celle de $B^{0} \rightarrow \rho^{ \pm} \pi^{\mp}$

${ }^{8}$ Cet effet a aussi été étudié dans la référence [33].
} 


\begin{tabular}{ccccc|c}
\hline \multicolumn{5}{c|}{ Configuration } & \multirow{2}{*}{$\sigma_{\alpha} \pm R M S$} \\
$T^{+-}$ & $T^{-+}$ & $T^{00}$ & $P^{+-}$ & $P^{-+}$ & \\
\hline 1.0 & 0.5 & 0.17 & 0 & 0 & $8.0 \pm 1.0$ \\
1.0 & 0.5 & 0.5 & 0 & 0 & $7.3 \pm 0.8$ \\
1.0 & 1.0 & 1.0 & 0 & 0 & $5.8 \pm 0.5$ \\
1.0 & 0.5 & 0.17 & 0.1 & 0.1 & $7.6 \pm 1.4$ \\
1.0 & 0.5 & 0.17 & 0.3 & 0.3 & $7.9 \pm 1.1$ \\
1.0 & 0.5 & 0.17 & $0.3 e^{i 20^{\circ}}$ & 0.3 & $8.4 \pm 1.4$ \\
1.0 & 0.47 & 0.14 & $-0.2 e^{i 29^{\circ}}$ & $0.15 e^{i 115^{\circ}}$ & $9.7 \pm 1.5$ \\
\hline
\end{tabular}

Tableau 6.6: Erreur sur $\alpha$ obtenue avec des simulations rapides dans la configuration sans bruits de fond, et pour le signal correctement reconstruit seulement, pour différentes valeurs de diagrammes en arbre et pingouins. La dernière ligne correspond au scénario de "large penguin" du BABAR book [59].

tableau 6.5). Nous introduisons une phase relative supplémentaire de $\pm 50^{\circ}$ autour de la valeur par défaut, et considérons aussi le cas extrême où la phase est décalée de $180^{\circ}$ : les biais observés sur $\alpha$ sont donnés dans le tableau 6.7 .

On observe que le biais maximal de $12.5^{\circ}$ est dû au $\rho(1450)$. Néanmoins, des phases fortes de quelques dizaines de degrés n'engendrent pas de biais importants.

\begin{tabular}{cc}
\hline Configuration & $\alpha$ \\
\hline$\beta \rightarrow \beta e^{+i 50}$ & $102.6 \pm 2.5$ \\
$\beta \rightarrow \beta e^{-i 50}$ & $100.7 \pm 2.2$ \\
$\beta \rightarrow \beta e^{i 180}$ & $112.5 \pm 2.6$ \\
$\gamma \rightarrow \gamma e^{+i 50}$ & $100.5 \pm 2.3$ \\
$\gamma \rightarrow \gamma e^{-i 50}$ & $100.0 \pm 2.4$ \\
$\gamma \rightarrow \gamma e^{i 180}$ & $105.2 \pm 2.8$ \\
\hline
\end{tabular}

Tableau 6.7: Effet systématique sur $\alpha$ de phases fortes relatives entre le $\rho(770)$ et les $\rho(1450)$ (paramétrée par $\beta$ ) et $\rho(1700)$ (paramétrée par $\gamma$ ) - cf. Eq. 6.5.1.3. On a généré $\alpha=100^{\circ}$.

Nous avons d'autre part essayé d'ajuster ces phases fortes dans les données: sur un échantillon de 500 événements, générés avec $\alpha=100^{\circ}$, nous obtenons

$$
\alpha=102.8 \pm 8.7^{\circ}
$$

quand les phases fortes sont fixées, et

$$
\begin{aligned}
\alpha & =105.8 \pm 9.7^{\circ} \\
\operatorname{phase}(\beta) & =19.1 \pm 19.0^{\circ} \\
\operatorname{phase}(\gamma) & =-2.2 \pm 18.3^{\circ}
\end{aligned}
$$

quand les phases fortes sont ajustées, avec une corrélation entre les phases de $\beta$ et $\gamma$ de $40 \%$, entre la phase de $\beta$ et $\alpha$ de $30 \%$, et entre la phase de $\gamma$ et $\alpha$ de moins de $10 \%$.

Nous disposons donc d'une sensibilité expérimentale pour ajuster les phases fortes sur les données: l'erreur sur $\alpha$ est dégradée de $12 \%$ environ, ce qui est bien inférieur à l'erreur systématique obtenue dans le tableau 6.7 . 


\subsubsection{Impact de la résolution sur $m^{2}\left(\pi^{ \pm} \pi^{0}\right)$}

Nous quantifions l'impact de la résolution du détecteur sur $m^{2}\left(\pi^{ \pm} \pi^{0}\right)$ pour les événements correctement reconstruits. La figure 6.5 montre la résolution sur la masse invariante. Nous générons par simulation rapide des échantillons tenant compte de la résolution sur la masse, puis ajustons ces échantillons sans tenir compte de la résolution: le biais observé sur $\alpha$ est inférieur à 1\%: nous en déduisons que l'effet de résolution sur les événements correctement reconstruits est négligeable.

L'effet de la résolution sur les événements mal reconstruits n'est pas encore évaluée à ce jour. Néanmoins, nous savons que le poids de ces événements dans le résultat final est faible, et donc ce point n'est pas critique.

\subsubsection{Impact des bruits de fond}

Nous avons réalisé des simulations rapides en incluant le bruit de fond continuum, et nous nous plaçons dans la configuration "large penguin" du BABAR book [59]: l'erreur sur $\alpha$ est augmentée de $25 \%$ par rapport au résultat donné dans le tableau 6.6. on passe donc d'une erreur de 9.7 à 12.1 .

En combinant les effets du bruit de fond continuum et $B$, on attend $\alpha$ avec une erreur d'environ $15^{\circ}$ dans la configuration "large penguin". Néanmoins, plus importants seront les effets systématiques, encore non-évalués à ce jour.

\subsection{Conclusion}

Les études préliminaires pour l'analyse Dalitz ont été menées: nous avons présenté la reconstruction et sélection de $B^{0} \rightarrow \pi^{+} \pi^{-} \pi^{0}$, et étudié les bruits de fond continuum et $B$. Nous avons examiné les étapes nécessaires à l'élaboration de la distribution des événements de signal dans le diagramme Dalitz: les distributions théoriques, les effets du détecteur, et de la sélection. Enfin, les premiers résultats utilisant cet environnement ont été donnés. Dans la configuration "large penguin", nous évaluons l'erreur statistique sur $\alpha$ à environ $15^{\circ}$ pour une luminosité intégrée de $81 \mathrm{fb}^{-1}$. Plus importantes sont les erreurs systématiques qui n'ont pas encore été évaluées. Nous avons néanmoins réalisé une étude des effets des $\rho(1450)$ et $\rho(1700)$ : l'erreur systématique sur $\alpha$ dues à une phase forte relative quelconque entre ces $\rho$ excités et le $\rho(770)$ est de $12^{\circ}$ maximum. On peut néanmoins déterminer directement ces phases sur les données, ce qui ne détériore l'erreur statistique sur $\alpha$ que de $12 \%$ (soit $1^{\circ}$ ). 


\section{Partie III}

\section{Contraintes sur la matrice CKM}





\section{Introduction}

Cette dernière partie de thèse concerne l'interprétation des observables reliées à la matrice CKM en terme de contraintes sur les éléments de cette dernière, ainsi que sur d'autres paramètres non prédits par le Modèle Standard et ses extensions.

Pour ce faire, nous avons développé un logiciel dénommé CKMfitter [15] permettant de réaliser une analyse globale de la matrice CKM au sein de différents environnements statistiques, dont l'approche fréquentiste Rfit (qui est celle utilisée dans cette thèse et dans les publications associées $[15,103,104,105,106,107,108])$.

La méthode statistique utilisée dans Rfit est décrite dans le chapitre 7. L'ajustement standard de la matrice CKM utilise uniquement les observables établies ( $c-\grave{a}-d$, dont les mesures sont publiées dans un journal scientifique) dont l'interprétation au sein du Modèle Standard ne souffre pas d'erreurs théoriques très controversées. C'est le cas de $\sin 2 \beta, \epsilon_{K}$, $\Delta m_{d},\left|V_{u b} / V_{c b}\right|$, etc. Une telle analyse standard est décrite dans le chapitre 8.

Les mesures reliées à $B \rightarrow \rho h$ décrites dans cette thèse ne rentrent pas dans la catégorie des observables standards, puisque comme expliqué dans la section 1.5.3 et le chapitre 2, l'interprétation de ces résultats en terme de l'angle $\alpha$ suppose, par exemple, de négliger des diagrammes considérés comme sous-dominant, ou fait appel à la symétrie $S U(3)$. Dans le chapitre 9 , nous interprétons les résultats reliés à $B \rightarrow \rho h$ dans le cadre de $S U(2)$ et $S U(3)$. 


\section{Chapitre 7}

\section{Méthode statistique}

\subsection{Introduction}

Il existe plusieurs méthodes statistiques permettant de quantifier l'accord entre mesures et modèles, regroupées en deux écoles de pensées: l'école fréquentiste (ou classique), et l'école Bayesienne. En plus des différences techniques détaillées plus loin, ces deux écoles diffèrent d'un point de vue "philosophique". Considérons que l'on souhaite déterminer le paramètre fondamental $\theta$ d'un modèle à partir d'une mesure $X$ (dont la prédiction au sein du modèle dépend de $\theta$ ):

- les fréquentistes considèrent que $\theta$ est une constante de la nature, et estiment la probabilité $p(X \mid \theta)$ d'observer la mesure $X$ pour une certaine valeur de $\theta$ : cette probabilité est donnée par la fréquence d'occurrence de la mesure $X$ dans la limite où l'on répète l'expérience (sous des conditions exactement identiques) un grand nombre de fois (d'où le nom d'approche "fréquentiste"). L'information sur $\theta$ disponible dans la mesure $X$ est contenue dans ce qu'on appelle en statistique un estimateur (noté $\hat{\theta}$ ). Des exemples d'estimateurs sont celui qui correspond au minimum des moindres carrés $\left(\chi^{2}\right)$, ou celui qui conduit au maximum de la fonction de vraisemblance $\mathcal{L}$. La fonction de vraisemblance est définie comme suit:

$$
\mathcal{L}(\theta)=\Pi_{i=1}^{N} p\left(X_{i} \mid \theta\right),
$$

où l'indice $i$ correspond aux $N$ expériences fournissant les mesures $X_{i}$ et $p\left(X_{i} \mid \theta\right)$ est la distribution de probabilité (Probability Distribution Function, en anglais, PDF) des mesures $X_{i}$ pour un $\theta$ donné.

On peut définir un "intervalle de confiance" (Confidence Level en anglais, CL) $\left[\theta_{1}, \theta_{2}\right]$ dans lequel la vraie valeur de $\theta$ se trouve avec un niveau de confiance donné. Le sens précis de cette proposition est le suivant: pour toute valeur $\theta$ dans l'intervalle $\left[\theta_{1}, \theta_{2}\right]$, la probabilité d'obtenir un résultat expérimental en "plus mauvais accord" que celui observé est supérieur ou égal au niveau de confiance.

Si la PDF de $X$ est une Gaussienne dont seule la valeur centrale dépend de $X$, alors l'intervalle est défini par:

$$
\ln \mathcal{L}(\theta) \geq \ln \mathcal{L}_{\text {max }}-\Delta \ln \mathcal{L}
$$

où la valeur de $\Delta \ln \mathcal{L}$ détermine le niveau de confiance associé à cet intervalle. Les limites de l'intervalle $\left[\theta_{1}, \theta_{2}\right]$ correspondent aux deux solutions où $\ln \mathcal{L}(\theta)=\ln \mathcal{L}_{\max }-$ 
$\Delta \ln \mathcal{L}$. Par exemple, l'intervalle à $68 \%$ de niveau de confiance correspond à $\Delta \ln \mathcal{L}=$ $1 / 2$.

- les Bayesiens considèrent le problème sous un angle radicalement opposé en introduisant le concept de probabilité $p(\theta \mid X)$ que $\theta$ ait une certaine valeur au vu de la mesure $X$ réalisée. Ils considèrent ainsi implicitement $\theta$ comme une variable aléatoire à laquelle une distribution de probabilité peut être assignée. Pour contourner cette notion curieuse d'une réalité aléatoire déterminée peu à peu par les mesures, le vocabulaire utilisé est différent. L'objet $p(\theta \mid X)$ dit représenter notre connaissance sur la vraie valeur de $\theta$. La $\mathrm{PDF} p(\theta \mid X)$ est obtenue à l'aide du théorème de Bayes:

$$
p(\theta \mid X)=\frac{p(X \mid \theta)}{p(X)} p(\theta),
$$

et nécessite d'estimer la distribution de probabilité a priori $p(\theta)$ du paramètres $\theta$, qui est modifiée par la mesure (correspondant au terme $p(X \mid \theta)$ ) pour donner la distribution de probabilité a posteriori $p(\theta \mid X)$. L'approche Bayesienne requiert donc une part de subjectivité, ce qui représente un point critique. Sous certaines hypothèse, l'eq. 7.3 peut s'exprimer en fonction de la fonction de vraisemblance $\mathcal{L}(\theta)$ :

$$
p(\theta \mid X)=\frac{\mathcal{L}(\theta) p(\theta)}{\int \mathcal{L}\left(\theta^{\prime}\right) p\left(\theta^{\prime}\right) d \theta^{\prime}}
$$

On peut définir un intervalle Bayesien $\left[\theta_{1}, \theta_{2}\right]$ ayant un niveau de confiance donné, correspondant à la probabilité intégrée sur cet intervalle:

$$
\mathrm{CL}(\theta)=\int_{\theta_{1}}^{\theta_{2}} p(\theta \mid X) d \theta
$$

Ces divergences de fond se traduisent aussi par des différences techniques entre les deux approches: imaginons qu'une observable $\mathcal{O}$ dépende de plusieurs paramètres théoriques $\mathcal{O}=f\left(\theta_{i}\right)$, alors l'utilisation d'une fonction de vraisemblance ou d'une PDF pour décrire cette observable peut mener à des résultats différents car les principes mathématiques derrière ces deux objets sont bien distincts. Alors que la fonction de vraisemblance de l'observable sera obtenue par simple multiplication des fonctions de vraisemblance des différents paramètres théoriques, sa PDF est quant à elle obtenue via une convolution, ce qui peut mener à de surprenants résultats: supposons par exemple que $\mathcal{O}=\Pi_{i}^{N} \theta_{i}$. Alors quelles que soient les formes des PDFs a priori des $\theta_{i}$, si ces dernières sont non-nulles à l'origine, la $\mathrm{PDF}$ de $\mathcal{O}$ présentera une singularité à $\mathcal{O}=0$ de la forme $(-\ln |\mathcal{O}|)^{N-1}$ (voir l'appendice A de la ref. [15]). L'utilisation de l'outil de convolution mène à la création d'information non-existante au départ (comme ce "pic" à l'origine dans l'exemple précédent): c'est la conséquence de l'attribution de PDF aux paramètres fondamentaux du modèle (de la Nature...).

Pour cette raison, et parce que le degré de subjectivité de l'approche Bayesienne est discutable, l'analyse présentée dans la suite suit l'approche fréquentiste.

Dans les sections suivantes, on construit la fonction de vraisemblance utilisée dans l'analyse d'ajustement des paramètres CKM. Les différentes phases de l'analyse sont ensuite détaillées: dans un premier temps, un test statistique est construit pour tester l'accord entre les données et le modèle théorique utilisé pour les décrire (en l'occurrence, le Modèle Standard). Si cet accord est bon, on procède à la phase métrologique où les paramètres CKM sont 
extraits, et diverses prédictions sont dérivées. Enfin, il est possible d'étudier des modèles au delà du Modèle Standard et contraindre de nouveaux paramètres fondamentaux introduits par ces modèles.

\subsection{La fonction de vraisemblance}

L'analyse d'ajustement des paramètres CKM implique différents protagonistes:

- un ensemble de $N_{\exp }$ mesures $x_{\exp }=x_{\exp }(1), \ldots, x_{\exp }\left(N_{\exp }\right)$ (correspondant aux “ $X$ " de la section précédente).

- les prédictions théoriques (par exemple, du Modèle Standard) correspondantes à ces mesures, $x_{\text {theo }}=x_{\text {theo }}(1), \ldots, x_{\text {theo }}\left(N_{\exp }\right)$. Ces prédictions théoriques dépendent de $N_{\text {mod }}$ paramètres de modèle $y_{\bmod }=y_{\bmod }(1), \ldots, y_{\bmod }\left(N_{\bmod }\right)$ (correspondant aux " $\theta$ " de la section précédente). Parmi eux, on compte:

- $N_{\text {theo }}$ paramètres fondamentaux du modèle (non-prédits par ce modèle). C'est le cas, dans le Modèle Standard, des quatre paramètres CKM, de la masse des quarks, etc. On les dénote $y_{\text {theo }}=y_{\text {theo }}(1), \ldots, y_{\text {theo }}\left(N_{\text {theo }}\right)$.

- $N_{\mathrm{QCD}}=N_{\text {mod }}-N_{\text {theo }}$ paramètres effectifs reliés à notre incapacité à calculer les quantités reliées à $\mathrm{QCD}$. On les dénote $y_{\mathrm{QCD}}=y_{\mathrm{QCD}}(1), \ldots, y_{\mathrm{QCD}}\left(N_{\mathrm{QCD}}\right)$.

La fonction de vraisemblance des paramètres du modèles, $y_{\text {mod }}$, reçoit deux types de contributions $\mathcal{L}_{\text {exp }}$ et $\mathcal{L}_{\text {theo }}:$

$$
\mathcal{L}\left(y_{\text {mod }}\right)=\mathcal{L}_{\text {exp }}\left(x_{\exp }-x_{\text {theo }}\left(y_{\text {mod }}\right)\right) \mathcal{L}_{\text {theo }}\left(y_{\mathrm{QCD}}\right) .
$$

La fonction de vraisemblance expérimentale $\mathcal{L}_{\exp }$ compare la mesure expérimentale $x_{\exp }$ à sa

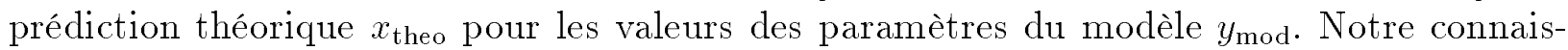
sance des paramètres QCD du modèle est contenue dans la fonction de vraisemblance dite "théorique" $\mathcal{L}_{\text {theo }}$. Ces deux contributions sont détaillées ci-dessous.

\subsubsection{La fonction de vraisemblance expérimentale $\mathcal{L}_{\exp }$}

Une mesure $x_{\exp }$ s'écrit de façon générale sous la forme $x_{\exp }=x_{0} \pm \sigma_{\text {gauss }} \pm \sigma_{\text {inter }}$ où $\sigma_{\text {gauss }}$ est une erreur de statistique Gaussienne, comme les erreurs de mesure due à la statistique limitée des évènements. La seconde erreur $\sigma_{\text {inter }}$ est une erreur non Gaussienne, dénommée erreur d'intervalle. Nous adoptons les mêmes notations pour les paramètres théoriques. Le deuxième type d'erreur $\sigma_{\text {inter }}$ rend compte des erreurs théoriques et des erreurs systématiques de mesure.

En l'absence de $\sigma_{\text {inter }}$, la fonction de vraisemblance est une simple Gaussienne de valeur centrale $x_{\text {theo }}$ et de variance $\sigma_{\text {gauss }}$. En présence de $\sigma_{\text {inter }}$, la fonction de vraisemblance contient:

- un intervalle de largeur égale à l'erreur d'intervalle:

$$
\mathcal{L}_{\text {inter }}= \begin{cases}1 & \text { quand }\left|x_{\text {exp }}-x_{\text {theo }}\right| \leq \sigma_{\text {inter }}, \\ 0 & \text { quand }\left|x_{\text {exp }}-x_{\text {theo }}\right|>\sigma_{\text {inter }}\end{cases}
$$


- une Gaussienne de variance $\sigma_{\text {gauss }}$ et de valeur centrale décalée par la valeur de l'intervalle $\sigma_{\text {inter: }}$

$$
\mathcal{L}_{\text {gauss }}=\frac{1}{\sqrt{2 \pi} \sigma_{\text {gauss }}} e^{-\frac{1}{2}\left(\frac{\left|x_{\text {exp }}-x_{\text {theo }}\right|-\sigma_{\text {inter }}}{\left.\sigma_{\text {gau }}\right)^{2}}\right.} .
$$

La fonction de vraisemblance expérimentale totale pour cette mesure particulière est alors le produit de $\mathcal{L}_{\text {inter }}$ et $\mathcal{L}_{\text {gauss }}{ }^{1}$.

\subsubsection{La fonction de vraisemblance théorique $\mathcal{L}_{\text {theo }}$}

La fonction de vraisemblance théorique est donnée par:

$$
\mathcal{L}_{\text {theo }}\left(y_{\mathrm{QCD}}\right)=\Pi_{i=1}^{N_{\mathrm{QCD}}} \mathcal{L}_{\text {theo }}(i)
$$

La même procédure que dans le cas d'une mesure est suivie pour la construction des fonctions de vraisemblance individuelles théoriques $\mathcal{L}_{\text {theo }}(i)$. Néanmoins, dans la plupart des cas, seule une erreur d'intervalle existe puisque les erreurs théoriques sont issues du jugement des théoriciens et non pas de processus statistiques. Les calculs sur réseaux constituent une exception, puisqu'il existe une erreur Gaussienne due à la statistique limitée du calcul.

\subsection{Test du Modèle Standard}

Le test du Modèle Standard ${ }^{2}$ consiste à calculer le minimum absolu de:

$$
\chi^{2}\left(y_{\text {mod }}\right)=-2 \ln \mathcal{L}\left(y_{\text {mod }}\right)
$$

par rapport aux paramètres $y_{\text {mod }}$, c'est à dire à trouver l'ensembles des paramètres du modèle qui reproduit le mieux les données. Nous dénotons cette valeur du $\chi^{2}: \chi_{\min ; y_{\text {mod }}}^{2}$.

Dans un cas Gaussien, nous pouvons assigner un niveau de confiance au Modèle Standard de la façon suivante:

$$
\mathrm{CL}(\mathrm{MS})=\operatorname{Prob}\left(\chi_{\min ; \mathrm{y}_{\mathrm{mod}}}^{2}, \mathrm{~N}_{\mathrm{ddl}}\right) \text {, }
$$

où $\mathrm{N}_{\text {ddl }}$ est le nombre de degrés de liberté donné par $\mathrm{N}_{\mathrm{ddl}}=\mathrm{N}_{\text {exp }}-\mathrm{N}_{\text {mod }}$, et la fonction Prob (qui tire son nom de la fonction de la bibliothèque informatique du CERN) vaut:

$$
\operatorname{Prob}\left(\chi_{\min ; y_{\text {mod }}}^{2}, N_{d d l}\right)=\frac{1}{\sqrt{2^{N_{d d l}}} \Gamma\left(N_{d d l} / 2\right)} \int_{\chi_{\min ; y_{\text {mod }}}^{\infty}}^{\infty} e^{-t / 2} t^{N_{d d l} / 2-1} d t
$$

Mais en la présence d'erreurs non Gaussiennes, il faut procéder au calcul de ce niveau de confiance par simulation Monte Carlo, dont les étapes sont les suivantes:

- on calcule les prédictions théoriques de chaque mesure pour l'ensemble des paramètres

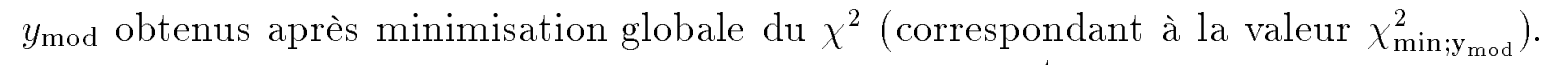
Nous dénotons cet ensemble de valeurs de paramètres $y_{\text {mod }}^{\text {opt }}$.

\footnotetext{
${ }^{1} \mathrm{~L}$ 'approche Bayesienne consisterait dans ce cas à convoler une PDF uniforme de largeur $\sigma_{\text {inter }}$ avec une Gaussienne, ce qui donne comme PDF globale une Gaussienne élargie, dans laquelle l'intervalle a disparu.

${ }^{2} \mathrm{Il}$ s'agit de l'équivalent du test de la qualité de l'ajustement pour d'une mesure, comme réalisé dans la section 4.12 .3 pour l'analyse $B^{0} \rightarrow \rho h$.
} 
- on génère ensuite un ensemble de mesures $x_{\exp }(i)$ en suivant la distribution des fonctions de vraisemblance individuelles $\mathcal{L}_{\exp }(i)$ avec $x_{\exp }(i)=x_{\text {theo }}(i)$, où $x_{\text {theo }}(i)$ a été calculé avec les $y_{\text {mod }}^{\mathrm{opt}}$.

- La fonction de vraisemblance théorique $\mathcal{L}_{\text {theo }}$ est calculée avec les valeurs originales des paramètres $y_{\text {mod }}$ (car ces valeurs ne sont pas des variables aléatoires, mais des paramètres contribuant à la définition de la fonction de vraisemblance).

- comme cela était fait sur les données, on minimise le $\chi^{2}$ par rapport à $y_{\text {mod }}$ sur ces mesures générées.

- on répète plusieurs fois la minimisation du $\chi^{2}$, et l'on obtient une distribution de minimum de $\chi^{2}, \mathcal{F}\left(\chi^{2}\right)$, que l'on normalise à l'unité.

Ainsi, on calcule la limite supérieure du niveau de confiance du Modèle Standard à partir de cette distribution par:

$$
\mathrm{CL}(\mathrm{MS})=\int_{\chi_{\min ; y_{\bmod }}^{\infty}}^{\infty} \mathcal{F}\left(\chi^{2}\right) \mathrm{d} \chi^{2}
$$

\subsection{Métrologie}

Si le niveau de confiance calculé en 7.13 est satisfaisant (par exemple, supérieur à 5\%), nous pouvons procéder à l'extraction des paramètres fondamentaux du Modèle Standard à partir des données.

Pour extraire les niveaux de confiance dans le plan $(\rho, \eta)$, par exemple, on minimise le $\chi^{2}$ par rapport à tous les autres paramètres, c'est à dire qu'en chaque point du plan $(\rho, \eta)$, on choisit les valeurs des autres paramètres qui maximisent l'accord avec les données. Puisque l'hypothèse du Modèle Standard a été acceptée, on corrige du minimum global de $\chi^{2}\left(\chi_{\min : y_{\bmod }}^{2}\right)$, afin que le point où se produise ce minimum ait un niveau de confiance de $100 \%$ :

$$
\Delta \chi^{2}(\rho, \eta)=\chi_{\min }^{2}(\rho, \eta)-\chi_{\min ; y_{\bmod }}^{2}
$$

Le niveau de confiance au point $(\rho, \eta)$ est calculé par:

$$
\mathrm{CL}(\rho, \eta)=\operatorname{Prob}\left(\Delta \chi^{2}(\rho, \eta), \mathrm{N}_{\mathrm{ddl}}\right) .
$$

On peut remplacer $(\rho, \eta)$ par n'importe quel autre jeu de paramètres du modèle que l'on souhaite contraindre $(\gamma, \sin 2 \beta, \sin 2 \alpha$, le paramètre de Jarlskog, $\ldots)$.

\subsection{Physique au delà du Modèle Standard}

Si le niveau de confiance du Modèle Standard est faible, on peut alors essayer de décrire les données avec un modèle alternatif de nouvelle physique. Dans le cas contraire, on peut poser des limites sur les paramètres de modèles au delà du Modèle Standard. Ces modèles doivent néanmoins être suffisamment prédictifs et ne pas contenir un nombre important de nouveaux paramètres (par exemple, un modèle générique de supersymétrie possède 43 nouvelles phases violant $C P$ ). 


\subsection{Conclusion}

L'approche statistique utilisée dans le logiciel CKMfitter est basée sur l'école de pensée fréquentiste, et se déroule en 3 phases: nous commençons par tester la qualité de l'ajustement des données avec le Modèle Standard. Si le niveau de confiance de ce dernier est bon, nous pouvons alors procéder à l'extraction des paramètres non-prédits par le Modèle Standard de façon numérique et graphique. Enfin, on peut explorer des modèles de physique au delà du Modèle Standard et contraindre les espaces de paramètres présents dans ces modèles. 


\section{Chapitre 8}

\section{Ajustement standard des paramètres CKM}

\subsection{Ingrédients de l'ajustement standard}

Les ingrédients pour l'ajustement standard de la matrice CKM sont détaillés dans cette section. Ils comportent des observables reliées aux éléments de matrice CKM, ainsi qu'à la violation de $C P$.

\subsubsection{Les éléments de matrice CKM}

Un certain nombre d'éléments de matrice CKM sont mesurés avec une bonne précision ( $\left|V_{u d}\right|$ à $\simeq 0.1 \%,\left|V_{u s}\right|$ à $\simeq 1 \%,\left|V_{c d}\right|,\left|V_{c s}\right|$ et $\left|V_{c b}\right|$ entre $\simeq 3$ et $5 \%$ ) et permettent de déterminer les paramètres CKM $\lambda$ et $A$. La mesure de $\left|V_{u b}\right|$, reliée à $\rho$ et $\eta$, est plus délicate.

Les derniers résultats de BABAR et Belle sur $\left|V_{c b}\right|$ et $\left|V_{u b}\right|$ (datant de la conférence Moriond 2003) ne sont pas inclus dans l'ajustement standard décrit ici.

$\left|\boldsymbol{V}_{u \boldsymbol{d}}\right|$ Trois méthodes sont utilisées pour mesurer $\left|V_{u d}\right|$ : les désintégrations nucléaires $\beta$ super-permises ${ }^{1}$ [109], les désintégrations $\beta$ du neutron $\left(n \rightarrow p e^{-\bar{\nu}}\right)$ [110], et celles du pion $\left(\pi^{+} \rightarrow \pi^{0} e^{+} \nu\right)$ [111]. On utilise la moyenne de ces trois résultats: $\left|V_{u d}\right|=0.97394 \pm 0.00089$.

$\left|\boldsymbol{V}_{u s}\right|$ La mesure de $\left|V_{u s}\right|$ détermine le paramètre $\lambda$ avec une précision $\simeq 1 \%$. Deux méthodes sont utilisées pour mesurer $\left|V_{u s}\right|$ : les désintégrations semi-leptoniques des kaons $\left(K^{+} \rightarrow \pi^{0} l^{+} \nu\right.$ et $\left.K_{L}^{0} \rightarrow \pi^{-} l^{+} \nu\right)$ [111], et celles des hypérons dont les erreurs théoriques ne sont pas sous contrôle [112]. Seul le premier résultat est donc utilisé: $\left|V_{u s}\right|=0.2200 \pm$ $0.0017_{\exp } \pm 0.0018_{\text {theo }}$.

$\left|\boldsymbol{V}_{\boldsymbol{c} \boldsymbol{b}}\right|$ L'élement de matrice $\left|V_{c b}\right|(\propto A)$ est mesuré à l'aide des désintégrations semileptoniques exclusives et inclusives des mésons $B$ en états finals charmés.

- Désintégrations semi-leptoniques exclusives $B \rightarrow D^{(*)} l \bar{\nu}$ : l'élément de matrice de la désintégration exclusive $B \rightarrow D^{(*)} l \bar{\nu}$ est paramétré par deux facteurs de forme

\footnotetext{
${ }^{1}$ Cette valeur dévie de la valeur obtenue à partir de la relation d'unitarité $\left|V_{u d}\right|^{2}+\left|V_{u s}\right|^{2}+\left|V_{u b}\right|^{2}=1$, en utilisant les valeurs mesurées de $\left|V_{u s}\right|$ et $\left|V_{u b}\right|$. On utilise donc la correction proposée par le PDG [44]: $\left|V_{u d}\right|=0.9740 \pm 0.0010$
} 
correspondant à l'hadronisation des quarks. Grâce à la présence des quarks $b$ et $c$ lourds, ces deux facteurs de forme sont reliés à la fonction universelle d'Isgur-Wise (dans la limite où $\left.m_{b}, m_{c} \rightarrow \infty\right)$. Ainsi, les taux de désintégrations de $B \rightarrow D^{(*)} l \bar{\nu}$ calculés dans le cadre de la théorie effective des quarks lourds ("Heavy Quark Effective Theory" en anglais, HQET [113]) peuvent s'écrire schématiquement par:

$$
\frac{d \Gamma\left(B \rightarrow D^{(*)} l \bar{\nu}\right)}{d w}=\left(\text { facteurs connus) } | V _ { c b } | ^ { 2 } \left\{\begin{array}{ll}
\left(w^{2}-1\right)^{1 / 2} \mathcal{F}_{*}^{2}(w) & \text { pour } B \rightarrow D^{*} \\
\left(w^{2}-1\right)^{3 / 2} \mathcal{F}^{2}(w) & \text { pour } B \rightarrow D
\end{array}\right.\right.
$$

où $w=\left(m_{B}^{2}+m_{D^{(*)}}^{2}-q^{2}\right) /\left(2 m_{B} m_{D^{(*)}}\right)$ avec $q$ l'impulsion transférée par le $\mathrm{W}$, et où $\mathcal{F}(w), \mathcal{F}_{*}(w)$ sont les facteurs de forme. Quand le $D^{(*)}$ a un recul nul (au repos dans le centre de masse du $B$, soit $w=1$ ), alors $\mathcal{F}_{(*)}(1)=1\left(\right.$ pour $\left.m_{b}, m_{c} \rightarrow \infty\right)$, ce qui permet de déterminer $\left|V_{c b}\right|$ de façon indépendante de modèle. Les corrections perturbatives dues à la masse finie du $b$ et $c$ sont calculées sur réseau et mènent à $\mathcal{F}_{*}(1)=0.91 \pm 0.04[114]$. Les mesures de $\left|V_{c b}\right| \mathcal{F}_{*}(1)$ par les expériences du LEP et CLEO sont obtenues par extrapolation du spectre expérimental de $\mathcal{F}_{*}(w)$ vers $w=1$, et valent:

$$
\left(\left|V_{c b}\right| \mathcal{F}_{*}(1)\right) \times 10^{-3}= \begin{cases}43.1 \pm 1.3 \pm 1.8 & \text { CLEO [116] } \\ 38.2 \pm 1.1 & \text { LEP }[117]\end{cases}
$$

- Désintégrations semi-leptoniques inclusives: dans la désintégration inclusive, on évite le problème du calcul non perturbatif de l'hadronisation en faisant l'hypothèse de la dualité quark-hadron, qui permet de relier les taux inclusifs hadroniques aux désintégrations au niveau des partons. Les taux inclusifs $\bar{B} \rightarrow X_{c} l \bar{\nu}$ sont calculés grâce à HQE ("Heavy Quark Expension"), qui est un développement en séries de puissance en $\Lambda_{\mathrm{QCD}} / m_{b}$ et $\alpha_{s}$, de façon indépendante de modèle pour des mesures suffisamment inclusives. Le paramètre $\left|V_{c b}\right|$ est alors donné par

$$
\Gamma(b \rightarrow c)=\frac{G_{F}^{2}\left|V_{c b}\right|^{2} m_{b}^{5}}{192 \pi^{3}}\left(f\left(\frac{m_{c}}{m_{b}}\right)+\sum_{n} C_{n}\left(\frac{\alpha_{S}}{\pi}\right)^{n}+\sum_{m} D_{m}\left(\frac{\Lambda_{\mathrm{QCD}}}{m_{b}}\right)^{m}\right),
$$

où les paramètres $C_{n}$ et $D_{m}$ correspondent respectivement aux corrections radiatives et non-perturbatives, et sont fonctions de $m_{c} / m_{b}, \lambda_{1}$ (énergie cinétique du quark $b$ dans le méson $B$ ) et $\lambda_{2}$ (couplage chromo-magnétique, proportionnel à $m_{B^{*}}^{2}-m_{B}^{2}$ ).

La moyenne des expériences du LEP est la suivante [117]:

$$
\left|V_{c b}\right|=(40.7 \pm 1.0 \pm 2.2) \times 10^{-3} \text {. }
$$

où les erreurs théoriques proviennent des termes d'ordre supérieur dans le développement 8.1.1, de l'incertitude sur la masse du $b$ (qui entre à la puissance 5 dans le taux de désintégration), et de $\lambda_{1}$.

La combinaison de la mesure du taux inclusif avec des analyses permettant de déterminer $m_{b}$ (ou l'énergie de liaison $\bar{\Lambda}=m_{B}-m_{b}+\mathcal{O}\left(1 / m_{b}\right)$ ) et $\lambda_{1}$ permet de réduire les erreurs théoriques. La collaboration CLEO a réalisé une telle analyse utilisant les moments de la masse hadronique invariante $[120,119]$ et le spectre du photon de $B \rightarrow X_{s} \gamma[121]$, et obtient $\left|V_{c b}\right|=(40.3 \pm 1.3) \times 10^{-3}[122]$.

La moyenne de $\left|V_{c b}\right|$ utilisée dans l'ajustement est:

$$
\left|V_{c b}\right|=(40.4 \pm 1.3 \pm 0.9) \times 10^{-3} .
$$


$\left|\boldsymbol{V}_{u b}\right|$ Mesurer $\left|V_{u b}\right|$ est une tache bien plus difficile que mesurer $\left|V_{c b}\right|$ puisque le taux de désintégration de $b \rightarrow u$ est environ 100 fois plus petit que celui de $b \rightarrow c$, ce dernier constituant donc un bruit de fond important.

- Désintégrations semi-leptoniques exclusives $B \rightarrow \rho / \pi l \bar{\nu}$ : Parce que le quark $u$ est léger, la désintégration exclusive $B \rightarrow \rho l \bar{\nu}$ ne bénéficie pas de la symétrie des quarks lourds qui permettait de relier le facteur de forme à la fonction d'Isgur-Wise. Le facteur de forme est donc calculé sur réseau, mais en utilisant l'approximation de "quenching", où seuls les quarks de valence du hadron sont traités de façon exacte, alors que la mer de quarks est modélisée de façon simplifiée. En utilisant la moyenne des facteurs de forme obtenus avec 5 modèles différents, CLEO a mesuré $\left|V_{u b}\right|=\left(3.25_{-0.32}^{+0.25} \exp \pm\right.$ $\left.0.55_{\text {theo }}\right) \times 10^{-3}[123]$.

- Désintégrations semi-leptoniques inclusives: le principe théorique est le même que pour $\left|V_{c b}\right|$ inclusif, mais des problèmes apparaissent lorsque qu'on applique des coupures cinématiques pour réduire le bruit de fond provenant de $b \rightarrow c$. Ces coupures sont de trois types: l'énergie du lepton $E_{l}>\left(m_{B}^{2}-m_{D}^{2}\right) /\left(2 m_{B}\right)$, la masse invariante hadronique $m_{X}<m_{D}$ [125], ou la masse invariante du dilepton $q^{2} \equiv\left(p_{l}+p_{\nu}\right)^{2}>$ $\left(m_{b}+m_{D}\right)^{2}[126]$ (les efficacités des coupures sont $10 \%, 80 \%$ et $20 \%$ respectivement). Les deux premières coupures isolent des régions de l'espace de phase où HQE ne converge plus, et donc où les erreurs théoriques grandissent. En sélectionnant les événements avec plusieurs coupures combinées, LEP a mesuré $\left|V_{u b}\right|=\left(4.09 \pm 0.61_{\exp } \pm 0.42_{\text {theo }}\right) \times 10^{-3}$ [127].

Comme pour $\left|V_{c b}\right|$ inclusif, les erreurs théoriques peuvent être réduites en utilisant, par exemple, le spectre du photon de $B \rightarrow X_{s} \gamma$. La collaboration CLEO a réalisé cette analyse, en utilisant la coupure sur l'énergie du lepton et a obtenu $\left|V_{u b}\right|=(4.08 \pm$ $\left.0.73_{\exp } \pm 0.29_{\text {theo }}\right) \times 10^{-3}[128]$.

Dans l'ajustement final, nous utilisons le produit des fonctions de vraisemblance associées à ces différents résultats.

$\left|\boldsymbol{V}_{c \boldsymbol{d}}\right|$ et $\left|\boldsymbol{V}_{\boldsymbol{c} s}\right| \quad$ La mesure de $\left|V_{c d}\right|$, comme celle de $\left|V_{u s}\right|$, détermine $\lambda$, mais avec une moins bonne précision expérimentale $(\simeq 6 \%)$. A la fois les éléments de matrice $\left|V_{c d}\right|$ et $\left|V_{c s}\right|$ sont mesurés dans la production de dimuons dans les collisions profondément inélastiques (CPI) de neutrinos et anti-neutrinos sur les nucléons, menant à $\left|V_{c d}\right|=0.224 \pm 0.014$ [111] et $V_{c s}=$ $1.04 \pm 0.16$ [44]. En plus des CPI, $\left|V_{c s}\right|$ peut être mesuré dans les désintégrations $D \rightarrow \bar{K} e^{+} \nu$, les désintégrations charmées du $W\left(W^{+} \rightarrow c \bar{q}\right)$, les désintégrations hadroniques du $W$ (ce dernier utilisant les relations d'unitarité, il s'agit d'une détermination indirecte) [111], et enfin les désintégrations $W \rightarrow X_{c} X[129]$.

\subsubsection{Les observables $C P$}

$\epsilon_{K} \quad$ La violation de $C P$ dans le mélange $K^{0}-\bar{K}^{0}$ est mesurée par $\epsilon_{K}$ :

$$
\epsilon_{K}=\frac{2}{3} \eta_{+-}+\frac{1}{3} \eta_{00}=(2.271 \pm 0.017) \times 10^{-3}[44],
$$

où $\eta_{+-}=A\left(K_{L} \rightarrow \pi^{+} \pi^{-}\right) / A\left(K_{S} \rightarrow \pi^{+} \pi^{-}\right)$et $\eta_{00}=A\left(K_{L} \rightarrow \pi^{0} \pi^{0}\right) / A\left(K_{S} \rightarrow \pi^{0} \pi^{0}\right)$. 
La prédiction théorique de $\epsilon_{K}$ est calculée grâce au développement en produit d'opérateurs ("Operator Product Expansion" en anglais, OPE), et s'écrit:

$$
\begin{gathered}
\epsilon_{K}=\frac{G_{F}^{2} m_{W}^{2} m_{K} f_{K}^{2}}{12 \sqrt{2} \pi^{2} \Delta m_{K}} B_{K}\left(\eta_{c c} S\left(\frac{m_{c}^{2}}{m_{W}^{2}}, \frac{m_{c}^{2}}{m_{W}^{2}}\right) \operatorname{Im}\left[\left(V_{c s} V_{c d}^{*}\right)^{2}\right]+\eta_{t t} S\left(\frac{m_{t}^{2}}{m_{W}^{2}}, \frac{m_{t}^{2}}{m_{W}^{2}}\right) \operatorname{Im}\left[\left(V_{t s} V_{t d}^{*}\right)^{2}\right]\right. \\
\left.+2 \eta_{c t} S\left(\frac{m_{c}^{2}}{m_{W}^{2}}, \frac{m_{t}^{2}}{m_{W}^{2}}\right) \operatorname{Im}\left[V_{c s} V_{c d}^{*} V_{t s} V_{t d}^{*}\right]\right)
\end{gathered}
$$

où $f_{K}$ et $B_{K}$ sont respectivement la constante de désintégration et le facteur de "sac" (non perturbatif) du kaon, et sont issus du calcul de l'élément de matrice hadronique $\left\langle\bar{K}^{0}\right|\left(\bar{s} \gamma^{\mu}(1-\right.$ $\left.\left.\gamma^{5}\right) d\right)^{2}\left|K^{0}\right\rangle=8 / 3 m_{K}^{2} f_{K}^{2} B_{K}$. Le paramètre $f_{K}$ est précisément mesuré dans les désintégrations leptoniques des kaons: $f_{K}=\left(159.8 \pm 1.4_{\left|V_{u s}\right|} \pm 0.44_{\text {théo }}\right) \mathrm{MeV}$ [44]. Le paramètre $B_{K}$ est calculé sur réseau, avec l'approximation "quenched" et en utilisant la symétrie $S U(3): B_{K}=$ $0.87 \pm 0.06 \pm 0.14_{\text {quench. }}$ Il fourni la principale source d'erreur de la prédiction théorique de $\epsilon_{K}$.

Les fonctions d'Inami-Lim [130] $S\left(x_{i}, x_{j}\right)\left(x_{i}=m_{i}^{2} / m_{W}^{2}\right)$ représentent les contributions électrofaibles en boucle:

$$
\begin{aligned}
S(x) \equiv S\left(x_{i}, x_{j}\right)_{i=j}=x\left(\frac{1}{4}\right. & \left.+\frac{9}{4(1-x)}-\frac{3}{2(1-x)^{2}}\right)-\frac{3}{2}\left(\frac{x}{1-x}\right)^{3} \ln (\mathrm{x}), \\
S\left(x_{i}, x_{j}\right)_{i \neq j}=x_{i} x_{j}[ & \left(\frac{1}{4}+\frac{3}{2\left(1-x_{i}\right)}-\frac{3}{4\left(1-x_{i}\right)^{2}}\right) \frac{1}{x_{i}-x_{j}} \ln \left(\mathrm{x}_{\mathbf{i}}\right) \\
& \left.+\left(x_{i} \leftrightarrow x_{j}\right)-\frac{3}{4} \frac{1}{\left(1-x_{i}\right)\left(1-x_{j}\right)}\right] .
\end{aligned}
$$

Les corrections QCD de courte distance de ces fonctions sont inclues dans les paramètres $\eta_{c c}, \eta_{c t}, \eta_{t t}$.

$\Delta \boldsymbol{m}_{\boldsymbol{d}} \quad$ L'observable $\Delta m_{d}$ est la différence de masse entre $B_{H}$ et $B_{L}(c f$. section 1.3.2), $c-\grave{a}-d$ la valeur de la fréquence d'oscillations entre $B^{0}-\bar{B}^{0}$ (Eq. 1.29). La mesure a été réalisée avec une précision de 1.2\%: $\Delta m_{d}=0.503 \pm 0.006$ [135] par un grand nombre d'expériences. La prédiction théorique de $\Delta m_{d}$ est simplifiée par le fait que la boucle contenant le quark $t$ domine sur celles contenant les quarks $u$ et $c$ :

$$
\Delta m_{d}=\frac{G_{\mathrm{F}}^{2}}{6 \pi^{2}} \eta_{B} m_{B_{d}} f_{B_{d}}^{2} B_{d} m_{W}^{2} S\left(x_{t}\right)\left|V_{t d} V_{t b}^{*}\right|^{2}
$$

Contrairement au système des $K$, la constante de désintégration des $B, f_{B_{d}}$, n'est pas pas encore mesurée et doit être calculée tout comme le facteur de sac $B_{d}$ sur réseau: avec des valeurs respectives de $f_{B_{d}}=(175 \pm 20) \mathrm{MeV}$ et $B_{d}=1.30 \pm 0.12 \pm 0.13_{\text {quench }}$ [131], ces deux paramètres fournissent la principale erreur de la prédiction théorique. Les corrections QCD à la fonction d'Inami-Lim $S\left(x_{t}\right)$ sont incluses dans $\eta_{B}=0.55 \pm 0.01$ [22].

$\Delta \boldsymbol{m}_{\boldsymbol{s}}$ L'observable $\Delta m_{s}$ n'a qu'une faible dépendance dans les paramètres CKM, mais le rapport $\Delta m_{s} / \Delta m_{d}$ introduit une contrainte plus forte que $\Delta m_{d}$ seul, car les erreurs systématiques du calcul de $f_{B} \sqrt{B}$ se compensent partiellement dans le rapport $\xi=f_{B_{s}} \sqrt{B_{s}} / f_{B_{d}} \sqrt{B_{d}}=$ $1.16 \pm 0.03 \pm 0.05_{\text {quench }}[131,132]$. D'autre part, le rapport $\Delta m_{s} / \Delta m_{d}$ ne dépend plus de $\eta_{B}$ et $m_{t}$. 
La fréquence de l'oscillation $B_{s}^{0}-\bar{B}_{s}^{0} n$ 'a pas encore été mesurée (seule une limite inférieure existe). Afin de simplifier la combinaison de résultats provenant de plusieurs expériences (en particulier pour tenir compte des effets systématiques), la mesure est réalisée selon la méthode de l'amplitude [133]: l'amplitude $\mathcal{A}$ est introduite devant le terme en cosinus de la probabilité d'oscillation $B_{s}^{0}-\bar{B}_{s}^{0}$ :

$$
\mathcal{P}(\Delta t) \propto 1 \pm \mathcal{A} \cos \left(\Delta m_{s} \Delta t\right)
$$

On ajuste ensuite $\mathcal{A}$ sur les données pour chaque valeur de $\Delta m_{s}$ : pour une statistique infinie, $\mathcal{A}$ vaut 0 pour $\Delta m_{s} \neq \Delta m_{s}^{\text {vrai }}$, et 1 pour $\Delta m_{s}=\Delta m_{s}^{\text {vrai }}$. Les valeurs de $\Delta m_{s}$ pour lesquelles $\left(\mathcal{A}+1.645 \sigma_{\mathcal{A}}\right)<1$ sont exclues à un niveau de confiance de $95 \%$ : cette limite vaut actuellement $14.4 \mathrm{ps}^{-1}[135]$.

Il existe plusieurs méthodes permettant d'exploiter l'information contenue dans le spectre d'amplitude (voir la discussion page 242 de la Ref. [15]). Dans le travail qui suit, la méthode fréquentiste de la ref. [136] est utilisée. La figure 8.1 montre les courbes de $\chi^{2}$ pour différentes méthodes (pour plus de détails, cf. la discussion page 242 de la Ref. [15]): la méthode de l'amplitude modifiée [15], la méthode du rapport des fonctions de vraisemblance [134], et la méthode fréquentiste de la ref. [136].

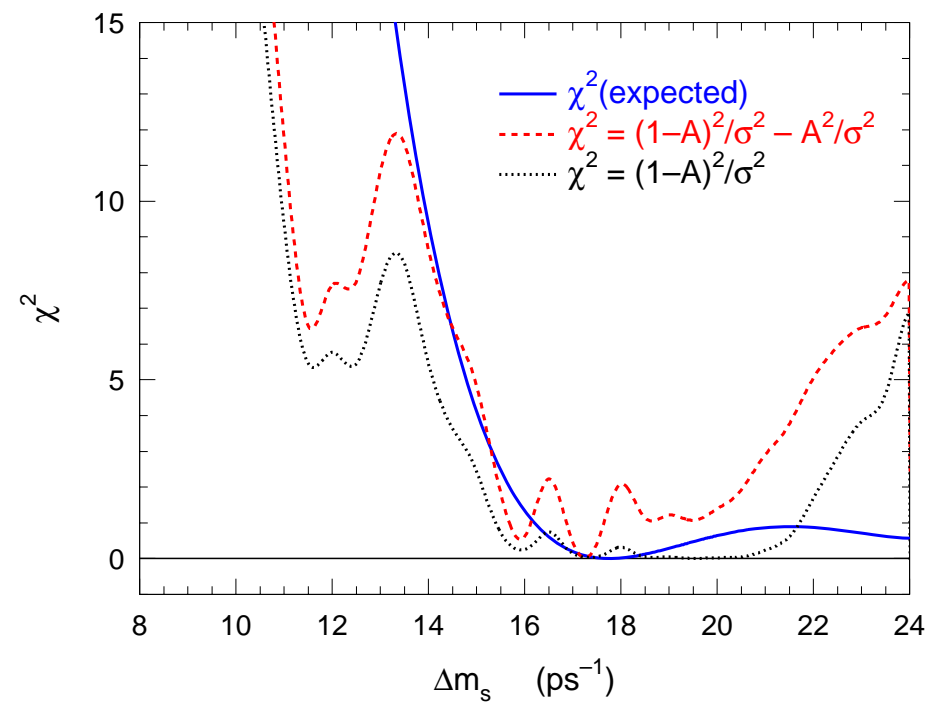

Figure 8.1: Distributions du $\chi^{2}$ pour diverses interprétations du spectre de $\Delta m_{s}$ : la méthode de l'amplitude modifiée [15] (ligne pointillée), la méthode du rapport des fonctions de vraisemblance [134] (ligne pointillée-tirets), et la méthode fréquentiste de la ref. [136] (ligne pleine). Pour plus de détails, cf. la discussion page 242 de la Ref. [15].

$\sin 2 \boldsymbol{\beta}$ Le principe de la mesure de $\sin 2 \beta$ est décrite dans l'introduction théorique de cette thèse ( $c f$. section 1.5.1). La valeur moyenne mondiale vaut actuellement $0.734 \pm 0.054$ [14].

\subsubsection{Résumé des ingrédients}

Le tableau 8.1 résume les valeurs et erreurs des ingrédients de l'ajustement standard de la matrice CKM décrits dans cette section. 


\begin{tabular}{|c|c|}
\hline Paramètre & Valeur \pm Erreur $(\mathrm{s})$ \\
\hline $\begin{array}{l}\left|V_{u d}\right| \\
\left|V_{u s}\right| \\
\left|V_{u b}\right| \text { (LEP incl.) } \\
\left|V_{u b}\right| \text { (CLEO incl.) } \\
\left|V_{u b}\right| \text { (CLEO excl.) } \\
\left|V_{c d}\right| \\
\left|V_{c s}\right| \\
\left|V_{c b}\right|\end{array}$ & $\begin{array}{c}0.97394 \pm 0.00089 \\
0.2200 \pm 0.0025 \\
(4.08 \pm 0.61 \pm 0.47) \times 10^{-3} \\
(4.08 \pm 0.56 \pm 0.40) \times 10^{-3} \\
(3.25 \pm 0.29 \pm 0.55) \times 10^{-3} \\
0.224 \pm 0.014 \\
0.969 \pm 0.058 \\
(40.4 \pm 1.3 \pm 0.9) \times 10^{-3}\end{array}$ \\
\hline $\begin{array}{l}\left|\epsilon_{K}\right| \\
\Delta m_{d} \\
\Delta m_{s} \\
\sin 2 \beta_{\mathrm{WA}}\end{array}$ & $\begin{array}{c}(2.271 \pm 0.017) \times 10^{-3} \\
(0.496 \pm 0.007) \mathrm{ps}^{-1} \\
\text { Voir texte } \\
0.734 \pm 0.054\end{array}$ \\
\hline $\begin{array}{l}m_{c} \\
m_{t}(\overline{\mathrm{MS}}) \\
m_{K} \\
\Delta m_{K} \\
m_{B_{d}} \\
m_{B_{s}} \\
m_{W} \\
G_{F} \\
f_{K}\end{array}$ & $\begin{array}{c}\left(1.3 \pm 0.1_{\text {theo }}\right) \mathrm{GeV} \\
(166.0 \pm 5.0) \mathrm{GeV} \\
(493.677 \pm 0.016) \mathrm{MeV} \\
(3.4885 \pm 0.0008) \times 10^{-15} \mathrm{GeV} \\
(5.2794 \pm 0.0005) \mathrm{GeV} \\
(5.3696 \pm 0.0024) \mathrm{GeV} \\
(80.419 \pm 0.056) \mathrm{GeV} \\
(1.16639 \pm 0.00001) \times 10^{-5} \mathrm{GeV}^{-2} \\
(159.8 \pm 1.5) \mathrm{MeV}\end{array}$ \\
\hline $\begin{array}{l}B_{K} \\
\eta_{c c} \\
\eta_{c t} \\
\eta_{t t} \\
\eta_{B}(\overline{\mathrm{MS}}) \\
f_{B_{d}} \sqrt{B_{d}} \\
\xi\end{array}$ & $\begin{array}{c}0.87 \pm 0.06 \pm 0.13 \\
1.38 \pm 0.53_{\text {theo }} \\
0.47 \pm 0.04_{\text {theo }} \\
0.574 \pm 0.004_{\text {theo }} \\
0.55 \pm 0.01_{\text {theo }} \\
(230 \pm 28 \pm 28) \mathrm{MeV} \\
1.16 \pm 0.03 \pm 0.05\end{array}$ \\
\hline
\end{tabular}

Tableau 8.1: Observables et paramètres utilisés dans l'ajustement standard de la matrice CKM. Lorsque deux erreurs sont données, la première est statistique, et la deuxième théorique (traitée comme un intervalle dans $\mathrm{R} f \mathrm{ft}$ ). Lorsqu'une seule erreur est donnée, il s'agit d'une erreur statistique, sauf mention contraire. 


\subsection{Niveau de confiance global de l'ajustement standard}

Le niveau de confiance de l'ajustement global au sein du Modèle Standard de toutes les observables décrites dans la section 8.1 est déterminé à partir de la valeur du minimum de $\chi^{2}$ absolu, $\chi_{\min ; y_{\bmod }}^{2}$, par la méthode décrite dans la section 7.3. La figure 8.2 montre la distribution de $\chi_{\min ; y_{\bmod }}^{2}$ obtenue par simulation Monte Carlo, et la flèche indique la valeur obtenue dans l'ajustement standard. L'intégration de la distribution par la droite jusqu'à la valeur indiquée par la flèche nous donne un niveau de confiance de $57 \%$. Nous en déduisons donc que le Modèle Standard est une théorie valable pour décrire l'ensemble des observables actuelles, et nous pouvons passer à la phase de métrologie.

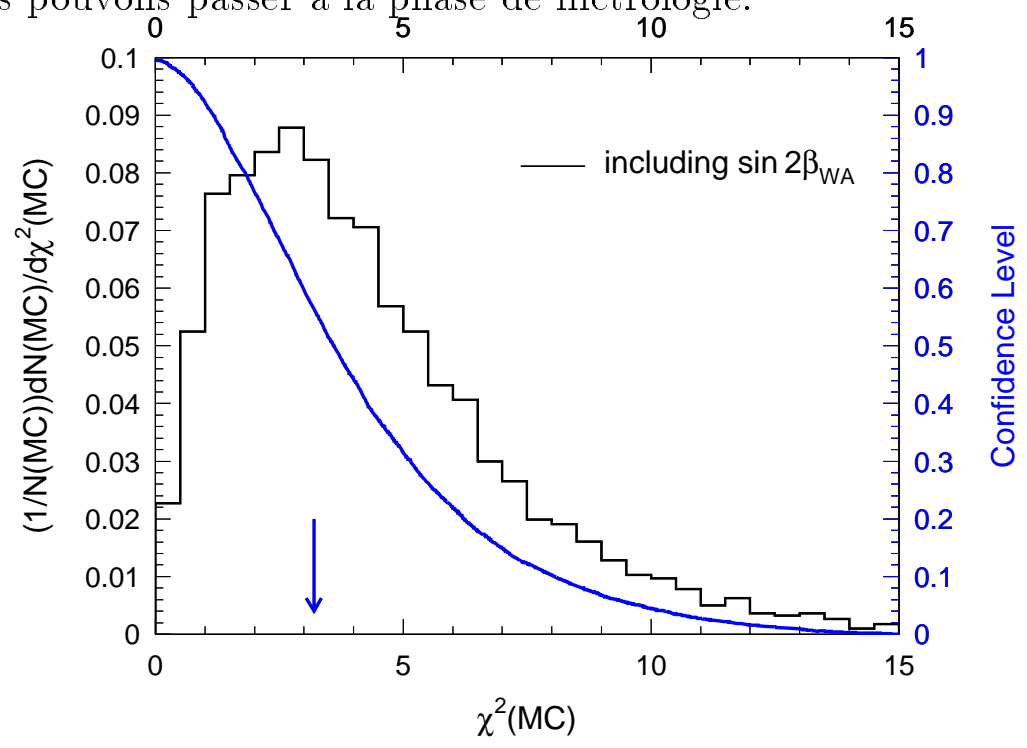

Figure 8.2: Distribution de $\chi_{\min ; \mathrm{y}_{\mathrm{m}} \mathrm{d}}^{2}$ obtenue par simulation Monte Carlo. La flèche indique la valeur obtenue dans l'ajustement standard. L'intégration de la distribution par la droite jusqu'à la valeur indiquée par la flèche nous donne un niveau de confiance de $57 \%$.

\subsection{Métrologie}

Nous représentons graphiquement les résultats de l'ajustement standard dans différents espaces bi- et uni-dimensionnels, et donnons des résultats numériques pour les principaux paramètres.

\subsubsection{Plan $(\bar{\rho}-\bar{\eta})$}

La figure 8.3 montre les principales contraintes individuelles dans le plan $(\bar{\rho}-\bar{\eta})$, et le résultat de l'ajustement global incluant ou pas la contrainte de $\sin 2 \beta$ (menant à quatre solutions miroirs pour $\beta$ ). On voit que l'une des solutions miroirs pour $\beta$ est en parfait accord avec la zone favorisée par les autres contraintes, et que l'inclusion de la mesure de $\sin 2 \beta$ améliore très nettement la contrainte globale dans le plan $(\bar{\rho}-\bar{\eta})$.

\subsubsection{Plan $(\sin 2 \alpha-\sin 2 \beta)$ et $(\sin 2 \beta-\gamma)$}

La figure 8.4 montre les contraintes individuelles et le résultat de l'ajustement final dans les plans $(\sin 2 \alpha-\sin 2 \beta)$ et $(\sin 2 \beta-\gamma)$ sans l'utilisation de la contrainte sur $\sin 2 \beta$. On voit 
que la contrainte obtenue sur $\sin 2 \alpha$ est faible, et que les valeurs $\gamma<90^{\circ}$ sont privilégiées.
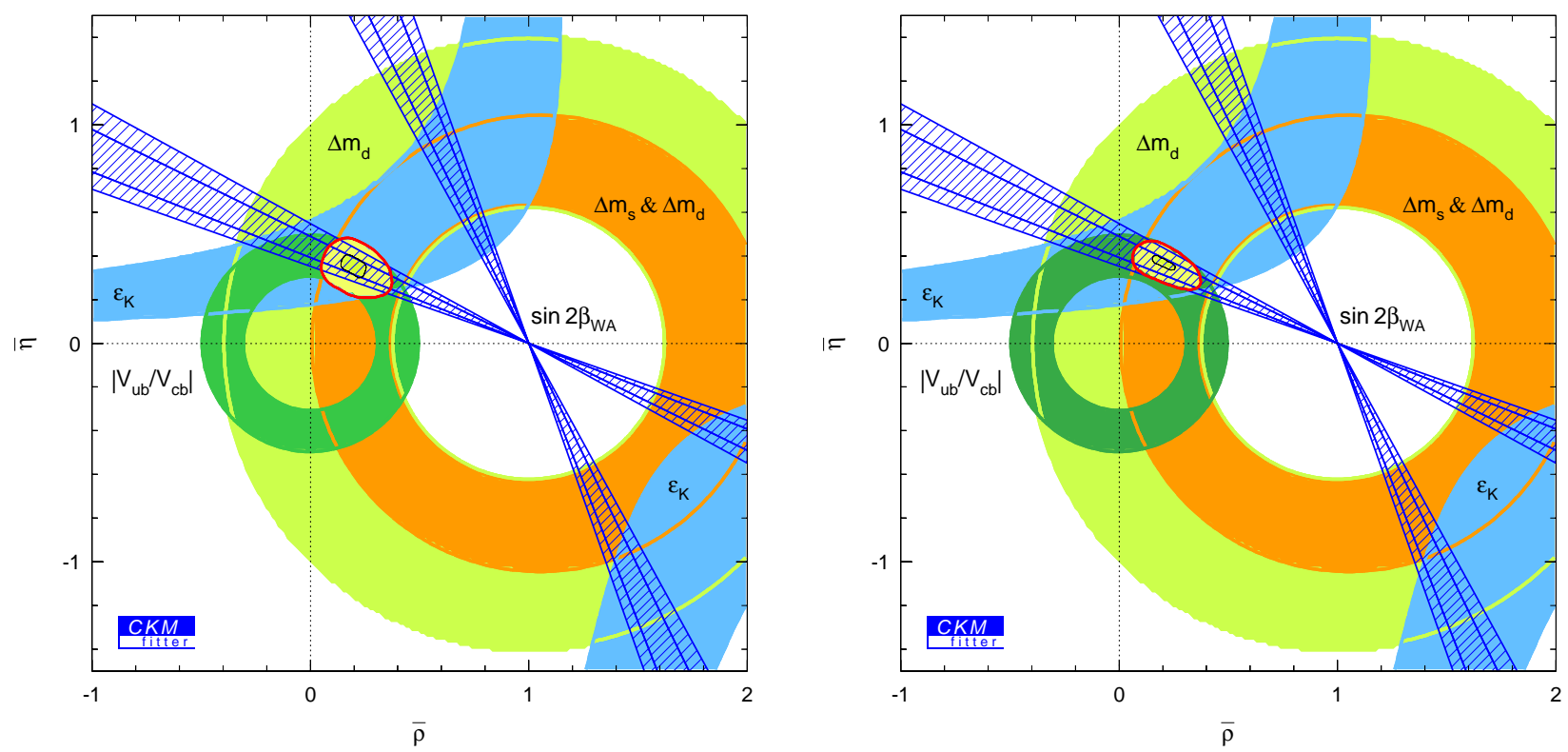

Figure 8.3: Principales contraintes individuelles dans le plan $(\bar{\rho}-\bar{\eta})$ : les zones contenant les points de plus de $5 \%$ de niveau de confiance sont dessinées. Le résultat de l'ajustement global incluant (figure de droite) ou pas (figure de gauche) la contrainte de $\sin 2 \beta$ est indiqué par la zone jaune cerclée de rouge: cette dernière contient les points ayant plus de $5 \%$ de niveau de confiance, et la ligne noire, ceux ayant plus de $90 \%$ de niveau de confiance.
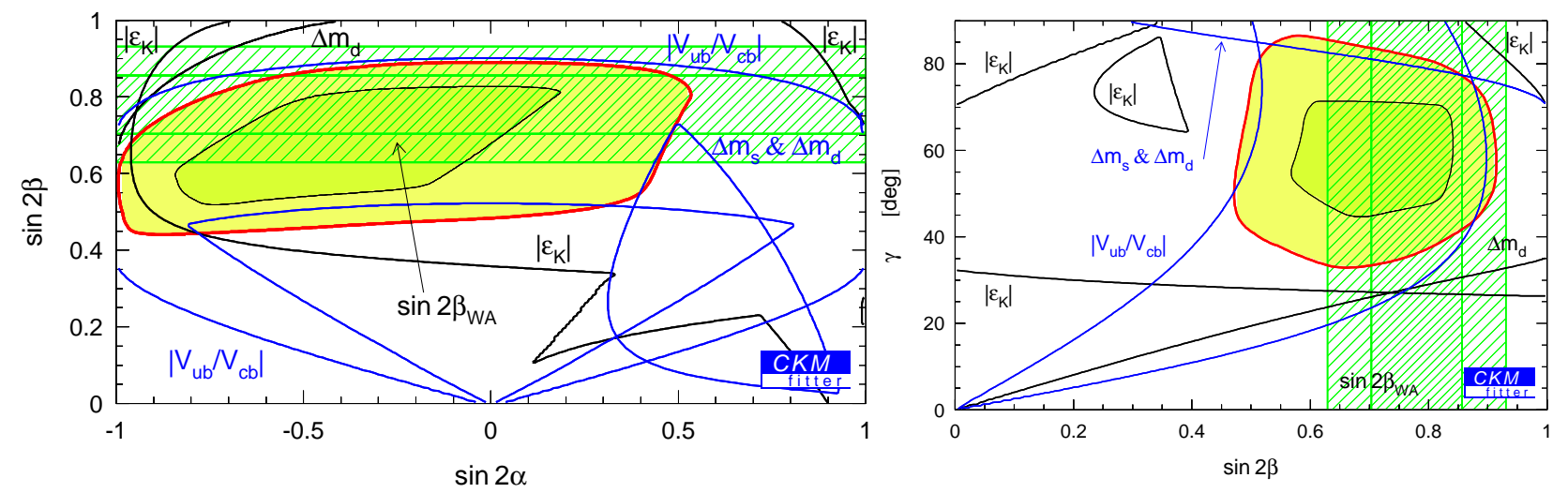

Figure 8.4: Principales contraintes individuelles dans le plan ( $\sin 2 \alpha-\sin 2 \beta)$ (gauche) et $(\sin 2 \beta-\gamma)$ (droite), ainsi que le résultat de l'ajustement global (cf. légende de la figure 8.3 pour la signification des couleurs) n'incluant pas la contrainte de $\sin 2 \beta$. (Note: la valeur de la contrainte de $\sin 2 \beta$ superposée sur ces figures est plus ancienne que la valeur actuelle, et vaut $0.78 \pm 0.08$. Néanmoins, le reste des contraintes correspond à ce qui a été décrit dans la section 8.1.) 


\subsubsection{Contraintes uni-dimensionnelles}

La figure 8.5 montre les distributions uni-dimensionnelles de niveau de confiance pour les paramètres de Wolfenstein $\lambda, A, \bar{\rho}$ et $\bar{\eta}$, ainsi que les trois angles du triangle d'Unitarité $\alpha$, $\beta$ et $\gamma$, et le paramètre de Jarlskog $J_{C P}$.

Les contributions des erreurs théoriques se traduisent par la présence d'un plateau dans la courbe de niveau de confiance: elles sont significatives dans la plupart des prédictions obtenues. Seuls les paramètres $\lambda$ et $\beta$ sont dominés par les erreurs statistiques. L'épaule présente dans certaines contraintes $(\bar{\rho}, \alpha$ et $\gamma)$ est due à la limite sur $\Delta m_{s}$.
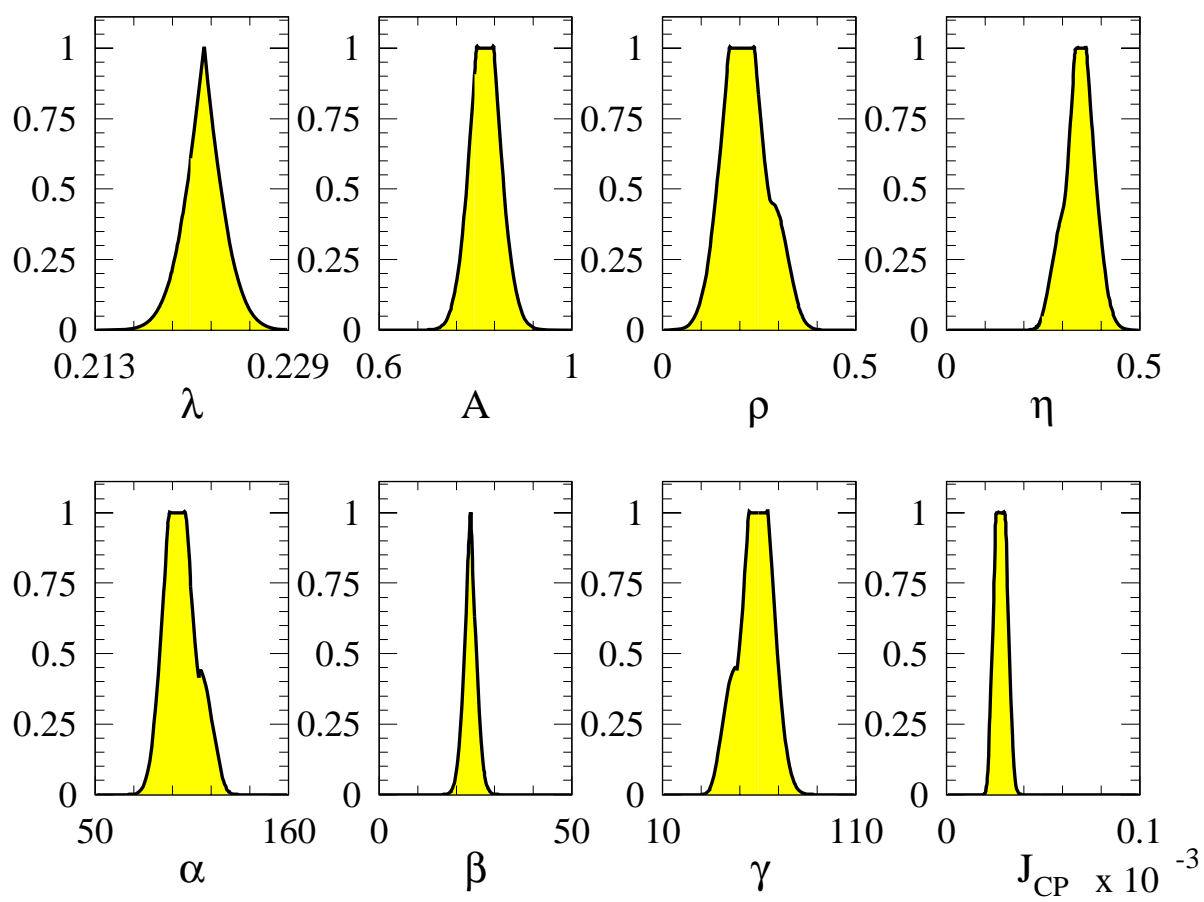

Figure 8.5: Distribution uni-dimensionnelles de niveau de confiance pour les paramètres de Wolfenstein (haut): $\lambda, A, \bar{\rho}$ et $\bar{\eta}$, ainsi que les paramètres $C P$ (bas): les trois angles du triangle d'Unitarité $\alpha, \beta$ et $\gamma$, et le paramètre de Jarlskog $J_{C P}$.

Le tableau 8.2 donne les intervalles contenant les valeurs des paramètres CKM et quantités dérivées ayant un niveau de confiance supérieur à $32 \%$ et $90 \%$. Les trois dernières lignes donnent les valeurs de paramètres théoriques choisis: dans ce cas, l'ajustement est réalisé dans tenir compte de la contrainte d'entrée sur ce paramètre théorique. Les intervalles obtenus sont plus larges que les intervalles d'entrée, ce qui montre que ces paramètres ne sont pas indirectement déterminés par les autres contraintes.

\subsection{Conclusions}

Le Modèle Standard permet de rendre compte de toutes les observables reliées à la matrice CKM avec un niveau de confiance de $57 \%$. La mesure précise de $\sin 2 \beta$ par BABAR et Belle permet de confirmer que le mécanisme KM est très probablement la source dominante de violation $C P$ dans les transitions changeant la saveur des quarks. D'autre part, cette mesure apporte une contrainte importante sur les paramètres CKM: les intervalles de $\lambda, A, \bar{\rho}$ et $\bar{\eta}$ 


\begin{tabular}{|c|c|c|c|c|}
\hline Paramètre & $\geq 32 \% \mathrm{CL}$ & demi-largeur & $\geq 5 \% \mathrm{CL}$ & demi-largeur \\
\hline$\lambda$ & \multicolumn{2}{|c|}{$0.2220 \pm 0.0021$} & \multicolumn{2}{|c|}{$0.2220 \pm 0.0042$} \\
\hline$A$ & $0.771-0.869$ & 0.048 & $0.742-0.900$ & 0.080 \\
\hline $\bar{\rho}$ & $0.125-0.317$ & 0.09 & $0.082-0.363$ & 0.13 \\
\hline $\bar{\eta}$ & $0.284-0.402$ & 0.06 & $0.247-0.435$ & 0.09 \\
\hline$J\left(10^{-5}\right)$ & $2.227-3.341$ & 0.54 & $2.108-3.567$ & 0.65 \\
\hline$\alpha$ & $85^{\circ}-115^{\circ}$ & $14^{\circ}$ & $78^{\circ}-123^{\circ}$ & $20^{\circ}$ \\
\hline$\beta$ & \multicolumn{2}{|c|}{$23.61 \pm 2.25$} & \multicolumn{2}{|c|}{$23.61 \pm 4.05$} \\
\hline$\gamma$ & $42^{\circ}-72^{\circ}$ & $15^{\circ}$ & $36^{\circ}-75^{\circ}$ & $19^{\circ}$ \\
\hline $\sin \theta_{12}$ & \multicolumn{2}{|c|}{$0.2200 \pm 0.0020$} & \multicolumn{2}{|c|}{$0.2200 \pm 0.0042$} \\
\hline $\sin \theta_{13}\left(10^{-3}\right)$ & $3.4-4.0$ & 0.35 & $3.1-4.3$ & 0.6 \\
\hline $\sin \theta_{23}\left(10^{-3}\right)$ & $38.2-42.6$ & 2.2 & $37.0-43.8$ & 3.4 \\
\hline$V_{u d}$ & \multicolumn{2}{|c|}{$0.9751 \pm 0.0005$} & \multicolumn{2}{|c|}{$0.9751 \pm 0.0010$} \\
\hline$V_{u s}$ & \multicolumn{2}{|c|}{$0.2220 \pm 0.0021$} & \multicolumn{2}{|c|}{$0.2220 \pm 0.0042$} \\
\hline$V_{u b}\left(\times 10^{-3}\right)$ & \multicolumn{2}{|c|}{$3.7 \pm 0.3$} & \multicolumn{2}{|c|}{$3.7 \pm 0.6$} \\
\hline$V_{c d}$ & \multicolumn{2}{|c|}{$0.2219 \pm 0.0021$} & \multicolumn{2}{|c|}{$0.2219 \pm 0.0042$} \\
\hline & \multicolumn{2}{|c|}{$0.9742 \pm 0.0005$} & \multicolumn{2}{|c|}{$0.9742 \pm 0.0010$} \\
\hline & \multicolumn{2}{|c|}{$0.0404 \pm 0.0022$} & \multicolumn{2}{|c|}{$0.0404 \pm 0.0034$} \\
\hline$V_{t d}\left(\times 10^{-3}\right)$ & \multicolumn{2}{|c|}{$7.7 \pm 1.1$} & \multicolumn{2}{|c|}{$7.7 \pm 1.5$} \\
\hline$V_{t s}$ & \multicolumn{2}{|c|}{$0.0398 \pm 0.0021$} & \multicolumn{2}{|c|}{$0.0398 \pm 0.0033$} \\
\hline$V_{t b}$ & \multicolumn{2}{|c|}{$0.9992 \pm 0.0001$} & \multicolumn{2}{|c|}{$0.9992 \pm 0.0001$} \\
\hline$f_{B_{d}} \sqrt{B_{d}}(\mathrm{MeV})$ & $202.1-273.0$ & 35.5 & $192.8-288.1$ & 47.6 \\
\hline$B_{K}$ & $0.581-1.448$ & 0.434 & $0.513-1.822$ & 0.655 \\
\hline$m_{t}(\mathrm{GeV})$ & $112.8-281.3$ & 84.2 & $98.3-375.9$ & 138.8 \\
\hline
\end{tabular}

Tableau 8.2: Résultats de l'ajustement global: nous donnons les intervalles contenant les valeurs des paramètres ayant un niveau de confiance supérieur à $32 \%$ et $90 \%$. Les trois dernières lignes donnent les valeurs de paramètres théoriques choisis (dans ce cas, on ne tient alors pas compte de la contrainte d'entrée). 
contenant les valeurs ayant plus de $32 \%$ de niveau de confiance sont les suivants:

$$
\begin{aligned}
\lambda & =0.2220 \pm 0.0021 \\
A & =0.771-0.869 \\
\bar{\rho} & =0.125-0.317 \\
\bar{\eta} & =0.284-0.402 .
\end{aligned}
$$




\section{Chapitre 9}

\section{Interprétation des résultats expérimentaux sur $B \rightarrow \rho h$}

\subsection{Introduction}

Nous discutons les implications de l'analyse en deux corps de $B^{0} \rightarrow \rho^{ \pm} \pi^{\mp}$ décrite dans le chapitre 4 en terme de contraintes sur l'angle $\alpha$ en utilisant les symétries de saveur $S U(2)$ (et en supposant que les pingouins électrofaibles sont négligeables) et $S U(3)$ ( $c f$. la section 1.5.3 et le chapitre 2).

Nous réalisons les quatre analyses suivantes:

- Hypothèse de pingouins nuls: ce cas académique nous permet d'estimer le pouvoir statistique de l'analyse en deux-corps de $B^{0} \rightarrow \rho^{ \pm} \pi^{\mp}$ dans le cas où les erreurs théoriques dues à la pollution des pingouins étaient nulles.

- Limite sur $\left|\alpha-\alpha_{\text {eff }}^{ \pm}\right|$grâce à SU(2), telle que décrite dans la section 2.2.5, et en particulier l'eq. 2.21.

- Extrapolations futures de l'analyse d'isospin $S U(2)$ complète, telle que décrite dans la section 2.2.3.

- Contraintes actuelles et futures sur $\alpha$ à partir de la symétrie $S U(3)$ ( $c f$. section 2.3).

La présence du logo $R \& D$ sur certaines figures de cette section signifie que l'on utilise des hypothèse théoriques (telles que la symétrie $S U(3)$ par exemple) pour obtenir les contraintes montrées sur les figures. Les incertitudes reliées à ces hypothèse théoriques ne sont générallement pas prises en compte, ou ne sont pas sous contrôle.

\subsection{Ingrédients pour les analyses $S U(2)$ et $S U(3)$}

Le tableau 9.1 résume les résultats obtenus dans la partie II, ainsi que les autres mesures de rapports d'embranchement et d'asymétries $C P$ pour les modes $B \rightarrow \rho \pi$ neutres et chargés, $B^{0} \rightarrow K^{*+} \pi^{-}$et $B^{0} \rightarrow \rho^{-} K^{+}$, réalisées par BABAR, Belle et CLEO. Dans les analyses décrites par la suite, nous utilisons la moyenne mondiale de ces mesures. 


\begin{tabular}{|c|c|c|c|c|}
\hline Mesure & $B A B A R$ & Belle & CLEO & Moyenne mondiale \\
\hline $\mathcal{B}^{ \pm \mp}$ & $22.6 \pm 1.8 \pm 2.2[39]$ & $20.8_{-6.3-3.1}^{+6.0}+2.8[46]$ & $27.6_{-7.4}^{+8.4} \pm 4.2[45]$ & $22.8 \pm 2.5$ \\
\hline$A_{C P}$ & $-0.18 \pm 0.08 \pm 0.03[39]$ & - & - & $-0.18 \pm 0.08$ \\
\hline$C_{\rho \pi}$ & $0.36 \pm 0.18 \pm 0.04[39]$ & - & - & $0.36 \pm 0.18$ \\
\hline$\Delta C_{\rho \pi}$ & $0.28_{-0.19}^{+0.18} \pm 0.04[39]$ & - & - & $0.28 \pm 0.19$ \\
\hline$S_{\rho \pi}$ & $0.19 \pm 0.24 \pm 0.03[39]$ & - & - & $0.19 \pm 0.24$ \\
\hline$\Delta S_{\rho \pi}$ & $0.15 \pm 0.25 \pm 0.03[39]$ & - & - & $0.15 \pm 0.25$ \\
\hline$A_{\rho \pi}^{+-}$ & $-0.11_{-0.17}^{+0.16} \pm 0.04[39]$ & - & - & $-0.11_{-0.16}^{+0.17}$ \\
\hline$A_{\rho \pi}^{-+}$ & $-0.62_{-0.28}^{+0.24} \pm 0.06[39]$ & - & - & $-0.62_{-0.29}^{+0.25}$ \\
\hline $\mathcal{B}^{+0}$ & - & - & $<43[45]$ & $?$ \\
\hline$A_{\rho \pi}^{+0}$ & - & 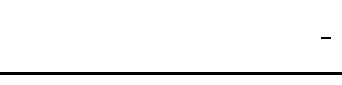 & - & - \\
\hline $\mathcal{B}^{0+}$ & $24 \pm 8 \pm 3[137]$ & $8.0_{-2.0}^{+2.3} \pm 0.7[46]$ & $10.4_{-3.4}^{+3.3} \pm 2.1[45]$ & $9.5 \pm 2.0$ \\
\hline$A_{\rho \pi}^{0+}$ & - & - & - & - \\
\hline $\mathcal{B}^{00}$ & cf. chapitre 5 & $<5.3[46]$ & $<5.5[45]$ & $?$ \\
\hline$C_{\rho \pi}^{00}$ & - & - & - & - \\
\hline$S_{\rho \pi}^{00}$ & - & - & - & - \\
\hline $\mathcal{B}_{\rho K}^{-+}$ & $7.3_{-1.2}^{+1.3} \pm 1.3[39]$ & $11.2_{-5.6}^{+5.9}{ }_{-1.8}^{+1.9}[138]$ & $16.0_{-6.4}^{+7.6} \pm 2.8[45]$ & $8.1 \pm 1.7$ \\
\hline$A_{\rho K}^{-+}$ & $0.28 \pm 0.17 \pm 0.08[39]$ & - & - & $0.28 \pm 0.19$ \\
\hline $\mathcal{B}_{K^{*} \pi}^{+-}$ & & $0_{-3.6}^{+3.9}{ }_{-1.8}^{+2.0}{ }_{-6.1}^{+6.9}[138]$ & $16_{-5}^{+6} \pm 2[139]$ & $16.0_{-4.3}^{+5.0}$ \\
\hline$A_{K^{*} \pi}^{+-}$ & - & & - & - \\
\hline
\end{tabular}

Tableau 9.1: Rapports d'embranchement et asymétries $C P$ et de dilution pour les modes $B \rightarrow \rho \pi$ et $B^{0} \rightarrow K^{*+} \pi^{-}, B^{0} \rightarrow \rho^{-} K^{+}$mesurés par BABAR, Belle et CLEO.

Certains des ingrédients de l'analyse $S U(2)$ n'ont pas encore été mesurés: c'est le cas du rapport d'embranchement et de l'asymétrie $C P$ directe de $B^{+} \rightarrow \rho^{+} \pi^{0}{ }^{1}$, de l'asymétrie $C P$ de $B^{+} \rightarrow \rho^{0} \pi^{+}$, des asymétries $C P$ et du rapport d'embranchement de $\mathrm{B}^{0} \rightarrow \rho^{0} \pi^{0}$ (seule une limite existe pour ce dernier). Nous ne pouvons donc pas à l'heure actuelle réaliser l'analyse d'isospin complète.

\footnotetext{
${ }^{1}$ Il existe une limite de CLEO pour $\mathcal{B}^{+0}\left(B^{+} \rightarrow \rho^{+} \pi^{0}\right)$ [45] bien au delà du rapport d'embranchement attendu, probablement dû à une pollution de l'échantillon par d'autres modes non-charmés souvent sousestimés dans les analyses anciennes.
} 


\subsection{Les pingouins dans $B^{0} \rightarrow \rho^{ \pm} \pi^{\mp}$}

\subsubsection{Hypothèse de pingouins nuls}

Le niveau de confiance d'avoir des amplitudes pingouins nulles étant données les valeurs pour les mesures obtenues dans le tableau 9.1 est de $3.8 \%$, ce qui est faible. Néanmoins, nous nous plaçons ici dans le cas académique où ces amplitudes pingouins sont nulles: ceci nous permet d'évaluer la contrainte qu'on obtiendrait sur $\alpha$ si les erreurs théoriques associées à la contamination des pingouins étaient nulles ( $c$ - ̀̀- $d$ évaluer la contrainte purement statistique sur $\alpha$ de nos mesures).

La figure 9.1 montre la contrainte sur $\alpha$ ainsi obtenue, comparée à la contrainte indirecte obtenue par l'ajustement standard de la matrice CKM ( $c f$. section 8). Nous voyons que l'angle $\alpha$ est déterminé à 8 solutions miroirs prêt entre 0 et $\pi$. Ces solutions ne sont plus qu'au nombre de 2 si on fixe aussi la phase forte relative $\hat{\delta}_{T}$ des diagrammes en arbre $T^{+-}$ et $T^{-+}$.

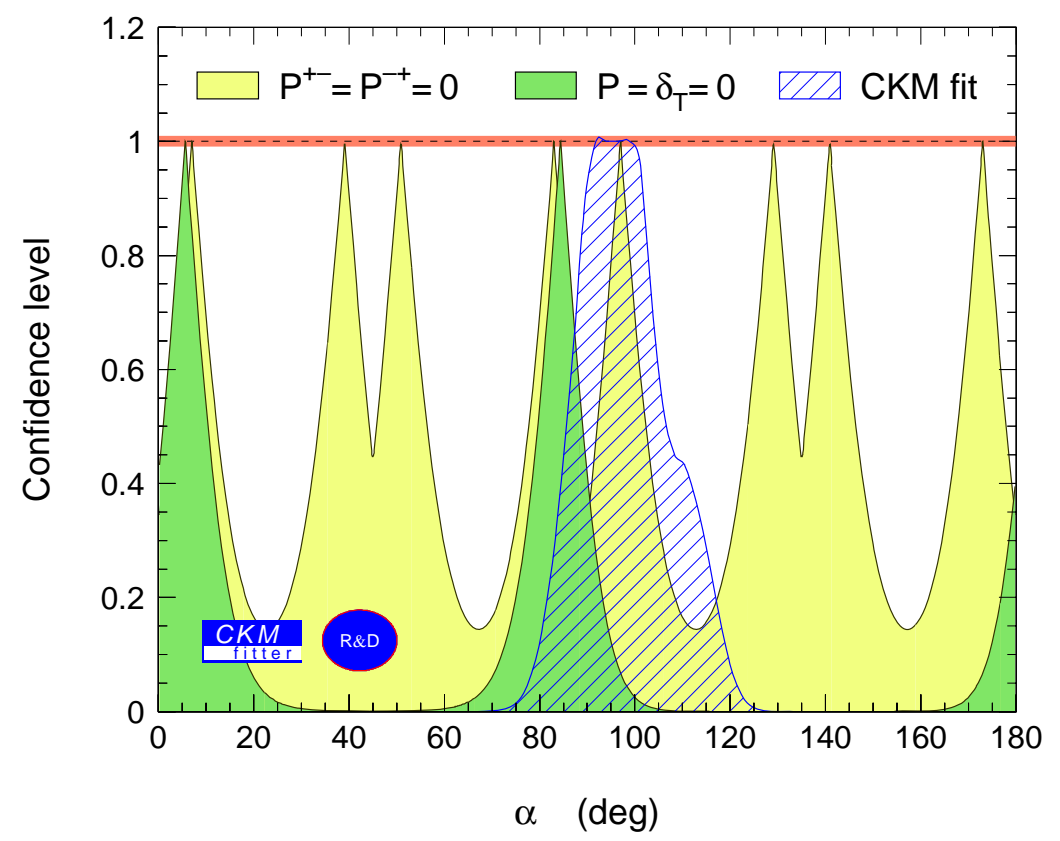

Figure 9.1: La zone claire montre la contrainte sur $\alpha$ obtenue à partir du rapport d'embranchement et des paramètres $C P$ et de dilution de $B^{0} \rightarrow \rho^{ \pm} \pi^{\mp}$, en faisant l'hypothèse que les amplitudes pingouins sont nulles. La zone foncée indique la contrainte obtenue en fixant en plus la phase relative $\hat{\delta}_{T}$ entre les arbres $T^{+-}$et $T^{-+}$à zéro. Enfin, la zone hachurée montre la contrainte sur $\alpha$ obtenue à partir de l'ajustement standard.

On voit qu'en l'absence de pingouins et avec $\hat{\delta}_{T}$ fixé à zéro, $\alpha$ est déterminé avec une erreur de $7^{\circ}$ (à une ambiguïté prêt entre 0 et $\pi$ ), soit une erreur bien inférieure à celle de la détermination indirecte par 1'ajustement standard (qui est de 14º cf. tableau 8.2). En laissant $\hat{\delta}_{T}$ libre, néanmoins, aucune valeur de $\alpha$ ne peut être exclue.

La figure 9.2 montre les niveaux de confiance dans le plan $\hat{\delta}_{T}-\alpha$, montrant l'éloignement des solutions miroirs dans ce plan. 


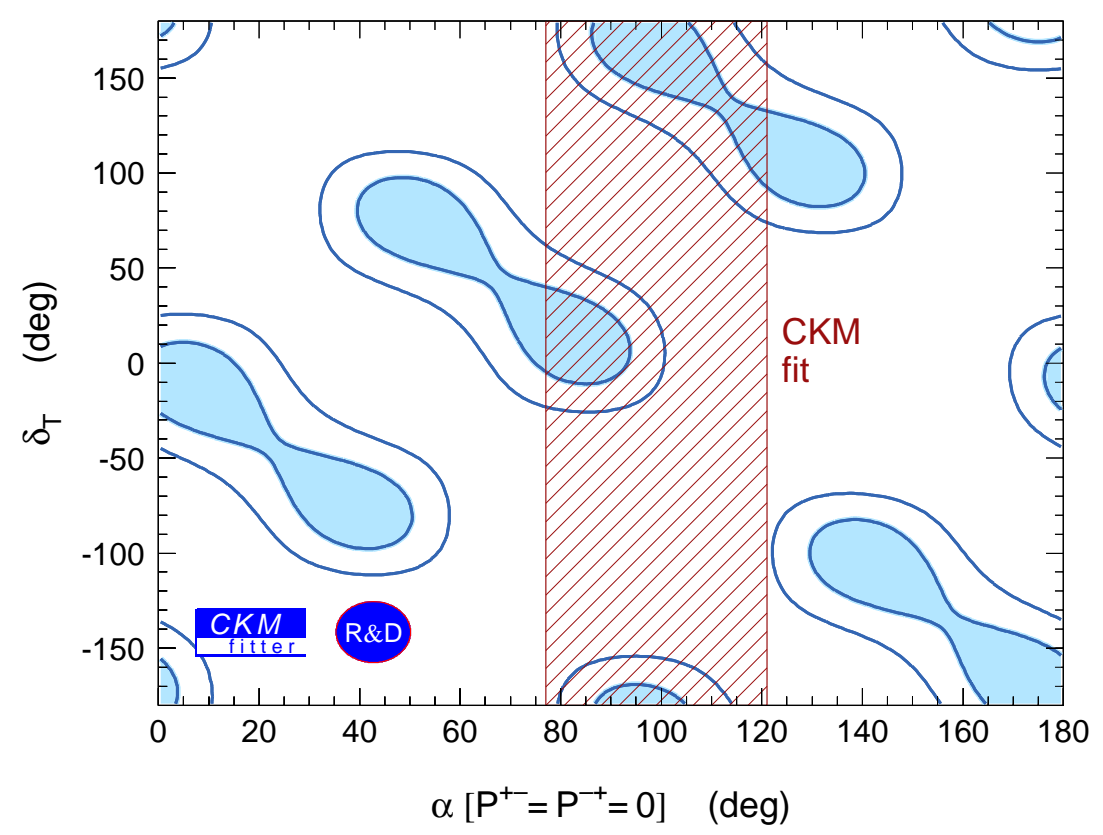

Figure 9.2: Niveaux de conflance dans le plan $\left(\hat{\delta}_{T}-\alpha\right)$ en supposant les pingouins nuls. Les zones ombrées contiennent les points ayant un niveau de confiance de plus de $32 \%$, et les zones blanches cerclées de noir, ceux ayant un niveau de confiance de plus de 5\%. La zone hachurée correspond à la contrainte (>10\%CL) obtenue par l'ajustement standard.

\subsubsection{Contraintes sur les rapports pingouin/arbre utilisant l'ajus- tement standard}

En utilisant l'ajustement standard pour contraindre $\alpha$, on peut déduire des contraintes sur les rapports des amplitudes pingouins et arbre de $B^{0} \rightarrow \rho^{ \pm} \pi^{\mp}$ à partir des mesures reliées à ce mode données dans le tableau 9.1.

La figure 9.3 montre les niveaux de confiance ainsi obtenus dans les plans $\delta_{P T^{+--}}{ }_{P T^{+-}}$ et $\delta_{P T^{+-}-r_{P T^{+-}}}$(ces paramètres sont définis juste après l'eq. 1.72).

Les asymétries $C P$ directe $\left(A_{\rho \pi}^{+-}\right.$et $\left.A_{\rho \pi}^{-+}\right)$étant négatives, les différences de phases fortes obtenues sont positives ( $c f$. eq. 1.71 et 1.72 ). Puisque $A_{\rho \pi}^{-+}$exhibe une violation de $C P$ directe plus importante que $A_{\rho \pi}^{+-}$, les différences de phases fortes reliées aux amplitudes $A^{-+}$et $\bar{A}^{+-}$ sont plus grandes que celles reliées aux deux autres amplitudes.

\subsection{Contraintes sur $\alpha$ utilisant $S U(2)$}

\subsubsection{Limites sur $\left|\alpha-\alpha_{\text {eff }}^{ \pm}\right|$utilisant $S U(2)$}

La section 2.2.5 décrit comment obtenir une limite sur $\left|\alpha-\alpha_{\text {eff }}^{ \pm}\right|$(où $\alpha_{\text {eff }}^{ \pm}$est défini dans l'eq. 2.22) à partir de $\mathcal{B}^{00}, \mathcal{B}^{ \pm}$et $A_{\rho \pi}^{ \pm}$(cf. eq. 2.21).

Nous avons calculé la contrainte sur $\mathcal{B}^{ \pm}$et $A_{\rho \pi}^{ \pm}$apportée par les mesures décrites dans le tableau 9.1. Ces contraintes, montrées sur la figure 9.4, sont très faibles: en effet, l'analyse en deux-corps de $B^{0} \rightarrow \rho^{ \pm} \pi^{\mp}$ n'est pas sensible aux interférences entre $\rho^{+} \pi^{-}$et $\rho^{-} \pi^{+}$entrant dans la définition des deux paramètres $\mathcal{B}^{ \pm}$et $A_{\rho \pi}^{ \pm}$. 

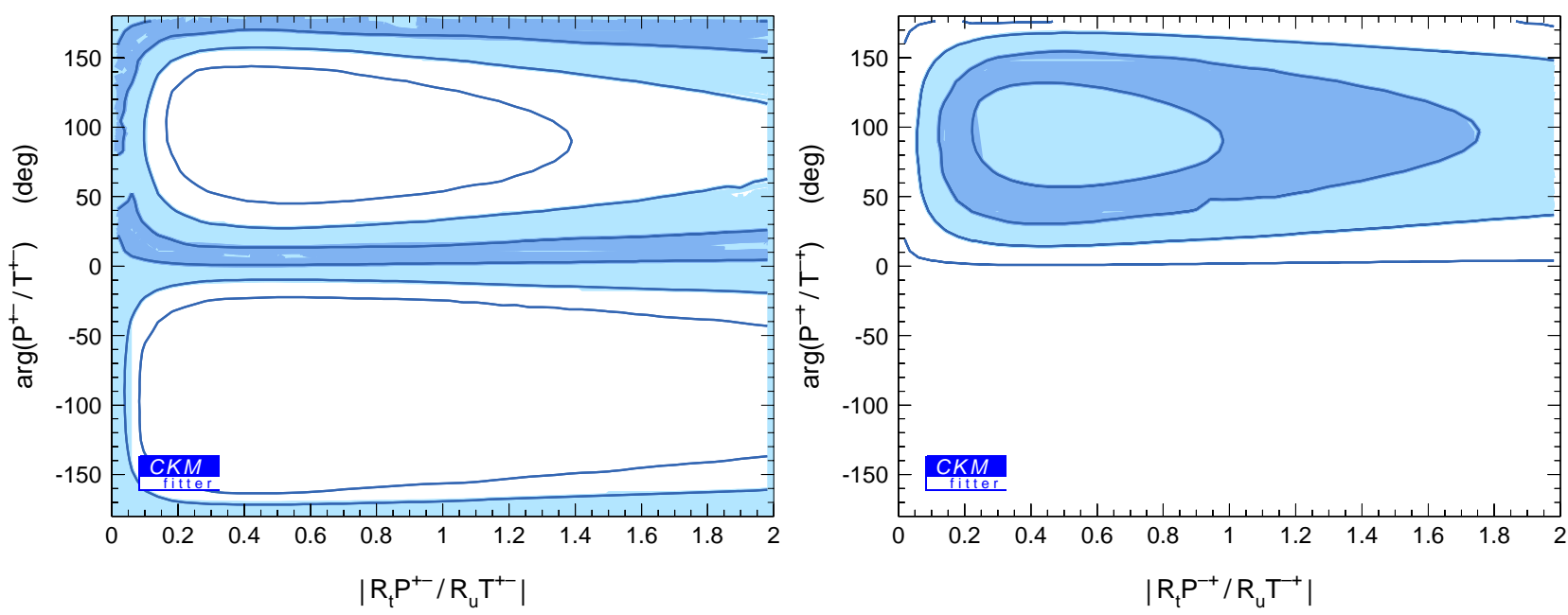

Figure 9.3: Niveaux de confiance dans les plans $\delta_{P T^{+-}-r_{P T^{+-}}}$(gauche) et $\delta_{P T^{+-}-r_{P T^{+-}}}$ (droite) utilisant l'ajustement standard pour déterminer $\alpha$. Les trois niveaux de couleur indiquent (du plus clair au plus foncé) les régions contenant les points avec plus de 5, 32 et $90 \%$ de niveau de confiance.
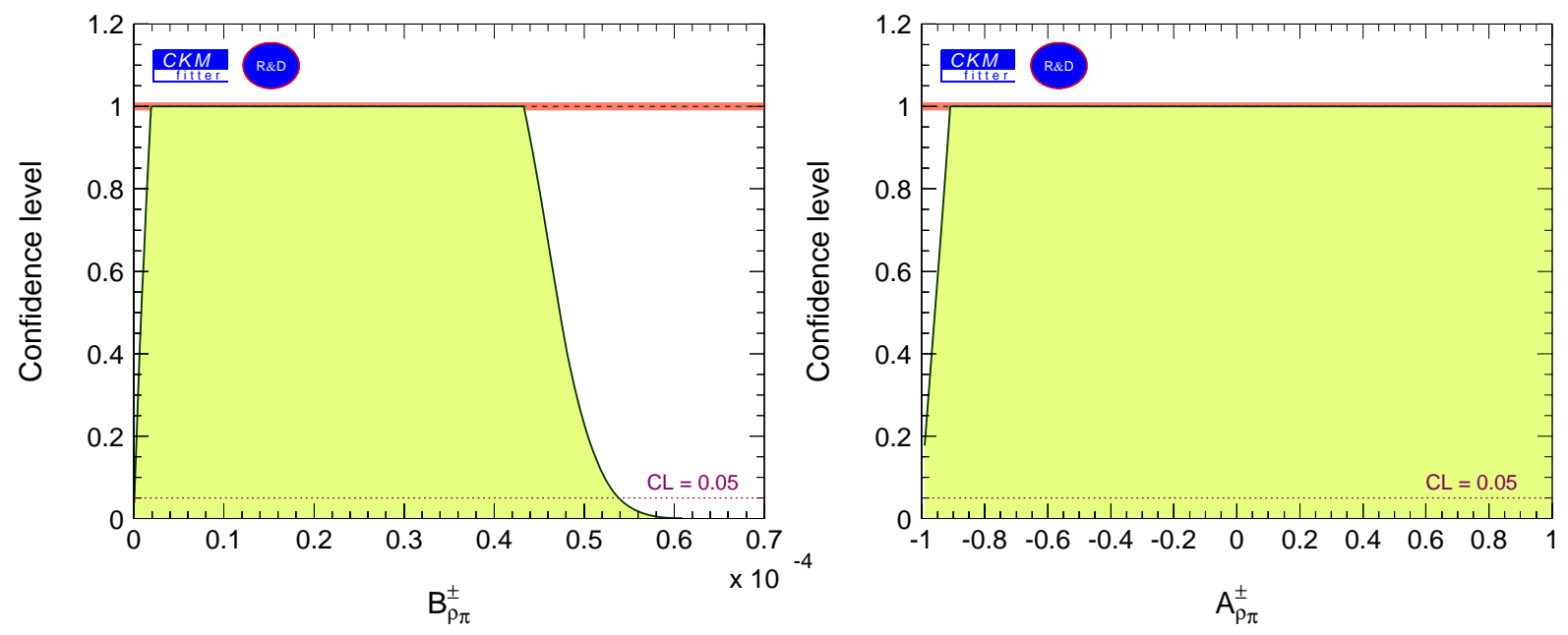

Figure 9.4: Niveaux de confiance pour le rapport d'embranchement $\mathcal{B}^{ \pm}$(gauche) et l'asymétrie $A_{\rho \pi}^{ \pm}$(droite) définis dans les eqs. 2.25 et 2.24, calculés à partir des mesures expérimentales du tableau 9.1. 
L'intervalle

$$
0.15<\mathcal{B}^{ \pm}<52 \cdot 10^{-6}
$$

contient les valeurs de $\mathcal{B}^{ \pm}$ayant un niveau de confiance de plus de $10 \%$ de niveau de confiance. L'intervalle défini de façon similaire pour $A_{\rho \pi}^{ \pm}$couvre toute la région physique.

Nous en déduisons que nous n'avons actuellement aucune sensibilité avec l'analyse en deux-corps de $B^{0} \rightarrow \rho^{ \pm} \pi^{\mp}$ pour obtenir une contrainte sur $\left|\alpha-\alpha_{\text {eff }}^{ \pm}\right|$.

\subsubsection{Extrapolations futures de l'analyse d'isospin complète}

Nous nous proposons dans cette section de réaliser des extrapolations futures de l'analyse d'isospin complète.

Nous choisissons un ensemble arbitraire d'amplitudes arbre et pingouin, ainsi qu'une valeur de l'angle $\alpha$ permettant de reproduire les mesures du tableau 9.1 (il existe une infinité de tels ensembles):

$$
\begin{aligned}
\alpha & =90^{\circ}, & \beta & =23.6^{\circ}, \\
T^{+-} & =0.0051, & T^{-+} & =0.0040 e^{-i 167^{\circ}}, \\
P^{+-} & =0.0013 e^{i 13^{\circ}}, & P^{-+} & =0.0014 e^{-i 78^{\circ}}, \\
T^{00} & =0.00002 e^{-i 79^{\circ}}, & T^{+0} & =0.0038 e^{i 61^{\circ}} .
\end{aligned}
$$

Nous obtenons à partir de ces amplitudes les valeurs suivantes pour les observables:

$$
\begin{aligned}
\mathcal{B}^{ \pm \mp} & =(22.6 \pm 1.0) \cdot 10^{-6}, A_{C P}=-0.18 \pm 0.04, \quad C_{\rho \pi}^{00}=-0.03 \pm 0.35, \\
\mathcal{B}^{00} & =(0.9 \pm 0.3) \cdot 10^{-6}, \quad C_{\rho \pi}=0.36 \pm 0.08, \quad S_{\rho \pi}^{00}=0.03 \pm 0.50, \\
\mathcal{B}^{+0} & =(9.0 \pm 1.0) \cdot 10^{-6}, \quad \Delta C_{\rho \pi}=0.28 \pm 0.08, \quad A_{\rho \pi}^{+0}=0.03 \pm 0.07, \\
\mathcal{B}^{0+} & =(11.0 \pm 0.5) \cdot 10^{-6}, \quad S_{\rho \pi}=0.19 \pm 0.10, \quad A_{\rho \pi}^{0+}=0.29 \pm 0.05, \\
\Delta S_{\rho \pi} & =0.15 \pm 0.11,
\end{aligned}
$$

où les erreurs sont extrapolées pour une luminosité intégrée de $500 \mathrm{fb}^{-1}$.

La figure 9.5 montre la contrainte sur $\alpha$ pour une luminosité intégrée de $500 \mathrm{fb}^{-1}$ ( $B A B A R$ et Belle à l'hiver 2005), $2000 \mathrm{fb}^{-1}$ (à la fin du programme des usines à $B$ de premières génération) et $10000 \mathrm{fb}^{-1}$ (auprès de "super" usines à $B$ de seconde génération). Les erreurs - à la fois statistiques et systématiques - sont optimistiquement supposées s'améliorer par un

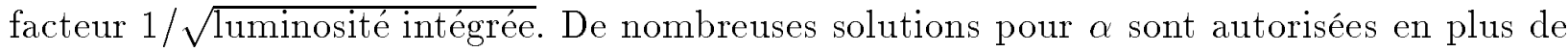
la vraie solution à $\alpha=90^{\circ}$.

La figure 9.6 montre la comparaison de la contrainte sur $\alpha$ pour une luminosité intégrée de $500 \mathrm{fb}^{-1}$ avec ou sans l'utilisation de la contrainte provenant de $\left|V_{u b}\right| /\left|V_{c b}\right|$ donnée par:

$$
\frac{\left|V_{u b}\right|}{\left|V_{c b}\right|}=\frac{\lambda}{\sqrt{\rho^{2}+\eta^{2}}}=\left(\frac{\lambda}{1-\lambda^{2} / 2}\right) \frac{\sin \beta}{\sin \alpha}=0.091 \pm 0.011,
$$

où l'on a utilisé les mesures de $\left|V_{u b}\right|$ et $\left|V_{c b}\right|$ décrites dans la section 8.1 pour obtenir la valeur numérique. On voit que l'utilisation de $\left|V_{u b}\right| /\left|V_{c b}\right|$ sélectionne un sous-ensemble de solutions miroirs sur $\alpha$.

La figure 9.7 montre la contrainte sur $\alpha$ obtenue à partir de l'analyse d'isospin complète pour une luminosité intégrée de $500 \mathrm{fb}^{-1}, 2000 \mathrm{fb}^{-1}$ et $10000 \mathrm{fb}^{-1}$, en faisant l'hypothèse que le rapport d'embranchement de $B^{0} \rightarrow \rho^{0} \pi^{0}$ reste toujours en dessous de la sensibilité 


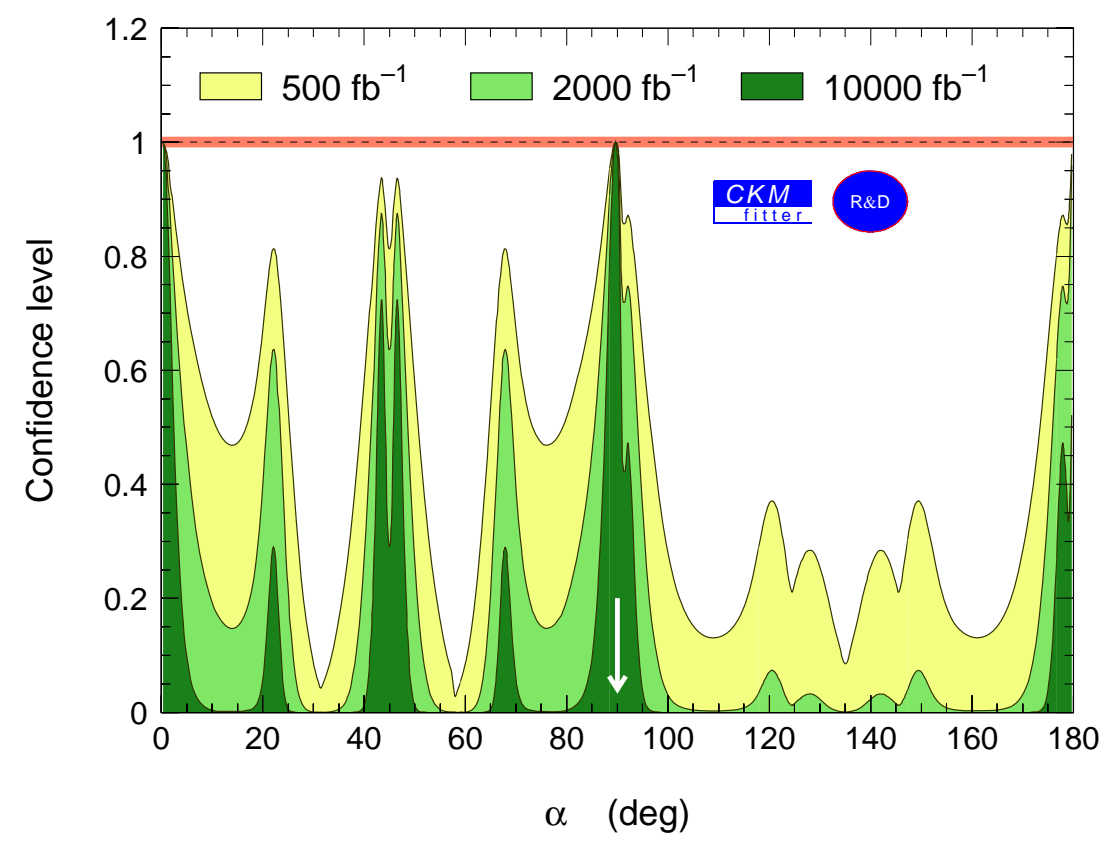

Figure 9.5: Contrainte sur $\alpha$ obtenue à partir de l'analyse d'isospin complète pour une luminosité intégrée de $500 \mathrm{fb}^{-1}, 2000 \mathrm{fb}^{-1}$ et $10000 \mathrm{fb}^{-1}$. La flèche indique la valeur de a générée. Cet exemple particulier d'analyse utilise les amplitudes données dans l'eq. 9.2 permettant de reproduire les observables du tableau 9.1.

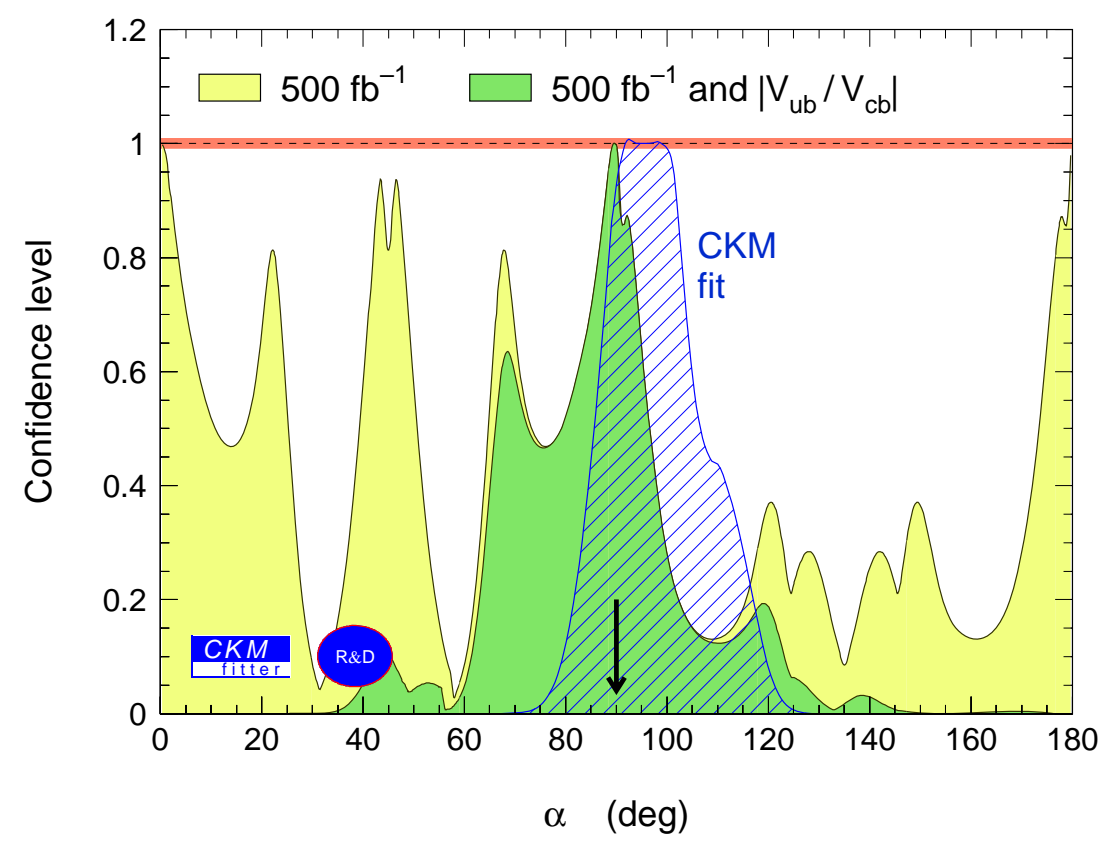

Figure 9.6: Contrainte sur $\alpha$ obtenue à partir de l'analyse d'isospin complète pour une luminosité intégrée de $500 \mathrm{fb}^{-1}$ avec (zone foncée) ou sans (zone claire) l'utilisation de la contrainte provenant de $\left|V_{u b}\right| /\left|V_{c b}\right|$. La contrainte indirecte sur $\alpha$ donnée par l'ajustement standard de la matrice CKM est indiquée par les hachures. La flèche indique la valeur de $\alpha$ générée. 


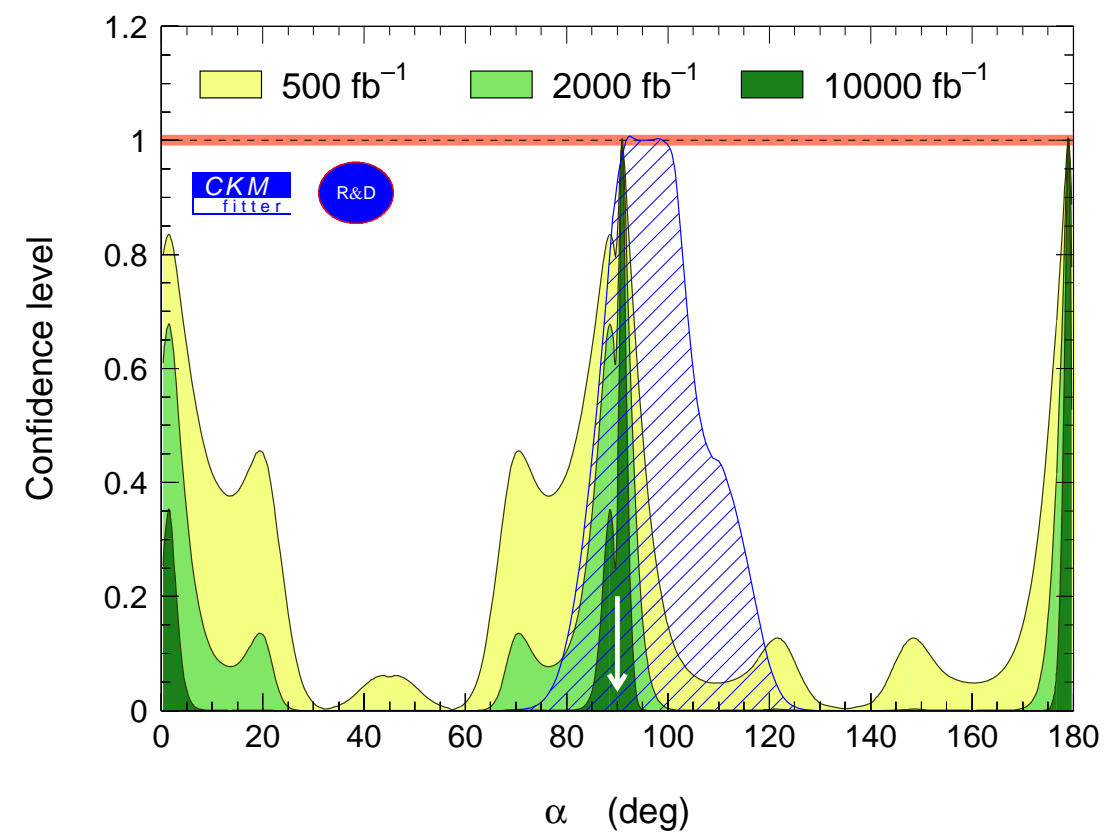

Figure 9.7: Contrainte sur $\alpha$ obtenue à partir de l'analyse d'isospin complète pour une luminosité intégrée de $500 \mathrm{fb}^{-1}, 2000 \mathrm{fb}^{-1}$ et $10000 \mathrm{fb}^{-1}$, en faisant l'hypothèse que le rapport d'embranchement de $B^{0} \rightarrow \rho^{0} \pi^{0}$ reste toujours en dessous de la sensibilité expérimentale: la contrainte apportée par l'eq. 2.21 est alors importante. La contrainte indirecte sur $\alpha$ donnée par l'ajustement standard de la matrice CKM (cf. chapitre 8) est indiquée par les hachures. La flèche indique la valeur de a générée.

expérimentale: dans la configuration étudiée, la contrainte apportée par l'eq. 2.21 est alors plus importante.

Ainsi, dans la configuration étudiée, à moins que le rapport d'embranchement de $B^{0} \rightarrow$ $\rho^{0} \pi^{0}$ ne reste en dessous de la sensibilité expérimentale, l'analyse $S U(2)$ ne permet pas d'obtenir des contraintes significatives sur $\alpha$ avec la statistique accumulée aux usines à $B$ de première génération. La généralisation de ce résultat pour toutes les configurations possibles est délicate: il est probablement possible de trouver des configurations menant à une conclusion opposée à celle exposée ci-dessus. Nous nous gardons donc de généraliser cette conclusion au delà du cas étudié ici.

\subsection{Contraintes sur $\alpha$ utilisant $S U(3)$}

\subsubsection{Contraintes actuelles}

Les eqs. 2.29 et 2.30 permettent de déduire des contraintes sur $\left|\alpha-\alpha_{\text {eff }}^{+-}\right|$et $\left|\alpha-\alpha_{\text {eff }}^{-+}\right|$en utilisant les paramètres $\mathcal{B}_{K^{*} \pi}^{+-}, \mathcal{B}_{\rho K}^{-+}, \mathcal{B}^{+-}, \mathcal{B}^{-+}$et les asymétries $A_{\rho \pi}^{+-}$et $A_{\rho \pi}^{-+}$(cf. section 1.5.3 et chapitre 2).

A partir des mesures reliées à $B^{0} \rightarrow \rho^{ \pm} \pi^{\mp}$ données dans le tableau 9.1, nous calculons $\mathcal{B}^{+-}, \mathcal{B}^{-+}$:

$$
\mathcal{B}^{+-}=\frac{1}{2}\left(1+\Delta C_{\rho \pi}+A_{C P} C_{\rho \pi}\right)=(13.9 \pm 2.7) \cdot 10^{-6},
$$




$$
\mathcal{B}^{-+}=\frac{1}{2}\left(1-\Delta C_{\rho \pi}-A_{C P} C_{\rho \pi}\right)=(8.9 \pm 2.5) \cdot 10^{-6},
$$

où la corrélation entre ces deux paramètres est de $-39 \%$, et où les erreurs incluent les incertitudes statistiques et systématiques. Les niveaux de confiance dans le plan $\mathcal{B}^{-+} \mathcal{B}^{+-}$ sont montrés sur la figure 9.8 à gauche.
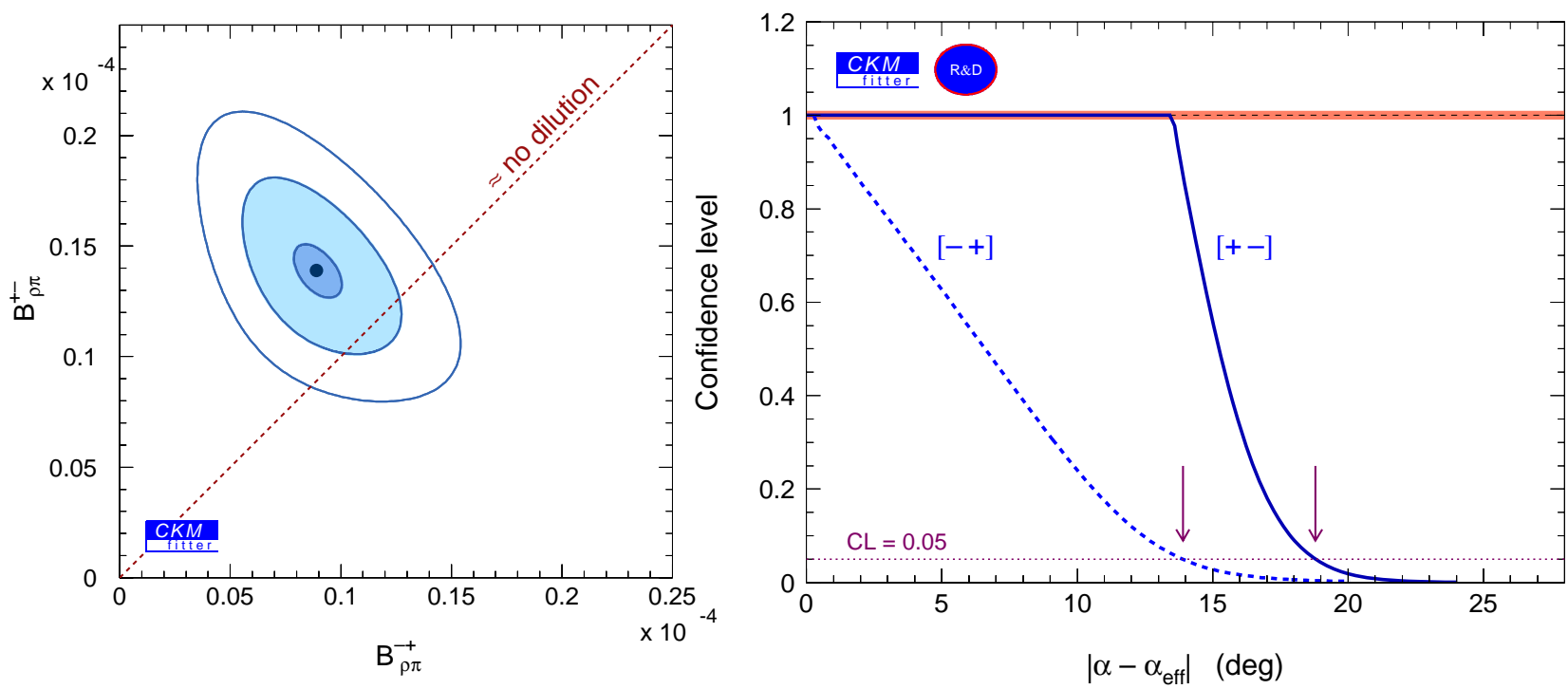

Figure 9.8: Figure de gauche: niveaux de confiance dans le plan $\mathcal{B}^{-+}-\mathcal{B}^{+-}$. Les trois niveaux de couleurs correspondent (du plus clair au plus foncé) aux régions ayant des points à plus de 5, 32 et $90 \%$ de niveau de confiance. La line pointillée diagonale correspond à une dilution minimale, soit $\Delta C=0$. Figure de droite: niveaux de confiance pour les limites sur $\left|\alpha-\alpha_{\mathrm{eff}}\right|$ des eqs. 2.29 et 2.30 utilisant les mesures du tableau 9.1.

Utilisant les mesures du tableau 9.1, on obtient les niveaux de confiance pour $\left|\alpha-\alpha_{\text {eff }}\right|$ tels que montrés sur la figure 9.8 à droite. La contrainte due à $\mathcal{B}_{\rho K}^{-+}$sur $\left|\alpha-\alpha_{\text {eff }}^{-+}\right|$est meilleure que celle due à $\mathcal{B}_{K^{*} \pi}^{+-}$sur $\left|\alpha-\alpha_{\text {eff }}^{+-}\right|$, puisque son rapport d'embranchement est plus petit.

Nous obtenons les intervalles suivants:

$$
\left|\alpha-\alpha_{\text {eff }}^{+-}\right|<18.8^{\circ}, \quad\left|\alpha-\alpha_{\text {eff }}^{-+}\right|<13.9^{\circ},
$$

contenant les valeurs ayant un niveau de confiance de plus de $5 \%$.

La figure 9.9 à gauche montre les contraintes sur $\alpha$ utilisant la symétrie $S U(3)$ : la région claire est obtenue en laissant toutes les inconnues libres de flotter dans l'ajustement, et la région foncée, en supposant que la phase relative entre $A^{+-}$et $A^{-+}, \hat{\delta}$, est connue et vaut zéro ( $\hat{\delta}$ est défini juste après l'eq. 1.76). On voit que lorsque toutes les inconnues sont libres, la contrainte permet tout juste de résoudre les huit solutions miroirs.

La figure 9.9 à droite montre les niveaux de confiance dans le plan $\hat{\delta}-\alpha$ En utilisant la contrainte sur $\alpha$ provenant de l'ajustement standard, nous déterminons les contraintes suivantes sur $\hat{\delta}(\mathrm{CL}>0.05)$, montrée sur la figure 9.10 à gauche:

$$
\hat{\delta} \in\left[-180^{\circ} \text { à }-87^{\circ}\right],\left[-55^{\circ} \text { à } 30^{\circ}\right],\left[148^{\circ} \text { à } 180^{\circ}\right] \text {. }
$$

On conclut que les valeurs $\hat{\delta}=0, \pi$ sont privilégiées: ce résultat est en conformité avec l'hypothèse de factorisation naïve. 

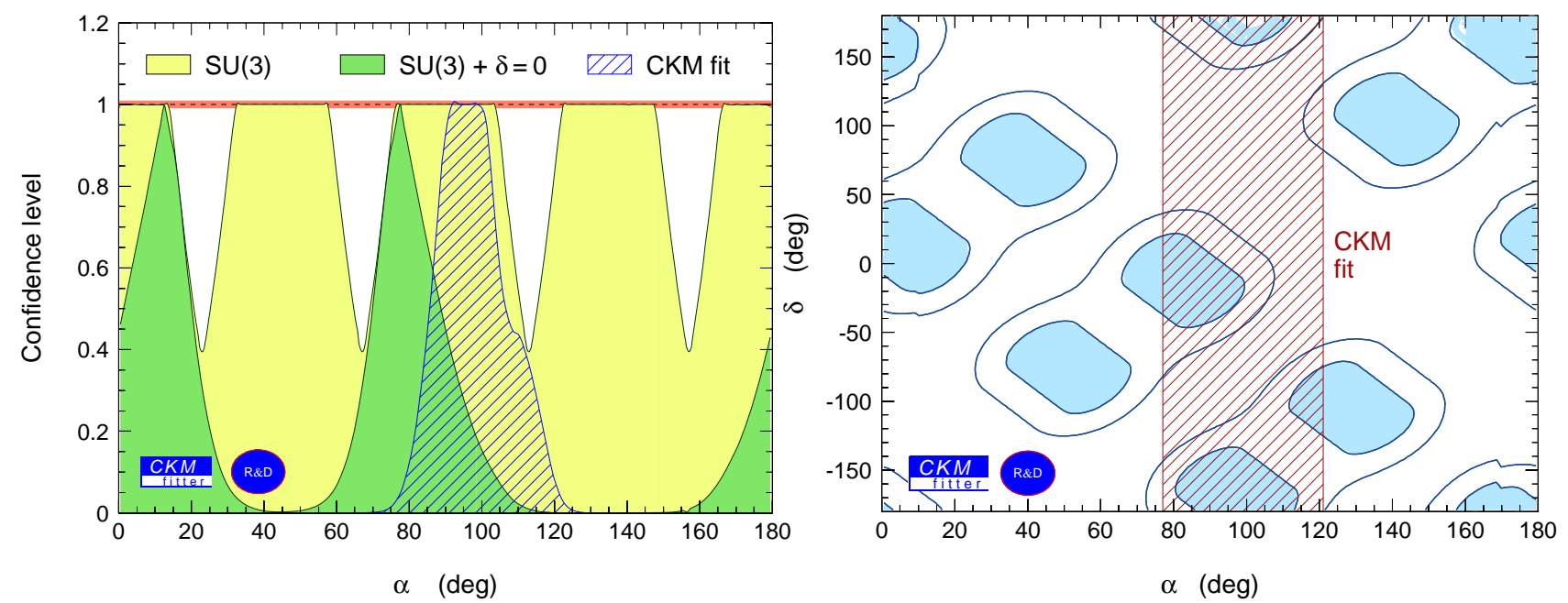

Figure 9.9: Figure de gauche: contraintes sur $\alpha$ obtenues en utilisant la symétrie SU(3). La région claire est obtenue en laissant toutes les inconnues libres de flotter dans l'ajustement, et la région foncée, en supposant que la phase forte relative $\hat{\delta}$ est connue et vaut zéro ( $\hat{\delta}$ est défini juste après l'eq. 1.76). La région hachurée correspond à la contraintre obtenue à partir de l'ajustement standard. Figure de droite: niveaux de confiance dans le plan $\hat{\delta}-\alpha$. La zone hachurée correspond à la contrainte provenant de l'ajustement standard (CL > 10\%).

Pour obtenir une grande asymétrie de $C P$ directe (visible dans les paramètres $A_{\rho \pi}^{+-}$et $\left.A_{\rho \pi}^{-+}\right)$, il faut une contribution significative des diagrammes pingouins. On inverse maintenant l'utilisation des limites 2.29 et 2.30 pour prédire les rapports d'embranchement $\mathcal{B}_{K^{*} \pi}^{+-}$et $\mathcal{B}_{\rho K}^{-+}$ à partir des asymétries $A_{\rho \pi}^{+-}$et $A_{\rho \pi}^{-+}$et autres mesures entrant dans ces limites. Le résultat est montré sur la figure 9.10 à droite. Comme on s'y attend, la grande valeur d'asymétrie $A_{\rho \pi}^{-+}$ entraîne la nécessité d'avoir une grande valeur du rapport d'embranchement de $B^{0} \rightarrow \rho^{-} K^{+}$. La mesure actuelle de ce rapport d'embranchement est en accord avec ces prédictions à seulement $17 \%$ niveau de confiance. Il sera intéressant de voir l'évolution de ce désaccord dans le futur.

Enfin, la figure 9.11 montre les contraintes sur les asymétries de $C P$ directes $A_{\rho \pi}^{+-}$ver-

sus $A_{\rho \pi}^{-+}$obtenues à partir des mesures des rapports d'embranchement et de la symétrie $S U(3)$ seulement. Puisque les rapports d'embranchement ne sont pas sensibles au signe de la violation de $C P$ directe, la contrainte est symétrique autour de zéro.

Nous excluons à $90 \%$ de niveau de confiance les domaines suivants:

$$
\left(\left|A_{\rho \pi}^{+-}\right|-0.33\right)\left(\left|A_{\rho \pi}^{-+}\right|-0.23\right)>\text { const },
$$

où const $\simeq 76$.

\subsubsection{Contraintes futures}

Nous nous intéressons maintenant aux contraintes futures sur $\alpha$ utilisant la symétrie $S U(3)$. La figure 9.12 montre les contraintes obtenues pour la luminosité intégrée actuelle de $82 \mathrm{fb}^{-1}$ et des luminosités intégrées futures de $500 \mathrm{fb}^{-1}, 2000 \mathrm{fb}^{-1}$ et $10000 \mathrm{fb}^{-1}$ en mettant les amplitudes pingouins à zéro: on voit que les pics obtenus peuvent être résolus à partir d'une luminosité intégrée de $2000 \mathrm{fb}^{-1}$.

En laissant les contributions pingouins libres dans l'ajustement, on obtient les contraintes montrées sur la figure 9.13: la largeur des plateaux $\left|\alpha-\alpha_{\text {eff }}^{+-}{ }^{-+}\right|$reste la même puisque les 

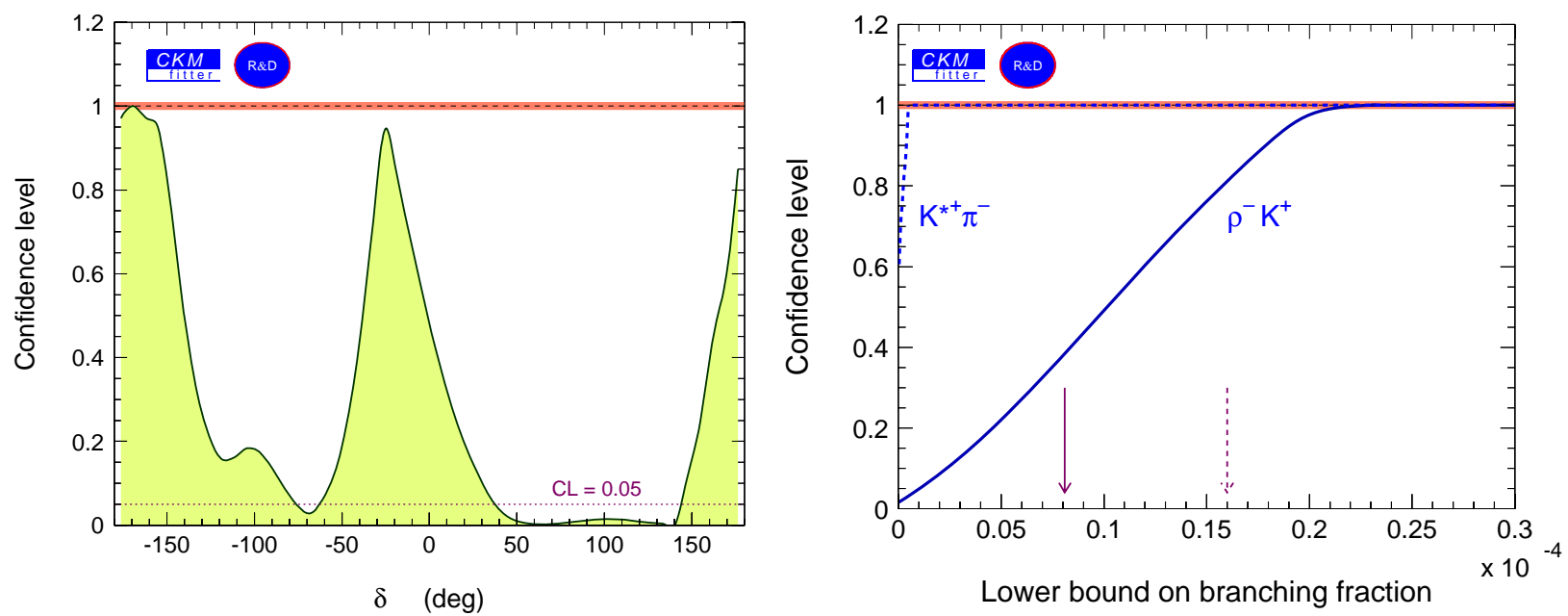

Figure 9.10: Figure de gauche: niveaux de confance pour la phase relative $\hat{\delta}$ obtenue à partir de l'analyse SU(3) et en prenant $\alpha$ de l'ajustement standard. Figure de droite: limites inférieures sur les rapports d'embranchement $\mathcal{B}_{K^{*} \pi}^{+-}$(ligne pointillée) et $\mathcal{B}_{\rho K}^{-+}$(ligne pleine) obtenues en utilisant la symétrie $S U(3)$ et en prenant $\alpha$ de l'ajustement standard. Les flèches indiquent les valeurs expérimentales.

valeurs centrales des limites ne changent pas, mais les pentes des plateaux augement, et les vallées se creusent quand la statistique augmente.

\subsection{Conclusion}

Nous avons interprété les résultats obtenus dans cette thèse sur $B^{0} \rightarrow \rho^{ \pm} \pi^{\mp}$ et $B^{0} \rightarrow \rho^{-} K^{+}$ en utilisant les symétries de saveur $S U(2)$ et $S U(3)$. Le but des différentes approches exposées est de contraindre les amplitudes pingouins dans l'amplitude de $B^{0} \rightarrow \rho^{ \pm} \pi^{\mp}$. Les données actuelles excluent l'hypothèse que les diagrammes pingouins soitent nuls à $96 \%$ de niveau de confiance.

Les conclusions des analyses présentées ici sont les suivantes:

- dans la configuration étudiée ici, l'analyse d'isospin $S U(2)$ ne permet pas de contraindre $\alpha$ significativement avec la statistique qui sera accumulé auprès des usines à $B$ de première génération $\left(\simeq 2000 \mathrm{fb}^{-1}\right.$ accumulés par $B A B A R$ et Belle en 2005) si le rapport d'embranchement de $B^{0} \rightarrow \rho^{0} \pi^{0}$ est accessible expérimentalement. Si ce dernier reste inaccessible, alors des contraintes significatives peuvent être obtenues sur $\alpha$. Ce résultat ne peux néanmoins pas être facilement généralisé à toutes les configurations possibles.

- puisque les modes $B^{0} \rightarrow \rho^{-} K^{+}$et $B^{\rightarrow} K^{*+} \pi^{-}$sont plus sensibles aux diagrammes pingouins que $B^{0} \rightarrow \rho^{0} \pi^{0}$, les contraintes sur $\alpha$ utilisant la symétrie $S U(3)$ sont meilleures que celles obtenus par $S U(2)$. Il existe un désaccord entre la petite valeur du rapport d'embranchement de $B^{0} \rightarrow \rho^{-} K^{+}$(suggérant des amplitudes pingouins faibles) et la grande valeur des asymétries $C P$ directes (suggérant des amplitudes pingouins importantes). Il sera intéressant de voir l'évolution de ce désaccord avec plus de statistique.

- en utilisant la symétrie $S U(3)$, nous obtenons les limites $\left|\alpha-\alpha_{\text {eff }}^{+-}\right|<18.8^{\circ}$ et $\mid \alpha-$ $\alpha_{\text {eff }}^{-+} \mid<13.5^{\circ}$ avec $C L>0.05$. 


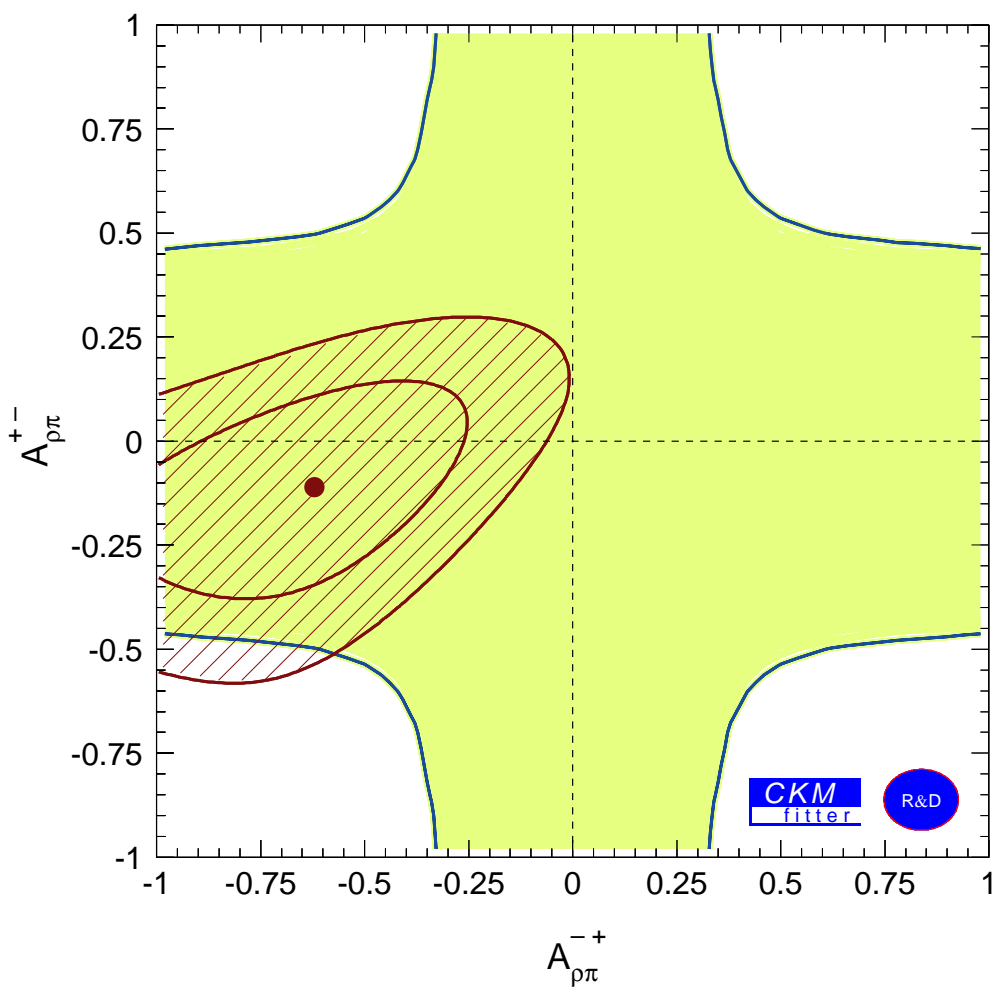

Figure 9.11: Contraintes sur les asymétries de CP directes $A_{\rho \pi}^{+-}$versus $A_{\rho \pi}^{-+}$obtenues à partir des mesures des rapports d'embranchement et de la symétrie SU(3) seulement. La région hachurée correspond aux contours à 1 et $2 \sigma$ des mesures expérimentales (cf. tableau 9.1).

- l'utilisation de $S U(3)$ et de la contrainte sur $\alpha$ obtenue par l'ajustement standard permet de privilégier de faibles phases relatives entre les amplitudes $A^{+-}$et $A^{-+}$. 


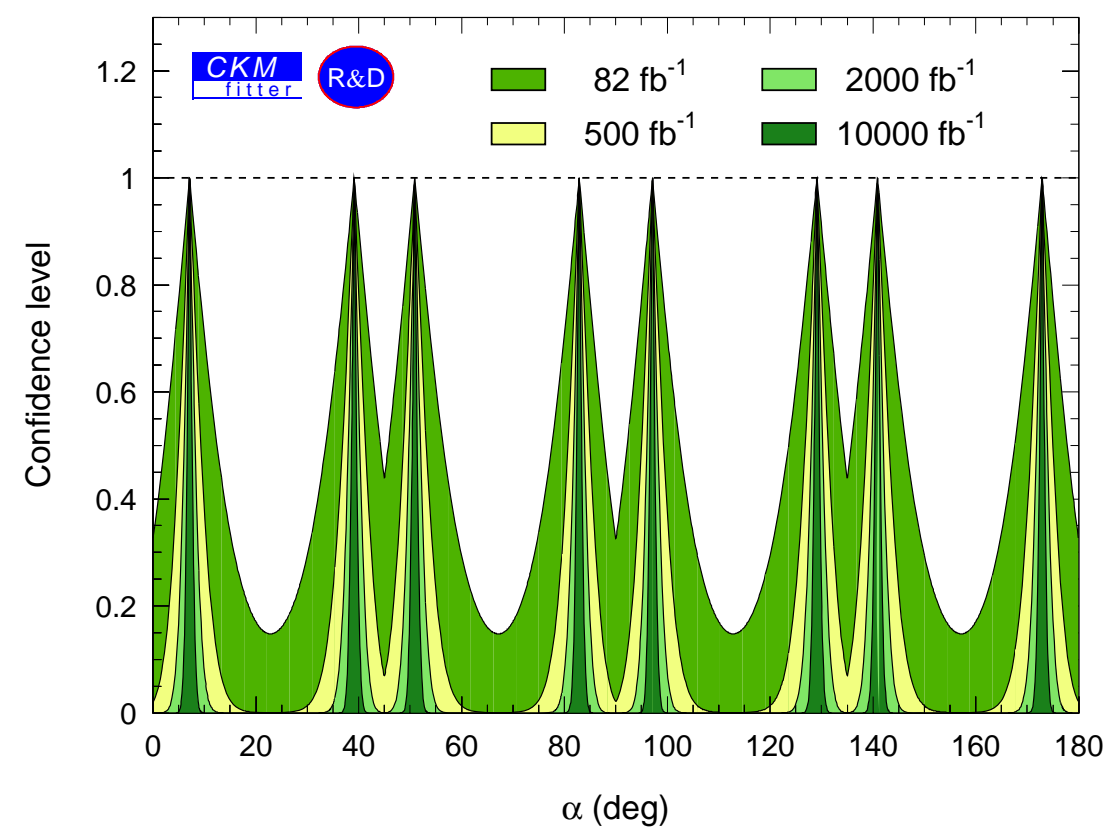

Figure 9.12: Contraintes sur $\alpha$ pour des scénarii futurs, dans une configuration de pingouins nuls.

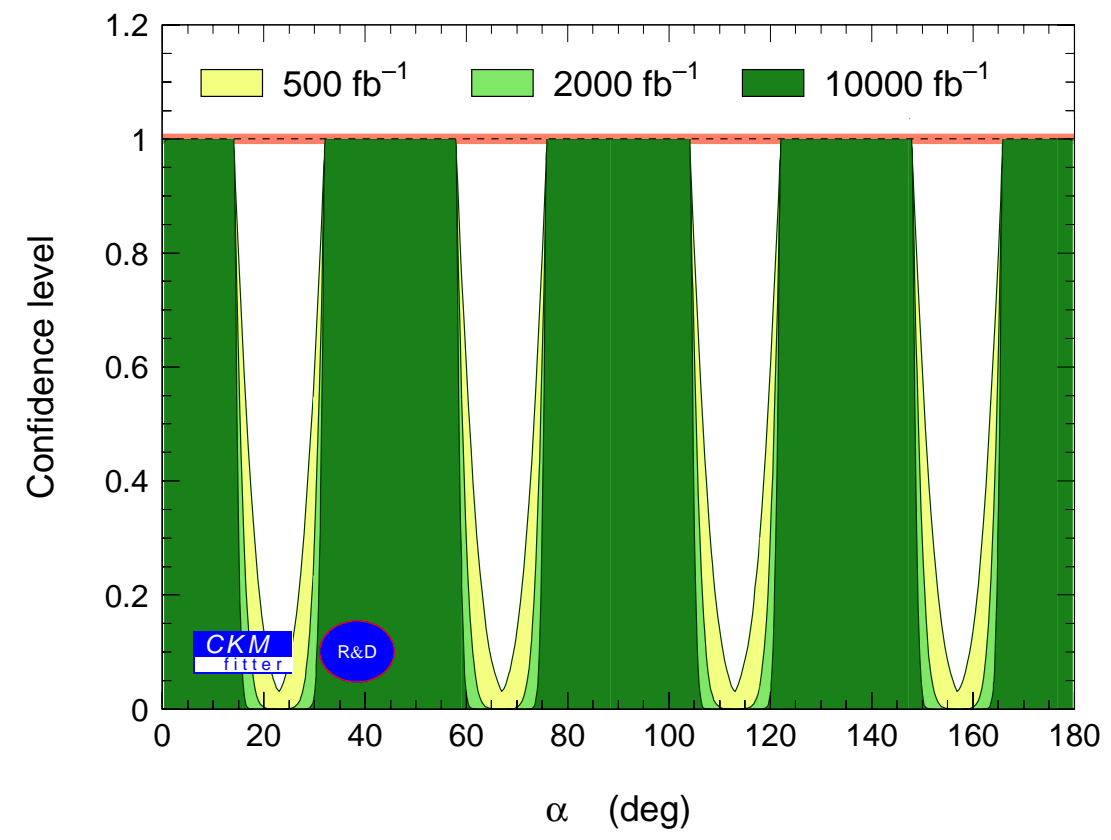

Figure 9.13: Contraintes sur $\alpha$ pour des scénarii futurs, en utilisant la symétrie $S U(3)$ pour contrôler les amplitudes pingouins. . 


\section{Chapitre 10}

\section{La matrice CKM au delà du Modèle Standard}

\subsection{Introduction}

Dans ce dernier chapitre, nous sortons du cadre du Modèle Standard pour nous intéresser à des modèles de nouvelle physique et voir comment une analyse globale de la matrice CKM permet de contraindre de tels modèles. Pour illustrer ce propos, nous nous concentrons sur l'exemple de l'asymétrie semi-leptonique, $A_{\mathrm{SL}}$.

Cette observable, définie dans l'éq. 1.39, permet de mesurer la violation de $C P$ dans le mélange $B^{0}-\bar{B}^{0}$, qui dépend de la phase relative entre les parties absorptives et dispersives de l'amplitude de mélange de l'éq. 1.23 [164]:

$$
A_{\mathrm{SL}}=\operatorname{Im}\left(\Gamma_{12} / M_{12}\right) \text {. }
$$

Dans le Modèle Standard, cette asymétrie est très petite, à cause de deux facteurs de suppression: d'abord, la magnitude du rapport est petite, $\left|\Gamma_{12} / M_{12}\right|=\mathcal{O}\left(m_{b}^{2} / m_{t}^{2}\right) \ll 1$, et ensuite, la phase est faible, $\arg \left(\Gamma_{12} / M_{12}\right)=\mathcal{O}\left(m_{c}^{2} / m_{b}^{2}\right) \ll 1$. Le premier facteur de suppression est indépendant de modèle, puisque, d'une part, on s'attend à ce que les contributions de physique au delà du Modèle Standard à $\Gamma_{12}$ soient petites (car $\Gamma_{12}$ correspond à la partie absorptive mettant en jeu des états intermédiaires réels), et d'autre part $\left|M_{12}\right|$ est mesuré par $\Delta m_{d}$. Par contre, le deuxième facteur de suppression peut facilement être influencé par la nouvelle physique, augmentant ainsi $A_{\mathrm{SL}}$ par un facteur $\mathcal{O}(10)$. L'observable $A_{\mathrm{SL}}$ est donc une variable sensible à la nouvelle physique.

La moyenne mondiale des mesures de $A_{\mathrm{SL}}$ vaut $[165,166,167,168]$ :

$$
A_{\mathrm{SL}}^{\exp }=(0.2 \pm 1.4) \times 10^{-2} \text {. }
$$

Avec une précision expérimentale de $1 \%$, ces mesures permettent de contraindre des contributions de nouvelle physique sur le mélange $B^{0}-\bar{B}^{0}$. Nous nous plaçons dans des modèles de nouvelle physique générique, ainsi que dans la classe de modèles dite de "violation de saveur minimale". 


\subsection{Prédiction de $A_{\mathrm{SL}}$ dans le Modèle Standard}

La prédiction de $A_{\mathrm{SL}}$ au sein du Modèle Standard est obtenue à partir de la référence [105]. A l'ordre dominant, elle est donnée par:

$$
A_{\mathrm{SL}}^{0}=-\kappa \operatorname{Im}\left(\frac{V_{c b} V_{c d}^{*}}{V_{t b} V_{t d}^{*}}\right)
$$

où

$$
\kappa=4 \pi \frac{m_{b}^{2}}{m_{W}^{2}} \frac{K_{1}+K_{2}}{\bar{\eta}_{B} S_{0}\left(m_{t}^{2} / m_{W}^{2}\right)} z, \quad z \equiv m_{c}^{2} / m_{b}^{2} .
$$

Dans cette expression, $K_{1}$ et $K_{2}$ sont des coefficients de Wilson, $\bar{\eta}_{B}$ est un facteur de correction QCD, et $S_{0}$ est la fonction d'Inami-Lim [130] (cf. Eq. 8.8). La dépendance en les paramètres CKM peut s'exprimer en fonction des paramètres $\bar{\rho}$ et $\bar{\eta}$ de la façon suivante:

$$
\operatorname{Im}\left(\frac{V_{c b} V_{c d}^{*}}{V_{t b} V_{t d}^{*}}\right)=\frac{\bar{\eta}}{(1-\bar{\rho})^{2}+\bar{\eta}^{2}} .
$$

Dans la référence [105], les corrections à l'ordre dominant sont calculées en fonction des petits paramètres $z, 1 / m_{b}, \alpha_{S}$ :

$$
A_{\mathrm{SL}}^{\mathrm{SM}}=A_{\mathrm{SL}}^{0}\left(1+a_{\mathrm{SL}}^{z}+a_{\mathrm{SL}}^{1 / m_{b}}+a_{\mathrm{SL}}^{\alpha_{s}}\right)
$$

En utilisant les ingrédients de la référence [105], on obtient la contrainte sur $A_{\mathrm{SL}}$ montrée sur la figure 10.1 , soit l'intervalle de $C L>10 \%$ suivant:

$$
-1.28 \times 10^{-3}<A_{\mathrm{SL}}<-0.48 \times 10^{-3} .
$$

Le modèle Standard prédit donc $A_{\mathrm{SL}}<0$ et de l'ordre de $10^{-3}$. L'erreur est dominée par la méconnaissance des corrections radiatives NLO (Next to Leading Order) et la masse du quark $b$.

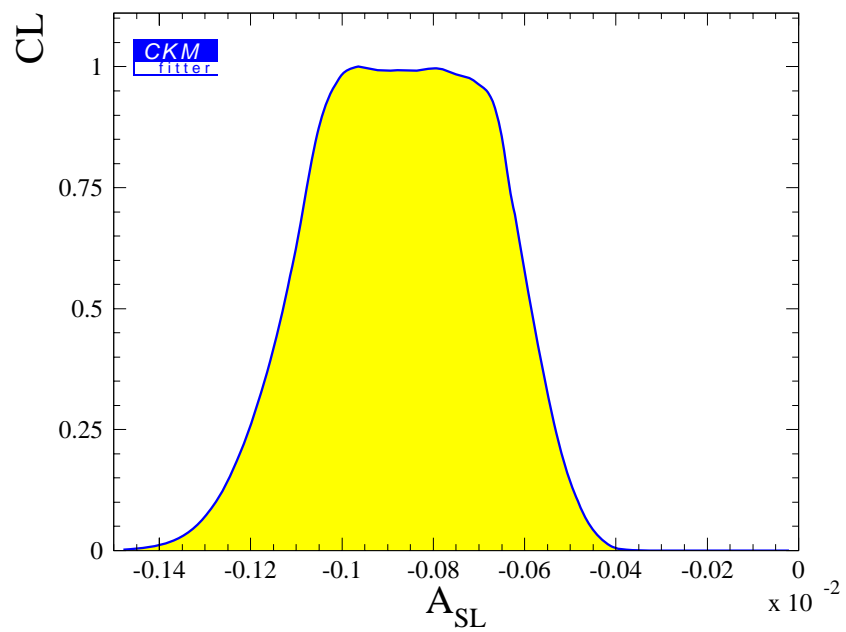

Figure 10.1: Niveau de confiance pour $A_{\mathrm{SL}}$ dans le Modèle Standard.

Les contraintes dans le plan $\bar{\rho}-\bar{\eta}$ pour la valeur actuelle de $A_{\mathrm{SL}}$ et une valeur future de $A_{\mathrm{SL}}=(-1 \pm 3) \times 10^{-3}$ sont montrées sur la figure 10.2. La valeur centrale future est choisie pour être en accord avec la contrainte de l'ajustement standard, et l'erreur correspond à celle obtenue avec la statistique intégrée en 2005 par BABAR et Belle. On voit que dans les prochaines années, $A_{\mathrm{SL}}$ ne fournira pas une contrainte utile dans le plan $\bar{\rho}-\bar{\eta}$ dans le contexte du Modèle Standard si le résultat demeure en accord avec la prédiction de ce dernier. 

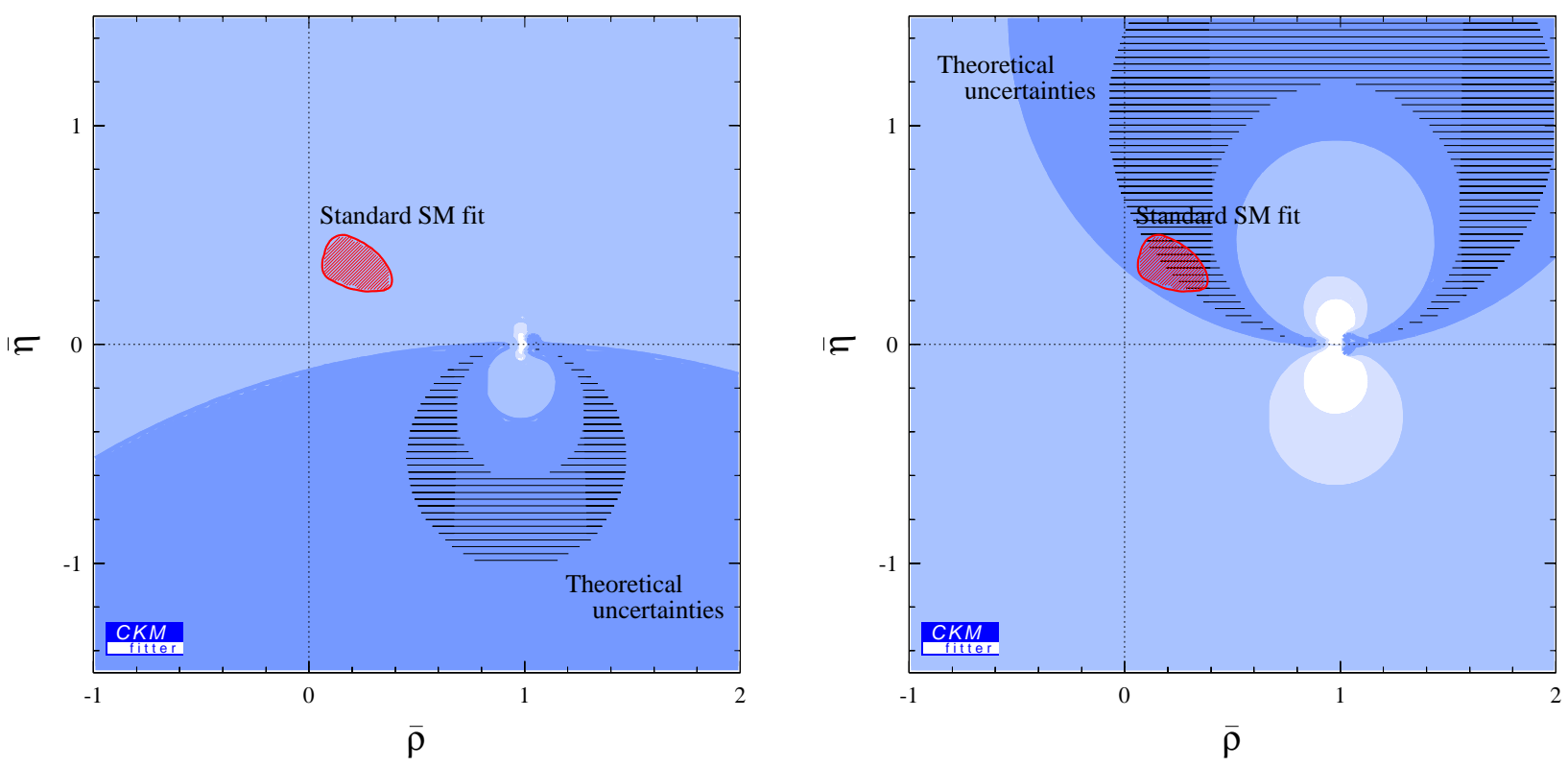

Figure 10.2: Contraintes dans le plan $\bar{\rho}-\bar{\eta}$ obtenues avec les mesures actuelles de $A_{\mathrm{SL}}$ dont la moyenne est donnée dans l'éq. 10.2 (figure de gauche), et avec une valeur hypothétique $A_{\mathrm{SL}}=(-1 \pm 3) \times 10^{-3}$ (figure de droite) dont la valeur centrale est choisie pour être en accord avec la contrainte de l'ajustement standard, et dont l'erreur correspond la statistique intégrée en 2005 par BABAR et Belle. Les zones claires, moyennes et foncées contiennent les points avec plus de $10 \%, 32 \%$ et $90 \%$ respectivement. Les hachures indiquent la contribution des erreurs théoriques.

\section{3 $A_{\mathrm{SL}}$ dans les modèles de nouvelle physique}

\subsubsection{Modèle de nouvelle physique dans le mélange $B^{0}-\bar{B}^{0}$}

On s'intéresse à un modèle générique de nouvelle physique dans le mélange $B^{0}-\bar{B}^{0}$ possédant les propriétés suivantes:

- la matrice CKM $3 \times 3$ est unitaire.

- Les processus en arbre sont dominés par le Modèle Standard. Ceci implique $\Gamma_{12}=\Gamma_{12}^{\mathrm{SM}}$.

La nouvelle physique entre alors seulement dans la matrice de masse $M_{12}$. Dans le système des mésons $B_{d}{ }^{1}$, on peut paramétrer un tel modèle par deux nouveaux paramètres $\theta_{d}$ et $r_{d}$ définis de la manière suivante:

$$
M_{12}=r_{d}^{2} e^{2 i \theta_{d}} M_{12}^{\mathrm{SM}} .
$$

Cette modification entraîne des modifications dans les observables standard liées au mélange $B^{0}-\bar{B}^{0}: \Delta m_{d}$ et l'asymétrie donnant lieu à la mesure de $\sin 2 \beta$ dans le cadre du Modèle Standard, notée $a_{c \bar{c} s}$ dans la suite:

$$
\Delta m_{d}=r_{d}^{2} \Delta m_{d}^{\mathrm{SM}}, \quad \text { et } \quad a_{c \bar{c} s}=\sin \left(2 \beta+2 \theta_{d}\right) .
$$

\footnotetext{
${ }^{1} \mathrm{Si}$ l'on s`intéresse aussi aux mésons $B_{s}$ et $K$, on doit alors introduire une nouvelle paire de paramètres pour chaque système, ce qui rend le modèle beaucoup moins prédictif. Nous nous concentrons donc ici uniquement sur les mésons $B_{d}$.
} 
La modification sur $A_{\mathrm{SL}}$ est la suivante:

$$
A_{\mathrm{SL}}=-\operatorname{Re}\left(\frac{\Gamma_{12}}{M_{12}}\right)^{\mathrm{SM}} \frac{\sin 2 \theta_{d}}{r_{d}^{2}}+\operatorname{Im}\left(\frac{\Gamma_{12}}{M_{12}}\right)^{\mathrm{SM}} \frac{\cos 2 \theta_{d}}{r_{d}^{2}} .
$$

Le facteur $\operatorname{Re}\left(\Gamma_{12}\right)$ présent dans le premier terme est supérieur à $A_{\mathrm{SL}}^{\mathrm{SM}}$ par un facteur $m_{b}^{2} / m_{c}^{2}$, et peut donc entraîner une augmentation de $A_{\mathrm{SL}}$ par un ordre de grandeur. Ceci peut arriver si les contributions de la nouvelle physique à $M_{12}$ possède une phase large. Le deuxième terme est supprimé par un facteur $m_{c}^{2} / m_{b}^{2}$ par rapport au premier, néanmoins il peut en principe devenir important dans la région où $\sin 2 \theta_{d} \approx 0$ et $r_{d}^{2} \ll 1$ correspondant au cas où les contributions à $\Delta m_{d}$ du Modèle Standard et de la nouvelle physique se compensent. On trouve cependant que ce facteur demeure numériquement négligeable.

La figure 10.3 montre le niveau de confiance sur $A_{\text {SL }}$ pour des valeurs de $r_{d}$ et $\theta_{d}$ arbitraires obtenu en utilisant les contraintes de $\left|V_{u b}\right| /\left|V_{c b}\right|, \Delta m_{d}$ et $a_{c \bar{c} s}$ La contrainte obtenue par la mesure de $A_{\mathrm{SL}}$ est superposée: elle est plus forte que les contraintes indirectes des autres mesures, montrant que $A_{\mathrm{SL}}$ permet de sélectionner certaines valeurs de $r_{d}$ et $\theta_{d}$.

Il est intéressant de noter que si $A_{\mathrm{SL}}$ est négatif, alors la nouvelle physique ne permet d'augmenter sa valeur (vers des valeurs encore plus négatives) que par un petit facteur, alors que si $A_{\mathrm{SL}}$ est positif, l'augmentation peut être très importante. La raison de cette situation est expliquée ci-dessous.

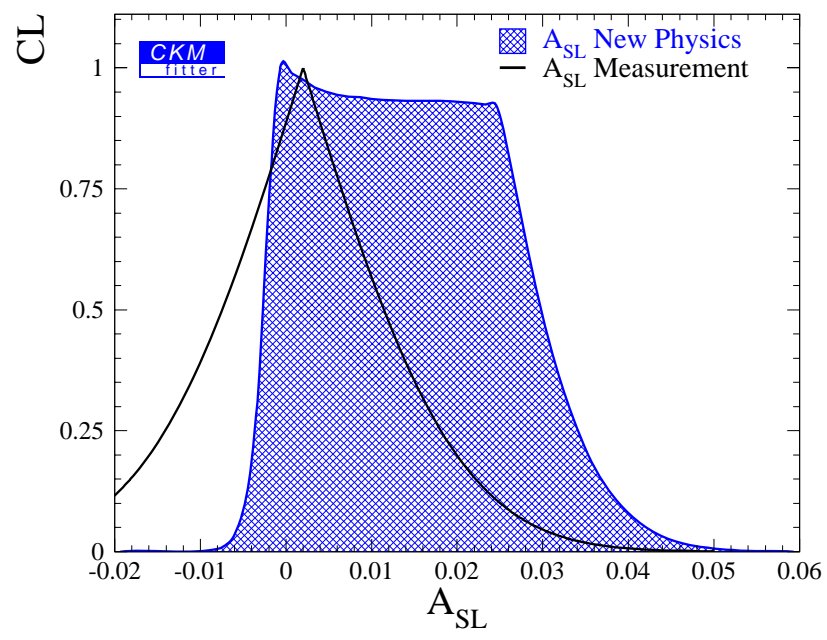

Figure 10.3: Niveau de confiance sur $A_{\mathrm{SL}}$ pour des valeurs de $r_{d}$ et $\theta_{d}$ arbitraires, utilisant les contraintes de $\left|V_{u b}\right| /\left|V_{c b}\right|, \Delta m_{d}$ et $a_{c \bar{c} s}$ (zone hachurées). La ligne noire correspond à la valeur moyenne des mesures de $A_{\mathrm{SL}}$ donnée dans l'éq. 10.2.

La figure 10.4 montre les contraintes dans le plan $r_{d}^{2}$ et $2 \theta_{d}$ dans diverses configurations: la figure en haut à gauche est obtenue en utilisant seulement les valeurs actuelles de $\left|V_{u b}\right| /\left|V_{c b}\right|$, $\Delta m_{d}$, et $a_{c \bar{c} s}$. On obtient les contraintes suivantes avec un $C L>10 \%$ :

$$
0.2<r_{d}^{2}<6.4, \quad-0.4<2 \theta_{d}<3.6 \mathrm{rad} .
$$

Cette figure permet d'expliquer pourquoi la nouvelle physique permet d'augmenter plutôt les valeurs positives de $A_{\mathrm{SL}}$ : d'une part, $a_{c \bar{c} s}>0$ privilégie les valeurs positives de $\sin 2 \theta_{d}$, ce qui mène à une valeur de $A_{\mathrm{SL}}$ positive ( $c f$. Eq. 10.10, en se souvenant que $\operatorname{Re}\left(\Gamma_{12} / M_{12}\right)^{\mathrm{SM}}$ est négatif). D'autre part, quand $\sin 2 \theta_{d}$ est positif, $r_{d}^{2}$ est petit, augmentant encore la valeur de $A_{\mathrm{SL}}$. 
La figure 10.4 en haut à droite montre les contraintes obtenues en rajoutant la mesure de $A_{\mathrm{SL}}$ : les faibles valeurs de $r_{d}^{2}(\lesssim 0.5)$ sont désormais défavorisées. La figure en bas à gauche montre la contrainte obtenue à partir d'une valeur hypothétique future $A_{\mathrm{SL}}=(-1 \pm 3) \times 10^{-3}$. Une telle mesure permettrait d'exclure une grande partie de l'espace des paramètres où $r_{d}^{2}<1$ (correspondant aux contributions à $\Delta m_{d}$ du Modèle Standard et de la nouvelle physique s'annulant), sauf pour $2 \theta_{d}$ proche de 0 ou $\pi$. Cette contrainte est peu sensible à l'amélioration de l'erreur sur $a_{c \bar{c} s}$, mais par contre est plus sensible à l'amélioration des erreurs sur $\left|V_{u b}\right|$ et $\Delta m_{d}$, comme montré sur la figure en bas à droite où l'erreur de $\left|V_{u b}\right|$ est choisie égale à $10 \%$, celle de $f_{B}$ à $10 \mathrm{MeV}$, et celle de $a_{c \bar{c} s}$ à 0.04 .
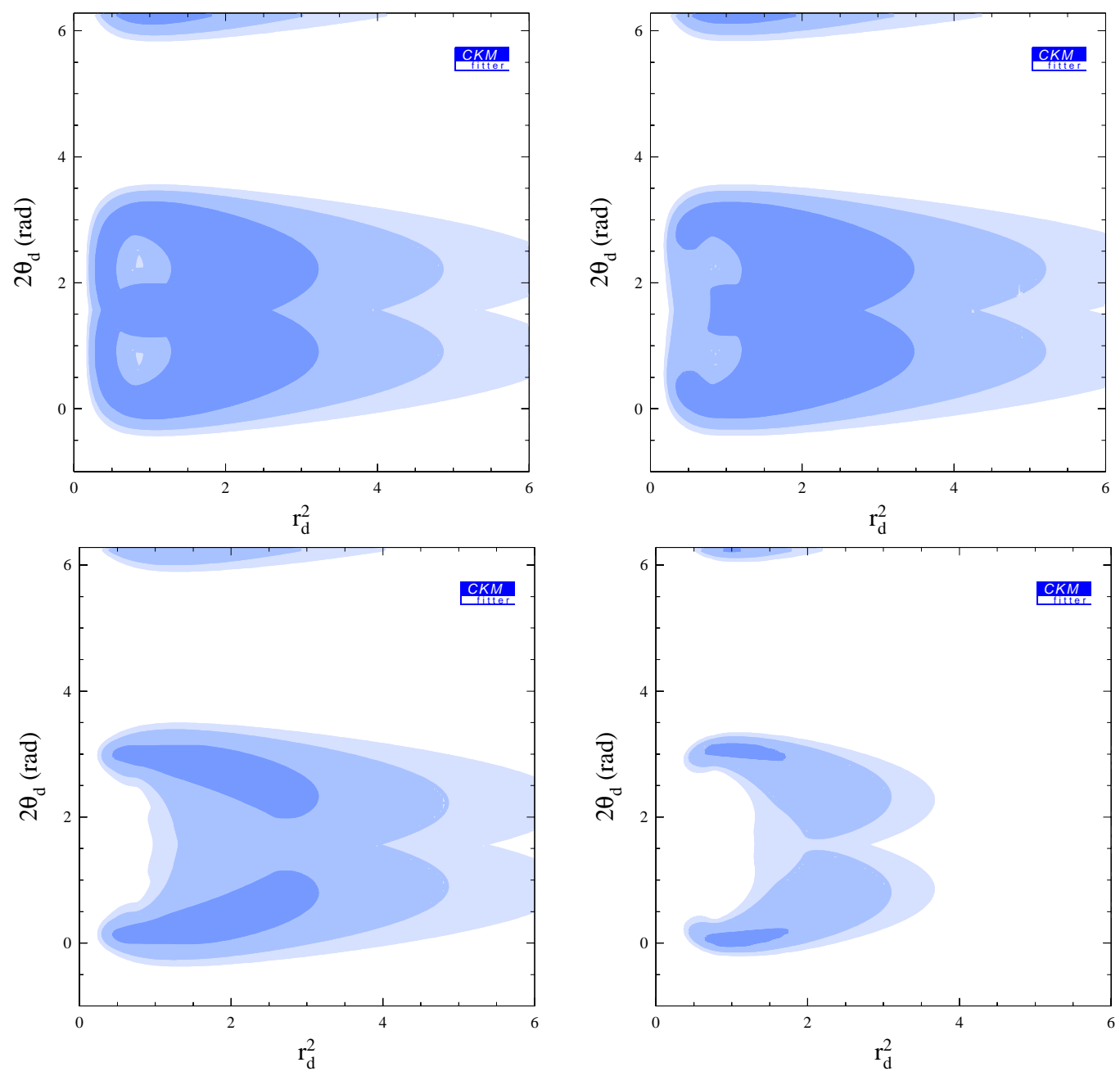

Figure 10.4: Niveau de confiance dans le plan $r_{d}^{2}-2 \theta_{d}$ utilisant les valeurs actuelles de $\left|V_{u b}\right| /\left|V_{c b}\right|, \Delta m_{d}$, et $a_{c \bar{c} s}$ (haut, gauche), ainsi que $A_{\mathrm{SL}}$ (haut, droite). La figure en bas à gauche montre la contrainte obtenue avec une valeur hypothétique de $A_{\mathrm{SL}}=(-1 \pm 3) \times 10^{-3}$, et celle en bas à droite fait l'hypothèse d'erreurs améliorées sur les autres observables: $\left|V_{u b}\right|$ $(10 \%), f_{B}(10 \mathrm{MeV})$ et $a_{c \bar{c} s}(0.04)$.

\subsubsection{Modèles avec violation de saveur minimale}

La classe des modèles avec violation de saveur minimale (Minimal Flavour Violation en anglais, "MFV") recouvrent les modèles de nouvelle physique possédant les propriétés sui- 
vantes $[169,170,171,172,173,174,175]$ :

- pas de nouveaux opérateurs par rapport à ceux présents dans le Modèle Standard.

- les transitions changeant la saveur sont gouvernées par la matrice CKM, et il n'existe pas de phase au delà de la phase Kobayashi-Maskawa.

Les contributions de nouvelle physique sont alors décrites par un seul nouveau paramètre réel, $F_{t t}$. On peut voir les modèles MFV comme un cas particulier du modèle générique exposé dans la section 10.3 .1 avec $r_{d}^{2}=\left|F_{t t}\right| / S_{0}$ et $2 \theta_{d}=0(\pi)$ pour $F_{t t}>0(<0)$ avec les contraintes additionnelles (ii) et (v) ci-dessous:

(i) Les désintégrations semi-leptoniques des $B$ dépendent de $\left|V_{u b} / V_{c b}\right|$ de la même façon que dans le Modèle Standard.

(ii) Le rapport $\Delta m_{d} / \Delta m_{s}$ dépend de $\left|V_{t d} / V_{t s}\right|$ de la même façon que dans le Modèle Standard.

(iii) L'asymétrie $a_{c \bar{c} s}$ dépend du signe de $F_{t t}[173]$ :

$$
a_{c \bar{c} s}=\operatorname{sign}\left(F_{t t}\right) \sin 2 \beta \text {. }
$$

(iv) La différence de masse $\Delta m_{d}$ dépend de $\left|F_{t t}\right|$ :

$$
\Delta m_{d}=\left(\Delta m_{d}\right)^{\mathrm{SM}} \frac{\left|F_{t t}\right|}{S_{0}} .
$$

Les corrections QCD dans les modèles MFV peuvent en principe différer de $\eta_{B}$, mais la modification est identique pour $\Delta m_{d}, \Delta m_{s}$ et pour la contribution du quark top dans $\epsilon_{K}$ : on peut donc l'absorber dans $F_{t t}[172]$.

(v) La contribution à $\epsilon_{K}$ qui dans le Modèle Standard est proportionnelle à $\operatorname{Im}\left[\left(V_{t s} V_{t d}^{*}\right)^{2}\right]$ est multipliée par $F_{t t}$, alors que les autres contributions (impliquant des quarks de faible masse) restent inchangées:

$$
\epsilon_{K}=\epsilon_{t t}^{\mathrm{SM}} F_{t t}+\epsilon_{c t}^{\mathrm{SM}}+\epsilon_{c c}^{\mathrm{SM}} .
$$

(vi) La dépendance en $F_{t t}$ de $A_{\mathrm{SL}}$ est la suivante: l'amplitude de mélange $B^{0}-\bar{B}^{0}$ est modifiée par $M_{12}=\left(M_{12}\right)^{\mathrm{SM}} F_{t t} / S_{0}$, et $\Gamma_{12}$ demeure inchangé. On a donc:

$$
A_{\mathrm{SL}}^{\mathrm{MFV}}=A_{\mathrm{SL}}^{\mathrm{SM}} \frac{S_{0}}{F_{t t}} .
$$

On peut penser naïvement que le signe de $A_{\mathrm{SL}}$ peut être opposé à celui du Modèle Standard, puisque $\operatorname{sign}\left(A_{\mathrm{SL}}^{\mathrm{MFV}}\right) \propto \operatorname{sign}\left(F_{t t}\right)$. Néanmoins, ce n'est pas le cas car $\operatorname{sign}\left(A_{\mathrm{SL}}^{\mathrm{SM}}\right)=$ $-\operatorname{sign}(\bar{\eta})$, et donc:

$$
\operatorname{sign}\left(A_{\mathrm{SL}}^{\mathrm{MFV}}\right)=-\operatorname{sign}\left(\bar{\eta} F_{t t}\right)
$$

Le produit $\bar{\eta} F_{t t}$ (en combinaison avec $\left|V_{u b} / V_{c b}\right|$ qui implique $\bar{\rho}<1$ ) détermine aussi le signe de $a_{c \bar{c} s}$ :

$$
\operatorname{sign}\left(a_{c \bar{c} s}^{\mathrm{MFV}}\right)=\operatorname{sign}\left(\bar{\eta} F_{t t}\right)
$$

et est donc expérimentalement positif. On en déduit donc que si le signe de $A_{\mathrm{SL}}$ est mesuré positif, alors à la fois le Modèle Standard et la classe de modèles MFV sont exclus.

La figure 10.5 à gauche montre les niveaux de confiance pour $F_{t t}$ : la courbe obtenue est très sensible à la valeur de $f_{B}$. En particulier, des valeurs élevées de $f_{B}$ font disparaître la 
solution négative pour $F_{t t}$. Pour la valeur de $f_{B}$ nominale $\left(f_{B}=200 \pm 30 \mathrm{MeV}\right)$, on obtient l'intervalle suivant pour $F_{t t}(\mathrm{CL}>10 \%)$ :

$$
-7.3<F_{t t}<-2.4 \quad \text { et } \quad 1.2<F_{t t}<7.3 \text {. }
$$

Le niveau de confiance pour $A_{\mathrm{SL}}$ dans les modèles MFV est montré sur la figure 10.5 à droite: la valeur de $A_{\mathrm{SL}}$ n'est pas significativement augmentée par rapport à celle du Modèle Standard. L'intervalle contenant les valeurs avec un CL de plus de 10\% est le suivant:

$$
-1.77 \times 10^{-3}<A_{\mathrm{SL}}<-0.33 \times 10^{-3}
$$

Si la valeur de $A_{\mathrm{SL}}$ est grande, on peut donc exclure à la fois le Modèle Standard et les modèles MFV.
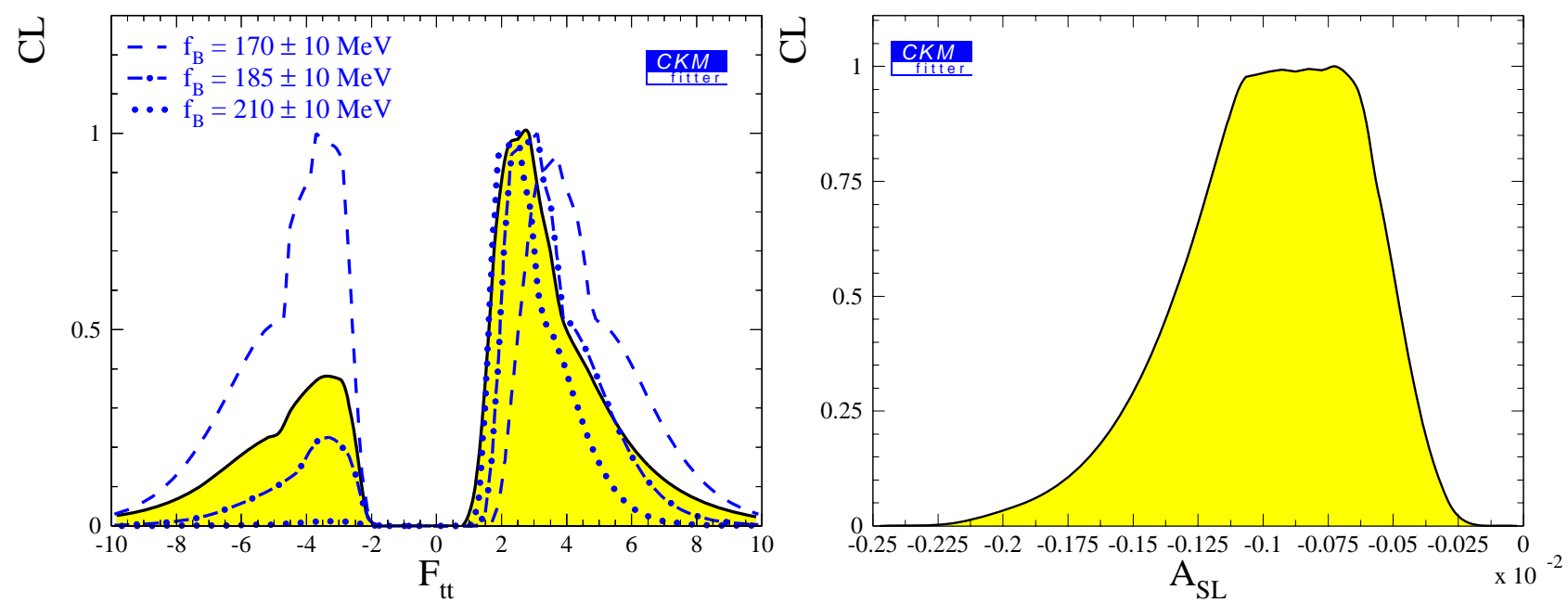

Figure 10.5: Figure de gauche: niveaux de confiance pour $F_{t t}$ pour diverses valeurs de $f_{B}$ : $f_{B}=200 \pm 30 \mathrm{MeV}$ (ligne pleine) $f_{B}=170 \pm 10 \mathrm{MeV}$ (ligne pointillée), $f_{B}=190 \pm 10 \mathrm{MeV}$ (ligne pointillée-tirets) et $f_{B}=210 \pm 10 \mathrm{MeV}$ (ligne pointillée). Dans ce dernier cas, la solution $F_{t t}<0$ est exclue. Figure de droite: niveaux de confiance de $A_{\mathrm{SL}}$ dans les modèles MFV.

La figure 10.6 montre les niveaux de confiance dans le plan $\bar{\rho}-\bar{\eta}$ dans les modèles MFV: la solution $\bar{\eta}<0$ est autorisée, mais avec un niveau de confiance plus bas que $\bar{\eta}>0$, reflétant le fait que la solution $F_{t t}<0$ a un niveau de confiance plus faible que $F_{t t}>0$.

\subsection{Conclusion}

L'exemple de $A_{\mathrm{SL}}$ illustre les possibilités offertes par l'analyse globale de la matrice CKM pour contraindre les modèles de nouvelle physique. Deux classes de modèles ont été considérées: les modèles génériques de nouvelle physique dans le mélange $B^{0}-\bar{B}^{0}$, paramétrés par $\theta_{d}$ et $r_{d}$, et les modèles de violation de saveur minimale, paramétrés par $F_{t t}$.

Dans la première classe de modèles, la valeur de $A_{\mathrm{SL}}$ peut être significativement augmentée par rapport aux prédictions du Modèle Standard, et en particulier, peut changer de signe. La mesure présente de $A_{\mathrm{SL}}$ pose déjà des contraintes dans l'espace des paramètres $\theta_{d}$ et $r_{d}$ : les petites valeurs de $r_{d}$ (correspondant au cas où les contributions à $\Delta m_{d}$ du Modèle Standard et de la nouvelle physique se compensent) sont défavorisées. 


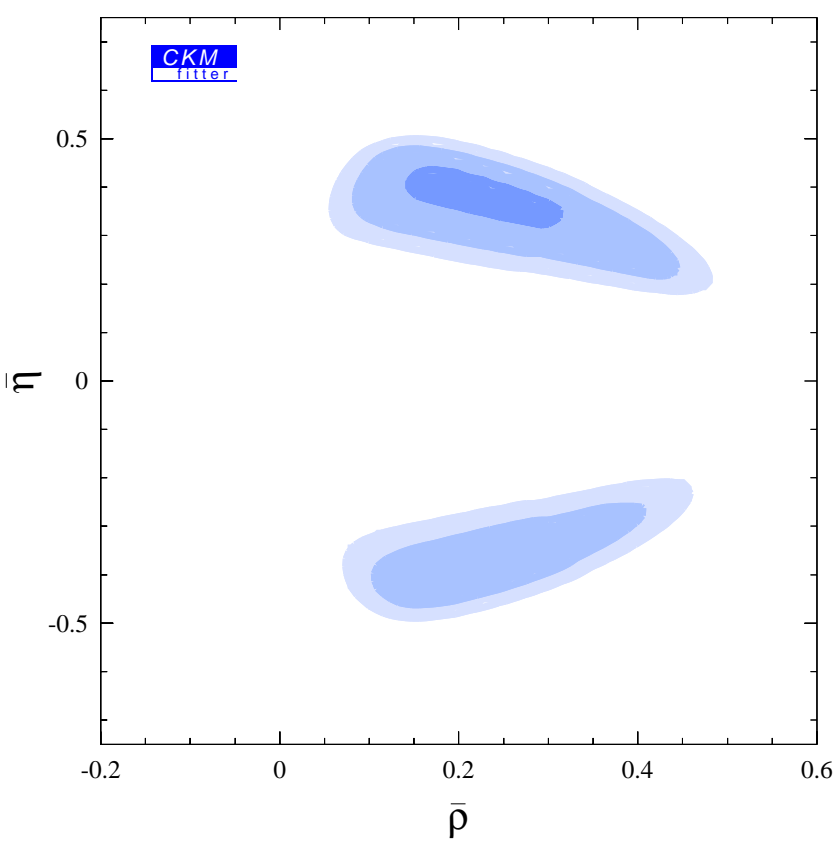

Figure 10.6: Niveaux de confiance dans le plan $\bar{\rho}-\bar{\eta}$ dans les modèles MFV. Les zones claires, moyennes et foncées contiennent les points avec plus de $10 \%, 32 \%$ et $90 \%$ respectivement.

Dans la seconde classe de modèles, la valeur de $A_{\mathrm{SL}}$ est du même ordre de grandeur et du même signe que la valeur prédite dans le Modèle Standard. Si on mesure une grande valeur de $A_{\mathrm{SL}}$, ou de signe positif, alors à la fois le Modèle Standard et les modèles MFV sont exclus. Dans cette classe de modèle, la solution $\bar{\eta}<0$ est permise, mais son niveau de confiance est très sensible à la valeur de $f_{B}$. 


\section{Conclusion}

La mise en service des usines à $B$ en 1999, puis la prise de données des expériences $B A B A R$ et Belle, se sont remarquablement déroulées, créant ainsi un environnement particulièrement propice pour la physique des saveurs lourdes et de la violation de $C P$ dans lequel s'est effectué le travail de thèse exposé dans ce document.

\section{Les désintégrations $B^{0} \rightarrow \rho \pi, B^{0} \rightarrow \rho K$}

Grâce à l'accumulation de plus de 88 millions de paires $\overline{B B}$ à l'été 2002 , une première analyse $C P$ dépendante du temps des désintégrations non-charmées $B^{0} \rightarrow \rho^{ \pm} \pi^{\mp}$ et $B^{0} \rightarrow \rho^{-} K^{+}$a été réalisée. Nous avons mesuré les asymétries $C P$ directe intégrées sur le temps:

$$
A_{\mathrm{CP}}^{\rho \pi}=-0.18 \pm 0.08_{\text {stat }} \pm 0.03_{\mathrm{syst}}, \quad A_{\mathrm{CP}}^{\rho K}=-0.28 \pm 0.17_{\mathrm{stat}} \pm 0.08_{\text {syst }},
$$

et les asymétries $C P$ dépendantes du temps:

$$
C=-0.36 \pm 0.18_{\text {stat }} \pm 0.04_{\text {syst }}, \quad S=-0.19 \pm 0.24_{\text {stat }} \pm 0.03_{\text {syst }},
$$

où $C$ est relié à la violation de $C P$ directe, et $S$, à la violation de $C P$ due à l'interférence entre les désintégrations avec et sans mélange. La signification statistique d'observation de violation de $C P$ directe $\left(c\right.$-à- $d$ liée aux paramètres $A_{\mathrm{CP}}^{\rho \pi}$ et $C$ ) est de $2.4 \sigma$. Celle de l'observation de violation $C P$ quelle qu'elle soit (incluant le paramètres $S$ ), est de $2.3 \sigma$.

Nous avons aussi mesuré les paramètres de dilution, reliés qualitativement aux asymétries entre la production du $\rho$ par le $W$ ou par le quark spectateur dans le diagramme en arbre de la désintégration:

$$
\Delta C=0.28 \pm 0.19_{\text {stat }} \pm 0.04_{\text {syst }}, \quad \Delta S=0.15 \pm 0.25_{\text {stat }} \pm 0.03_{\text {syst }} .
$$

Les erreurs systématiques de tous ces paramètres sont faibles devant les erreurs statistiques. Qui plus est, elles sont dominées par le bruit de fond dû aux autres désintégrations du $B$, et vont donc diminuer dans le futur lorsque les modes contaminants seront mesurés.

Nous avons aussi mesuré les rapports d'embranchement suivants:

$$
\begin{aligned}
\mathcal{B}\left(B^{0} \rightarrow \rho^{ \pm} \pi^{\mp}\right) & =\left(22.6 \pm 1.8_{\text {stat }} \pm 2.2_{\text {syst }}\right) \times 10^{-6}, \\
\mathcal{B}\left(B^{0} \rightarrow \rho^{-} K^{+}\right) & =\left(7.3 \pm 1.3_{\text {stat }} \pm 1.3_{\text {syst }}\right) \times 10^{-6},
\end{aligned}
$$

où les erreurs systématiques sont dominées par la reconstruction des $\pi^{0}$, le bruit de fond dû aux autres désintégrations du $B$, et aux corrélations résiduelles non prises en compte dans l'ajustement final. Ces erreurs vont aussi diminuer dans le futur. La signification statistique de ces résultats est de $20.3 \sigma$ pour $B^{0} \rightarrow \rho^{ \pm} \pi^{\mp}$ et $8.0 \sigma$ pour $B^{0} \rightarrow \rho^{-} K^{+}$. Grâce à l'optimisation de l'analyse, en particulier concernant la contamination des bruits de fond issus 
de la production de paires quark-antiquark et issus de la désintégration d'autres modes $B$, et grâce à la bonne efficacité de sélection du signal, les erreurs statistiques obtenues sont deux fois plus faibles que celles extrapolées à partir de la mesure précédente la plus précise (réalisée par la collaboration Belle). Les erreurs systématiques sont aussi inférieures à celles des précédentes analyses (de $25 \%$ pour $B^{0} \rightarrow \rho^{ \pm} \pi^{\mp}$, et de $45 \%$ pour $B^{0} \rightarrow \rho^{-} K^{+}$), malgré le fait que les erreurs dues aux bruits de fond $B$ étaient alors fortement sous-estimées.

Nous avons également mesuré le rapport d'embranchement du mode supprimé de couleur $B^{0} \rightarrow \rho^{0} \pi^{0}:$

$$
\mathcal{B}\left(B^{0} \rightarrow \rho^{0} \pi^{0}\right)=\left(1.24 \pm 0.57_{\text {stat }} \pm 0.42_{\text {syst }}\right) \times 10^{-6},
$$

dont l'erreur systématique expérimentale est dominée par les mêmes sources que pour les modes $B^{0} \rightarrow \rho^{ \pm} \pi^{\mp}$ et $B^{0} \rightarrow \rho^{-} K^{+}$. La signification statistique de ce résultat n'étant que de $2.7 \sigma$, nous calculons une limite supérieure sur le rapport d'embranchement, $\mathcal{B}\left(B^{0} \rightarrow \rho^{0} \pi^{0}\right)<$ $2.7 \times 10^{-6}$ à $90 \% \mathrm{CL}$, incluant les incertitudes statistiques et systématiques.

Nous avons interprété ces résultats en terme de contraintes présentes et futures sur l'angle $\alpha$ utilisant les symétries de saveur $S U(2)$ et $S U(3)$. Dans la configuration utilisée dans ces études, on conclut que la symétrie $S U(2)$, utilisée avec les désintégrations $B^{0, \pm} \rightarrow \rho \pi$, ne permet pas de contraindre $\alpha$ avec la statistique finale attendue des premières générations d'usines à $B$ à moins que le rapport d'embranchement de $B^{0} \rightarrow \rho^{0} \pi^{0}$ ne soit bien plus faible que la valeur attendue.

La symétrie $S U(3)$ permet d'obtenir de meilleures contraintes sur $\alpha$. Notons que les valeurs actuelles relativement grande d'asymétrie $C P$ directe pour $B^{0} \rightarrow \rho^{ \pm} \pi^{\mp}$ impliquent d'importants diagrammes pingouins. A l'inverse, le faible rapport d'embranchement de $B^{0} \rightarrow$ $\rho^{-} K^{+}$implique des diagrammes pingouins peu importants. Il sera intéressant de suivre l'évolution future de cette incompatibilité (d'une signification de $17 \%$ à l'heure actuelle).

Enfin, en utilisant l'ensemble des contraintes de l'ajustement standard de la matrice CKM et l'hypothèse de la symétrie $S U(3)$, on privilégie de faibles phases relatives entre les amplitudes de désintégrations de $B^{0} \rightarrow \rho^{+} \pi^{-}$et de $B^{0} \rightarrow \rho^{-} \pi^{+}$, résultat en faveur de la factorisation naïve.

Enfin, toujours dans le cadre des modes $B^{0} \rightarrow \rho h$, nous avons développé le coeur de l'analyse complète de $B^{0} \rightarrow \pi^{+} \pi^{-} \pi^{0}$ qui exploite toute l'information présente dans le diagramme de Dalitz. Des estimations de sensibilité expérimentale pour $\alpha$ ont été effectuées.

\section{Le mode $B^{0} \rightarrow a_{0}^{ \pm} \pi^{\mp}$}

Nous avons réalisé la mesure du rapport d'embranchement du mode $B^{0} \rightarrow a_{0}(\rightarrow \eta \pi) \pi$ sur une luminosité intégrée plus réduite de $20 \mathrm{fb}^{-1}$ :

$$
\mathcal{B}\left(B^{0} \rightarrow a_{0}^{ \pm} \pi^{\mp}\right) \times \mathcal{B}\left(a_{0} \rightarrow \eta \pi\right)=\left(6.2_{-2.5}^{+3.0} \text { stat } \pm 1.1_{\text {syst }}\right) \times 10^{-6},
$$

dont la signification statistique est de $3.7 \sigma$. Ce mode était originellement candidat pour une analyse du diagramme de Dalitz similaire à celle de $B^{0} \rightarrow \pi^{+} \pi^{-} \pi^{0}$ pour la détermination de $\alpha$. Nous avons montré que cette analyse est en fait impossible, puisque les limites cinématiques de la désintégration coupent la majorité des effets d'interférences.

Nous avons mené des études phénoménologiques sur la faisabilité d'une analyse $C P$ d'isospin de $B^{0} \rightarrow a_{0}^{ \pm} \pi^{\mp}$ traité en deux-corps. La suppression de certains des diagrammes de la désintégration survient à cause de l'absence des courants de second classe dans le Modèle Standard. Dans le cadre de la factorisation naïve, ces suppressions entraînent une 
simplification de l'analyse d'isospin par rapport à celle du mode $B^{0} \rightarrow \rho^{ \pm} \pi^{\mp}$. Il est aussi possible de mesurer les corrections éventuelles à la factorisation naïve dans ce mode.

\section{Le système de protection contre les radiations du Silicon Vertex Tracker}

Ma contribution au dispositif expérimental de $B A B A R$ a pris place au sein du système de protection contre les radiations du Silicon Vertex Tracker, dénommé SVTRAD. L'étude de ce système de protection, lorsque l'on m'en a confié la responsabilité pendant plusieurs mois à SLAC, a mis en évidence des points faibles au niveau du logiciel de protection en temps réel associé durant les périodes d'injection des faisceaux. J'ai donc amélioré ce dernier dans deux directions: d'une part, le nombre de coupures de faisceaux durant les périodes d'injection a été limité, car ces coupures étaient cô̂teuses en temps et entraînaient un surcroit de radiations, et d'autre part, j'ai mis en place un algorithme de protection incontournable inhibant les injections lorsque la dose intégrée depuis de l'injection est trop élevée.

Ce nouveau système a été adopté par PEP-II et BABAR, et s'est montré tout à fait satisfaisant en terme de protection et de contrôle des doses reçues par le SVT.

\section{Analyse globale de la matrice CKM}

En marge de la collaboration BABAR, nous avons développé un logiciel, dénommé CKMfitter, permettant de réaliser une analyse globale de la matrice CKM au sein d'une approche statistique fréquentiste. Utilisant toutes les observables reliées à la matrice CKM, le logiciel permet de tester la validité du Modèle Standard (ou de modèles au delà du Modèle Standard) et de poser des contraintes sur les paramètres non-prédits par le Modèle Standard: les quatre paramètres de Wolfenstein de la matrice CKM, mais aussi les masses des quarks, les paramètres de QCD, les rapports d'embranchement de modes rares, etc.. Les intervalles contenant les valeurs ayant plus de $32 \%$ de niveau de confiance pour les principaux paramètres reliés à la violation de $C P$ sont:

$$
\begin{aligned}
& \bar{\rho}=0.13-0.32, \quad \bar{\eta} \quad=0.28-0.40, \\
& \alpha=85^{\circ}-115^{\circ}, \quad \beta=21.4-25.9 \text {, } \\
& \gamma=42^{\circ}-72^{\circ}, \quad J\left(10^{-5}\right)=2.23-3.34 \text {. }
\end{aligned}
$$

En plus des résultats numériques, des contraintes graphiques peuvent être tracées dans divers plans tels que $\bar{\rho}-\bar{\eta}, \beta-\alpha$, etc., et des contraintes sur des paramètres de modèles de nouvelle physique peuvent être obtenues. Notre logiciel est aujourd'hui largement diffusé et nos résultats fréquemment utilisés dans la communauté scientifique. 


\section{Annexe A}

\section{Le mode $B^{0} \rightarrow a_{0}^{ \pm} \pi^{\mp}$ : théorie et mesure du rapport d'embranchement}

Cette annexe expose un travail phénoménologique et expérimental sur le mode $B^{0} \rightarrow a_{0}^{ \pm} \pi^{\mp}$ où $a_{0}^{ \pm} \rightarrow \eta \pi^{ \pm}$. Ce mode a été mis en avant par Dighe et Kim [35] comme devant permettre l'extraction de l'angle $\alpha$ de façon similaire que dans $B^{0} \rightarrow \rho^{ \pm} \pi^{\mp}$. L'étude phénoménologique [36] résumée dans la section A.1 montre que ce mode est en fait très différent d'un point de vue théorique de $B^{0} \rightarrow \rho^{ \pm} \pi^{\mp}$. Nous avons aussi réalisé une étude expérimentale de ce mode ( $c f$. section A.2) en vue de mesurer son rapport d'embranchement. Chronologiquement, cette mesure a été réalisée avant celles de $B^{0} \rightarrow \rho^{ \pm} \pi^{\mp}, B^{0} \rightarrow \rho^{-} K^{+}$et $B^{0} \rightarrow \rho^{0} \pi^{0}$ ( $c f$. partie II), sur une luminosité plus réduite de $20 \mathrm{fb}^{-1}$. Cette analyse étant plus simple que celle de $B^{0} \rightarrow \rho h$, nous ne résumons ici que le résultat final, sans détailler les étapes intermédiaires [42].

\section{A.1 Théorie relative au mode $B^{0} \rightarrow a_{0}^{ \pm} \pi^{\mp}$}

\section{A.1.1 Analyse Dalitz de $B^{0} \rightarrow a_{0}^{ \pm} \pi^{\mp}$}

L'observation [36] du diagramme Dalitz de $B^{0} \rightarrow a_{0}^{ \pm} \pi^{\mp}$, montré sur la figure A.1, révèle que la zone d'interférences entre le $a_{0}^{+}$et $a_{0}^{-}$est située à l'extérieur des limites cinématiques du diagramme de Dalitz $B^{0} \rightarrow \eta \pi \pi$. Ceci est dû à la masse élevée du $\eta$ par rapport à celle du $\pi^{0}$, ce qui pousse l'extrémité en bas à gauche du diagramme Dalitz de 0.85 à $1.7 \mathrm{GeV}$.

On peut calculer l'amplitude des interférences résiduelles grâce à la formule:

$$
\epsilon=\frac{\left|\sum_{i=1}^{3} f_{i}\right|^{2}}{\sum_{i=1}^{3}\left|f_{i}\right|^{2}-1},
$$

où $f_{i}$ sont les Breit-Wigner des résonances dans le Dalitz, tenant compte de la distribution angulaire des produits de désintégration. En utilisant des Breit-Wigner non relativistes, on obtient des valeurs typiques pour $|\epsilon|$ de l'ordre de $\simeq 1 \%$, alors que les valeurs sont plutôt autour de 15 à $20 \%$ dans le diagrammes de Dalitz de $B^{0} \rightarrow \rho^{ \pm} \pi^{\mp}$.

Nous en concluons que l'analyse Dalitz n'est pas réalisable dans cette désintégration. 


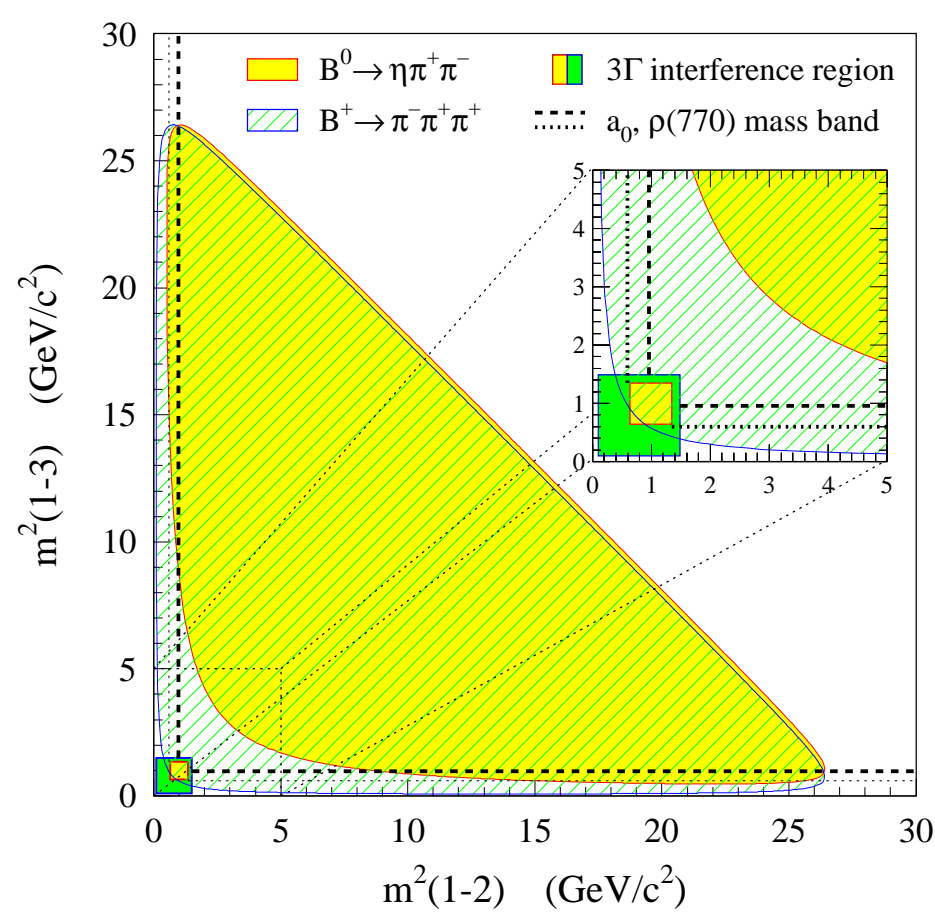

Figure A.1: Limites cinématiques du diagramme Dalitz pour les désintégrations $B^{0} \rightarrow \eta \pi \pi$ et $B^{0} \rightarrow \pi^{0} \pi^{+} \pi^{-}$. Les lignes en tirets (pointillés) montrent les bandes des masses du $a_{0}(\rho)$. La région d'interférence principale définie comme la zone à l'intérieur de $3 \Gamma$ de la masse de la résonance est indiquée par les carrés colorés à l'intersection des bandes de masse. Pour le $a_{0}$, cette zone est à l'extérieur du diagramme Dalitz $B^{0} \rightarrow \eta \pi \pi$.

\section{A.1.2 Analyse en deux-corps de $B^{0} \rightarrow a_{0}^{ \pm} \pi^{\mp}$}

L'analyse du diagramme de Dalitz de $B^{0} \rightarrow a_{0}^{ \pm} \pi^{\mp}$ n'étant pas réalisable, nous avons étudié la faisabilité de l'analyse en deux-corps pour l'extraction de $\alpha$. Nous avons utilisé les propriétés particulières du $a_{0}$ pour proposer une analyse simplifiée en deux corps.

\section{A.1.2.1 Absence des courants de seconde classe dans le Modèle Standard}

Le $a_{0}$ possède les nombres quantiques suivants: $J^{P C}=0^{++}$. Ainsi, si l'on veut produire un $a_{0}$ par le $W$, c'est la partie vecteur $V$ du courant électrofaible qui intervient. Or le courant vecteur possède une $\mathrm{G}$-parité de +1 , alors que le $a_{0}$ possède une G-parité de -1 : le $a_{0}$ ne peut donc pas être produit par le $W$.

On appelle "courants de seconde classe" (interdits dans le Modèle Standard), les courants qui ne possèdent pas les associations de nombres quantiques suivantes:

$$
\begin{aligned}
& G=-1 \quad \text { à } \quad P^{I}=0^{-}, 1^{+}, \ldots \quad \text { (spin - parité naturelle), } \\
& G=+1 \quad \text { à } \quad P^{I}=0^{+}, 1^{-}, \ldots \quad \text { (spin - parité non naturelle). }
\end{aligned}
$$

Il existe par ailleurs un autre argument, indépendant de la G-parité, pour la non-production du $a_{0}$ par le $W$ : la conservation du courant vecteur (dénommée CVC dans la littérature) due à la conservation du nombre quantique d'isospin. La production d'une particule $M$ par le courant électrofaible s'écrit: $\left\langle 0\left|J_{\mu}\right| M\right\rangle=q_{\mu} f_{M}$ où $q$ est la quadri-impulsion de $M, f_{M}$ sa constante de couplage, et le courant $J_{\mu}=J_{\mu}^{V}+J_{\mu}^{A}$ est composée d'une partie vecteur $V$ et axiale $A$. L'argument de CVC impose que $\partial^{\mu} J_{\mu}^{V}=m_{u}-m_{d} \simeq 0$ (et la conservation 
partielle du courant axial, PCAC, que $\partial^{\mu} J_{\mu}^{A}=m_{u}+m_{d} \simeq 2 m_{u}$ ). On obtient donc que $f_{M}\left(M=a_{0}\right)=0$, et que le $a_{0}$ n'est donc pas produit par le courant vecteur.

\section{A.1.2.2 Simplification de l'analyse en deux-corps de $B^{0} \rightarrow a_{0}^{ \pm} \pi^{\mp}$}

Avec l'hypothèse de factorisation naïve, le fait que le $a_{0}$ ne soit pas produit par le $W$ se traduit par le fait que le diagramme en arbre où le $a_{0}$ est issu du $W$ (pour les désintégrations $B^{0} \rightarrow a_{0}^{+} \pi^{-}$et $\bar{B}^{0} \rightarrow a_{0}^{-} \pi^{+}$) est nul: $T^{+-}=0$.

Nous avons ainsi:

$$
\begin{aligned}
& A\left(B^{0} \rightarrow a_{0}^{+} \pi^{-}\right)=A^{+-}=P^{+-} \\
& A\left(\overline{B^{0}} \rightarrow a_{0}^{-} \pi^{+}\right)=\bar{A}^{-+}=P^{+-}
\end{aligned}
$$

et donc

$$
A\left(B^{0} \rightarrow a_{0}^{+} \pi^{-}\right)=A\left(\overline{B^{0}} \rightarrow a_{0}^{-} \pi^{+}\right) .
$$

Si l'égalité A.6 n'est pas vérifiée expérimentalement, on doit alors remettre en question l'hypothèse de factorisation naïve.

Le nombre d'inconnues dans l'analyse en deux-corps est donc réduit par rapport à l'analyse en deux-corps de $B^{0} \rightarrow \rho^{ \pm} \pi^{\mp}$, comme montré dans le tableau A.1 donnant le nombre d'inconnues et d'observables pour les analyses en deux-corps de $B^{0} \rightarrow a_{0}^{ \pm} \pi^{\mp}, B^{0} \rightarrow \mathrm{a}_{0}^{ \pm} \rho^{\mp}$, $B^{0} \rightarrow \rho^{ \pm} \pi^{\mp}$ et $B^{0} \rightarrow \pi^{+} \pi^{-}$(le cas de $B^{0} \rightarrow \rho^{ \pm} \pi^{\mp}$ est aussi discuté dans le tableau 2.1). La désintégration $B^{0} \rightarrow \mathrm{a}_{0}^{ \pm} \rho^{\mp}$ possède d'avantage d'observables que $B^{0} \rightarrow a_{0}^{ \pm} \pi^{\mp}$, car on peut réaliser une analyse en temps du mode $\mathrm{B}^{0} \rightarrow a_{0}^{0} \rho^{0}$, chose impossible pour $B^{0} \rightarrow a_{0}^{0} \pi^{0}$ (il faut au moins une trace chargée dans l'état final pour reconstruire $\Delta t$ ).

\section{A.1.2.3 Au delà de la factorisation naïve}

Des corrections à l'hypothèse de factorisation naïve amènent à introduire un "pseudo" diagramme en arbre $T^{+-}$représentant les contributions de ces corrections ${ }^{1}$.

Notons que si ces corrections sont significatives, alors l'égalité A.6 ne tient plus: on peut donc expérimentalement vérifier la taille de ces corrections, et il s'agit là en fait du premier test à réaliser sur les données avant de se lancer dans l'analyse $C P$ en deux corps de $B^{0} \rightarrow a_{0}^{ \pm} \pi^{\mp}$ simplifiée.

Nous pouvons néanmoins évaluer quantitativement l'influence des corrections non perturbatives à l'amplitude de $B^{0} \rightarrow a_{0}^{ \pm} \pi^{\mp}$ sur l'analyse $C P$, et évaluer le biais sur $\alpha$. Pour ceci, nous considérons des corrections égales à $10 \%$ de l'amplitude $T^{+-}$de la désintégration $B^{0} \rightarrow \rho^{ \pm} \pi^{\mp}$ telle que calculée dans le scénario "Large penguin" du BABAR book [59]. Cela revient à introduire le $T^{+-}$effectif suivant:

$$
T^{+-}\left(B^{0} \rightarrow a_{0}^{ \pm} \pi^{\mp}\right)=f T^{+-}\left(B^{0} \rightarrow \rho^{ \pm} \pi^{\mp}\right) .
$$

Pour évaluer le biais sur $\alpha$, nous réalisons l'étude Monte Carlo suivante: nous produisons un lot de 1500 événements $B^{0} \rightarrow a_{0}^{ \pm} \pi^{\mp}$ (correspondant environ à une luminosité de $500 \mathrm{fb}^{-1}$ pour une efficacité de sélection de $10 \%$, et pour un rapport d'embranchement de l'ordre de grandeur de celui de $B^{0} \rightarrow \rho^{ \pm} \pi^{\mp}$ ) avec des valeurs d'amplitudes arbre et pingouin égales

\footnotetext{
${ }^{1}$ En effet, les corrections à la factorisation naïve correspondent à d'éventuels diagrammes, différents de $T^{+-}$, subissant des interactions dans l'état final - qui est donc en fait un état intermédiaire - de telle sorte qu’ils mènent à l'état final $a_{0}^{+} \pi^{-}$. On peut modéliser cet effet en introduisant un pseudo $T^{+-}$.
} 


\begin{tabular}{|c|c|c|c|c|c|c|c|c|c|}
\hline \multirow{2}{*}{$\begin{array}{c}\text { Mode } \\
\text { Ex: } B \rightarrow a_{0} \pi\end{array}$} & \multirow{2}{*}{$\begin{array}{c}\text { Amplitudes } \mathrm{T} \& \mathrm{P} \\
\text { contribuant }\end{array}$} & \multicolumn{2}{|c|}{$a_{0} \pi$} & \multicolumn{2}{|c|}{$a_{0} \rho$} & \multicolumn{2}{|c|}{$\rho \pi$} & \multicolumn{2}{|c|}{$\pi \pi$} \\
\hline & & $\mathcal{O}$ & $\mathcal{U}$ & $\mathcal{O}$ & $\mathcal{U}$ & $\mathcal{O}$ & $\mathcal{U}$ & $\mathcal{O}$ & $\mathcal{U}$ \\
\hline$B^{0} \rightarrow a_{0}^{+} \pi^{-}$ & $e^{-i \alpha} T^{+-}+P^{+-}$ & \multirow{6}{*}{$\begin{array}{c}3_{t} \\
3_{t} \\
-1 \\
-1 \\
\end{array}$} & 5 & \multirow[b]{2}{*}{$3_{t}$} & 5 & \multirow[b]{2}{*}{$3_{t}$} & 5 & \multirow[b]{2}{*}{$3_{t}$} & \multirow{2}{*}{$\begin{array}{l}5 \\
-\end{array}$} \\
\hline$\overline{B^{0}} \rightarrow a_{0}^{+} \pi^{-}$ & $e^{+i \alpha} T^{-+}+P^{-+}$ & & 4 & & 4 & & 4 & & \\
\hline$B^{0} \rightarrow a_{0}^{-} \pi^{+}$ & $e^{-i \alpha} T^{-+}+P^{-+}$ & & - & 3 & - & $3 t$ & - & - & - \\
\hline$B^{0} \rightarrow a_{0}^{-} \pi^{+}$ & $e^{+i \alpha} T^{+-}+P^{+-}$ & & - & & & & & & \\
\hline \multicolumn{2}{|c|}{ Norm. \& phase } & & -2 & -1 & -2 & \multirow[t]{2}{*}{-1} & -2 & \multirow[t]{2}{*}{-1} & \multirow[t]{2}{*}{-2} \\
\hline \multicolumn{2}{|c|}{$T^{+-}=0$} & & -2 & -1 & -2 & & & & \\
\hline \multicolumn{2}{|c|}{ Total utilisant $B^{0}$} & \multicolumn{2}{|c|}{4 4vs 5} & \multicolumn{2}{|c|}{4 4vs 5} & \multicolumn{2}{|c|}{5 vs 7} & \multicolumn{2}{|c|}{2 2vs 3} \\
\hline$B^{0} \rightarrow a_{0}^{0} \pi^{0}$ & $e^{-i \alpha} T^{00}+P^{00}$ & \multirow{3}{*}{$\begin{array}{l}1_{i} \\
1_{i}\end{array}$} & 4 & \multirow{3}{*}{$3_{t}$} & \multirow{3}{*}{$\begin{array}{c}4 \\
- \\
-2 \\
\end{array}$} & \multirow{3}{*}{$3_{t}$} & \multirow{3}{*}{$\begin{array}{c}4 \\
- \\
-2 \\
\end{array}$} & \multirow{3}{*}{$\begin{array}{l}1_{i} \\
1_{i}\end{array}$} & \multirow{3}{*}{$\begin{array}{c}4 \\
- \\
-2 \\
\end{array}$} \\
\hline$\overline{B^{0}} \rightarrow a_{0}^{0} \pi^{0}$ & $e^{+i \alpha} T^{00}+P^{00}$ & & - & & & & & & \\
\hline \multicolumn{2}{|c|}{ Relations d'isospin } & & -2 & & & & & & \\
\hline \multicolumn{2}{|c|}{ Total avec état final neutre } & \multicolumn{2}{|c|}{6 vs 7} & \multicolumn{2}{|c|}{7 vs 7} & \multicolumn{2}{|c|}{8 vs 9} & \multicolumn{2}{|c|}{4 vs 5} \\
\hline$B^{+} \rightarrow a_{0}^{+} \pi^{0}$ & $e^{-i \alpha} T^{+0}+P^{+0}$ & \multirow{5}{*}{$\begin{array}{l}1_{i} \\
1_{i} \\
1_{i} \\
1_{i}\end{array}$} & 4 & $1_{i}$ & 4 & $1_{i}$ & 4 & $1_{i}$ & 4 \\
\hline$B^{+} \rightarrow a_{0}^{0} \pi^{+}$ & $e^{-i \alpha} T^{0+}+P^{0+}$ & & 4 & $1_{i}$ & 4 & $1_{i}$ & 4 & - & - \\
\hline$B^{-} \rightarrow a_{0}^{-} \pi^{0}$ & $e^{+i \alpha} T^{+0}+P^{+0}$ & & & $1_{i}$ & & $1_{i}$ & & $1_{i}$ & \\
\hline$B^{-} \rightarrow a_{0}^{0} \pi^{-}$ & $e^{+i \alpha} T^{0+}+P^{0+}$ & & & $1_{i}$ & & $1_{i}$ & & - & \\
\hline Relati & s d'isospin & & -6 & & -6 & & -6 & & -4 \\
\hline Total er & joutant $B^{ \pm}$ & 10 & s 9 & 11 & us 9 & 12 & $s 11$ & & \\
\hline
\end{tabular}

Tableau A.1: Nombre d'inconnues $(\mathcal{U})$ et d'observables $(\mathcal{O})$ dans les analyses en deux-corps des modes de $B^{0} \rightarrow a_{0}^{ \pm} \pi^{\mp}, B^{0} \rightarrow \mathrm{a}_{0}^{ \pm} \rho^{\mp}, B^{0} \rightarrow \rho^{ \pm} \pi^{\mp}$ et $B^{0} \rightarrow \pi^{+} \pi^{-}$. L'analyse en temps des désintégrations de $B$ neutres amène trois observables (indiqué par l'indice "t"), alors que les analyses non-temporelles amènent deux observables (indiqué par l'indice "i"). La suppression de $T^{+-}=0$ s'applique aux modes $B^{0} \rightarrow a_{0}^{ \pm} \pi^{\mp}$ et $B^{0} \rightarrow \mathrm{a}_{0}^{ \pm} \rho^{\mp}$.

à celle du scénario "Large penguin" pour $B^{0} \rightarrow \rho^{ \pm} \pi^{\mp}$ calculée dans le BABAR book [59], et la valeur de $T^{+-}$donnée en Eq. A.7. L'analyse est réalisée comme indiquée dans la section A.1.2.2, $c$ - $\grave{a}-d$ en considérant ce diagramme comme nul.

La figure A.2 montre les effets des corrections non factorisables sur l'analyse $C P$ : la figure du haut représente la distribution de $-2 \ln \mathcal{L}$ pour $f=0$ (factorisation naíve) et $f=0.1$ (10\% de correction): dans la configuration choisie, il existe une solution miroir à $\alpha \simeq 2.2$ quasiment dégénérée avec la vraie solution $\alpha=1.35$ pour $f=0$, mais qui devient plus profonde pour $f=0.1$. La figure $\mathrm{du}$ bas montre le biais sur $\alpha$ (obtenu comme la différence entre la valeur de $\alpha$ générée et celle du minimum de $-2 \ln \mathcal{L}$ le plus proche de cette valeur ${ }^{2}$ ) en fonction de $f$.

Pour $f=0.1$, le biais est de $0.18 \mathrm{rad}$, c'est-à-dire d'un ordre de grandeur comparable à l'erreur statistique sur $\alpha$, qui vaut $0.23 \mathrm{rad}$.

\footnotetext{
${ }^{2}$ Néanmoins, dans une configuration malchanceuse comme celle montrée sur le haut de la figure A.2, le minimum correspondant à la vraie solution d' $\alpha$ n'est plus le minimum le plus profond. Le biais est alors plus élevé, puisqu'il correspond à la différence entre la valeur d' $\alpha$ générée et celle correspondant au minumum le plus profond.
} 

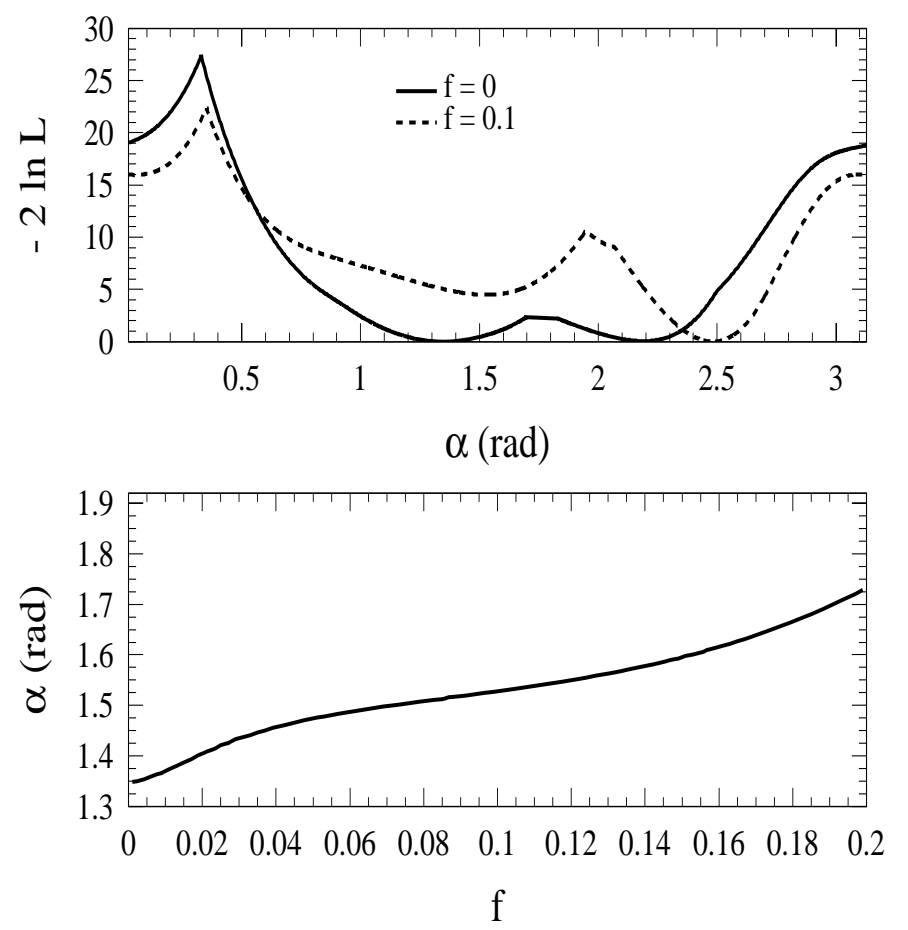

Figure A.2: Figure du haut: distribution de $\chi^{2}(\alpha)=-2 \ln \mathcal{L}$ pour $f=0$ et $f=10 \%$. Figure du bas: biais sur $\alpha$ en fonction de $f$.

\section{A.1.3 Conclusions}

Les contraintes imposées par l'absence des courants de seconde classe dans le Modèle Standard ouvrent de nouvelles opportunités d'analyses pour le mode $B^{0} \rightarrow a_{0}^{ \pm} \pi^{\mp}$ : dans le cadre de la factorisation naïve, l'analyse $C P$ en deux corps dépendante du temps est simplifiée par rapport à celle de $B^{0} \rightarrow \rho^{ \pm} \pi^{\mp}$. Ceci impose néanmoins de maîtriser les effets non factorisables, qui peuvent être évalués expérimentalement en vérifiant la présence de violation de $C P$ directe entre les modes $B^{0} \rightarrow a_{0}^{+} \pi^{-}$et $\vec{B}^{0} \rightarrow a_{0}^{-} \pi^{+}$. Grâce à une étude Monte Carlo basée sur $500 \mathrm{fb}^{-1}$, nous évaluons que le biais sur $\alpha$ engendré par des corrections à la factorisation naïve de l'ordre de $10 \%$ comme étant du même ordre de grandeur que l'erreur statistique. Néanmoins, ce biais peut en réalité être plus important à cause de la présence de solutions miroirs pour $\alpha$.

\section{A.2 Mesure du rapport d'embranchement}

L'analyse menant à la mesure du rapport d'embranchement est décrite en détail dans les références [42] et [43], seul le résultat de cette analyse, utilisant $22.7 \cdot 10^{6}$ paires $\overline{B B}$, est résumé ici.

Nous reconstruisons le $a_{0}$ dans sa désintégration dominante, $\eta \pi$. L'autre désintégration du $a_{0}$ en $K K$ possède un rapport d'embranchement négligeable. La sélection est similaire à celle du mode $B^{0} \rightarrow \rho^{ \pm} \pi^{\mp}$, une fois le $\rho$ remplacé par le $a_{0}$, qui est une particule scalaire dont la largeur plus petite que celle du $\rho\left(\Gamma_{a_{0}} \simeq 50 \mathrm{MeV}\right.$ [44]), et une fois le $\pi^{0}$ remplacé par le $\eta$ $(m(\eta)=547 \mathrm{MeV})$. La sélection du $a_{0}$ se fait en demandant $0.9<m(\eta \pi)<1.08 \mathrm{GeV} / \mathrm{c}^{2}$, et 
celle du $\eta$, reconstruit uniquement en $\gamma \gamma^{3}$, en demandant $0.470<m(\gamma \gamma)<0.615 \mathrm{GeV} / \mathrm{c}^{2}$. Parce que la masse du $\eta$ est plus élevée que celle $d u \pi^{0}$, les photons issus du $\eta$ sont plus énergétiques, et donc mieux reconstruits.

L'efficacité de sélection finale est de 32.8\%. Cette analyse ayant été réalisée avant celle de $B^{0} \rightarrow \rho h$ qui a introduit les différentes composantes de signal correctement et mal reconstruit, seule une composante totale de signal est considérée par la suite ${ }^{4}$.

La lutte contre le bruit de fond continuum se fait en entraînant un réseau de neurones sur 12 variables topologiques utilisé dans l'ajustement par maximum de vraisemblance. Ce dernier utilise les variables $m_{\mathrm{ES}}, \Delta E, m(\eta)$ et $x_{\mathrm{NN}}$. La masse du $a_{0}$ n'est pas utilisée afin de l'utiliser comme variable de contrôle pour le signal. Le bruit de fond provenant d'autres désintégrations $B$ est bien plus faible que pour $B^{0} \rightarrow \rho^{ \pm} \pi^{\mp}$, puisque le $a_{0}$ a une plus petite largeur, et les photons issus du $\eta$ sont plus énergétiques.

Parmi les 9248 événements de données on-peak passant les coupures de sélection, on trouve $16.2_{-7.2}^{+8.6}$ événements de signal. Le niveau de confiance de l'ajustement est de $50 \%$. On en déduit donc le rapport d'embranchement suivant:

$$
\mathcal{B}\left(B^{0} \rightarrow a_{0}^{ \pm} \pi^{\mp}\right) \times \mathcal{B}\left(a_{0} \rightarrow \eta \pi\right)=\left(6.2_{-2.5}^{+3.0} \text { stat } \pm 1.1_{\text {syst }}\right) \times 10^{-6},
$$

où l'incertitude systématique est dominée par la reconstruction des $\eta$ et la contamination du mode $B^{0} \rightarrow a_{0} K$. La signification statistique du résultat est de $3.7 \sigma$. Dans les critères de $B A B A R$, elle n'est pas suffisante pour prétendre à une observation, et nous posons donc la limite supérieure sur le rapport d'embranchement suivante:

$$
\mathcal{B}\left(B^{0} \rightarrow a_{0}^{ \pm} \pi^{\mp}\right)<11.5 \times 10^{-6} \quad \text { à } \quad 90 \% \quad \mathrm{CL},
$$

incluant les incertitudes statistiques et systématiques.

La figure A.3 de gauche montre la projection de la variable

$$
x=\frac{\mathcal{L}(\text { signal })-\mathcal{L}(\mathrm{bdf})}{\mathcal{L}(\text { signal })+\mathcal{L}(\mathrm{bdf})}
$$

pour laquelle le signal pique à $x=+1$ et le bruit de fond à $x=-1$. La figure du bas montre un zoom dans la région de signal, où l'on voit un excès à $x=+1$ par rapport aux attentes de bruit de fond évaluées grâce aux données off-peak. La figure de droite montre la projection de la fonction de vraisemblance sur la masse du $a_{0}$, non utilisée dans l'ajustement final. On y voit un clair excès d'événements par rapport aux attentes de bruit de fond (dont la distribution est uniforme pour cette variable).

\footnotetext{
${ }^{3}$ Le $\eta$ se désintègre en $\gamma \gamma 39.43 \%$ du temps. Les autres modes de désintégration du $\eta$ incluent majoritairement $3 \pi^{0}$ et $\pi^{+} \pi^{-} \pi^{0}$.

${ }^{4} \mathrm{On}$ s'attendrait de toute façon à une fraction d'événements mal reconstruits bien plus faible que pour $B^{0} \rightarrow \rho^{ \pm} \pi^{\mp}$ puisque les photons sont plus énergétiques, et puisque la zone dans le diagramme de Dalitz où ces événements s'accumulaient pour $B^{0} \rightarrow \rho^{ \pm} \pi^{\mp}$ est en dehors des limites cinématiques pour cette désintégration.
} 

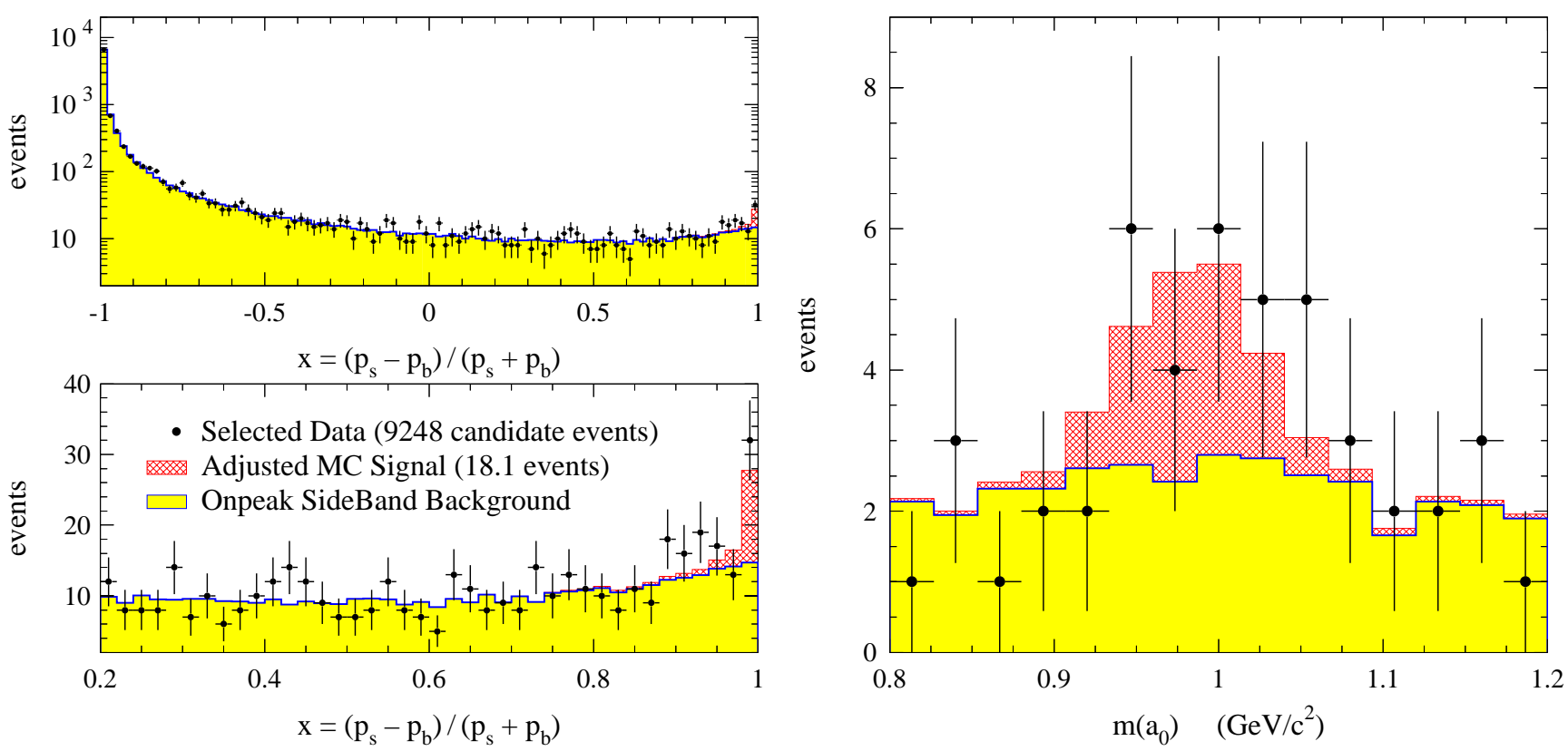

Figure A.3: Figure de gauche: projection de la variable $x$ (définie dans l'éq. A.10) dans les données on-peak (points avec barres d'erreur). Les contributions du bruit de fond continuum évaluées à partir des données off-peak sont montrées par l'histogramme jaune. La contribution de signal est colorée en rouge. La figure du bas montre un zoom dans la région de signal de celle du haut. Figure de droite: projection de la fonction de vraisemblance sur la masse du $a_{0}$. La contribution de bruit de fond est indiquée en jaune, et est uniforme pour cette variable. La contribution du signal est indiquée en rouge. 


\section{Annexe B}

\section{Variables pour la lutte contre le bruit de fond $q \bar{q}$}

Les variables considérées dans l'analyse $B^{0} \rightarrow \rho^{ \pm} h^{\mp}$ sont décrites dans la section 4.6. Celles non retenues sont décrites dans cette annexe. Les raisons pour lesquelles ces variables n'ont pas été retenues sont exposées.

\section{B.1 Variables cinématiques}

Les variables cinématiques considérées initiallement pour la lutte contre le bruit de fond continuum, mais non retenues dans l'analyse $B^{0} \rightarrow \rho^{ \pm} h^{\mp}$ sont les suivantes:

- $m\left(\pi^{+} \pi^{-}\right)$, la masse invariante des traces chargées. Cette variable est corrélée avec $\cos \theta_{H}(\rho)$ (voir Fig. 4.18)qui a été retenue dans l'analyse.

- $p_{\text {fast }}^{*}, p_{\text {slow }}^{*}$, respectivement les impulsions de la trace célibataire et du pion issu du $\rho$. Ces variables sont corrélées avec $m_{\mathrm{ES}}$ utilisée dans l'ajustement final.

- $\cos \left(\theta_{B_{T}, \pi \text { (slow) }}\right), \cos \left(\theta_{\left.B_{T}, \pi \text { (fast) }\right)}\right)$ cosinus de l'angle entre la direction du $B$ et la trace célibataire et issue du $\rho$, respectivement. La même remarque que pour $p_{\text {fast }}^{*}, p_{\text {slow }}^{*}$ s'applique quant à la corrélation avec $m_{\mathrm{ES}}$.

- $\Delta z$, distance entre les vertex des deux $B:$ nous utilisons déjà $\Delta t$ dans l'ajustement final.

\section{B.2 Variables topologiques}

Les variables topologiques considérées initialement pour la lutte contre le bruit de fond continuum, mais non retenues dans l'analyse $B^{0} \rightarrow \rho^{ \pm} h^{\mp}$ sont les suivantes:

- Les moments Super-Fox-Wolfram (SFW) (VpG), introduits par la collaboration Belle [140], sont définis par:

$$
R_{\mathrm{SFW}}=r_{0}+\sum_{\ell=2,4} \alpha_{\ell} R_{\ell}^{S O}+\sum_{\ell=1,2,3,4} \beta_{\ell} R_{\ell}^{O O}
$$

avec

$$
R_{\ell}^{X_{1} X_{2}}=\frac{H_{\ell}^{X_{1} X_{2}}}{H_{0}}
$$


où les exposants $S$ et $O$ représentent les traces du candidat $B$ et les traces du ROE, respectivement. Il s'agit de discriminants linéaires dont les coefficients $\alpha_{\ell}$ et $\beta_{\ell}$ sont optimisés pour l'analyse considérée. Les moments de Fox-Wolfram [141] $H_{\ell}^{X_{1} X_{2}}$ sont construits avec les polynômes de Legendre $P_{\ell}$ d'ordre $\ell$ :

$$
H_{\ell}^{X_{1} X_{2}}=\sum_{i \in X_{1}, j \in X_{2}}\left|p_{i}\right|\left|p_{j}\right| \times P_{\ell}\left(\cos \theta_{i j}\right) .
$$

La définition de $R_{\ell}^{S O}$ est proche d'une version de Legendre des monômes $L_{n}$, avec la différence cruciale que l'impulsion entre explicitement dans la définition du premier (VpG), alors que le second utilise la direction de la poussée du $B$ (VG): ainsi, les moments impairs $R_{\ell=1,3, \ldots}^{S O}$ sont corrélés de façon significative avec $m_{\mathrm{ES}}$ et $\Delta E$. Après optimisation de l'éq. (B.3) pour le Monte Carlo $\rho^{ \pm} \pi^{\mp}$ et les données continuum on-peak (en dehors de la région de signal), on obtient les valeurs des coefficients suivantes:

$$
\begin{array}{r}
r_{0}=0.64, \quad \alpha_{2}=-4.24, \quad \alpha_{4}=-0.26, \\
\beta_{1}=-0.01, \quad \beta_{2}=-0.36, \quad \beta_{3}=1.00, \quad \beta_{4}=1.21 .
\end{array}
$$

Les moments SFW correspondant aux coefficients précédents (B.4) sont représentés sur la figure 4.17 . Les performances de $R_{\mathrm{SFW}}$ sont dues principalement au deuxième moment (son coefficient est le plus important).

- Les 9 cônes de CLEO ( $\mathrm{VnG}$ ) mesurent l'énergie du ROE dans 9 cônes de 10 degrés chacun autour de l'axe de poussée du $B$ :

$$
\mathcal{C}^{j}=\sum_{i}^{\text {roe }} p_{i} \times \delta_{i}^{j}\left(\cos \theta_{i}\right)
$$

où $\cos \theta_{i}$ est le cosinus de l'angle entre la $i$-ème trace du ROE et l'axe de poussée du $B, p_{i}$ est l'impulsion de la $i$-ème trace, et $\delta_{i}^{j}$ prend la valeur 1 quand la $i$-ème trace est à l'intérieur du $j$-ème cône, et 0 sinon.

- $\left|\cos _{\max }\left(\tilde{\theta}_{T}\right)\right|(\mathrm{VpG})$, le maximum de la valeur absolue du cosinus de l'angle entre la trace célibataire ou le candidat $\rho$ et l'axe de poussée du ROE. Elle est corrélée à $99.9 \%$ avec $\left|\cos \left(\theta_{T}\right)\right|$, et l'utilisation simultanée de ces deux variables dans une analyse multidimensionnelle crée des corrélations avec $m_{\mathrm{ES}}$.

- La trace de plus grande, et deuxième plus grande impulsion du ROE, $p_{\max , \mathrm{ROE}}^{*}, p_{\max 2, \mathrm{ROE}}^{*}$ $(\mathrm{VnG})$. 


\section{Annexe C}

\section{Lutte contre le bruit de fond $B$ non charmé}

Nous exposons dans cette section une stratégie alternative à celle de l'analyse finale retenue dans $B A B A R$ qui consiste à élaborer un réseau de neurones de lutte contre le bruit de fond $B$ (en fait, un pour les 3 -corps, et un pour les 4-corps), et couper sur sa sortie pour rejeter ce bruit de fond. Le but est de réduire l'erreur systématique associée au bruit de fond $B$ en diminuant le nombre de modes $B$ à considérer. L'amélioration de l'erreur systématique se fait néanmoins au détriment de l'erreur statistique, puisque la coupure sur le réseau de neurones de lutte contre le bruit de fond $B$ diminue l'efficacité de sélection du signal. L'analyse étant actuellement dominée par les erreurs statistiques, nous n'avons pas retenu la stratégie présentée ici.

On a vu dans la section 4.3.3 que le bruit de fond $B$ en 2-corps peut être efficacement rejeté en coupant sur $m\left(\pi^{+} \pi^{-}\right)$. Le nombre restant d'événements des $B$ en 2-corps est faible et bien connu (voir tableau 4.13). Nous ne nous soucions donc pas de la lutte contre ce bruit de fond ici.

\section{C.1 Elaboration des réseaux de neurones}

Les figures C.1 et C.2 montrent les distributions des 11 variables retenues pour discriminer les bruits de fond $B$ en 3 - et 4-corps du signal: $m\left(\rho^{ \pm}\right), m\left(\pi^{0}\right), \cos \theta_{H}(\rho), \cos \theta_{H}\left(\pi^{0}\right), L_{0}, L_{2}$, $\cos \theta(B, z), m\left(\pi^{+} \pi^{-}\right), p_{\max 2, \mathrm{ROE}}^{*}, \Sigma_{\mathrm{pT}}, \cos \left(\theta_{B_{T}, \pi \text { (slow) }}\right){ }^{1}$.

Nous entraînons deux réseaux de neurones avec ces 11 variables sur un échantillon de signal $B^{0} \rightarrow \rho^{ \pm} \pi^{\mp}$ et de bruits de fond respectivement en 3 - et 4-corps. Le graphe de gauche de la figure C.3 montre l'efficacité de sélection du signal pour une coupure sur les sorties de ces deux réseaux de neurones, comparée à l'efficacité de sélection pour une coupure sur $\Delta E$ : les performances du réseau de neurones entraîné contre le bruit de fond 3-corps (4-corps) sont meilleures (similaires) à celles de $\Delta E$.

\footnotetext{
${ }^{1}$ Ces variables sont définies dans les sections 4.6.1 et 4.6.2, ainsi que dans l'Annexe B. Les critères de choix des variables sont les mêmes que pour la lutte contre le bruit de fond continuum.
} 

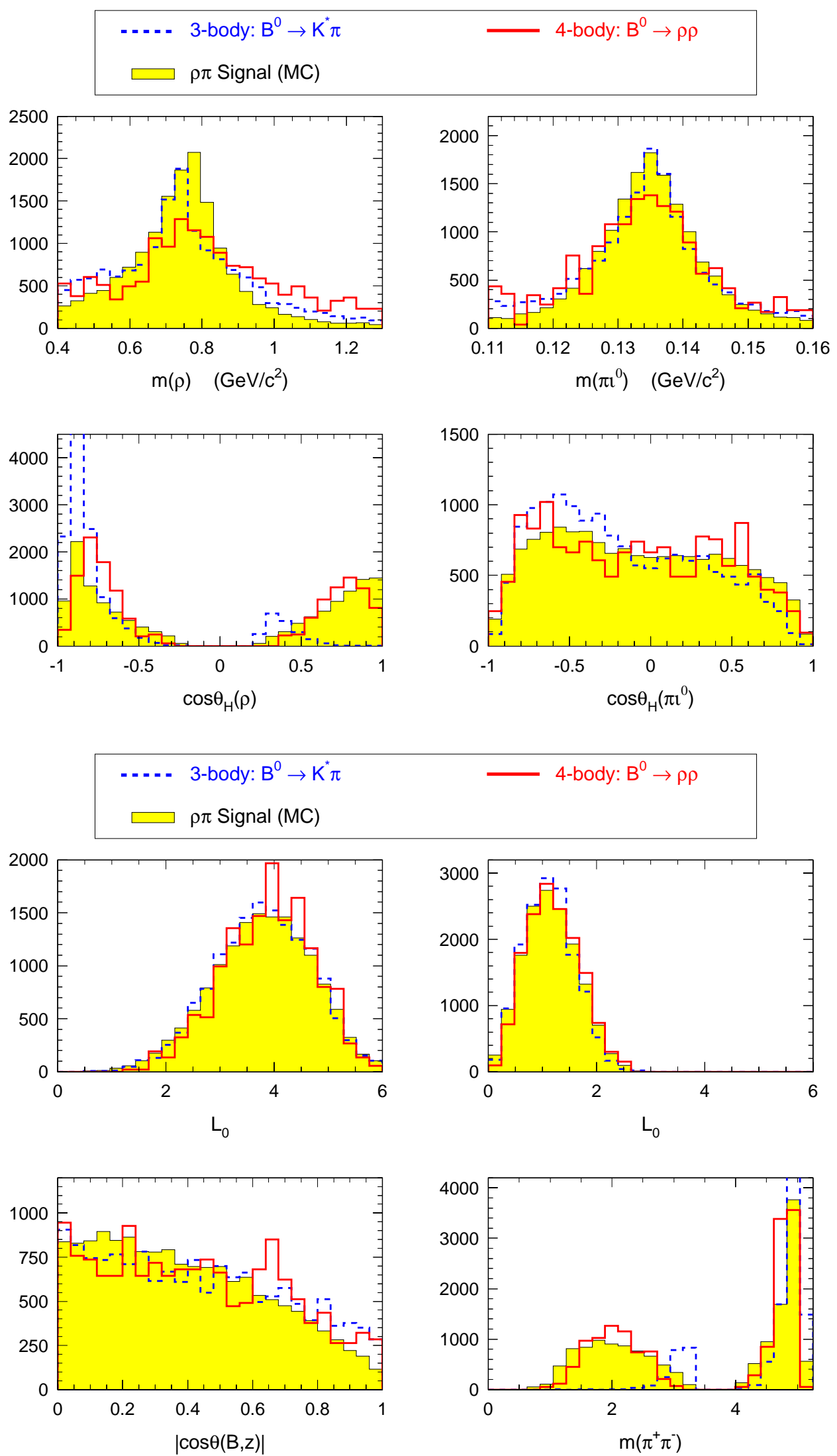

Figure C.1: Distributions de $m\left(\rho^{ \pm}\right), m\left(\pi^{0}\right), \cos \theta_{H}(\rho)$, et $\cos \theta_{H}\left(\pi^{0}\right)$ (figures du haut) et $L_{0}, L_{2}, \cos \theta(B, z)$ et $m\left(\pi^{+} \pi^{-}\right)$pour le signal $B^{0} \rightarrow \rho^{ \pm} \pi^{\mp}$ et les bruits de fond 3$\left(B^{0} \rightarrow K^{*+}\left(K^{+} \pi^{-}\right) \pi^{-}\right)$et 4 -corps $\left(B^{0} \rightarrow \rho^{+} \rho^{-}\right)$. 

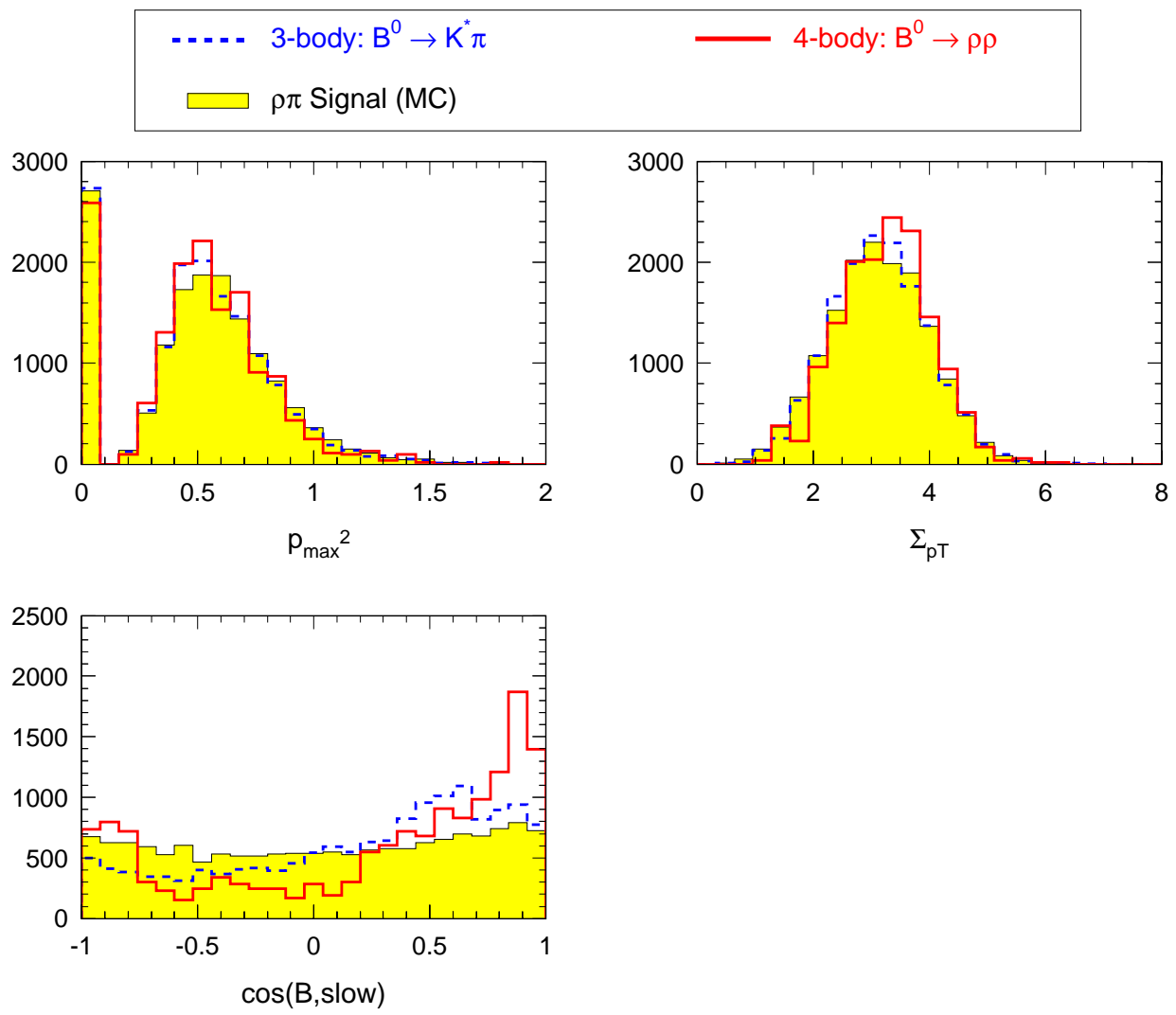

Figure C.2: Distributions de $p_{\max , \mathrm{ROE}}^{*}, \Sigma_{\mathrm{pT}}$ et $\cos \left(\theta_{B_{T}, \pi(\text { slow })}\right)$ pour le signal $B^{0} \rightarrow \rho^{ \pm} \pi^{\mp}$ et les bruits de fond $3-\left(B^{0} \rightarrow K^{*+}\left(K^{+} \pi^{-}\right) \pi^{-}\right)$et 4-corps $\left(B^{0} \rightarrow \rho^{+} \rho^{-}\right)$.

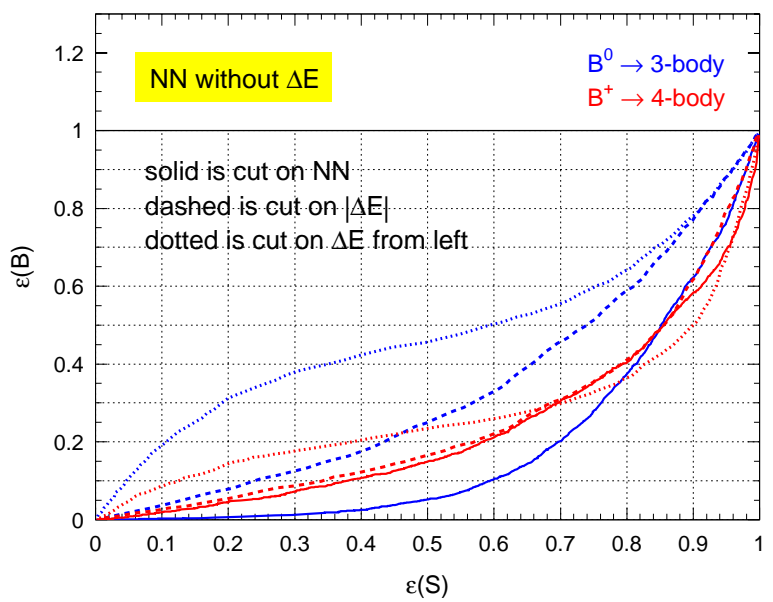

Figure C.3: Efficacité de sélection du signal $B^{0} \rightarrow \rho^{ \pm} h^{\mp}$ pour une coupure sur les sorties de ces deux réseaux de neurones (ligne pleine), comparée à l'efficacité de sélection pour une coupure sur $\Delta E$ soit symétrique (ligne en tirets), soit en partant de la gauche (ligne pointillée). 


\section{C.2 Incertitudes systématiques}

Nous avons réalisé des simulations rapides pour comparer les performances des deux méthodes suivantes:

- Scénario 1: pas d'utilisation des réseaux de neurones contre le bruit de fond $B$. On coupe sur la sortie du réseau de neurones contre le bruit de fond continuum à 0.08 .

- Scénario 2: utilisation des réseaux de neurones contre le bruit de fond $B$. Pour obtenir la même efficacité que dans le scénario 1, la coupure sur le réseau de neurones continuum est relâchée à -0.8 .

Les efficacités de sélection sont données dans le tableau C.1, ainsi que le nombre d'événements on-peak et $B^{0} \rightarrow \rho^{+} \rho^{-}$pour les deux scénarios envisagés.

\begin{tabular}{|c|c|c|c|c|}
\hline Scénario & $\epsilon(\rho \pi)(\%)$ & $\epsilon(\rho K)(\%)$ & $N_{\text {onpeak }}$ & $N_{\rho^{+} \rho^{-}}$ \\
\hline 1 & 29.82 & 26.9 & 39178 & 96.5 \\
2 & 29.9 & 26.3 & 94027 & 62.8 \\
\hline
\end{tabular}

Tablean C.1: Efficacités de sélection pour $B^{0} \rightarrow \rho^{ \pm} \pi^{\mp}$ et $B^{0} \rightarrow \rho^{-} K^{+}$, ainsi que le nombre d'événements onpeak et $B^{0} \rightarrow \rho^{+} \rho^{-}$pour les deux scénarios envisagés: sans (scénario 1 ) ou avec (scénario 2) les réseaux de neurones de lutte contre le bruit de fond $B$.

Nous calculons les erreurs systématiques pour les deux modes de bruits de fond dominants 3- et 4-corps: $B^{0} \rightarrow \rho^{+} \rho^{-}$long. et $B^{0} \rightarrow K^{*+}\left(K^{+} \pi^{0}\right) \pi^{-}$. L'erreur systématique est la différence entre une simulation rapide d'un ajustement de référence (dans lequel les nombres d'événements et autres coefficients reliés aux bruits de fond $B$ sont fixés aux valeurs centrales nominales pour la génération et l'ajustement), et celle d'un ajustement biaisé (dans lequel les coefficients de bruit de fond varient à l'intérieur de leurs erreurs).

Les résultats sont donnés dans le tableau C.2. L'erreur systématique est exprimée relativement à l'erreur statistique.

Les erreurs systématiques des paramètres $C P$ et de dilution peuvent être réduites de 30 à $40 \%$, et celles sur les nombres d'événements de $20 \%$ pour le mode $B^{0} \rightarrow \rho^{+} \rho^{-}$, mais ne sont pas réduites significativement pour $B^{0} \rightarrow K^{*+}\left(K^{+} \pi^{0}\right) \pi^{-}$. Ces diminutions des erreurs systématiques ne sont pas suffisantes ( $c f$. tableau 4.27: même avec ces diminutions, cela reste l'incertitude dominante de plusieurs paramètres). Pour obtenir une diminution conséquente, il faut sacrifier l'efficacité de sélection (comme indiqué sur la figure C.3), ce qui n'est pas souhaitable. 


\begin{tabular}{|c|c|c|c|c|c|c|c|c|c|c|}
\hline & $\begin{array}{c}\mathrm{Br} \\
+1 \sigma\end{array}$ & $\begin{array}{c}\mathrm{Br} \\
-1 \sigma\end{array}$ & $\begin{array}{l}C^{+} \\
\times 2 \\
\end{array}$ & $\begin{array}{l}C^{+} \\
=0\end{array}$ & $\begin{array}{l}C^{-} \\
\times 2 \\
\end{array}$ & $\begin{array}{l}C^{-} \\
=0\end{array}$ & $\begin{aligned} & S^{+} \\
= & -1\end{aligned}$ & $\begin{array}{c}S^{+} \\
=+1\end{array}$ & $\begin{aligned} & S^{-} \\
= & -1\end{aligned}$ & $\begin{array}{c}S^{-} \\
=+1\end{array}$ \\
\hline \multicolumn{11}{|l|}{ Scen.1 } \\
\hline \multicolumn{11}{|l|}{$B^{0} \rightarrow \rho^{+} \rho^{-}$} \\
\hline$C^{-}$ & 6.7 & 2.6 & 0.0 & 0.0 & 2.1 & 2.2 & 0.0 & 0.0 & 0.5 & 0.3 \\
\hline$C^{+}$ & 4.8 & 0.0 & 3.3 & 3.9 & 0.0 & 0.0 & 1.0 & 0.5 & 0.1 & 0.0 \\
\hline$S^{-}$ & 1.5 & 0.4 & 0.0 & 0.1 & 0.3 & 0.0 & 0.2 & 0.1 & 10.9 & 12.7 \\
\hline$S^{+}$ & 2.1 & 1.2 & 0.6 & 0.3 & 0.0 & 0.0 & 11.7 & 11.4 & 0.2 & 0.0 \\
\hline$N_{\rho \pi}$ & 21.2 & 19.7 & 0.3 & 0.0 & 0.0 & 0.1 & 0.1 & 0.4 & 0.2 & 0.6 \\
\hline$N_{\rho K}$ & 0.5 & 0.4 & 0.0 & 0.0 & 0.0 & 0.0 & 0.0 & 0.1 & 0.0 & 0.1 \\
\hline \multicolumn{11}{|l|}{ Scen.2 } \\
\hline \multicolumn{11}{|l|}{$B^{0} \rightarrow \rho^{+} \rho^{-}$} \\
\hline$C^{-}$ & 2.5 & 0.3 & 0.0 & 0.2 & 0.8 & 0.4 & 1.1 & 0.3 & 0.5 & 1.0 \\
\hline$C^{+}$ & 0.5 & 1.5 & 1.2 & 1.2 & 0.5 & 0.3 & 2.4 & 0.5 & 0.0 & 0.1 \\
\hline$S^{-}$ & 0.3 & 1.5 & 0.0 & 0.0 & 0.1 & 0.1 & 1.4 & 0.2 & 7.7 & 7.6 \\
\hline$S^{+}$ & 3.2 & 1.1 & 0.0 & 0.1 & 0.4 & 0.1 & 0.2 & 6.3 & 0.1 & 0.1 \\
\hline$N_{\rho \pi}$ & 16.5 & 15.4 & 0.1 & 0.1 & 0.9 & 5.8 & 4.3 & 0.7 & 0.2 & 0.5 \\
\hline$N_{\rho K}$ & 0.6 & 0.3 & 0.0 & 0.0 & 0.5 & 0.3 & 0.0 & 0.1 & 0.1 & 0.1 \\
\hline
\end{tabular}

\begin{tabular}{|c|cc|cc|}
\hline & $\begin{array}{c}\mathrm{Br} \\
+1 \sigma\end{array}$ & $\begin{array}{c}\mathrm{Br} \\
-1 \sigma\end{array}$ & $\begin{array}{c}A_{C P} \\
-0.5\end{array}$ & $\begin{array}{c}A_{C P} \\
+0.5\end{array}$ \\
\hline \hline Scen.1 & & & & \\
\hline$B^{0} \rightarrow K^{*+}\left(K^{+} \pi^{0}\right) \pi^{-}$ & & & & \\
$C^{-}$ & 2.7 & 2.3 & $\mathbf{9 . 8}$ & 7.7 \\
$C^{+}$ & 1.9 & 3.8 & $\mathbf{8 . 8}$ & $\mathbf{8 . 4}$ \\
$S^{-}$ & 0.6 & 0.2 & 0.8 & 0.4 \\
$S^{+}$ & 0.0 & 1.8 & 1.6 & 0.7 \\
$N_{\rho \pi}$ & $\mathbf{1 0 . 9}$ & $\mathbf{1 1 . 3}$ & 0.5 & 1.1 \\
$N_{\rho K}$ & 4.5 & 4.9 & 0.1 & 0.4 \\
\hline Scen.2 $K^{*+}\left(K^{+} \pi^{\mathbf{0}}\right) \pi^{-}$ & & & & \\
$C^{-}$ & 0.2 & 2.7 & $\mathbf{6 . 7}$ & 0.0 \\
$C^{+}$ & 1.4 & 2.1 & 7.6 & 7.5 \\
$S^{-}$ & $\mathbf{0 . 1}$ & 0.3 & 1.7 & $\mathbf{0 . 1}$ \\
$S^{+}$ & $\mathbf{0 . 1}$ & $\mathbf{0 . 4}$ & $\mathbf{0 . 0}$ & 1.0 \\
$N_{\rho \pi}$ & $\mathbf{1 0 . 3}$ & $\mathbf{1 0 . 4}$ & $\mathbf{0 . 6}$ & 1.2 \\
$N_{\rho K}$ & 5.1 & 5.3 & $\mathbf{0 . 2}$ & $\mathbf{0 . 6}$ \\
\hline \hline$B^{0} \rightarrow K^{-}$
\end{tabular}

Tablea1 C.2: Incertitudes systématiques relatives (en \% de l'erreur statistique), obtenues par la comparaison d'une simulation rapide d'un ajustement de référence (dans lequel la génération et l'ajustement sont cohérents), avec celle d'un ajustement biaisé (dans lequel la génération et l'ajustement ne sont pas cohérents - voir le texte) pour les deux modes dominants de bruits de fond $B: B^{0} \rightarrow \rho^{+} \rho^{-}$long. (tableau du haut) et $B^{0} \rightarrow K^{*+}\left(K^{+} \pi^{0}\right) \pi^{-}$ (tableau du bas). Deux scenarii sont étudiés: sans (scénario 1) et avec (scénario 2) coupures sur les réseaux de neurones de lutte contre le bruit de fond $B$. 


\section{Annexe D}

\section{Rapport d'embranchement des bruits de fond $B$ non charmés}

La détermination des rapports d'embranchement des modes de bruits de fond $B$ non charmés est une étape essentielle pour la crédibilité du résultat de l'analyse $B^{0} \rightarrow \rho h$ : l'erreur systématique sur les rapports d'embranchement et les paramètres $C P$ et de dilution est en effet dominée par le bruit de fond $B$ (cf. section 4.14.7). D'autre part, ce bruit de fond soustrait 67.8 événements au signal $B^{0} \rightarrow \rho^{ \pm} \pi^{\mp}$ (soit 13.6\%), et 51.4 événements au signal $B^{0} \rightarrow \rho^{-} K^{+}$(soit $30 \%$ ). Il est donc important de maîtriser leurs rapports d'embranchement.

L'évaluation de ceux-ci est détaillée ci-dessous:

- Le rapport d'embranchement de $B^{+} \rightarrow \pi^{0} \rho^{+}$est estimé à partir de ceux de $B^{+} \rightarrow \pi^{+} \rho^{0}$ $\left((9.5 \pm 2.0) \cdot 10^{-6}\right)[142,143,144]$ et $B^{0} \rightarrow \pi^{ \pm} \rho^{\mp}$ en utilisant la symétrie d'isospin qui mène à la relation ( $c f$. Eq. 2.5$)$ :

$$
\sqrt{2}\left(A^{+0}+A^{0+}\right)=2 A^{00}+A^{+-}+A^{-+} \text {. }
$$

On fait l'hypothèse que $A^{00} \sim 0$, et on néglige les interférences entre amplitudes. On obtient alors la relation grossière: $\mathcal{B}\left(B^{+} \rightarrow \pi^{0} \rho^{+}\right)+\mathcal{B}\left(B^{+} \rightarrow \pi^{+} \rho^{0}\right) \simeq 1 / 2 \mathcal{B}\left(B^{0} \rightarrow\right.$ $\left.\pi^{ \pm} \rho^{\mp}\right)$. On utilise donc l'intervalle $\mathcal{B}\left(B^{+} \rightarrow \pi^{0} \rho^{+}\right) \sim(5-25) \cdot 10^{-6}$.

- On peut estimer le rapport d'embranchement de $B^{0} \rightarrow \rho^{+} \rho^{-}$de plusieurs façons:

- à partir de $\mathcal{B}\left(B^{0} \rightarrow \rho^{ \pm} \pi^{\mp}\right)$ en utilisant le rapport des constantes de désintégration du $\rho$ et du $\pi:\left(f_{\rho} / f_{\pi}\right)^{2} \sim 2.4$. C'est une bonne approximation quand le $\rho / \pi$ est issu du vertex du $W$, et dans la limite de la factorisation naïve. Cette approximation ne s'applique pas très bien pour le rapport $\mathcal{B}\left(B^{0} \rightarrow \rho^{+} \pi^{-}\right) / \mathcal{B}\left(B^{0} \rightarrow \pi^{+} \pi^{-}\right)$qui vaut 4.7: ceci est interprété comme étant dû aux interférences destructives entre les diagrammes en arbre et pingouins dans l'amplitude du mode $B^{0} \rightarrow \pi^{+} \pi^{-}$.

- en utilisant la symétrie d'isospin, et en négligeant la contribution de $B^{0} \rightarrow \rho^{0} \rho^{0}$ : on obtient $\mathcal{B}\left(B^{0} \rightarrow \rho^{+} \rho^{-}\right) \sim 2 \cdot \mathcal{B}\left(B^{+} \rightarrow \rho^{+} \rho^{0}\right)$. Deux mesures de $\mathcal{B}\left(B^{+} \rightarrow \rho^{+} \rho^{0}\right)$ sont disponibles: $\mathcal{B}\left(B^{+} \rightarrow \rho^{+} \rho^{0}\right)=\left(10.8_{-2.8}^{+2.9} \pm 3.3\right) \cdot 10^{-6}$ (par BABAR, résultat non publié) [145] et $\mathcal{B}\left(B^{+} \rightarrow \rho^{+} \rho^{0}\right)=\left(38.5 \pm 10.9_{-5.4-7.5}^{+5.9+2.5}\right) \cdot 10^{-6}$ (par Belle) [146], ce qui mène à l'intervalle $\mathcal{B}\left(B^{0} \rightarrow \rho^{+} \rho^{-}\right) \sim(20-80) \cdot 10^{-6}$. Encore une fois, l'approximation précédente n'est pas vérifiée avec une grande précision dans le cas de la désintégration $B^{0} \rightarrow \pi \pi$ où l'on a plutôt: $\mathcal{B}\left(B^{0} \rightarrow \pi^{+} \pi^{-}\right) \simeq 0.9$. $\mathcal{B}\left(B^{+} \rightarrow \pi^{+} \pi^{0}\right)$, suggérant que les contributions des diagrammes pingouins sont non négligeables. 
En tenant compte de ces arguments, nous utilisons l'intervalle $\mathcal{B}\left(B^{0} \rightarrow \rho^{+} \rho^{-}\right)=(40 \pm$ $30) \cdot 10^{-6}$.

Pour le mode $B^{+} \rightarrow \rho^{+} \rho^{0}$, nous augmentons les erreurs expérimentales des rapports d'embranchement donnés ci-dessus, afin de tenir compte de leur compatibilité marginale. Nous utilisons $(22.5 \pm 17.5) \cdot 10^{-6}$.

Enfin, $B^{0} \rightarrow \rho^{0} \rho^{0}$ est attendu supprimé de couleur, et nous utilisons l'intervalle (07) $\cdot 10^{-6}$.

- Les modes $B \rightarrow a_{1} \pi$ ( $B$ chargé et neutre) n'ont pas encore été observés. Le $a_{1}$ se désintègre pour $50 \%$ en $\rho^{0} \pi^{+} \rightarrow 2 \pi^{+} \pi^{-}$et pour $50 \%$ en $\rho^{+} \pi^{0} \rightarrow \pi^{+} 2 \pi^{0}$. Le facteur de forme du $a_{1}$ est plus grand que celui du $\rho$. On suppose donc l'intervalle $(10-50) \cdot 10^{-6}$ pour $B^{+} \rightarrow\left(a_{1} \pi\right)^{+}$et $B^{0} \rightarrow\left(a_{1} \pi\right)^{0}$.

- Les mode $B \rightarrow \eta^{\prime} X$ sont dominés par les diagrammes pingouins. Le $\eta$ peut se désintégrer en $\eta^{\prime} \rightarrow \rho \gamma(\mathcal{B}=(29.9 \pm 1.0) \%$, en incluant la contribution non résonante $\pi^{+} \pi^{-} \gamma$ ) [44], ce qui mène à un état final proche de celui de notre signal. Expérimentalement, on observe que le taux du mode $B^{+} \rightarrow \eta^{\prime} K^{+}$est (anormalement) 6 fois plus grand que celui du mode $B^{+} \rightarrow \pi^{0} K^{+1}$. En ce qui concerne les autres modes, la plupart ont été observés, ou une limite a été posée. On utilise les moyennes mondiales prises des références [149].

- Les modes $B \rightarrow \eta \pi / \rho$ ont été mesurés [149, 154]. Seuls ceux impliquant un $\rho$ produisent un bruit de fond suffisamment important pour être considérés.

- Nous supposons les modes $B \rightarrow K \pi, B \rightarrow K^{*} \pi, B \rightarrow K \rho, B \rightarrow K^{*} \rho$ dominés par les diagrammes pingouins, impliquant $\Delta I=I_{f}-I_{i}=1 / 2$, où $I_{i}\left(I_{f}\right)$ est l'isospin de l'état initial (final). On peut ainsi relier ces modes entre eux:

$$
\begin{aligned}
\mathcal{B}\left(B^{0} \rightarrow K^{+} \pi^{-}\right) & =\mathcal{B}\left(B^{+} \rightarrow K^{0} \pi^{+}\right) \\
& =2 \times \mathcal{B}\left(B^{+} \rightarrow K^{+} \pi^{0}\right) \\
& =2 \times \mathcal{B}\left(B^{0} \rightarrow K^{0} \pi^{0}\right),
\end{aligned}
$$

et de même pour les modes où l'on a remplacé $K \rightarrow K^{*}$ et $\pi \rightarrow \rho$. On suit la hiérarchie observée dans les modes $\mathcal{B}\left(B^{0} \rightarrow K^{+} \pi^{-}\right)>\mathcal{B}\left(B^{0} \rightarrow \pi^{+} \pi^{-}\right)>\mathcal{B}\left(B^{0} \rightarrow K^{+} K^{-}\right)$, pour déduire des intervalles de rapports d'embranchement aux modes où $K \rightarrow K^{*}$ et $\pi \rightarrow \rho$.

- Les modes qui impliquent deux kaons, $B \rightarrow K^{(*)} K^{(*) \prime}$ sont produits uniquement par diagrammes d'échange (pour les $B$ neutres) et/ou d'annihilation (pour les $B$ chargés) qui sont supprimés par un facteur $\left(f_{B} / m_{b}\right)^{2} \sim 0.002$, ou à travers des interactions dans l'état final. Aucun de ces modes n'a encore été observé, et la meilleure limite supérieure à ce jour est $\mathcal{B}\left(B \rightarrow K^{+} K^{-}\right)<0.6 \cdot 10^{-6}(B A B A R)$ [147]. D'autre part, l'efficacité de sélection de ces modes est faible, et ils ne contaminent donc pas notre signal.

- Les rapports d'embranchement des désintégrations radiatives $B \rightarrow K^{*} \gamma$ ont été mesurés [148]: $\mathcal{B}\left(B^{+} \rightarrow K^{*+} \gamma\right)=(40.8 \pm 3.8) \cdot 10^{-6}$ et $\mathcal{B}\left(B^{0} \rightarrow K^{* 0} \gamma\right)=(40.2 \pm 2.7) \cdot 10^{-6}$. Ils ne contaminent pas notre signal non plus.

\footnotetext{
${ }^{1}$ Plusieurs explications sont avancées: singlet de saveur [150,151], termes reliés au charme plus grand que prévu [152], ou des interférences avec le diagramme $b \rightarrow K$ [153].
} 
- Certains des modes $B \rightarrow K^{*} \pi$ mènent au même état final que le signal $B^{0} \rightarrow \rho^{-} K^{+}$: $B^{0} \rightarrow K^{*+}\left(\rightarrow K^{+} \pi^{0}\right) \pi^{-}$et $B^{0} \rightarrow K^{* 0}\left(\rightarrow K^{+} \pi^{-}\right) \pi^{0}$. Ces modes peuvent aussi contaminer $B^{0} \rightarrow \rho^{ \pm} \pi^{\mp}$ si le kaon est identifié comme un pion. Deux des modes $B \rightarrow K^{*} \pi$ ont été mesurés: $\mathcal{B}\left(B^{+} \rightarrow K^{* 0}\left(\rightarrow K^{+} \pi^{-}\right) \pi^{+}\right)=(10.3 \pm 2.6) \cdot 10^{-6}$ (BABAR [156]), $\mathcal{B}\left(B^{+} \rightarrow K^{* 0}\left(\rightarrow K^{+} \pi^{-}\right) \pi^{+}\right)=(12.9 \pm 5.4) \cdot 10^{-6}$ (Belle [159]), et $\mathcal{B}\left(B^{0} \rightarrow K^{*+}(\rightarrow\right.$ $\left.\left.K^{+} \pi^{0}\right) \pi^{-}\right)=(8.7 \pm 3.0) \cdot 10^{-6}$ (Belle [157]). On utilise l'éq. D.2 pour déduire les rapports de branchement des autres modes à partir de ces mesures. Notons que cette équation relie aussi les deux modes mesurés entre eux, mais les mesures ne la respectent que marginalement. L'existence du $\kappa(797)$ peut modifier l'interprétation de ces mesures ( $c f$. Ref. [158]). Pour ces raisons, nous assignons des incertitudes généreuses sur les rapports d'embranchements déduits de ces mesures.

- Les désintégrations du $B$ comportant une résonance kaonique de masse élevée ( $c$-à$d$, les $K^{*}(1430)$ et $K^{*}(1680)$, que l'on dénotera par $K_{X}^{(* *)}$ ) peuvent aussi contaminer $B^{0} \rightarrow \rho^{-} K^{+}$. Généralement, ces résonances se désintègrent en cascade jusqu'à l'état le plus stable, comme dans $K_{2}^{*+}(1430) \rightarrow K^{*+} \pi^{0}, K^{*+} \rightarrow K^{+} \pi^{0}$ avec $\mathcal{B}\left(K_{2}^{*}(1430) \rightarrow\right.$ $\left.K^{*} \pi\right)=(24.7 \pm 1.5) \%$ [44]. Dans ce cas, l'état final est à 4-corps (ou plus), et peuple donc la région $\Delta E<0$. Mais certaines de ces résonances kaoniques, comme par exemple les $K_{0}^{*}(1430), K_{2}^{*}(1430)$ et $K^{*}(1680)$, se désintègrent directement vers l'état le plus stable: e.g., $\mathcal{B}\left(K_{2}^{*}(1430) \rightarrow K \pi\right)=(49.9 \pm 1.2) \%$ [44]. Dans ce cas, l'état final obtenu est le même que dans $B^{0} \rightarrow \rho^{-} K^{+}$.

Il existe très peu d'information expérimentale ou théorique sur les rapports de branchement des modes $B \rightarrow K_{X}^{(* *)} \pi$. Notre point de départ est la mesure des taux inclusifs $B^{0} \rightarrow K^{+} \pi^{-} \pi^{0}$ et $B^{+} \rightarrow K^{+} \pi^{-} \pi^{+}$:

$$
\begin{aligned}
\mathcal{B}\left(B^{0} \rightarrow K^{+} \pi^{-} \pi^{0}\right) & =(35.6 \pm 9.6) \cdot 10^{-6}(\text { Belle }[157]), \\
\mathcal{B}\left(B^{+} \rightarrow K^{+} \pi^{-} \pi^{+}\right) & =(55.6 \pm 9.6) \cdot 10^{-6}(\text { Belle }[159]), \\
\mathcal{B}\left(B^{+} \rightarrow K^{+} \pi^{-} \pi^{+}\right) & =(59.1 \pm 5.0) \cdot 10^{-6}(\text { BABAR }[160]) .
\end{aligned}
$$

Le taux inclusif $B^{0} \rightarrow K^{+} \pi^{-} \pi^{0}$ est la somme des rapports d'embranchement des modes $B^{0} \rightarrow K_{X}^{(* *)} \pi$ et des modes autres que $B^{0} \rightarrow K_{X}^{(* *)} \pi$, dont la plupart ont été mesurés (voir tableau 4.11). Parmi eux, les modes $B^{0} \rightarrow K^{*} \pi$ ont été discutés au point précédent. Le signal $B^{0} \rightarrow \rho^{-} K^{+}$contribue aussi ${ }^{2}$. Par soustraction, nous arrivons à l'estimation suivante:

$$
\mathcal{B}\left(B^{0} \rightarrow K_{X}^{(* *)} \pi \rightarrow K^{+} \pi^{-} \pi^{0}\right)=(20 \pm 15) \cdot 10^{-6},
$$

où nous avons assigné une erreur systématique généreuse. La contamination des modes $B^{0} \rightarrow K_{X}^{(* *)} \pi \rightarrow K^{+} \pi^{-} \pi^{0}$ vers $B^{0} \rightarrow \rho^{ \pm} \pi^{\mp}$ est faible, puisque les masses des résonances $K_{X}^{(* *)}$ sont beaucoup plus élevées que la masse du $\rho$. Quand le mode $B^{0} \rightarrow K_{X}^{(* *)} \pi \rightarrow$ $K^{+} \pi^{-} \pi^{0}$ est reconstruit en $B^{0} \rightarrow \rho^{-} K^{+}$, le candidat $\rho$ est composé d'un pion provenant de la désintégration du $K_{X}^{(* *)}$, et un autre pion célibataire. Dans ce cas, le fait que la masse du $K_{X}^{(* *)}$ soit égale à $1400 \mathrm{GeV} / c^{2}$ ou $1700 \mathrm{GeV} / c^{2}$, de même que sa valeur de spin importent peu.

\footnotetext{
${ }^{2}$ Comme notre mesure de $B^{0} \rightarrow \rho^{-} K^{+}$dépend du taux de bruit de fond $B$ que nous lui assignons, nous devrions procéder itérativement à l'estimation de $B \rightarrow K_{X}^{(* *)} \pi$ par soustraction. Néanmoins, comme sa contribution ne représente qu'une petite fraction du taux inclusif total, nous nous contentons d'utiliser la mesure déjà existante de $B^{0} \rightarrow \rho^{-} K^{+}[49]$.
} 
Nous devons aussi tenir compte du bruit de fond provenant des modes $B^{0} \rightarrow K_{X}^{(* *)} \pi \rightarrow$ (état 3-corps autre que $K^{+} \pi^{-} \pi^{+}$) et $B^{0} \rightarrow K_{X}^{(* *)} \pi \rightarrow$ (> état 3-corps), que l'on dénotera de façon collective par $B^{0} \rightarrow K_{X}^{(* *)} \pi \rightarrow$ (autre que $K^{+} \pi^{-} \pi^{+}$). On leur attribue un taux à partir de celui de $\mathcal{B}\left(B^{0} \rightarrow K_{X}^{(* *)} \pi \rightarrow K^{+} \pi^{-} \pi^{0}\right)$. L'incertitude sur $\mathcal{B}\left(B^{0} \rightarrow\right.$ $K_{X}^{(* *)} \pi \rightarrow$ (autre que $\left.K^{+} \pi^{-} \pi^{+}\right)$) provient de l'incertitude sur $\mathcal{B}\left(B^{0} \rightarrow K_{X}^{(* *)} \pi \rightarrow\right.$ $K^{+} \pi^{-} \pi^{0}$ ) et sur l'abondance relative des différentes résonances $K_{X}^{(* *)}$, puisque les rapports d'embranchement des différentes désintégrations du $K_{X}^{(* *)}$ dépendent de la résonance considérée. Pour l'ajustement nominal, on suppose que les seules résonances $K_{X}^{(* *)}$ contribuant à $B^{0} \rightarrow K_{X}^{(* *)} \pi$ sont le $K_{0}^{*}(1430)$, le $K_{2}^{*}(1430)$ et le $K^{*}(1680)$, et on suppose qu'elles contribuent avec le même poids. Suivant la hiérarchie dictée par l'éq. D.2, on suppose que $\mathcal{B}\left(B^{0} \rightarrow K_{X}^{(* *)+} \pi^{-}\right)=2 \cdot \mathcal{B}\left(B^{0} \rightarrow K_{X}^{(* *) 0} \pi^{0}\right)$. En utilisant les rapports d'embranchement des désintégrations des $K_{0}^{*}(1430), K_{2}^{*}(1430)$ et $K^{*}(1680)$ [44], on obtient

$$
\mathcal{B}\left(B^{0} \rightarrow K_{X}^{(* *)} \pi \rightarrow\left(\text { autre que } K^{+} \pi^{-} \pi^{+}\right)\right)=(52 \pm 52) \cdot 10^{-6} .
$$

Les distributions de référence pour $B^{0} \rightarrow K_{X}^{(* *)} \pi$ sont obtenues à partir des Monte Carlo de $B^{0} \rightarrow K_{0}^{(* *)+}(1430) \pi^{-}, K_{0}^{(* *) 0}(1430) \pi^{0}, B^{0} \rightarrow K_{2}^{(* *)+}(1430) \pi^{-}, K_{2}^{(* *) 0}(1430) \pi^{0}$ et $B^{0} \rightarrow K^{(* *)+}(1680) \pi^{-}, K^{(* *) 0}(1680) \pi^{0}$.

Pour traduire le taux inclusif de $B^{+} \rightarrow K^{+} \pi^{-} \pi^{+}$en une estimation du bruit de fond pour $B^{+} \rightarrow K_{X}^{(* *)} \pi$, nous procédons de la même manière que pour $B^{0} \rightarrow K_{X}^{(* *)} \pi$, à la différence qu'il existe une famille de résonances supplémentaire: $B^{+} \rightarrow f_{0}(980)(\rightarrow$ $\left.\pi^{+} \pi^{-}\right) K^{+}$et $B^{+} \rightarrow f_{X}(1300)\left(\rightarrow \pi^{+} \pi^{-}\right) K^{+}$. Les analyses des Refs. [157] et [160] obtiennent un taux inclusif de $B^{+} \rightarrow K^{+} \pi^{-} \pi^{+}$, mais aussi des contributions des résonances $\rho^{0} K^{+}, K^{* 0} \pi^{+}, K_{X}^{(* *) 0} \pi^{+}, f_{0}(980) K^{+}$et $f_{X}(1300) K^{+}$. Notre modèle (voir tableau 4.11) est basé sur ces résultats et l'hypothèse que seules les résonances $K_{0}^{*}(1430), K_{2}^{*}(1430)$ et $K^{*}(1680)$ contribuent en abondance relative égale. Les contributions des désintégrations autres que $K_{X}^{(* *) 0} \rightarrow K^{+} \pi^{-}$sont estimées de la même façon que dans le cas de $B^{0} \rightarrow K_{X}^{(* *)} \pi$

- La référence [145] donne une mesure du rapport d'embranchement $\mathcal{B}\left(B^{+} \rightarrow \rho^{0} K^{*+}\right)=$ $\left(10.1_{-2.2}^{+2.5} \pm 2.3\right) \cdot 10^{-6}$, avec l'hypothèse que les contributions des polarisations longitudinales et transverses sont de $50 \%$ chacune. Pour tenir compte de l'incertitude sur cette hypothèse, nous utilisons un intervalle élargi: $\mathcal{B}\left(B^{+} \rightarrow \rho^{0} K^{*+}\right) \sim(0-18) \cdot 10^{-6}$. Les taux des états finals possédant des combinaisons de charge différentes sont déduits de cet intervalle en utilisant l'éq. D.2.

- Pour estimer le bruit de fond de $B \rightarrow K_{X}^{(* *)} \rho$, nous supposons que $\mathcal{B}\left(B^{0} \rightarrow K_{X}^{(* *)} \rho\right)=$ $\mathcal{B}\left(B^{0} \rightarrow K_{X}^{(* *)} \pi\right)$ et $\mathcal{B}\left(B^{+} \rightarrow K_{X}^{(* *)} \rho\right)=\mathcal{B}\left(B^{+} \rightarrow K_{X}^{(* *)} \pi\right)$, et assignons une incertitude de $100 \%$ sur les taux obtenus. Seules les désintégrations de $K_{X}^{(* *)}$ en $K^{+} \pi^{-} \pi^{0}$ et $K^{+} \pi^{-} \pi^{+}$sont prises en compte. Le nombre d'événements de bruit de fond attendu pour ces modes est très petit (voir tableau 4.11 ).

- Des contributions non résonantes peuvent être présentes pour tous les états finals des désintégrations $B \rightarrow 3 / 4$ - corps. Les efficacités de sélection sont en général beaucoup plus faibles que celles des contributions résonantes. En supposant un rapport d'embranchement pour ces contributions compris entre 0 et $1 \cdot 10^{-5}$, nous ne trouvons aucune contribution significative provenant des modes non résonants. 


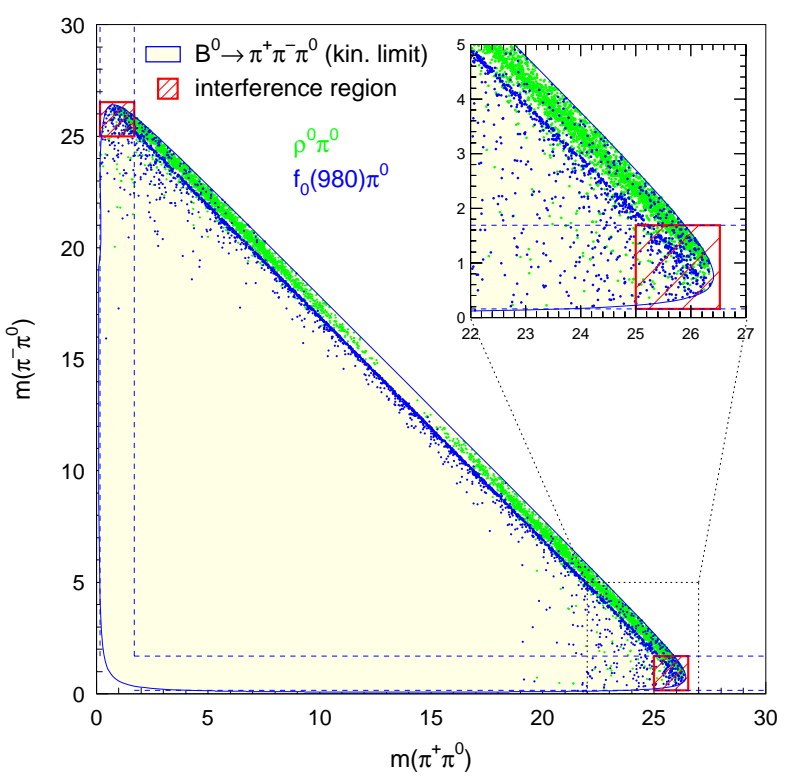

Figure D.1: Diagramme Dalitz pour la désintégration $B^{-} \rightarrow \pi^{+} \pi^{-} \pi^{0}$. La zone ombrée montre les limites cinématiques. Les points verts correspondent aux événements $B^{0} \rightarrow \rho^{0}(\rightarrow$ $\left.\pi^{+} \pi^{-}\right) \pi^{0}$, et les points bleus à ceux de $B^{0} \rightarrow f_{0}(980)\left(\rightarrow \pi^{+} \pi^{-}\right) \pi^{0}$.

- Des modes avec des résonances scalaires peuvent aussi contribuer à l'état final $\pi^{+} \pi^{-} \pi^{0}$ (cf. chapitre 6), comme $B^{0} \rightarrow f_{0}(980)\left(\rightarrow \pi^{+} \pi^{-}\right) \pi^{0}$, qui n'a pas encore été observé. Une autre résonance scalaire candidate est la résonance large $f_{0}(400-1200)$, (souvent appelée $\sigma$ dans la littérature). La collaboration E791 [161] rapporte des contributions significatives des résonances $f_{0}(980,1270,1370)$ dans les désintégrations des mésons charmés $D_{s}^{+} \rightarrow \pi^{+} \pi^{-} \pi^{+}$et $D^{+} \rightarrow \pi^{+} \pi^{-} \pi^{+}$. Ils obtiennent aussi un meilleur ajustement du diagramme Dalitz quand environ la moitié des événements $D^{+} \rightarrow \pi^{+} \pi^{-} \pi^{+}$est supposée provenir de la résonance intermédiaire $\sigma(500)$. Ils mesurent $m(\sigma)=478_{-23}^{+24} \pm$ $17 \mathrm{MeV} / c^{2}$ et $\Gamma(\sigma)=324_{-40}^{+42} \pm 21 \mathrm{MeV} / c^{2}$.

Les contributions scalaires $f_{0}$ et $\sigma$ sont moins dangereuses que les contributions vecteurs (comme le $\left.\rho^{0}\right)$ : comme indiqué sur la figure D.1, l'hélicité nulle des résonances scalaires ne les pousse pas à peupler les coins du plan Dalitz peuplés par les $\rho^{ \pm}$(comme c'est le cas pour le $\rho^{0}$ ) avec lesquelles elles pourraient interférer. Nous observons que l'efficacité $\epsilon\left(\rho^{0} \pi^{0}\right)=2.8 \%$ est plus élevée que $\epsilon\left(f_{0}(980) \pi^{0}\right)=1.5 \%$. Cette dernière efficacité est proche de celle de la contribution non résonante $B^{0} \rightarrow \pi^{+} \pi^{-} \pi^{0}$. Nous nous servons donc de la limite supérieure posée sur cette contribution dans la Ref. [89] pour estimer l'erreur systématique associée à une contribution scalaire. 


\section{Bibliographie}

[1] C. Geweniger et al., Phys. Lett. B 48, 487 (1974); A. Apostolakis et al., Phys. Lett. B 458, 545 (1999); A. Angelopoulos et al., Phys. Lett. B 420, 191 (1998); J. H. Christenson et al., Phys. Rev. Lett. 43, 1209 (1979)

[2] M. Kobayashi, T. Maskawa, Prog. Theor. Phys 49, 652 (1973)

[3] V.A. Kuzmin, V.A. Rubakov et M.E. Shaposhnikov, Phys. Lett. B 155, 36 (1985);

S.M. Barr, G. Segre et H.A. Weddon, Phys. Rev. D 20, 2494 (1979)

[4] Petit Robert, dictionnaire de la langue française

[5] N. Cabibbo, Phys. Rev. Lett. 10, 531 (1963)

[6] C. Jarlskog, Phys. Rev. Lett. 55, 1039 (1985)

[7] L. Wolfenstein, Phys. Rev. Lett. 51, 1945 (1983)

[8] A.J. Buras, M.E. Lautenbacher and G. Ostermaier,Phys. Rev. D 50, 3433 (1994)

[9] R. Aleksan, proceedings de l'école d'été de physique des particules de Gif, 1991

[10] S.L. Glashow, J. Iliopoulos et L. Maiani,Phys. Rev. D 2, 1285 (1970)

[11] A. Einstein, B. Podolsky, N. Rosen, Phys. Rev. 41, 777 (1935)

[12] Voir les deux revues suivantes, et les références à l'intérieur: H. Quinn, conférence pour la Médaille Dirac, hep-ph/0110050; M. Dine, Cours de TASI 2000, hep-ph/0011376.

[13] Collaboration KTeV, A. Alavi-Harati et al., hep-ex/0208007, soumit à Phys.Rev.D; Collaboration NA48, G. Unal et al., hep-ex/0209064

[14] B. Aubert et al., Collaboration BABAR, hep-ex/0207042, soumit à Phys.Rev.Lett.; K. Abe et al., Collaboration Belle, hep-ex/0208025, soumis à Phys.Rev.D

[15] A. Höcker, H. Lacker, S. Laplace et F. Le Diberder, Eur.Phys.J. C 21, 225 (2001), et résultats récents sur la page "http://www.slac.stanford.edu/ laplace/ckmfitter.html"

[16] B. Aubert et al., Collaboration BABAR, hep-ex/0207070; pour la collaboration Belle, voir Ref. [14]

[17] G. Hiller, Phys. Rev. D 66, 071502 (2002)

[18] K. Abe et al., Collaboration Belle, hep-ex/0301032

[19] B. Aubert et al., Collaboration BABAR, hep-ex/0207055 
[20] L. Roos et al., BABAR Analysis Document \#519;

"http: //www.slac.stanford.edu/laplace/ckmfitter/ckm_charmless.html"

[21] S. Gardner, Phys. Rev. D 59, 077502 (1999)

[22] G. Buchalla, A.J. Buras, M.E. Lautenbacher, Rev. Mod. Phys 68, 1125 (1996)

[23] Y. Grossman, H. Quinn, Phys. Rev. D 56, 7259 (1997)

[24] J. Charles, Thèse, Université de Paris-Sud, 1999

[25] G. Zweig, CERN report 8419/th, 412 (1964); S. Okubo, Phys. Lett. B 5, 165 (1965);

I. Tizuka, Prog. Theor. Phys. Suppl. 37, 21 (1966); S. Okubo, Phys. Rev. D 16, 2336 (1977)

[26] J. Sun, G. Zhu, D. Du, hep-ph/0211154; M. Yang, Y. Yang, Phys. Rev. D 62, 114019 (2000); R. Aleksan et al., hep-ph/0301165

[27] M. Gronau, D. London, Phys. Rev. Lett. 65, 3381 (1990)

[28] H.J. Lipkin, Y. Nir, H.R. Quinn, A. Snyder, Phys. Rev. D 44, 1454 (1991)

[29] A. Snyder, H. Quinn, Phys. Rev. D 48, 2139 (1993)

[30] "http://www.slac.stanford.edu/laplace/ckmfitter/charmless.html";

[31] "http: //www.slac.stanford.edu/econf/C010630/papers/E214.PDF"

[32] B. Aubert et al., Collaboration BABAR, hep-ex/0207063

[33] S. Versillé, Thèse de l'Université Paris Sud soutenue le 15 avril 1999. Copie disponible sur "http: //www-lpnhep.in2p3.fr/babar/public/versille/Thesis/"

[34] J. Charles, Phys. Rev. D 59, 054007 (1999), où des estimations de $\left|P_{\pi \pi} / T_{\pi \pi}\right|$ sont réalisées à partir des rapports de branchement de $B^{0} \rightarrow K^{+} \pi^{-}$et $B^{0} \rightarrow \pi^{+} \pi^{-}$. Les estimations de $\left|P_{\rho \pi} / T_{\rho \pi}\right|$ suivent le même modèle.

[35] A.S. Dighe, C.S. Kim , Phys. Rev. D 62, 111302 (2000)

[36] S. Laplace, V. Shelkov, Eur.Phys.J. C 22, 431 (2001)

[37] M. Bauer, B Stech, M. Wirbel, Z. Phys. C 34, 103 (1987)

[38] B. Aubert et al., Collaboration BABAR, "Search for CP violation in $B^{0} / \bar{B}^{0}$ decays to $\pi^{+} \pi^{-} \pi^{0}$ and $K^{ \pm} \pi^{\mp} \pi^{0}$ in regions dominated by the $\rho^{ \pm}$resonance", hep-ex/020706.

[39] R. Aleksan et al., BABAR Analysis Document \#350 (2002); B. Aubert et al., Collaboration $B A B A R$, publication de l'analyse $B^{0} \rightarrow \rho^{ \pm} \pi^{\mp}, B^{0} \rightarrow \rho^{-} K^{+}$en préparation

[40] A. Höcker et al., BABAR Analysis Document \#494 (2003)

[41] S. Laplace, présentation réunion de collaboration BABAR, décembre 2002;

A. Höcker, "Time-dependent B0 -> pi + pi-pi0 analysis", présentation au workshop Dalitz, décembre 2002, SLAC

[42] A. Höcker et al., BABAR Analysis Document \#141 (2001)

[43] M. Bona pour la collaboration BABAR, hep-ex/0111017, proceedings de "International Europhysics Conference on High-Energy Physics", Budapest, Hongrie, 2001. 
[44] K. Hagiwara et al., Particle Data Group, Phys. Rev. D 66, 010001 (2002)

[45] C. Jessop et al., Collaboration CLEO, Phys. Rev. Lett. 85, 2881 (2000)

[46] A. Gordon et al., Collaboration Belle,Phys. Lett. B 542, 183 (2002)

[47] C.P. Jessop et al., Collaboration CLEO, hep-ex/9908018

[48] B. Aubert et al., Collaboration BABAR, hep-ex/0107058

[49] H. Ozaki pour la collaboration Belle, proceedings de "International Symposium on Heavy Flavour Physics", Caltech, Pasadena, 2001. "http: //3w.hep.caltech.edu/HF9/programdetail.html"

[50] B. Aubert et al., Collaboration BABAR, Nucl. Instrum. Methods A 479, 1 (2002)

[51] B. Gobbo et al., Nucl. Instrum. Methods A 386, 52 (1997); C. Bozzi et al., Nucl. Instrum. Methods A 435, 25 (1999); C. Bozzi et al., Nucl. Instrum. Methods A 453, $78(2000)$

[52] B. Aubert et al., collaboration BABAR, Phys. Rev. D 66, 032003 (2002)

[53] P. Billoir, Nucl. Instrum. Methods A 225, 225 (1984)

[54] B. Brabson et al., Nucl. Instrum. Methods A 332, 419 (1993); S. Otto, Diplomarbeit, Technische Universitäte Dresden, Allemagne (2000)

[55] A. Drescher et al., Nucl. Instrum. Methods A 237, 464 (1985)

[56] R. Sinkus, T. Voss, Nucl. Instrum. Methods A 391, 360 (1997)

[57] Reference SVTRAD (page web, these Tim )

[58] V. Re et al., IEEE Trans.Nucl.Sci.49, 3284,2002

[59] P.H. Harrison, H. Quinn, "The BABAR physics book"

[60] "http://www.slac.stanford.edu/BFROOT/www/Physics/Analysis/AWG/EHBDOC/postcpf

[61] T. Sjostrand, Comp. Phys. Comm. 82, 74 (1994)

[62] A. Ryd et al., Note interne BABAR

[63] CERN, GEANT, Program Library long write-up W5013

[64] "http: //wwwinfo.cern.ch/asd/geant4/geant4.html"

[65] J.H. Kühn et A. Santamaria, Z. Phys. C 48, 445 (1990)

[66] R. Barate et al., Collaboration ALEPH, Z. Phys. C 76, 15 (1997)

[67] D. Bisello et al., Collaboration DM2, Phys. Lett. B 220, 321 (1989)

[68] S. Rahatlou, Thèse, 2003, Université de San Diego, Etats-Unis.

[69] U. Langenegger, BABAR Analysis Document \#90 (2001);

"http: //www.slac.stanford.edu/BFROOT/www/Physics/Tools/Pid/Electrons/ cutbasedSelector.html" 
[70] S.M. Spanier, G. Mancinelli, BABAR Analysis Document \#116 (2001)

[71] "http://www.slac.stanford.edu/BFROOT/www/Physics/Tools/Pid/Protons/description.html"

[72] J. Smith et al., BABARNote \#497 (1999)

[73] J. Beringer, BABAR Analysis Document \#119

[74] J. Beringer, BABAR Analysis Document \#118

[75] H. Hu et al., BABAR Analysis Document \#103

[76] C.H. Cheng et al., BABAR Analysis Document \#13 (2001)

[77] M. Carpinelli et al., BABAR Analysis Document \#102 (2000)

[78] M. Pivk, Thèse soutenue le 5 mai 2003, Université Paris 7

[79] A. Höcker et al., BABAR Analysis Document \#141 (2001)

[80] R.A. Fisher, Annals of Eugenics, 179 (1936)

[81] Il existe de nombreux types de perceptrons multi-couches. Celui utilisé dans cette thèse provient de la référence P. Gay, B. Michel, J. Proriol, and O. Deschamps, New computing techniques in physics research, 725 (1995)

[82] K.S. Cranmer, , ALEPH 99-144 (1999) (voir aussi http://www-wisconsin.cern.ch/cranmer/keys.html)

[83] T. Skwarnicki, Thèse, DESY-F31-86-02 (1986)

[84] A. Albrecht et al., Collaboration ARGUS, Phys. Lett. B 185, 218 (1987)

[85] A. Farbin et al., BABAR Analysis Document \#224

[86] F. Bellini, F. Di Lodovico, BABAR Analysis Document \#378 et "http: //www.slac.stanford.edu/BFROOT/www/Physics/Analysis/AWG/Neutrals/validation/results

[87] M.A. Baak, O. Long, BABAR Analysis Document \#572

[88] P. Harrison, BABAR Analysis Document \#159

[89] P. Harrison et al., BABAR Analysis Document \#256

[90] A. Farbin et al., BABAR Analysis Document \#357

[91] O. Leitner et al., hep-ph/0211003

[92] Collaborations ALEPH, DELPHI, L3 et OPAL, groupe de travail pour la recherche du boson de Higgs, CERN-EP/98-046

[93] "http://www.slac.stanford.edu/BFROOT/www/Physics/Tools/Pid/PidKilling/PidKilling.html"

[94] B. Aubert et al., Collaboration BABAR, hep-ex/0207089; H. Muramatsu et al., Collaboration CLEO, Phys. Rev. Lett. 89, 251802 (2002); E.M. Aitala et al., Collaboration E791, Phys. Rev. Lett. 89, 121801 (2002); S. Kopp et al., Collaboration CLEO, Phys. Rev. D 63, 092001 (2001); P.L. Frabetti et al., Collaboration E687, Phys. Lett. B 407, $79(1997)$ 
[95] T.G. Trippe, Phys. Rev. D 66, 010001 (2002); I.V. Ajinenko, hep-ex/0205027; M.I. Martinez, Mod.Phys.Lett. A 16, 2197 (2001); V.Yu. Batusov, Nucl. Phys. B 513, 3 (1998)

[96] A. Höcker, Thèse de l'Université Paris-Sud, Avril 1998

[97] M. Davier et al., hep-ph/0208177, et références à l'intérieur

[98] J.H. Kühn, A. Santamaria, Z. Phys. C 48, 445 (1990)

[99] G.J. Gounaris, J.J. Sakourai, Phys. Rev. Lett. 21, 244 (1968)

[100] L.M. Barkov et al., Collaboration LOYA, CMD, Nucl. Phys. B 256, 365 (1985)

[101] M. Benayoun et al., Z. Phys. C 58, 31 (1993)

[102] M. Bona, présentation à International Europhysics Conference on High Energy Physics, 2001, Budapest, Hongrie, hep-ex/011017

[103] A. Hocker, H. Lacker, S. Laplace et F. Le Diberder, LAL-01-14, Prepared for 4th International Conference on B Physics and CP Violation (BCP 4), Ago Town, Mie Prefecture, Japan, 19-23 Feb 2001

[104] A. Hocker, H. Lacker, S. Laplace et F. Le Diberder, AIP Conf. Proc. 618, 27 (2002), hep-ph/0112295.

[105] S. Laplace, Z. Ligeti, Y. Nir et G. Perez, Phys. Rev. D 65, 094040 (2002), hep$p h / 0202010$.

[106] S. Laplace, hep-ph/0209188.

[107] L. Roos et al., BABAR Analysis Document \#519

[108] A. Höcker et al., BABAR Analysis Document en préparation sur l'interpretation du résultat de $\rho h$

[109] I.S. Towner, J.C. Hardy, nucl-th/9809087

[110] H. Abele, Nucl. Instrum. Methods A440, 499 (2000); S. Arzumanov, Nucl. Instrum. Methods A440, 511 (2000)

[111] M. Bargiotti et al., hep-ph/0001293

[112] J.F. Donoghue, B.R. Holstein, S.W. Klimt, Phys. Rev. D 35, 934 (1997); R. FloresMendieta, A. Garcia, G. Sanchez-Colon, Phys. Rev. D 54, 6855 (1996)

[113] N. Isgur, M. Wise, Phys. Lett. B 232, 113 (1989)

[114] Z. Ligeti, rapport de la conférence "International Europhysics Conference on HEP", Budapest, 12 au 18 juillet 2001, hep-ph/0112089

[115] K. Abe et al., Collaboration Belle, Phys. Rev. Lett. 88, 231803 (2001)

[116] R.A. Briere et al., Collaboration CLEO, hep-ph/0203032

[117] groupe de travail $V_{c b}$ LEP, "http//lepvcb.web.cern.ch/LEPVCB"

[118] K. Abe et al., Collaboration Belle, hep-ex/0208033 
[119] M. Gremm, A. Kapustin, Phys. Rev. D 55, 6924 (1997)

[120] A.F. Falk, M. Luke, M.J. Savage, Phys. Rev. D 53, 2491 (1996), Phys. Rev. D 53, 6316 (1996)

[121] A. Kapustin, Z. Ligeti, Phys. Lett. B 355, 318 (1995); Z. Ligeti, M. Luke, A.V. Manohar, M.B. Wise, Phys. Rev. D 60, 034019 (1999)

[122] D. Cronin-Hennessy et al., Collaboration CLEO, hep-ph/0108033, S. Chen et al., Collaboration CLEO, hep-ph/0108032

[123] B.H. Behrens, Collaboration CLEO, Phys. Rev. D 61, 052001 (2000)

[124] B. Aubert et al., Collaboration BABAR, hep-ex/0207080

[125] A.F. Falk, Z. Ligeti, M.B. Wise, Phys. Lett. B 406, 225 (1997); I. Bigi, R.D. Dikeman, N. Uraltsev, Eur.Phys.J. C 4, 453 (1998)

[126] C.W. Bauer, Z. Ligeti, M. Luke, Phys. Lett. B 479, 395 (2000)

[127] Groupe de travail $\left|V_{u b}\right|$ de LEP, "http//battagl.home.cern.ch/battagl/vub/vub.html"

[128] A. Bornheim et al., Collaboration CLEO, Phys. Rev. Lett. 88, 231803 (2002)

[129] G. Abbiendi et al., Collaboration OPAL, Phys. Lett. B 490, 71 (2000)

[130] T. Inami et C.S. Lim, Prog. Theor. Phys 65, 297 (1981) Erratum-ibid. 65 (1981) 1772

[131] C. Bernard, Nucl. Phys. Proc. Suppl. 94, 159 (2001)

[132] M. Ciuchini et al., hep-ph/0012308

[133] H.G. Moser, A. Roussarie, Nucl. Instrum. Methods A 384, 491 (1997); D. Abbaneo, G. Boix, JHEP 08, 004 (1999)

[134] M. Ciuchini et al., JHEP 0107: 013 (2001)

[135] Groupe de travail des oscillations du $B$ de LEP, "http: //lepbosc.web.cern.ch/LEPBOSC"

[136] F. Le Diberder, CERN CKM workshop, Frevrier 2002, "http: //ckmworkshop.web.cern.ch/ckm-workshop/"

[137] B. Auber et al., Collaboration BABAR, hep-ex/0008058 (2000)

[138] P. Chang et al., Collaboration Belle, présentation à ICHEP'02 (2002)

[139] Collaboration CLEO, hep-ex/0206024

[140] Collaboration Belle, hep-ex/0104030

[141] G.C. Fox and S. Wolfram, Phys. Rev. Lett. 41, 1581 (1978)

[142] B. Aubert et al., Collaboration BABAR hep-ex/0008058

[143] K. Abe et al., Collaboration Belle, hep-ex/0207007

[144] Collaboration CLEO, hep-ex/0006008 
[145] L.M. Mir et A. Gritsan, BABAR Analysis Document \#381

[146] Collaboration Belle, A. Gordon, présentation donnée à ICHEP'02

[147] B. Aubert et al., Collaboration BABAR hep-ex/0207055

[148] B. Aubert et al., Collaboration BABAR hep-ex/0110065

[149] B. Aubert et al., Collaboration BABAR hep-ex/0107037; BABAR Analysis Document \#412; K.F. Chen, Collaboration Belle, présentation donnée à ICHEP'02 Collaboration CLEO, hep-ex/9912059

[150] C.W. Chiang, J.L. Rosner, Phys. Rev. D 65, 074035 (2002)

[151] M.Z. Yang, Y.D. Yang, Nucl. Phys. B 609, 469 (2001)

[152] E.V.Shuryak, A. Zhitnisky, Phys. Rev. D 57, 2001 (1998)

[153] M. Beneke, M.Ñeubert, hep-ph/0210085

[154] T. Ford et al., BABAR Analysis Document \#237

[155] Collaboration CLEO, Phys. Rev. Lett. 85, 2881 (2000)

[156] B. Aubert et al., Collaboration BABAR hep-ph/0109007

[157] Collaboration Belle, BELLE-CONF-0115

[158] Voir la discussion sur les contributions potentielles de $B^{0} \rightarrow \kappa(797)^{+} \pi^{-}$par P. Chang pour la collaboration Belle, présentation donnée à ICHEP'02;

Pour plus d'information sur le $\kappa(797)^{+}$, voir:

Collaboration E791, Phys. Rev. Lett. 89, 121801 (2002)

[159] Collaboration Belle, Phys. Rev. D 65, 092005 (2002)

[160] J. Back, BABAR Analysis Document \#505;

W. Bhimji et al., BABAR Analysis Document \#362

[161] Collaboration E791, Phys. Rev. Lett. 86, 770 (2001), Phys. Rev. Lett. 86, 765 (2001)

[162] Voir par exemple S. Gardner et U.G. Meissner, Phys. Rev. D 65, 094004 (2002)

[163] Collaboration BABAR hep-ex/0203007

[164] J. S. Hagelin, Nucl. Phys. B 193, 123 (1981); J. S. Hagelin and M. B. Wise, Nucl. Phys. B 189, 87 (1981); A. J. Buras, W. Slominski and H. Steger, Nucl. Phys. B 245, $369(1984)$

[165] G. Abbiendi et al., Collaboration OPAL, Eur.Phys.J. C 12, 609 (2000)

[166] D. E. Jaffe et al., Collaboration CLEO, Phys. Rev. Lett. 86, 5000 (2001)

[167] R. Barate et al., Collaboration ALEPH, Eur.Phys.J. C 20, 431 (2001)

[168] B. Aubert et al., Collaboration BABAR, Phys. Rev. Lett. 88, 231801 (2002)

[169] M. Ciuchini, G. Degrassi, P. Gambino et G. F. Giudice, Nucl. Phys. B 534, 3 (1998)

[170] A. Ali et D. London, Eur.Phys.J. C 9, 687 (1999) 
[171] A. J. Buras, P. Gambino, M. Gorbahn, S. Jäger et L. Silvestrini, Phys. Lett. B 500, $161(2001)$

[172] A. J. Buras et R. Buras, Phys. Lett. B 501, 223 (2001)

[173] A. J. Buras et R. Fleischer, Phys. Rev. D 64, 115010 (2001)

[174] A. J. Buras, hep-ph/0101336

[175] S. Bergmann et G. Perez, Phys. Rev. D 64, 115009 (2001) 


\section{Abstract}

The BABAR experiment, at the PEP-II collider at SLAC, has been studying since 1999 $\mathrm{CP}$ violation in the $\mathrm{B}$ meson system. After the precise measurement of $\sin 2 \beta$, one is now concentrating on measuring the angles $\alpha$ and $\gamma$ of the unitarity triangle. The work presented in this thesis concerns the measurement of the angle $\alpha$ in the $B^{0} \rightarrow \rho \pi$ mode.

We realized a $C P$ time-dependant analysis, as well of the branching ratio measurements of the $B^{0} \rightarrow \rho^{ \pm} \pi^{\mp}$ and $B^{0} \rightarrow \rho^{-} K^{+}$modes. The results obtained on an integrated luminosity of $80.9 \mathrm{fb}^{-1}$ are the following:

$$
\begin{aligned}
& \mathcal{B}\left(B^{0} \rightarrow \rho^{ \pm} \pi^{\mp}\right)=(22.6 \pm 1.8 \pm 2.2) \times 10^{-6}, \\
& \mathcal{B}\left(B^{0} \rightarrow \rho^{-} K^{+}\right)=(7.3 \pm 1.3 \pm 1.3) \times 10^{-6} \text {, } \\
& A_{\mathrm{CP}}^{\rho \pi}=-0.18 \pm 0.08 \pm 0.03, \quad A_{\mathrm{CP}}^{\rho K}=-0.28 \pm 0.17 \pm 0.08, \\
& C_{\rho \pi}=-0.36 \pm 0.18 \pm 0.04, \quad S_{\rho \pi}=-0.19 \pm 0.24 \pm 0.03, \\
& \Delta C_{\rho \pi}=0.28 \pm 0.19 \pm 0.04, \quad \Delta S_{\rho \pi}=0.15 \pm 0.25 \pm 0.03 \text {. }
\end{aligned}
$$

We also measured the branching ratio of $B^{0} \rightarrow \rho^{0} \pi^{0}$ with a significance of $2.7 \sigma$. We therefore put the following upper limit at $90 \% \mathrm{CL}$ :

$$
\mathcal{B}\left(B^{0} \rightarrow \rho^{0} \pi^{0}\right)<2.7 \times 10^{-6} \text { à } 90 \% \mathrm{CL} .
$$

Finally, we built the heart of a complete Dalitz plot analysis of $B^{0} \rightarrow \pi^{+} \pi^{-} \pi^{0}$, and estimated the experimental sensibility on $\alpha$.

The results obtained on the $B^{0} \rightarrow \rho \pi$ modes are interpreted in terms of constraints on the angle $\alpha$ within methods using $\mathrm{SU}(2)$ and $\mathrm{SU}(3)$ symmetries.

We also measured the branching ratio of $B^{0} \rightarrow a_{0} \pi$ using a reduced luminosity of $B^{0} \rightarrow$ $a_{0} \pi$, leading to the result:

$$
\mathcal{B}\left(B^{0} \rightarrow a_{0}^{ \pm} \pi^{\mp}\right)=\left(6.2_{-2.5}^{+3.0} \pm 1.1\right) \times 10^{-3} .
$$

Some phenomenological studies have been performed to infer the feasibility of a $C P$ analysis to determine the angle $\alpha$ thanks to this mode.

Finally, we describe the improvements made on the SVT radiation protection system for the injection periods.

Outside the BABAR collaboration scope, we developed a package called CKMftter allowing us to perform a global analysis of the CKM matrix, within a frequentist statistical approach.

\section{KEY WORDS:}

$B A B A R$

$\alpha$

CKM matrix

$C P$ violation

$$
\begin{aligned}
& B^{0} \rightarrow \rho \pi \\
& \text { Unitarity triangle } \\
& B \text { mesons }
\end{aligned}
$$




\section{Résumé}

L'expérience BABAR, dont la prise de données a débuté en 1999 auprès du collisionneur PEP-II a SLAC, a pour objectif principal l'étude de la violation de la symétrie $C P$ dans le système des mésons $B$. Après avoir précisémment mesuré $\sin 2 \beta$, notre attention se porte sur les deux autres angles $\alpha$ et $\gamma$ du triangle d'unitarité. Les travaux présentés dans cette thèse concernent la mesure de l'angle $\alpha$ dans le mode $B^{0} \rightarrow \rho \pi$.

Nous avons réalisé une analyse $C P$ dépendante du temps, ainsi que la mesure des rapports d'embranchement des modes $B^{0} \rightarrow \rho^{ \pm} \pi^{\mp}$ et $B^{0} \rightarrow \rho^{-} K^{+}$. Les résultats obtenus avec une luminosité intégrée de $80.9 \mathrm{fb}^{-1}$ sont les suivants:

$$
\begin{aligned}
& \mathcal{B}\left(B^{0} \rightarrow \rho^{ \pm} \pi^{\mp}\right)=(22.6 \pm 1.8 \pm 2.2) \times 10^{-6}, \\
& \mathcal{B}\left(B^{0} \rightarrow \rho^{-} K^{+}\right)=(7.3 \pm 1.3 \pm 1.3) \times 10^{-6} \text {, } \\
& A_{\mathrm{CP}}^{\rho \pi}=-0.18 \pm 0.08 \pm 0.03, \quad A_{\mathrm{CP}}^{\rho K}=-0.28 \pm 0.17 \pm 0.08, \\
& C_{\rho \pi}=-0.36 \pm 0.18 \pm 0.04, \quad S_{\rho \pi}=-0.19 \pm 0.24 \pm 0.03 \text {, } \\
& \Delta C_{\rho \pi}=0.28 \pm 0.19 \pm 0.04, \Delta S_{\rho \pi}=0.15 \pm 0.25 \pm 0.03 \text {. }
\end{aligned}
$$

Nous avons également mesuré le rapport d'embranchement de $B^{0} \rightarrow \rho^{0} \pi^{0}$, avec une signification statistique de $2.7 \sigma$. Nous calculons une limite supérieure sur ce rapport d'embranchement:

$$
\mathcal{B}\left(B^{0} \rightarrow \rho^{0} \pi^{0}\right)<2.7 \times 10^{-6} \text { à } 90 \% \mathrm{CL} .
$$

Enfin, nous avons developpé le coeur de l'analyse complète de $B^{0} \rightarrow \pi^{+} \pi^{-} \pi^{0}$ qui exploite toute l'information présente dans le diagramme de Dalitz. Des estimations de sensibilité expérimentale pour $\alpha$ ont été effectuées.

Les résultats obtenus pour les modes $B^{0} \rightarrow \rho \pi$ sont interprétés en terme de contraintes sur l'angle $\alpha$ au sein de méthodes utilisant la symétrie $\mathrm{SU}(2)$ et les modes $B^{+} \rightarrow \rho \pi$, ou la symétrie $\mathrm{SU}(3)$ et les modes $B^{0} \rightarrow K^{*} \pi$ et $B^{0} \rightarrow \rho K$.

Nous avons aussi réalisé la mesure du rapport d'embranchement du mode $B^{0} \rightarrow a_{0} \pi$ sur une luminosité plus réduite de $20 \mathrm{fb}-1$, menant au résultat:

$$
\mathcal{B}\left(B^{0} \rightarrow a_{0}^{ \pm} \pi^{\mp}\right)=\left(6.2_{-2.5}^{+3.0} \pm 1.1\right) \times 10^{-3} .
$$

Des études phénoménologiques sur la faisabilité d'une analyse $C P$ et la détermination de l'angle $\alpha$ grâce à ce mode ont aussi été accomplies.

Enfin, nous décrivons les améliorations apportées au système de protection du SVT durant les périodes d'injection.

En dehors du champ de la collaboration $B A B A R$, nous avons développé un logiciel, dénomé CKMfitter permettant de réaliser une analyse globale de la matrice CKM au sein d'une analyse fréquentiste.

\section{MOTS CLES:}

$\begin{array}{ll}B A B A R & B^{0} \rightarrow \rho \pi \\ \alpha & \text { Triangle d'unitarité } \\ \text { Matrice CKM } & \text { Mésons } B \\ \text { Violation de } C P & \end{array}$

